NASA Conference Publication 2115

\title{
Radio Interferometry Techniques for Geodesy
}

Proceedings of a conference

held at the Massachusetts Institute

of Technology, Cambridge, Massachusetts

and the Northeast Radio Observatory

Corporation Haystack Observatory,

Westford, Massachusetts

June 19-21 1979 
NASA Conference Publication 2115

\section{Radio Interferometry Techniques for Geodesy}

Proceedings of a conference sponsored by the National Aeronautics and Space Administration and the American Geophysical Union in cooperation with Special Study Group 2.51 of the International Association of Geodesy and held at the Massachusetts Institute of Technology, Cambridge, Massachusetts, and the Northeast Radio Observatory Corporation Haystack Observatory, Westford, Massachusetts, June 19-21, 1979

\section{N/SA \\ National Aeronautics and Space Administration \\ Sclentific and Technical Information Otfice}


The requirement for the use of the International System of Units (SI) has been waived for this document under the authority of NPD 2220.4, paragraph 5.d. 


\section{CONTENTS}

\section{SESSION A \\ SURVEYS OF THE SEVENTIES}

Geodetic Measurements With a Mobile VLBI System . . . . . . . . . . . . . . .

A. E. Niell, E. S. Claflin, T. G. Lockhart, P. F. MacDoran, D. D. Morabito,

K. M. Ong, and G. M. Resch

NOS/NGS Activities To Support Development of Radio Interferometric

Surveying Techniques . . . . . . . . . . . . . . . . . . . . .

W. E. Carter, J. F. Dracup, L. D. Hothem, D. S. Robertson, and W. E. Strange

Long Baseline Vector Determinations and Intercomparisons. . . . . . . . . . .

East Coast VLBI Group

VLBI Measurements of Baselines, UT and Polar Motion With Deep Space

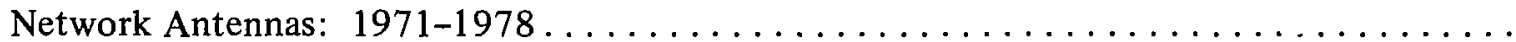

J. B. Thomas, J. L. Fanselow, E. J. Cohen, G. H. Purcell, D. A. Rogstad,

O. J. Sovers, L. J. Skjerve, and D. J. Spitzmesser

Polar Motion and UT1: Comparison of VLBI, Lunar Laser, Satellite Laser,

Satellite Doppler, and Conventional Astrometric Determinations . . . . . . . . . . .

D. S. Robertson, T. A. Clark, R. J. Coates, C. Ma, J. W. Ryan, B. E. Corey,

C. C. Counselman, R. W. King, I. I. Shapiro, H. F. Hinteregger, C. A. Knight,

A. E. E. Rogers, A. R. Whitney, J. G. Pigg, and B. R. Schupler

A Review of Connected Element Radio Interferometry Directed at

Establishing An Almost Inertial Reference Frame. . . . . . . . . . . . . . . . K. J. Johnston

Progress Report on The USNO/NRL Green Bank Interferometer Program . . . . . . . . . . .

W. J. Klepczynski, G. H. Kaplan, D. D. McCarthy, F. J. Josties,

R. L. Branham, K. J. Johnston, and J. S. Spencer

Geodetic Long Baseline Interferometry Research In Canada . . . . . . . . . . . . . . .

Phase Coherent VLBI Using A Satellite Link. . . . . . . . . . . . . . . .

W. B. Waltman, S. H. Knowles, J. L. Yen, D. N. Fort,

G. W. Swenson, Jr., W. H. Cannon, and J. Galt 


\section{CONTENTS (continued)}

\section{SESSION B \\ MOVEMENTS TERRESTRIAL AND CELESTIAL}

Scandinavia Studies of Recent Crustal Movements and

The Space Geodetic Basclinc Network $\ldots \ldots \ldots \ldots \ldots \ldots \ldots \ldots \ldots \ldots \ldots \ldots \ldots$

Allen Joel Anderson

Geodetic Strategies Following Large Dip-Slip Earthquakes .

J. J. Melosh, A. Raefsky, and M. A. Slade

The Measurement of Earth Rotation on a Deformable Earth

W. H. Cannon

Crustal Deformation at Very Long Baseline Interferometry Sites Due to

Seasonal Air-Mass and Ground Water Variations

A. Stoltz and D. R. Larden

Geophysical and Astronomical Models Applied in The Analysis of

Very Long Baseline Interferometry

Chopo Ma, James W. Ryan, and Bruce R. Schupler

Relativistic Formulation of The VLBI Observable.

S. A. Gourevitch, R. Epstein, and I. I. Shapiro

Radio Source Positions and Astronomical Constants.

T. A. Clark

VLBI Measurements of Radio Source Positions at The Jet Propulsion Laboratory . . . . . . .

G. H. Purcell, Jr., J. L. Fanselow, J. B. Thomas, E. J. Cohen,

D. H. Rogstad, O. J. Sovers, L. J. Skjerve, and D. J. Spitzmesser

A Very Long Baseline Interferometry Sky Survey

R. A. Preston and D. D. Morabito

Astrometry Using Basic Mark III Very Long Baseline Interferometry

J. H. Spencer, E. B. Waltman, K. J. Johnston, and D. C. Backer

Differential Radio Astronomy of Galactic Objects

D. C. Backer 
Source Structure Corrections To The Geodetic Very Long

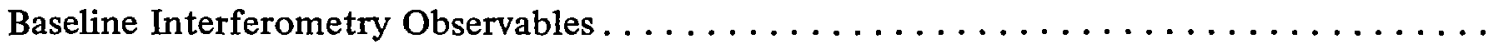
William D. Cotton

Effects of Source Structure on Geodetic Measurements

K. Johnson

\section{SESSION C \\ DEGREES KELVIN AND DEGREES OF PHASE}

Hydrogen Maser Frequency Standard

R. F. C. Vessot

The Performance of NASA Research Hydrogen Masers. . . . . . . . . . . . . . .

Victor S. Reinhardt and Lauren J. Rueger

Ultra-Low-Noise Microwave Amplifiers

Robert C. Clauss and David L. Trowbridge

Phase and Group Delay Calibration of a Very Long Baseline

Interferometer by East Coast VLBI Group.

A. E. E. Rogers

Use of a Digital Tone Extractor for Real-Time Phase Analysis

Elliott H. Sigman and Gary S. Parks

Considerations in the Placement of Phase Calibrator Tones.

L. E. Young

The Sensitivity of a Very Long Baseline Interferometer

A. E. E. Rogers and the East Coast VLBI Group

\section{SESSION D \\ THE MARK III VLBI SYSTEM}

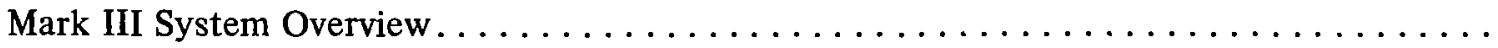

T. A. Clark

Automation of The Mark III Field System ....................... 291

N. R. Vandenberg, T. A. Clark, L. N. Foster, A. R. Whitney, and G. Lampe

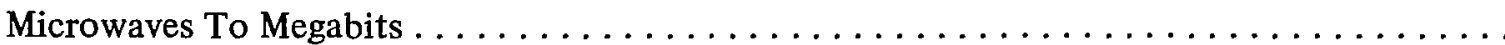

A. E. E. Rogers 


\section{CONTENTS (continued)}

The Mark III Wideband Digital Recorder in Perspective . . . . . . . . . . . . . . . . . 305 Hans F. Hinteregger

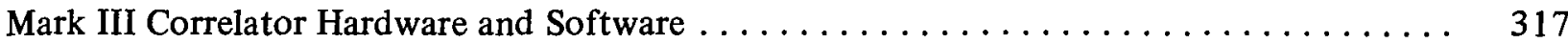
Alan R. Whitney

Correlator Computer Interface and Module Implementation - Mark III Processor . . . . . . . Edwin F. Nesman

Mark III VLBI System - Tropospheric Calibration Subsystems . . . . . . . . . . .

G. M. Resch

Mark III Real-Time Fringe-Detection System . . . . . . . . . . . . . . . . . . .

J. I. Levine and A. R. Whitney

The Mark III Data Base Handler .

James W. Ryan, Chopo Ma, and Bruce R. Schupler

Mark III Interactive Data Analysis System

James W. Ryan, Chopo Ma, and Bruce R. Schupler

\section{SESSION E \\ WAVES OF THE FUTURE AND OTHER EMISSIONS}

Water Vapor as an Error Source in Microwave Geodetic Systems:

Background and Survey of Calibration Techniques . . . . . . . . . . . . . . . . .

E. S. Claflin and G. M. Resch

The Estimation of the Propagation Delay Through the

Troposphere from Microwave Radiometer Data

$J$. M. Moran and B. R. Rosen

Microwave Radiometry as a Tool to Calibrate Tropospheric Water-Vapor Delay. . . . . . . .

G. M. Resch and E. S. Claflin

Phase Versus Delay in Geodetic VLBI James Campbell

Phase Delay Astrometry and Its Geodetic Applications. 
CONTENTS (continued)

Satellite Emission Radio Interferometric Earth Surveying (SERIES)

P. F. MacDoran

Backpack VLBI Terminal with Subcentimeter Capability

409

C. C. Counselman III, I. I. Shapiro, R. L. Greenspan, and D. B. Cox, Jr.

SESSION F

ADHERENCE AND COHERENCE IN NETWORKS AND PLANS

The European VLBI Network $\ldots \ldots \ldots \ldots \ldots \ldots \ldots \ldots \ldots \ldots \ldots \ldots \ldots \ldots$

R. T. Schilizzi

NASA Geodetic Applications of the Mark III VLBI System Robert Coates

On the VLBI-Satellite Laser Ranging “Iron Triangle" Intercomparison Experiment

Yehuda Bock, Ivan I. Mueller, and Erricos Pavlis

The Deep Space Network Capabilities for Radio Interferometry, . . . . . . . . . . .

J. L. Fanselow

The Jet Propulsion Laboratory/California Institute of Technology

Block II VLBI Processor . . . . . . . . . . . . . . . . . . . . . . . . . . .

D. H. Rogstad

Project Polaris: A Status Report. . . . . . . . . . . . . . . . . . . . .

W. E. Carter

Plans for Phase Coherent Long Baseline Interferometry for Geophysical Applications

Using the Anik-B Communications Satellite $\ldots \ldots \ldots \ldots \ldots \ldots \ldots \ldots \ldots$

W. H. Cannon, W. T. Petrachenko, J. L. Yen, J. A. Galt,

W. B. Waltman, S. H. Knowles, and J. Popelar

NASA Geodynamics Program $\ldots \ldots \ldots \ldots \ldots \ldots \ldots \ldots \ldots \ldots \ldots \ldots \ldots \ldots \ldots$

E. A. Flinn 

SESSION A

\section{SURVEYS OF THE SEVENTIES}





\title{
GEODETIC MEASUREMENTS WITH A MOBILE VLBI SYSTEM*
}

\author{
A. E. Niell, E. S. Claflin, T. G. Lockhart, P. F. MacDoran, \\ D. D. Morabito, K. M. Ong, and G. M. Resch \\ Jet Propulsion Laboratory \\ California Institute of Technology
}

\begin{abstract}
The Project ARIES 9-meter transportable antenna has been used as one element of a very long baseline interferometer (VLBI) to begin monitoring locations of six sites in California relative to large diameter fixed antennas at the NASA Deep Space Network, Goldstone, California, and at the Caltech Owens Valley Radio Observatory, Big Pine, California. An accuracy of about $6 \mathrm{~cm}$ in the horizontal components has been demonstrated by comparison with measurements of the National Geodetic Survey. The RMS scatter of the lengths of the baselines between any pair of antennas is about $3 \mathrm{~cm}$ except for the Goldstone-JPL (Pasadena) baseline. In the period August 1974 to August 1977 the length of this baseline increased by $15 \pm 5 \mathrm{~cm}$ as JPL moved westward relative to Goldstone at the rate of $6 \pm 2 \mathrm{~cm} /$ year. The baseline lengths are unaffected by the uncertainties of UT1, polar motion, and tropospheric water vapor, which are the limitations to present three-dimensional vector accuracies.

Initial demonstrations have been made with a highly mobile 3.7 -m-diameter antenna, but marginal system sensitivity has limited the accuracy. Planned improvements such as a low-noise receiver and wide-band Mark III data system will provide baseline precisions of a few centimeters for about two sites per week beginning about summer 1981 .
\end{abstract}

*This paper presents the results of one phase of research carried out at the Jet Propulsion Laboratory, California Institute of Technology, under Contract No. NAS 7-100, sponsored by the National Aeronautics and Space Administration. 


\begin{tabular}{|c|c|c|}
\hline ANALYSIS & INSTRUMENTATION & $\begin{array}{l}\text { GEOPHYSICAL } \\
\text { REQUIREMENTS }\end{array}$ \\
\hline $\begin{array}{l}\text { E. S. Claflin } \\
\text { T. E. Erickson } \\
\text { H. F. Fliegel } \\
\text { A. Y. Lam } \\
\text { T. G. Lockhart } \\
\text { P. F. Ma } \\
\text { R. B. Mi } \\
\text { K. M. Ong } \\
\text { A. E. Nie } \\
\text { J. S. Spitler } \\
\text { C. J. Steinberg } \\
\text { K. S. Wallace }\end{array}$ & $\begin{array}{l}\text { M. S. Ewing (CALTECH) } \\
\text { D. W. Fite } \\
\text { B. B. Johnson (FACC) } \\
\text { E. T. Lobdell (FACC) } \\
\text { Joran } \\
\text { er } \\
\text { M. G. Newsted (FACC) } \\
\text { S. R. Paine } \\
\text { G. S. Parks } \\
\text { L J. Skjerve } \\
\text { D. J. Spitzmesser } \\
\text { h } \\
\text { L. Tanida } \\
\text { N. I. Yamane }\end{array}$ & $\begin{array}{l}\text { SEISMOLOGICAL } \\
\text { LABORATORY, CALTECH } \\
\text { J. H. WhitCOMb, CIRES, } \\
\text { UNIV. OF COLORADO }\end{array}$ \\
\hline
\end{tabular}

FACC - Ford Aerospace Communications Corporation

Figure 1. ARIES project team.

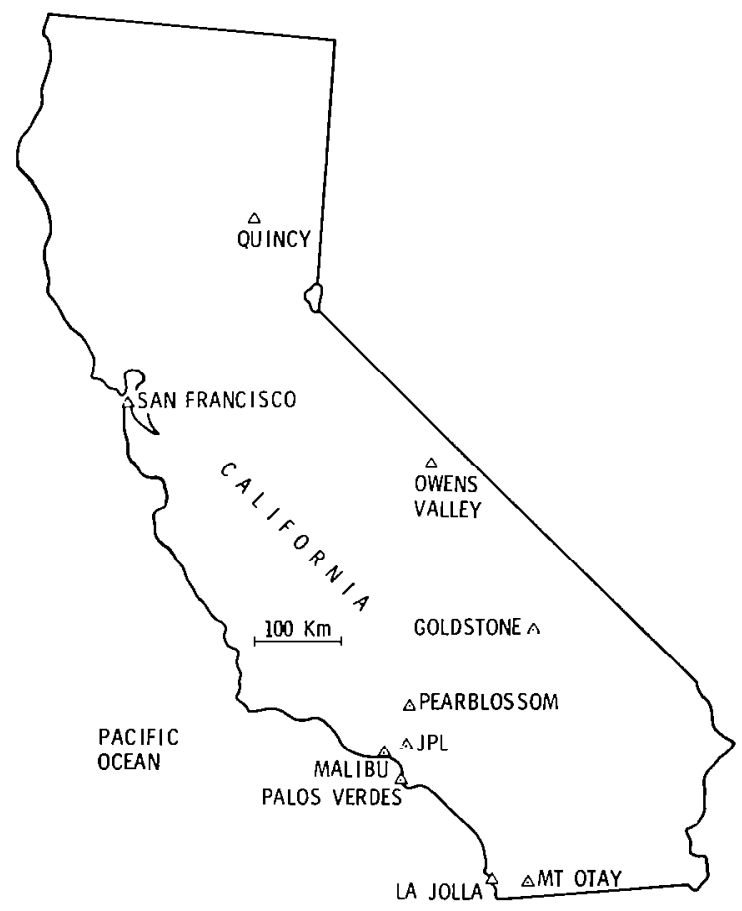

Figure 2. ARIES network. 
STATIONS

- ARIES - 9m DIAM TRANSPORTABLE ANTENNA

- OVRO - 40m DIAM RADIO TELESCOPE - BIG PINE, CALIFORNIA

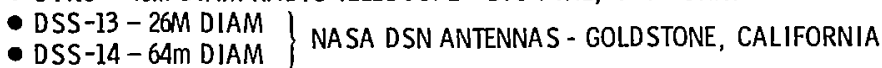

FREQUENCIES

- S-BAND: $2.3 \mathrm{GHZ}-40 \mathrm{OR} 80 \mathrm{MHZ}$ BANDWIDTH SYNTHESIS

- X-BAND: $8.4 \mathrm{GHz}$ - 8O MHz BANDWIDTH SYNTHESIS

FREQUENCY AND TIME REFERENCE

- HYDROGEN MASERS USUALLY

- RUBIDIUM CLOCK WHEN MASER NOT AVA ILABLE

TROPOSPHERE CALIBRATION

- DRY COMPONENT - SURFACE PRESSURE AND TEMPERATURE

- WET COMPONENT - WATER VAPOR RADIOMETER OR SURFACE MEASUREMENTS

PHASE CALIBRATOR - NOT USED

Figure 3. Instrumentation ARIES VLBI network.

UNCERTA INTIES WITHIN INDIVIDUAL EXPERIMENT ( 24 HOURS)

- DELAY MEASUREMENT UNCERTA INTY

- 30-500 PICOSEC (1-15 CM)

- SOURCE POSITIONS

- $\quad 0.03-0.1$ APRIORI

- tROPOSPHERE CALIBRATION

- $\leqslant 6$ CM PER OBSERVATION

- CLOCK DIFFERENCE MODELLING (IMPLICIT IN X ${ }^{2}$ ADJUSTMENT)

$-\quad \leqslant 3 \mathrm{CM}$ (IMPLICIT IN X2 ADJUSTMENT)

SYSTEMATIC ERRORS FOR EACH EXPERIMENT

- earth Rotation parameters

- $\quad 2$ CM PER $100 \mathrm{KM}$

- USE OF GREEN BOOK FOR NUTATION. PRECESSION

- $\quad$ I CM PER $100 \mathrm{KM}$

- tROPOSPHERE bIas

- $\quad$ CM ILOCAL VERTICALI

- location of transporta ble antenNa

$-\quad<1 \mathrm{CM}$ UNMODELLED EFFECTS

- EARTH TIDES AND OCEAN LOADING

- $\quad \leqslant 3$ CM $(<1$ CM FOR 24 HOURS)

- IONOSPHERE

- $\quad<2 \mathrm{CM}(1977)$

- planetary perturbations on earth'S VELOCITY

- $~ I$ CM PER $100 \mathrm{KM}$

- gravitational bending

$-\quad ?$

Figure 4. Error sources ARIES VLBI network (BLE500 km). 


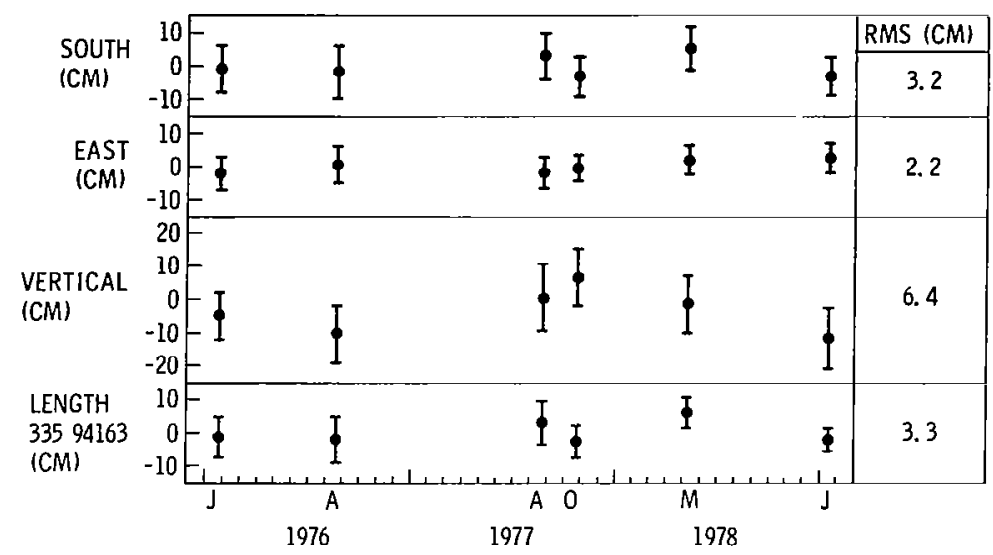

Figure 5. OVRO/ARIES (JPL) baseline vector. Relative local coordinates at JPL; OVRO assumed fixed. UT1: BIH; polar motion: NSWC (Doppler).

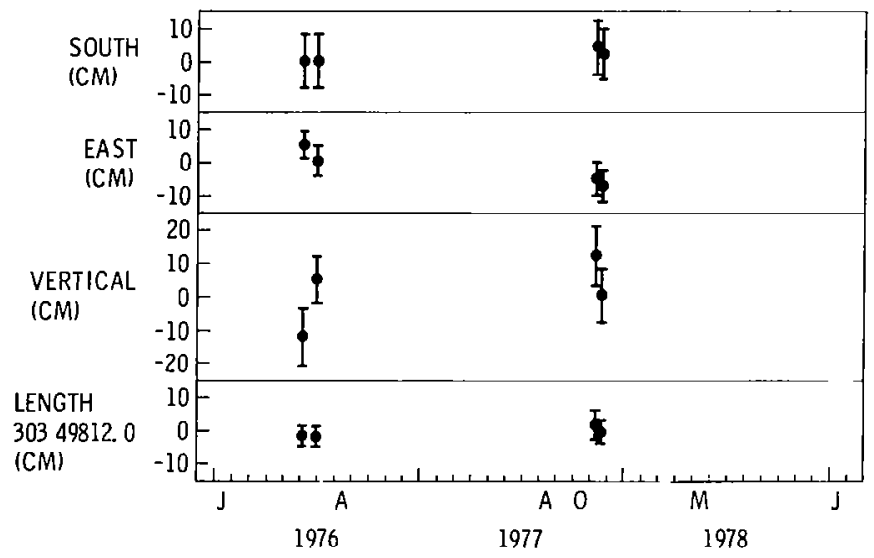

Figure 6. OVRO/ARIES (Pearblossom) baseline vector. (Relative local coordinates at Pearblossom; OVRO assumed fixed.) UT1: BIH; polar motion: NSWC (Doppler). 


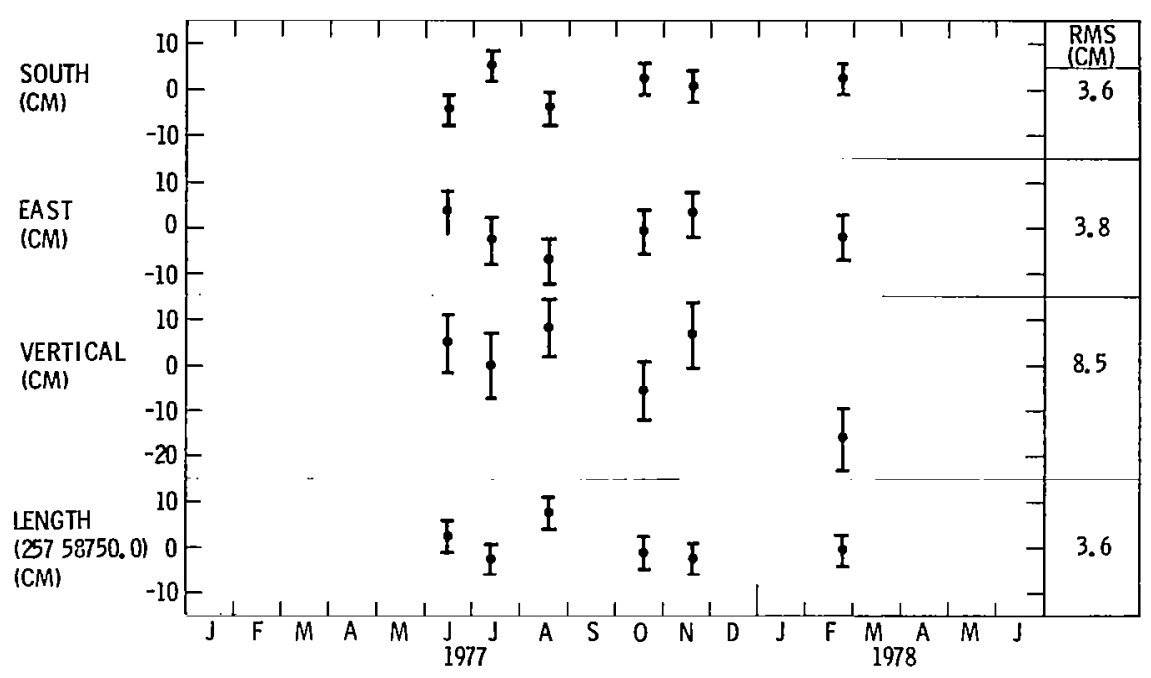

Figure 7. Goldstone (DSS-13)/OVRO baseline vector. (Relative local coordinates at OVRO; DSS-13 assumed fixed.) UT1: BIH; polar motion: NSWC (Doppler).

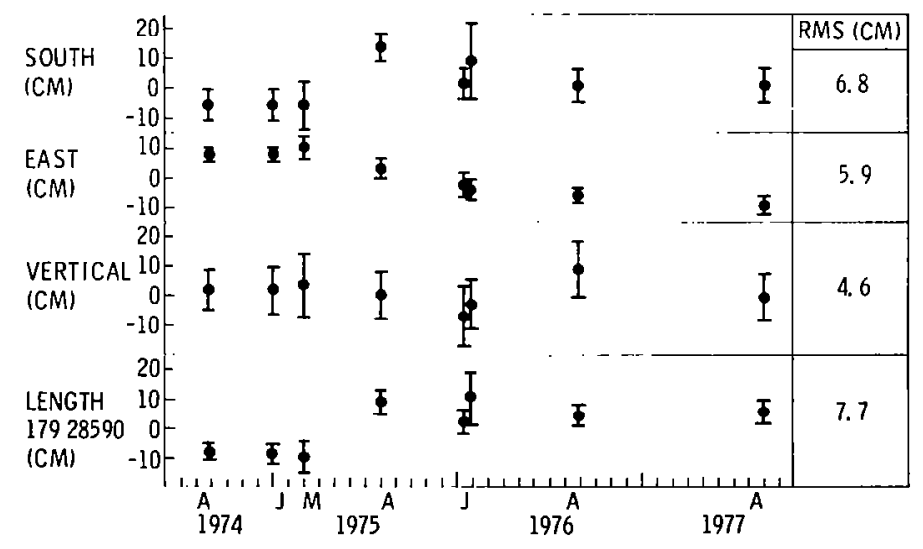

Figure 8. Goldstone (DSS-14/ARIES (JPL) baseline vector. (Relative local coordinates at JPL; DSS-14 assumed fixed.) UT1: BIH; polar motion: NSWC (Doppler).
- FREQUENCY DISTRIBUTION SYSTEM STABILITYI CALI BRATION

- TROPOSPHERE CALIBRATION

- EARTH ORIENTATION PARAMETERS

Figure 9. Major challenges. 
V 


\title{
NOS/NGS ACTIVITIES TO SUPPORT DEVELOPMENT OF RADIO INTERFEROMETRIC SURVEYING TECHNIQUES
}

\author{
W. E. Carter, J. F. Dracup, L. D. Hothem, \\ D. S. Robertson, W. E. Strange \\ National Oceanic and Atmospheric Administration \\ National Ocean Survey, National Geodetic Survey
}

\begin{abstract}
An important part of the NOAA National Ocean Survey/National Geodetic Survey (NOS/NGS) mission is to develop improved geodetic surveying methods. Radio interferometry is producing some extraordinary experimental results, and NGS is working closely with researchers in other organizations to develop operational survey systems based on the technique. One aspect of this work involves the performance of special surveys using appropriately selected methods, which are already considered operational, to provide supplemental and comparative data for the analysis and evaluation of radio interferometric surveying (RIS) projects. In this paper, we review these NGS activities, including descriptive information about the field procedures, data reduction and analysis, and the results obtained to date.
\end{abstract}




\section{INTRODUCTION}

The new vitality infused in geodynamics by the development and refinement of plate tectonics theories has created demands for geodetic data of unprecedented spatial and temporal resolutions. Since present methods of geodetic surveying cannot provide the required resolution, more accurate methods must be implemented.

Independent clock astronomical radio interferometry, commonly referred to as very long baseline interferometry (VLBI), has produced some extraordinary experimental results during the past few years (e.g., Robertson et al., 1978; Rogers et al., 1978; Niell et al., 1979). NOS/NGS is working with researchers at academic institutions and other government agencies, particularly the National Aeronautics and Space Administration (NASA), on the development of operational radio interferometric surveying (RIS) systems.

Before a new surveying method can be considered fully operational, studies must be conducted comparing the new method with current operational methods. Such tests serve a multiplicity of purposes: They broaden the interface between the developmental and operational communities, stimulate flow of information, and ensure a high confidence level in the new method. In addition they provide information about the compatibility of the new data with historical data and they often uncover operational limitations, deficiencies, or inconveniences in the new system that were previously overlooked or ignored.

NOS/NGS plans to use RIS methods to define and maintain a highly stable reference frame and to establish a national geodynamics control network. The former application involves the founding of a new generation polar motion and Earth rotation monitoring system, to be known as the POLARIS system. The status of the POLARIS project and some of the results of supporting studies are reported in other papers presented at this conference, i.e., "Project POLARIS: A Status Report" (W. E. Carter, 1979) and "Polar Motion and UT1 Comparisons of VLBI, Lunar Laser, Satellite Laser, Satellite Doppler, and Conventional Astrometric Determinations," (Robertson et al., 1979).

The establishment of a national geodynamics control network requires the measurement of a network of vector baselines designed to detect and separate local, regional, and continental scale motions of the Earth's crust. NASA and the Jet Propulsion Laboratory (JPL) have been developing a mobilc RIS system for such applications. In support of this, NOS/NGS has performed special surveys of selected vector baselines in order to provide a basis for quantitative evaluation and comparisons of the experimental RIS systems. Some of the results of these special surveys are reviewed.

\section{Background}

The surveying techniques that will provide the best determination of a vector baseline depend primarily on the length of the baseline and, to a lesser extent, on factors such as the height, latitude and longitude, terrain, climate, obstructions, and local clutter which are peculiar to each station and, in turn, to each vector baseline. 
In table 1, the baselines are divided into three general categories, according to length, and the most appropriate survey methods, approximate accuracies which are presently attainable, and examples of NOS/NGS surveys in each category, are listed.

Table 1

Summary of Methods and Accuracies of NGS Vector Baseline Surveys

\begin{tabular}{|l|l|l|l|l|}
\hline Classifications & \multicolumn{1}{|c|}{$\begin{array}{c}\text { Baseline } \\
\text { Length }\end{array}$} & \multicolumn{1}{|c|}{$\begin{array}{c}\text { Survey } \\
\text { Method }\end{array}$} & \multicolumn{1}{|c|}{ Accuracy } & \multicolumn{1}{c|}{$\begin{array}{c}\text { Examples } \\
\text { Actual Surveys }\end{array}$} \\
\hline $\begin{array}{l}\text { Short } \\
\text { Baselines }\end{array}$ & $\begin{array}{l}\text { Local to a few } \\
\text { kilometers }\end{array}$ & $\begin{array}{l}\text { 3-dimensional } \\
\text { geodesy }\end{array}$ & $\begin{array}{l}\text { Few millimeters } \\
\text { to about a centi- } \\
\text { meter }\end{array}$ & $\begin{array}{l}\text { ARIES site surveys, } \\
\text { Goldstone phase I } \\
\text { Haystack-Westford }\end{array}$ \\
\hline $\begin{array}{l}\text { Intermediate } \\
\text { Length }\end{array}$ & $\begin{array}{l}\text { Few tens of } \\
\text { kilometers }\end{array}$ & $\begin{array}{l}\text { 3-dimensional } \\
\text { geodesy }\end{array}$ & $\begin{array}{l}\text { Few centimeters } \\
\text { to about a deci- } \\
\text { meter }\end{array}$ & $\begin{array}{l}\text { Goldstone phase II, } \\
\text { McDonald-Ft. Davis } \\
\text { baseline } \\
\text { Malibu-Palos Verdes } \\
\text { baseline }\end{array}$ \\
\cline { 2 - 5 } & $\begin{array}{l}\text { Few tens to few } \\
\text { hundred kilometers }\end{array}$ & $\begin{array}{l}\text { Doppler } \\
\text { Relative Mode }\end{array}$ & $\begin{array}{l}\text { About 25 } \\
\text { centimeters }\end{array}$ & $\begin{array}{l}\text { ARIES inter- } \\
\text { comparison }\end{array}$ \\
\hline $\begin{array}{l}\text { Long } \\
\text { Baselines }\end{array}$ & $\begin{array}{l}\text { Several hundred } \\
\text { to thousands of } \\
\text { kilometers }\end{array}$ & $\begin{array}{l}\text { Doppler point } \\
\text { positioning mode }\end{array}$ & $\begin{array}{l}\text { About 50 } \\
\text { centimeters }\end{array}$ & $\begin{array}{l}\text { Space systems } \\
\text { International Inter- } \\
\text { comparison }\end{array}$ \\
\hline
\end{tabular}

The survey methods fall into two general categories: three-dimensional geodesy and Doppler satellite positioning. By three-dimensional geodesy, we refer to methods in which measurements of the astronomic positions, azimuths, zenith distances, and line lengths (and with certain assumptions, height differences determined by leveling) are combined in a three-dimensional least-squares adjustment to determine the components of the vector baseline (Vincenty, 1979; Heiskanen and Moritz, 1967; Rapp, 1975). Uncertainties in the angular and distance measurements caused by instrumental, observer, and atmospheric modeling deficiencies limit the useful range of these methods to a few tens of kilometers. As the distance grows beyond this limit, the uncertainties soon become excessive and the costs prohibitive.

Two modes of operation are used in Doppler satellite positioning: the relative mode and the point positioning mode. The relative mode is used to determine baselines a few hundred kilometers or less in length where it is possible to observe the satellite passes simultaneously from both endpoints. Certain errors, or portions thereof, become systematic biases that cause nearly equal shifts in the estimated positions of both stations. The difference in the positions is unaffected by the biases; there- 
fore, the baseline components can be determined with improved accuracy. As it is impossible to collect a set of well-distributed simultaneous observations for longer baselines, the station biases become more disparate. The observing schedules are optimized for each station individually, and the reductions are performed in the point positioning mode. The baseline components are determined by simple differencing of the coordinates of the "independently" determined endpoint stations. For the highest degree of accuracy, the observations should be collected during the same date interval in order to minimize the disparateness of the biases.

\section{Short Baselines: The Haystack-Westford Survey}

Rogers et al. (1978) reported the results of 11 RIS measurements of the Haystack-Westford baseline from October 1974 to January 1976. The scatter of the length, height, and two horizontal components was only $3,7,5$ and $3 \mathrm{~mm}$, respectively, in a Haystack altitude-azimuth frame of reference.

The vector baseline was defined to originate at the Haystack VLBI reference point and terminate at the Westford VLBI reference point. Figure 1 is a schematic sketch of the Haystack telescope. Figure 2 is a similar schematic sketch of the Westford telescope. Notice that the Westford telescope is an altitude-azimuth mounted instrument with offset (nonintersecting) axes.

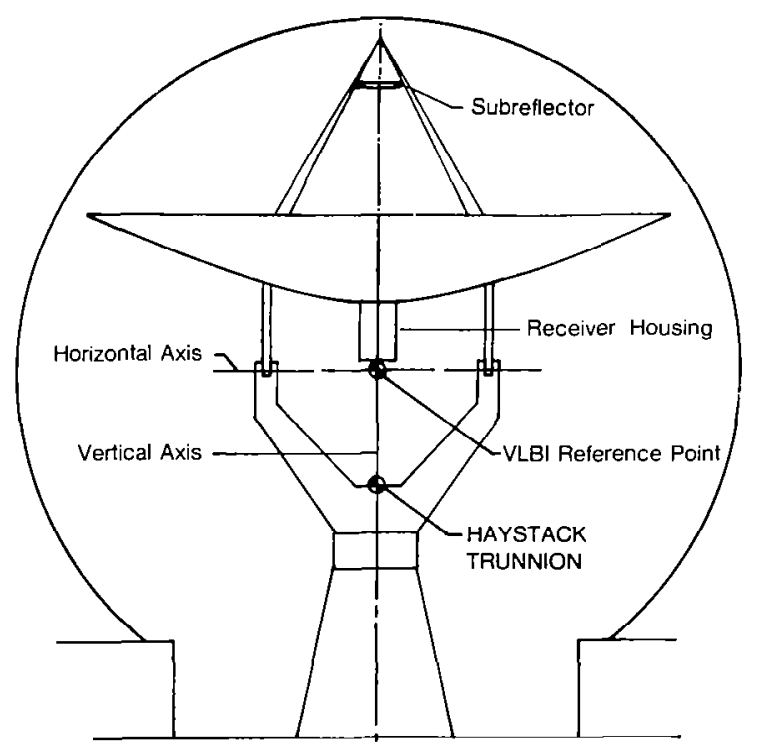

Figure 1. Haystack radio telescope. 


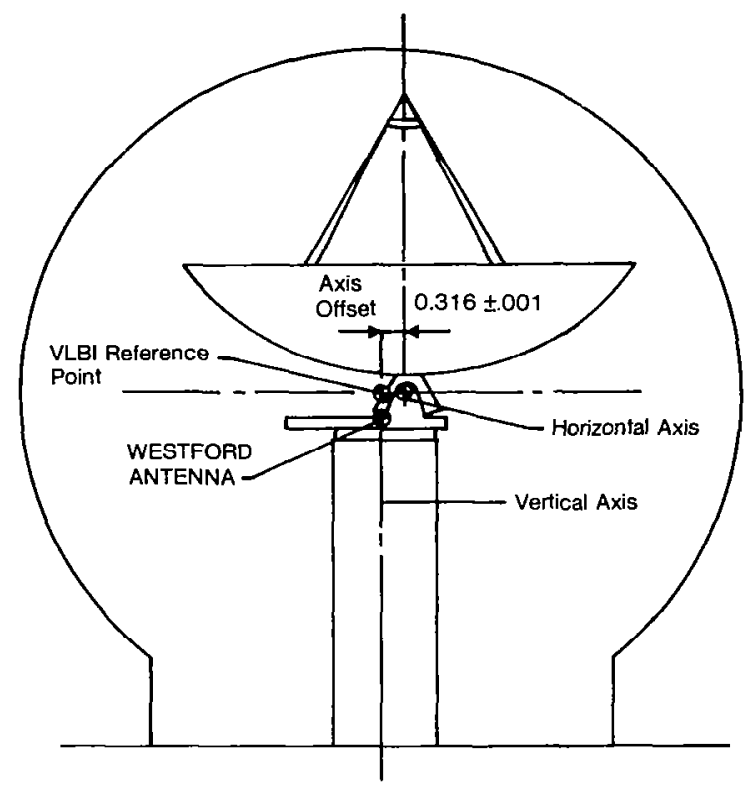

Figure 2. Westford radio telescope.

A high-accuracy three-dimensional geodetic survey of the 1.24-km Ilaystack-Westford RIS vector baseline was conducted to determine the components with uncertainties of a few millimeters. Because local terrain, vegetation, and obstructions associated with the radio telescopes and the enclosing structures made direct measurement of the vector baseline impossible, it was necessary to use a network (figure 3). A detailed account of the survey, including complete listings of the input and output of the adjustment is contained in Carter et al. (1979). Table 2 has been extracted from that publication. Notice that the length and two horizontal components of the baseline, as determined by the three-dimensional survey, agree to within a few millimeters with the RIS values. Only the difference in the height componcnt, $\Delta Z$, is significantly larger than might be expected from the quoted uncertainties. Subsequent studies have determined that gravitational flexure of the Haystack telescope is the major source of the discrepancy. $\Lambda$ joint NOS/MIT/Haystack paper reporting these results is in preparation. Table 3, extracted from the draft of that paper, shows that the discrepancy is still largest in $\Delta \mathrm{Z}$ but is less than $1 \mathrm{~cm}$.

Table 2

Components of the Haystack-Westford Vector Baseline. All Units are Meters.

\begin{tabular}{|c|c|c|c|}
\hline Component & $\begin{array}{c}\text { 3-Dimensional } \\
\text { Survey }\end{array}$ & RIS & $\Delta$ \\
\hline $\mathrm{B}$ & $1239.390 \pm .001$ & $1239.394 \pm .003$ & -.004 \\
$\Delta \mathrm{X}$ & $-1149.592 \pm .002$ & $-1149.594 \pm .003$ & +.002 \\
$\Delta \mathrm{Y}$ & $-462.196 \pm .003$ & $-462.200 \pm .005$ & +.004 \\
$\Delta \mathrm{Z}$ & $-30.024 \pm .004$ & $-30.005 \pm .007$ & -.019 \\
\hline
\end{tabular}




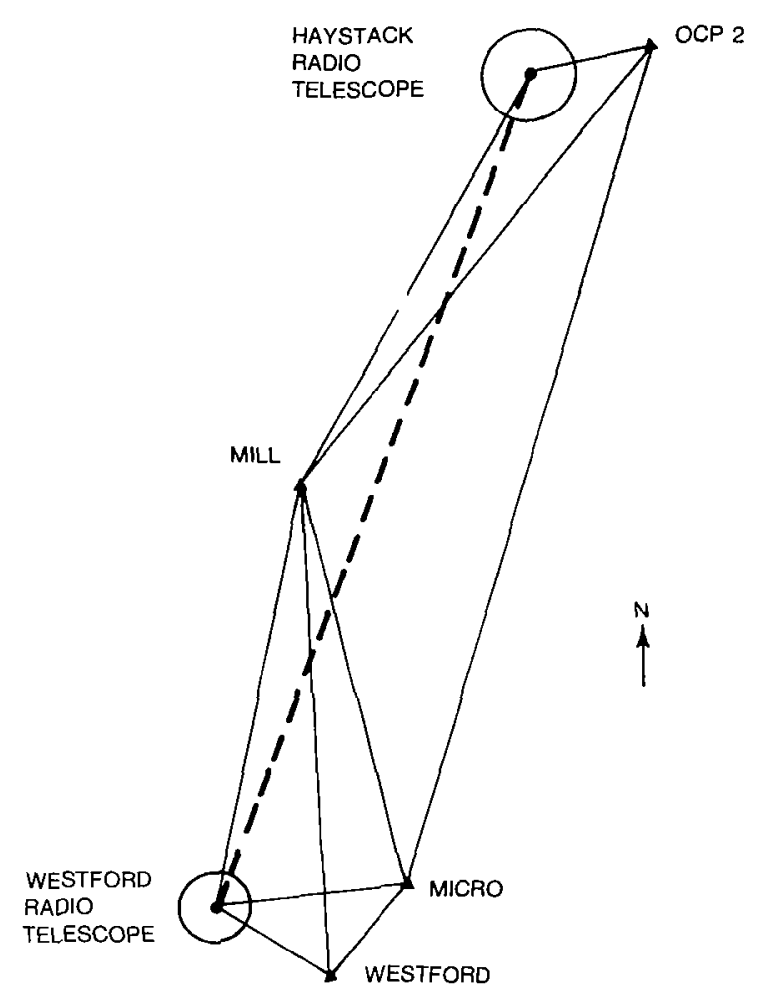

Figure 3. Haystack-Westford survey scheme.

Table 3

Differences in Components of Haystack-Westford Baseline After Correcting RIS Values for Presently Known Flexures of the Telescopes and the Measured Offset of Westford Axes

\begin{tabular}{|l|c|c|c|c|}
\hline & $\Delta \mathrm{X}_{\mathrm{H}}$ & $\Delta \mathrm{Y}_{\mathrm{H}}$ & $\Delta \mathrm{Z}_{\mathrm{H}}$ & $\mathrm{B}$ \\
\cline { 2 - 4 } $\begin{array}{l}\text { Survey - VLBI } \\
\text { (Units are Millimeters) }\end{array}$ & +2 & +4 & -6 & -4 \\
\hline
\end{tabular}

\section{Intermediate Length Baselines}

For baselines a few tens of kilometers in length, three-dimensional geodetic surveys can achieve accuracies of a few centimeters and, at the present state-of-the-art of RIS, provide a useful standard for comparisons. The maximum useful range depends on the local terrain and the resulting complexity of the network required to span the baseline. The MALIBU-PALOS VERDE survey and the Goldstone Validation Survey - Phase II represent extremes in complexity. 


\section{MALIBU-PALOS VERDE SUrvey}

The JPL ARIES transportable RIS system was used to determine two vector baselines: OWENS VALLEY-PALOS VERDE and OWENS VALLEY-MALIBU. By differencing the two, the MALIBUPALOS VERDE baseline was synthesized. This baseline is approximately 42 kilometers long and runs across the Santa Monica Bay in southern California. An unobstructed line of sight exists along the baseline allowing (except for the vertical offsets associated with the survey towers) the length and orientation to be measured directly. The only complications arise from the peculiar atmospheric conditions caused by the bay.

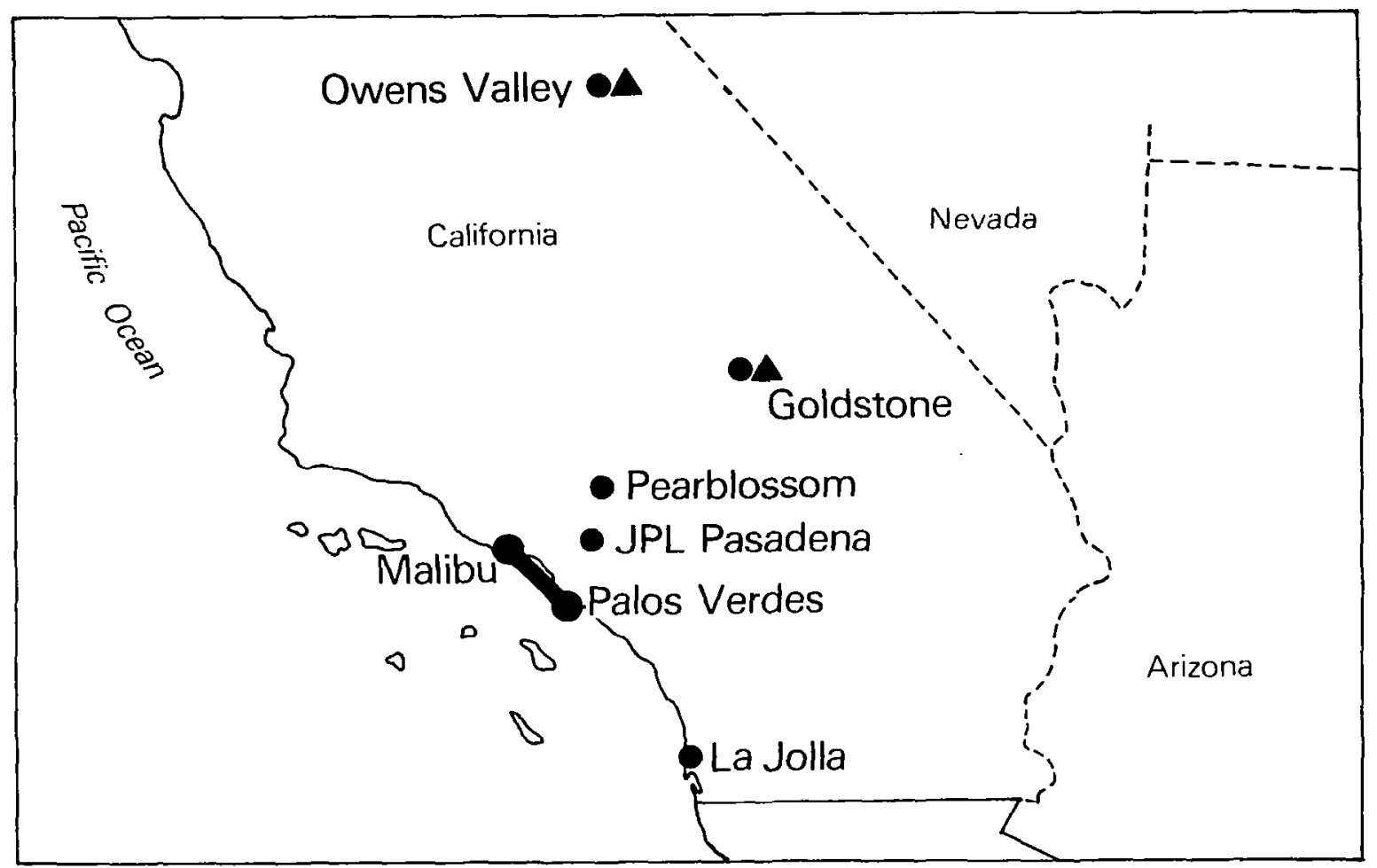

Figure 4. The MALIBU-PALOS VERDE baseline.

The NOS/NGS measured the length and azimuth of the baseline during July 1977. As the meteorological conditions were rather poor, the results must be considered less than optimal. Nevertheless, the RIS and three-dimensional survey results agree within $6 \mathrm{~cm}$ in length and 0.5 arc seconds in azimuth. The vertical component of the baseline was not determined with sufficient precision by the geodetic survey to provide a useful comparison. A more detailed account of this study can be found in Niell et al. (1979). NOS/NGS and JPL plan to perform additional measurements of the MALIBUPALOS VERDE baseline using more accurate techniques to measure and remove the atmospheric effects. 


\section{Goldstone Validation Survey - Phase II}

The Goldstone Deep Spacc Communications Complex includes scveral antennas that are scattered throughout an area several kilometers wide and about 25 kilometers long. The MARS site is located at onc extreme of the complex; the VENUS site at the other. The MARS site has been used extensively for RIS developmental work. NOS/NGS performed a three-dimensional geodetic survey to support the intercomparison studics discussed later in this paper. The results are contained in Carter and Pettey (1978). NASA plans to make extensive use of the VENUS site for RIS activities, particularly as a base station for use with the transportable ARIES system. Also, the MARS-VENUS baseline will be used to study RIS capabilities over distances of a few tens of kilometers. NOS/NGS, with JPL funding, is performing a high-accuracy three-dimensional survey between the MARS and VENUS sites. The objective of the survey is to determine the components of the baseline with uncertainties of less the $5 \mathrm{~cm}$.

Unfortunately, the terrain within the Goldstone complex is rugged and the antennas are located in low "pockets" where they are sheltered by surrounding hills. As a result, the survey scheme is relatively complex (figure 5), and achieving the desired accuracies will be challenging. The field portion of this survey is now in progress; it will be several months (early 1980) before the results are available. This survey approaches the limits, in terms of complexity and cost, over which it is reasonable to use the three-dimensional geodetic survey methods - relative mode Doppler satellite methods rapidly become more attractive for more complex surveys.

NOS/NGS has only recently begun to explore the potentials of the relative mode of Doppler satellite surveying. The accuracy figure quoted in table $1(25 \mathrm{~cm})$ is only a rough estimate based on the experiences of those who have used the relative mode and an extrapolation of our results with the point positioning mode.

NOS/NGS and Defense Mapping Agency (DMA) teams recently completed the field portion of a multi-phased relative mode Doppler satellite survey of "space systems" sites in California. There were a total of four phases of data collection. Figure 6 shows the network occupied during Phase 4 . The vector baselines included in this study range in length from the 42-kilometer MALIBU-PALOS VERDE baseline to the 780-kilometer UKIAH-LA JOLLA baseline. Table 4 summarizes the observational data collected during each of the four phases.

We had hoped to present the initial results of our reductions and analysis of these data today, but equipment failure has caused unavoidable delays in processing the data.

\section{Long Baselines}

During 1978, NASA and NOS/NGS, with the cooperation of several other government agencies and academic institutions, sponsored and executed an intercomparison study of satellite laser ranging, RIS, and Doppler satellite methods over the three-station network formed by the Haystack, Owens 


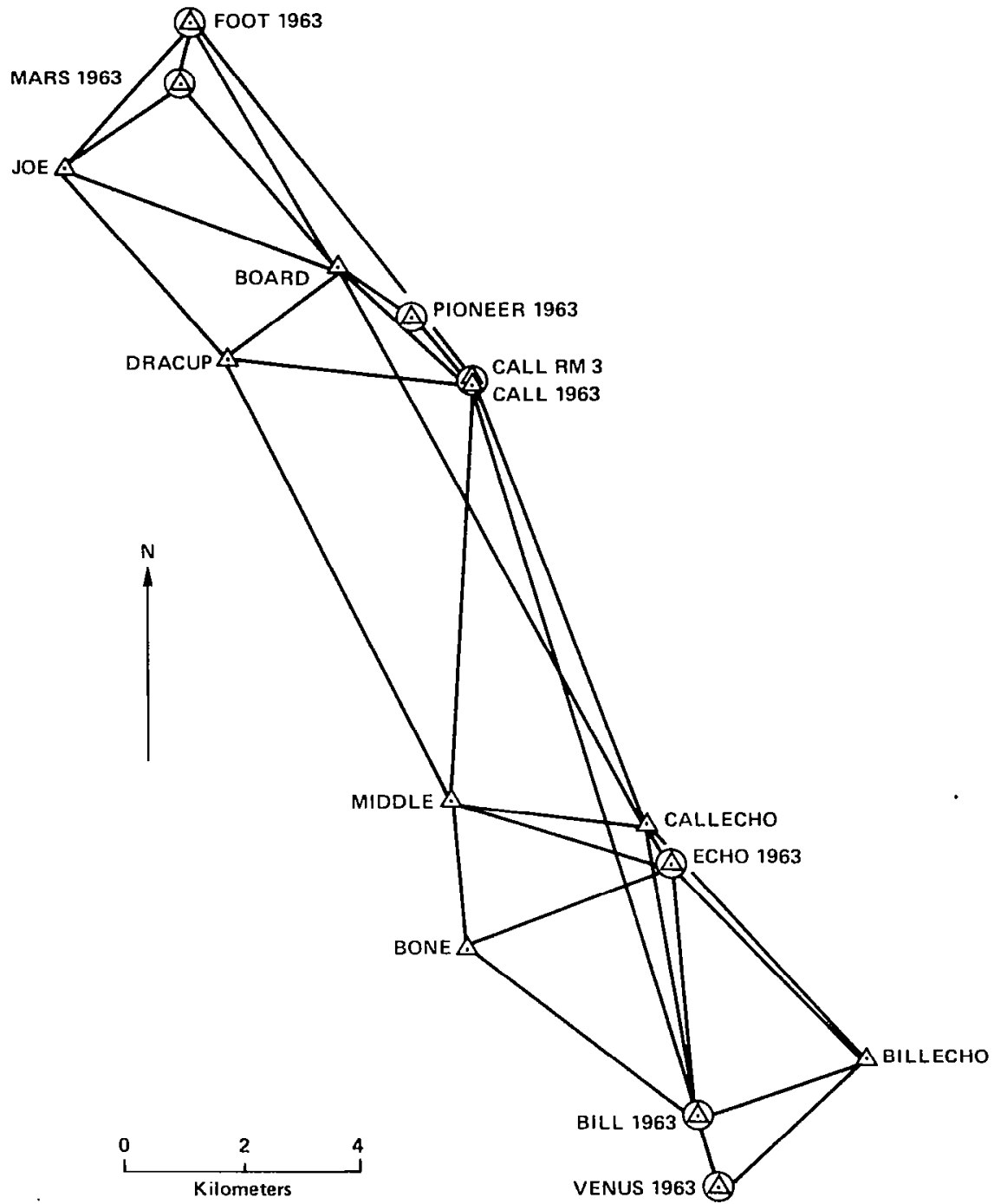

Figure 5. Goldstone validation survey - Phase II. 


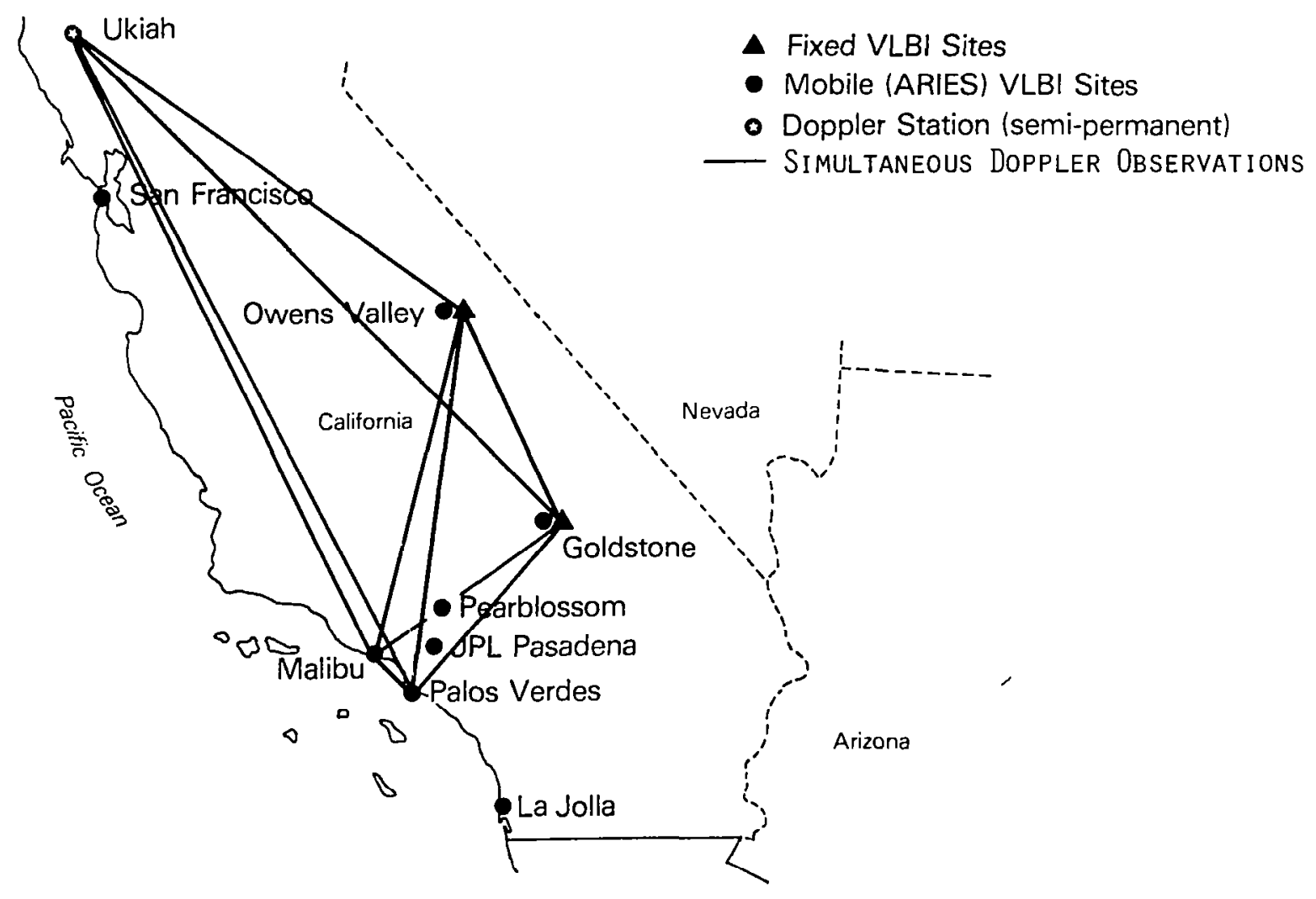

Figure 6. Doppler observation campaign - Phase IV (November 1978 - February 1979). 
Table 4

Doppler Observation Campaign ARIES VLBI Network

(November 1978 - February 1979)

\begin{tabular}{|l|c|c|c|c|}
\multicolumn{1}{c|}{ Station } & \multicolumn{4}{|c|}{ Passes Observed } \\
\cline { 2 - 5 } & $\begin{array}{c}\text { Phase I } \\
(11 / 6-11 / 22 / 78)\end{array}$ & $\begin{array}{c}\text { Phase II } \\
(11 / 28-12 / 12 / 78)\end{array}$ & $\begin{array}{c}\text { Phase III } \\
(1 / 5-1 / 26 / 79)\end{array}$ & $\begin{array}{c}\text { Phase IV } \\
(1 / 30-2 / 19 / 79)\end{array}$ \\
\hline Ukiah & 192 & 168 & 164 & 166 \\
Owens Valley & 157 & 150 & 162 & 161 \\
Goldstone & - & 174 & 191 & 166 \\
San Francisco & 202 & - & - & - \\
La Jolla & 159 & - & 190 & - \\
Pasadena & - & 158 & 174 & - \\
Pearblossom & - & - & - & 231 \\
Malibu & - & - & - & 209 \\
Palos Verdes & - & - & - & - \\
\hline
\end{tabular}

Valley, and Goldstone radio observatories. Various combinations of the systems were also operated at other sites.

Preliminary results of this intercomparison were reported by Hothem (1979); the figures and tables cited have been excerpted (with slight modifications) from that paper.

Figure 7 shows the deployment of the various systems. Table 5 contains a summary of comparisons between the Doppler and Mark I (VLBI) RIS determinations. Table 6 compares the Doppler and Mark II (VLBI) RIS results. The "raw" Doppler values had been corrected $-0.4 \mathrm{ppm}$ in scale for known deficiencies in the computations of the precise ephemerides of the TRANSIT satellites.

Differences in the orientations of the baselines, expressed in terms of their equatorial altitude (declination) and equatorial azimuth (Green wich hour angle) indicate no significant bias in the former, but a bias of about 0.8 arc second in the latter. These numbers agree with previous studies which suggested that the Doppler coordinate system is aligned to the CIO, but has a significant offset in its origin of longitude.

NOS/NGS is also cooperating with researchers of other nations to study very long baselines that are several thousands of kilometers in length. 


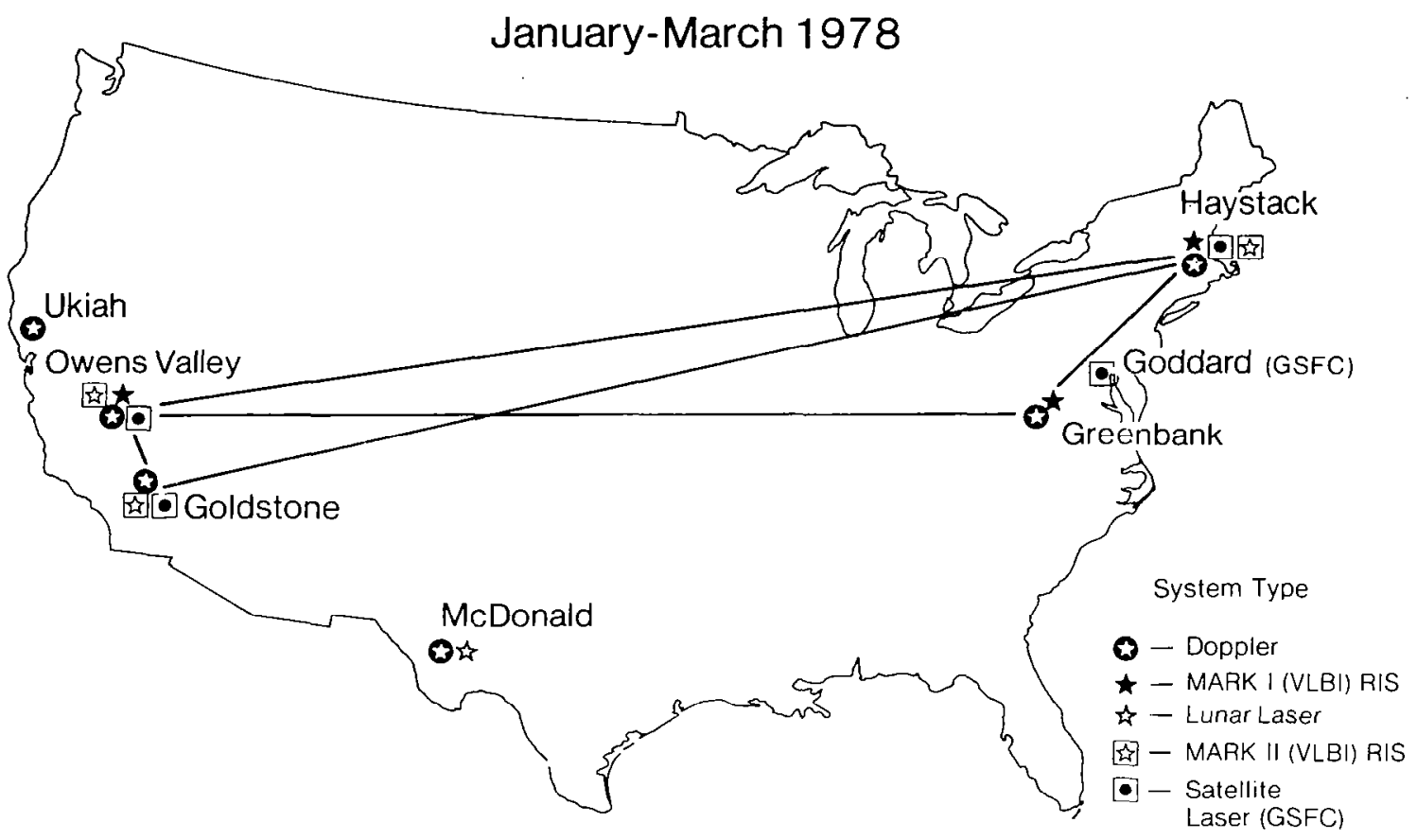

Figure 7. Deployment of systems within the United States for the 1978 long baseline intercomparison studies.

Table 5

Comparison of Doppler Satellite and Mark I (VLBI) RIS Results

\begin{tabular}{|c|c|c|c|c|c|}
\hline \multirow[b]{2}{*}{ FROM ${ }^{\text {STATIONS }}$ TO } & \multicolumn{3}{|c|}{ BASELINE } & \multirow{2}{*}{$\begin{array}{l}\text { EQUATORIAL } \\
\text { ALTITUDE } \\
\text { DIFFERENCE } \\
\text { (SEC) }\end{array}$} & \multirow{2}{*}{$\begin{array}{c}\text { EQUATORIAL } \\
\text { AZIMUTH } \\
\text { DIFFERENCE } \\
\text { (SEC) }\end{array}$} \\
\hline & $\begin{array}{l}\text { DOPPLER* } \\
(M)\end{array}$ & $\begin{array}{l}\text { VLBI } \\
(M)\end{array}$ & $\begin{array}{l}\text { DIFFERENCE } \\
\text { (CM) (PPM) }\end{array}$ & & \\
\hline \multicolumn{6}{|c|}{ PRE-1978 DOPPLER WITH PRE-1978 VLBI DATA } \\
\hline $\begin{array}{l}\text { HAYSTACK-GOLDSTONE } \\
\text { HAYSTACK-OWENS VALLEY } \\
\text { GREENBANK-OWENS VALLEY }\end{array}$ & $\begin{array}{l}3899844.74 \\
3929896.88 \\
3325637.47\end{array}$ & $\begin{array}{l}3899844.87 \\
3929896.72 \\
3325637.10\end{array}$ & $\begin{array}{r}-13-0.03 \\
+16+0.04 \\
+37+0.11\end{array}$ & $\begin{array}{l}+0.024 \\
+0.044 \\
+0.018\end{array}$ & $\begin{array}{l}-0.801 \\
-0.728 \\
-0.828\end{array}$ \\
\hline \multicolumn{6}{|c|}{1978 DOPPLER AND PRE-1978 VLBI DATA } \\
\hline $\begin{array}{l}\text { HAYSTACK-GOLDSTONE } \\
\text { HAYSTACK-OWENS VALLEY } \\
\text { GREENBANK-OWENS VALLEY }\end{array}$ & $\begin{array}{l}3899844.68 \\
3929896.69 \\
3325637.09\end{array}$ & $\begin{array}{l}3899844.87 \\
3929896.72 \\
3325637.10\end{array}$ & $\begin{array}{l}-19-0.05 \\
=\quad 3-0.01 \\
-\quad 1-0.00\end{array}$ & $\begin{array}{l}+0.049 \\
+0.080 \\
+0.066\end{array}$ & $\begin{array}{l}-0.824 \\
-0.738 \\
-0.849\end{array}$ \\
\hline \multicolumn{6}{|c|}{1978 DOPPLER AND 1978 VLBI DATA *ᄎ } \\
\hline HAYSTACK-OWENS VALLEY & 3929896.69 & 3929896.78 & $-9-0.02$ & -0.022 & -0.784 \\
\hline
\end{tabular}

* DOPPLER DISTANCES CORRECTED FOR SCALE OF -0.4 PPM.

* VLBI DATA OBSERVED FEBRUARY 24,1978 , SOLUTIONS PRELIMINARY. 
Table 6

Comparison of Doppler Satellite and Mark II (VLBI) RIS Results

\begin{tabular}{|c|c|c|c|c|c|}
\hline \multirow[b]{2}{*}{ FROM $^{\text {STATIONS }}$ TO } & \multicolumn{3}{|c|}{ BASELINE } & \multirow{2}{*}{$\begin{array}{l}\text { EQUATORIAL } \\
\text { ALTITUDE } \\
\text { DIFFERENCE } \\
\text { (SEC) }\end{array}$} & \multirow{2}{*}{$\begin{array}{l}\text { EQUATORIAL } \\
\text { AZIMUTH } \\
\text { DIFFERENCE } \\
\text { (SEC) }\end{array}$} \\
\hline & $\begin{array}{l}\text { DOPPLER* } \\
(M)\end{array}$ & $\begin{array}{l}\text { VLBI**} \\
(M)\end{array}$ & $\begin{array}{l}\text { DIFFERENCE } \\
\text { (CM) (PPM) }\end{array}$ & & \\
\hline HAYSTACK-GOLDSTONE & 3899844.68 & 3899845.17 & $-49-0.13$ & +0.033 & -0.795 \\
\hline HAYSTACK-OWENS VALLEY & 3929896.69 & 3929896.82 & $-13-0.03$ & +0.034 & -0.804 \\
\hline OWENS VALLEY-GOLDSTONE & 237191.43 & 237191.21 & +22 & -0.055 & -0.487 \\
\hline
\end{tabular}

* FROM 1978 DATA, SOLUTIONS PRELIMINARY, DISTANCES CORRECTED FOR SCALE OF -0.4 PPM.

** DATA OBSERVED FEBRUARY 24,1978 , SOLUTIONS PRELIMINARY.

\section{Concluding Remarks}

The intercomparison studies, past, present, and planned, include vector baselines ranging in length from a few hundred meters to several thousand kilometers. When all these baseline studies have been completed, there should be no doubt about the validity and power of the geodetic applications of radio interferometric surveying methods.

\section{REFERENCES}

Carter, W. E., 1979: "Project POLARIS: a status report." Presented at Mass. Inst. of Tech. and Northeast Radio Observatory Corp. Haystack Observatory Meeting on Radio Interferometry - Techniques for Geodesy, June 18-22.

Carter, W. E. and Pcttcy, J. E., 1978: "Goldstone Validation Survcy Phase I," NOAA Technical Memorandum NOS NGS 15, 39 pp. National Technical Information Service, Springfield, Va. 22161

Carter, W. E., Fronczek, C. J., and Pettcy, J. E., 1979: "Haystack-Westford Survey," NOAA Technical Memorandum (in press).

Heiskanen, W. A. and Moritz, H. 1967: Physical Geodesy, Freeman and Co., San Francisco and London, $364 \mathrm{pp}$.

Hothem, L. D., 1979: "Determination of Accuracy, Orientation and Scale of Satellite Doppler PointPositioning Coordinates," Proceedings of 2nd International Geodetic Symposium on Satellite Doppler Positioning Austin, Texas (in press). 
Neill, A. E., Ong, K. M., MacDoran, P. E., Resch, G. M., Fite, D. W., Skjerve, L. T., Spitzmesser, D. J., Morabito, D. D., Tanida, L., Claflin, E. S., Johnson, B. B., Newsted, M. G., Ganisch, A., and Dracup, J. F., 1979: "Comparison of a Radio Interferometric Differential Base Line Measurement with Conventional Geodesy," In: C. A. Whitten, R. Green and B. K. Meade (Editors) Recent Crustal Movements 1977. Tectonophysics, 52.

Rapp, R. H., 1975: Geometric Geodesy Notes, Vol. II, Ohio State University, Columbus, pp. 111134.

Robertson, D. S., Carter, W. E., Corey, B. E., Cotton, W. D., Counselman, C. C., Shapiro, I. I., Wittelo, J. J., Hinteregger, H. F., Knight, C. A., Rigers, A. E. E., Ryan, J. W., Clark, T. A., Coates, R. J., Ma, C., and Moran, J. M., 1978: "Recent Results of Radio Interferometric Determinations of a Transcontinental Base Line, Polar Motion, and Earth Rotation," International Astronomical Union Symposium No. 82, Cadiz, Spain In: Time and the Earth Rotation Edited D. S. McCarthy and John D. Pilkington, 1979.

Robertson, D. S., Clark, T. A., Coates, R. J., Ma, C., Ryan, J. W., Corey, B. E., Counselman, C. C., King, R. W., Shapiro, I. I., Hinteregger, H. F., Knight, C. A., Rogers, A. E. E., Whitney, A. R., Pigg, J. C., and Schupler, B. R., 1979: "Polar motion and UT1 comparisons of VLBI, lunar lascr, satellite laser, satcllite Doppler, and conventional astronometric determinations." Prcsented at Mass. Inst. of Tech. and Northeast Radio Observatory Corp. Haystack Observatory Meeting on Radio Interferometry - Techniques for Geodesy, June 18-22, Boston.

Rogers, A. E. E., Knight, C. A., Hinteregger, H. F., Whitney, A. R., Counselman, C. C., Shapiro, I. I., Gourevitch, S. A., and Clark, T. A., 1978: "Geodesy by Radio Interferometry: Determination of 1.24-km. Base Line Vector with $5 \mathrm{mmm}$ Repeatability," Journal of Geophysical Research Vol. 83, B1, pp. 325-334.

Vincenty, T., 1979: The HAVAGO three-dimensional adjustment program. NOAA Technical Memorandum NOS NGS 17, 18 pp. National Technical Information Service, Springfield, Va. 2261 . 


\title{
LONG BASELINE VECTOR DETERMINATIONS AND INTERCOMPARISONS
}

\author{
By East-Coast VLBI Group* \\ Presented by Curtis A. Knight
}

Haystack Observatory

The East Coast VLBI group has performed about 60 experiments over the last 10 years in the process of developing and exploiting advanced VLBI techniques. I will report on the most recent 14 experiments. About 2 years ago, we began a series of baseline measurements between the Haystack 37-m antenna, the OVRO 40-m antenna, and the Green Bank 43-m antenna (figure 1). Several technical improvements were made at the beginning of this series. The most significant of these was the introduction of wide bandwidth receivers so as to exploit the bandwidth synthesis technique more effectively. Phase calibration devices were used, as they had been in the past, to insure that the delay stability of the interferometer would be limited by the Hydrogen Maser frequency standards and not some other part of the receiver chain. The phase calibrators include a cable measurement system so that changes in the length of the cable between the Hydrogen Maser and the phase calibrator injection point can be compensated in the data processing. This cable compensation was not included in any of the data presented. The wideband receivers were in use at Haystack and OVRO from the beginning, but were used at Green Bank for only the last experiment.

Each of these experiments was of 1 or 2 days duration. Each day's work produced about 150 measurements of interferometric delay, and delay rate per baseline spread over about 10 radio sources. From these measurements, it is possible to estimate all three components of each baseline vector and all the source coordinates except the right ascension of one source, which must be fixed in order to define the origin of the right ascension coordinate. The baseline vector components are expressed in a "crust-fixed" coordinate system defined by the BIH circular D values for polar motion and AT-UT1 with McClure's formulation for diurnal polar motion and Dahlen's values for the Love numbers for the solid Earth tides, with tidal potentials calculated directly from the solar system geometry. (These models are discussed further in another paper.) Unfortunately, these models are not sufficiently accurate to permit an interpretation of a change in the angular position of the baseline vector in terms of a local motion of the Earth's crust. A variation of time in AT-UT1 of only 0.001 second of time is sufficient to change the equatorial components of the Haystack-OVRO baseline by 30 centimeters. This provides both a problem and an opportunity. The opportunity will be discussed in another paper, and I will avoid the problem by means of the observation that there is a quantity associated with the baseline vector that is independent of rotations - its length.

\footnotetext{
*East-Coast VLBI Group-Participating Organizations: Goddard Space Flight Center, Haystack Observatory, Massachusetts Institute of Technology, National Geodetic Survey, National Radio Astronomy Observatory.
} 


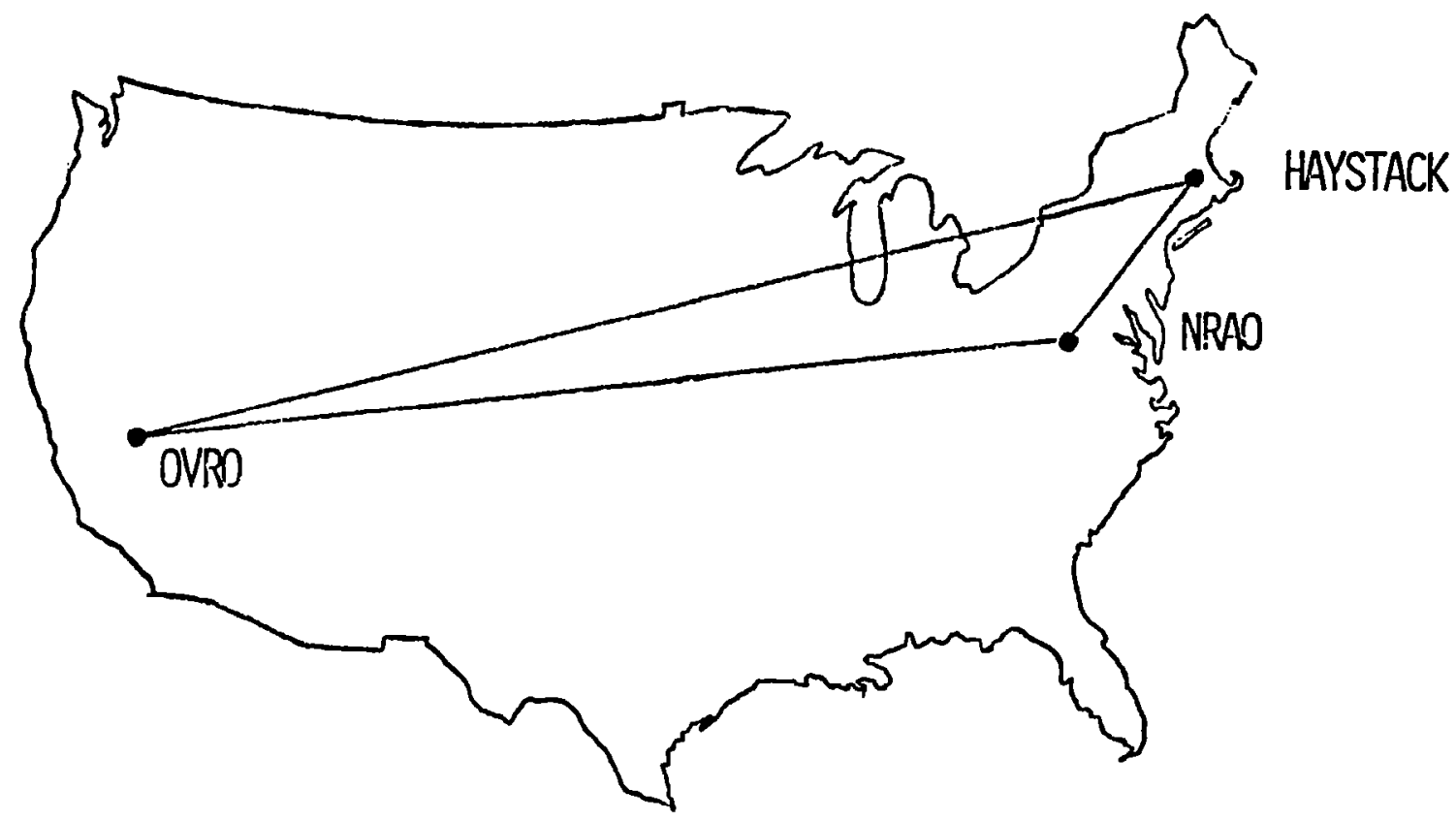

Figure 1.

Figure 2 displays preliminary, nearly independent determinations of the Haystack-OVRO baseline length obtained from data collected in 14 sessions of observations distributed over almost 2 years. These results are preliminary and not quite independent because, for example, the radio source coordinates were fixed at values determined from an average of those obtained from analysis of the data from each session of observations separately. When the source coordinates are allowed to vary in each baseline determination, the effect is to increase the scatter in haseline length by about 50 percent. This set of measurements is remarkable in that it demonstrates that for this period there has been no change in the baseline length of more than a few centimeters per year.

In figure 3, similar determinations of the Haystack-Green Bank baseline length are displayed. Measurements from older series of experiments have been included to extend the time spanned to almost 5 years. Note that the wideband receivers were used only in the last experiment. The limit on the rate of change in length of this baseline is also a few centimeters per year. 


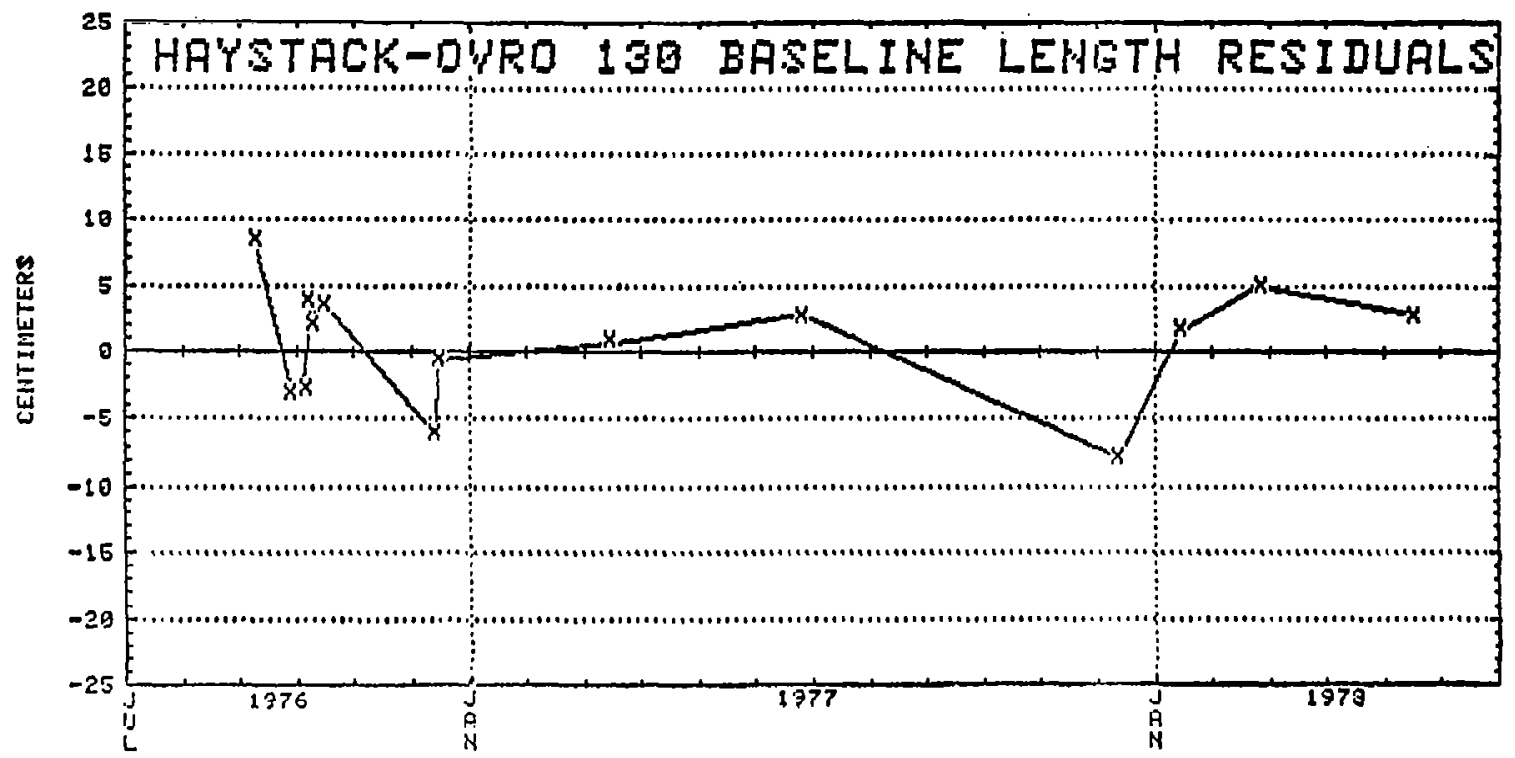

Figure 2.

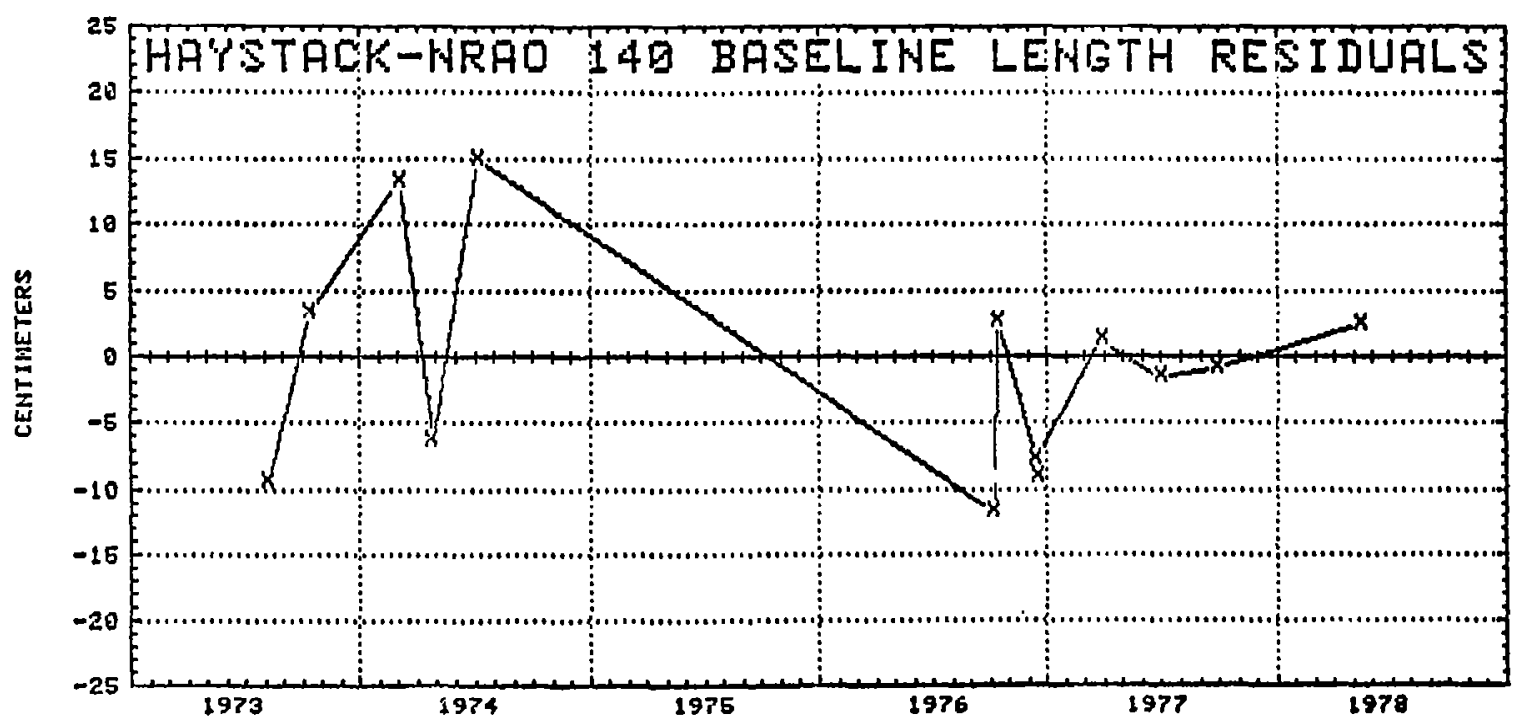

Figure 3. 
How can we improve upon these results in the future? When deployed world-wide, the Mark-III VLBI system currently under development by the East-Coast VLBI group will provide the sensitivity to observe radio sources in all parts of the sky. This will eliminate the scheduling constraints associated with the Mark-I system, which could see relatively few sources. The multiplicity of baselines available will permit the observation of angular changes in one baseline relative to another, since the rotation of the Earth will be measured simultaneously with the baseline vectors to high accuracy. (This assumes that an adequate definition for 'crust-fixed' coordinates can be found in the age of centimeter level geodesy.) Water vapor line radiometers being developed at the Jet Propulsion Laboratory will, when placed at the Mark-III sites, permit the inference of the atmospheric delay in the line of sight to the radio sources. In short, we may look forward in the near future to a significant increase in the quantity and quality of geodetic measurements. Their interpretation should provide excitement for years to come. 


\title{
VLBI MEASUREMENTS OF BASELINES, UT AND POLAR MOTION WITH DEEP SPACE NETWORK ANTENNAS: 1971 - 1978
}

\author{
J. B. Thomas, J. L. Fanselow, E. J. Cohen, G. H. Purcell, D. A. Rogstad, \\ O. J. Sovers, L. J. Skjerve, D. J. Spitzmesser
}

Jet Propulsion Laboratory

\begin{abstract}
From 1971 through 1978, numerous VLBI measurements have been carried out with Deep Space Network antennas in Goldstone, California; Madrid, Spain; and Canberra, Australia. Analysis of these experiments has produced measurements of the California/Spain and California/Australia baselines as well as measurements of UT and polar motion.
\end{abstract}


BA SELINES:

(1) GOLDSTONE, CALIFORNIA

- CANBERRA, AUSTRALIA

(2) GOLDSTONE, CALIFORNIA

- MADRID, SPAIN

(3) OWENS VALLEY, CALIFORNIA

- MASSACHUSETTS

RADIO SOURCES: 66 SEPARATE SOURCES WHERE $-45^{\circ} \leqq \delta \lesssim+71^{\circ}$ AND $0 \leqq a \leqq 24$ HOURS

OBSERVATIONS: 3I SEPARATE SESSIONS CONTAINING A TOTAL OF ABOUT 1260 OBSERVATIONS

OBSERVA BLES: DELAY AND DELAY RATE OR DELAY RATE ONLY WHERE

$0.4 \mathrm{nsec} \leqq \sigma_{\tau} \lesssim 3.0 \mathrm{nsec}$ AND $0.6 \times 10^{-13} \lesssim \sigma_{\dot{\tau}} \lesssim 1.0 \times 10^{-12}$

OBSERVING FOR $\dot{\tau}$ ONLY, 250 OBS AT S-BAND. FOR $\tau$ AND $\dot{\tau}, 245$ OBS AT S-BAND;

FREQUENCIES: $\quad 365$ OBS ATX-BAND; 345 OBS WITH SIX

Figure 1. Summary of 1971-1978 VLBI experiments.

\begin{tabular}{|c|c|c|c|}
\hline & GEOMETRY & $\begin{array}{l}\text { TIME AND } \\
\text { FREQUENCY }\end{array}$ & $\begin{array}{l}\text { PROPAGATION } \\
\text { EFFECTS }\end{array}$ \\
\hline $\begin{array}{l}\text { PARAMETERS } \\
\text { FITTED }\end{array}$ & $\begin{array}{l}\text { STATION LOCATIONS } \\
\text { SOURCE POSITIONS } \\
\text { UTI - UTC } \\
\text { POLAR MOTION }\end{array}$ & $\begin{array}{l}\text { CLOCK EPOCH } \\
\text { DIFFERENCES } \\
\text { CLOCK RATE } \\
\text { DIFFERENCES }\end{array}$ & TROPOSPHERE \\
\hline $\begin{array}{l}\text { PARAMETERS } \\
\text { NOT FITTED }\end{array}$ & $\begin{array}{l}\text { PRECESSION } \\
\text { NUTATION } \\
\text { SOLID EARTH TIDES }\end{array}$ & & IONOSPHERE \\
\hline $\begin{array}{l}\text { EFFECTS } \\
\text { NOT MODELED }\end{array}$ & $\begin{array}{l}\text { DIURNAL POLAR MOTION } \\
\text { LIQUID CORE } \\
\text { GENERAL RELATIVITY }\end{array}$ & $\begin{array}{l}\text { HIGHER ORDER } \\
\text { TERMS }\end{array}$ & \\
\hline
\end{tabular}

Figure 2. Components of the delay model. 


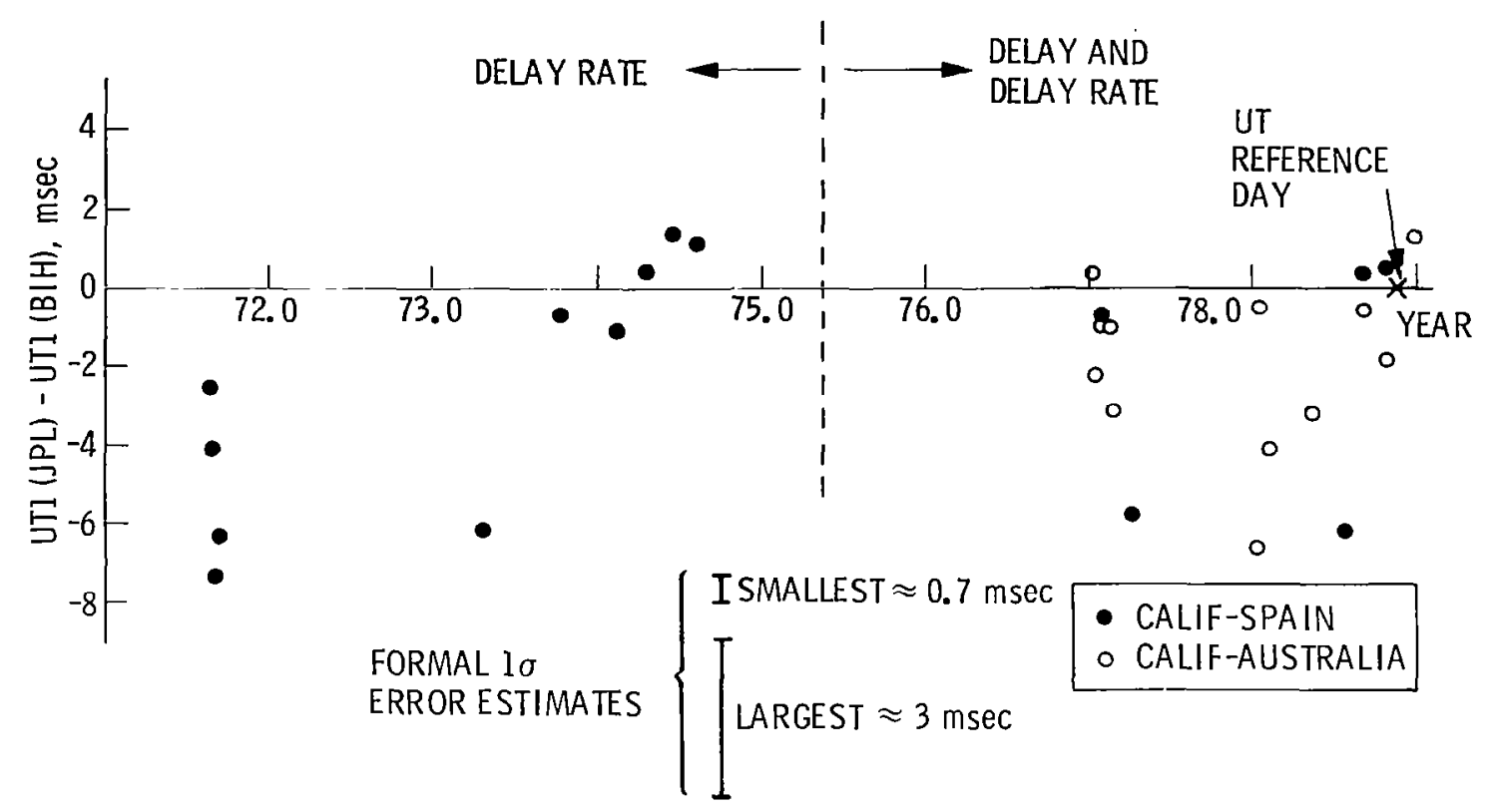

Figure 3. UT1 results from 1971-1978 VLBI data.

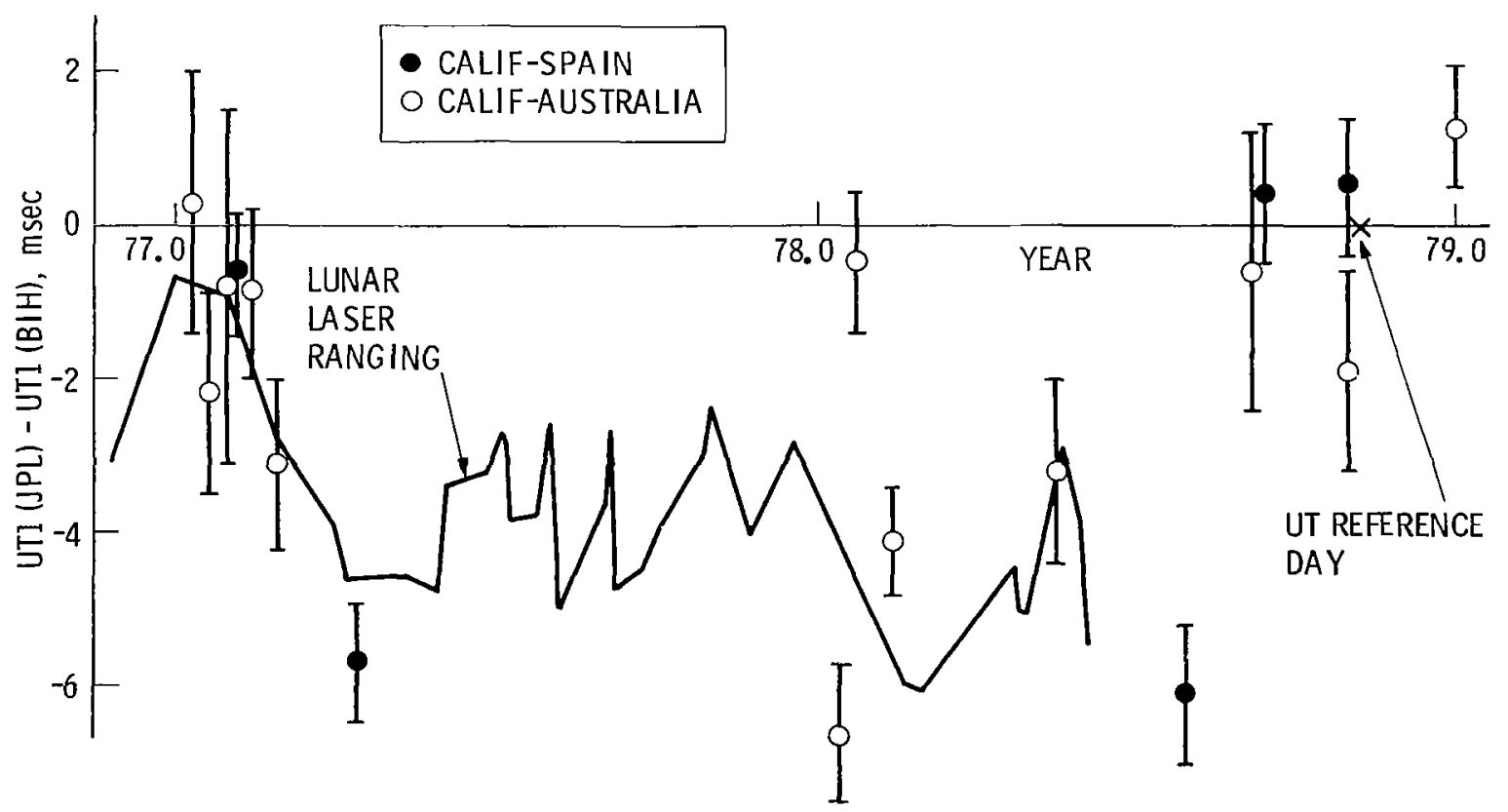

ERROR BARS ARE FORMAL VLBI UNCERTAINTIES

Figure 4. UT1 results from 1977-1978 VLBI data. 


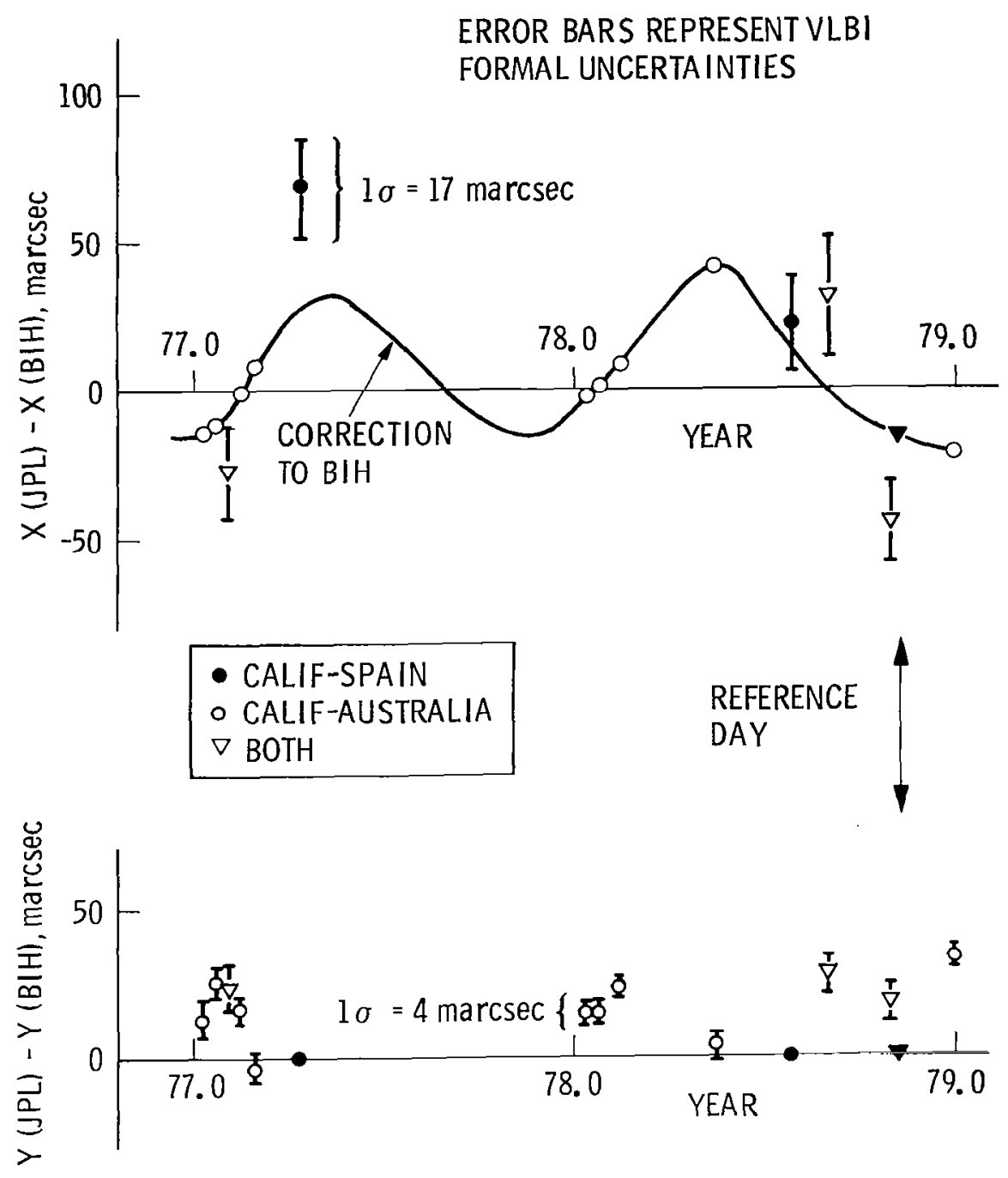

Figure 5. Polar motion results from 1977-1978 VLBI data. 


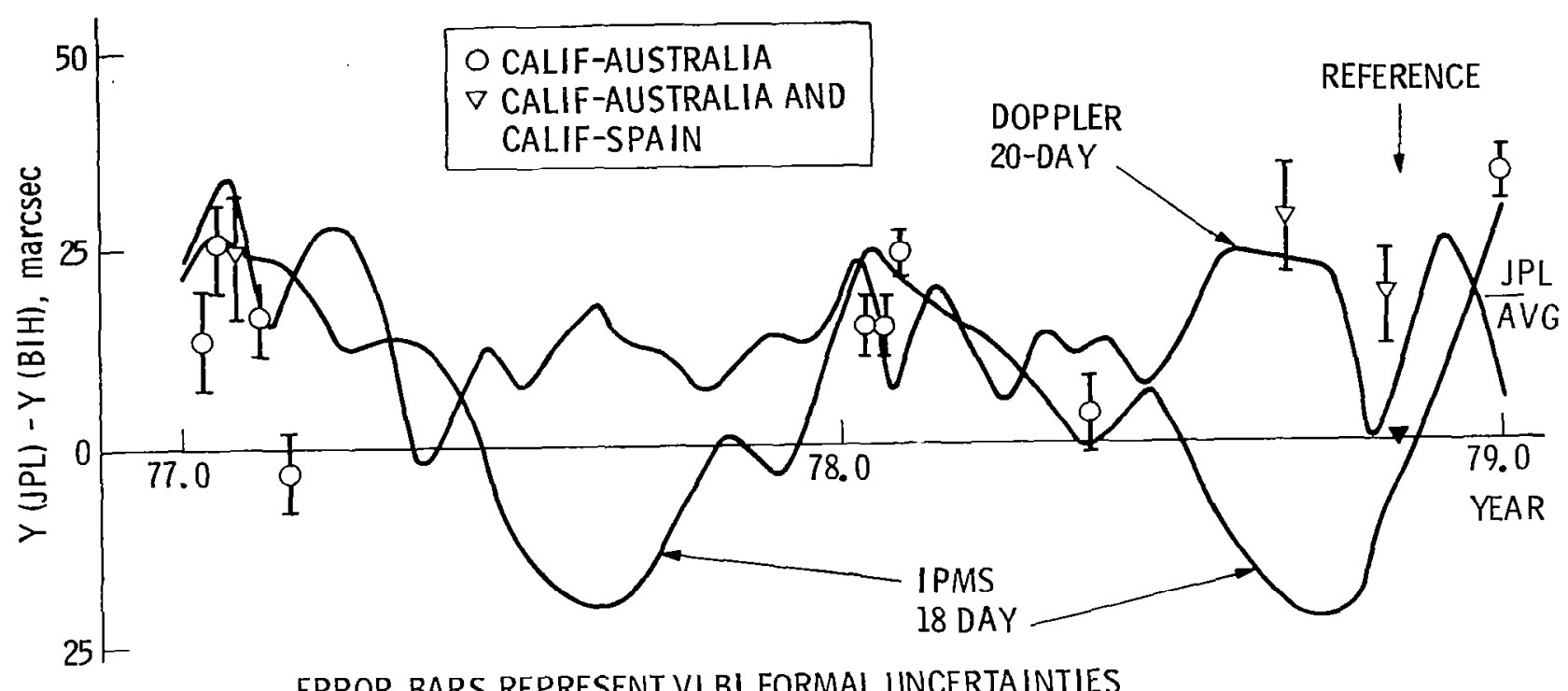

ERROR BARS REPRESENT VLBI FORMAL UNCERTAINTIES

Figure 6. Results for Y polar motion from 1977-1978 VLBI data. 
GOLDSTONE, CALIFORNIA (DSS14) TO CANBERRA, AUSTRALIA (DSS43)

\begin{tabular}{|l|l|}
\hline EQUATORIAL LENGTH & $7,620,842.07 \pm 0.12 \mathrm{~m}$ \\
\hline EQUATORIAL LONGITUDE & $106.052288 \pm 0.47 \mathrm{~m}$ \\
\hline POLAR PROJECTION & $-7,351,800.10 \pm 0.37 \mathrm{~m}$ \\
\hline LENGTH & $10,588,965.89 \pm 0.26 \mathrm{~m}$ \\
\hline
\end{tabular}

GOLDSTONE, CALIFORNIA (DSS14) TO MADRID, SPAIN (DSS63)

\begin{tabular}{|l|l|}
\hline EQUATORIAL LENGTH & $8,378,986.60 \pm 0.16 \mathrm{~m}$ \\
\hline EQUATORIAL LONGITUDE & $30.726471 \pm 0.45 \mathrm{~m}$ \\
\hline POLAR PROJECTION & $438,056.09 \pm 0.50 \mathrm{~m}$ \\
\hline LENGTH & $8,390,429.64 \pm 0.16 \mathrm{~m}$ \\
\hline
\end{tabular}

OWENS VALLEY, CALIFORNIA (OVRO) TO MASSACHUSETTS (HO)

\begin{tabular}{|l|l|}
\hline EQUATORIAL LENGTH & $3,902,062.53 \pm 0.05 \mathrm{~m}$ \\
\hline EQUATORIAL LONGITUDE & $0.309553 \pm 0.15 \mathrm{~m}$ \\
\hline POLAR PROJECTION & $458,278.21 \pm 0.14 \mathrm{~m}$ \\
\hline LENGTH & $3,928,881.64 \pm 0.04 \mathrm{~m}$ \\
\hline
\end{tabular}

"ALL ERRORS REPRESENT VLBI FORMAL UNCERTA INTIES

Figure 7. Baseline vector results from 1971-1978 VLBI data. 


\title{
POLAR MOTION AND UT1: COMPARISON OF VLBI, LUNAR LASER, SATELLITE LASER, SATELLITE DOPPLER, AND CONVENTIONAL ASTROMETRIC DETERMINATIONS
}

\author{
D. S. Robertson \\ National Geodetic Survey, NOS, NOAA \\ T. A. Clark, R. J. Coates, C. Ma, J. W. Ryan \\ NASA, Goddard Space Flight Center \\ B. E. Corey, C. C. Counselman, R. W. King, I. I. Shapiro \\ Department of Earth and Planetary Sciences \\ and Department of Physics, \\ Massachusetts Institute of Technology \\ H. F. Hinteregger, C. A. Knight, A. E. E. Rogers, A. R. Whitney \\ Haystack Observatory \\ J. C. Pigg, B. R. Schupler \\ Computer Sciences Corporation
}

\begin{abstract}
Very-long-baseline interferometry (VLBI) observations made with a $3900 \mathrm{~km}$ baseline interferometer (Haystack Observatory in Massachusetts to Owens Valley Observatory in California) have been used to estimate changes in the X-component of the position of the Earth's pole and in UT1. These estimates are compared with corresponding ones from lunar laser ranging, satellite laser ranging, satellite Doppler, and stellar observations.
\end{abstract}




\section{INTRODUCTION}

Significant progress is being made in the technology of measuring the motion of the Earth's pole and the irregularities in the Earth's rotation. Very-long-baseline interferometry (VLBI), lunar laser ranging, satellite laser ranging, and satellite Doppler observations all have the potential of measuring the Earth's orientation with a precision far better than than obtainable with classical techniques. In this paper we compare the VLBI determinations of polar motion and Earth rotation with the corresponding results from other techniques and attempt to assess the current state of the art of such measurements.

\section{SENSITIVITY OF VLBI MEASUREMENTS TO POLAR MOTION AND UT1}

VLBI delay observations of extra-galactic radio sources are insensitive to translations of the VLBI baseline, but are sensitive to some changes in the orientation of that baseline with respect to the (planar) radio wavefronts. To specify orientation in space, three angles are generally required; however, a baseline is one-dimensional and its orientation in space is specified by two angles. A rotation about an axis parallel to the baseline does not change the orientation of the baseline, but rather produces at most a translation of that baseline. VLBI observations are therefore insensitive to rotations about any axis parallel to the baseline. This result can be expressed analytically as follows: Define a cartesian coordinate system fixed to the rigid Earth, with the Z-axis in the direction of the reference pole, the $\mathrm{X}$ axis perpendicular to $\mathrm{Z}$ in the direction of the Greenwich meridian, and the $\mathrm{Y}$ axis completing a right-hand triad. The changes in the coordinates of the baseline vector caused by polar motion and variations in UT1 can be written as:

$$
\begin{aligned}
& \Delta X=-\theta Y-x Z \\
& \Delta Y=\theta X+y Z \\
& \Delta Z=x X-y Y
\end{aligned}
$$

where $\theta$ is UT1-UTC in radians, and $\mathrm{x}$ and $\mathrm{y}$ are the coordinates of the instantaneous pole, also in radians. Non-zero values for $\Delta \mathrm{X}$ and $\Delta \mathrm{Y}$ will introduce a sinusoidal signature in the delays observed for a non-polar source because of the rotation of the Earth. A non-zero value for $\Delta \mathrm{Z}$ will introduce a time-independent change in the delays, whose magnitude will depend on the declination of the source being observed. For the case of a polar baseline $(X=0, Y=0)$ the sensitivity to $\theta$ vanishes, and a change in pole position introduces a sinusoid in the delay observations whose amplitude and phase can be used to estimate the components of this change in pole position. For the case of an equatorial baseline oriented parallel to the plane of the Greenwich meridian $(Y=0, Z=0)$ the sensitivity to the $\mathrm{y}$ component of the pole position vanishes. $\theta$ will introduce a sinusoidal variation in the observed delays, and $x$ will introduce a constant offset in those delays. For $Y \neq 0$, the VLBI observations will be sensitive to a change in the component of the pole that is in the direction of the 
meridian whose plane is parallel to the baseline, and insensitive to a change in the component perpendicular to that plane. Thus for an equatorial baseline, the effects of polar motion and UT1 are separable.

The VLBI measurements discussed in this paper were obtained in 14 separate experiments spread between September 1976 and May 1978. These experiments utilized the 37-meter-diameter antenna at the Haystack Observatory in Massachusetts, and the 40-meter-diameter antenna at the Owens Valley Radio Observatory in California. This baseline is nearly parallel to the Earth's equatorial plane, having a declination of less than $7^{\circ}$, and is even closer to parallel to the plane of the Greenwich meridian, making an angle of less than $0^{\circ} .5$ with that plane. The interferometric observations are therefore sensitive to changes in the $\mathrm{X}$-component of the position of the pole and to changes in UT1 and are practically insensitive to changes in the Y-component of the pole.

\section{POLAR MOTION}

VLBI determinations of the X-component of the pole are shown in figure 1, displayed as differences from the corresponding values determined by the Bureau International de l'Heure (BIH). The VLBI values show peak-to-valley excursions from the $\mathrm{BIH}$ values of about $80 \mathrm{msec}$ of arc, or a little more than two meters. The error bars shown are the formal standard errors, based on an adjustment of the measurement errors to yield an rms value of unity for the postfit residuals. Further analysis of these data, including detailed studies of effects of clock and atmosphere errors, as well as inclusion of some additional data from NRAO and Onsala (Sweden), may change these values by amounts up to a few times the formal standard errors.

VLBI measurements alone are sensitive only to changes in the pole position and therefore the zero point of figure 1 is necessarily arbitrary. It was set by fixing the value of the X-component of the pole position at the BIH value for the experiment of October 4-5, 1976.

Figure 2 shows the two-day average values of the X-component of the pole position determined from satellite Doppler observations by the Defense Mapping Agency Hydrographic and Topographic Control (DMAHTC). Again, the values are differenced from those of the BIH and are plotted on the same scale as before. A clear systematic trend is present which appears to have an annual period with an amplitude of about $30 \mathrm{msec}$ of arc; the point-to-point (short-period) scatter has an RMS of about $25 \mathrm{msec}$. In order to better exhibit the systematic trend, we smoothed these data by convolution with a Gaussian function whose full-width at half-maximum was 10 days. The result is shown in figure 3.

Figure 4 shows the corresponding determinations of the X-component of the position of the pole from laser ranging observations of the LAGEOS satellite. These values were supplied to us by D. Smith of NASA's Goddard Space Flight Center. Figure 5 shows these values smoothed by the same filter used to produce figure 3. Again, an annual trend seems to be present. 


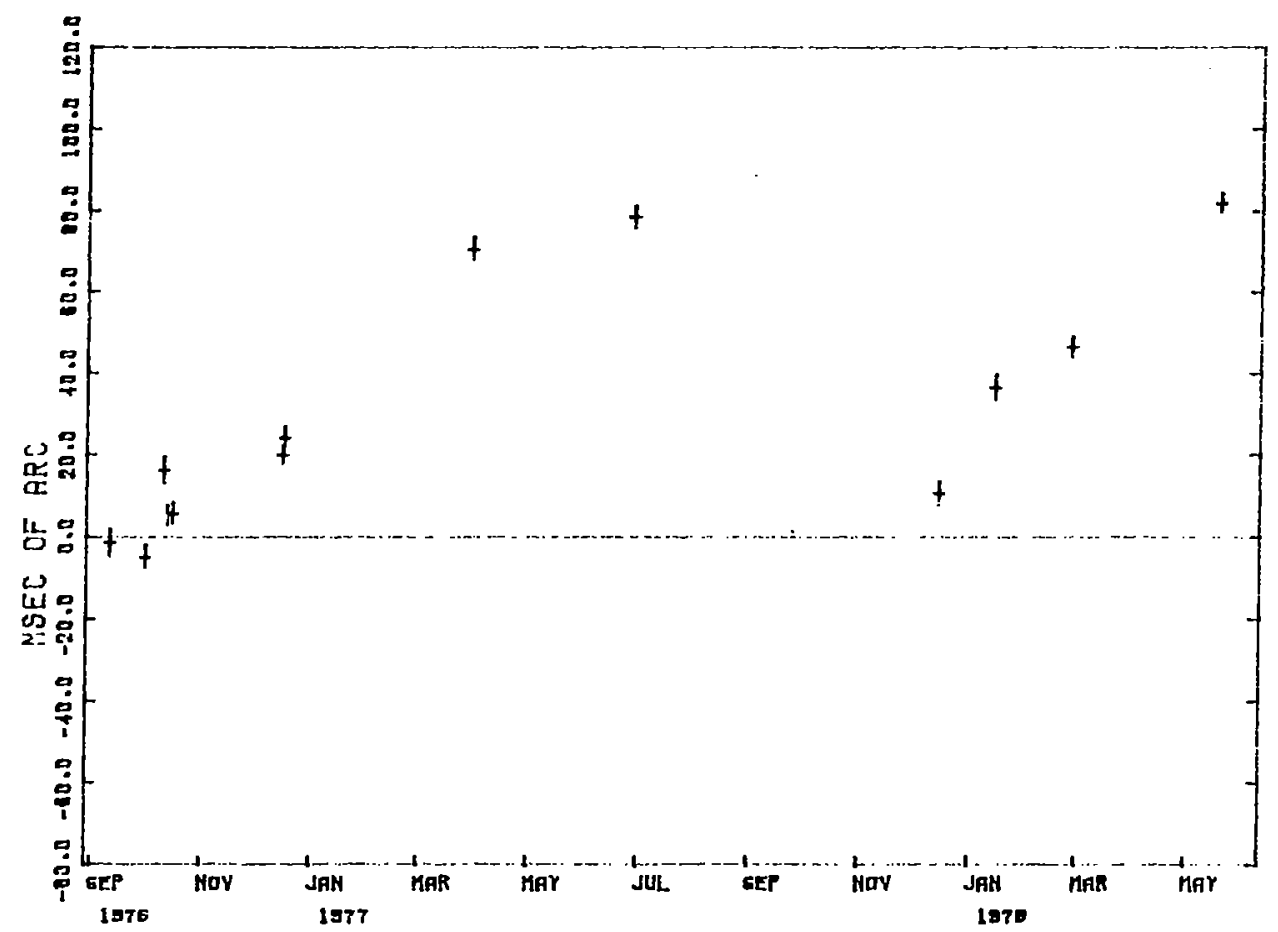

Figure 1.

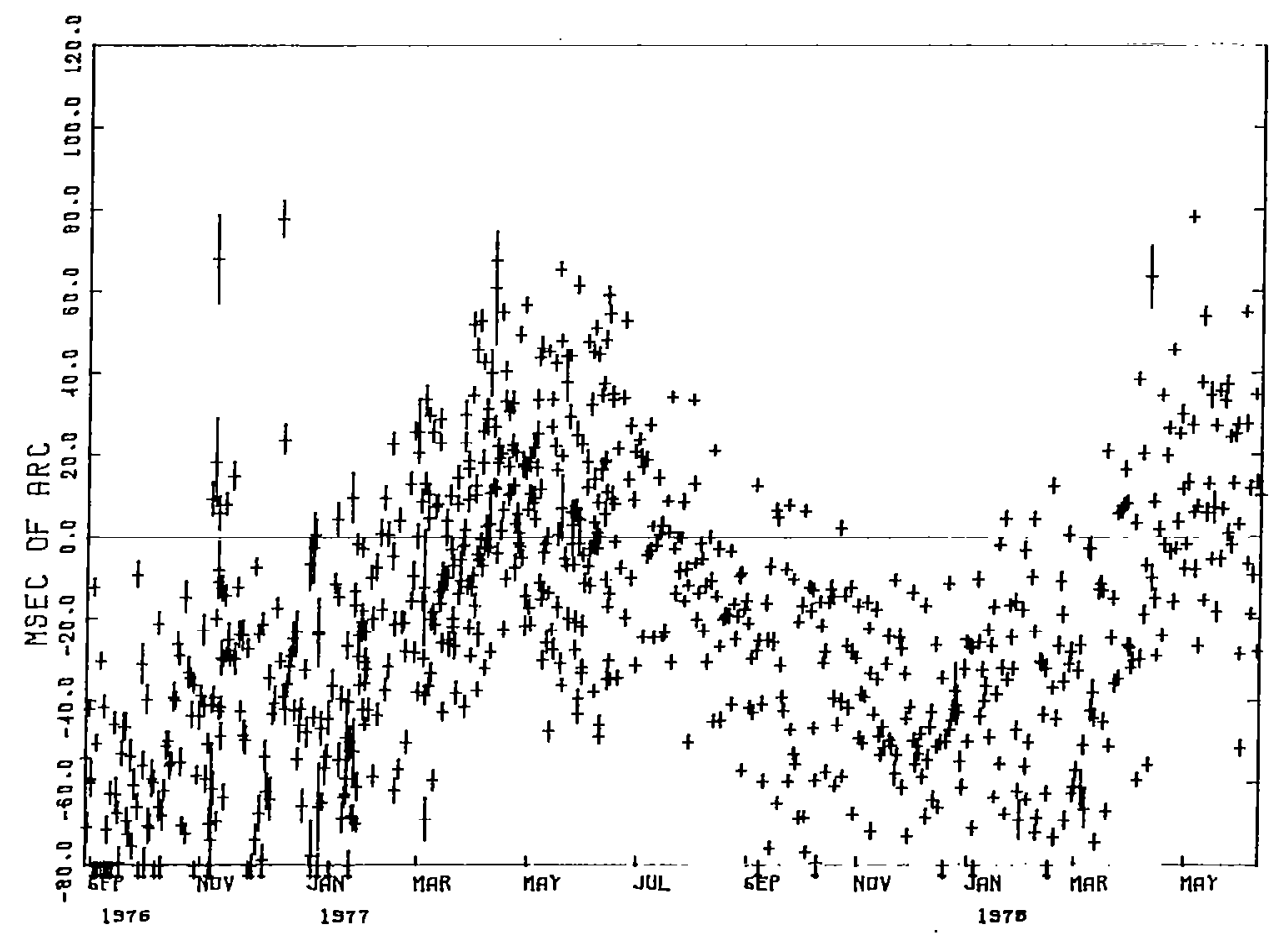

Figure 2 . 


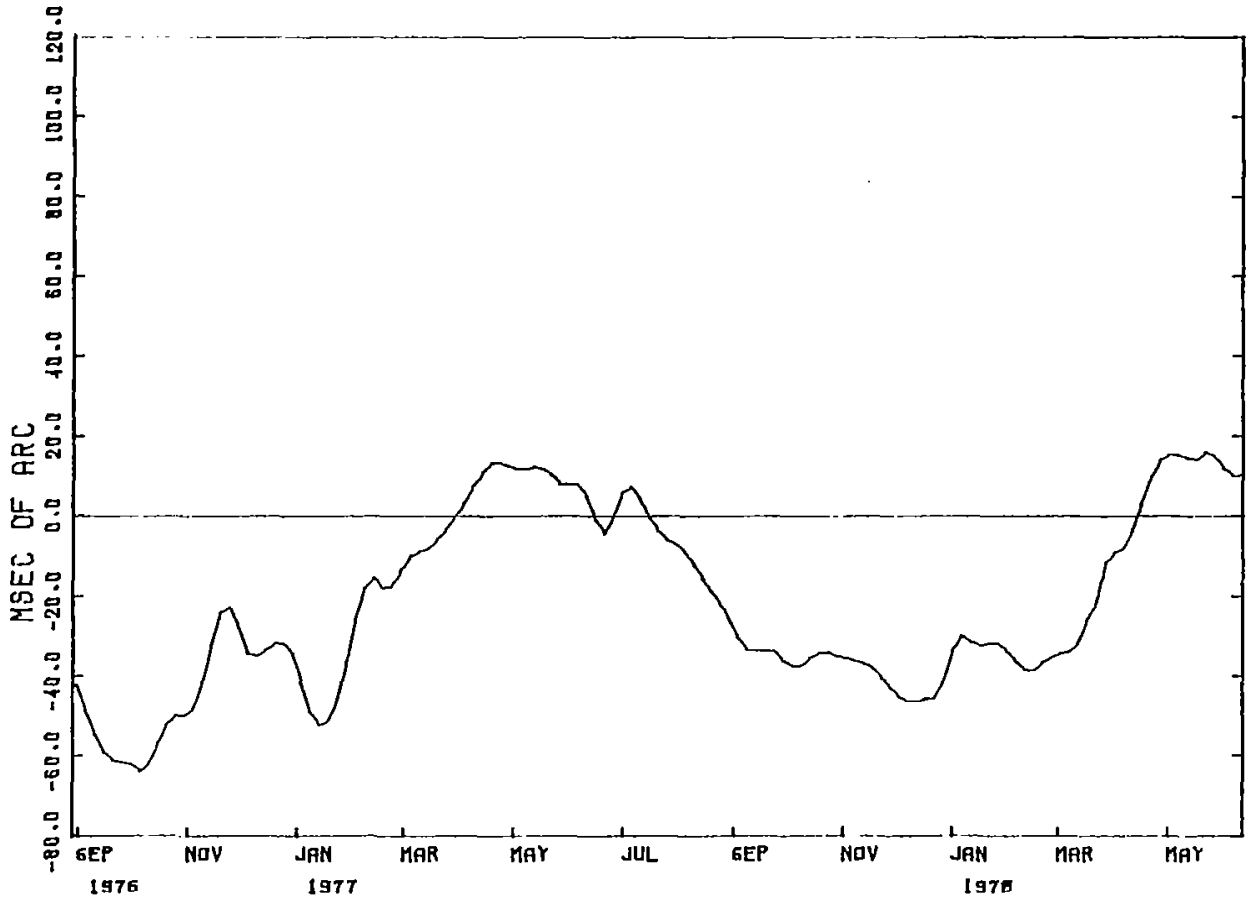

Figure 3.

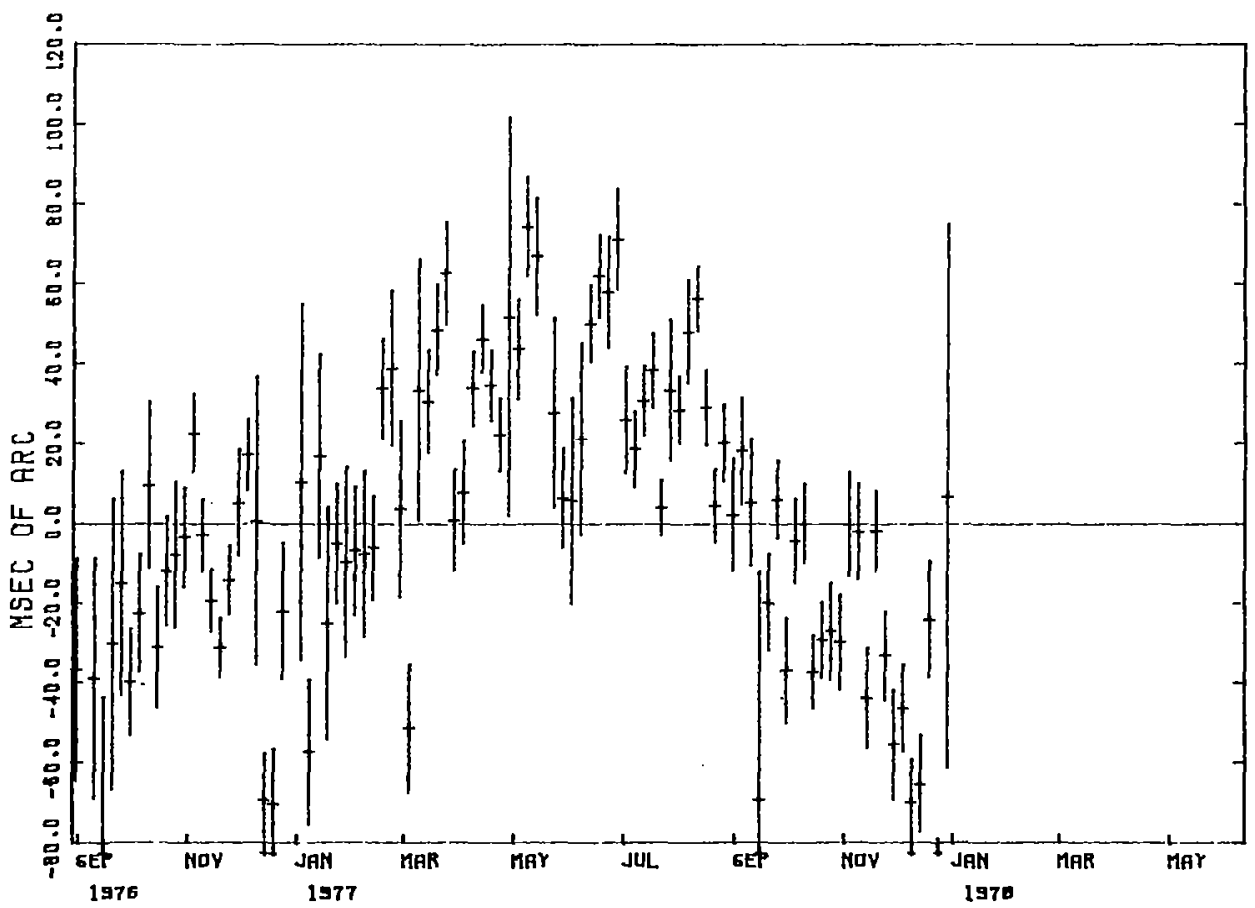

Figure 4 . 


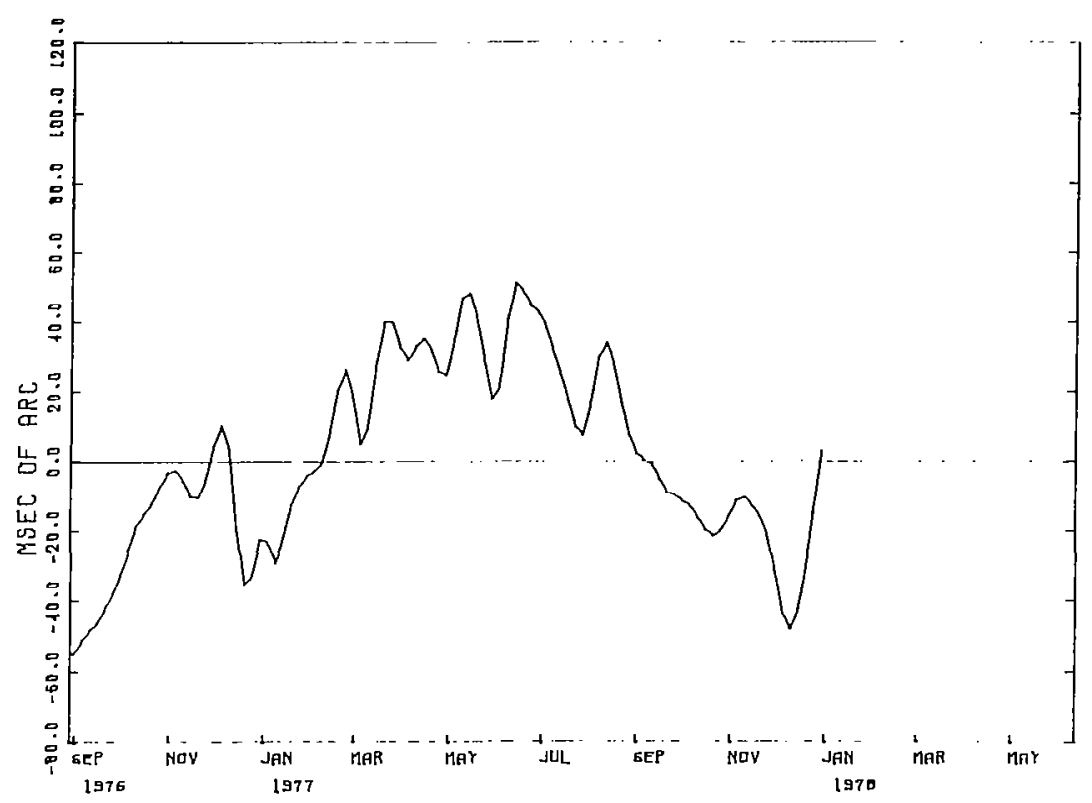

Figure 5.

Figure 6 shows the corresponding determination of the X-component of the position of the pole as determined by the International Polar Motion Service (IPMS) in Misuzawa, Japan. The IPMS employs only classical optical observations (PZT, VZT, etc.). Even here, the annual trend away from the BIH is clear.

Each of the figures of differences in estimates of pole position has a different bias. This bias can be removed by re-defining the "zero point." (In fact, this procedure was used for the Doppler, satellite laser, and IPMS values, but for different spans of data.) Shifting each set of differences shown in our figures by the mean value of its difference from the corresponding values from the Doppler set yields figure 7 . The consistency of the amplitudes and phases of the annual terms is quite clear.

In order to evaluate quantitatively the short-term differences between the results from the different techniques, we can calculate the RMS deviation about the mean of the differences between the corresponding members of each pair of results, for the dates in common. The results of these calculations are tabulated in matrix form in table 1 . The first value in the matrix indicates a 9 msec RMS difference between the VLBI and the Doppler estimates of the position of the pole. Since the expected standard error in the Doppler estimates of $\sim 10 \mathrm{msec}$, and the average formal standard error of the VLBI estimates is $\sim 3 \mathrm{msec}$, the RMS difference is about what one would expect. Although, admittedly, there are only 13 VLBI estimates, this RMS difference does indicate that the systematic errors in the Doppler estimates are not contributing significantly to the total errors. The other values in the table are consistent with the errors in the satellite laser ranging estimates of the pole position being $\sim 12 \mathrm{msec}$, the corresponding IPMS errors $\sim 15 \mathrm{msec}$, and the BIH errors $\sim 25 \mathrm{msec}$. 


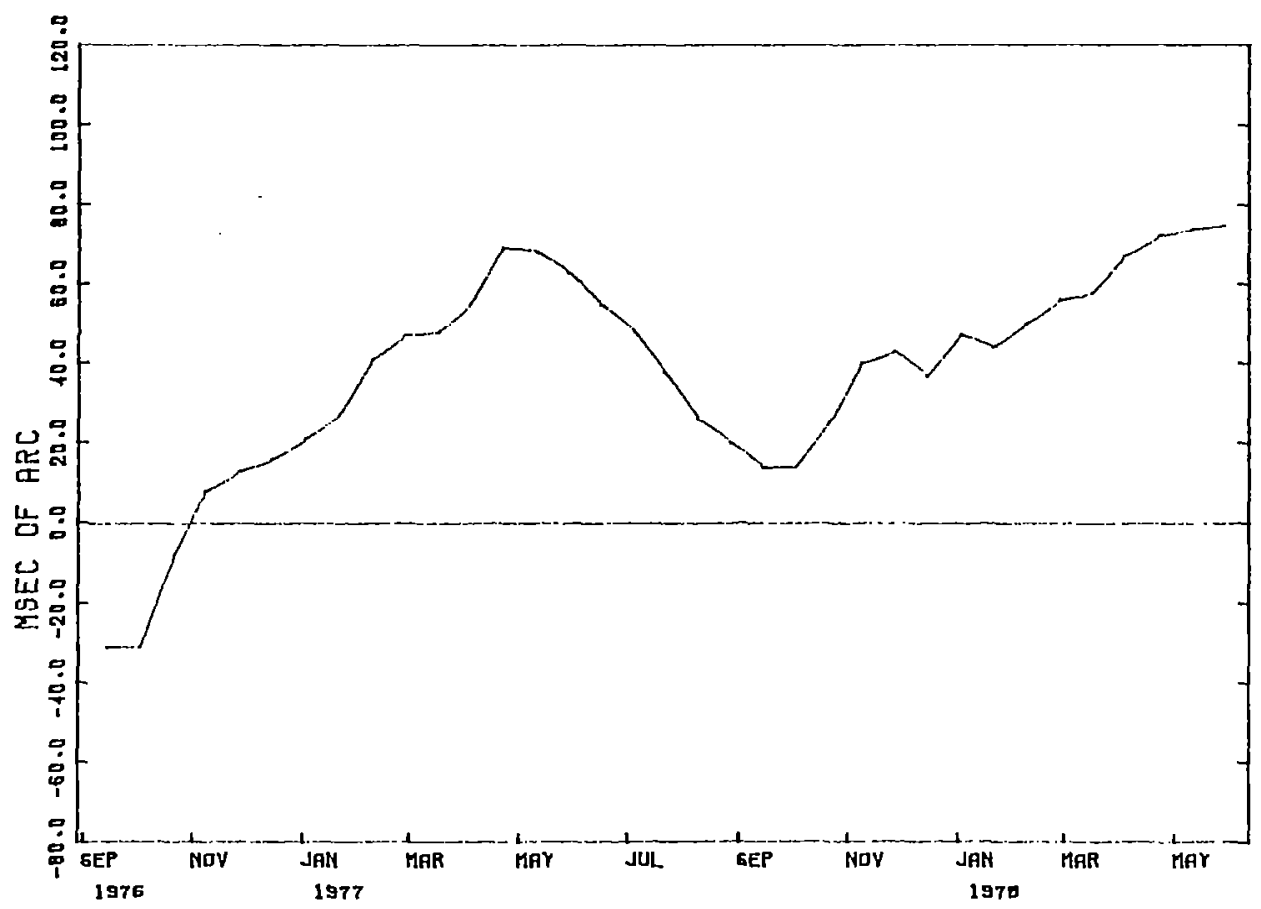

Figure 6.

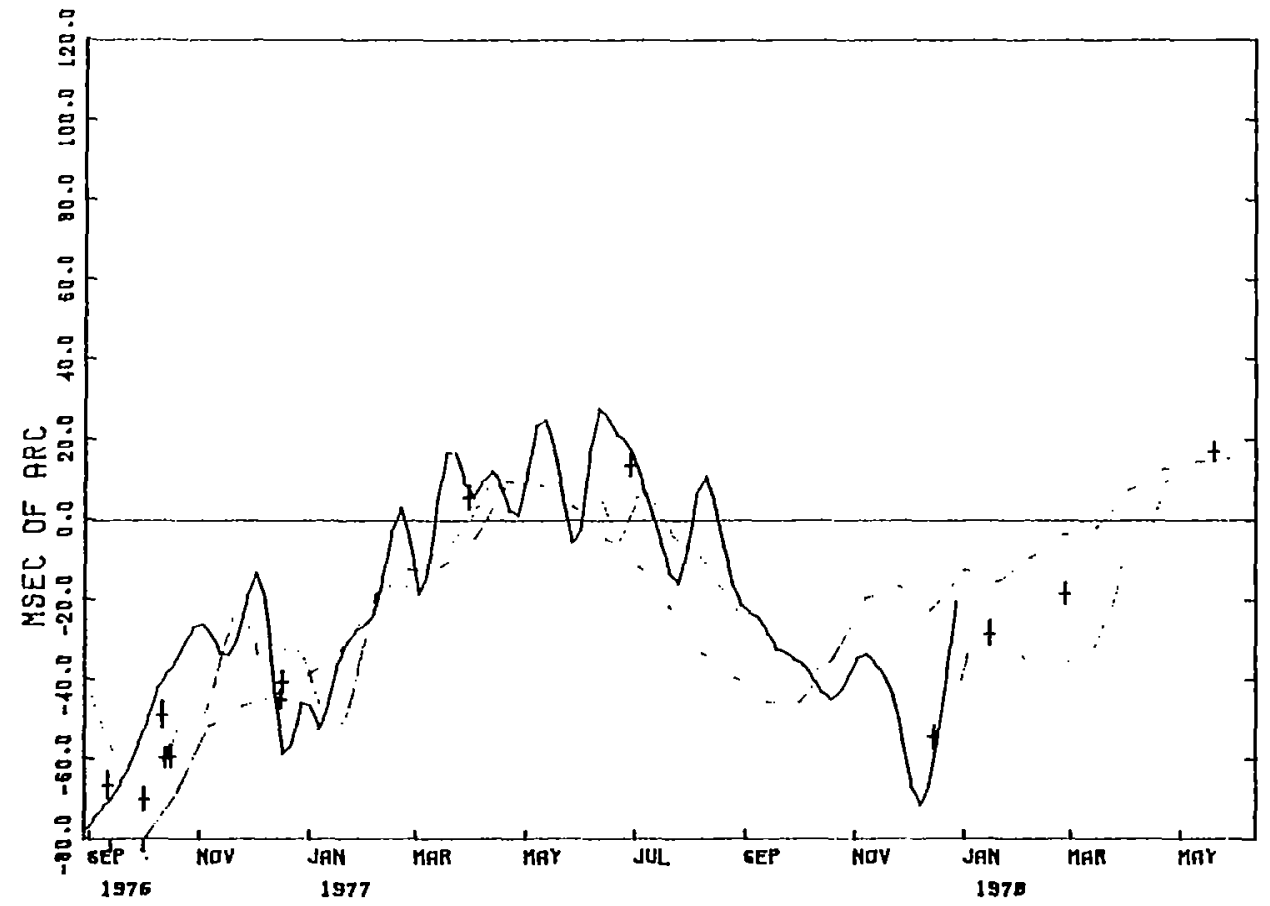

Figure 7. 
The anomalously large RMS difference between the VLBI and the BIH estimates is due to the distribution of the VLBI values in time. The epochs of most of the VLBI observations were close to the extrema of the annual error in the BIH estimates.

Table 1

RMS Deviations about the Mean of the Differences between the Determinations of the X-Component of the Pole by Various Monitoring Techniques

\begin{tabular}{|l|c|c|c|c|c|}
\hline & VLBI & Doppler & $\begin{array}{c}\text { Satellite } \\
\text { Laser }\end{array}$ & IPMS & BIH \\
\hline VLBI & - & & & & \\
Doppler & $9 \mathrm{~ms}$ & - & - & & \\
Satellite Laser & $12 \mathrm{~ms}$ & $14 \mathrm{~ms}$ & $18 \mathrm{~ms}$ & - & \\
IPMS & $16 \mathrm{~ms}$ & $15 \mathrm{~ms}$ & $24 \mathrm{~ms}$ & $27 \mathrm{~ms}$ & - \\
BIH & $30 \mathrm{~ms}$ & $23 \mathrm{~ms}$ & $17 \mathrm{~ms}$ & $19 \mathrm{~ms}$ & $15 \mathrm{~ms}$ \\
Corrected BIH & $16 \mathrm{~ms}$ & $11 \mathrm{~ms}$ & $17 \mathrm{~ms}$ \\
\hline
\end{tabular}

In May 1979, the BIH altered this picture by announcing that it would introduce an ad hoc correction to its X-component values in the form of an annual term and a semi-annual term. The form of the correction is displayed in figure 8. After application of this correction, the RMS difference between the BIH estimates and those from the other techniques are displayed in the last line of table 1. The errors in the BIH estimates of the X-component of the position of the pole appear to have been reduced by the correction to about the level of the errors in the IPMS values.

\section{UT1}

Figure 9 displays the differences between the VLBI determinations of (UT1-UTC) and the BIH determinations. As with the values of pole position, completion of a more detailed analysis of the VLBI observations may lead to changes in these estimates of (UT1-UTC) by amounts up to several times the formal standard errors. Nonetheless, the general trends in the differences from the BIH estimates are probably significant.

Lunar laser ranging data have also been employed to determine variations in UT1. The UT1 variations were modeled as a piece-wise linear function, as described by King et al. (1978). To compare the lunar laser ranging values with the VLBI values, the laser values were converted to UT1 from UT0 at the McDonald Observatory by using the estimate of the position of the Earth's pole as determincd from the Doppler observations. Figure 10 shows the lunar lascr values compared to the VLBI values which have been adjusted to allow for a different value used for the precession constant in the lunar laser program. 


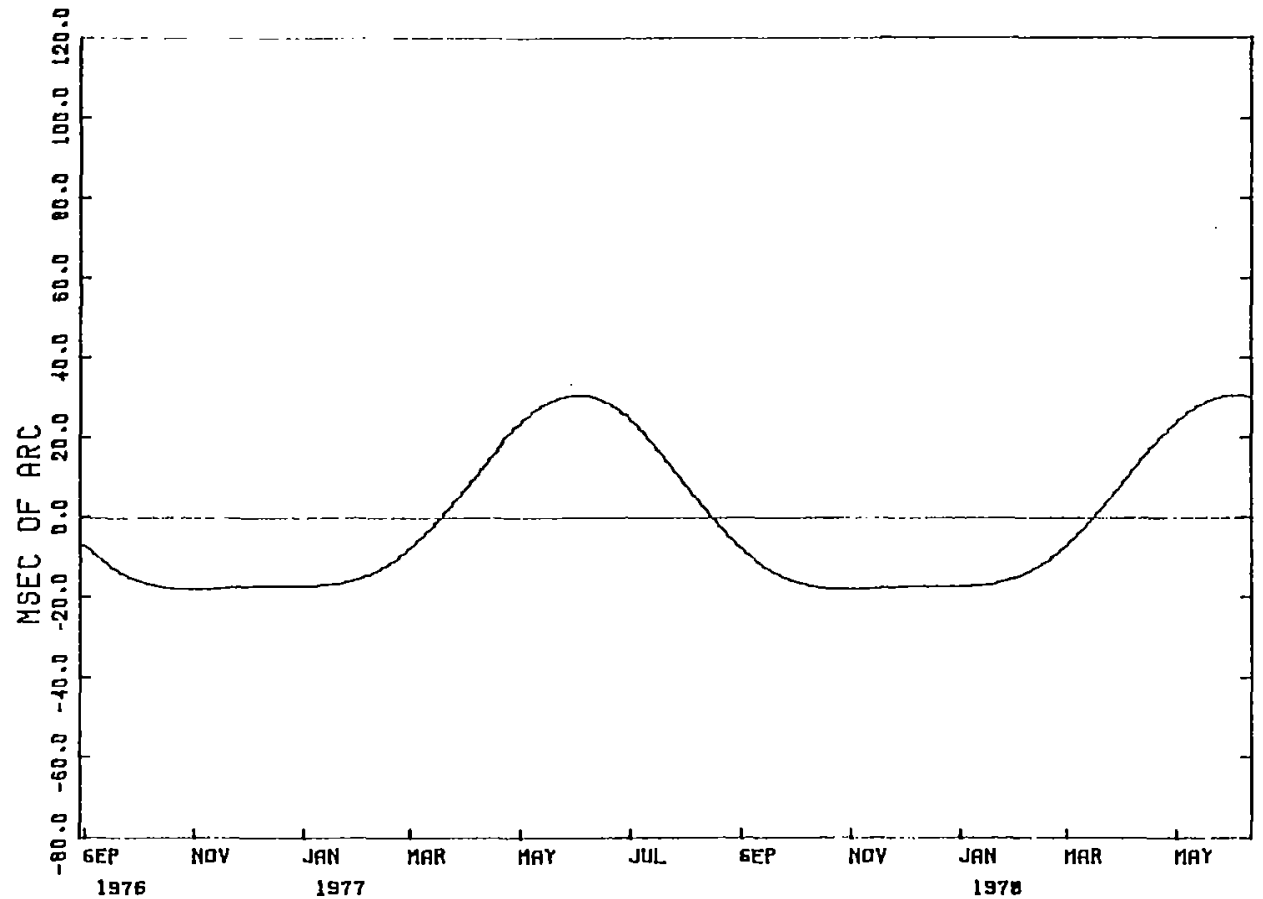

Figure 8.

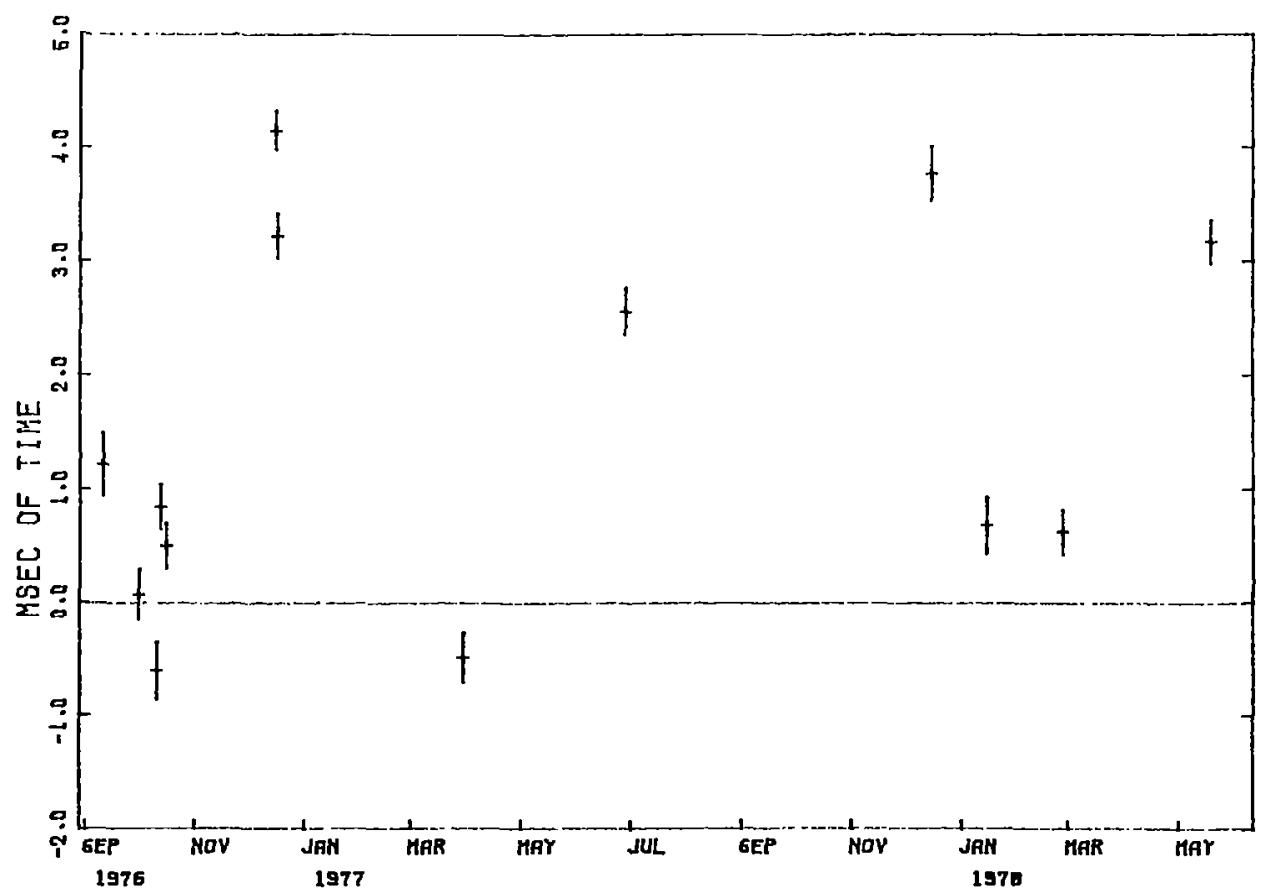

Figure 9 . 


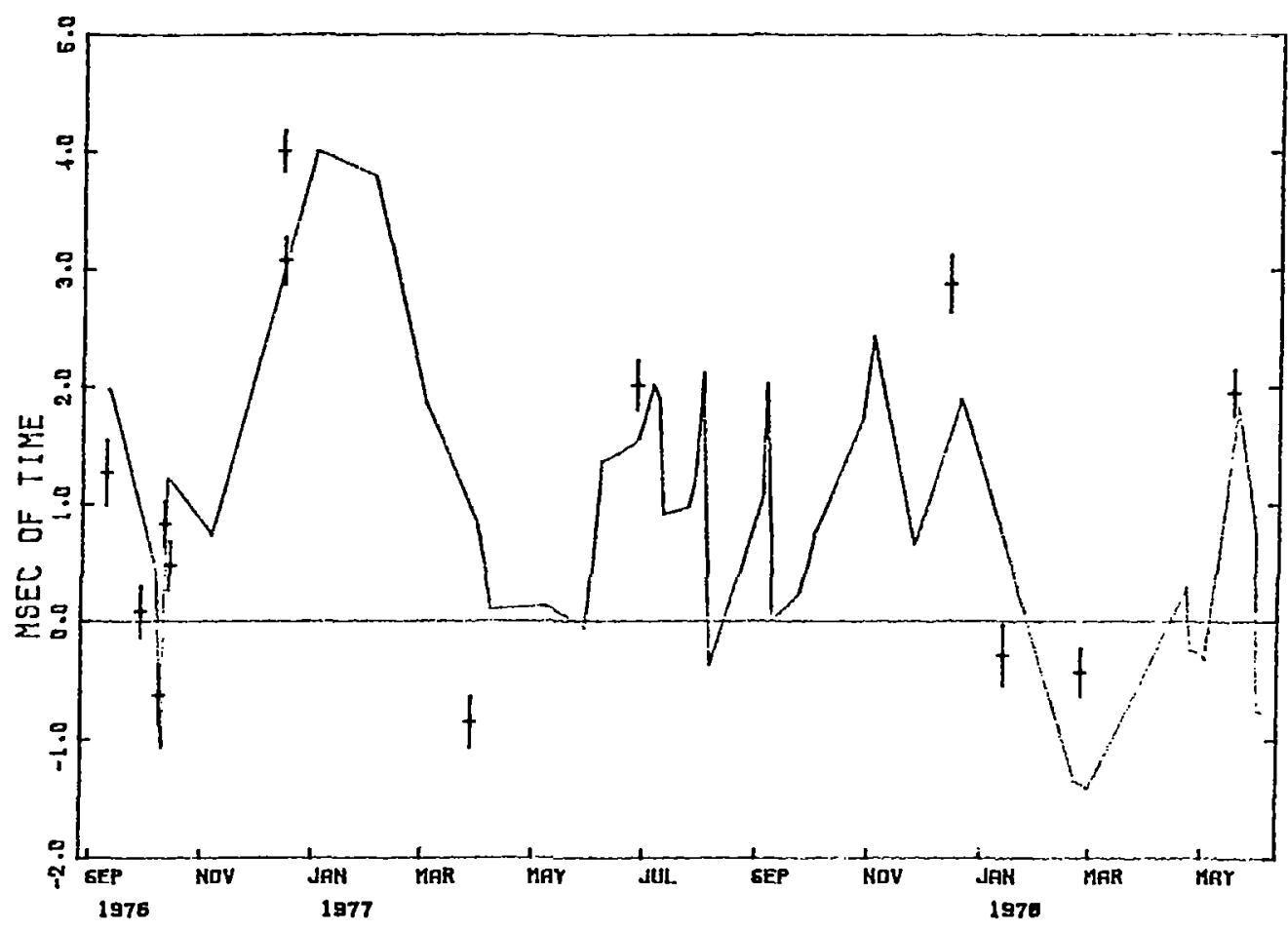

Figure 10.

The RMS differences between the lunar laser and the BIH values and between the VLBI and the $\mathrm{BIH}$ values are both $1.6 \mathrm{msec}$, but the difference between the VLBI and the lunar laser values is only $0.8 \mathrm{msec}$.

Although the general agreement between the VLBI and the lunar laser values is good, more regular monitoring of Earth rotation with VLBI is necessary to establish the significance of any small $(>2 \mathrm{msec})$ differences between the BIH values and those from VLBI and lunar laser ranging.

The BIH has also published an annual and a semi-annual correction term for its UT1 values. The form of this correction is shown in figure 11. With this correction included, the RMS differences between the BIH and the VLBI and lunar laser results are $1.3 \mathrm{msec}$ and $1.1 \mathrm{msec}$, respectively, still larger than the corresponding difference between the VLBI and the lunar laser ranging estimates of UT1. 


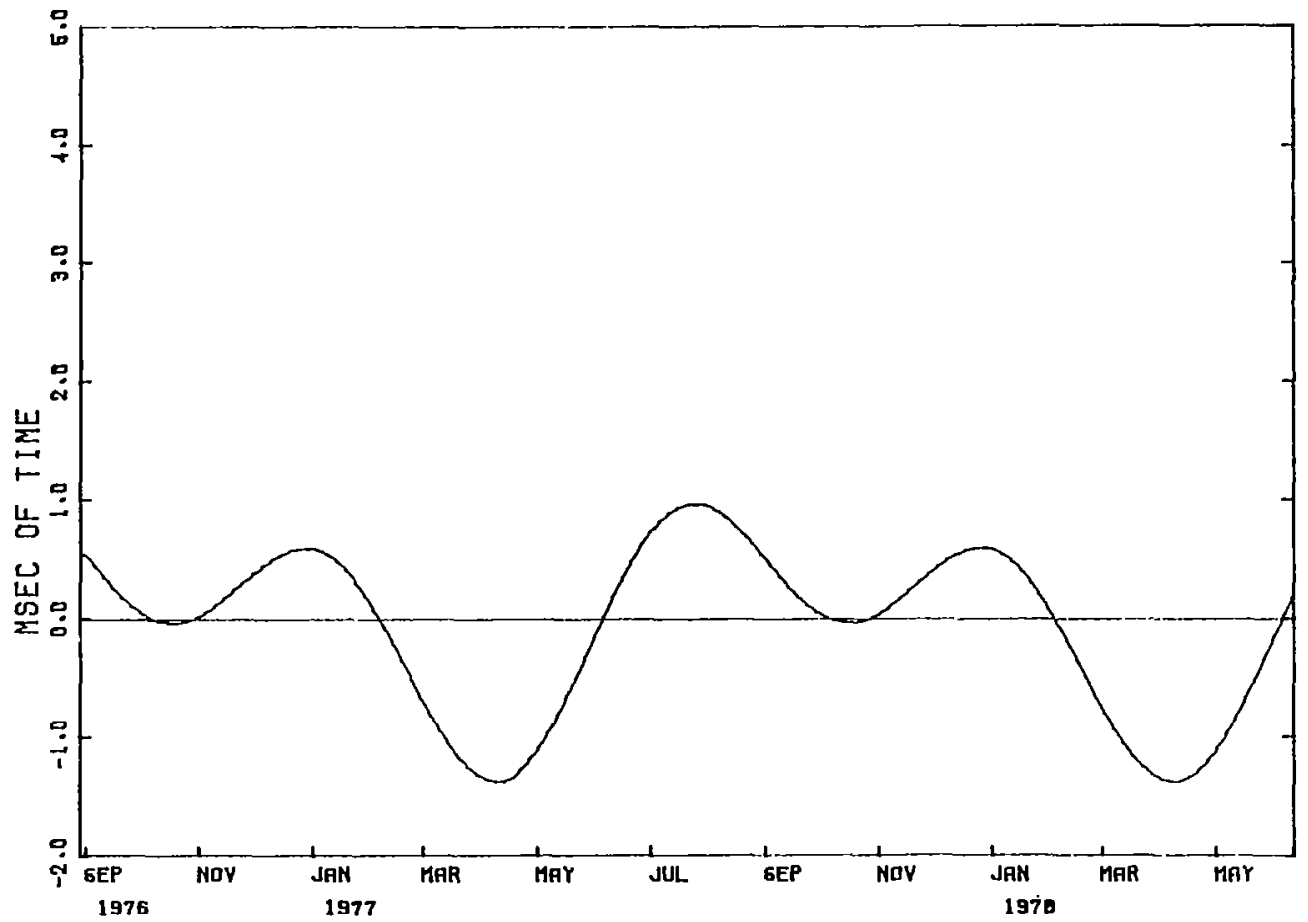

Figure 11.

\section{CONCLUSIONS}

The determination of the position of the Earth's pole from satellite Doppler and satellite laser ranging observations are approaching an accuracy of $\sim 10 \mathrm{msec}$ of arc $(30 \mathrm{~cm})$. The VLBI determinations may have better accuracy, although a determination of whether the VLBI accuracy is indeed better, and by how much, must await the collection and analysis of a far larger set of VLBI data.

The comparison of lunar laser ranging and VLBI determinations of UT1 suggests that the RMS of the uncertainties in each set are under $1 \mathrm{msec}$.

All of these comparisons have been hampered by the paucity of VLBI data. To rectify this problem, the National Geodetic Survey has undertaken Project POLARIS whose object is to obtain VLBI data at least several times per week, starting in 1982. 


\section{REFERENCE}

King, R. W., C. C. Counselman, and I. I. Shapiro, Universal Time: Results from Lunar Laser Ranging, J. Geophys. Res., 83, 3377-3381, 1978. 


\title{
A REVIEW OF CONNECTED ELEMENT RADIO INTERFEROMETRY DIRECTED AT ESTABLISHING AN ALMOST INERTIAL REFERENCE FRAME
}

\author{
K. J. Johnston \\ E. O. Hulburt Center for Space Research \\ Naval Research Laboratory
}

\begin{abstract}
The present status of connected element radio interferometry towards establishing an accurate grid of positions of extragalactic radio sources is reviewed. Many of the problems being encountered are, in general, also faced by very long baseline interferometry (VLBI).
\end{abstract}




\section{INTRODUCTION}

One of the prime objectives of radio interferometry as applied to geophysics is establishing a grid of extragalactic radio sources against which to measure motions of the earth. The stability of this grid is of prime importance as this directly affects the accuracy of the geodetic measurements. Using radio interferometric techniques, the positions of these objects may be measured quite precisely. In this paper, the use of connected element interferometry will be emphasized. The very long baseline (VLBI) techniques are the subject of many other papers at this meeting and therefore will be mentioned only as they are relevant to establishing a reference frame of radio sources.

\section{Connected Element Interferometry}

In connected element interferometry, a common local oscillator is fed to all the antennas comprising the interferometer. This naturally limits the spacing of the antennas to several tens of kilometers. Antennas are linked together by cable as in the Cambridge interferometer, by radio link as in the Green Bank interferometer, or via waveguide as in the very large array (VLA) in Socorro, New Mexico. At present, experiments are being carried out to link antennas separated by thousands of kilometers via satellites. Since the antennas have a common local oscillator, the interferometer phase and amplitude are the prime observables. This differs from VLB, in which the prime observables are delay or fringe rate and amplitude. As you probably know, the delay and fringe rate are simply the frequency and time derivatives, respectively, of phase. This is the reason connected element interfcrometry has been able in the past to achieve similar accuracies in the measurement of the positions of celestial sources as VLB despite the shorter baselines used. We shall hear several talks at this meeting concerning the use of phase by VLB observers.

Highly accurate astrometric work in connectcd clement interferometry was done by Elsmore and Ryle (1976) at Cambridge using an east-west interferometer of length $5 \mathrm{~km}$ at a frequency of $5 \mathrm{GHz}$. They achieved an accuracy of $\sim 0 . " 03$ for a source at declination $45^{\circ}$. This celestial position accuracy roughly corresponds to measuring the baseline length to an accuracy of $0.3 \mathrm{~mm}$. This was followed by the work of Wade and Johnston (1977) who used the Green Bank interferometer with a baseline of $35 \mathrm{~km}$ at a frequency of $2.7 \mathrm{GHz}$ to measure the positions of celestial sources to $0 . " 03$ at a declination of $40^{\circ}$. Since this instrument has a baseline which is along an azimuth of $\sim 205^{\circ}$, it has a considerable north-south component. This allowed the positions of sources south of the equator to be measured with high accuracy. Here again, the $35-\mathrm{km}$ baseline must be measured to an accuracy of a millimeter.

The chief cause of inaccuracy in these catalogs is believed to be caused by inhomogeneities in the water vapor content of the troposphere which give rise to irregularities in the instrumental phase. The time scale of these irregularities is a few minutes to many hours and of amplitude, a few millimeters in differential phase path between the antennas (Hinder 1970; Johnston 1979a). Repeated observations are used to smooth out these irregularities. Elsmore and Ryle (1976) calibrate their interferometer and then observe a single source, horizon to horizon. Wade and Johnston (1977) 
observe all sources $(\sim 25)$ every day at $\sim 5$ hour angles distributed over the sky. In this way, they calibrate their instrument as they observe.

\section{Radio Source Catalogs}

The quoted catalog errors versus declination are shown as a function of declination in figure 1. Here, one can easily see the accuracy of source position in declination decrease as one approaches the equator. Also shown in this figure are the VLB catalogs of Clark et al. (1976) and Fanslow (1978). One can easily see a trend towards increasing accuracy as a function of time.

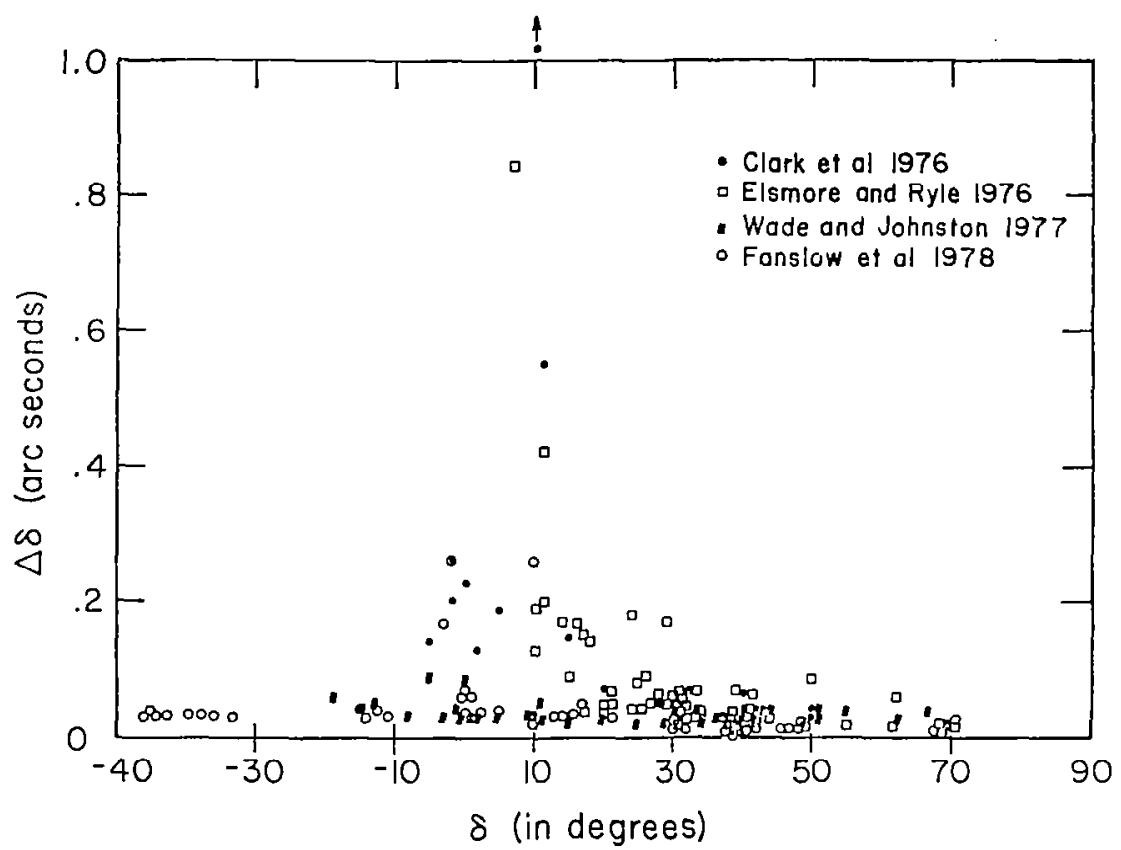

Figure 1. Error in source declination as a function of declination. Note the errors in the earlier observations increase substantially near the equator.

Comparison of these catalogs was also encouraging. Table 1 shows the weighted mean differences for sources common to various catalogs for comparison. The zero point in right ascension is not significant since each catalog uses a different zero point. Clark et al. (1976), Wade and Johnston (1977), and Spencer et al. (1979) use 3C273B; Elsmore and Ryle (1976) use $\beta$ Persei; and Fanslow (1978)* uses NRAO140.

*J. Fanslow, private communication, 1978. 
Table 1

Weighted Mean Differences

Wade and Johnston (1977) - Other Catalogs

\begin{tabular}{|l|c|c|c|}
\hline \multicolumn{1}{|c|}{ Catalog } & $\begin{array}{c}\text { Sources in } \\
\text { Common }\end{array}$ & $\Delta \alpha$ & $\Delta \delta$ \\
\hline Clark et al. (1976) & 17 & $-0.0001 \pm 0.0006$ & $0.002 \pm 0.009$ \\
Elsmore \& Ryle (1976) & 17 & $-0.0060 \pm 0.0015$ & $0.049 \pm 0.016$ \\
Fanslow (1978) & 10 & $-0.0032 \pm 0.0026$ & $0.012 \pm 0.017$ \\
This paper & 10 & $0.0002 \pm 0.0020$ & $0.000 \pm 0.016$ \\
\hline
\end{tabular}

\section{Sources of Disagreement}

There are discrepancies in position in these catalogs which exceed the quoted errors. These discrepancies are due to source structure, baseline geometry, and the use of different astronomical constants employed in the data reduction process. The last difficulty can be easily removed if fulure catalogs are made using the adopted astronomical constants and stating this explicitly in the catalog.

The beams of the connected element interferometer are quite large by VLB standards; i.e., $\sim 3$ " for the Cambridge telescope and 0."6 for the Green Bank telescope. This can and will cause problems. The maps of 3C345 made by Readhead et al. (1978), displayed in figure 2, show how source structure changes versus frequency. Since these are VLB maps in which the same antennas are usually employed at all frequencies, the resolution varies with frequency. We are faced with the problem that at different frequencies the source structure may appear quite different. The extended structure ranges in position angle from $-76^{\circ}$ to $-57^{\circ}$ going from highest to lowest resolution $(\sim 1$ to 10 milliarcseconds). A VLA map (Perley and Johnston, 1979) of 3C345 is displayed in figure 3 along with the spectra of the two major components. There is a resolved extended component at a position angle of $-31^{\circ}$ apparent with a resolution of 1 arcsecond.

Radio catalogs have been made with a large number of beamwidths and at frequencies varying from $2-8 \mathrm{GHz}$. The sensitivity to structure is of the order of a synthesized beamwidth. This can be seen to be a problem for the larger beamwidth, low frequency operation of connected element interferometry.

All of the catalogs published at present are from observations made at a single frequency, and some effectively made over a single baseline such as the catalog of Wade and Johnston (1977). There was an increase in accuracy in the published work about 1976, for which most of the data was obtained 


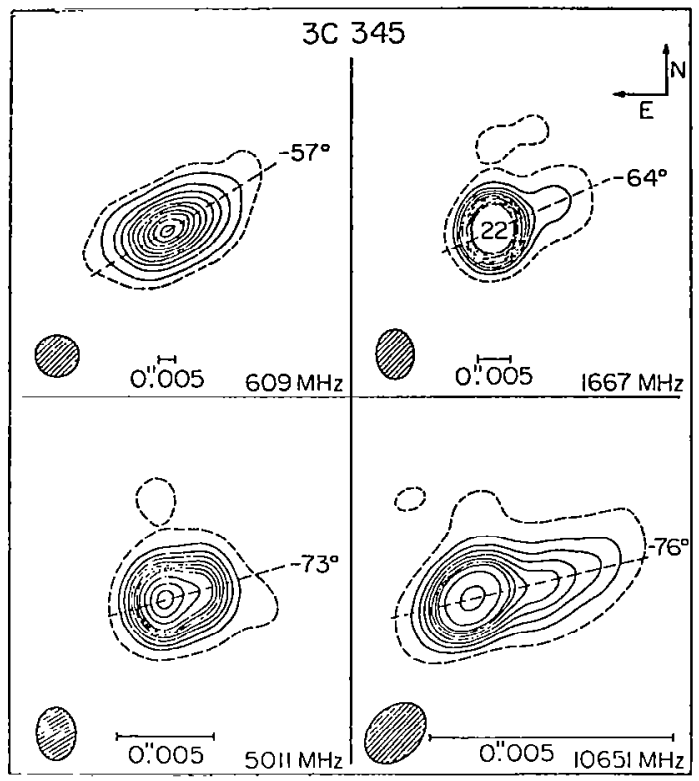

Figure 2. Hybrid radio maps of $3 \mathrm{C} 345$ at 609 , 1667, 5011, and $10651 \mathrm{MHz}$. Note the systematic rotation of the source structure at the different frequencies. The dashed ellipses show the size (FWHM) of the restoring beams (after Readhead et al., 1978).

at solar minimum. Now that solar maximum is here (October 1979), the effects of the ionosphere will be $\sim 100$ times greater. For example, in the 1974 to 1976 data of Wade and Johnston (1977), who used a $35-\mathrm{km}$ baseline, there was no need for a differential phase pathlength correction because this effect did not exceed $0.1 \mathrm{~cm}$ at a wavelength of $11.1 \mathrm{~cm}$. However, in the present operation of the Green Bank interferometer by the United States Naval Observatory, ionospheric effects have been observed to contribute a differential phase pathlength of over $10 \mathrm{~cm}$. Therefore, at times of high solar activity, dual frequency observations are necessary for accurate astrometric work (i.e., 0.01 ) especially at low radio frequencies and intermediate range baselines $(\sim 100 \mathrm{~km})$. One other aspect concerns ionospheric effects on observations over a single baseline. The effects of the ionosphere cannot be removed from the data without some a priori knowledge of the radio source structure. Observations over several baselines at two frequencies are necessary to separate these effects since the effects of source structure will be displayed in the lack of phase closure among three or more baselines. 


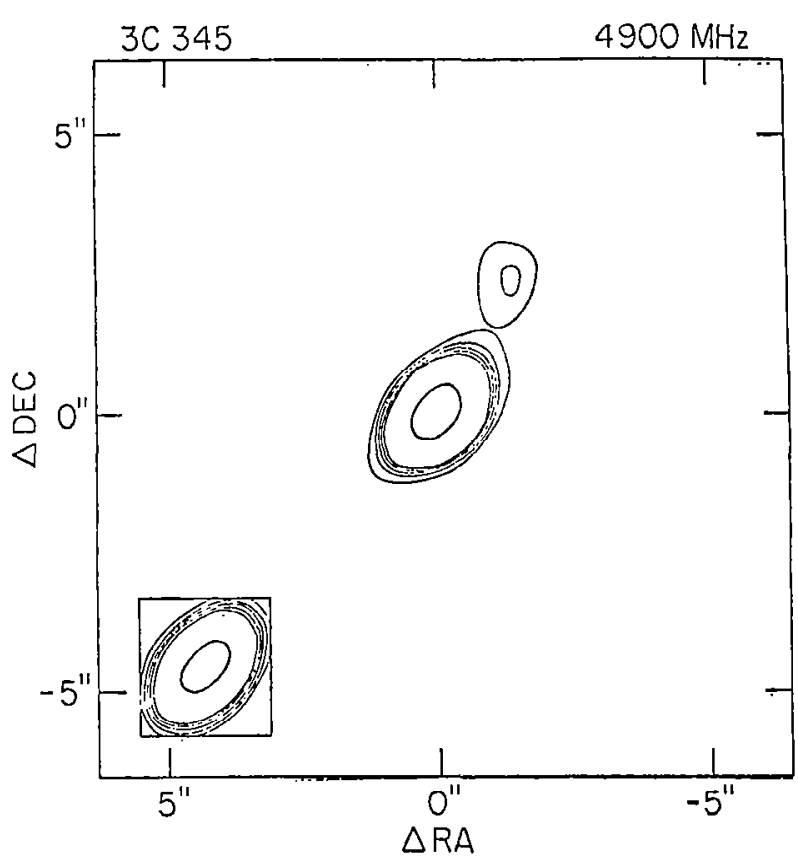

(a)

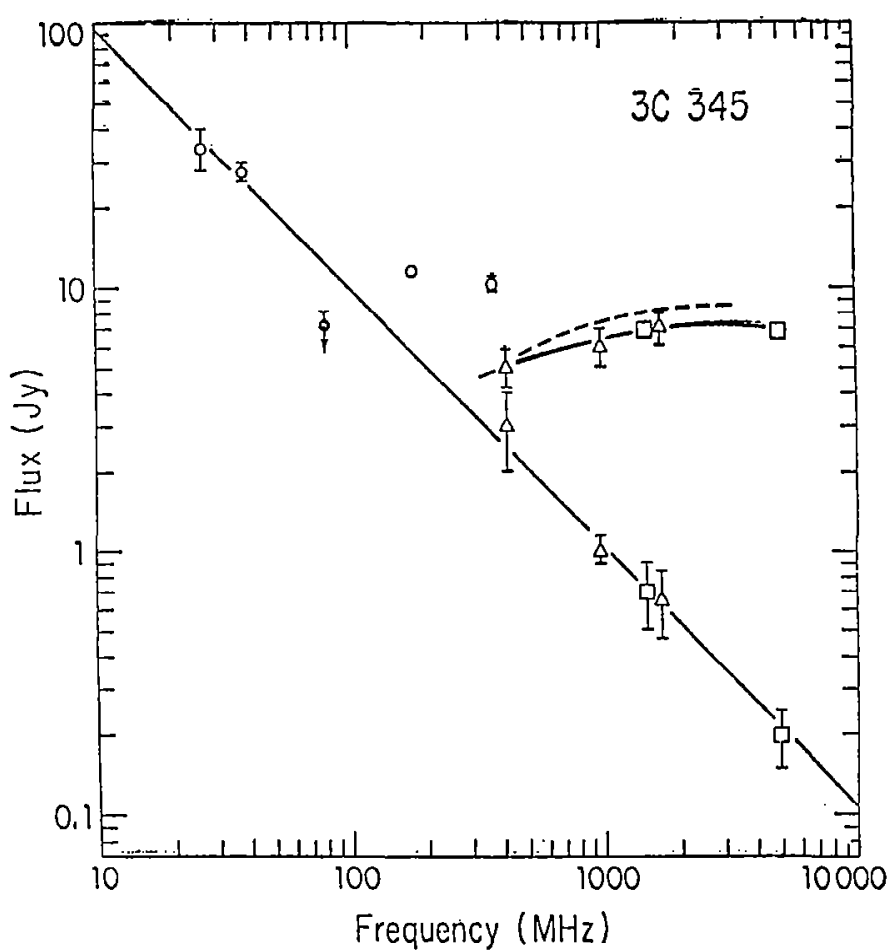

(b)

Figure 3. (a) Radio maps of $3 \mathrm{C} 345$ at $4990 \mathrm{MHz}$ made with the VLA. The contour intervals are $1 \%$ of the peak brightness to the $5 \%$ level, with the $50 \%$ contour added to show the half-power beamwidth. The clean beam is displayed in the lower left corner (after Perley and Johnston, 1979). (b) The radio spectrum of 3C345 (after Perley and Johnston, 1979). The spectrum of the compact "astrometric" source $(--)$ is flat while that of the extended component $(-)$ is very steep. 


\section{A Source Catalog}

With these problems in mind, the ideal radio source for astrometry is one with little or no structure in its radiation. To establish a reference frame, a large number of sources are needed. Optical catalogs contain over a thousand stars; i.e., the FK4 contains 1535 fundamental stars. Johnston (1979b) estimates from the number of radio sources with flat spectra found in radio surveys that there will be approximately 1500 sources of intensity $\geq 0.6 \mathrm{Jy}$ distributed over the celestial sphere containing compact components. Many of these sources may contain complex structure. Optical astronomers would like to identify compact radio sources which have optical counterparts in order to use the superior accuracy of the radio source position to reduce the zonal systematic errors in the optical catalogs.

With these views in mind, a working group for the Identification of Radio/Optical Sources was established at IAU Colloquium No. 48. This report was presented at the IAU General Assembly in August 1979. Since this report has a very limited distribution among the geophysical community, it has been added as Appendix A to this paper. It contains a primary list of sources having positions exceeding $0 . " 1$ in accuracy. The secondary list contains sources that are not useful because of radio structure or they have a poor position. This list is a first attempt at establishing, through a coordinated effort, an extragalactic reference frame from QSO's and other radio sources. These lists should be viewed as changeable as more information becomes known about individual sources. Anyone suggesting changes in this source catalog should inform a member of this working group.

At present, the accuracy of radio source catalogs from papers presented at this meeting is approaching 0.01 . For improved accuracy beyond this figure, the structure of radio sources on the milliarcsecond scale must be taken into account. This structure is frequently complex as in 3C84 or may have a simple, two-component structure as in BL Lacetae. Since these sources radiate via the synchrotron mechanism, a lower limit of about a milliarcsecond on the angular size is set by energy losses due to inverse Compton scattering. Therefore, it is very important to identify those radio sources with the simplest structure. No mention has been made of the variability of this structure. This will also have to be evaluated, as the most compact sources are the most time variable.

\section{Conclusion}

The establishment of a reference frame based upon extragalactic radio sources is fast approaching. Connected element interferometry has not produced a new catalog of source positions since 1977. However in the near future, work presently being done on the VLA and the Green Bank interferometer should result in new catalogs. The VLBI work reported in this meeting by G. Purcell (JPL) and T. Clark (Goddard) shows that the accuracy in source position is approaching $0 . " 01$. The number of sources with precise position excceds one hundred. There only remains to refine this data, as well as is possible, to define a reference frame and make it available to those interested in geodetic applications. 


\section{REFERENCES}

Clark, T. A., Hutton, L. K., Marandino, G. E., Counselman, III, C. C., Robertson, D. S., Shapiro, I. I., Wittels, J. J., Hinteregger, H. F., Knight, C. A., Rogers, A. E. E., Whitney, A. R., Niel, A. E., Rönnäng, B. O., and Rydbeck, O. E. H., 1976, Astron. J., 81, p. 599.

Elsmore, B. and Ryle, M., 1976, M.N.R.A.S., 174, p. 111.

Hinder, R. A., 1970, Nature, 225, p. 614.

Johnston, K. J., 1979a, I.A.U. Symposium \#82, "Time and The Earth's Rotation," edited by D. D. McCarthy and J. D. Pilkington, published by D. Reidel, Dordrecht, Holland, p. 183.

Johnston, K. J., 1979b, I.A.U. Symposium \#48, “Modern Astrometry," edited by R. F. Prochazka and R. H. Tucker, published by University Observatory, Vienna, p. 171.

Perley, R. A. and Johnston, K. J., 1979, Astron. J., 84, p. 1247.

Readhead, A. C. S., Cohen, M. H., and Pearson, T. J., 1978, Nature, 276, p. 768.

Spencer, J. H., Waltman, E. B., Johnston, K. J., and Backer, D. C., NASA CP 2115, "Radio Interferometry-Techniques for Geodesy," 1980.

Wade, C. M. and Johnston, K. J., 1977, Astron. J., 82, p. 791. 


\section{APPENDIX A \\ Report of IAU Commission 24, Working Group on the Identification of Radio/Optical Astrometric Sources}

The primary objective of this working group is to select a preliminary list of suitable celestial sources that may be employed as a benchmark in establishing an inertial reference frame based upon extragalactic sources. Space astrometry programs in the next decade should have a precision capability in the milliarcsecond range; therefore the reference frame needs to be established to that accuracy. Advances in astrometry at radio frequencies make objects that radiate at these frequencies the best candidates at present for establishing this reference frame. Therefore, the working group has first attempted to identify suitable radio sources. In order to use this reference frame of extragalactic radio sources to improve already established optical and dynamical reference frames, as well as to determine the origin of the improved frame, suitable radio sources with optical counterparts are required.

Radio sources should be chosen that do not have significant large scale (greater than $1^{\prime \prime}$ ) structure in the radio range above $1.4 \mathrm{GHz}$. This criterion would eliminate almost all sources which have precise published positions. However the radio structure of a large number of sources is not yet known, so this criterion cannot now be strictly applied. Extensive additional observations are required to definitely establish these sources, and it is hoped that the list presented with this report can serve as a starting point in the search for these sources.

Not all sources listed have known optical counterparts, but these are listed in the hope that these counterparts may yet be identified. Ideally the optical magnitude of the sources should be brighter than 18 , and it should be constant to 0.5 magnitude. The object should be stellar-like, free of nebulosity, and have no nearby companion (less than $1 "$ ). The sky coverage should be as uniform as possible, although it is recognized that at present the lack of observatories in the southern hemisphere restricts the coverage at high negative declinations, and the zone of avoidance restricts primarily the right ascension coverage.

At present, because it is impossible to definitely identify the best sources, the list of candidate sources has been divided into a primary and a secondary list. The primary list contains those sources which at this time are known to be good sources that should be used in establishing the reference frame. The secondary sources are those for which there is inadequate knowledge, i.e. poor radio or optical position, uncertain identification, lack of knowledge of radio or optical structure, etc. In addition to these lists, there is a third list of galactic objects which display radio emissions and are bright optically. These objects include binary stars, infrared objects, masers, etc.

Unfortunately, the optical astrometry contains possible systematic errors, perhaps as large as 0.1 . For instance, among the sources on the USNO optical program that are on the above primary list and have more than one position published in the recent, high-precision radio and optical lists, a radio mean point has both an internal and an external error of about $0 . ' 05$, indicating no systematic radio errors at that level. However, whereas an optical mean point has an internal mean error 
also around $0 . " 05$, it has an external error of about 0.08 , indicating a formal systematic error of about $0 . " 06$. This could be due, for instance, to catalog errors in one or more of the catalogs to which the positions are ultimately referred, or it could be due to a magnitude error in one or more of the telescopes used in the cascading process, or it could be due to one or more other, unforeseen, causes. Before the optical reference frame can be adequately referred to the radioframe, at the precisions now possible, these systematic errors must be identified and eliminated.

A secondary objective of this working group, therefore, is to prepare a core list of objects that would be suitable for use in trying to solve the systematic error problem. The objects on this list must be from the above primary list, in order that we can be sure the radio positions are suitable standards. They must meet all of the above optical characteristics, particularly as to magnitude, in order that they will be bright enough to be observed on all participating programs. (NRAO 512 does not meet this criterion, but it is in the same field as 3C345 and therefore is of special interest.) The list must be short enough but have adequate coverage to permit all observatories to observe all objects a sufficient number of times. Therefore, we present a fourth list of objects which, for the most part, have a significant observational history, but which should continue to be observed routinely for calibration purposes. These objects are denoted by an * on the primary list.

The working group makes the following specific recommendations:

(1) The objects on the primary list should receive the maximum possible optical and radio attention, to insure the highest-quality positions and information on structure, which will therefore make these objects suitable as an inertial reference frame at the milliarcsecond level for space astrometry.

(2) That the publication of radio catalogs contain clear references to the radio frequency, epoch of observation, the equator and equinox and the values of the principal astronomical constants used in the reduction process.

(3) The objects on the secondary list should continue to be surveyed, both to improve knowledge of structure and to improve positional, and therefore identification, information. These objects may become of quality to be on the primary list.

(4) Additional objects should be observed for radio structure and optical counterparts as facilities permit, for possible later inclusion on either of the above lists.

(5) The core list should be observed as intensively as possible by all groups carrying out either optical or radio astrometry of these sources, to make possible a complete analysis of the systematic errors present before the space astrometry programs go into operation. 


\section{Members of the Working Group}

\begin{tabular}{|c|c|c|}
\hline \multirow{8}{*}{ Chairman: } & A. N. Argue & University of Cambridge \\
\hline & B. Elsmore & Cavandish Laboratory \\
\hline & R. S. Harrington & U.S. Naval Observatory \\
\hline & K. J. Johnston & Naval Research Laboratory \\
\hline & I. Kumkova & Pulkova Observatory \\
\hline & C. DeVegt & Hamburger Stern warte \\
\hline & H. Walter & Astronomisches Rechen-Institut \\
\hline & A. Witzel & Max-Planck Institut für Radioastronomie \\
\hline
\end{tabular}

The lists of primary and secondary sources are displayed in Table Ia and Ib. The columns are:
Column 1 Source Source designation in IAU coordinates. * denotes core list of objects

Column 2

$a_{(1950)}$

Column 3

$\delta_{(1950)}$

Column 4

$\mathrm{S}(5 \mathrm{GHz})$

Column 5

I.D.

Column 6

$\mathrm{m}_{\mathrm{v}}$

Column 7
Right ascension based upon epoch 1950.

Declination based upon 1950 epoch.

Radio flux at a frequency of $5 \mathrm{GHz}$ in Janskys. Thus flux for most sources is variable, and this flux should be taken as a very imprecise source flux for measurements at any particular epoch.

Source identification:

$$
\begin{aligned}
& \text { Q-quasar } \\
& \text { G-galaxy } \\
& \text { BSO-blue stellar object } \\
& \text { Lac-BL Lacertae object }
\end{aligned}
$$

Visual magnitude. Again since these sources are quite variable in their visual luminosity, this number should be taken as a very imprecise indication of the visual magnitude at any epoch.

Redshift. 
Table Ia

Primary Sources

\begin{tabular}{|c|c|c|c|c|c|c|}
\hline $\begin{array}{c}\text { (1) } \\
\text { Source }\end{array}$ & $\begin{array}{c}(2) \\
a_{(1950)}\end{array}$ & $\begin{array}{c}(3) \\
\delta_{(1950)}^{(3)}\end{array}$ & $\begin{array}{c}(4) \\
S \\
(5 \mathrm{GHz})\end{array}$ & $\begin{array}{l}\text { (5) } \\
\text { I.D. }\end{array}$ & $\begin{array}{l}(6) \\
m_{v}\end{array}$ & $\begin{array}{l}(7) \\
Z\end{array}$ \\
\hline $0016+731$ & $00^{\mathrm{h}} 16^{\mathrm{m}} 54^{\mathrm{s}} 14$ & $+73^{\circ} 10^{\prime} 51^{\prime \prime} 6$ & 1.72 & $\mathrm{Q}$ ? & 18.0 & \\
\hline $0106+013$ & $\begin{array}{llll}01 & 06 & 04.523\end{array}$ & $\begin{array}{lll}+01 & 19 & 01.06\end{array}$ & 3.7 & $Q$ & 18.4 & 2.107 \\
\hline $0133+476$ & $\begin{array}{lll}01 & 33 & 55.11\end{array}$ & $+47 \quad 36 \quad 13.0$ & 2.0 & $\mathrm{Q}$ ? & 19.0 & \\
\hline 0153 & $\begin{array}{lll}01 & 53 & 04.25\end{array}$ & $+74 \quad 2805.5$ & 1.09 & $\mathrm{Q} ?$ & 16.0 & \\
\hline $0212+735$ & $\begin{array}{lll}02 & 12 & 49.87\end{array}$ & +733540.2 & 2.24 & $\mathrm{Q} ?$ & 19.0 & \\
\hline $0237-027$ & $\begin{array}{lll}02 & 37 & 13.71\end{array}$ & $-0247 \quad 32.5$ & 0.88 & Q & 19.5 & \\
\hline 0237-233* & $0237 \quad 52.8$ & $\begin{array}{lll}-23 & 22 & 06.4\end{array}$ & 3.33 & Q & 16.6 & 2.224 \\
\hline $0300+471$ & $\begin{array}{llll}03 & 00 & 10.12\end{array}$ & $+47 \quad 0433.7$ & 2.20 & $Q$ & & \\
\hline $0316+413^{*}$ & $\begin{array}{lll}03 & 16 & 29.566\end{array}$ & $+41 \quad 1951.90$ & 50.0 & G & 12.7 & 0.018 \\
\hline $0331-403$ & $\begin{array}{lll}03 & 32 & 25.23\end{array}$ & $\begin{array}{lll}-40 & 18 & 23.4\end{array}$ & 1.47 & Q & 18.5 & 1.445 \\
\hline $0333+321^{*}$ & $\begin{array}{lll}03 & 33 & 22.406\end{array}$ & $+3208 \quad 36.65$ & 2.4 & Q & 17.0 & 1.263 \\
\hline 0336-019* & $03 \quad 36 \quad 58.954$ & $\begin{array}{lll}-01 & 56 & 16.86\end{array}$ & 2.2 & Q & 17.5 & 0.852 \\
\hline $0338-214$ & $\begin{array}{lll}03 & 38 & 23.27\end{array}$ & $\begin{array}{lll}-21 & 29 & 07.5\end{array}$ & 1.28 & G & 18.0 & 0.048 \\
\hline $0402-362$ & $\begin{array}{lll}04 & 02 & 02.59\end{array}$ & $\begin{array}{lll}-36 & 13 & 11.8\end{array}$ & 1.0 & Q & 16.0 & 1.417 \\
\hline $0420-015$ & $04 \quad 2043.55$ & $\begin{array}{lll}-01 & 27 & 28.7\end{array}$ & 2.0 & Q & 18.0 & 0.915 \\
\hline $0422-380$ & $04 \quad 22 \quad 56.16$ & $\begin{array}{lll}-38 & 03 & 09.0\end{array}$ & 1.08 & Q & 50.78 & \\
\hline $0438-436$ & 043843.18 & $-43 \cdot 38 \quad 53.5$ & 5.0 & Q & 19.8 & 2.852 \\
\hline $0454+844^{*}$ & $04 \quad 54 \quad 57.02$ & $+84 \quad 2753.1$ & 1.57 & $\mathrm{G} ?$ & 16.5 & \\
\hline $0518+165$ & $\begin{array}{llll}05 & 18 & 16.526\end{array}$ & +163526.85 & 4.0 & Q & 19.0 & \\
\hline $0537-441$ & $\begin{array}{lll}05 & 37 & 21.07\end{array}$ & $-44 \quad 06 \quad 45.0$ & 4.20 & Q & 15.5 & 0.894 \\
\hline $0552+398$ & $\begin{array}{lll}05 & 52 & 01.408\end{array}$ & $+39 \quad 48 \quad 21.93$ & 5.0 & Q & 18.0 & 2.365 \\
\hline $0615+820$ & $\begin{array}{lll}06 & 15 & 32.80\end{array}$ & $+8203 \quad 56.5$ & 0.99 & Q & 17.5 & \\
\hline $0636+680$ & $06 \quad 3647.64$ & $\begin{array}{lll}+68 & 01 & 27.2\end{array}$ & 0.52 & Q & 19.0 & \\
\hline $0642+449$ & $06 \quad 42 \quad 53.020$ & +445430.85 & 0.9 & Q & 18.0 & \\
\hline $0736+017$ & $0736 \quad 42.51$ & $+01 \quad 4400.1$ & 2.13 & Q & 18.0 & 0.191 \\
\hline $0738+313$ & $\begin{array}{lll}07 & 38 & 00.18\end{array}$ & $+31 \quad 1902.1$ & 2.0 & Q & 17.5 & 0.630 \\
\hline $0814+425$ & $\begin{array}{lll}08 & 14 & 51.672\end{array}$ & $+42 \quad 32 \quad 07.68$ & 1.7 & Q & 18.5 & \\
\hline $0828+494$ & $\begin{array}{lll}08 & 28 & 47.94\end{array}$ & $+49 \quad 23 \quad 33.4$ & 1.4 & Q & 18.5 & \\
\hline $0831+557^{*}$ & $\begin{array}{lll}08 & 31 & 04.379\end{array}$ & +554441.36 & 5.6 & G & 17.5 & \\
\hline $0839+187$ & $\begin{array}{lll}08 & 39 & 14.076\end{array}$ & +184627.20 & 1.4 & BSO & 16.5 & \\
\hline $0859+470$ & $\begin{array}{lll}08 & 59 & 39.99\end{array}$ & $+47 \quad 02 \quad 56.9$ & 1.9 & Q & 18.7 & \\
\hline $0923+392 *$ & $09 \quad 2355.318$ & +391523.57 & 7.4 & $\mathrm{Q}$ & 17.0 & 0.698 \\
\hline $0954+253$ & $09 \quad 53 \quad 59.742$ & +252933.55 & 1.6 & Q & 17.5 & 0.712 \\
\hline $0954+556$ & $\begin{array}{lll}09 & 54 & 14.34\end{array}$ & $+5537 \quad 16.6$ & 2.3 & $\mathrm{Q}$ & 17.5 & \\
\hline $0954+658$ & $\begin{array}{lll}09 & 54 & 27.86\end{array}$ & $+6548 \quad 15.5$ & 0.8 & Q & 18.7 & \\
\hline $0955+326$ & $09 \quad 55 \quad 25.403$ & +323823.05 & 1.0 & Q & 16.0 & \\
\hline
\end{tabular}


SURVEYS OF THE SEVENTIES

Table Ia (continued)

\begin{tabular}{|c|c|c|c|c|c|c|}
\hline $\begin{array}{c}\text { (1) } \\
\text { Source }\end{array}$ & $\begin{array}{c}(2) \\
a_{(1950)}^{(2)}\end{array}$ & $\begin{array}{c}(3) \\
\delta_{(1950)}^{(3)}\end{array}$ & $\begin{array}{c}(4) \\
\mathrm{S} \\
(5 \mathrm{GHz})\end{array}$ & $\begin{array}{l}\text { (5) } \\
\text { I.D. }\end{array}$ & $\begin{array}{l}(6) \\
m_{v}\end{array}$ & $\begin{array}{c}(7) \\
Z\end{array}$ \\
\hline $1030+415$ & $10^{\mathrm{h}} 30^{\mathrm{m}} 07^{\mathrm{s}} 79$ & $+41^{\circ} 31^{\prime} 34^{\prime \prime} 9$ & 0.9 & $\mathrm{Q} / \mathrm{Lac}$ & 18.2 & \\
\hline $1034-293$ & $1034 \quad 55.82$ & $\begin{array}{lll}-29 & 18 & 26.9\end{array}$ & 1.78 & $\mathrm{Q}$ & 18.0 & \\
\hline $1104-445$ & $\begin{array}{lll}11 & 04 & 50.37\end{array}$ & $\begin{array}{lll}-44 & 32 & 52.7\end{array}$ & 2.54 & $\mathrm{Q}$ & 18.0 & \\
\hline $1127-145^{*}$ & $\begin{array}{lll}11 & 27 & 35.68\end{array}$ & $-14 \quad 3254.8$ & 5.39 & $Q$ & 16.9 & 1.187 \\
\hline $1143-245$ & $1143 \quad 36.37$ & $\begin{array}{lll}-24 & 30 & 52.7\end{array}$ & 1.16 & $\mathrm{Q}$ & 18.5 & 1.95 \\
\hline $1148-001$ & $1148 \quad 10.13$ & $\begin{array}{lll}-00 & 07 & 13.2\end{array}$ & 2.5 & $\mathrm{Q}$ & 17.6 & 1.982 \\
\hline $1155+251$ & 115551.641 & +250659.86 & 0.9 & G & 17.5 & \\
\hline $1219+285$ & $\begin{array}{lll}12 & 19 & 01.102\end{array}$ & +283036.49 & 1.6 & Q & 14.5 & \\
\hline $1226+023 *$ & $12 \quad 2633.246$ & +021943.38 & 33.5 & $\mathrm{Q}$ & 13.0 & 0.158 \\
\hline $1252+119$ & $1252 \quad 07.70$ & $+11 \quad 5721.3$ & 0.97 & $\mathrm{Q}$ & 16.6 & 0.87 \\
\hline $1253-055^{*}$ & $1253 \quad 35.832$ & $-05 \quad 3108.01$ & 14.0 & $\mathrm{Q}$ & 16.8 & 0.536 \\
\hline $1328+254$ & $\begin{array}{lll}13 & 28 & 15.924\end{array}$ & +252437.58 & 3.3 & $\mathrm{Q}$ & 18.0 & 1.055 \\
\hline $1328+307$ & $\begin{array}{lll}13 & 28 & 49.660\end{array}$ & +304558.70 & 7.4 & $Q$ & 17.0 & 0.846 \\
\hline $1404+286^{*}$ & $1404 \quad 45.615$ & $+2841 \quad 29.23$ & 3.0 & G & 14.0 & 0.077 \\
\hline $1442+101$ & $14 \quad 42 \quad 50.476$ & $+10 \quad 11 \quad 11.89$ & 1.2 & $\mathrm{ST}$ & 18.5 & \\
\hline $1502+106$ & $\begin{array}{lll}15 & 02 & 00.159\end{array}$ & $+1041 \quad 17.71$ & 2.2 & Q & 19.4 & 1.833 \\
\hline $1510-089^{*}$ & $\begin{array}{lll}15 & 10 & 08.92\end{array}$ & $\begin{array}{lll}-08 & 54 & 48.0\end{array}$ & 1.2 & $\mathrm{Q}$ & 17.8 & 0.361 \\
\hline $1519-273$ & $\begin{array}{lll}15 & 19 & 37.23\end{array}$ & $\begin{array}{lll}-27 & 19 & 29.6\end{array}$ & 2.0 & $Q$ & 18.5 & \\
\hline $1555+001$ & $\begin{array}{lll}15 & 55 & 17.688\end{array}$ & $+00 \quad 0643.54$ & 2.1 & $\mathrm{Q}$ & 19.0 & \\
\hline $1611+343$ & $16 \quad 1147.912$ & $+34 \quad 2019.83$ & 2.3 & $\mathrm{Q}$ & 18.0 & 1.404 \\
\hline $1616+063$ & $1616 \quad 36.55$ & +062013.9 & 0.9 & $\mathrm{Q}$ & 19.0 & \\
\hline $1634+628$ & $\begin{array}{lll}16 & 34 & 01.057\end{array}$ & +625141.83 & 1.6 & Q & & \\
\hline $1638+398 *$ & 163848.173 & $+3952 \quad 30.09$ & 0.6 & $\mathrm{Q}$ & 17.0 & \\
\hline $1641+399 *$ & $1641 \quad 17.608$ & $+3954 \quad 10.82$ & 7.2 & $\mathrm{Q}$ & 16.3 & \\
\hline $1642+690$ & $1642 \quad 18.03$ & $\begin{array}{lll}+69 & 02 & 13.2\end{array}$ & 1.6 & $\mathrm{Lac}$ & 19.2 & \\
\hline $1656+571$ & $16 \quad 5626.48$ & $+57 \quad 1026.0$ & 0.6 & Q & 17.4 & \\
\hline $1705+456$ & $\begin{array}{lll}17 & 05 & 50.42\end{array}$ & $+45 \quad 40 \quad 01.9$ & 0.4 & Q & 17.4 & 0.646 \\
\hline $1726+455$ & $\begin{array}{lll}17 & 26 & 01.03\end{array}$ & $+45 \quad 33 \quad 05.4$ & 0.9 & $\mathrm{Q}$ & 18.5 & \\
\hline $1730-130$ & $\begin{array}{lll}17 & 30 & 13.536\end{array}$ & $\begin{array}{lll}-13 & 02 & 45.93\end{array}$ & 5.2 & $\mathrm{Q}$ & 18.5 & \\
\hline $1741-038$ & $17 \quad 4120.619$ & $\begin{array}{lll}-03 & 48 & 49.02\end{array}$ & 2.2 & Q & 18.5 & \\
\hline $1749+701$ & $\begin{array}{lll}17 & 49 & 03.38\end{array}$ & $+70 \quad 06 \quad 39.5$ & 2.1 & Q & 17.5 & \\
\hline $1807+698^{*}$ & $\begin{array}{lll}18 & 07 & 18.547\end{array}$ & +694857.07 & 1.9 & Lac & 14.2 & 0.051 \\
\hline $1921-293$ & 192142.18 & $\begin{array}{lll}-29 & 20 & 24.9\end{array}$ & 6.1 & $\mathrm{Q}$ ? & 17.5 & \\
\hline $1928+738 *$ & $\begin{array}{lll}19 & 28 & 49.34\end{array}$ & $+73 \quad 5144.7$ & 3.11 & $\mathrm{Q}$ & 15.5 & \\
\hline $1954+513$ & $19 \quad 54 \quad 22.44$ & +512346.6 & 1.3 & Q & 18.5 & 1.230 \\
\hline $2005+403$ & $\begin{array}{lll}20 & 05 & 59.560\end{array}$ & +402102.80 & 4.5 & $\mathrm{Q}$ & 19.5 & 1.736 \\
\hline 2008-159 & $\begin{array}{lll}20 & 08 & 25.90\end{array}$ & $-15 \quad 55 \quad 37.6$ & 1.0 & Q & 18.0 & \\
\hline
\end{tabular}


Table Ia (continued)

\begin{tabular}{|c|c|c|c|c|c|c|}
\hline $\begin{array}{c}\text { (1) } \\
\text { Source }\end{array}$ & $\begin{array}{c}(2) \\
a_{(1950)}\end{array}$ & $\delta_{(1950)}^{(3)}$ & $\begin{array}{c}(4) \\
\mathrm{S} \\
(5 \mathrm{GHz})\end{array}$ & $\begin{array}{l}(5) \\
\text { I.D. }\end{array}$ & $\begin{array}{l}(6) \\
m_{v}\end{array}$ & $\begin{array}{l}(7) \\
Z\end{array}$ \\
\hline $2134+004$ & $21^{\mathrm{h}} 34^{\mathrm{m}} 05^{\mathrm{s}} 19$ & $+00^{\circ} 28^{\prime} 25^{\prime \prime} 3$ & 10.6 & BSO & 18.0 & \\
\hline $2200+420 *$ & $\begin{array}{llll}22 & 00 & 39.363\end{array}$ & +420208.58 & 3.0 & Lac & 14.0 & 0.07 \\
\hline $2201+315$ & $\begin{array}{lll}22 & 01 & 01.432\end{array}$ & +313105.87 & 1.5 & Q & 14.5 & \\
\hline $2223-052$ & $22 \quad 23 \quad 11.08$ & -051217.8 & 3.6 & Q & 18.4 & 1.404 \\
\hline $2230+114 *$ & $\begin{array}{lll}22 & 30 & 07.810\end{array}$ & +112822.76 & 4.0 & Q & 17.3 & 1.037 \\
\hline $2251+158 *$ & $\begin{array}{lll}22 & 51 & 29.522\end{array}$ & +155254.31 & 9.0 & Q & 16.1 & 0.859 \\
\hline $2345-167$ & $\begin{array}{lll}23 & 45 & 29.691\end{array}$ & $\begin{array}{lll}-16 & 47 & 52.79\end{array}$ & 2.3 & Q & 15.0 & 0.6 \\
\hline $2352+495$ & $\begin{array}{lll}23 & 52 & 37.790\end{array}$ & +493326.76 & 1.8 & G & 19.0 & 0.237 \\
\hline
\end{tabular}

Table Ib

Secondary Sources

\begin{tabular}{|c|c|c|c|c|c|c|}
\hline $\begin{array}{c}\text { (1) } \\
\text { Source }\end{array}$ & $\begin{array}{c}(2) \\
a_{(1950)}\end{array}$ & $\delta_{(1950)}^{(3)}$ & $\begin{array}{c}(4) \\
S \\
(5 \mathrm{GHz})\end{array}$ & $\begin{array}{l}\text { (5) } \\
\text { I.D. }\end{array}$ & $\begin{array}{l}(6) \\
m_{v}\end{array}$ & $\begin{array}{c}(7) \\
Z\end{array}$ \\
\hline $0116-219$ & $01^{\mathrm{h}} 16^{\mathrm{m}} 32.48$ & $-21^{\circ} 57^{\prime} 17^{\prime \prime} 5$ & 0.49 & $\mathrm{Q} ?$ & 19.0 & \\
\hline $0134+329$ & 013449.826 & +325420.52 & 5.6 & $\mathrm{Q}$ & 16.5 & \\
\hline $0150-334$ & $01 \quad 50 \quad 56.95$ & $\begin{array}{lll}-33 & 25 & 09.6\end{array}$ & 0.74 & $\mathrm{Q}$ & 16.5 & 0.610 \\
\hline $0224+671$ & $02 \quad 2441.164$ & $+6707 \quad 39.70$ & 2.0 & $\mathrm{EF}$ & & \\
\hline $0229+132$ & $\begin{array}{lll}02 & 29 & 02.52\end{array}$ & +130940.4 & 2.0 & $\mathrm{Q}$ & 18.0 & 2.065 \\
\hline $0430+052$ & $0430 \quad 31.602$ & +05 $14 \quad 59.58$ & 6.0 & $\mathrm{~S}$ & 15.0 & 0.032 \\
\hline $0529+075$ & $\begin{array}{lll}05 & 29 & 56.39\end{array}$ & +07 3040.5 & 1.53 & $Q$ & 19.0 & \\
\hline $0607-157$ & $\begin{array}{lll}06 & 07 & 25.98\end{array}$ & $\begin{array}{lll}-15 & 42 & 03.4\end{array}$ & 2.35 & $\mathrm{Q}$ & 17.0 & \\
\hline $0735+178$ & $\begin{array}{lll}07 & 35 & 14.13\end{array}$ & +174909.3 & 2.0 & $\mathrm{Lac}$ & 16.0 & \\
\hline $0945+408$ & $0945 \quad 50.08$ & $+40 \quad 53 \quad 43.5$ & 1.6 & $\mathrm{Q}$ & 17.5 & 1.252 \\
\hline $0952+179$ & $09 \quad 52 \quad 11.795$ & $+1757 \quad 44.47$ & 0.9 & $\mathrm{Q}$ & 18.0 & \\
\hline $0959-443$ & 095958.76 & $-44 \quad 23 \quad 30.0$ & 0.44 & $\mathrm{Q}$ & 17.0 & 0.840 \\
\hline $1055+018$ & $1055 \quad 55.31$ & $+01 \quad 50 \quad 03.7$ & 2.79 & $\mathrm{Q}$ & 18.0 & 0.888 \\
\hline $1215+303$ & 121521.141 & $+30 \quad 23 \quad 39.93$ & 0.4 & $\mathrm{Q}$ & 14.5 & \\
\hline $1311+678$ & 131145.06 & +675142.2 & 0.92 & BF & & \\
\hline $1430-178$ & $1430 \quad 10.65$ & $\begin{array}{lll}-17 & 48 & 24.1\end{array}$ & 0.9 & $\mathrm{Q}$ & 19.0 & \\
\hline $1451-375$ & $\begin{array}{lll}14 & 51 & 18.28\end{array}$ & -373522.6 & 1.0 & $\mathrm{Q}$ & 17.0 & 0.314 \\
\hline $1514+197$ & $\begin{array}{lll}15 & 14 & 40.981\end{array}$ & $+1943 \quad 10.80$ & 0.5 & $G$ & 19.5 & \\
\hline $1517+204$ & $\begin{array}{lll}15 & 17 & 50.614\end{array}$ & $+20 \quad 2653.03$ & 0.8 & $\mathrm{G}$ & 19.5 & \\
\hline $1629+680$ & $16 \quad 2950.82$ & $\begin{array}{lll}+68 & 03 & 38.7\end{array}$ & 0.4 & $\mathrm{Q}$ & 18.7 & \\
\hline $1637+626$ & 163755.286 & $+6240 \quad 34.24$ & 1.4 & G & 22.5 & \\
\hline
\end{tabular}


Table Ib (continued)

\begin{tabular}{|c|c|c|c|c|c|c|}
\hline $\begin{array}{c}\text { (1) } \\
\text { Source }\end{array}$ & $\begin{array}{c}(2) \\
a_{(1950)}^{(2)}\end{array}$ & $\delta_{(1950)}^{(3)}$ & $\begin{array}{c}(4) \\
S \\
(5 \mathrm{GHz})\end{array}$ & $\begin{array}{l}(5) \\
\text { I.D. }\end{array}$ & $\begin{array}{l}(6) \\
m_{v}\end{array}$ & $\begin{array}{c}(7) \\
\mathrm{Z}\end{array}$ \\
\hline $1716+686$ & $17^{\mathrm{h}} 16^{\mathrm{m}} 27^{\mathrm{s}} 82$ & $+68^{\circ} 39^{\prime} 48^{\prime \prime} 3$ & 0.7 & $\mathrm{Q}$ & 18.5 & \\
\hline $1739+522$ & $\begin{array}{lll}17 & 39 & 29.00\end{array}$ & $\begin{array}{lll}+52 & 13 & 10.4\end{array}$ & 1.5 & $\mathrm{Q}$ & 18.5 & \\
\hline $1749+096$ & $\begin{array}{lll}17 & 49 & 10.39\end{array}$ & t093942.7 & 1.6 & Lac & 18.0 & \\
\hline $1821+107$ & 182141.65 & +104244.0 & 1.4 & $\mathrm{Q}$ & 16.0 & \\
\hline $1823+568$ & $18 \quad 23 \quad 14.99$ & $+5649 \quad 18.0$ & 1.5 & $\mathrm{Q}$ & 18.4 & \\
\hline $1849+670$ & $\begin{array}{llll}18 & 49 & 16.48\end{array}$ & $\begin{array}{lll}+67 & 02 & 07.8\end{array}$ & 0.5 & $\mathrm{Q}$ & 18.0 & \\
\hline $1901+319$ & $\begin{array}{lll}19 & 01 & 02.313\end{array}$ & +315513.81 & 1.8 & $\mathrm{Q}$ & 17.5 & \\
\hline $1933-400$ & $1933 \quad 51.11$ & $\begin{array}{llll}-40 & 04 & 47.1\end{array}$ & 1.0 & $Q$ & 19.0 & \\
\hline $2227-399$ & $\begin{array}{lll}22 & 27 & 44.99\end{array}$ & $\begin{array}{lll}-39 & 58 & 16.9\end{array}$ & 0.7 & $\mathrm{Q}$ & 18.0 & 0.323 \\
\hline $2254+074$ & $22 \quad 54 \quad 45.965$ & +072708.40 & 0.5 & BSO & 15 & \\
\hline $2331-240$ & $\begin{array}{lll}23 & 31 & 17.85\end{array}$ & $\begin{array}{lll}-24 & 00 & 13.3\end{array}$ & 1.0 & $G$ & 16.5 & 0.048 \\
\hline
\end{tabular}

The list of galactic objects is displayed in Table II. The columns are:

Column 1 Source Source name - usually variable star designation.

Column $2 \quad a_{(1950)} \quad$ Right ascension at epoch 1950 in hours, minutes, and seconds.

Column $3 \quad \delta_{(1950)} \quad$ Declination at epoch 1950 in degrees, minutes, and seconds.

Column $4 \quad \mathrm{~S}(5 \mathrm{GHz}) \quad$ Flux at a radio frequency of $5 \mathrm{GHz}$ in Janskys. The fluxes reported in the literature for most of these stars is usually the maximum radio flux emitted by this object. Note again most of these stars are extremely variable in their radio flux.

Column 5 I.D.

Source identification:

$$
\begin{aligned}
& 1=\text { Flare star } \\
& 2=\text { Normal single star } \\
& 3=\text { Double or multiple stars } \\
& 5=\text { X-ray stars } \\
& 6=\text { peculiar stars (dwarfs, magnetic stars) } \\
& 7=\text { shell stars or other emission line stars }
\end{aligned}
$$

Column $6 \quad \mathrm{~m}_{\mathrm{v}} \quad$ Visual magnitude

Column $7 \quad d_{p c} \quad$ Distance in parsecs

Column $8 \quad$ Comments 
Table II

Galactic Objects

\begin{tabular}{|c|c|c|c|c|c|c|c|}
\hline $\begin{array}{c}\text { (1) } \\
\text { Source }\end{array}$ & $\begin{array}{c}(2) \\
a_{(1950)}^{(2)}\end{array}$ & $\delta_{(1950)}^{(3)}$ & $\begin{array}{c}(4) \\
S \\
(5 \mathrm{GHz})\end{array}$ & $\begin{array}{l}\text { (5) } \\
\text { I.D. }\end{array}$ & $\begin{array}{l}(6) \\
m_{v}\end{array}$ & $\begin{array}{l}\text { (7) } \\
d \\
p c\end{array}$ & $\begin{array}{c}(8) \\
\text { Comments }\end{array}$ \\
\hline UU Psc & $00^{\mathrm{h}} 12^{\mathrm{m}} 24.12$ & $08^{\circ} 32^{\prime} 36^{\prime \prime} 6$ & 0.01 & 3 & 5.9 & 71 & \\
\hline UV Psc & $\begin{array}{lll}01 & 14 & 18.59\end{array}$ & $06 \quad 3253.6$ & 0.0 & 3 & 9.1 & 125 & \\
\hline HR 407 & $01 \quad 2251.41$ & $\begin{array}{lll}23 & 15 & 07.6\end{array}$ & 0.005 & 3 & 6.0 & 90 & \\
\hline $\mathrm{Ls}+61^{\circ} 303$ & 023640.60 & $\begin{array}{lll}61 & 00 & 54.0\end{array}$ & & 5 & $11.4 *$ & $>1,000$ & \\
\hline$\beta$ Per & $\begin{array}{lll}03 & 04 & 54.36\end{array}$ & $40 \quad 45 \quad 52.5$ & $1.0-0.01$ & 3 & 2.2 & 25 & FK4-1 11 \\
\hline CC Cas & $\begin{array}{lll}03 & 10 & 07.40\end{array}$ & $\begin{array}{lll}59 & 22 & 38.5\end{array}$ & 0.01 & 3 & 7.1 & 1,000 & \\
\hline UX Ari & $\begin{array}{lll}03 & 23 & 33.15\end{array}$ & $\begin{array}{lll}28 & 32 & 31.7\end{array}$ & $0.10-0.01$ & 3 & 6.5 & 50 & \\
\hline HR 1099 & $\begin{array}{lll}03 & 34 & 13.13\end{array}$ & $\begin{array}{lll}00 & 25 & 31.0\end{array}$ & $1.0-0.05$ & 3 & 5.9 & 35 & \\
\hline T53B & $\begin{array}{lll}03 & 46 & 15.00\end{array}$ & $24 \quad 08 \quad 20$ & & 1 & 15.5 & 126 & \\
\hline $\mathrm{DM}+09^{\circ} 549$ & $\begin{array}{lll}04 & 10 & 50.08\end{array}$ & $\begin{array}{lll}10 & 05 & 11.9\end{array}$ & & 2 & 6.2 & 126 & \\
\hline b Per & $\begin{array}{llll}04 & 14 & 28.44\end{array}$ & $\begin{array}{lll}50 & 10 & 28.6\end{array}$ & 0.01 & 3 & 4.6 & 56 & \\
\hline $\mathrm{DM}+18^{\circ} 734$ & $\begin{array}{lll}04 & 45 & 46.28\end{array}$ & $\begin{array}{llll}18 & 37 & 40.3\end{array}$ & $<0.0005$ & 3 & 6.8 & 83 & \\
\hline V371 Ori & $\begin{array}{lll}05 & 31 & 10.00\end{array}$ & $\begin{array}{lll}01 & 54 & 53.0\end{array}$ & & 1 & 11.7 & 15 & \\
\hline$a$ Ori & $\begin{array}{lll}05 & 52 & 27.78\end{array}$ & $\begin{array}{lll}07 & 23 & 57.7\end{array}$ & 0.005 & 2 & 1.9 & 200 & FK4-224 \\
\hline$\pi$ Aur & $\begin{array}{lll}05 & 56 & 13.36\end{array}$ & $\begin{array}{lll}45 & 56 & 04.2\end{array}$ & 0.01 & 7 & 4.5 & 167 & \\
\hline$\sigma \mathrm{Gem}$ & $\begin{array}{lll}07 & 40 & 11.39\end{array}$ & $\begin{array}{lll}29 & 00 & 22.3\end{array}$ & 0.01 & 3 & 4.3 & 59 & \\
\hline Ross 882 & $\begin{array}{lll}07 & 42 & 04.00\end{array}$ & $\begin{array}{lll}03 & 40 & 48.0\end{array}$ & 0.05 & 1 & 11.8 & 5 & \\
\hline $54 \mathrm{Cam}$ & $\begin{array}{lll}07 & 58 & 32.15\end{array}$ & $\begin{array}{lll}57 & 24 & 51.2\end{array}$ & 0.01 & 3 & 6.4 & 38 & \\
\hline$\zeta$ Pup & $\begin{array}{lll}08 & 01 & 49.55\end{array}$ & $\begin{array}{lll}-39 & 51 & 41.0\end{array}$ & & 2 & 2.3 & 450 & \\
\hline$\gamma$ Vel & $\begin{array}{lll}08 & 07 & 59.45\end{array}$ & $\begin{array}{llll}-47 & 11 & 18.3\end{array}$ & 0.036 & 3 & 1.8 & 350 & \\
\hline RU Cnc & 083433.73 & $\begin{array}{llll}23 & 44 & 12.8\end{array}$ & 0.006 & 3 & 11.6 & 190 & \\
\hline TY Рyx & $08 \quad 57 \quad 34.04$ & $\begin{array}{lll}-27 & 37 & 10.5\end{array}$ & & 3 & 7.5 & 55 & \\
\hline$\kappa$ Dra & $\begin{array}{llll}12 & 31 & 21.43\end{array}$ & $\begin{array}{lll}70 & 03 & 48.7\end{array}$ & 0.02 & 6 & 3.8 & 100 & FK4-472 \\
\hline HR 5110 & $13^{\mathrm{h}} 32^{\mathrm{m}} 33^{\mathrm{s}} .92$ & $37^{\circ} 26^{\prime} 16^{\prime \prime} 6$ & & 3 & 5.0 & 46 & FK4-502 \\
\hline ZZ Boo & $\begin{array}{lll}13 & 53 & 51.82\end{array}$ & $\begin{array}{lll}26 & 09 & 46.1\end{array}$ & 0.009 & 3 & 6.8 & 116 & \\
\hline Cir X-1 & $\begin{array}{lll}15 & 16 & 48.30\end{array}$ & $\begin{array}{lll}-56 & 59 & 14.0\end{array}$ & & 5 & $\sim 16$ & $>10,000$ & \\
\hline$\sigma$ CrB-A & $\begin{array}{lll}16 & 12 & 48.27\end{array}$ & $\begin{array}{lll}33 & 59 & 02.2\end{array}$ & 0.016 & 3 & 5.8 & 23 & \\
\hline Sco X-1 & $\begin{array}{lll}16 & 17 & 04.47\end{array}$ & $\begin{array}{lll}-15 & 31 & 15.8\end{array}$ & 0.033 & 5 & 12.5 & 500 & \\
\hline$a \mathrm{Sco}$ & $\begin{array}{lll}16 & 26 & 20.21\end{array}$ & $\begin{array}{lll}-26 & 19 & 22.0\end{array}$ & 0.01 & 3 & 1.1 & 130 & FK4-616 \\
\hline Ry Sct & $\begin{array}{lll}18 & 22 & 42.71\end{array}$ & $\begin{array}{lll}-12 & 43 & 09.2\end{array}$ & 0.1 & 3 & 9.7 & 2,100 & \\
\hline$\beta \mathrm{Lyr}$ & $\begin{array}{lll}18 & 48 & 13.96\end{array}$ & $\begin{array}{lll}33 & 18 & 12.2\end{array}$ & 0.01 & 3 & 3.4 & 260 & FK4-705 \\
\hline SS 43319 & $\begin{array}{lll}19 & 09 & 21.32\end{array}$ & $\begin{array}{lll}04 & 53 & 52.9\end{array}$ & 0.5 & 1 & 14.2 & 3,500 & \\
\hline Cyg X-1 & $\begin{array}{llll}19 & 56 & 28.87\end{array}$ & $\begin{array}{lll}35 & 03 & 55.0\end{array}$ & 0.02 & 5 & 8.9 & 2,000 & \\
\hline HD 192163 & $\begin{array}{llll}20 & 10 & 17.30\end{array}$ & $\begin{array}{lll}38 & 12 & 14.9\end{array}$ & 0.003 & 7 & 7.2 & & \\
\hline P Cyg & $\begin{array}{llll}20 & 15 & 56.54\end{array}$ & $\begin{array}{lll}37 & 52 & 35.2\end{array}$ & & 7 & 4.7 & 1,800 & \\
\hline $\mathrm{DM}+43^{\circ} 3571$ & $20 \begin{array}{lll}20 & 18 & 46.71\end{array}$ & $\begin{array}{llll}43 & 41 & 42.9\end{array}$ & & 3 & 7.7 & 1,600 & \\
\hline
\end{tabular}


Table II (continued)

\begin{tabular}{|c|c|c|c|c|c|c|c|}
\hline $\begin{array}{c}(1) \\
\text { Source }\end{array}$ & $\begin{array}{c}(2) \\
a_{(1950)}^{(2)}\end{array}$ & $\begin{array}{c}(3) \\
\delta_{(1950)}\end{array}$ & $\begin{array}{c}(4) \\
\mathrm{S} \\
(5 \mathrm{GHz})\end{array}$ & $\begin{array}{l}\text { (5) } \\
\text { I.D. }\end{array}$ & $\begin{array}{l}(6) \\
m_{v}\end{array}$ & $\begin{array}{c}\text { (7) } \\
\mathrm{d} \\
\mathrm{pc}\end{array}$ & $\begin{array}{c}(8) \\
\text { Comments }\end{array}$ \\
\hline Cyg X-2 & $\begin{array}{lll}21 & 42 & 36.50\end{array}$ & $\begin{array}{llll}38 & 00 & 21.9\end{array}$ & 0.003 & 5 & 14.0 & & \\
\hline RT Lac & $\begin{array}{lll}21 & 59 & 28.63\end{array}$ & $\begin{array}{lll}43 & 38 & 56.0\end{array}$ & 0.02 & 3 & 8.8 & 200 & \\
\hline AR Lac & $\begin{array}{lll}22 & 06 & 39.41\end{array}$ & $\begin{array}{lll}45 & 29 & 46.2\end{array}$ & 0.01 & 3 & 6.1 & 50 & \\
\hline $\mathrm{DM}+54^{\circ} 2846$ & $\begin{array}{lll}22 & 42 & 05.68\end{array}$ & $\begin{array}{llll}55 & 19 & 35.1\end{array}$ & & 6 & 8.8 & & \\
\hline EV Lac & $\begin{array}{lll}22 & 44 & 39.98\end{array}$ & $\begin{array}{lll}44 & 04 & 35.5\end{array}$ & & 1 & 10.2 & 5 & \\
\hline HD 216489 & $\begin{array}{lll}22 & 50 & 34.48\end{array}$ & $\begin{array}{lll}16 & 34 & 31.9\end{array}$ & 0.033 & 3 & 5.7 & 112 & \\
\hline HR 8752 & $\begin{array}{lll}22 & 57 & 58.20\end{array}$ & $\begin{array}{llll}56 & 40 & 36.8\end{array}$ & 0.01 & 7 & 5.0 & 1,700 & \\
\hline SZ Psc & $\begin{array}{lll}23 & 10 & 50.54\end{array}$ & $\begin{array}{llll}02 & 24 & 08.9\end{array}$ & & 3 & 7.7 & 100 & \\
\hline$\lambda$ And & $\begin{array}{lll}23 & 35 & 06.61\end{array}$ & $\begin{array}{llll}46 & 11 & 10.9\end{array}$ & 0.065 & 3 & 3.9 & 23 & \\
\hline HD 224085 & $\begin{array}{lll}23 & 52 & 29.08\end{array}$ & $\begin{array}{llll}28 & 21 & 17.9\end{array}$ & 0.08 & 3 & 7.5 & 29 & \\
\hline
\end{tabular}


. 


\title{
PROGRESS REPORT ON THE USNO/NRL GREEN BANK INTERFEROMETER PROGRAM
}

\author{
W. J. Klepczynski, G. H. Kaplan, D. D. McCarthy, \\ F. J. Josties, and R. L. Branham \\ U.S. Naval Observatory \\ K. J. Johnston and J. S. Spencer \\ Naval Research Laboratory
}

\section{INTRODUCTION}

The connected-element radio interferometer of the National Radio Astronomy Observatory (NRAO) in Green Bank, West Virginia, is being used by the U.S. Naval Observatory (USNO) and the Naval Research Laboratory (NRL) in a joint program to apply radio interferometric techniques to the determination of improved source coordinates, astronomical constants, and variations in Earth rotation parameters. This is the first time that a radio interferometer has been applied to astrometric work on a full-time, continuous basis.

Radio interferometry was first used for the determination of Earth rotation parameters by Elsmore (1973) and by Shapiro, et al. (1974) with accuracies approaching $3 \mathrm{~ms}$. Observations by Wade and Johnston (1977) indicated that the Green Bank interferometer also could be used effectively in this pursuit. With the construction of the very large array (VLA) by NRAO, the Green Bank interferometer became available for continuous use for astrometric work in October 1978. It was decided to initiate a series of observations to evaluate connected-element radio interferometry for the above purposes.

The primary observables and operational aspects of this program are described elsewhere (Johnston, et al., 1978).

\section{The Green Bank Interferometer}

The Green Bank instrument has been described by Hogg, et al. (1969). This instrument consists of three 26-meter antennas which are located along an azimuth of $242^{\circ}$. In the present operational mode, the two movable antennas are separated by 1.5 and $2.4 \mathrm{~km}$ from the fixed antenna. A 14meter antenna is located approximately $35 \mathrm{~km}$ from the fixed antenna at an azimuth of $204^{\circ}$. The operating frequencies are 2695 and $8085 \mathrm{MHz}$ with an IF bandwidth of $30 \mathrm{MHz}$ and a total system temperature of about $125 \mathrm{~K}$. The IF signals from the remote antenna are transmitted to the Green Bank site via a phase-stable microwave link. 
In the initial phase of the program, two radio sources which pass close to the zenith at Green Bank were observed extensively over a large range of hour angles. These two sources (NRAO 150 and 3C345) were the primary sources for the determination of UTO until July 1979. Another 19 sources distributed over a declination range of $-30^{\circ}$ to $+70^{\circ}$ were used primarily to determine polar motion. These sources were observed at least five times daily at various hour angles, with each observation being at least 5 minutes long. From October 1978 through mid-March 1979, the observations were made only at $2695 \mathrm{MHz}$. After this time, all observations were made at both observing frequencies in order to eliminate the ionospheric contribution to the interferometer phase noise.

The data, in the form of 30-second averages of fringe amplitude and phase, are recorded on magnetic tape and sent to the USNO daily. They are reduced using a set of programs which apply a correction for differential path delay in the atmosphere that is based upon a standard model atmosphere and ground-level weather data, along with corrections for relativistic effects, and drifts in the local oscillator. The phases also are rotated to correspond to the apparent places of the sources observed, computed using the adopted IAU values for precession and the nutation series. No corrections were applied for ionospheric effects, differential solid Earth tides, source structure effects, or for errors in the nutation series.

\section{Source Positions}

The list of sources observed in the Universal Time/Polar Motion (UT/PM) program is derived from the catalog of Wade and Johnston (1977), with a few additional sources at high and low declinations. Updated positions were obtained for most of the sources from S-band $(2695 \mathrm{MHz})$ observations which were made during several weeks of excellent observing weather in October and November 1978. At this point, several sources which showed exceptionally large postfit residuals were eliminated from the observing schedule. (See table 1.)

Because of a degradation in the quality of the observations during the winter of 1978-79, several tests on equipment and of observing procedures were begun in February 1979. It was found that ionospheric effects contributed to the short-term $(\sim 0.5$ hour to $\sim 4$ hours $)$ phase drifts at S-band. Dual frequency observations were begun in mid-March 1979. A set of observations, obtained by using the S- and X-band observations to eliminate the effect of the ionosphere, produced interferometer phases with about half the intrinsic scatter of the pure S-band phases. This result implied, that, at the current solar maximum, the differential ionosphere over our $35-\mathrm{km}$ baseline is important at S-band at the $0.5-\mathrm{cm}$ level, with significant variations occurring over time spans as short as several minutes.

Therefore, it seems apparent that dual-frequency observations are necessary in order to achieve angular measurements with accuracies approaching the 0."01 level. However, the data reduction procedure for combining the $\mathrm{S}$ - and $\mathrm{X}$-band data is laborious because of the many lobe ambiguities in the X-band data which must be resolved. Because of current manpower constraints, less than a month of dual-frequency data has been fully reduced at this time, but this relatively small data set has permitted further position improvements for a number of sources. 
Table 1

Source Position Catalog

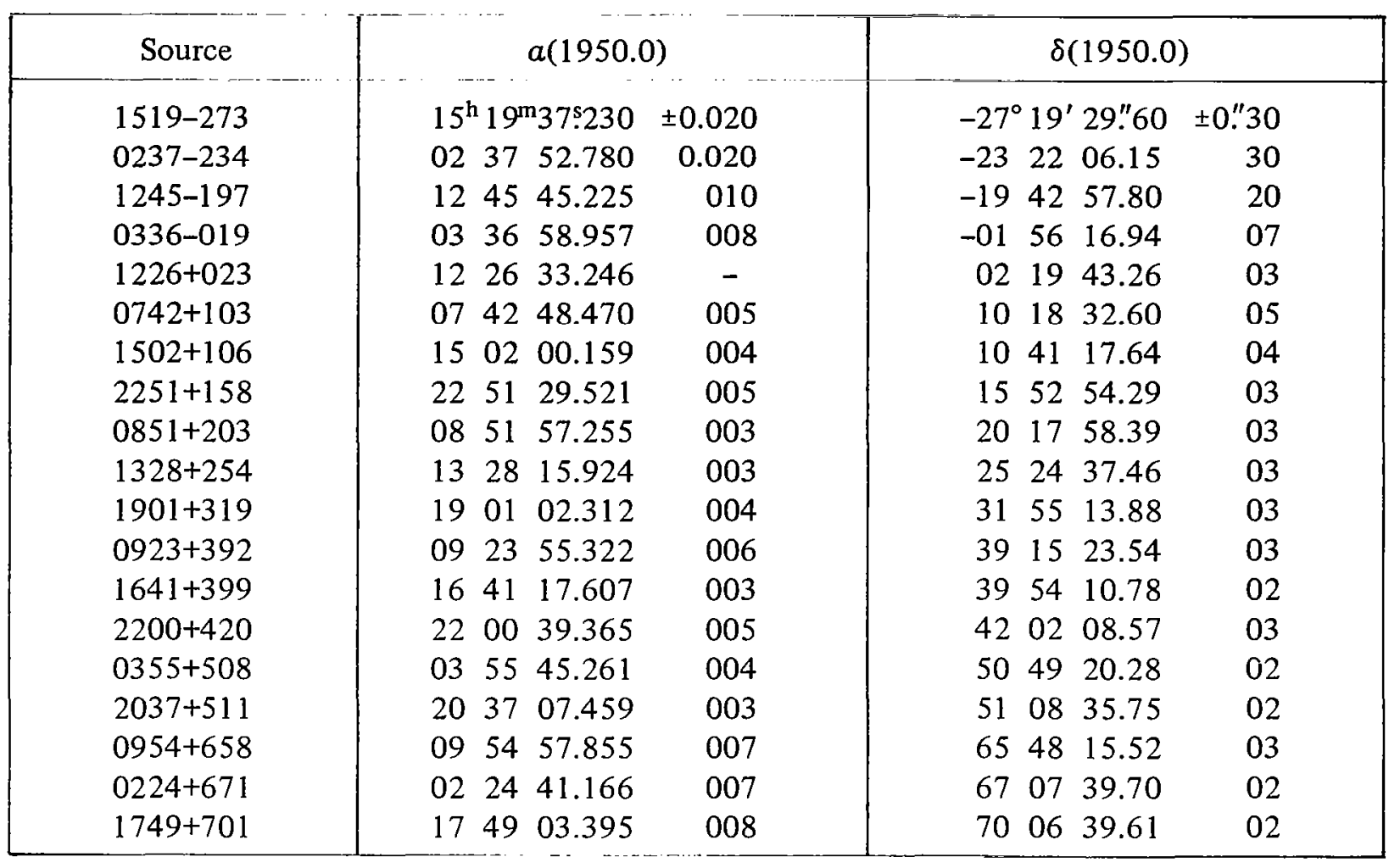

Figure 1 gives an indication of the sensitivity of the $35-\mathrm{km}$ baseline to errors in source position. The figure shows the error in source position (either E-W or N-S) that is needed to produce a $30^{\circ}$ phase error at S-band at some time during the observing day, as a function of source declination. The limits of the observing day are determined by two conditions: (1) the source must be at an altitude of $20^{\circ}$ or more and (2) the source must be less than 6 hours from the local meridian. It can be seen that the interferometer's sensitivity to source position errors deteriorates rapidly below the equator, especially in the N-S direction (declination). The situation below the equator is a little worse that the figure indicates since right ascension and declination errors become increasingly correlated as the hour-angle coverage decreases.

\section{Earth Rotation Parameters}

It is to be emphasized that the results and data presented below on Earth rotation parameters have not yet been corrected for the effects of the ionosphere, source structure, or solid Earth tides. Figure 2 shows the components $(\mathrm{Bx}, \mathrm{By}$, and $\mathrm{Bz}$ ) of the baseline vector as determined from 2-day averages of interferometer data for the representative period from April 11 to May 30, 1979. The width of each curve represents twice the probable error of its determination, which ranges from 4 


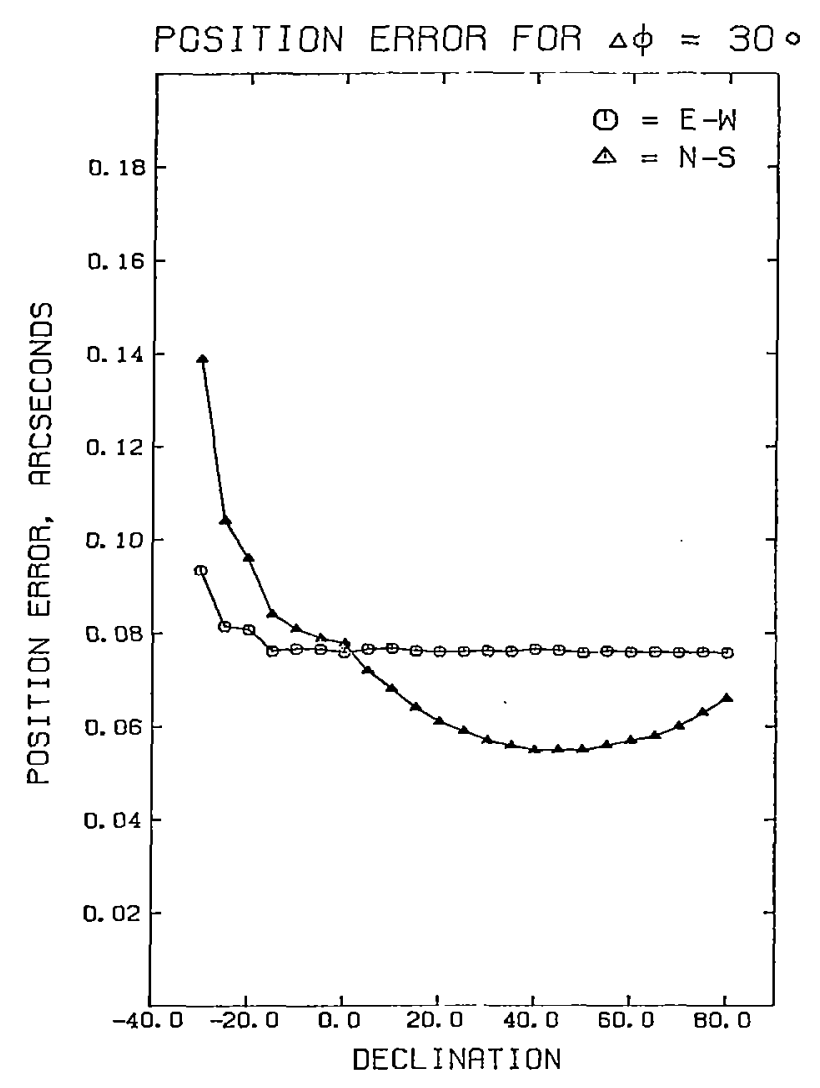

Figure 1. Sensitivity of the $35-\mathrm{km}$ baseline to errors in source position. Source position error (ordinate) needed to produce a $30^{\circ}$ phase error at S-band as a function of source declination (abscissa).

to $24 \mathrm{ps}$, corresponding to 1.2 to $7.2 \mathrm{~mm}$. The top curve represents the length of the baseline which should be invariant under a rotation of the baseline vector. To achieve a goal of a precision of $2 \mathrm{~ms}$ in time, the baseline must be determined with a precision of approximately 5 ps which corresponds to about $2 \mathrm{~mm}$ in baseline length.

The components of the baseline vector $(\mathrm{Bx}, \mathrm{By}$, and $\mathrm{Bz})$ are expected to vary due to the motion of the instantaneous axis of rotation with respect to some reference point and to variations in the Earth's rate of rotation. In order to insure the accuracy of the delay tracking of the interferometer, the sidereal time used in controlling the telescope is derived by using values of UT1-UTC, provided by the Rapid Service of the Bureau International de l'Heure (BIH). Thus, the baseline components shown in figure 2 have the effects of a first approximation to variations in the Earth's rotation rate removed from them but are not corrected for the effects of polar motion. 


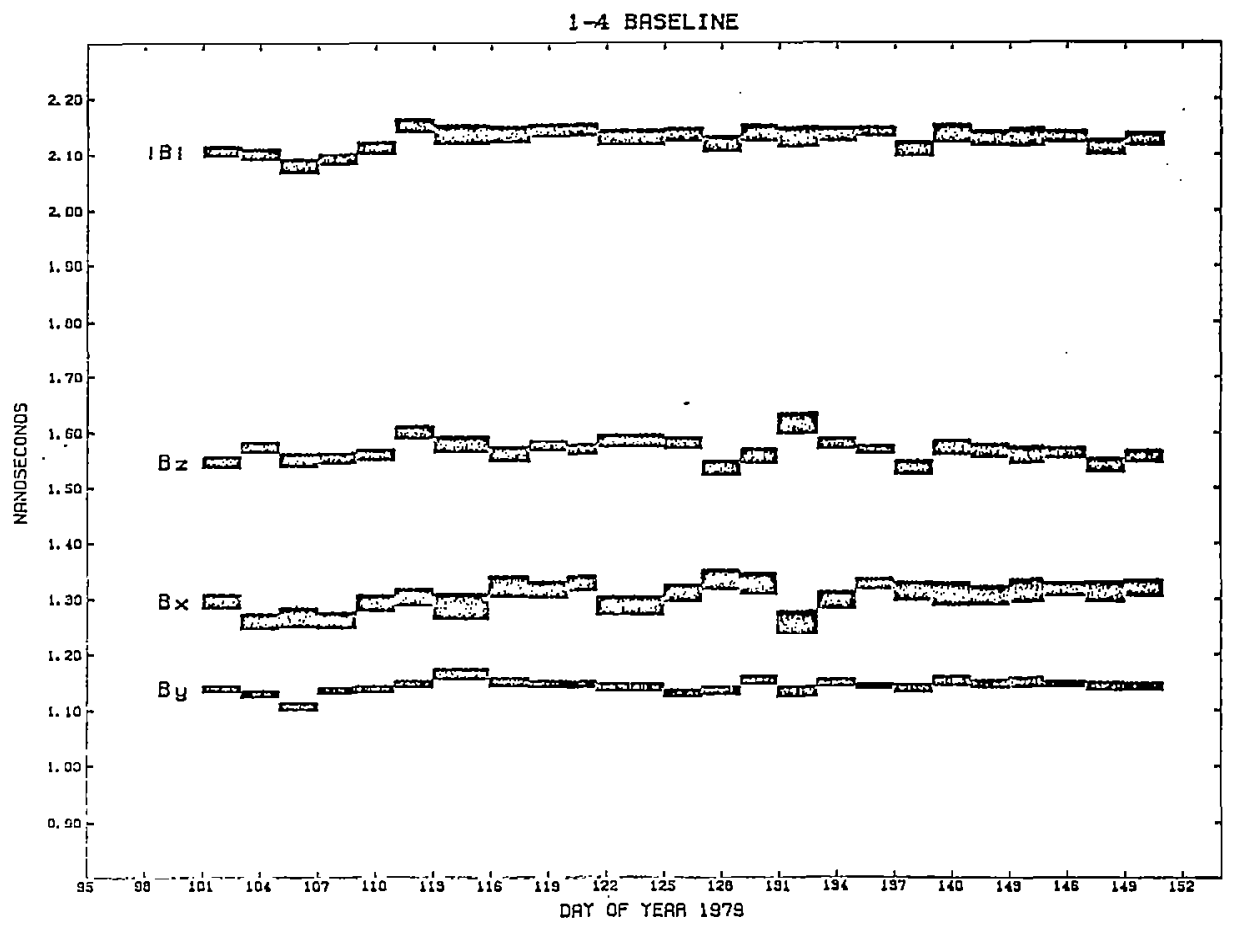

Figure 2. Normalized changes in the baseline components $(B x, B y, B z)$ for the period April 11 to May 30. Width of the curves is twice the formal probable error. The top curve represents the change in baseline length $(|B|)$.

For the current configuration of the Green Bank interferometer, the equations relating changes in baseline components to the motion of the pole and changes in the Earth's rate of rotation are:

$$
\left[\begin{array}{c}
\Delta \mathrm{B}_{\mathrm{x}} \\
\Delta \mathrm{B}_{\mathrm{y}} \\
\Delta \mathrm{B}_{\mathrm{z}}
\end{array}\right]=\left[\begin{array}{ccc}
-\mathrm{B}_{\mathrm{z}} \cos \lambda & -\mathrm{B}_{\mathrm{z}} \sin \lambda & \mathrm{B}_{\mathrm{y}} \\
\mathrm{B}_{\mathrm{z}} \sin \lambda & -\mathrm{B}_{\mathrm{z}} \cos \lambda & -\mathrm{B}_{\mathrm{x}} \\
\left(\mathrm{B}_{\mathrm{x}} \cos \lambda-\mathrm{B}_{\mathrm{y}} \sin \lambda\right) & \left(\mathrm{B}_{\mathrm{x}} \sin \lambda+\mathrm{B}_{\mathrm{y}} \cos \lambda\right) & 0
\end{array}\right]\left[\begin{array}{c}
\Delta \mathrm{x} \\
\Delta \mathrm{y} \\
\mathrm{T}
\end{array}\right]
$$

where

$\mathrm{Bx}, \mathrm{By}, \mathrm{Bz}$ are the components of the reference baseline in a local left-handed system;

$\Delta \mathrm{B}_{\mathrm{x}}, \Delta \mathrm{B}_{\mathrm{y}}, \Delta \mathrm{B}_{\mathrm{z}}$ are observed changes in the baseline components;

$\Delta \mathrm{x}, \Delta \mathrm{y}$ are changes in the polar coordinates from those of the reference data; 
$\mathrm{T}$ is the change in UT1-UTC from the value assumed in the observations;

$\lambda$ is the adopted longitude of the interferometer $(5 \mathrm{~h} \quad 19 \mathrm{~m} 20 \mathrm{~s} 000)$.

Since the determinant of the matrix formed by the coefficients of the right-hand side of these equations is zero, the matrix cannot be inverted. In other words, the single baseline can only be used to determine two combinations of the three quantities of interest. In the future, it is hoped, with the cooperation of the National Science Foundation (NSF) and NRAO, to establish a second long baseline nearly orthogonal to the current one to overcome this difficulty. Until that time, it has been decided to publish values of UTO-UTC and variations in the component of polar motion determined from variations in the component of the interferometer baseline (McCarthy, et al., 1979). Table 2 lists the values of UTO-UTC obtained for the more recent period of observations preceding this conference (April 11 to May 30) and their probable errors. The formal probable errors for the quantity UTO-UTC are about $2 \mathrm{~ms}$ for 2-day averages and, in some instances, approach $1 \mathrm{~ms}$.

Table 2

Values of UTO-UTC as Determined by the $35-\mathrm{km}$ Interferometer

\begin{tabular}{|c|c|c|}
\hline Date & MJD & UTO-UTC \\
\hline 79412 & 43975.000 & $0.2819 \quad 0.0015$ \\
\hline 79414 & 43977.000 & $0.2791 \quad 0.0016$ \\
\hline 79416 & 43979.000 & $\begin{array}{lll}0.2687 & 0.0019\end{array}$ \\
\hline 79418 & 43981.000 & $0.2662 \quad 0.0017$ \\
\hline 79420 & 43983.000 & $\begin{array}{lll}0.2560 & 0.0017\end{array}$ \\
\hline 79422 & 43985.000 & 0.24920 .0018 \\
\hline 79425 & 43987.500 & $0.2449 \quad 0.0026$ \\
\hline 79427 & 43990.000 & $0.2302 \quad 0.0023$ \\
\hline 79429 & 43992.000 & $0.2234 \quad 0.0018$ \\
\hline 7951 & 43994.000 & $\begin{array}{lll}0.2419 & 0.0017\end{array}$ \\
\hline 7954 & 43997.000 & $\begin{array}{ll}0.2085 & 0.0019\end{array}$ \\
\hline 7956 & 43999.000 & 0.19910 .0018 \\
\hline 7958 & 44001.000 & $\begin{array}{lll}0.1905 & 0.0021\end{array}$ \\
\hline 79510 & 44003.000 & 0.18740 .0022 \\
\hline 79512 & 44005.000 & $\begin{array}{lll}0.1888 & 0.0024\end{array}$ \\
\hline 79514 & 44007.000 & $0.1805 \quad 0.0020$ \\
\hline 79516 & 44009.000 & $0.1712 \quad 0.0013$ \\
\hline 79518 & 44011.000 & 0.16640 .0020 \\
\hline 79520 & 44013.000 & 0.16290 .0008 \\
\hline 79522 & 44015.000 & $0.1571 \quad 0.0021$ \\
\hline 79524 & 44017.000 & $0.1495 \quad 0.0024$ \\
\hline 79526 & 44019.000 & $\begin{array}{lll}0.1450 & 0.0017\end{array}$ \\
\hline 79526 & 44021.000 & 0.13960 .0020 \\
\hline 79530 & 44023.000 & 0.13340 .0018 \\
\hline
\end{tabular}


In order to test the accuracy of our UTO-UTC data, they may be compared with the values derived from the Earth rotation parameters of the BIH Rapid Service. Using the values for $\Delta x, \Delta y$, and UT1-UTC from the BIH Rapid Service, values for UT0-UTC are computed for the dates listed in table 2. The differences between the computed values for UT0-UTC and those determined by the interferometer are shown in figure 3. The values are given in the BIH 1979 System and have been adjusted by a constant so that there is no difference between the observed and computed values on the first date. Figure 4 shows the same comparison but for a period covering the initial months of operation from October 19 to December 12, 1978. These data were computed in the BIH 1968 System and also adjusted to make the difference between observed and computed values on the first day equal to zero. Because of the brevity and discontinuity of the data presented here, no reliable conclusions regarding the accuracy of the data can be drawn.

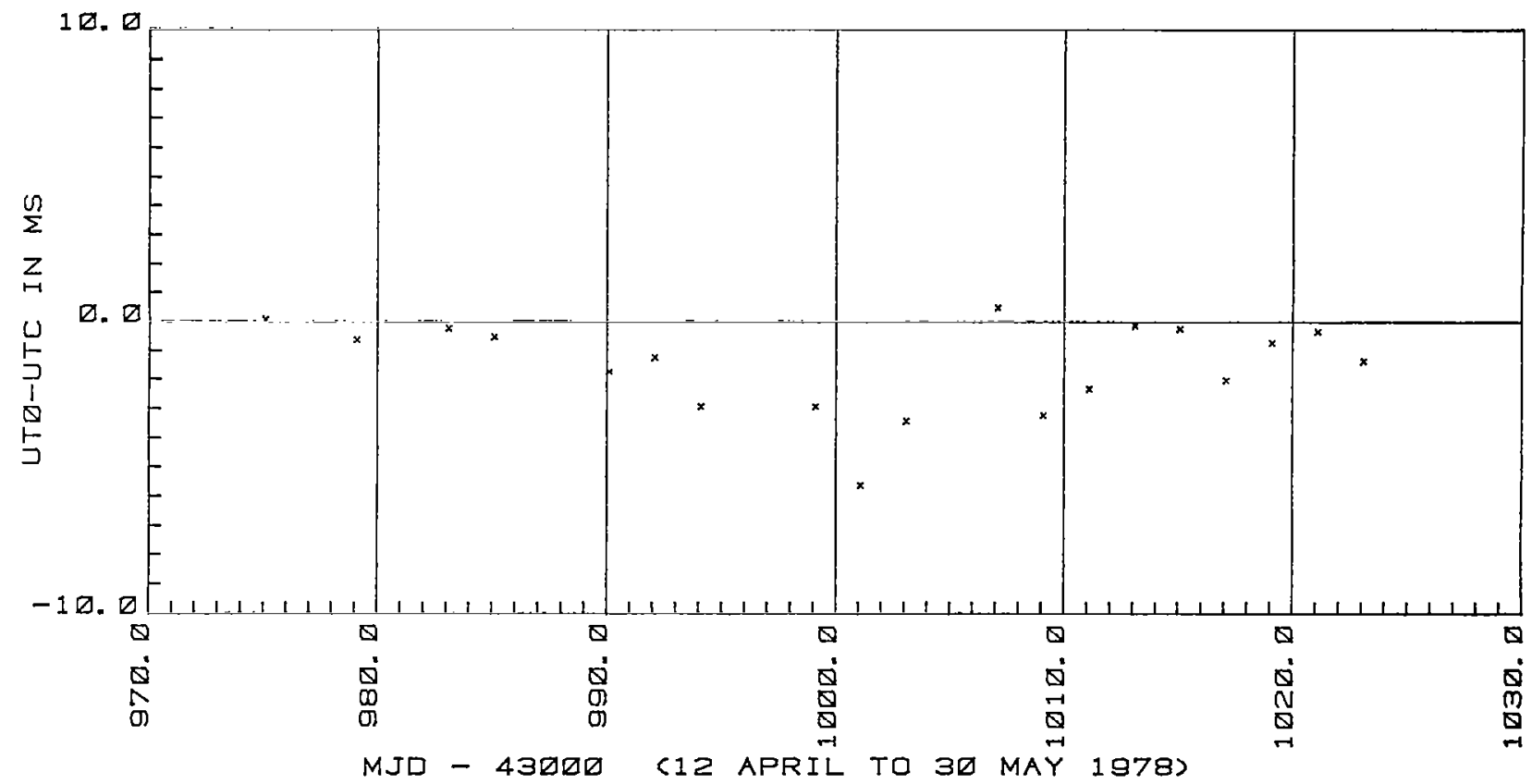

Figure 3. The difference in UTO-UTC between that observed and that computed from the BIH Rapid Service data (referred to the BIH 1979 System), for the period April 12 to May 30. 


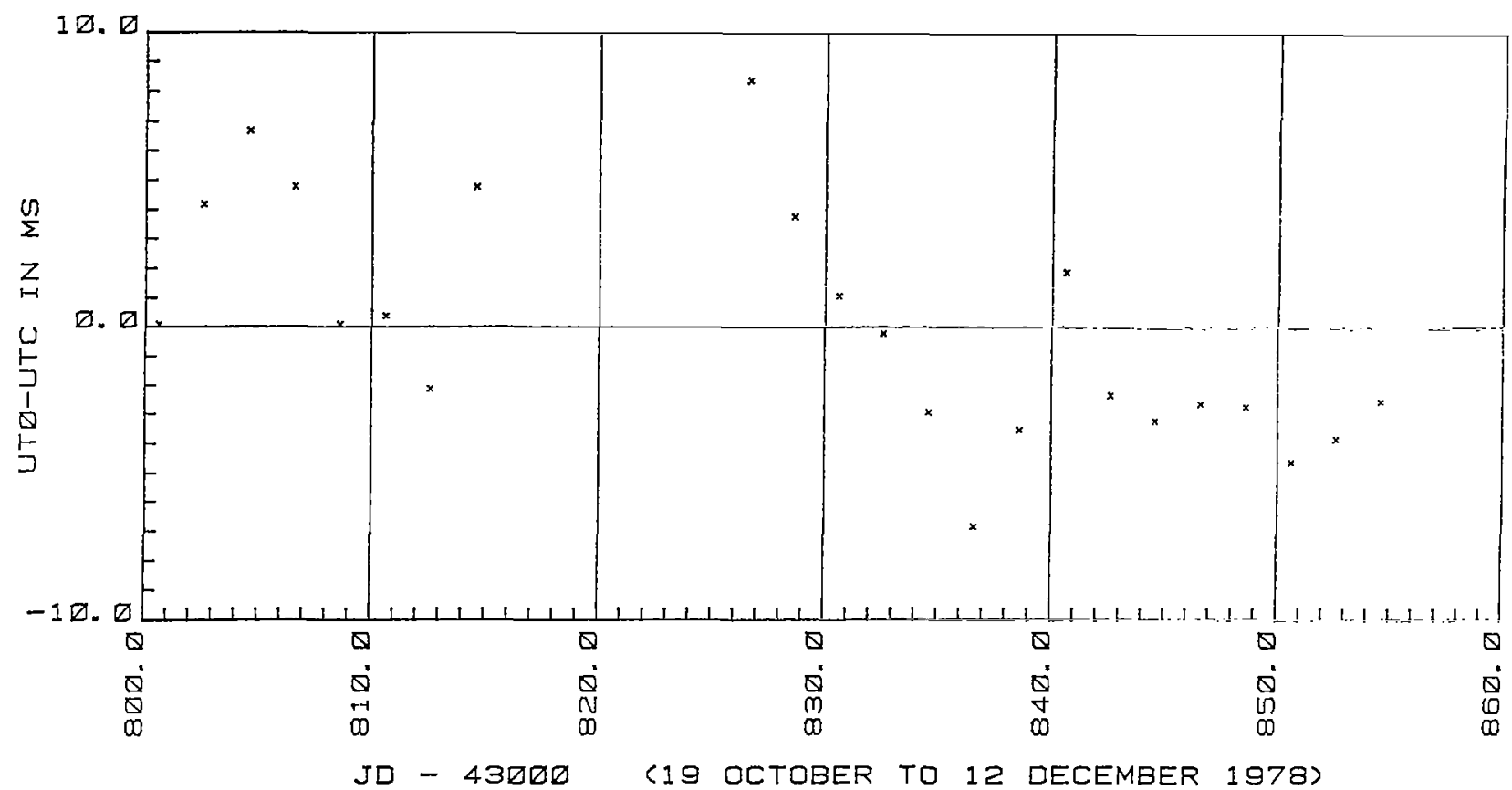

Figure 4. The difference in UTO-UTC between that observed and that computed from the BIH Rapid Service data (referred to the BIH 1968 System), for the period October 19 to December 12.

\section{REFERENCES}

Elsmore, B., Nature, 244, 423 (1973).

Hogg, D. E., Macdonald, G. H., Conway, R. G., and Wade, C. M., Astron. J., 74, 1206 (1969).

Johnston, K. J., Spencer, J., Mayer, C., Klepczynski, W. J., Kaplan, G. H., McCarthy, D. D., and Westerhout, G., Modern Astrometry (IAU Coll. 48), 1978.

McCarthy, D. D., Klepczynski, W. J., Kaplan, G. H., Josties, F. J., Westerhout, G., Johnston, K. J., and Spencer, J. H., Annual Report for 1978 (Bureau International de l'Heure).

Shapiro, I. I., Robertson, D. S., Knight, C. A., Counselman, C. C., Rogers, A. E. E., Hinteregger, H. F., Lippincott, S., Whitney, A. R., Clark, T. A., Niell, A. E., and Spitzmesser, D. J., Science, $\underline{186}, 920$ (1974).

Wade, C. M. and Johnston, K. J., Astron. J., 82, 791 (1977). 


\title{
GEODETIC LONG BASELINE INTERFEROMETRY RESEARCH IN CANADA
}

\author{
R. B. Langley,* W. T. Petrachenko and W. H. Cannon \\ Department of Physics and CRESS \\ York University
}

\begin{abstract}
Long baseline interferometry (LBI) has proved itself to be an invaluable geodetic tool. In this paper, the objectives and results of several studies using the Canadian LBI system will be presented. Radio telescopes at the Algonquin Radio Observatory (ARO), Lake Traverse, Ontario; the Owens Valley Radio Observatory (OVRO), Big Pine, California; and the Chilbolton Observatory (CHIL), Chilbolton, England, are being used to obtain precise positions of a number of extragalactic radio sources and to determine the components of the baselines connecting the radio telescopes with submeter precision. For example, the standard deviation of the weighted mean of the equatorial component of the AROCHIL baseline from five observing sessions between March 1973 and January 1978 is only $31 \mathrm{~cm}$.

Since LBI is insensitive to the uncertainty in the geocentric gravitational constant, GM, it is a very useful technique for determining the scales of the coordinate systems used by other precise techniques. Beginning in May 1977, a number of LBI observing sessions were accompanied by simultaneous satellite Doppler observations. The baseline components obtained from the satellite Doppler observations were compared to the LBI values. The weighted mean scale bias of the NSWC 9Z-2 satellite Doppler coordinate system relative to the LBI system was found to be $0.42 \pm 0.05$ PPM. The weighted mean difference in the origin of longitude was found to be $0.87 \pm 0$ ". 01 while the difference in declination origin was found to be $0.06 \pm 0.01$.

A pilot project to use LBI to make high-accuracy measurements of earth rotation parameters is also under way. The major innovation setting this system apart from other similar efforts is the inclusion of a scheme to accurately monitor local oscillator drifts using two-way transmissions through the Anik B communications satellite. The purpose of this innovation is to eliminate the troublesome possibility of mistakenly interpreting oscillator drifts as real geophysical effects.
\end{abstract}

*Now at Department of Earth and Planetary Sciences, Massachusetts Institute of Technology, Cambridge, MA 02139 


\section{INTRODUCTION}

Although originally designed for astrophysical purposes, the Canadian Long Baseline Interferometry (LBI) system has been used to obtain a number of geodetically useful results [Petrachenko, 1976; Cannon et al., 1979a; Langley et al., 1979]. In this paper, we present the results of further studies using this system and briefly discuss plans for a project to make high-accuracy measurements of earth rotation parameters using the Anik B communications satellite to accurately monitor local oscillator drifts.

\section{VECTOR BASELINE AND SOURCE COORDINATE DETERMINATIONS}

Between February 1975 and January 1978, seven LBI observing sessions were carried out using the $46 \mathrm{~m}$ antenna at the Algonquin Radio Observatory (ARO), Lake Traverse, Ontario; the 40m antenna at the Owens Valley Radio Observatory (OVRO), Big Pine, California; and the $25 \mathrm{~m}$ antenna at the Chilbolton Observatory (CHIL), Chilbolton, England. The dates on which observations were made and the frequencies used are listed in table 1 . Thus far, only the data from the observing sessions of May 1977 and January 1978 (considered to be the best) have been analyzed for their geodetic and astrometric content.

Table 1

Dates of Observations

\begin{tabular}{|c|c|c|c|c|}
\hline \multirow{2}{*}{ Observing Session } & \multirow{2}{*}{$\begin{array}{c}\text { Frezuency } \\
\text { (MHz) }\end{array}$} & \multicolumn{3}{|c|}{ Antenna } \\
\cline { 3 - 5 } & & ARO & CHIL & OVRO \\
\hline 4- 6 February 1975 & 10680 & $\mathrm{x}$ & $\mathrm{x}$ & \\
21 - 25 June 1975 & 10680 & $\mathrm{x}$ & $\mathrm{x}$ & $\mathrm{x}$ \\
13-16 May 1977 & 10680 & $\mathrm{x}$ & $\mathrm{x}$ & $\mathrm{x}$ \\
15-18 July 1977 & 10680 & $\mathrm{x}$ & $\mathrm{x}$ & $\mathrm{x}$ \\
15 - 18 September 1977 & 10660 & $\mathrm{x}$ & $\mathrm{x}$ & $\mathrm{x}$ \\
9-13 November 1977 & 10660 & $\mathrm{x}$ & $\mathrm{x}$ & $\mathrm{x}$ \\
17- 20 January 1978 & 10660 & $\mathrm{x}$ & $\mathrm{x}$ & $\mathrm{x}$ \\
\hline
\end{tabular}

A description of the Canadian LBI system, the observing strategy and the analysis technique has been given by Langley [1979]. Only a few of the details will be repeated here.

The observations were first corrected for the effect of the neutral atmosphere. Hopfield's model [Hopfield, 1971] was implemented using an extension of the algorithm of Yionoulis [1970]. Surface measurements of pressure, temperature, and relative humidity used as input to the model were taken at or near each of the stations during the observations. The observations were not corrected for the effect of the ionosphere. At the approximately $10 \mathrm{GHz}$ observing frequency, the maximum contributions of the ionosphere to fringe frequency and delay are about $0.3 \mathrm{MHz}$ and $1 \mathrm{~ns}$ respectively and that for most of the observations the contributions will be less than $0.1 \mathrm{MHz}$ and $0.3 \mathrm{~ns}$. 
Since the standard deviations of the fringe frequency and delay residuals were expected to be about $2 \mathrm{MHz}$ and $20 \mathrm{~ns}$ respectively, the ionospheric effect was considered to be fairly insignificant.

For each observing session, the corrected delays and fringe frequencies from all three baselines were simultaneously fitted to a model [Langley, 1979] for which the following parameters were estimated: the $\mathrm{x}, \mathrm{y}$, and $\mathrm{z}$ components of each baseline; the right ascension and declination of the sources observed (with the exception of $3 \mathrm{C} 273 \mathrm{~B}$ ); and the coefficients of clock polynomials for fringe frequency and delay. The right ascension and declination of 3C273B were not estimated as they were used as a fiducial point for determining the coordinates of the other sources and, in part, the orientation of the baselines. It is usually the case when precise delay observations are available that only the right ascension of the fiducial source must be held fixed. However, when fringe frequency observations alone are used or when the fringe frequency observations dominate the delay observations when both types of observables are used (as in the present case), both the right ascension and declination of the fiducial source must be fixed [Cannon, 1978; Shapiro, 1976]. The coordinates adopted for 3C273B are those of Clark et al. [1976]. The estimated uncertainty in the position for $3 \mathrm{C} 273 \mathrm{~B}$ is \pm 0.01 and \pm 0 " 13 for the right ascension and declination respectively.

The orientation of the LBI coordinate system was determined in part by the position of the earth's rotation pole and the differences UT1-UTC during the observing sessions as published a posteriori by the Bureau International de l'Heure (BIH) [1978a, 1978b]. These data are expressed in the 1968 BIH System.

The IAU system of astronomical constants that was introduced in 1968 was adhered to in the model including the value of the speed of light of $299792.5 \mathrm{~km} \cdot \mathrm{s}^{-1}$.

Because the Canadian LBI system has a relatively narrow recorded bandwidth $(\sim 4 \mathrm{MHz})$, the fringe frequency observations are much more sensitive to source position and the equatorial components of the baselines than are the delay observations. The fringe frequency observations therefore dominate the delay observations in the model fitting as far as these parameters are concerned. However, the fringe frequency observable is essentially insensitive to the $z$ component of the baseline, and the delay observations provide the only information for estimating this component.

The weighted mean values of the source positions resulting from the model fits to the May 1977 and January 1978 data are shown in table 2. The weights used were the formal standard deviations derived from the post-fit residuals and the parameter variance-covariance matrix. Half of the sources were observed in only one of the observing sessions. For these sources, the single result from the individual model fit has been entered. The E-terms of annual aberration have been included in the coordinates in table 2. This conforms with normal cataloging procedures for the epoch 1950.0.

The baseline components from the individual fits are shown in tables 3 and 4, while the weighted mean values of the baseline components are shown in table 5. Again, the weights used were the formal standard deviations derived from the post-fit residuals and the parameter variance-covariance 
Table 2

Weighted Mean Values of Source Positions from May 1977 and January 1978 Observing Sessions

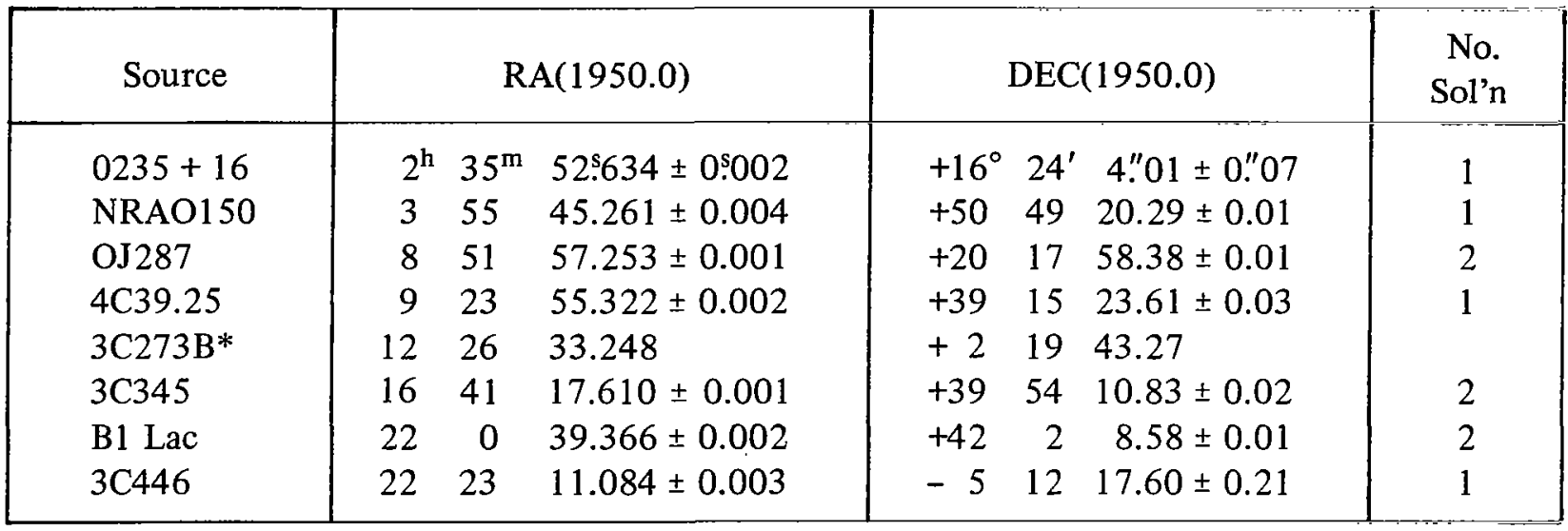

*3C273B was used as the fiducial source; see text

matrix. The standard deviations of the weighted mean $\mathrm{z}$ components of the baselines have anomalously small values, in light of the fact that the formal standard deviations of the individual determinations of the $\mathrm{z}$ components are of the order of $4 \mathrm{~m}$. No explanation is offered for the remarkable agreement between the values of the $\mathrm{z}$ components from the two observing sessions other than that supported by the (slim) odds of probability. It should be noted however that the agreement between the $\mathrm{z}$ components of the baselines of table 5 and those determined by satellite Doppler observations (to be discussed shortly) is about 1 to $2 \mathrm{~m}$ ! Also, because the $\mathrm{z}$ components of the baselines are relatively small, the errors in $\mathrm{z}$ components do not contribute appreciably to the overall errors in the total lengths of the baselines.

The standard deviations of the post-fit fringe frequency and delay residuals for the May 1977 and January 1978 observing sessions are $2.0 \mathrm{MHz}, 15.2 \mathrm{~ns}$ and $1.9 \mathrm{MHz}, 13.1 \mathrm{~ns}$ respectively.

Preliminary results of the baseline and source position determinations from the May 1977 observing session alone have been published previously [Langley et al., 1979]. The results presented here differ slightly from the preliminary results for two reasons: (1) some (poor) observations of the source NRAO150 were used in the preliminary analysis but not in the final model fit for the May 1977 observations, and (2) the model used for the preliminary analysis did not include the effect of earth tides on the observations.

The equatorial components of the ARO-CHIL baseline obtained from the May 1977 and January 1978 observing scssions have becn compared to values obtained from the analysis of threc obscrving sessions in 1973 from which only fringe frequency data were obtained [Petrachenko, 1976]. The five values are shown in figure 1 . The weighted mean of the five values is $5251087.68 \mathrm{~m}$ with a standard deviation of $0.31 \mathrm{~m}$. 


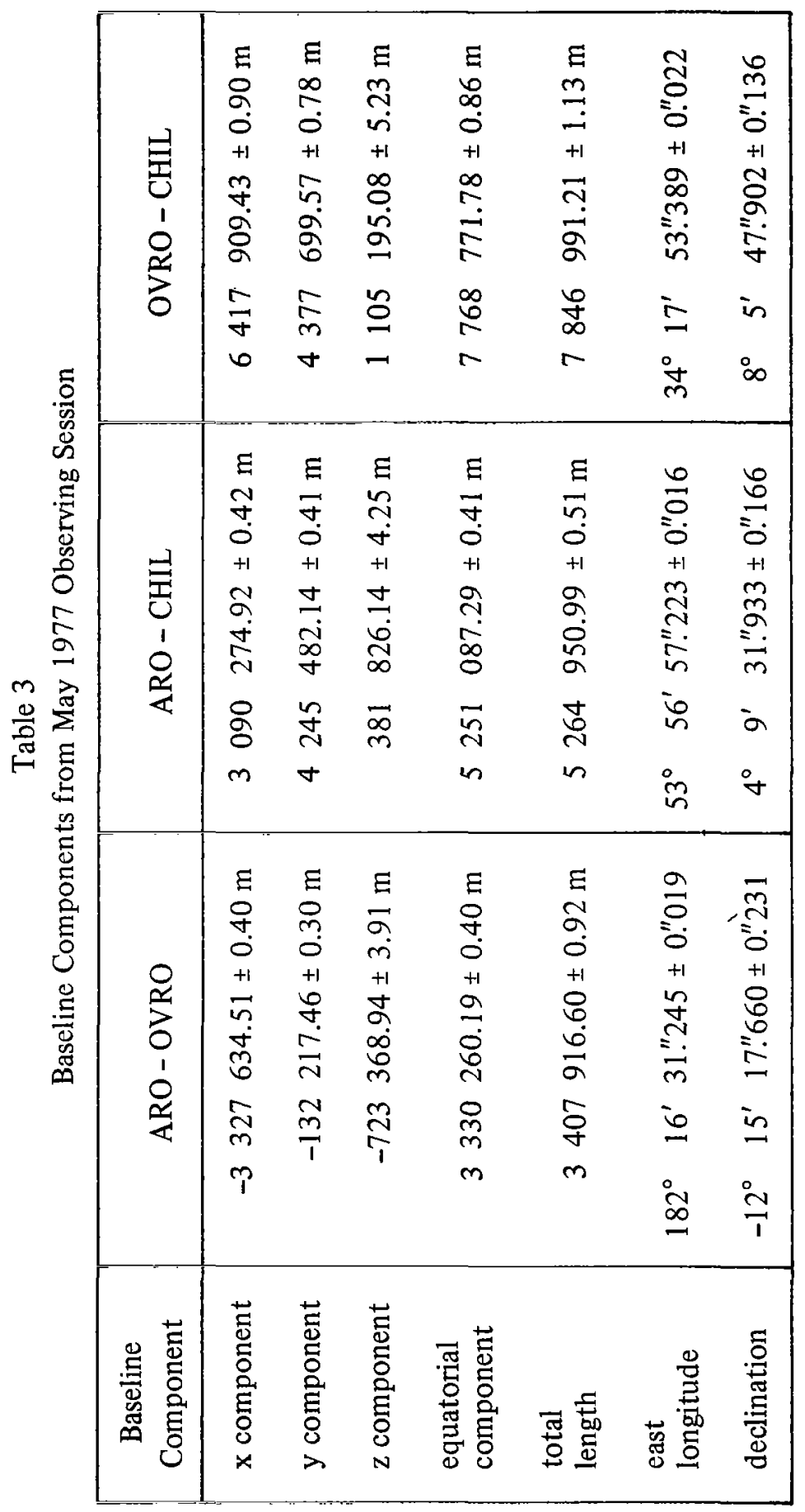




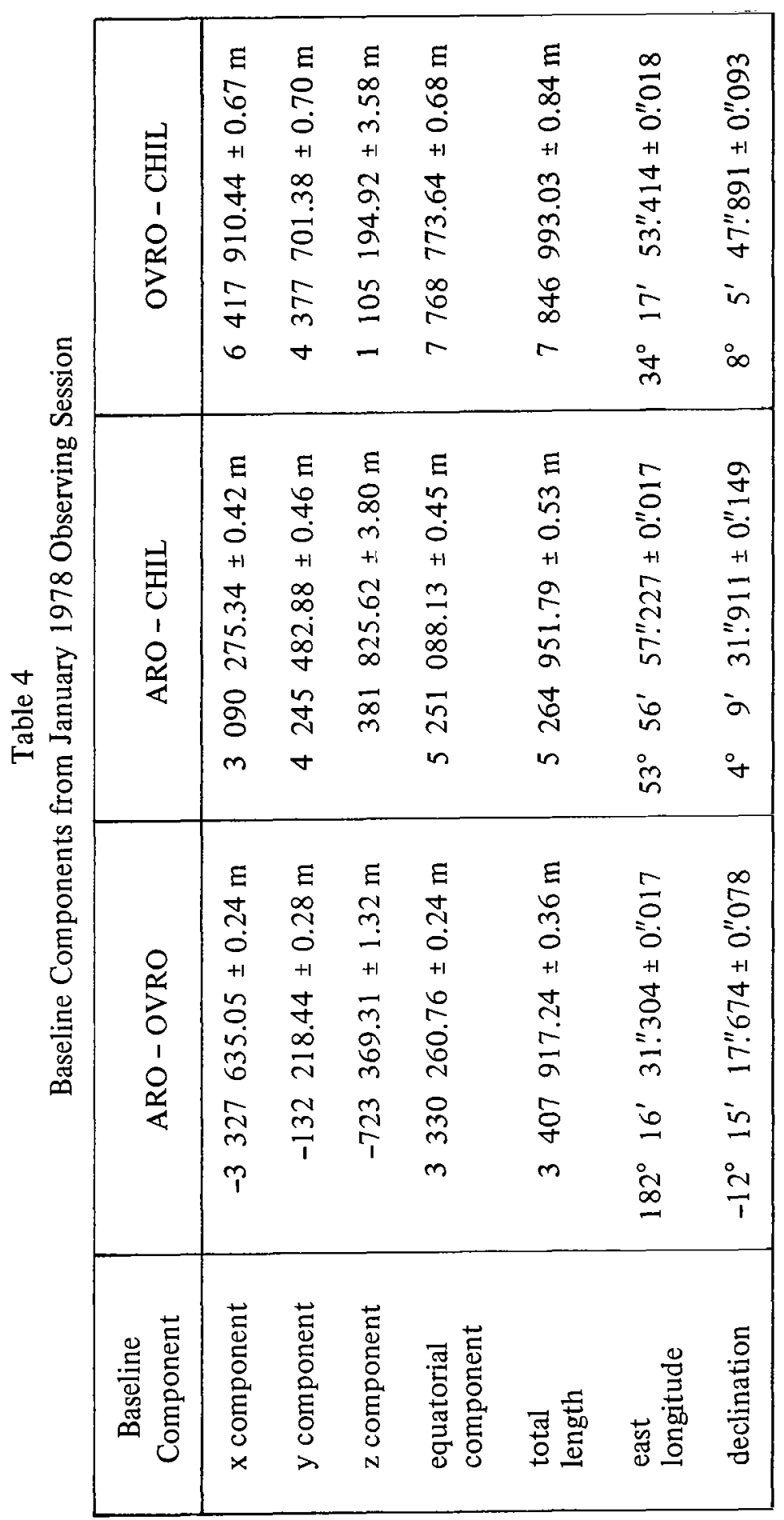




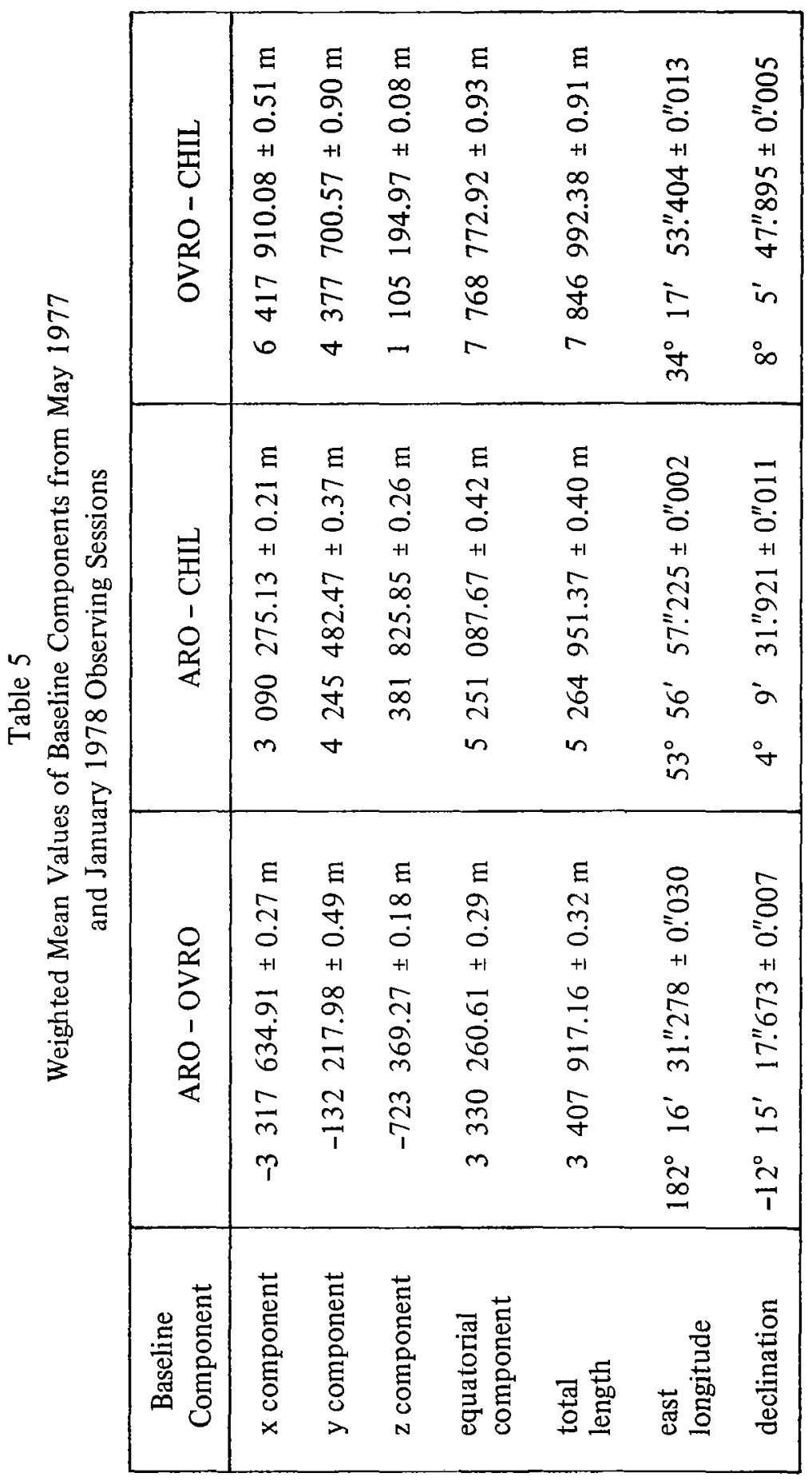




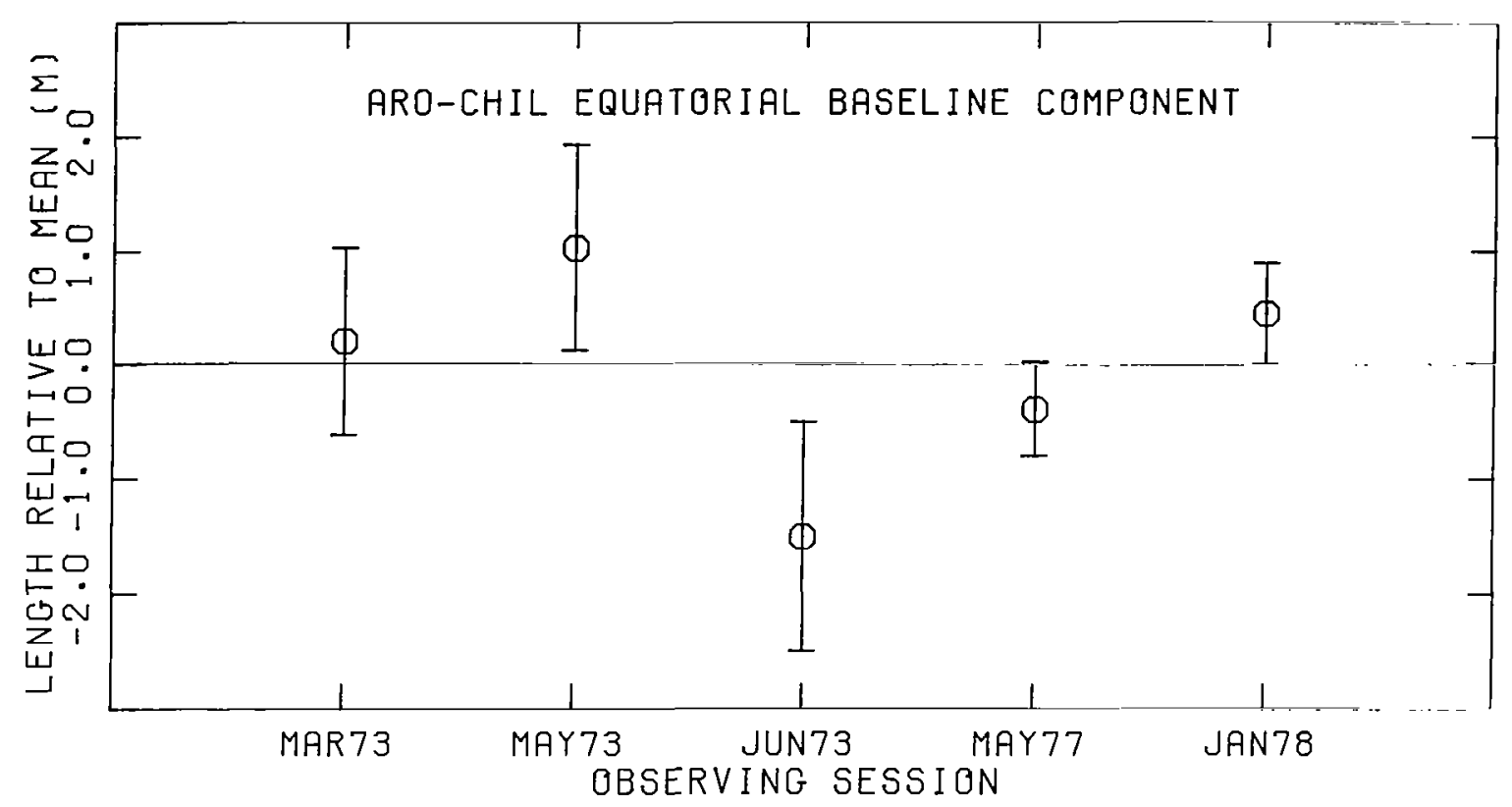

Figure 1. Five determinations of the equatorial component of the ARO-CHIL baseline. The weighted mean is $5251087.68 \pm 0.31 \mathrm{~m}$.

\section{COMPARISON OF LBI BASELINE DETERMINATIONS WITH SATELLITE DOPPLER RESULTS}

During the May 1977 and January 1978 observing sessions, satellite Doppler receiving stations were set up near the antennas used for the LBI observations. The object of these simultaneous LBIsatellite Doppler observing sessions was to compare the determinations of the vector baselines between each of the LBI antennas by the two techniques; hence, to derive the differences in scale and orientation of the coordinate systems employed.

For the May 1977 observing session, satellite Doppler receivers were installed within about $100 \mathrm{~m}$ of the LBI antennas at each of the observatories. For the January 1978 observing session, there was no satellite Doppler receiver at the Chilbolton Observatory. However, for both observing sessions, satellite Doppler observations were available from the TRANET permanent tracking station at Barton Stacey which is only a few kilometers away from the Chilbolton Observatory.

The satellite Doppler observations were conducted between May 9 and 15,1977, and between January 16 and 21, 1978. The observations at ARO were made by the Geodetic Survey of Canada, those at OVRO by the National Geodetic Survey of the U. S. Department of Commerce, and those at CHIL by the University of Nottingham and the Ordnance Survey of Great Britain. The TRANET station is operated by the Directorate of Military Survey of the United Kingdom. 
The satellite Doppler observations were reduced by Jan Kouba of the Earth Physics Branch of the Department of Energy, Mines, and Resources using the GEODOP computer program [Kouba and Boal, 1976] and a precise ephemeris supplied by the U.S. Defense Mapping Agency Topographic Center (DMATC). The number of satellite passes used to obtain the satellite Doppler station positions varied from 25 for the May 1977 observations at the Barton Stacey TRANET station to 48 for the May 1977 observations at ARO and OVRO. Although as many as five TRANSIT satellites were observed at each station, only the observations of satellites 68 and 77 were used in the data reduction.

The satellite Doppler and LBI observations refer to different positions. The satellite Doppler reference point is the electrical center of the Doppler antenna. The LBI reference point is the point where the azimuth axis of the LBI antenna intersects the horizontal plane in which the altitude axis rotates. The differences in the reference points for each observing site were determined by conventional surveys.

The derived Doppler station positions were combined with the survey differences to obtain the baseline components for the LBI antennas. These are presented separately for each observing session in tables 6 and 7. The components are expressed in the NSWC 9Z-2 coordinate system. The first entry in table 6 for each component of the ARO-CHIL and OVRO-CHIL baselines was derived from the observations at the Chilbolton Observatory station. The second entry was derived from the observations at the Barton Stacey TRANET station; only the least significant figures are given. In each case, the two results for the baseline components of the ARO-CHIL and OVRO-CHIL baselines are within the estimated accuracies.

The differences between the LBI-derived baseline components and those derived from the combination of satellite Doppler and ground surveys are presented in tables 8 and 9. The differences are given in the sense: LBI result minus satellite Doppler result. The accuracý estimates were obtained by combining the uncertainties of the LBI results with the uncertainties of the satellite Doppler results.

The scale bias of the NSWC 9Z-2 coordinate system as determined from the total baseline lengths is given in table 10 for each baseline from both observing sessions together with the differences in east longitude and declination.

The scale difference ranges from $0.26 \pm 0.17$ to $0.70 \pm 0.41$ PPM with a weighted mean of $0.42 \pm$ 0.05 PPM

The differences in the east longitudes of the baselines, which can be interpreted as a difference in definition of the longitude origins of the coordinate systems, have a weighted mean of $0 . " 87 \pm 0 . " 01$. This is in agreement with a previous result [Cannon et al., 1979a].

The differences in the declinations of the baselines as determined by the two techniques have a weighted mean of $0 . " 06 \pm 0 . " 01$. This small difference between the two techniques may, in part, be due to the adopted declination of source 3 C273B which defines the declination origin of the LBI 


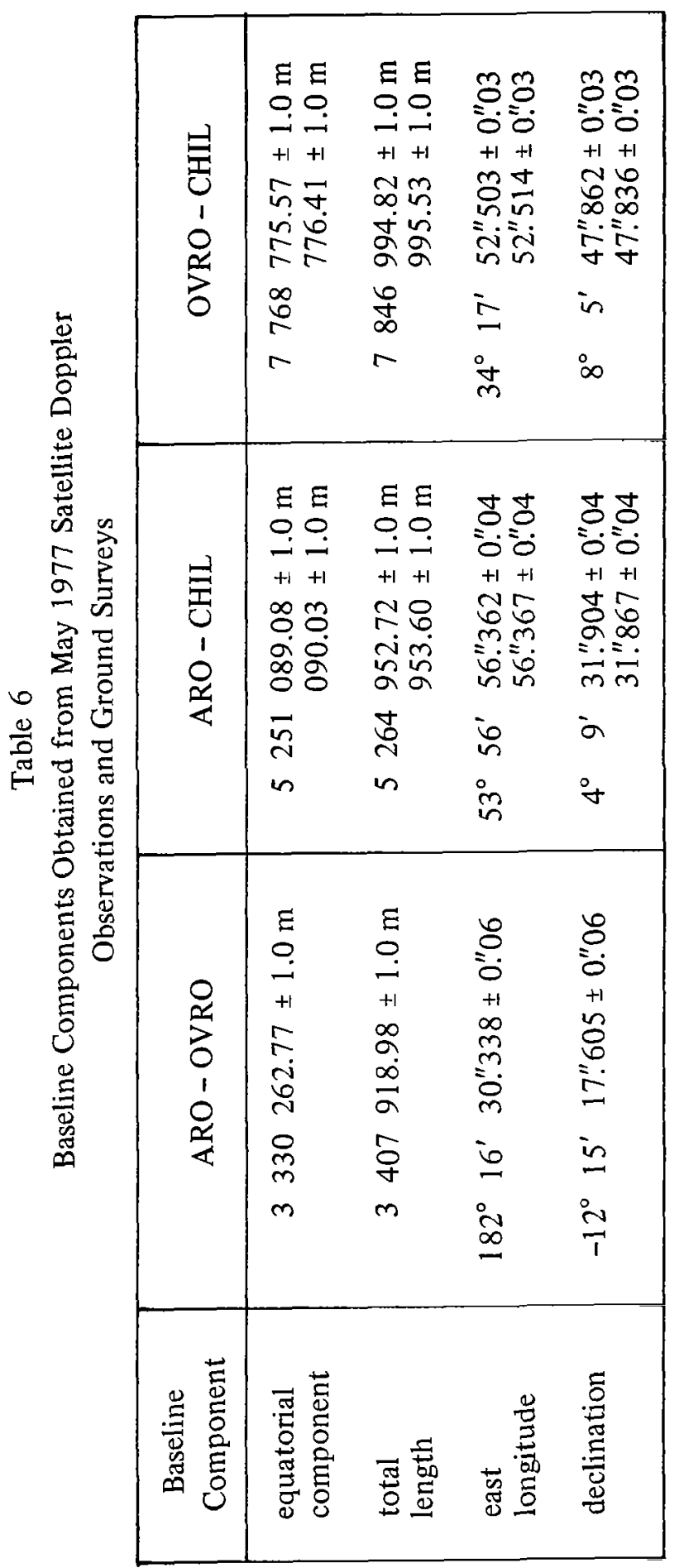




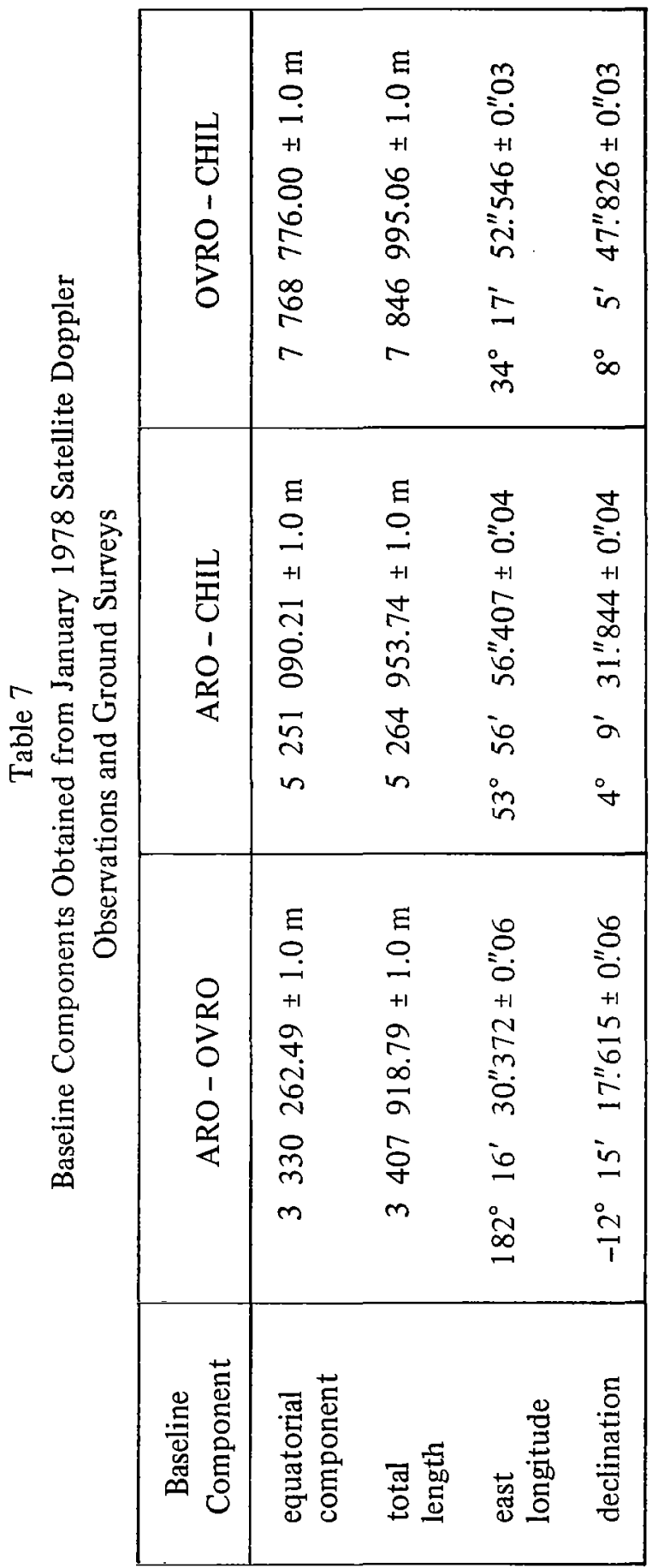




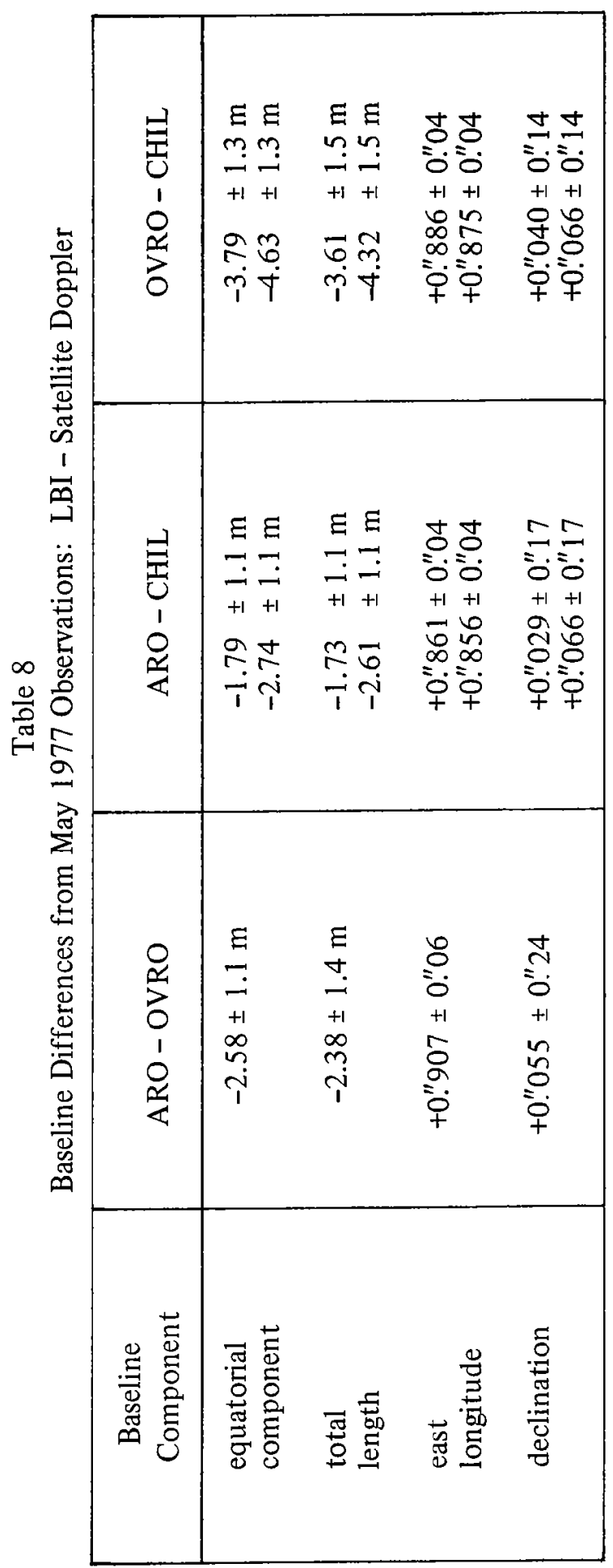




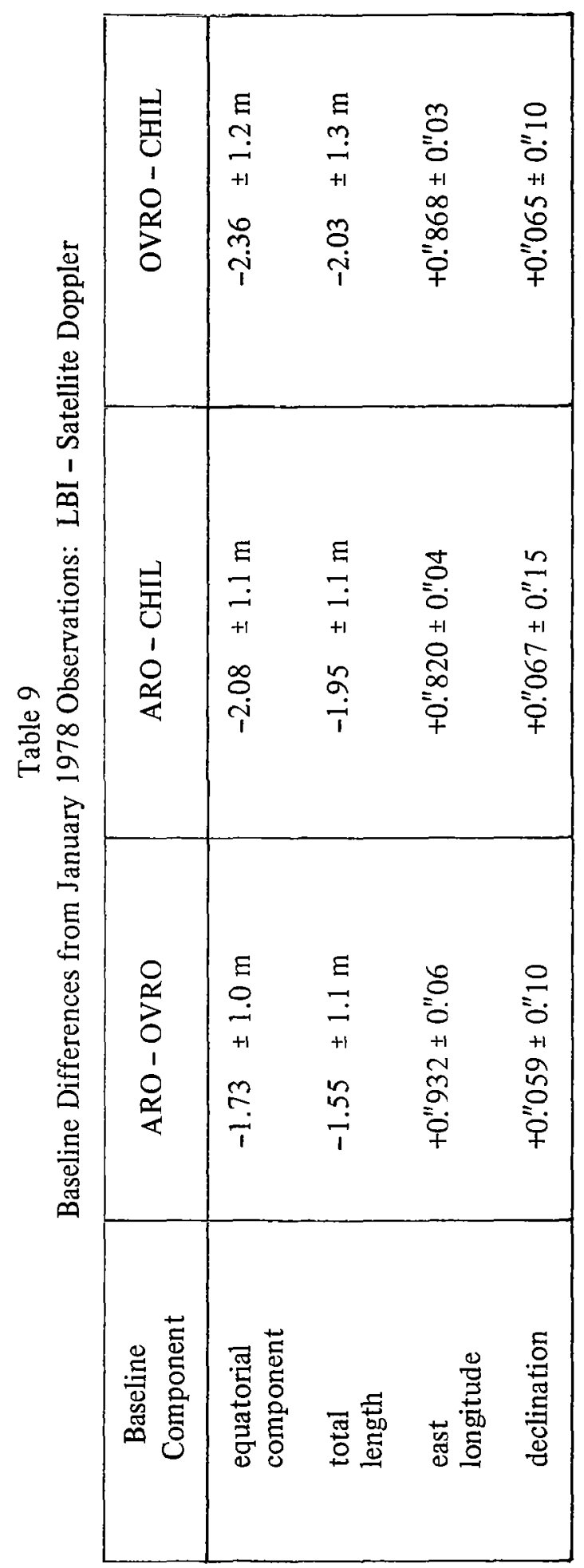


Table 10

Scale, Longitude, and Declination Differences

\begin{tabular}{|l|c|c|c|}
\hline \multicolumn{1}{|c|}{ Baseline } & \multicolumn{1}{c|}{$\begin{array}{c}\text { Scale } \\
(\mathrm{PPM})\end{array}$} & $\begin{array}{c}\text { East } \\
\text { Longitude (") }\end{array}$ & $\begin{array}{c}\text { Declination } \\
(\prime)\end{array}$ \\
\hline May 1977 & & & $0.055 \pm 0.24$ \\
ARO-OVRO & $0.70 \pm 0.41$ & $0.907 \pm 0.06$ & $0.029 \pm 0.17$ \\
ARO-CHIL & $0.33 \pm 0.21$ & $0.861 \pm 0.04$ & $0.066 \pm 0.17$ \\
ARO-CHIL* & $0.50 \pm 0.21$ & $0.856 \pm 0.04$ & $0.040 \pm 0.14$ \\
OVRO-CHIL & $0.46 \pm 0.19$ & $0.886 \pm 0.04$ & $0.066 \pm 0.14$ \\
OVRO-CHIL* & $0.55 \pm 0.19$ & $0.875 \pm 0.04$ & $0.059 \pm 0.10$ \\
& & & $0.067 \pm 0.15$ \\
Jan 1978 & $0.45 \pm 0.32$ & $0.932 \pm 0.06$ & $0.065 \pm 0.10$ \\
ARO-OVRO & $0.37 \pm 0.32$ & $0.820 \pm 0.04$ & $0.06 \pm 0.01$ \\
ARO-CHIL & $0.26 \pm 0.17$ & $0.868 \pm 0.03$ & \\
OVRO-CHIL & & & \\
Weighted Mean & $0.42 \pm 0.05$ & $0.87 \pm 0.01$ & \\
\hline
\end{tabular}

*Based on satellite Doppler observations at Barton Stacey.

coordinate system and the difference between the BIH pole position values used in the analysis of the LBI data and the DMATC pole position values used in the analysis of satellite Doppler data.

\section{EARTH ROTATION MEASUREMENTS BY SATELLITE LINK LBI}

A program to investigate the ability of a long baseline interferometer to measure UT1, pole position, and precession-nutation has recently been undertaken. This program is discussed in detail by Cannon et al. [1979b], and only a brief overview will be given here.

UT1, pole position, and precession-nutation have traditionally been monitored using optical instruments such as photographic zenith tubes (PZTs) and polar telescopes. Recently, the optical methods havc been supplemented by more modern techniques. Of these techniques, satellite Doppler is currently the most widely used. Satellite Doppler pole positions are now routinely determined. Although of higher precision than the optical pole positions they are unfortunately tied to the reference frame of the satellite orbits which slowly drift with respect to an inertial reference frame. Furthermore, satellite Doppler data are relatively insensitive to UT1. Due to these deficiencies in the satellite Doppler technique, LBI is being considered as an alternative technique for supplying accurate pole position and UT1 measurements. 
In Canada, the Earth Physics Branch of the Department of Energy, Mines, and Resources is responsible for the measurement of UT1 and pole position. It contributes both PZT and satellite data to the BIH determinations of these quantities. In March 1978, the Earth Physics Branch entered into a contract with a group of researchers including J. L. Yen of the University of Toronto, J. A. Galt of the Dominion Radio Astrophysical Observatory, S. K. Knowles and W. B. Waltman of the U. S. Naval Research Laboratory, and W. H. Cannon and W. T. Petrachenko of York University, with the collaboration of the Herzberg Institute of Astrophysics of the National Research Council of Canada. The terms of the contract require the coinvestigators to use a long baseline interferometer to measure UT1 with an accuracy at least equal to the accuracy of the best PZTs in existence; i.e., 2-3 ms. Potential for improvement is also expected to be shown along with an evaluation of the ability of a long baseline interferometer to measure pole position.

The use of a long baseline interferometer to measure earth rotation parameters is not a new idea. This potential usage was recognized even before the first successful implementation of LBI in 1967. However, technical problems have delayed the realization of this anticipated use. Since 1967, most of these technical problems have been solved, but the fact that in the operation of a long baseline interferometer, separate oscillators must be used at the ends of the baselines leads to the troublesome possibility of mistakenly interpreting oscillator drifts as real geophysical effects. In order to eliminate the effect of the drifting oscillators, it has been proposed that for this project, the phases of the oscillators be continuously compared via a satellite link. This is a new and significant innovation setting this system apart from other LBI systems attempting to measure earth rotation parameters.

Over the past year, phase link experiments have been performed using the Hermes Communications Technology Satellite. The results of these experiments were encouraging. It was apparent that the major corrupting effect, the satellite Doppler shift, had been reduced below the level of detection. However, small residual phase drifts presently under study still remain. Over measurement periods of about 1 day, these drifts limit the performance of the phase link to approximately the level of a rubidium clock.

On December 15, 1978, Anik B was launched. This is the satellite to be used during the forthcoming experiment to measure UT1. The satellite is now fully operational and the first phase link tests have just been performed (June 1979). Monthly shakedown experiments will follow over the next 3 months, with the first simultaneous use of the phase link with a long baseline interferometer taking place later this year. The interferometer will consist of stations at the Maryland Point Observatory, Maryland Point, Maryland; the Algonquin Radio Observatory, Lake Traverse, Ontario; and the Dominion Radio Astrophysical Observatory, Penticton, British Columbia.

\section{ACKNOWLEDGMENTS}

Many individuals assisted with the LBI and satellite Doppler observations and the correlation of the recorded LBI data. We would like to thank our Canadian colleagues at the Herzberg Institute of 
Astrophysics of the National Research Council of Canada, the Geodetic Survey of Canada, and the Earth Physics Branch of the Department of Energy, Mines, and Resources; our British colleagues at the Appleton Laboratory of the Science Research Council, the Ordnance Survey, the Directorate of Military Survey, and the University of Nottingham; and our American colleagues at the National Geodetic Survey and the Defense Mapping Agency Topographic Center.

The Algonquin Radio Observatory is operated as a national observing facility by the National Research Council of Canada. The Chilbolton Observatory is operated by the United Kingdom Science Research Council. The Owens Valley Radio Observatory is operated by the California Institute of Technology.

This work was supported by a National Research Council of Canada operating grant to W. H. Cannon. R. B. Langley is grateful for a National Research Council of Canada postgraduate scholarship. W. T. Petrachenko was assisted by York University and Province of Ontario postgraduate scholarships.

\section{REFERENCES}

Bureau International de l'Heure, Circular D, Paris, 1978a.

Bureau International de l'Heure, Rapport Annuel pour 1977, Paris, 1978b.

Cannon, W. H., "The Classical Analysis of the Response of a Long Baseline Radio Interferometer," Geophysical Journal of the Royal Astronomical Society, 53, 503-530, 1978.

Cannon, W. H., R. B. Langley, W. T. Petrachenko and J. Kouba, "Geodesy and Astrometry by Transatlantic Long Base Line Interferometry," Journal of Geophysical Research, 84, 229-236, 1979a.

Cannon, W. H., J. Galt, S. H. Knowles, W. 'T. Petrachenko, J. A. Popelar, W. B. Waltman, and J. L. Yen, "Plans for Phase Coherent VLBI for Geophysical Applications Using the ANIK-B Satellite," these proceedings, $1979 \mathrm{~b}$.

Clark, T. A., L. K. Hutton, G. E. Marandino, C. C. Counselman, D. S. Robertson, I. I. Shapiro, J. J. Wittels, H. F. Hinteregger, C. A. Knight, A. E. E. Rogers, A. R. Whitney, B. O. Rönnäng and D. E. H. Rydbeck, "Radio Source Positions from Very-Long-Baseline Interferometry Observations," Astronomical Journal, 81, 599-603, 1976.

Hopfield, H. S. "Tropospheric Effect on Electromagnetically Measured Range: Prediction from Surface Weather Data," Radio Science, 6, 357-367, 1971.

Kouba, J. and J. D. Boal, Program GEODOP, Department of Energy, Mines and Resources, Ottawa, April 1976. 
Langley, R. B., "Precision Geodesy and Astrometry with a Three Station Long Baseline Interferometer," Ph.D. Thesis, York University, Toronto, 1979.

Langley, R. B., W. H. Cannon, W. T. Petrachenko and J. Kouba, “LBI and Satellite Doppler: Baseline Comparisons," Proceedings of the 2nd International Geodetic Symposium on Satellite Doppler Positioning, Austin, Texas, 1979.

Petrachenko, W. T., "Geodetic and Astrometric Analysis of Fringe Frequency Data at $2.8 \mathrm{~cm}$ Wavelength from the Algonquin-Chilbolton Long Baseline Interferometer,' M.Sc. Thesis, York University, Toronto, 1976.

Shapiro, I. I., "Estimation of Astrometric and Geodetic Parameters," in Methods in Experimental Physics, Vol. 12, part C, ed. M. L. Meeks, Academic Press, New York, 1976.

Yionoulis, S. M., "Algorithm to Compute Tropospheric Refraction Effects on Range Measurements," Journal of Geophysical Research, 75, 7636-7637, 1970. 



\title{
PHASE COHERENT VLBI USING A SATELLITE LINK
}

\author{
W. B. Waltman and S. H. Knowles \\ E. O. Hulburt Center for Space Research \\ Naval Research Laboratory \\ J. L. Yen \\ University of Toronto \\ Canada \\ D. N. Fort \\ Herzberg Research Institute \\ National Research Council \\ Canada \\ G. W. Swenson, Jr. \\ University of Illinois \\ W. H. Cannon \\ York University \\ Canada \\ J. Galt \\ Dominion Radio Astronomical Observatory \\ Canada
}

\begin{abstract}
Results will be presented from experiments using the Hermes (formerly CTS) communications satellite to provide a local oscillator and data link between two antennas. The techniques used to compensate for the satellite motion and translation oscillator will be described. Plans for a series of three station experiments to measure UT and polar motion using the ANIK-B satellite to synchronize the local oscillators will be discussed.
\end{abstract}




\section{INTRODUCTION}

In November 1976, our group used the Hermes geosynchronous communications satellite (formerly known as CTS) as a data link for the first successful realtime VLBI experiment. ${ }^{(1)}$ Following this and other experiments, we began work to use the satellite link to obtain full phase coherence for the local oscillator signals as well as to provide a realtime data link. The ability to obtain direct phase data from the interferometer rather than group-delay or fringe-rate data should provide a substantial improvement in accuracy, particularly in the measurement of absolute positions, universal time (UT), and polar motion. It should also permit the use of crystal oscillator or rubidium-vapor frequency standards at the telescope sites in place of the more expensive hydrogen mascrs.

The primary effect which must be cancelled in the phase link is that due to the motion of the satellite. A geosynchronous satellite may exhibit a link delay change of more than $600 \mu \mathrm{sec}$ within a 24hour period because of its non-zero orbital inclination. This is equivalent to more than $10^{6}$ turns at an 18-cm observing frequency. A two-way link must be used to cancel this delay change. A second effect is that of the satellite translation oscillator. A block diagram of the CTS satellite is shown in figure 1. Incoming signals in the $14-\mathrm{GHz}$ uplink band are translated to the $12-\mathrm{GHz}$ downlink band by the crystal controlled oscillator on the satellite. In the Hermes satellite, the same oscillator also generates an $11.7-\mathrm{GHz}$ beacon signal which can be received at each end of the link and used to cancel the effect of the translation oscillator.

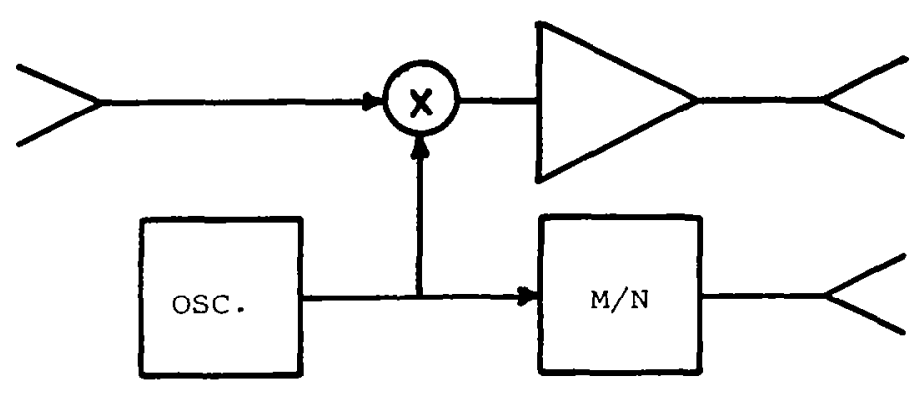

Figure 1. Hermes satellite.

\section{PHASE LINK EXPERIMENT}

The basic technique used for the phase link is shown in figure 2. Rather than generating an actual coherent local oscillator signal, the phase link is used in a separate experiment simultaneously with the VLBI to monitor the phase relationship between the frequency standards at the two sites. To cancel the effects of satellite motion, a dual link is used. The results from the remote site are sent over the data link to be subtracted from the local results giving an output independent of the length of the phase link path. The actual system used is shown in figure 3. It differs from the basic system in three ways. First, the beacon signal is received at each site and used to cancel the satellite translation oscillator as previously mentioned. (The translation oscillator frequency is $5 / 27$ of the beacon frequency.) Second, the pilot signal at the remote end is $14.09-\mathrm{GHz}$ while the pilot signal at the 


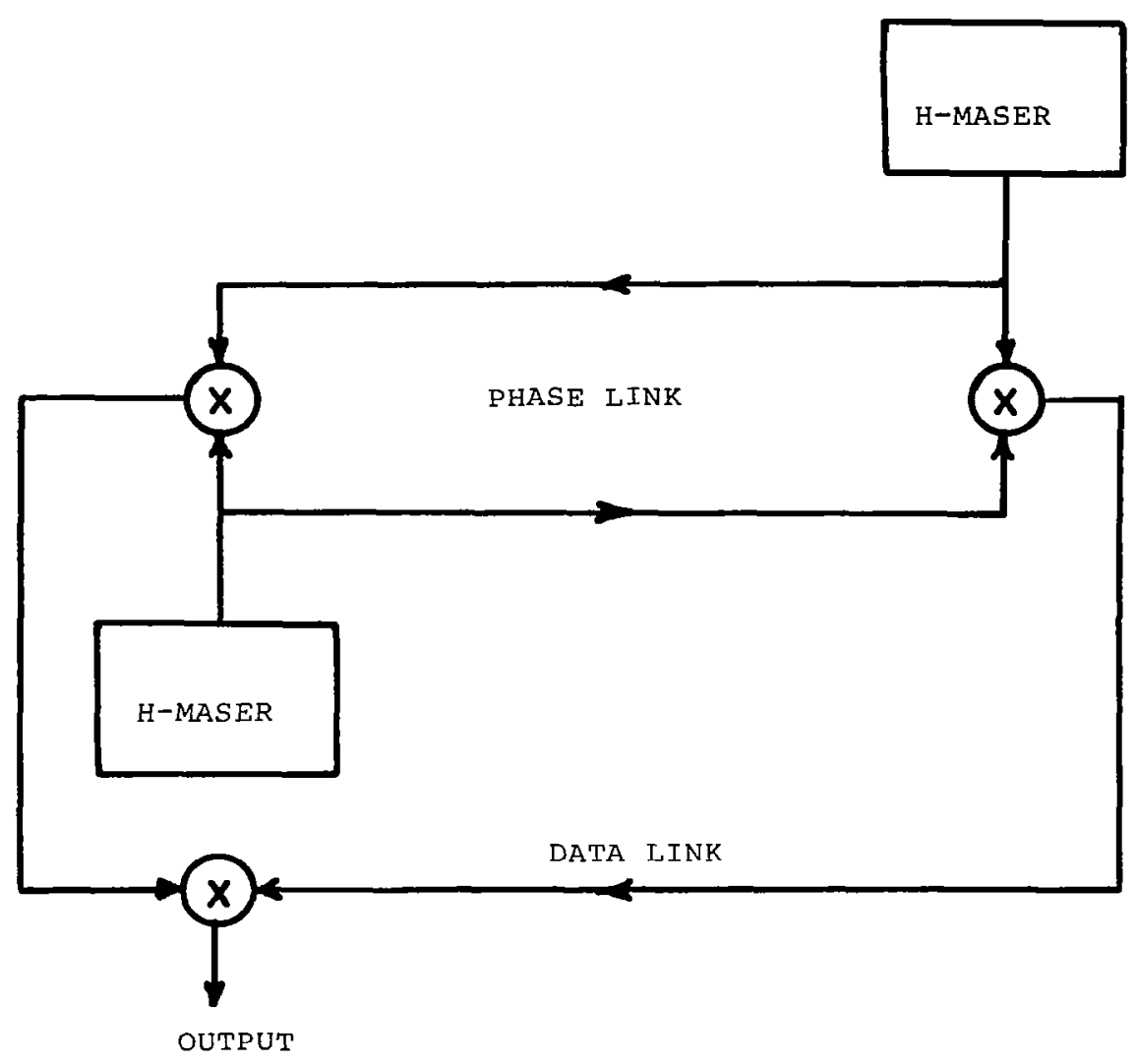

Figure 2. Basic phase measurement link.

local site is $14.23-\mathrm{GHz}$ due to satellite frequency band assignments. Third, the final outputs from the local and remote sites are separately recorded by the computer for later processing.

The computer post-processing interpolates over data gaps due to loss of data sync, antenna peaking, etc., multiplies the remote phase by $14.09 / 14.23$ to correct for the different pilot frequencies, and computes the difference between the local and remote phase values. An arbitrary phase zero is established at the beginning of each run and the phase is tracked for the duration of good data. The constant frequency offset of the two hydrogen masers is subtracted from all results.

\section{EXPERIMENTAL RESULTS}

This link was operated successfully in May 1978 and November 1978 between the Algonquin Radio Observatory, Lake Traverse, Ontario, and the Owens Valley Radio Observatory of California Institute of Technology, Big Pine, California. The May experiment yielded only short runs of data due to difficulties with the digital data link. The results of the November experiment are shown in figures 4 and 5 . These results show the measured difference in phase between the two masers, with the 


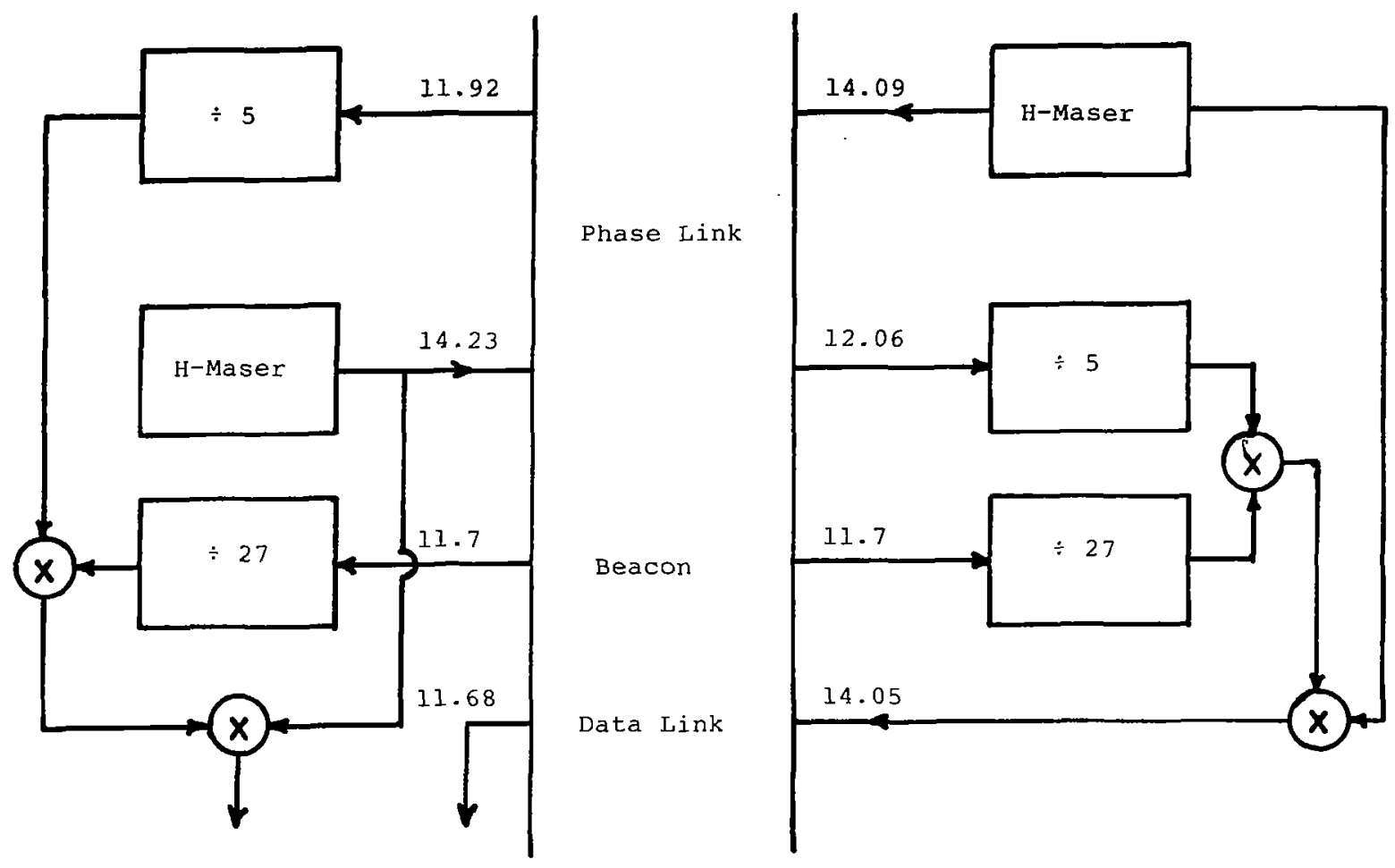

Figure 3. Hermes phase link.

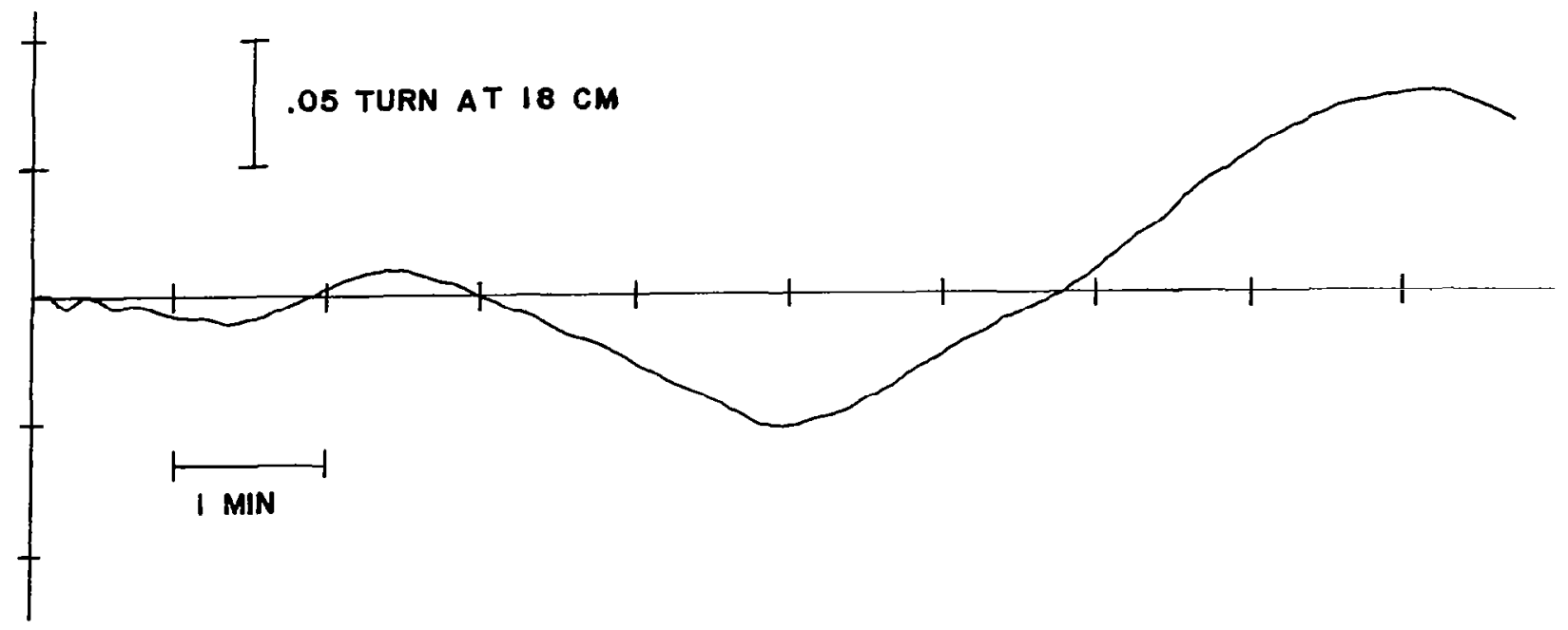

Figure 4. Hermes phase link results. 
DAY 329

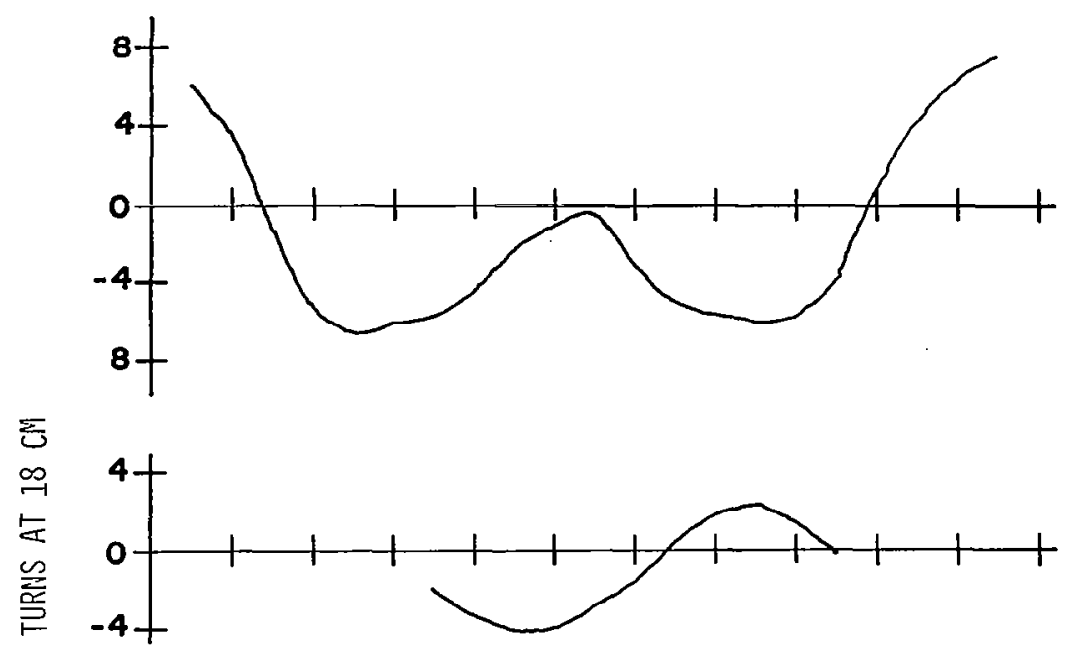

DAY 332

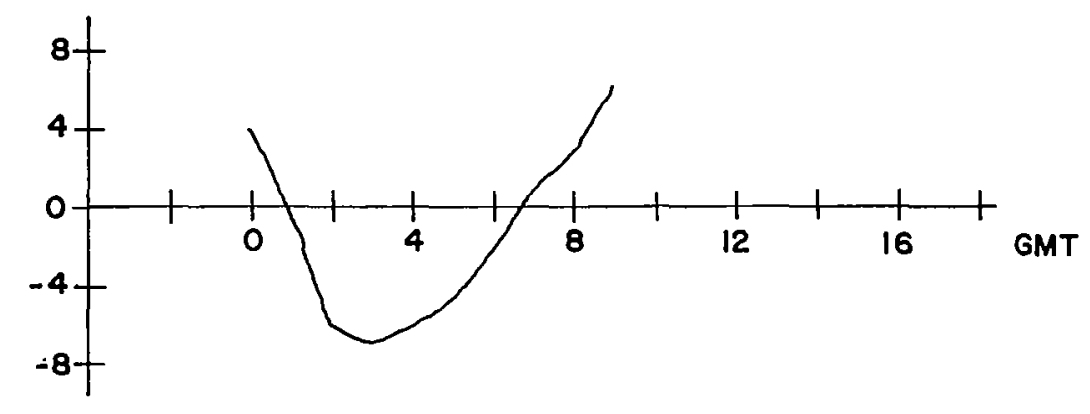

Figure 5. Hermes phase link results - Nov. 1978.

frequency offset removed, normalized to the $18 \mathrm{~cm}$ wavelength of the VLBI experiment. The short term data in figure 4 has been smoothed with a 300 -second filter.

The short term data show that the signal-to-noise of the link is sufficient, so only a good crystal oscillator, or at most a rubidium frequency standard, would be needed at each site. The long term data show no evidence of a 24-hour period indicating that the $10^{6}$ turns due to the satellite motion have been totally cancelled. The result obtained of \pm 6 turns over 18 hours $( \pm 3.5 \mathrm{nsec})$ is still not as good as we had hoped. We believe most of the observed phase change is probably in the cables and electronics used in the link. Figure 6 shows a comparison of the present phase link data with the laboratory performance of hydrogen masers and rubidium standards. We expect that with calibration and monitoring of the instrumental phase delays, the link performance will exceed that of the hydrogen maser for times greater than 1 day. 


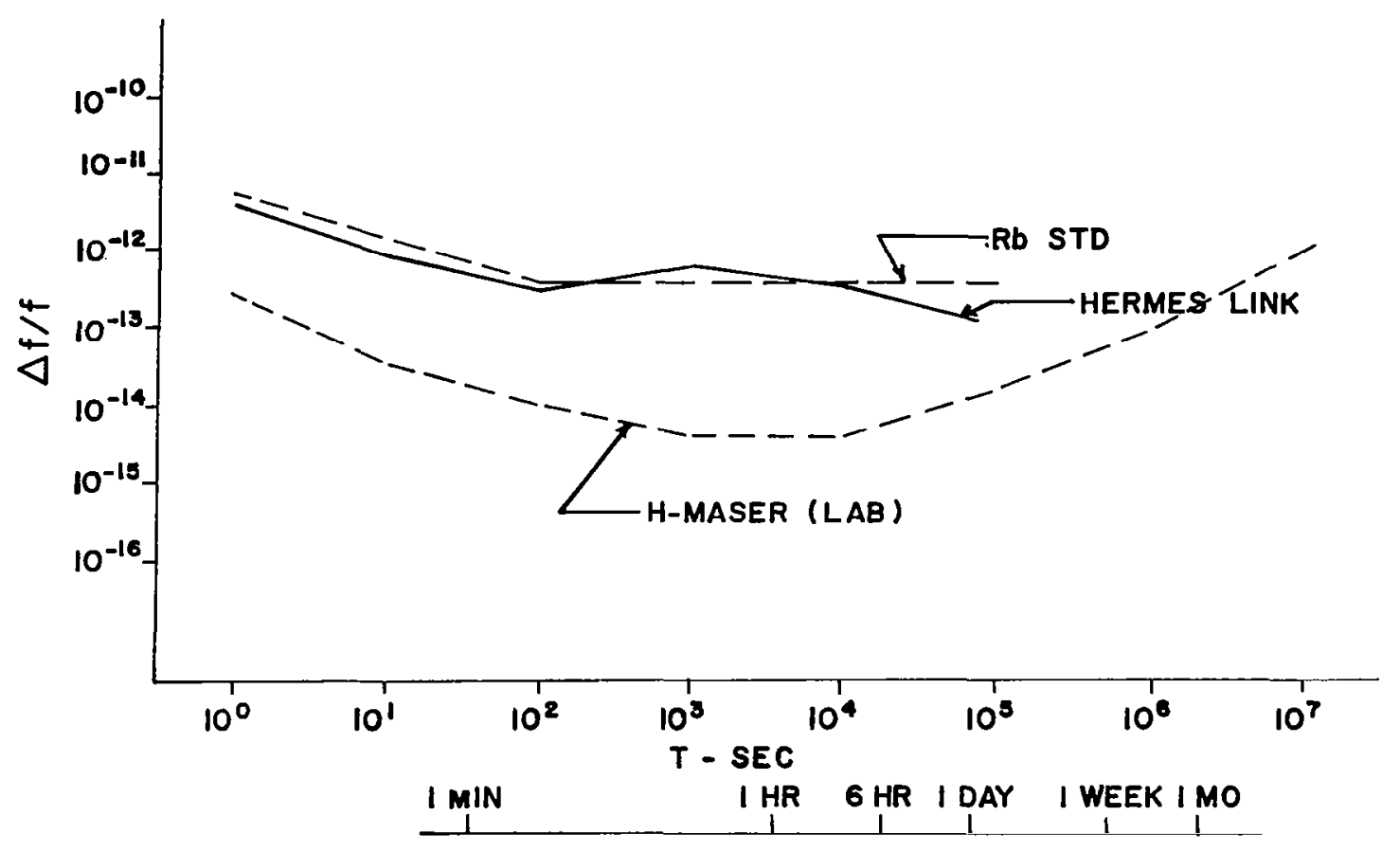

Figure 6. Comparison of phase stability.

\section{FUTURE PLANS}

This was our last opportunity to use the Hermes satellite; however, our efforts will continue with a program to use the ANIK-B satellite in a series of three station experiments to measure UT and polar motion. A detailed description of this system is given in another paper. ${ }^{(2)}$

\section{REFERENCES}

(1) J. L. Yen, K. I. Kellermann, Benno Rayhrer, G. W. Broten, D. N. Fort, S. H. Knowles, W. B. Waltman, and J. W. Swenson, Jr., Science, 198, 21 October 1977.

(2) W. H. Cannon, W. T. Petrachenko, J. L. Yen, J. A. Galt, W. B. Waltman, S. H. Knowles, J. Popelar, "Plans for Phase Coherent Long Baseline Interferometry for Geophysical Applications Using the ANIK-B Communications Satellite," Symposium on "Radio Interferometry: Techniques for Geodesy," MIT, June 19-21, 1979. 
SESSION B

MOVEMENTS TERRESTRIAL AND CELESTIAL 


\title{
SCANDINAVIA STUDIES OF RECENT CRUSTAL MOVEMENTS AND THE SPACE GEODETIC BASELINE NETWORK
}

\author{
Allen Joel Anderson \\ Institute of Geophysics \\ The University of Uppsala
}

\begin{abstract}
A brief review of crustal movements within the Fenno-scandia shield is given. Results from postglacial studies, projects for measuring active fault regions, and dynamic ocean loading experiments are presented.

The 1979 Scandinavian Doppler Campaign (SCANDOC I) Network is discussed. This network includes Doppler translocation baseline determination of future VLBI baselines to be measured in Scandinavia. Intercomparison of earlier Doppler translocation measurements with a high precision terrestrial geodetic baseline in Scandinavia has yielded internal agreement of $6 \mathrm{~cm}$ over $887 \mathrm{~km}$. This is a precision of better than 1 part in $10^{7}$.
\end{abstract}




\section{INTRODUCTION}

Scandinavia is an ideal place for testing theories of recent crustal movements. Historically, there is a detailed record of the Fenno-scandia uplift provided for by local water level observations carefully recorded for periods of over one thousand years. Furthermore, apart from this well studied phenomenon, the Fenno-scandia shield is known to be one of the most stable plates on the earth, with a seismic background far below that of most other parts of the crust.

For these reasons, Heiskanen (1958), Cathles (1975), and others have used the Fenno-scandia uplift data to provide the best overall measurements of the viscosity and behavior of the earth's mantle to surface loads. The relaxation time, or $1 / \mathrm{e}$ value, has been shown to be about $10^{4}$ years. Detailed models using various hypotheses have been generated for the region. Predictions on the uplift phenomenon can be very accurate over the entire shield area.

Detailed records of earthquake activity have also been kept. The largest earthquake in recent time occurred in the Oslo fjord in the year 1904. Its estimated Richter magnitude was 6.5. Since that year, a seismograph has been in continual operation at Uppsala. This earthquake and the seismicity of Scandinavia have been the subject of papers by Husebye, Ringdal, and Lande (1976), Båth (1977), and others.

Two large aperture seismic array stations are operated in Scandinavia; one in Norway, one in Swcden. The station at Hagfors in Sweden has been used for the basis of a recent book on the detection of underground nuclear explosions (Dahlman and Israelsson, 1978).

It is clear then that the Scandinavian area provides a good control ground for testing theory against measurement for recent crustal movements.

\section{RECENT STUDIES OF DYNAMIC CRUSTAL MOVEMENT}

Figure 1 shows the extent of post-glacial uplift measured in Sweden using shore line data by Mörner (1979). These values agree well with precise levelling data obtained over the past 50 years by the Swedish Survey Office. The vertical rate approaches $1 \mathrm{~cm} / \mathrm{yr}$ at the upper part of the gulf of Bothnia. Vertical uplift gradients are sometimes very steep, leading to some local seismicity.

Figure 2 has plotted all earthquakes which have occurred in Sweden above magnitude 2.5 during the 1951-1976 period, with data from Båth (1977). It can be seen that the level of seismic activity is small and is rclated to two main areas, that along the coast of the gulf of Bothnia and that around Lake Vattern, an ancient fault in south central Sweden.

Crustal loading by the ocean tides has been the topic of a recent investigation (Anderson (1976). Figure 3 shows a time series obtained with a geodynamics recording gravity meter in central Finland. The spectral analysis of this record indicates a peculiar signal believed to be caused by the resonance 


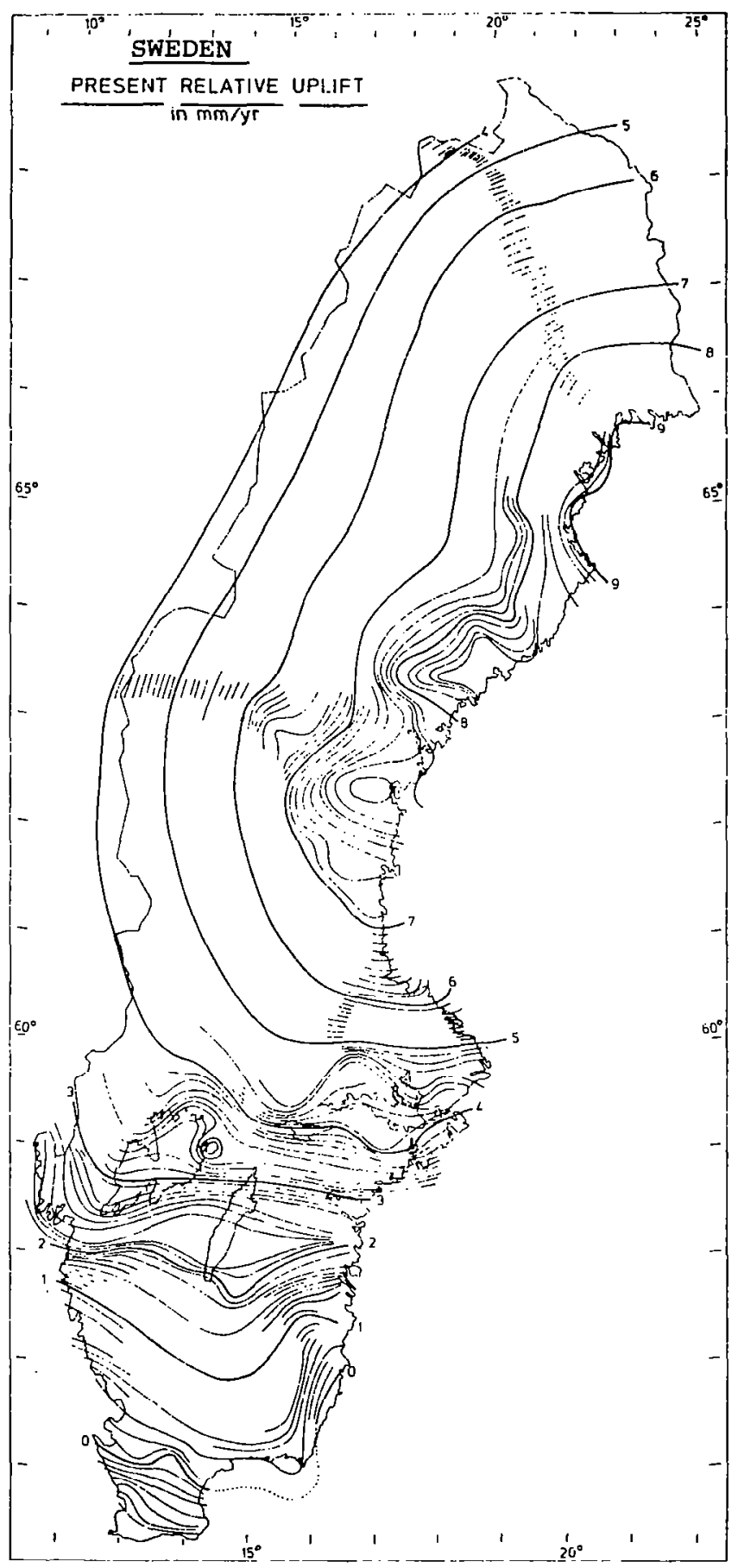

Figure 1. Present uplift in Scandinavia caused by post-glacial rebound (Mörner, 1979). 


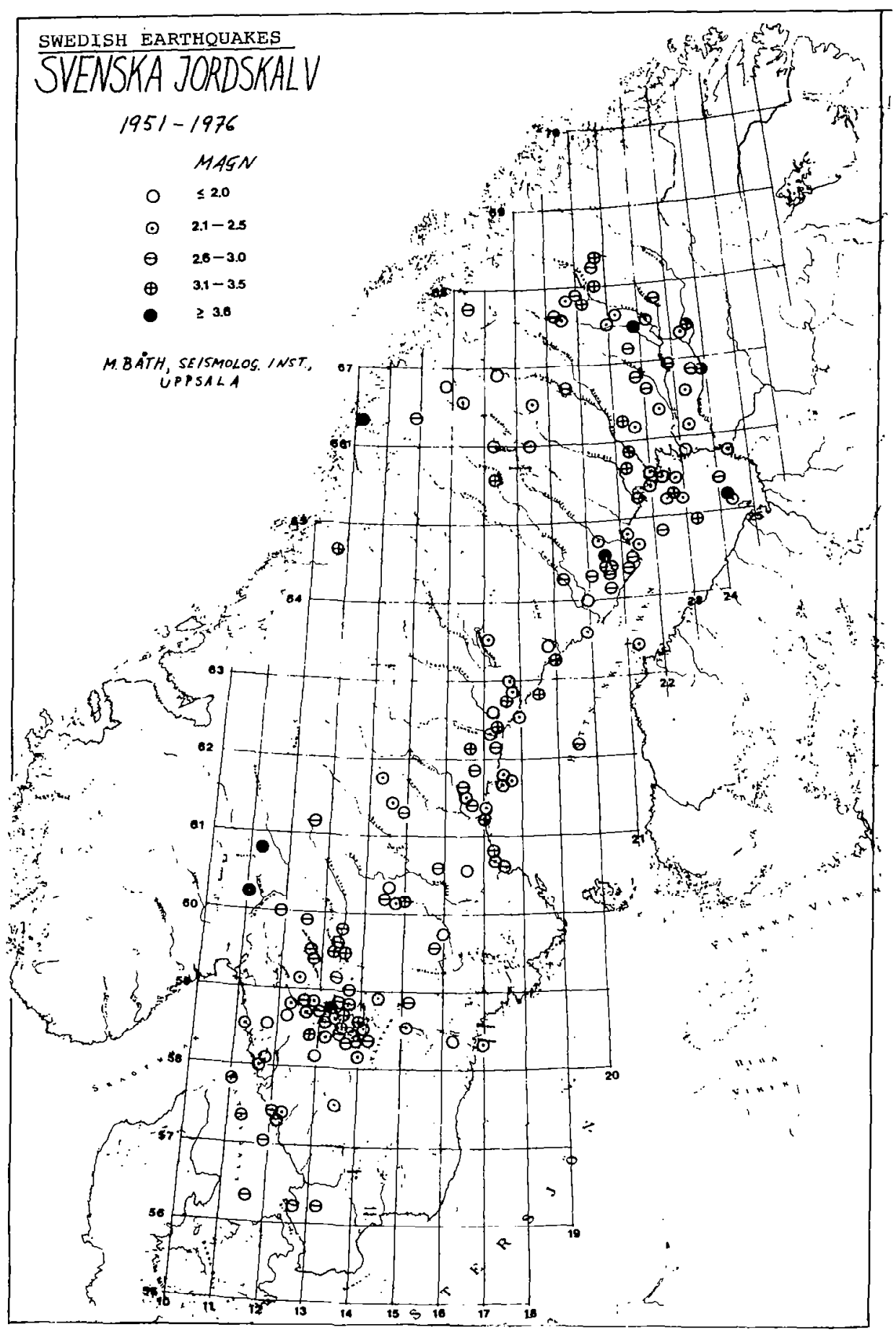

Figure 2. Recent earthquakes in Sweden (Bath, 1977). 

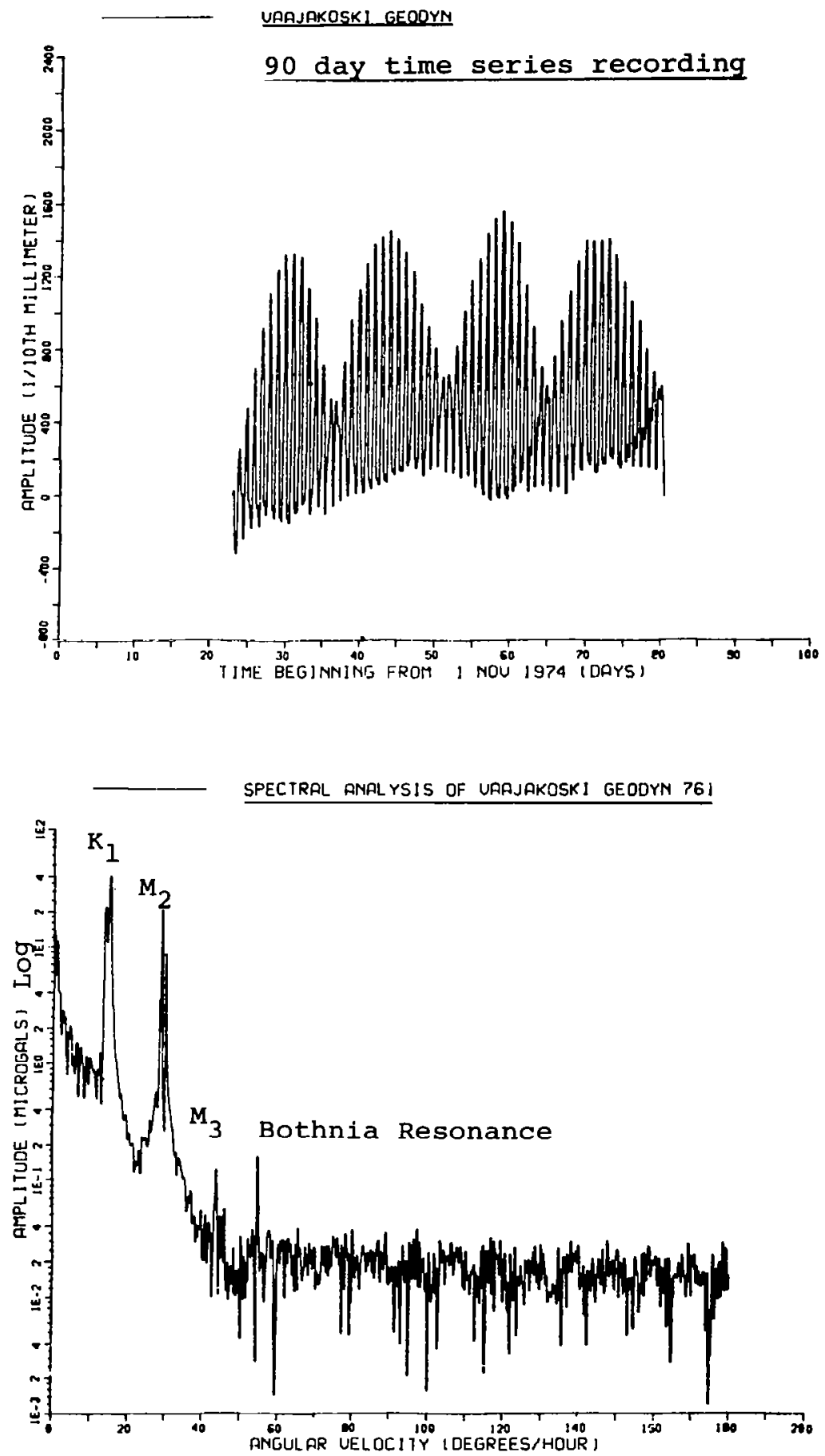

Figure 3. Solid-earth tidal gravity recording showing spectra and Bothnia Gulf resonance effect (Anderson, 1976). 
of the Gulf of Bothnia. This resonance causes areas perpendicular to the gulf to move up and down by several tenths of a centimeter with a period of 6.59 hours. A further study of horizontal tilt was made along the Scandinavian peninsula and models for the global ocean loading terms were considered (Anderson (1977)).

A study of the secular change of gravity in the area of uplift is being carried out by the Finnish Geodetic Institute and the Swedish Survey Office.

The Swedish Geological Survey is studying what are believed to be ancient active faults activated by post-glacial rebound.

\section{THE SPACE GEODETIC NETWORK}

To coordinate these activities on the study of recent crustal movements, Anderson (1978) presented a paper at the Nordic Geodetic Commission meeting in the hope that modern Doppler and VLBI methods would be able to provide the first confirmation of plate motion to the Scandinavian Shield area and provide a means of mapping local crustal displacements.

In coordination with the National Survey Offices of each Scandinavian country and in cooperation with the Finnish Geodetic Institute and the Institute of Geophysics at Uppsala, the Scandinavian Doppler Campaign for 1979 (SCANDOC I) was carried out in May 1979. Ten individual receivers were used in the translocation mode (relative Doppler). It is expected that such measurements shall be repeated and improved regularly throughout the 1980's.

Figure 4 shows the SCANDOC I network and indicates the future VLBI baselines expected to be measured in the 1980's. An earlier joint VLBI-Doppler experiment was carried out between Onsala and Bonn in 1975 (Campbell and Beyer (1978)), and a further one was repeated in 1978.

Comparison with the Finnish precision baseline and relative Doppler has been made during 1977-78. On the basis of 3 months of data, an agreement of $6 \mathrm{~cm}$ was obtained between the measured baseline results (Kakkuri, 1979) and the relative Doppler value (Mårtensson, 1978).

Figure 5 shows a polar projection of the VLBI baselines considered for study in this paper. An estimate of the expected horizontal displacement rate due to known geophysical phenomenon is given in table 1. Correction terms for land uplift are calculated, and it is shown that these terms are dominant for Scandinavian Plate baselines. As much as 26 percent in addition to the mid-Atlantic term needs to be taken into account for the Haystack-Sodankylä baseline. 


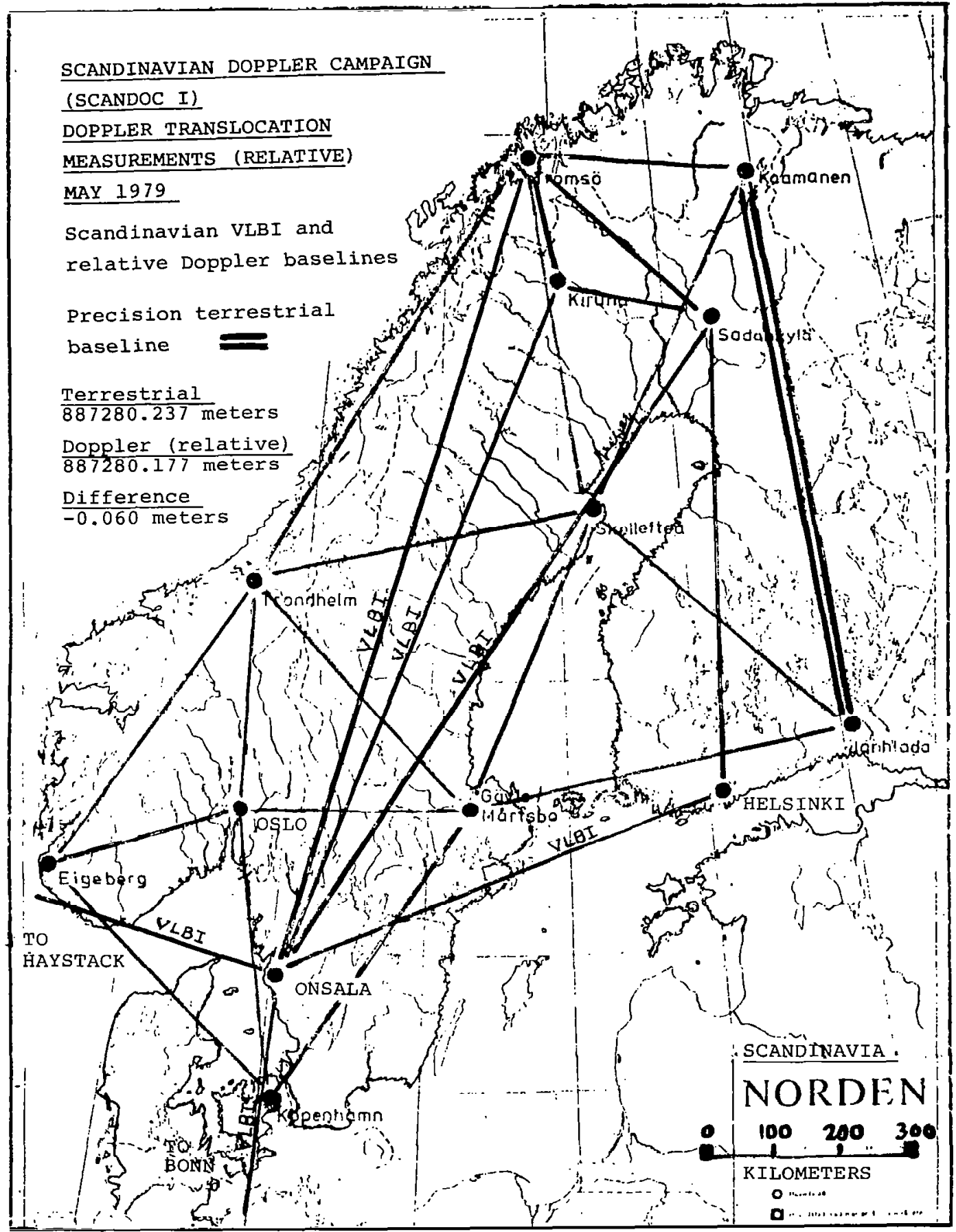

Figure 4. Scandinavian Space Geodetic Network showing SCANDOCI and future VLBI baselines. Comparison of precise terrestrial and relative Doppler made (Kakkuri, 1979). 


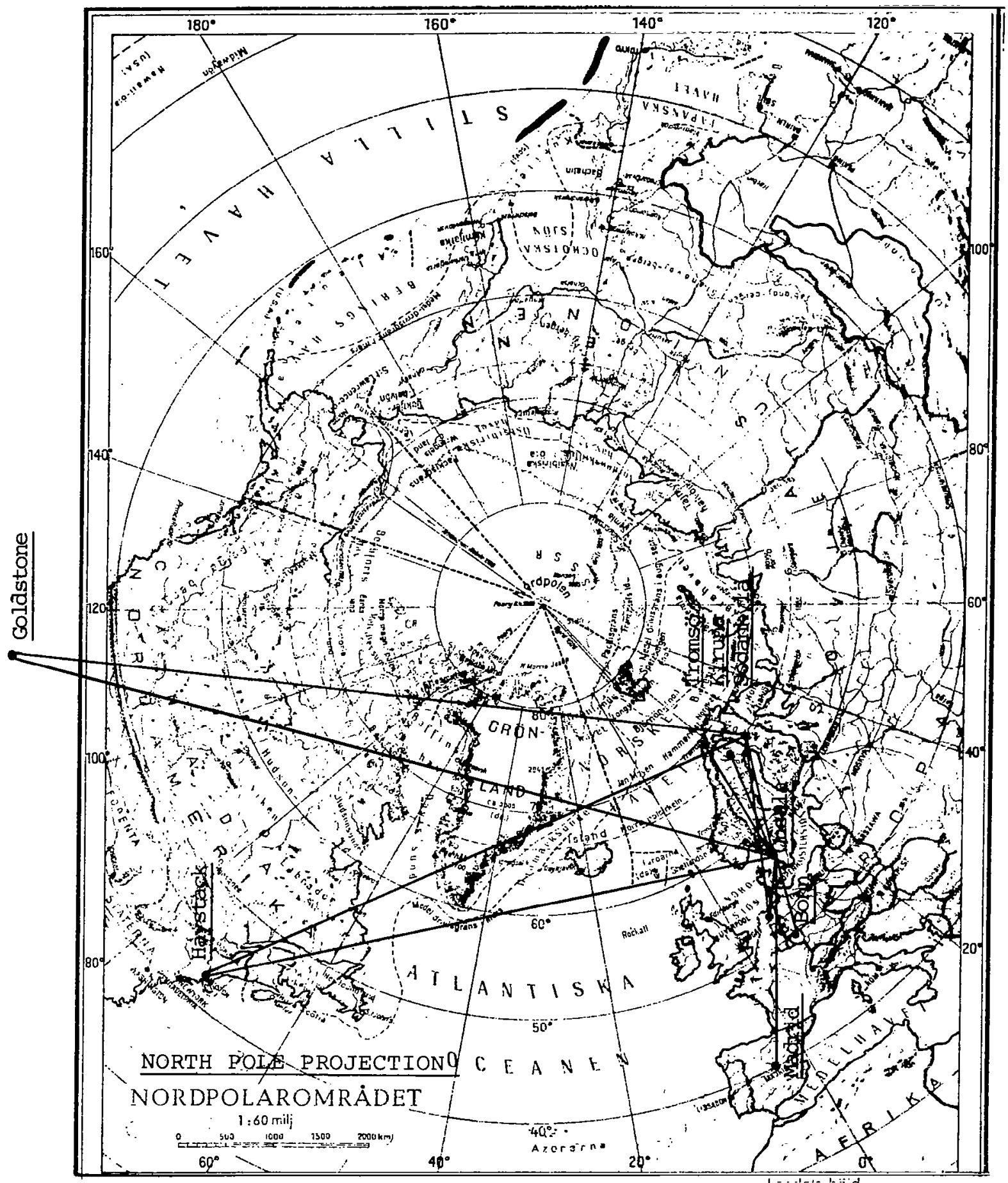

Figure 5. Polar projection of VLBI baselines planned for use with Scandinavian stations. Expected horizontal strain rates and uplift corrections are given in table 1. 
Table 1

Rates of Uplift, Baseline Rates, Estimated Horizontal Strain Release vs. Uplift Baseline Rates for Scandinavian VLBI Baselines

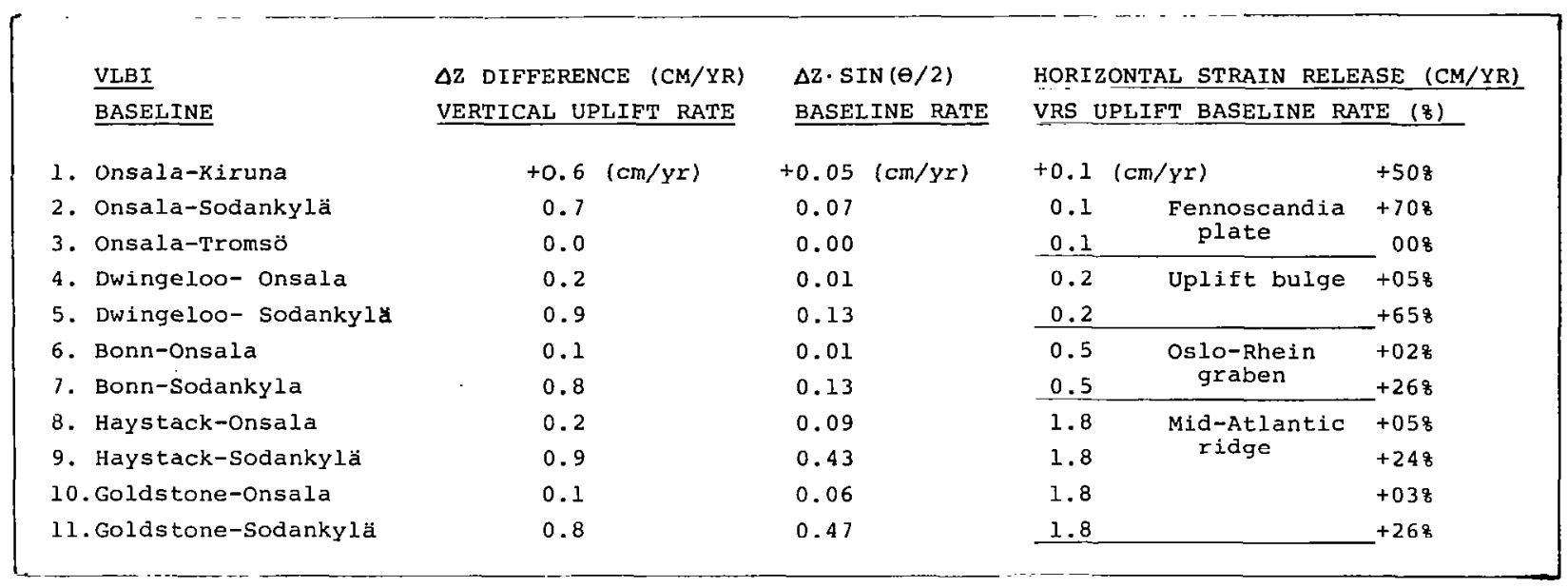

\section{CONCLUSIONS}

It has been demonstrated that the Scandinavia area provides an excellent area for testing theories of crustal motion. It provides the addition of a stable, well-studied crust complimented with long sets of geodynamic data and well-refined solutions. It also provides for sets of unique VLBI baselines with many of the world's observatories due to its proximity to the pole.

\section{REFERENCES}

Anderson, A. J. (1976) Geodynamic Studies using earth tide instruments. ACTA UNIVERSITATIS UPSALIENSIS, $\underline{396}$.

Anderson, A. J. (1977) Spectral separation of effects on solid-earth tidal records in Scandinavia, Geologiska Föreningens i Stockholm förhandingar, 99, 84-86.

Anderson, A. J. (1978) Satellite and ground based geodynamic measurements: A European Space Agency project for the 1980 's. In Proc. $8^{\text {th }}$ Nordic Geodetic Commission, Oslo.

Båth, M. (1977) Seismicity of Sweden and related problems, report from the Seismological Institute, Uppsala.

Campbell, J. and Beyer, W. (1978) Onsala-Effelsberg baseline components from January, 1974 GOOF-2 VLBI experiment. (personal communication). 
Cathles, L. M. (1975) The Viscosity of the Earth's Mantle, Princeton University Press.

Dahlman, O. and Israelsson, H. (1978) Seismic Methods of Detection of Underground Nuclear Explosions, Elsevier Publ. Co., Amsterdam.

Heiskanen, W. A. and Vening Meinesz (1958) The Earth and its Gravity Field, McGraw-Hill, Ncw York.

Husebye, E., Ringdal, F. and Lande, G. (1976) Jordskalvet i Oslofjörden-Nordens största i moderntid, Forskning och Framsteg, $\underline{76}, \underline{6}$.

Kakkuri, J. (1979) A recalculation of the precise Finnish Baseline of 887 Kilometers, Report from the Finnish Geodetic Institute, Helsinki.

Mårtensson, S. G. (1978) Jämförelse mellan utjämnade Translokations-mätningar och "point position" beräkningar för fyra Nordiska stationer, in Proc. $8^{\text {th }}$ Nordic Geodetic Commission, Oslo.

Mörner, N. A. (1979) Earth Rheology, Isostacy and Eustacy, John Wiley \& Sons, London. 


\section{GEODETIC STRATEGIES FOLLOWING LARGE DIP-SLIP EARTHQUAKES}

\section{J. J. Melosh, A. Raefsky, M. A. Slade}

Jet Propulsion Laboratory

Presented but not submitted. 


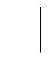




\title{
THE MEASUREMENT OF EARTH ROTATION ON A DEFORMABLE EARTH
}

\author{
W. H. Cannon* \\ Earth and Environmental Sciences \\ York University
}

\begin{abstract}
Until recently, the methods of geodetic positioning on the earth were limited to a precision of roughly one part in $10^{6}$. At this level of precision, the earth can be regarded as a rigid body since the largest departure of the earth from rigidity is manifested in the strains of the earth tides which are of the order of one part in $10^{7}$. Long baseline interferometry is expected to routinely provide global positioning to a precision of one part in $10^{8}$ or better. At this level of precision, all parts of the earth's surface must be regarded as being, at least potentially, in continual motion relative to the geocenter as a result of a variety of geophysical effects.
\end{abstract}

This paper discusses the general implications of this phenomenon for the theory of the earth's rotation and focuses particular attention on the question of the measurement of the "earth's rotation vector" on a deformable earth.

\footnotetext{
*Present! address until September 1, 1979: Jet Propulsion Laboratory, California Institute of Technology, 4800 Oak Grove Drive,
} Pasadena, California, 91103. 


\section{INTRODUCTION}

Almost 10 years ago, a paper entitled "Geodetic Ties Between Continents by Means of Radio Telescopes" [Jones, 1969] appeared in the journal Canadian Surveyor. The author, Harold Jones of the Geodetic Survey of Canada, reported on what was probably the first geodetic application of the technique of long baseline interferometry (LBI). Jones' solution for the $2400 \mathrm{~km}$ baseline vector between antennas at Prince Albert, Saskatchewan, and Algonquin Park, Ontario, yielded a "correction" of $30 \mathrm{~m} \pm 20 \mathrm{~m}$ to the value previously determined by ground-based survey methods.

Baseline vectors of comparable magnitude are now routinely determined by LBI to an accuracy of $\pm 10 \mathrm{~cm}$ to $\pm 100 \mathrm{~cm}$. This represents an improvement of two orders of magnitude in 10 years and stands as a testimonial to the determination and skill of those who have worked to develop the technique over the past decade. So dramatic has been this development that LBI measurements of the near future, with expected accuracies of $\pm 1 \mathrm{~cm}$ to $\pm 5 \mathrm{~cm}$ between continents, coupled with other known geophysical effects, such as continental drift, threaten to render obsolete our fundamental definitions of the body-fixed and space-fixed coordinate frames which serve as a basis for defining positions on the earth and their motion relative to inertial space. In discussing this issue, it is useful to present a brief historical description of the evolution of these concepts, for the present situation is best understood in terms of its past history. This past history is in turn often useful in indicating the direction of the next logical step in the development.

The rotation of the earth or the earth's orientation in space is specified by the time dependent transformation between a set of body-fixed basis vectors $\left(\widehat{\mathrm{e}}_{1}, \widehat{\mathrm{e}}_{2}, \widehat{\mathrm{e}}_{3}\right)$ conceived of as being "attached" to the earth and a set of space-fixed basis vectors $\left(\widehat{\mathrm{E}}_{1}, \widehat{\mathrm{E}}_{2}, \widehat{\mathrm{E}}_{3}\right)$ conceived of as being "attached" to space. In general, the time dependent transformation can be represented as

$$
\widehat{\mathrm{e}}_{\mathrm{i}}(\mathrm{t})=\mathrm{T}_{\mathrm{ij}}(\mathrm{t}) \widehat{\mathrm{E}}_{\mathrm{j}}
$$

where the transformation matrix $T_{i j}(t)$ expresses all that is known about the earth's rotation. (Explanatory Supplement to the Astronomical Ephemeris and the American Ephemeris and Nautical Almanac, 1960; Mueller, 1969). Our understanding of $\mathrm{T}_{\mathrm{ij}}(\mathrm{t})$, which has progressively improved historically, can be usefully classified into six distinct eras.

\section{Ancient Era}

Since ancient times, mankind has identified two great circles on the celestial sphere. The first was the equator whose location on the celestial sphere was defined by the diurnal rotation of the earth. The second was the ecliptic whose location on the celestial sphere was defined by the annual motion of the brightest object in the sky - the sun.

According to the ancients, these two great circles were fixed on the sky, providing reference directions and a celestial coordinate frame with which to develop elaborate sky maps populated with 
mythical characters. The zodiac, which is referenced to the ecliptic and the equator, dates from these early times.

If these early conceptions were to be updated into modern language, they could be described by the introduction of two sets of basis vectors with a common origin at the center of the earth consisting of:

(a) a space-fixed set of basis vectors, $\widehat{E}_{1}, \widehat{E}_{2}, \widehat{E}_{3}$, such that $\widehat{E}_{1}$ is contained by the equator and the ecliptic at the ascending node, $\widehat{\mathrm{E}}_{3}$ is oriented toward the pole of the ecliptic, and $\widehat{\mathrm{E}}_{2}$ makes up a right-handed orthogonal triad; and

(b) a body-fixed set of basis vectors, $\widehat{\mathrm{e}}_{1}, \widehat{\mathrm{e}}_{2}, \widehat{\mathrm{e}}_{3}$, such that $\widehat{\mathrm{e}}_{3}$ is parallel with the earth's rotation axis, $\widehat{e}_{1}$ is contained by the intersection of the equator and the prime meridian, and $\widehat{\mathrm{e}}_{2}$ makes up a right-handed orthogonal triad.

With the exception of the sun, the moon, and the five planets observable with the naked eye which were deified for their persistent anomalous behavior, the ancients described all motion by spinning the earth at a uniform rate about an axis fixed in the earth and fixed in space. The mathematical transformation between the space-fixed basis vectors and the body-fixed basis vectors could be represented by the introduction of a time dependent spin matrix $S_{i j}(t)$.

$$
\widehat{e}_{i}(t)=S_{i j}(t) \widehat{E}_{j}
$$

The quantities $d S_{i j} / d t$ were considered to be constants since the earth's rotation was held to be uniform.

\section{The Classical and Medieval Era (500 B.C. - 1600 A.D.)}

During this period, the precision with which astronomical observations could be made steadily improved, as did the mathematical models required to make astronomical predictions of eclipses, occultations, and other periodic celestial phenomena. During this era, no truly dynamic theories of celestial motions were introduced, and the algorithms on which calculations depended were, for the most part, highly geometrical. The principal advance in the knowledge of the earth's rotation which occurred in this era was the discovery by Hipparchus, circa 150 B.C., of the precession of the equinoxes.

Following Hipparchus' discovery, previously held conceptions of the space-fixed basis vectors $\widehat{\mathrm{E}}_{\mathrm{i}}$ were retained; however, it was necessary to redefine the space-fixed basis vectors as being the $\widehat{\mathrm{E}}_{\mathbf{i}}$ referenced to some celestial pole and equinox of a particular epoch. The body-fixed basis vectors $\widehat{\mathrm{e}}_{\mathrm{i}}$ retained their previous definitions. 
In this era, the practice of realizing the body-fixed basis vectors by assigning numerical coordinates to observatories fixed on the earth was developed. A similar practice of realizing the space-fixed basis vectors by assigning numerical coordinates to stars at a particular epoch led to the development of extensive star catalogues.

With the discovery of precession, the mathematical transformation between the space-fixed basis vectors and the body-fixed basis vectors required the introduction of the time dependent precession matrix $P_{i j}(t)$.

$$
\widehat{\mathrm{e}}_{\mathrm{i}}(\mathrm{t})=P_{\mathrm{ij}}(\mathrm{t}) \mathrm{S}_{j k}(\mathrm{t}) \widehat{\mathrm{E}}_{\mathrm{k}}
$$

The quantities $d S_{i j} / d t$ were still held to be constants.

\section{The Newtonian Era (1600 A.D. - 1800 A.D.)}

The Newtonian era was characterized by the rapid development of Newton's dynamical theory and its extensive application to geodynamics and problems of the earth's rotation. Around the year 1680 , Newton offered an explanation of the precession of the equinoxes and predicted the oblate figure for the earth on the basis of his theory of gravitation. At about the same time, Newton and Huyghens published a predicted value for the polar flattening of the earth.

These important geophysical developments were being matched by corresponding dramatic astronomical discoveries. In 1718, the astronomer Edmund Halley announced the discovery of stellar proper motion, and the researches of the astronomer James Bradley, circa 1728, led to his discovery of both stellar aberration and astronomical nutation. This was followed in 1735 by the geodetic surveys in Finland and Peru under Pierre Bouguer of the French Academy which verified Newton's prediction of polar flattening and confirmed the theory of precession and nutation.

The discoveries of the Newtonian era placed the definition of the space-fixed coordinate frame on a firm dynamical footing. As a result of the discovery of the periodic phenomenon of nutation, it became necessary to reference the space-fixed basis vectors $\widehat{\mathrm{E}}_{\mathbf{i}}$ to the mean equator and ecliptic of a particular epoch, averaging out the small periodic effects of nutation on the location of the equator.

In addition, the discoveries of the Newtonian era made it necessary to realize the space-fixed basis vectors $\widehat{E}_{i}$ by assigning coordinates, not to the stars themselves which were no longer regarded as being fixed, but to locations on the celestial sphere known as "mean stellar positions of epoch" which were related to the actual stellar positions by:

(a) the removal of the effects of proper motion since the epoch, and

(b) the averaging out of the periodic effect of annual aberration. The relatively constant contribution to the aberration arising from the effects of the eccentricity of the earth's orbit were retained in the stellar mean position. 
The body-fixed basis vectors retained their classical and medieval definition and continued to be realized by assigning coordinates to observatories fixed on the earth. The meridian through Greenwich was widely adopted as the prime geographic meridian at this time.

With the discovery of nutation, it became necessary to introduce a third time dependent matrix, the nutation matrix $\mathrm{N}_{\mathrm{ij}}(\mathrm{t})$, into the mathematical transformation between the space-fixed basis vectors $\widehat{E}_{i}$ and the body-fixed basis vectors $\widehat{\mathrm{e}}_{\mathbf{i}}$.

$$
\widehat{\mathrm{e}}_{i}(t)=P_{i j}(t) N_{j k}(t) S_{k \ell}(t) \widehat{E}_{\ell} .
$$

The quantities $\mathrm{dS}_{\mathrm{ij}} / \mathrm{dt}$ were still held to be constant.

\section{The Euler/Kelvin Era (1800 A.D. - 1900 A.D.)}

The Euler/Kelvin era was characterized by two major developments in the theory of the earth's rotation. The first was inspired by the work of Leonard Euler who in 1765 published his famous treatise on the dynamics of rigidly rotating bodies. Euler showed that for an oblate spheroid such as the earth, the rotation axis, the angular momentum axis, and the figure axis will not coincide in general and furthermore, that in the event that they do not coincide the rotation axis cannot remain fixed within the body.

As a consequence of Euler's work, a search was begun circa 1840 for evidence of the effects of Eulerian motion on the earth's rotation. This search persisted for 50 years before achieving success, and at one time or another involved the active participation of Peters, Bessel, Maxwell, Kelvin, Newcomb, Künster, and Chandler (Munk and MacDonald, 1960).

The second major development of this era was inspired by the work of Kelvin (W. Thompson, 1863) who in 1863 was the first to introduce in to theoretical geodynamics considerations of the deformability of the earth. Kelvin's seminal work on tidal deformations of the earth stimulated the growth of an entire branch of geodynamical research concerned with the general geodynamical effects of earth deformations which included the work of Darwin, Lamb, Love, and others and which is being vigorously pursued to this day.

These two developments were wed in a remarkable manner when, in 1891, the work of Künster and Chandler finally revealed the elusive Eulerian motion of the earth with a period 40 percent larger than that predicted by Euler's rigid body theory. This gross discrepancy between the theoretical and observed period of the wobble was reconciled the following year by Newcomb, who showed how the yielding of the deformable earth to the centrifugal forces of the wobble reduced its effective dynamical ellipticity and lengthened the wobble period to the observed value.

The Euler/Kelvin era ended in 1900 with the establishment of the five observatories of the International Latitude Service (ILS). 
The developments of the Euler/Kelvin era produced no essential modifications of the definition of the space-fixed coordinate frame spanned by the basis vectors $\widehat{E}_{\mathrm{i}}$. The equator of rotation and the equator of figure were now regarded as distinct and moving relative to each other. However, their relative motion was such that their mean positions respectively coincided with the equator of angular momentum, and since the basis vectors $\widehat{\mathrm{E}}_{\mathrm{i}}$ were already defined in terms of the mean equator and ecliptic of epoch, the space-fixed basis vectors $\widehat{\mathrm{E}}_{\mathrm{i}}$ retained to their previous Newtonian definitions and realizations.

The discoveries of the Euler/Kelvin era profoundly altered the definitions of the body-fixed coordinate frame spanned by the basis vectors $\widehat{e}_{i}$. In the first place, since the rotation axis could no longer be regarded as a body fixed axis, it could no longer serve as a reference axis for the $\widehat{e}_{3}$ basis vector. Consequently, $\widehat{e}_{3}$ was redefined as being parallel to the earth's figure axis. However, as a consequence of the demonstration of the earth's tidal deformability, the figure axis was not fixed within the earth. It was necessary to finally define the $\widehat{e}_{3}$ body-fixed basis vector as lying in the direction of the mean figure axis of the earth which is related to the instantaneous figure axis by averaging out the effects of deformations due to tides and polar motion. This averaging was actually carried out by ILS observatories over the years 1900 to 1905.

The $\widehat{\mathrm{e}}_{1}$ body-fixed basis vector was then defined as lying in the planes of the mean equator of figure and the meridian through Greenwich which has been adopted internationally as the prime meridian. The body-fixed basis vector $\widehat{\mathrm{e}}_{2}$ was defined to complete a right-handed orthogonal triad.

The body-fixed basis vectors $\widehat{\mathrm{e}}_{1}$ were now realized in practice by assigning coordinates, not to the observatories themselves which were no longer regarded as being fixed, but to their mean locations on the earth which were related to their actual locations by:

(a) averaging out latitude and longitude variations due to polar motion, and

(b) averaging out tidal displacements.

With the discovery of Eulerian motion, it became necessary to introduce a fourth time dependent matrix, the wobble matrix $\mathrm{W}_{\mathrm{ij}}(\mathrm{t})$, into the mathematical transformation between space-fixed basis vectors $\widehat{\mathrm{E}}_{\mathrm{i}}$ and body-fixed basis vectors $\widehat{\mathrm{e}}_{\mathrm{i}}$.

$$
\widehat{e}_{i}(t)=P_{i j}(t) N_{j k}(t) S_{k \ell}(t) W_{\ell m}(t) \hat{E}_{m}
$$

In this stage of the development of the theory of the earth's motion, the instantaneous rotation axis is taken as an observable reference axis and the orientation of the earth (spanned by $\widehat{e}_{i}$ ) in space (spanned by $\widehat{\mathrm{E}}_{\mathrm{i}}$ ) is expressed by (see figure 1):

(a) First, orienting the reference axis in space, accomplished by the matrices $P_{i j}(t), N_{i j}(t)$; and

(b) Second, orienting the earth relative to the reference axis, accomplished by the matrices $S_{i j}(t), W_{i j}(t)$. 


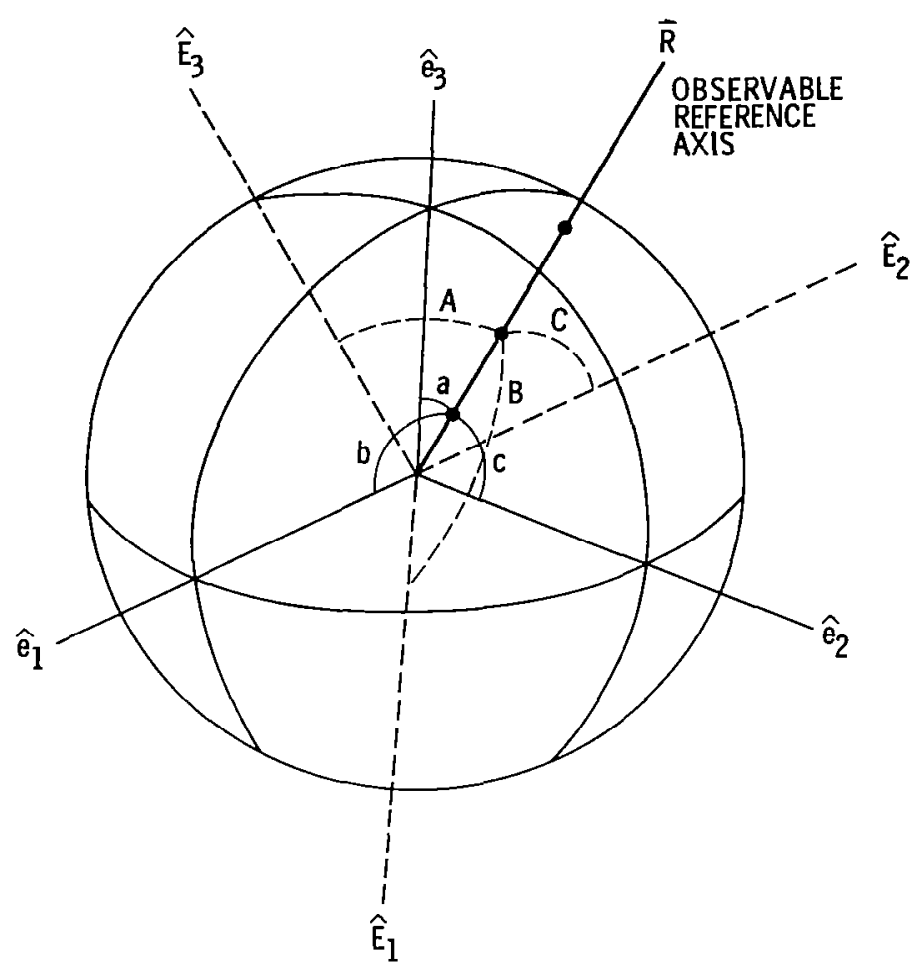

Figure 1 .

The matrix $S_{i j}(t)$, which describes the orientation in azimuth of the earth about the reference instantaneous rotation axis, no longer has the property that the quantities $\mathrm{dS}_{\mathrm{ij}} / \mathrm{dt}$ are constants. This is merely a consequence of the kinematical choice of reference axis and the fact that the chosen reference axis is itself moving within the body of the earth as is explained by Woolard (1953, pp.27, 163-165). However, while they are no longer constants, the kinematical quantities $\mathrm{dS}_{\mathrm{ij}} / \mathrm{dt}$ are precisely known from the dynamics, and consequently, the elements of the matrix $S_{i j}(t)$ are precisely predictable.

The matrix $W_{i j}(t)$ which describes the location in the body-fixed frame of the instantaneous axis of rotation can be decomposed in to two components:

(a) a predictable contribution $W_{i j}^{\circ}(t)$ due to the effects of the gravitational torques of the sun and moon and often referred to as the "dynamical variations of latitude," and

(b) an unpredictable part $\mathrm{W}_{\mathrm{ij}}^{\mathrm{G}}(\mathrm{t})$ due to a variety of internal geophysical effects. The unpredictable part of $\mathrm{W}_{\mathrm{ij}}(\mathrm{t})$ is determined observationally and published in arrears. 


\section{Early Twentieth Century (1900 A.D. - 1960 A.D.)}

The era extending from roughly 1900 A.D. to 1960 A.D. was characterized by a rapid and unprecedented expansion of geophysical research and international cooperation. Many important advances in instrumentation and observation techniques occurred which had bearing on the measurement of the earth's rotation. Some of these include the development of the zenith telescope (1912), the PZT (1915), the quartz crystal clock (1950), the dual rate moon camera (1954), and the atomic clock (1958).

Technological development resulted in a rapid sequence of startling geophysical discoveries in all branches of geophysics, oceans, atmosphere, and "solid" earth including the earth-moon system and "near space" as well as the two principal fields associated with the earth - the gravitational field and the electromagnetic field. The principal impact of this era on the theory of the earth's rotation came from the discovery by Stoyko in 1936 that the earth's rotation described by the matrix $S_{i j}(t)$ contained a variable component which was not predicted by the existing theory. This effect discovered by Stoyko was largely due to the seasonally varying atmospheric winds.

Throughout this era beginning with the work of the ILS and its successors, the IPMS and the BIH, the body-fixed basis vectors $\widehat{e}_{i}$ were referenced:

(a) $\widehat{e}_{3}$ to the mean figure axis of the earth as established by an average over the interval 1900 to 1905 , and

(b) $\widehat{e}_{1}$ to the meridian of the mean observatory and the plane of the mean figure axis of 1900 to 1905.

The "mean observatory" is a fictitious observatory occupying the meridian to which the time scales UT1 and UT2, published in the forms of UT1-UTC and UT2-UTC, are referred. In general, the meridian of the mean observatory does not contain the transit circle of the observatory at Greenwich but usually passes to the east or west of Greenwich by a few milliseconds of time depending on the particular mix of observatories and their respective weightings which have contributed to the data base from which the time scales UT1 and UT2 have been deduced. The concept of the "mean observatory" has been introduced to allow for variations in the least squares adjustment procedures by which UT1 and UT2 are obtained (Mueller, 1969).

During this era, the body-fixed basis vectors $\widehat{e}_{i}$ were realized by assigning coordinates to mean observatory positions which have been obtained from the actual observatory positions by:

(a) averaging out latitude and longitude variations due to polar motion,

(b) averaging out tidal displacements, and

(c) allowing for the effects of the least squares adjustment procedure. 
Throughout this era, the space-fixed basis vectors $\widehat{E}_{i}$ retained their previous Euler/Kelvin era definitions and realization.

The mathematical transformation from the space-fixed basis vectors $\widehat{\mathrm{E}}_{\mathbf{i}}$ to the body-fixed basis vectors $\widehat{\mathrm{e}}_{\mathrm{i}}$ is still expressed as it was in the Euler/Kelvin era,

$$
\widehat{e}_{i}(t)=P_{i j}(t) N_{j k}(t) S_{k \ell}(t) W_{\ell m}(t) \widehat{E}_{m}
$$

except that now both $S_{i j}(t)$ and $W_{i j}(t)$ consist of a combination of predictable and unpredictable contributions. In the case of $S_{i j}(t)$, the predictable contribution constitutes the majority of it with the unpredictable portion appearing as a small perturbation. In the case of $W_{i j}(t)$, the unpredictable contribution constitutes the majority of it with the predictable portion appearing as a small perturbation.

\section{The Present Day Era (1960 - )}

The year 1960 is identified here as the epoch marking the beginning of the present-day era of the study of earth rotations, for in the few years surrounding 1960, two scientific discoveries occurred which at the time seemed entirely unrelated to each other and to the question of earth rotation but may ultimately prove to be of fundamental importance in advancing our future understanding of earth rotation.

First was the geophysical discovery of what has come to be known as "global plate tectonics." This "discovery" actually spanned about a decade of time and, like most discoveries which involve a large number of participants, was the product of, and to some extent anticipated by, earlier work. Nevertheless, this decade of discovery spanned the years 1955 to 1965 and can be regarded as being made up of two somewhat distinct intervals:

(a) 1955 to 1960 during which the palaeo-magnetic evidence of large scale polar wander also confirmed the hypothesis of continental drift, and

(b) 1960 to 1965 during which the conception of "sea floor spreading" with rigid tectonic plates in relative motion at their boundaries emerged and was confirmed by the studies of the polarity reversals of the geomagnetic field and the magnetic striping on the ocean floor at the mid-ocean ridges.

These discoveries destroyed the validity of the concept of the "mean location" of an observatory and along with it destroyed the rigorous validity of the operational procedure by which the bodyfixed basis vectors $\widehat{e}_{i}$ are realized. According to the theory of plate tectonics, the observatories are moving secularly relative to each other and relative to the bulk of the earth in quasi-random directions and at rates which vary between 1 and $10 \mathrm{~cm} /$ year. Under these circumstances, the concept of an observatory's mean position has no inherent meaning, for it is a function of the averaging interval and epoch chosen. 
The discovery of plate tectonics injected a certain lack of rigor into the observational procedures which had developed historically for measuring earth rotation and created a vague discomfort in the minds of geophysicists who worried about such matters. However, it had little impact on the day to day operations of earth rotation measurement, because the effects being manifested by continental drift and plate tectonics were below the detectability threshold of the instruments of the instruments of the 1960's. This situation changed dramatically with the development of the long baseline interferometer.

The second event was the astrophysical discovery in 1960 of quasi-stellar objects often referred to as QSO's or quasars. By 1963, the quasars were known from their red shifts to be among the most distant objects in the universe. The long baseline interferometer was developed in 1967 specifically to study the radio emission from these objects.

It was the long baseline radio interferometer which linked the separate discoveries of sea floor spreading and quasars to the question of the earth's rotation. This was due to the fact that, in the operation of a long baseline interferometer, there was for the first time the potential to make measurements of the earth's rotation so accurately that the effects of the secular deformation rates implied by the theory of global plate tectonics could be detected. Confronted with this fact and the continued development of the long baseline interferometer as a device for making regular earth rotation measurements, it is rapidly becoming necessary to develop rigorous operational procedures and definitions which explicitly incorporate into the measurement of each rotation the secular deformability and continuing fracture of the earth's crustal structure.

\section{Strain in Deformable Bodies: The Separation of Rotations and Deformations}

We begin by considering two mass elements of a continuous deformable body which before deformation occupy points $P_{1}$ and $P_{2}$ given by position vectors $\vec{r}_{1}$ and $\vec{r}_{2}$ respectively and separated by a vector $d \vec{x}$. The geometry of this arrangement is illustrated in figure 2 . The body is then subjected to a deformation which carries the mass elements at $P_{1}$ and $P_{2}$ through displacements $\vec{u}_{1}$ and $\vec{u}_{2}$ to points $Q_{1}$ and $Q_{2}$ respectively. If the deformation field $\vec{u}(\vec{r})$ is a continuous and differentiable function of position, which is equivalent to asserting that no fracture has occurred, then for points $P_{1}$ and $P_{2}$ separated by an infinitesimal vector $d \vec{x}$, we have

$$
\overrightarrow{\mathrm{u}}_{2}=\overrightarrow{\mathrm{u}}_{1}+\nabla \overrightarrow{\mathrm{u}} \cdot \mathrm{d} \overrightarrow{\mathrm{x}}
$$

The relative displacement vector $\Delta \overrightarrow{\mathrm{u}}=\overrightarrow{\mathrm{u}}_{2}-\overrightarrow{\mathrm{u}}_{1}$ is given by

$$
\Delta \overrightarrow{\mathrm{u}}=\nabla \overrightarrow{\mathrm{u}} \cdot \mathrm{d} \overrightarrow{\mathrm{x}}
$$

where

$$
\nabla \overrightarrow{\mathrm{u}}=\frac{\partial \mathrm{u}_{\mathrm{i}}}{\partial \mathrm{x}_{\mathrm{j}}}
$$




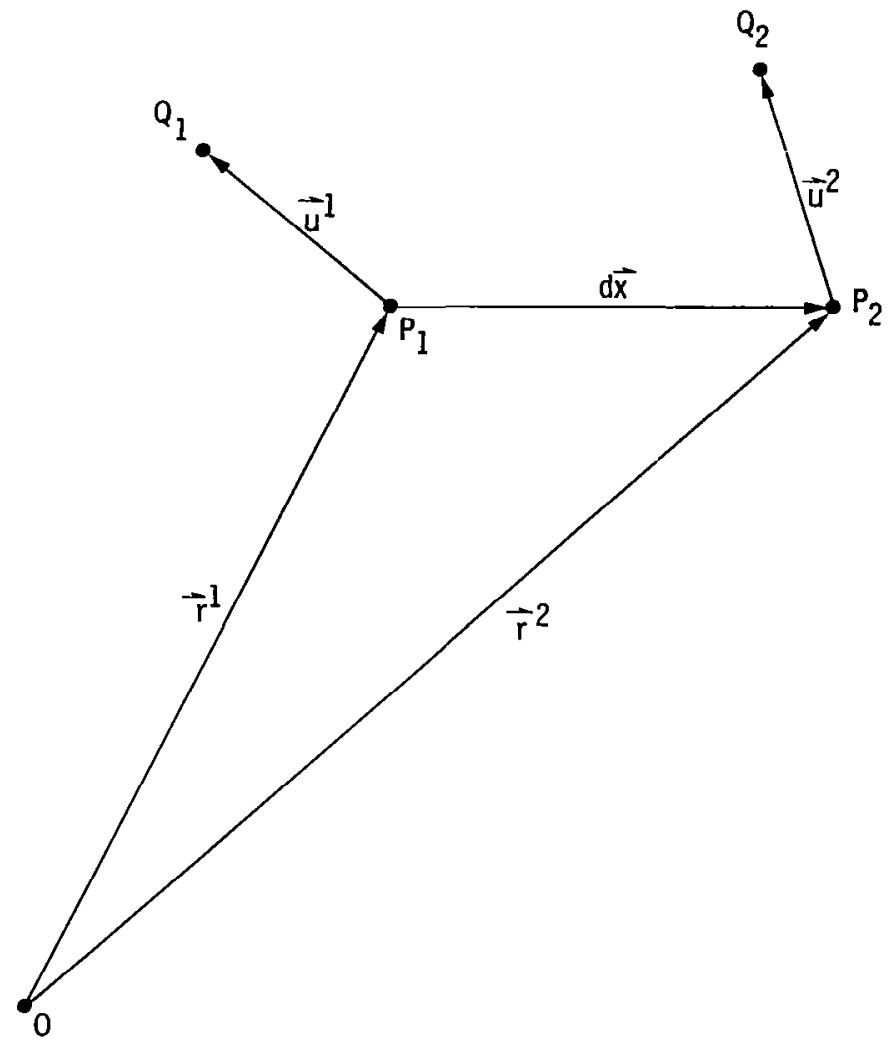

Figure 2.

The tensor $\Delta \overrightarrow{\mathrm{u}}$ can be split up into its symmetric and antisymmetric parts

$$
\nabla \overrightarrow{\mathrm{u}}=\frac{1}{2} \mathrm{e}_{\mathrm{ij}}+\frac{1}{2} \Omega_{\mathrm{ij}}
$$

where

$$
e_{i j}=\frac{\partial u_{i}}{\partial x_{j}}+\frac{\partial u_{j}}{\partial x_{i}}
$$

is symmetric and where

$$
\Omega_{i j}=\frac{\partial u_{i}}{\partial x_{j}}-\frac{\partial u_{j}}{\partial x_{i}}
$$

is antisymmetric, which allows the components of relative displacement $\Delta u_{i}$ to be written

$$
\Delta u_{i}=\frac{1}{2} e_{i j} d x_{j}+\frac{1}{2} \Omega_{i j} d x_{j}
$$


Like all antisymmetric tensors, $\Omega_{\mathrm{ij}}$ possesses a dual tensor $\Omega_{\mathrm{i}}$ given by

$$
\Omega_{\mathrm{i}}=\epsilon_{\mathrm{ijk}} \Omega_{\mathrm{jk}}
$$

where $\epsilon_{\mathrm{ijk}}$ is the alternating tensor defined by

$$
\epsilon_{\mathrm{ijk}}=\left\{\begin{array}{lll}
+1 & \mathrm{ijk} & \text { cyclic } 123 \\
0 & \mathrm{ijk} & \text { not all distinct } \\
-1 & \mathrm{ijk} & \text { noncyclic } 123 .
\end{array}\right.
$$

It can easily be shown from the property of $\epsilon_{\mathrm{ijk}}$ that

$$
\Omega_{\mathrm{ij}}=\epsilon_{\mathrm{kij}} \Omega_{\mathrm{k}},
$$

and, as a result, the components of relative displacement can be written

$$
\Delta u_{i}=\frac{1}{2} e_{i j} d x_{j}+\frac{1}{2} \epsilon_{k i j} \Omega_{k} d x_{j} .
$$

By introducing the axial vector $\vec{\Omega}$ given by

$$
\vec{\Omega}=\Omega_{\mathrm{i}} \mathrm{e}_{\mathrm{i}}=\epsilon_{\mathrm{ijk}} \Omega_{\mathrm{jk}} \mathrm{e}_{\mathrm{i}},
$$

we see that equation (16) can be written in coordinate free notation as

$$
\Delta \overrightarrow{\mathrm{u}}=\frac{1}{2} \tilde{\mathrm{e}} \cdot \mathrm{d} \overrightarrow{\mathrm{x}}-\frac{1}{2} \vec{\Omega} \times \mathrm{d} \overrightarrow{\mathrm{x}}
$$

We see from this result that, in general, the relative displacement of two mass elements of the medium separated by an infinitesimal vector $\mathrm{d} \overrightarrow{\mathrm{x}}$ can always be decomposed into the sum of a relative displacement $\Delta \overrightarrow{\mathrm{u}}_{\mathrm{D}}$,

$$
\Delta \overrightarrow{\mathrm{u}}_{\mathrm{D}}=\frac{1}{2} \tilde{\mathrm{e}} \cdot \mathrm{d} \overrightarrow{\mathrm{x}},
$$

arising solely from the effects of deformation (should any be present) and a relative displacement $\Delta \overrightarrow{\mathrm{u}}_{\mathrm{R}}$,

$$
\Delta \overrightarrow{\mathrm{u}}_{\mathrm{R}}=-\frac{1}{2} \vec{\Omega} \times \mathrm{d} \overrightarrow{\mathrm{x}},
$$


arising solely from the effects of rigid body rotation (should any be present). In this way, the effects of deformational and rotational contributions to the relative displacement field are separable.

The strain tensor $\tilde{e}$ whose elements are $e_{i j}$ has the property that if $d^{2}$ is the squared distance between the mass elements at the points $P_{1}$ and $P_{2}$ before deformation and $\mathrm{ds}^{\prime 2}$ is the squared distance between the same two mass elements after deformation, then

$$
\delta\left(d s^{2}\right)=d s^{\prime 2}-d s^{2}=e_{i j} d x_{i} d x_{j} .
$$

The rotation tensor $\widetilde{\Omega}$ whose elements are $\Omega_{\mathrm{ij}}$ has the property that the axial vector $\vec{\Omega}$ obtained from its dual gives the amount of rigid body rotation suffered by the separation vector $d \vec{x}$ as a result of the displacements.

An alternate and, in this instance, quite useful approach to the problem [Brillouin 1964, pp. 287 ff.] is to adopt the point of view that the mass elements of the medium are assigned unchanging coordinates whose numerical values are preserved under the material transformations which accompany the displacements. This requires the coordinate surfaces to be associated with the material medium and to be carried along and deformed with it as it moves. These deformed coordinate systems generally will be curvilinear.

In the usual treatment of this problem, the mass element at position $\vec{r}$ and coordinates $x_{i}$, related by

$$
\overrightarrow{\mathrm{r}}=\mathrm{x}_{1} \widehat{\mathrm{e}}_{1}+\mathrm{x}_{2} \widehat{\mathrm{e}}_{2}+\mathrm{x}_{3} \widehat{\mathrm{e}}_{3},
$$

gets displaced to a position $\vec{r}^{\prime}=\vec{r}+\vec{u}(\vec{r})$ with coordinates $X_{i}$ such that

$$
X_{i}=x_{i}+u_{i}
$$

and

$$
\overrightarrow{\mathrm{r}}^{\prime}=\mathrm{X}_{1} \widehat{\mathrm{e}}_{1}+\mathrm{X}_{2} \widehat{\mathrm{e}}_{2}+\mathrm{X}_{3} \widehat{\mathrm{e}}_{3}
$$

or equivalently

$$
\overrightarrow{\mathrm{r}}^{\prime}=\left(\mathrm{x}_{1}+\mathrm{u}_{1}\right) \widehat{\mathrm{e}}_{1}+\left(\mathrm{x}_{2}+\mathrm{u}_{2}\right) \widehat{\mathrm{e}}_{2}+\left(\mathrm{x}_{3}+\mathrm{u}_{3}\right) \widehat{\mathrm{e}}_{3} .
$$

In the alternate approach, the mass element at position $\overrightarrow{\mathrm{r}}$ and coordinates $\mathrm{x}_{\mathrm{i}}$ gets displaced to a position $\vec{r}^{\prime}=\vec{r}+\vec{u}(\vec{r})$ with coordinates $\bar{X}_{i}$, such that

$$
\overline{\mathrm{X}}_{\mathrm{i}}=\mathrm{x}_{\mathrm{i}}
$$

and

$$
\overrightarrow{\mathrm{r}}^{\prime}=\overline{\mathrm{X}}_{1} \widehat{\mathrm{e}}_{1}^{\prime}+\overline{\mathrm{X}}_{2} \widehat{\mathrm{e}}_{2}^{\prime}+\overline{\mathrm{X}}_{3} \widehat{\mathrm{e}}_{3}^{\prime}
$$

or equivalently

$$
\overrightarrow{\mathrm{r}}^{\prime}=\mathrm{x}_{1} \widehat{\mathrm{e}}_{1}^{\prime}+\mathrm{x}_{2} \widehat{\mathrm{e}}_{2}^{\prime}+\mathrm{x}_{3} \widehat{\mathrm{e}}_{3}^{\prime}
$$


where the basis vectors $\widehat{\mathrm{e}}_{1}^{\prime}, \widehat{\mathrm{e}}_{2}^{\prime}, \widehat{\mathrm{e}}_{3}^{\prime}$ locally spanning the $\overline{\mathrm{X}}$ coordinate frame are necessarily changed from the basis vectors $\widehat{\mathrm{e}}_{1}, \widehat{\mathrm{e}}_{2}, \widehat{\mathrm{e}}_{3}$ locally spanning the $\mathrm{X}$ coordinate frame in order to accommodate the fact that $\overrightarrow{\mathrm{r}} \neq \overrightarrow{\mathrm{r}}^{\prime}$

Without loss of generality, we can begin by assuming that the basis vectors $\widehat{\mathrm{e}}_{1}, \widehat{\mathrm{e}}_{2}, \widehat{\mathrm{e}}_{3}$ span a local Cartesian coordinate frame. The relationship between the coordinates $\mathrm{X}_{\mathrm{i}}$ referred to the basis vectors $\widehat{\mathrm{e}}_{1}, \widehat{\mathrm{e}}_{2}, \widehat{\mathrm{e}}_{3}$, and the coordinates $\overline{\mathrm{X}}_{\mathrm{i}}$ referred to the basis vectors $\widehat{\mathrm{e}}_{1}^{\prime}, \widehat{\mathrm{e}}_{2}^{\prime}, \widehat{\mathrm{e}}_{3}^{\prime}$ can be established by considering two mass elements at position $\vec{r}_{1}$ and $\vec{r}_{2}$ separated by the infinitesimal vector $d \vec{x}$, where

$$
d \vec{x}=\vec{r}_{2}-\vec{r}_{1} \text {. }
$$

As a result of the displacements and the motion of the mass elements, the vector $d \vec{x}$ is transformed into the vector $\mathrm{d} \overrightarrow{\mathrm{X}}$ :

$$
d \vec{X}=\vec{r}_{2}^{\prime}-\vec{r}_{1}^{\prime}=\vec{r}_{2}+\vec{u}\left(\vec{r}_{2}\right)-\vec{r}_{1}-\vec{u}\left(\vec{r}_{1}\right)
$$

or

$$
d \vec{X}=d \vec{x}+d \vec{u}
$$

where

$$
d \vec{u}=\vec{u}\left(\vec{r}_{2}\right)-\vec{u}\left(\vec{r}_{1}\right)
$$

Now, in component form, equation (31) gives

$$
\mathrm{dX}_{\mathrm{i}}=\mathrm{dx}_{\mathrm{i}}+\mathrm{du}_{\mathrm{i}}
$$

and the chain rule of differentiation gives

$$
\frac{d X_{i}}{d x_{k}}=\frac{d x_{i}}{d x_{k}}+\frac{\partial u_{i}}{\partial x_{j}} \frac{d x_{j}}{d x_{k}} .
$$

Recognizing that $\mathrm{dx}_{\mathrm{i}} / \mathrm{dx}_{\mathrm{j}} \equiv \delta_{\mathrm{ij}}$ where $\delta_{\mathrm{ij}}$ is the Kronecker delta, and that by the definition of equation (26) $\mathrm{d}_{\mathrm{k}}=\mathrm{dx}_{\mathrm{k}}$, we obtain the relationship that

$$
\mathrm{dX}_{\mathrm{i}}=\left[\delta_{\mathrm{ik}}+\frac{\partial u_{\mathrm{i}}}{\partial \mathrm{x}_{\mathrm{k}}}\right] \mathrm{d} \overline{\mathrm{X}}_{\mathrm{k}} .
$$

The use of equation (10) in equation (35) gives

$$
\mathrm{dX}_{\mathrm{i}}=\left[\delta_{\mathrm{ik}}+\frac{1}{2} \mathrm{e}_{\mathrm{ik}}+\frac{1}{2} \Omega_{\mathrm{ik}}\right] \mathrm{d} \overline{\mathrm{X}}_{\mathrm{k}}
$$


which states that the $\mathrm{X}$ and $\overline{\mathrm{X}}$ coordinate systems are locally transformed, one into the other, by the combination of (a) a rigid body rotation $\Omega_{i k}$ and (b) a deformation $e_{i k}$.

If we proceed to compute the squared distance $\mathrm{ds}^{2}$ between the two mass elements after the displacements on both the $\mathrm{X}$ coordinate frame and the $\overline{\mathrm{X}}$ coordinate frame, these two expressions must be equal since $d s^{2}$ is a scalar quantity whose value is independent of the choice of coordinates. In the $\mathrm{X}$ coordinate system, which has been assumed without loss of generality to be a local Cartesian coordinate frame with metric tensor $\mathrm{g}_{\mathrm{ij}}=\delta_{\mathrm{ij}}$, then

$$
d s_{X}^{2}=\delta_{i j} d X_{i} d X_{j}
$$

The equivalent expression in the $\overline{\mathrm{X}}$ coordinate frame is obtained from equation (36) as

$$
\mathrm{ds}_{\overline{\mathrm{X}}}^{2}=\delta_{\mathrm{ij}}\left[\delta_{\mathrm{ik}}+\frac{1}{2} \mathrm{e}_{\mathrm{ik}}+\frac{1}{2} \Omega_{\mathrm{ik}}\right]\left[\delta_{\mathrm{j} \ell}+\frac{1}{2} \mathrm{e}_{\mathrm{j} \ell}+\frac{1}{2} \Omega_{\mathrm{j} \ell}\right] \mathrm{d} \overline{\mathrm{X}}_{\mathrm{k}} \mathrm{d} \overline{\mathrm{X}}_{\ell}
$$

which, using the antisymmetric property of $\Omega_{\mathrm{ij}}$ reduces finally to

$$
\mathrm{ds}_{\mathrm{X}}^{2}=\left(\delta_{\mathrm{ij}}+\mathrm{e}_{\mathrm{ij}}\right) \mathrm{d} \overline{\mathrm{X}}_{\mathrm{i}} \mathrm{d} \overline{\mathrm{X}}_{\mathrm{j}}
$$

or

$$
\mathrm{ds}_{\overline{\mathrm{X}}}^{2}=\overline{\mathrm{g}}_{\mathrm{ij}} \mathrm{d} \overline{\mathrm{X}}_{\mathrm{i}} \mathrm{d} \overline{\mathrm{X}}_{\mathrm{j}}
$$

A comparison of equations (37) and (39) shows that a consequence of the theoretical development on which the displaced mass elements are allowed to retain their coordinate values is that the strain tensor $\mathrm{e}_{\mathrm{ij}}$ is simply absorbed into the geometric tensor $\overline{\mathrm{g}}_{\mathrm{ij}}$ of the deformed $\overline{\mathrm{X}}$ coordinate system. Whereas the basis vectors $\widehat{\mathrm{e}}_{1}, \widehat{\mathrm{e}}_{2}, \widehat{\mathrm{e}}_{3}$ are all orthogonal and of unit length, the basis vectors $\mathrm{e}_{1}^{\prime}, \mathrm{e}_{2}^{\prime}, \mathrm{e}_{3}^{\prime}$ are nonorthogonal and no longer of unit length. This is illustrated in figure 3.

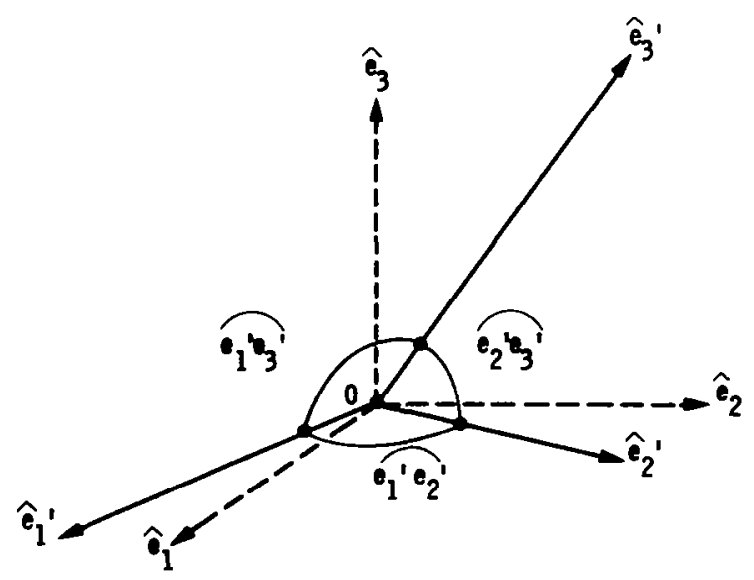

Figure 3 . 
The metric tensor $\overline{\mathrm{g}}_{\mathrm{ij}}$ of the $\overline{\mathrm{X}}$ coordinate frame is given by definition as

$$
\overline{\mathrm{g}}_{\mathrm{ij}}=\left[\begin{array}{lllll}
\left|\hat{\mathrm{e}}_{1}^{\prime}\right|^{2} & \left|\hat{\mathrm{e}}_{1}^{\prime}\right|\left|\hat{\mathrm{e}}_{2}^{\prime}\right| & \cos \widehat{\mathrm{e}_{1}^{\prime} \mathrm{e}_{2}^{\prime}} & \left|\widehat{\mathrm{e}}_{1}^{\prime}\right|\left|\widehat{\mathrm{e}}_{3}^{\prime}\right| & \cos \widehat{\mathrm{e}_{1}^{\prime} \mathrm{e}_{3}^{\prime}} \\
\left|\widehat{\mathrm{e}}_{2}^{\prime}\right|\left|\hat{\mathrm{e}}_{1}^{\prime}\right| & \cos \widehat{\mathrm{e}_{2}^{\prime} \mathrm{e}_{1}^{\prime}} & \left|\widehat{\mathrm{e}}_{2}^{\prime}\right|^{2} & \left|\widehat{\mathrm{e}}_{2}^{\prime}\right|\left|\widehat{\mathrm{e}}_{3}^{\prime}\right| & \cos \widehat{\mathrm{e}_{2}^{\prime} \mathrm{e}_{3}^{\prime}} \\
\left|\widehat{\mathrm{e}}_{3}^{\prime}\right|\left|\hat{\mathrm{e}}_{1}^{\prime}\right| & \cos \widehat{\mathrm{e}_{3}^{\prime} \mathrm{e}_{1}^{\prime}} & \left|\widehat{\mathrm{e}}_{3}^{\prime}\right|\left|\widehat{\mathrm{e}}_{2}^{\prime}\right| & \cos \widehat{\mathrm{e}_{3}^{\prime} \mathrm{e}_{2}^{\prime}} & \left|\widehat{\mathrm{e}}_{3}^{\prime}\right|^{2}
\end{array}\right]
$$

So, we see from equations (39), (40), and (41) that

$$
\begin{aligned}
& \left|\hat{\mathrm{e}}_{1}^{\prime}\right|=\sqrt{1+\mathrm{e}_{11}} \\
& \left|\widehat{\mathrm{e}}_{2}^{\prime}\right|=\sqrt{1+\mathrm{e}_{22}} \\
& \left|\widehat{\mathrm{e}}_{3}^{\prime}\right|=\sqrt{1+\mathrm{e}_{33}}
\end{aligned}
$$

and that

$$
\begin{aligned}
& \cos \widetilde{\mathrm{e}_{1}^{\prime} \mathrm{e}_{2}^{\prime}}=\frac{\mathrm{e}_{12}}{\sqrt{1+\mathrm{e}_{11}} \sqrt{1+\mathrm{e}_{22}}} \\
& \cos \overparen{\mathrm{e}_{1}^{\prime} \mathrm{e}_{3}^{\prime}}=\frac{\mathrm{e}_{13}}{\sqrt{1+\mathrm{e}_{11}} \sqrt{1+\mathrm{e}_{33}}} \\
& \cos \overparen{\mathrm{e}_{2}^{\prime} \mathrm{e}_{3}^{\prime}}=\frac{\mathrm{e}_{23}}{\sqrt{1+\mathrm{e}_{22}} \sqrt{1+\mathrm{e}_{33}}}
\end{aligned}
$$

If the $\left|e_{i j}\right|<<1$ then these relationships reduce to

$$
\begin{aligned}
& \left|\widehat{\mathrm{e}}_{1}^{\prime}\right| \cong 1+\frac{1}{2} \mathrm{e}_{11} \\
& \left|\widehat{\mathrm{e}}_{2}^{\prime}\right| \cong 1+\frac{1}{2} \mathrm{e}_{22} \\
& \left|\widehat{\mathrm{e}}_{3}^{\prime}\right| \cong 1+\frac{1}{2} \mathrm{e}_{33}
\end{aligned}
$$


and to

$$
\begin{aligned}
& \overparen{\mathrm{e}_{1}^{\prime} \mathrm{e}_{2}^{\prime}} \cong \frac{\pi}{2}-\mathrm{e}_{12} \\
& \widetilde{\mathrm{e}_{1}^{\prime} \mathrm{e}_{3}^{\prime}} \cong \frac{\pi}{2}-\mathrm{e}_{13} \\
& \widetilde{\mathrm{e}_{2}^{\prime} \mathrm{e}_{3}^{\prime}} \cong \frac{\pi}{2}-\mathrm{e}_{23}
\end{aligned}
$$

The entire preceding analysis is based on the components of the separation vector $d \vec{x}$ and the components of the relative displacement vector $\Delta \overrightarrow{\mathrm{u}}$. The direction of the separation vector $\mathrm{d} \overrightarrow{\mathrm{x}}$ is arbitrary and a reversal of the direction of $d \vec{x}$ simply reverses the sign of $\Delta \vec{u}$. The quantities in this theory have analogs in long baseline interferometry. The analog of the separation vector $d x^{\prime}$ is the interferometer baseline vector $\vec{\beta}$ separating the two antennas. The analog of the relative displacement vector $\Delta \overrightarrow{\mathrm{u}}$ is $\vec{\Delta}$ the change in the baseline vector which is observed to occur between two successive measurements of $\vec{\beta}$.

Next we shall attempt to apply these procedures to the analysis of long baseline interferometry data and to question of the measurement of earth rotation on a deformable earth. While these procedures are potentially useful in this context, they will also have certain limitations.

First, the above geometrical procedures will not give rise to tensor quantities when applied to the whole earth. Tensor quantities can only be defined in the neighborhood of a point or, for pairs of points, separated by infinitesimal separation vectors $d \vec{x}$. In long baseline interferometry, we will be concerned with relative displacement between points separated by thousands of kilometers. The analysis procedure described above will produce quantities which have many of the properties of tensors but, because they refer to pairs of points separated by a finite distance, will not possess the general transformation properties of tensors. In particular, these quantities will possess the mathematical properties of tensors only in Cartesian coordinate frames. To distinguish them from true tensors, we will refer to them as tensor analogs.

Second, the deformation field of the earth is not continuous and differentiable everywhere. Fracture is occurring on and within the earth in a complex, largely unknown pattern. This fact does not affect the validity of these procedures as applied to the earth insofar as we restrict ourselves to the discipline of kinematics or geometry. However, entering the realm of dynamics or geophysics and attempting to relate strain tensor analogs to stress tensors will be a risky procedure. Where there is fracture, there can be local strain without local stress. Without considerably more geologic knowledge, the complexity of the pattern of fracture affecting baselines of thousands of kilometers in length would make the inference of stress from strain suspect. The two possible exceptions to this might be: 
(a) The case of strain tensor analogs measured on very short baselines from a few tens to a few hundred kilometers in length, in which case, the material supporting the interferometer can either be considered homogeneous and free of fracture or can be mapped in sufficient geological detail to adequately account for the effects of fracture; and

(b) The case of a mean strain tensor analog obtained by a global average of many local strain tensor analogs, in which case, the random effects of geologic faulting affecting the local measurements have been averaged out and the residual mean strain tensor analog describes that global strain field which is deforming the earth coherently as though it were the neighborhood of a point.

\section{The Measurement of Earth Rotation on a Deformable Earth}

\section{Basic Definitions and Procedures}

We presuppose a global network of interferometer baseline vectors $\vec{\beta}^{k} k=1,2,3, \ldots N$ forming a polyhedron as shown in figure 4 which will be used for the purposes of:

(a) defining the body-fixed coordinate frame, and

(b) measuring earth rotation or equivalently defining the transformation from the body-fixed coordinate frame to the space-fixed coordinate frame.

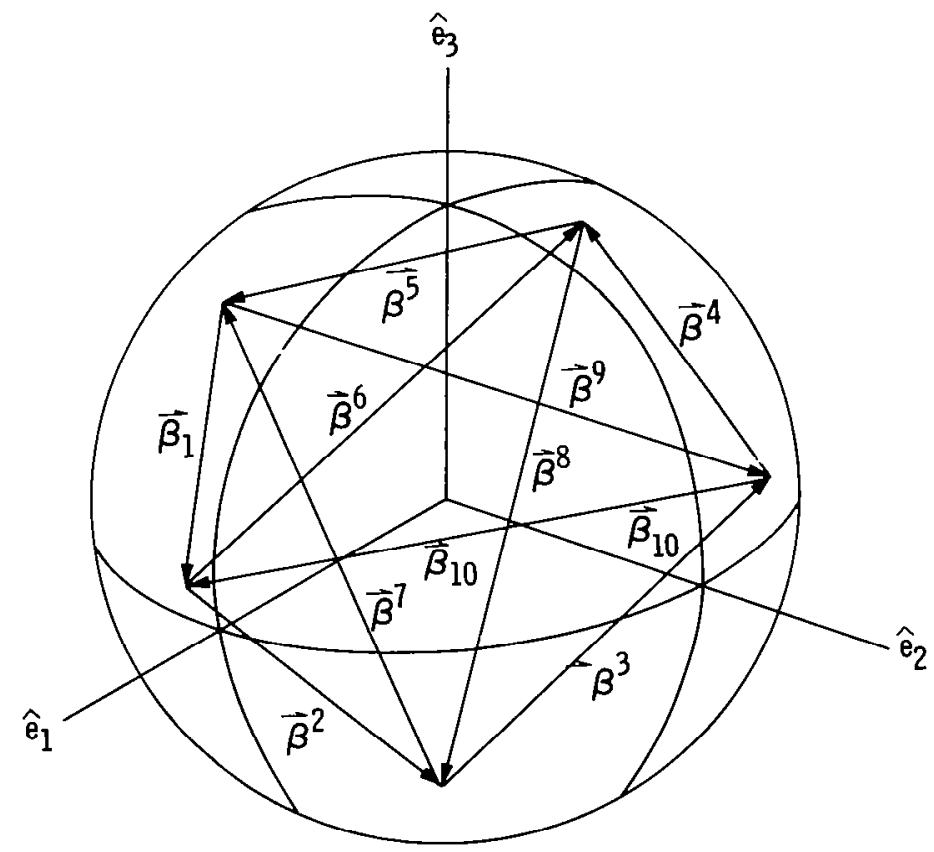

Figure 4 . 
These definitions must be operable in the face of the fact that as a result of the discoveries of plate tectonics, each point of the earth's surface must be regarded as possessing its own rotation axis. While points on the same tectonic plate might be expected to have nearly identical rotation axes as a consequence of Euler's theorem on rigid body motion on the surface of a sphere [McKenzie, 1972], different rotation axes will be associated with points on different tectonic plates. Furthermore, when the relative vertical motions, which occur within the plates and which can be as large as $1 \mathrm{~cm} /$ year, are also considered, it is clear that in general the concept of a rotation axis must be rigorously regarded as only applying locally on the earth.

Each of the time dependent interferometer baseline vectors $\vec{\beta}_{1}^{k}(t), k=1,2,3, \ldots N$ will possess space-fixed components $B_{i}^{k}(t), i=1,2,3$,

$$
\vec{\beta}^{k}(t)=B_{1}^{k}(t) \hat{E}_{1}+B_{2}^{k}(t) \hat{E}_{2}+B_{3}^{k}(t) \hat{E}_{3},
$$

and body-fixed components $b_{i}^{k}(t), i=1,2,3$,

$$
\vec{\beta}^{k}(t)=b_{1}^{k}(t) \widehat{e}_{1}+b_{2}^{k}(t) \widehat{e}_{2}+b_{3}^{k}(t) \widehat{e}_{3} .
$$

The transformation from space-fixed components to body-fixed components expresses all our knowledge of earth rotation and is currently expressed by equation (6) as

$$
b_{i}^{k}(t)=W_{i j}^{T}(t) S_{j k}^{T}(t) N_{k \ell}^{T}(t) P_{\ell m}^{T}(t) B_{m}^{k}
$$

where the superscript " $k$ " serves merely to identify the baseline and does not obey the Einstein summation and range convention. The superscript " $T$ " denotes a matrix transpose.

Repeated measurements of the baselines $\vec{\beta}^{k}(t)$ at times ... $t_{m-2} t_{m-1} t_{m} t_{m+1} t_{m+2} \ldots$ yield relative displacement vectors $\vec{\Delta} \mathrm{k}\left(\mathrm{t}_{\mathrm{m}}\right)$ given by

$$
\vec{\Delta}^{\mathrm{k}}\left(\mathrm{t}_{\mathrm{m}}\right)=\vec{\beta}^{\mathrm{k}}\left(\mathrm{t}_{\mathrm{m}}\right)-\vec{\beta}^{\mathrm{k}}\left(\mathrm{t}_{\mathrm{m}-1}\right) \text {. }
$$

The relative displacement vectors $\vec{\Delta}^{k}\left(t_{m}\right)$ will possess space-fixed components $D_{i}^{k}(t), i=1,2,3$,

$$
\vec{\Delta}^{k}\left(t_{m}\right)=D_{1}^{k}\left(t_{m}\right) \widehat{E}_{1}+D_{2}^{k}\left(t_{m}\right) \widehat{E}_{2}+D_{3}^{k}\left(t_{m}\right) \widehat{E}_{3}
$$

and body-fixed components $d_{i}^{k}\left(t_{m}\right), i=1,2,3$,

$$
\vec{\Delta}^{k}\left(t_{m}\right)=d_{1}^{k}\left(t_{m}\right) \widehat{e}_{1}+d_{2}^{k}\left(t_{m}\right) \widehat{e}_{2}+d_{3}^{k}\left(t_{m}\right) \widehat{e}_{3}
$$

also related one to the other, according to our present knowledge of earth rotation, by equation (6)

$$
d_{i}^{k}\left(t_{m}\right)=W_{i j}^{T}\left(t_{m}\right) S_{j k}^{T}\left(t_{m}\right) N_{k \ell}^{T}\left(t_{m}\right) P_{\ell m}^{T}\left(t_{m}\right) D_{m}^{k}\left(t_{m}\right) .
$$


The operation of the long baseline interferometer and the subsequent reduction of the data finally provide us with the quantities $b_{i}^{k}\left(t_{m}\right), d_{i}^{k}\left(t_{m}\right) i=1,2,3, k=1,2,3 \ldots N$ which together with the epochs $t_{m}, m=1,2,3 \ldots$ constitute the basic data with which we must achieve our two stated objectives.

We can begin creating analogs to continuum mechanics by defining, for the $\mathrm{k}^{\text {th }}$ baseline, a dimensionless quantity $c_{\mathrm{ij}}^{\mathrm{k}}$ given by

$$
c_{i j}^{k}=\frac{d_{i}^{k} b_{j}^{k}}{b_{\ell}^{k} b_{\ell}^{k}}
$$

which will serve as the analog of the tensor $\nabla \overrightarrow{\mathrm{u}}$ of equation (9). The tensor analog $c_{\mathrm{ij}}^{\mathrm{k}}$ is expanded in its symmetric and antisymmetric parts.

$$
\mathrm{c}_{\mathrm{ij}}^{\mathrm{k}}=\frac{1}{2} \mathrm{e}_{\mathrm{ij}}^{\mathrm{k}}+\frac{1}{2} \Omega_{\mathrm{ij}}^{\mathrm{k}}
$$

where

$$
e_{i j}^{k}=\left[c_{i j}^{k}+c_{j i}^{k}\right]=\frac{1}{b_{\ell}^{k} b_{\ell}^{k}}\left[d_{i}^{k} b_{j}^{k}+d_{j}^{k} b_{i}^{k}\right]
$$

is the strain tensor analog for the $\mathrm{k}^{\text {th }}$ interferometer baseline and where

$$
\Omega_{i j}^{k}=\left[c_{i j}^{k}-c_{j i}^{k}\right]=\frac{1}{b_{l}^{k} b_{l}^{k}}\left[d_{i}^{k} b_{j}^{k}-d_{j}^{k} b_{i}^{k}\right]
$$

is the rotation tensor analog for the $\mathrm{k}^{\text {th }}$ interferometer baseline. It can readily be shown that these definitions permit us to write

$$
d_{i}^{k}=\frac{1}{2} e_{i j}^{k} b_{j}^{k}+\frac{1}{2} \Omega_{i j}^{k} b_{j}^{k}
$$

Equations (54) through (57) are the analogs of equations (10) through (13) for the case of continuum mechanics.

To illustrate this procedure, we consider the case of an interferometer baseline $\vec{\beta}^{\mathbf{k}}$ with body-fixed components $b_{j}^{\mathrm{k}}, \mathrm{i}=1,2,3$, given by $\mathrm{x}, \mathrm{y}, \mathrm{z}$ and a relative displacement vector $\vec{\Delta}^{\mathrm{k}}$ with body-fixed components $d_{i}^{k}, i=1,2,3$, given by $\delta x, \delta y, \delta z$, then: 


$$
\begin{aligned}
& \mathrm{c}_{\mathrm{ij}}^{\mathrm{k}}=\frac{1}{\mathrm{x}^{2}+\mathrm{y}^{2}+\mathrm{z}^{2}}\left[\begin{array}{ccc}
\mathrm{x} \delta \mathrm{x} & \mathrm{y} \delta \mathrm{x} & \mathrm{z} \delta \mathrm{x} \\
\mathrm{x} \delta \mathrm{y} & \mathrm{y} \delta \mathrm{y} & \mathrm{z} \delta \mathrm{y} \\
\mathrm{x} \delta \mathrm{z} & \mathrm{y} \delta \mathrm{z} & \mathrm{z} \delta \mathrm{z}
\end{array}\right] \\
& \mathrm{e}_{\mathrm{ij}}^{\mathrm{k}}=\frac{1}{\mathrm{x}^{2}+\mathrm{y}^{2}+\mathrm{z}^{2}}\left[\begin{array}{ccc}
2 \mathrm{x} \delta \mathrm{x} & \mathrm{y} \delta \mathrm{x}+\mathrm{x} \delta \mathrm{y} & \mathrm{z} \delta \mathrm{x}+\mathrm{x} \delta \mathrm{z} \\
\mathrm{x} \delta \mathrm{y}+\mathrm{y} \delta \mathrm{x} & 2 \mathrm{y} \delta \mathrm{y} & \mathrm{z} \delta \mathrm{y}+\mathrm{y} \delta \mathrm{z} \\
\mathrm{x} \delta \mathrm{z}+\mathrm{z} \delta \mathrm{x} & \mathrm{y} \delta \mathrm{z}+\mathrm{z} \delta \mathrm{y} & 2 \mathrm{z} \delta \mathrm{z}
\end{array}\right] \\
& \Omega_{\mathrm{ij}}^{\mathrm{k}}=\frac{1}{\mathrm{x}^{2}+\mathrm{y}^{2}+\mathrm{z}^{2}}\left[\begin{array}{ccc}
0 & \mathrm{y} \delta \mathrm{x}-\mathrm{x} \delta \mathrm{y} & \mathrm{z} \delta \mathrm{x}-\mathrm{x} \delta \mathrm{z} \\
\mathrm{x} \delta \mathrm{y}-\mathrm{y} \delta \mathrm{x} & 0 & \mathrm{z} \delta \mathrm{y}-\mathrm{y} \delta \mathrm{z} \\
\mathrm{x} \delta \mathrm{z}-\mathrm{z} \delta \mathrm{x} & \mathrm{y} \delta \mathrm{z}-\mathrm{z} \delta \mathrm{y} & 0
\end{array}\right]
\end{aligned}
$$

To show that $\mathrm{e}_{\mathrm{ij}}^{\mathrm{k}}$ possesses the properties of a strain tensor insofar as the $\mathrm{k}^{\text {th }}$ baseline is concerned, we compute the variation in the squared distance $\mathrm{s}^{\mathrm{k}^{2}}$ between the two observatories defining the $\mathrm{k}^{\mathrm{th}}$ baseline which occurs as a result of the relative displacement. Before relative displacement

and after relative displacement

$$
s^{k^{2}}=b_{i}^{k} b_{i}^{k}
$$

$$
s^{k^{2}}=\left(b_{i}^{k}+d_{i}^{k}\right)\left(b_{i}^{k}+d_{i}^{k}\right) .
$$

The difference $\delta\left(\mathrm{s}^{2}\right)$ is given by

$$
\delta\left(\mathrm{s}^{\mathrm{k}^{2}}\right)=\left(\mathrm{b}_{\mathrm{i}}^{\mathrm{k}}+\mathrm{d}_{\mathrm{i}}^{\mathrm{k}}\right)\left(\mathrm{b}_{\mathrm{i}}^{\mathrm{k}}+\mathrm{d}_{\mathrm{i}}^{\mathrm{k}}\right)-\mathrm{b}_{\mathrm{i}}^{\mathrm{k}} \mathrm{b}_{\mathrm{i}}^{\mathrm{k}}
$$

or

$$
\delta\left(\mathrm{s}^{\mathrm{k}^{2}}\right)=2 \mathrm{~d}_{\mathrm{i}}^{\mathrm{k}} \mathrm{b}_{\mathrm{i}}^{\mathrm{k}}\left[1+0\left(\frac{\mathrm{d}}{\mathrm{b}}\right)\right] \simeq 2 \mathrm{~d}_{\mathrm{i}}^{\mathrm{k}} b_{\mathrm{i}}^{\mathrm{k}}
$$

neglecting terms of the order of $d / b<<1$. Equation (63) can be written

$$
\delta\left(s^{k^{2}}\right)=\left[\frac{d_{i}^{k} b_{j}^{k}+d_{j}^{k} b_{i}^{k}}{b_{\ell}^{k} b_{\ell}^{k}}\right] b_{i}^{k} b_{j}^{k}=e_{i j}^{k} b_{i}^{k} b_{j}^{k}
$$

which is the analog of equation (21) for the case of continuum mechanics.

A further demonstration that $e_{\mathrm{ij}}^{\mathrm{k}}$ possesses the properties of the strain tensor for the $\mathrm{k}^{\text {th }}$ baseline is provided by considering the special case of an earth subjected to a uniform dilatation. In this case, the observatories at position $\vec{r}_{1}$ and $\vec{r}_{2}$ which make up the $\mathrm{k}^{\text {th }}$ interferometer baseline $\vec{\beta}^{k}$ suffer displacements $\delta \overrightarrow{\mathrm{r}}_{1}$ and $\delta \overrightarrow{\mathrm{r}}_{2}$ given by 


$$
\begin{aligned}
\delta \vec{r}_{1} & =A \vec{r}_{1} \\
\delta \vec{r}_{2} & =A \vec{r}_{2}
\end{aligned}
$$

where $3 \mathrm{~A}$ is the magnitude of the dilatation. The relative displacement vector $\vec{\Delta}^{\mathrm{k}}$ is given by

$$
\vec{\Delta}^{\mathrm{k}}=\mathrm{A}\left(\overrightarrow{\mathrm{r}}_{2}-\overrightarrow{\mathrm{r}}_{1}\right)
$$

and will have body-fixed components $d_{i}^{k}$

$$
d_{i}^{k}=A b_{i}^{k}
$$

where $b_{i}^{k}$ are the body-fixed components of the $k^{\text {th }}$ baseline vector $\vec{\beta}^{k}=\vec{r}_{2}-\vec{r}_{1}$. The strain tensor analog for the $\mathrm{k}^{\text {th }}$ baseline in this case is

$$
e_{i j}^{k}=\frac{A}{b_{l}^{k} b_{l}^{k}}\left[b_{i} b_{j}+b_{j} b_{i}\right]
$$

and the trace of $e_{i j}^{k}, T_{r}\left(e_{i j}^{k}\right)$, in this case is

$$
T_{r}\left(e_{i j}^{k}\right)=2 A .
$$

This result should be compared to the well known result from continuum mechanics that

$$
\mathrm{T}_{\mathrm{r}}\left(\mathrm{e}_{\mathrm{ij}}\right)=2 \theta
$$

where $\theta$ is the dilatation.

To show that $\Omega_{\mathrm{ij}}^{\mathrm{k}}$ possesses the properties of a rotation tensor insofar as the $\mathrm{k}^{\text {th }}$ baseline is concerned, we compute the rigid body rotation $\vec{\omega}$ which is imparted to an interferometer baseline vector $\vec{\beta}$ as a result of a relative displacement $\vec{\Delta}$. The geometry of this situation is illustrated in figure 5 with the body-fixed basis vectors shown for reference. The rotation vector $\vec{\omega}$ can be expressed as

$$
\vec{\omega}=\Omega \widehat{\omega}
$$

where $\Omega$ is the magnitude of the angle between $\vec{\beta}$ and $\vec{\beta}+\vec{\Delta}$. Neglecting terms of the order of $\vec{\Delta} / \vec{\beta}$ where $|\vec{\Delta}|<<|\vec{\beta}|$, we can express the area of the shaded triangle in two equivalent forms and equate them to obtain

$$
\frac{1}{2} \Omega|\vec{\beta}|^{2}=\frac{1}{2}|\vec{\beta} \times \vec{\Delta}|
$$


From this it follows that

$$
\Omega=\frac{|\vec{\beta} \times \vec{\Delta}|}{|\vec{\beta}|^{2}}
$$

Since

$$
\omega=\frac{\vec{\beta} \times \vec{\Delta}}{|\vec{\beta} \times \vec{\Delta}|},
$$

equations (70) and (71) give

$$
\vec{\omega}=\frac{\vec{\beta} \times \vec{\Delta}}{|\vec{\beta}|^{2}}
$$

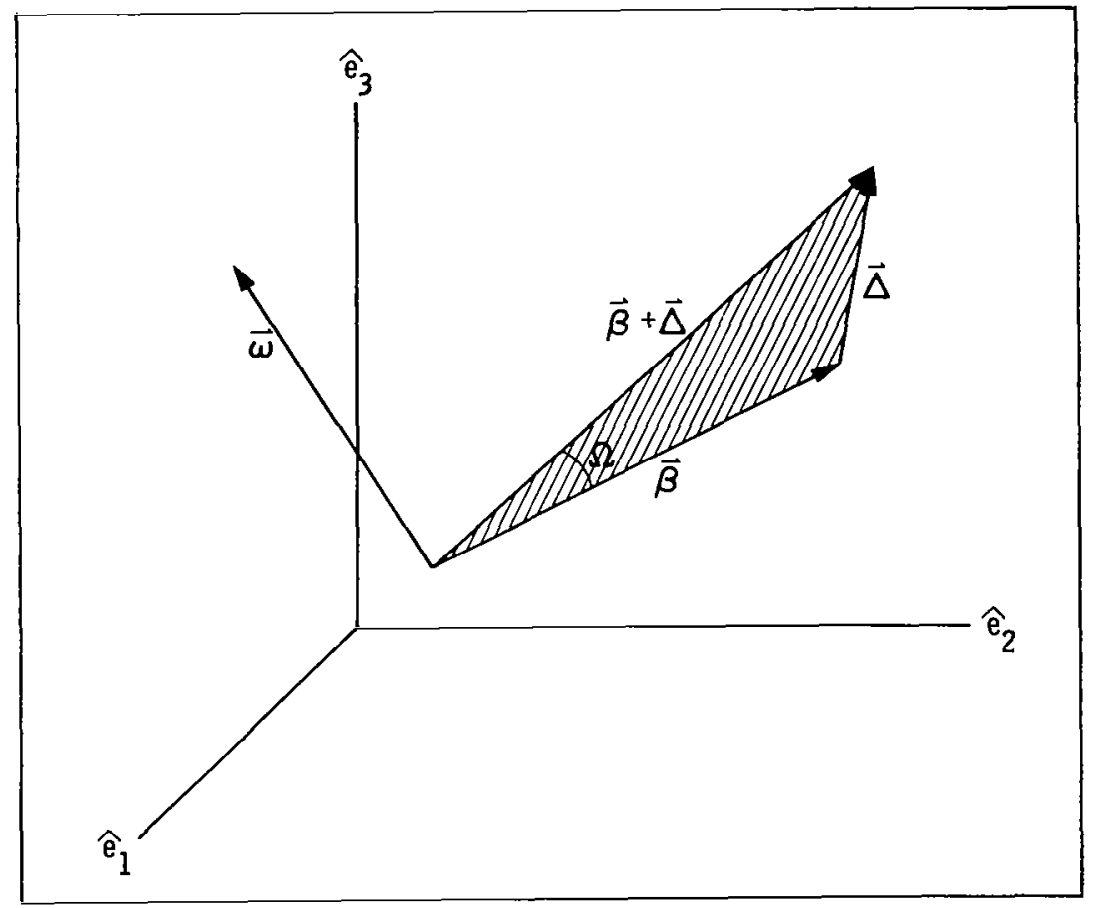

Figure 5 .

In the body-fixed frame,

$$
\begin{aligned}
& \vec{\omega}=\omega_{i} \widehat{\mathrm{e}}_{\mathrm{i}} \\
& \vec{\beta}=b_{i} \widehat{\mathrm{e}}_{\mathrm{i}} \\
& \vec{\Delta}=\mathrm{d}_{\mathrm{i}} \widehat{\mathrm{e}}_{\mathrm{i}}
\end{aligned}
$$


and so

$$
\omega_{i}=\frac{\epsilon_{i j k} b_{j} d_{k}}{b_{\ell} b_{\ell}}
$$

The antisymmetric tensor $\omega_{\mathrm{ij}}$ dual to the axial vector $\vec{\omega}$ is

$$
\omega_{\mathrm{ij}}=\epsilon_{\mathrm{kij}} \omega_{\mathrm{k}}
$$

or

$$
\omega_{i j}=\frac{\epsilon_{k i j} \epsilon_{k \ell m} b_{\ell} d_{m}}{b_{n} b_{n}} .
$$

Using the properties of the alternating tensor, this can be written

$$
\omega_{i j}=\left(\delta_{i \ell} \delta_{j m}-\delta_{i m} \delta_{j \ell}\right) \frac{b_{\ell} d_{m}}{b_{n} b_{n}}
$$

or

$$
\omega_{i j}=\frac{b_{i} b_{j}-b_{j} d_{i}}{b_{n} b_{n}} .
$$

A comparison of this result with the definition in equation (56) of the rotation tensor analog $\Omega_{\mathrm{ij}}^{\mathrm{k}}$ for the $\mathrm{k}^{\text {th }}$ baseline shows that

$$
\Omega_{\mathrm{ij}}^{\mathrm{k}}=-\omega_{\mathrm{ij}}
$$

As a consequence of this, we see that the body-fixed components $\Omega_{i}^{k}$ of the vector $\vec{\Omega}^{k}$ of the $k^{\text {th }}$ interferometer baseline occurring as a result of the rotation relative displacements $\vec{\Delta}^{\mathrm{k}}$ are obtained from the rotation tensor analog $\Omega_{\mathrm{ij}}^{\mathrm{k}}$ by

$$
\Omega_{\mathrm{i}}^{\mathrm{k}}=-\epsilon_{\mathrm{imn}} \Omega_{\mathrm{mn}}^{\mathrm{k}}
$$

\section{The Body-Fixed Coordinate Frame}

From observations on a global network of $\mathrm{N}$ interferometer baselines, we can define

$$
E_{i j}=\frac{1}{\sum_{k=1}^{N} W^{k}} \sum_{k=1}^{N} W^{k} e_{i j}^{k}
$$


and

$$
\Lambda_{\mathrm{ij}}=\frac{1}{\sum_{\mathrm{k}=1}^{\mathrm{N}} \mathrm{w}^{\mathrm{k}}} \sum_{\mathrm{k}=1}^{\mathrm{N}} \mathrm{w}^{\mathrm{k}} \Omega_{\mathrm{ij}}^{\mathrm{k}}
$$

where $\mathrm{W}^{\mathrm{k}} \mathrm{k}=1,2,3, \ldots \mathrm{N}$ are appropriate weighting factors. $\mathrm{E}_{\mathrm{ij}}$ is the weighted mean global strain tensor analog and $\Lambda_{\mathrm{ij}}$ is the weighted mean global rotation tensor analog. The strain tensor analog $\mathrm{e}_{\mathrm{ij}}^{\mathrm{k}}$ and rotation tensor analog $\Omega_{\mathrm{ij}}^{\mathrm{k}}$ on each interferometer baseline can then be expressed as the sum of the above global means and a "local" residual $\epsilon_{\mathrm{ij}}^{\mathrm{k}}$ and $\omega_{\mathrm{ij}}^{\mathrm{k}}$ respectively specific to the $\mathrm{k}^{\text {th }}$ baseline.

$$
\begin{gathered}
e_{i j}^{k}=E_{i j}+\epsilon_{i j}^{k} \\
\Omega_{i j}^{k}=\Lambda_{i j}+\omega_{i j}^{k}
\end{gathered}
$$

where, by definition, the weighted global means of the local residuals vanish.

$$
\begin{aligned}
& \frac{1}{\sum_{k=1}^{N} W^{k}} \sum_{k=1}^{N} W^{k} \epsilon_{i j}^{k}=0 \\
& \frac{1}{\sum_{k=1}^{N} W^{k}} \sum_{k=1}^{N} W^{k} \omega_{i j}^{k}=0 .
\end{aligned}
$$

If the global distribution of interferometer baselines is arranged to representatively sample the earth's crust, then $E_{i j}$ might be expected to reveal the deformation properties of the earth as a whole. Such measurements might be expected to reveal a number of global processes which are expected to be producing coherent deformation of the whole earth.

Earth expansion or contraction would be revealed by a significant departure of $T_{r}\left(E_{i j}\right)$ from zero. Overall earth expansion or contraction is associated with a number of outstanding problems in geophysics such as:

(a) the problem of the internal evolution of the earth and chemical phase changes possibly associated with the growth or decay of the solid inner or liquid outer cores,

(b) the problem of the effect on the earth's figure of the secular spin-down and the question of the finite strength of the mantle and the persistence or otherwise of a "fossil" equatorial bulge, and

(c) the problem of possible cosmological effects due to a non-zero value of $\dot{\mathrm{G}} / \mathrm{G}$ causing secular changes in the earth's size. 
The off-diagonal elements of $E_{i j}$ would be associated with global shear deformation and would reveal tidal-like effects on the earth. The eigenvectors of $E_{i j}$ would indicate the directions of principal global stress as evidenced by crustal observations. Their evolution with time might reveal information about processes in the earth's interior. Of particular interest would be a comparison of the evolution in time of the eigenvectors $E_{i j}$ as measured by long baseline interferometry and the evolution in time of the principal axes of inertia of the earth as evidenced by its rotational dynamics and the orbits of satellites.

For certain geophysical applications such as the study of plate tectonics, it may prove useful to introduce a material body-fixed coordinate frame $\bar{x}, \bar{y}, \bar{z}$ spanned by basis vectors $\bar{e}_{1}, \bar{e}_{2}, \bar{e}_{3}$ defined in the sense that for coherent global deformations $E_{i j}$, the body-fixed coordinate surfaces, deform along with the global motion of the material. This is easily accomplished using the observed mean global strain tensor analog $E_{i j}$ according to the procedure described above in the section on continuum mechanics.

At some epoch $t_{o}$, the earth's internal state is adopted as an arbitrary standard with $E_{i j}=E_{i j}^{o} \equiv 0$ and the material body-fixed basis vectors $\bar{e}_{1}, \bar{e}_{2}, \bar{e}_{3}$ coinciding with the basis vectors $\widehat{e}_{1}, \widehat{e}_{2}, \widehat{e}_{3}$ defined in equation (6). The material coordinates $\bar{x}, \bar{y}, \bar{z}$ are therefore identical to the Cartesian coordinates $x, y, z$ at the epoch. For times $t>t_{o}$ later than the epoch, the material body-fixed basis vectors $\bar{e}_{1}, \bar{e}_{2}, \bar{e}_{3}$ deform along with the mean global motion and are given by equations (44) and (45) as

$$
\begin{aligned}
& \left|\bar{e}_{1}\right|=1+\frac{1}{2} E_{11} \\
& \left|\bar{e}_{2}\right|=1+\frac{1}{2} E_{22} \\
& \left|\bar{e}_{3}\right|=1+\frac{1}{2} E_{33}
\end{aligned}
$$

and

$$
\begin{aligned}
& \overparen{\overline{\mathrm{e}}_{1} \overline{\mathrm{e}}_{2}}=\pi / 2-\mathrm{E}_{12} \\
& \overparen{\overline{\mathrm{e}}_{1} \overline{\mathrm{e}}_{3}}=\pi / 2-\mathrm{E}_{13} \\
& \overparen{\overline{\mathrm{e}}_{2} \overline{\mathrm{e}}_{3}}=\pi / 2-\mathrm{E}_{23}
\end{aligned}
$$

The coordinate system $\bar{x}, \bar{y}, \bar{z}$ spanned by basis vectors $\overline{\mathrm{e}}_{1}, \overline{\mathrm{e}}_{2}, \overline{\mathrm{e}}_{3}$ might offer certain advantages in studies of plate tectonics as the coordinate velocity, or changes in the coordinates of points on the earth referenced to this coordinate frame, will be due only to "local" tectonic effects. The effects of coherent global deformation are removed automatically by deformation of the coordinate frame. 
The strain tensor analog $E_{\mathrm{ij}}$ represents mean strain in the global network of interferometer baselines shown in figure 4. The residuals $\epsilon_{\mathrm{ij}}^{\mathrm{k}}$ given in equation (81) represent local deformations of the $\mathrm{k}^{\text {th }}$ baseline relative to the global network and are due to the effects of regional tectonic motion. The $\epsilon_{\mathrm{ij}}^{\mathrm{k}}$ will contain the information about regional tectonics on the earth including both inter- and intra-plate geologic processes.

If the global distribution of interferometer baselines is arranged to representatively sample the earth's crust, then the mean global rotation tensor analog $\Lambda_{\mathrm{ij}}$ and its dual axial vector $\vec{\Lambda}$ with bodyfixed components $\Lambda_{\mathrm{i}}$ given by

$$
\Lambda_{\mathrm{i}}=\epsilon_{\mathrm{ijk}} \Lambda_{\mathrm{ij}}
$$

represent a net mean rotation of the network of interferometer baselines shown in figure 4 relative to the body-fixed basis vectors $\widehat{\mathrm{e}}_{1}, \widehat{\mathrm{e}}_{2}, \widehat{\mathrm{e}}_{3}$ of equation (6). If the global network of interferometers is to be used to define the body-fixed coordinate frame spanned by $\widehat{e}_{1}, \widehat{e}_{2}, \widehat{e}_{3}$, it is a contradiction for such a coordinate frame to be rotating relative to itself. Hence, the body-fixed basis vectors $\widehat{e}_{1}$, $\widehat{e}_{2}, \widehat{e}_{3}$ must necessarily be defined in such a way that $\Lambda_{i j}$ vanishes. Since $\Lambda_{\mathrm{ij}}$ can be regarded as an infinitesimal rotation residual to the transformation of equation (6), the most direct way of defining $\widehat{e}_{1}, \widehat{e}_{2}, \widehat{e}_{3}$ so that $\Lambda_{i j}$ vanishes is to include the effects of the rotation of $\Lambda_{i j}$ directly into the transformation of equation (6) from the space-fixed to the body-fixed frame. This can be done by rewriting the transformation as

$$
\widehat{\mathrm{e}}_{\mathrm{i}}(\mathrm{t})=\left[\delta_{\mathrm{ij}}-\Lambda_{\mathrm{ij}}(\mathrm{t})\right] P_{j k}(\mathrm{t}) \mathrm{N}_{\mathrm{k} \ell}(\mathrm{t}) \mathrm{S}_{\ell m}(\mathrm{t}) \mathrm{W}_{\mathrm{m} n}(\mathrm{t}) \widehat{\mathrm{E}}_{\mathrm{n}}
$$

However, this is probably not the most useful procedure to follow since the measured values of $\Lambda_{\mathrm{ij}}(\mathrm{t})$ contain, in addition to the unknown effects of global deformability, the effects of errors in the models $\mathrm{P}_{\mathrm{ij}}$, $\mathrm{N}_{\mathrm{ij}}$, etc. for the known effects of precession, nutation, spin, and wobble. By observing the time dependence of the measured values of $\Lambda_{\mathrm{ij}}$, it will be possible to decide between the alternatives of modifications to the known transformation matrices $\mathrm{P}_{\mathrm{ij}}, \mathrm{N}_{\mathrm{ij}}, \mathrm{S}_{\mathrm{ij}}, \mathrm{W}_{\mathrm{ij}}$ and the addition of yct another transformation matrix $\mathrm{D}_{\mathrm{ij}}$ representing the rotational effects of global deformability.

Historical precedent indicates that the discovery of new geophysical or astronomical phenomena has been accompanied by the addition of a new matrix to the transformation from the space-fixed frame to the body-fixed frame. Howevcr, since the models for preccssion, nutation, ctc. are also expected to contain errors at the level of measurement anticipated by future long baseline interferometry, we should be prepared to excrcisc both options.

The residuals $\omega_{\mathrm{ij}}^{\mathrm{k}}$ given in equation (82) represent local rotations of the $\mathrm{k}^{\text {th }}$ baseline relative to the global network and, like the residuals $\epsilon_{\mathrm{ij}}^{\mathrm{k}}$ of equation (81), are due to the effects of regional tectonic motion. The $\omega_{\mathrm{ij}}^{\mathrm{k}}$, like the $\epsilon_{\mathrm{ij}}^{\mathrm{k}}$, will contain information about regional tectonics on the earth including both inter- and intra-plate geologic processes. 


\section{The Space-Fixed Coordinate Frame}

The present set of space-fixed basis vectors $\widehat{\mathrm{E}}_{1}, \widehat{\mathrm{E}}_{2}, \widehat{\mathrm{E}}_{3}$ are dynamically defined by the orbit of the earth about the sun and, while they play an essential role in the formulation of the theory of the orbital dynamics of the earth, they play no essential role in the formulation of the theory of the rotational dynamics of the earth. The basis vectors $\widehat{E}_{1}, \widehat{E}_{2}, \widehat{E}_{3}$ have served both purposes in the past as a matter of convenience. However, since translational (orbital) and rotational motions of the earth are dynamically independent, no great difficulty should arise if the two motions were referred to different space-fixed coordinate frames - should it prove convenient to do so. It is apparent that the application of long baseline interferometry to problems of earth rotation would be greatly facilitated by referencing the space-fixed basis vectors directly to the cosmic radio frame of the quasars. Besides being the most remote objects in the observable universe, hence expected to possess very little proper motion, the choice of the quasars to define the space-fixed frame offers some important measurement advantages.

The delay observable $\tau^{\mathrm{k}}$ is a scalar quantity rclated to the inner product of a unit vector $\widehat{\mathrm{s}}$ in the direction of the radio source and the baseline vector $\vec{\beta}^{\mathbf{k}}$

$$
\tau^{k}=\frac{1}{c} \widehat{s} \cdot \vec{\beta}^{k} .
$$

The delay observable can be written in two equivalent forms

$$
\begin{aligned}
\tau^{\mathrm{k}} & =\frac{1}{\mathrm{c}} \mathrm{s}^{\mathrm{i}} \beta_{\mathrm{i}}^{\mathrm{k}} \\
\tau^{\mathrm{k}} & =\frac{1}{\mathrm{c}} \mathrm{c}_{\mathrm{i}} \beta^{\mathrm{ik}}
\end{aligned}
$$

where $\mathrm{s}^{\mathrm{i}}, \mathrm{s}_{\mathrm{i}}$, are the contravariant and covariant components of the unit source vector $\widehat{\mathrm{s}}$ and $\beta^{i k}, \beta_{\mathrm{i}}^{\mathrm{k}}$ are the contravariant and covariant components of the $\mathrm{k}^{\text {th }}$ baseline vector $\vec{\beta}^{\mathrm{k}}$ both referred to a set of, as yet unspecified, basis vectors the usual choice for which is $\widehat{\mathrm{E}}_{1}, \widehat{\mathrm{E}}_{2}, \widehat{\mathrm{E}}_{3}$ or $\widehat{\mathrm{e}}_{1}, \widehat{\mathrm{e}}_{2}, \widehat{\mathrm{e}}_{3}$.

If we introduce a space-fixed coordinate frame whose fundamental directions are defined by three selected quasar radio sources $\mathrm{s}(1), \mathrm{s}(2), \mathrm{s}(3)$, then it is clear that the baseline vector $\vec{\beta}^{\mathrm{k}}$ has two representations given by

and

$$
\vec{\beta}^{k}=\beta_{1}^{k} \widehat{s}^{1}+\beta_{2}^{k} \widehat{s}^{2}+\beta_{3}^{k} \widehat{s}^{3}
$$

$$
\vec{\beta}^{\mathrm{k}}=\beta^{1 \mathrm{k}} \widehat{\mathrm{s}}_{1}+\beta^{2 \mathrm{k}} \widehat{\mathrm{s}}_{2}+\beta^{3 \mathrm{k}} \widehat{\mathrm{s}}_{3}
$$

where $\widehat{\mathrm{s}}^{1}, \widehat{\mathrm{s}}^{2}, \widehat{\mathrm{s}}^{3}$ are the contravariant quantities or so-called basis 1-forms [Misner, Throne, Wheeler, 1973, pp. 53 ff.] and $\widehat{s}_{1}, \widehat{s}_{2}, \widehat{s}_{3}$ are the covariant quantities or so-called basis vectors spanning the space-fixed frame appropriate to radio sources $s(1), s(2), s(3)$. 
An analysis of the operation of a long baseline interferometer [Thomas 1972, Cannon 1978] shows that the delay observable yields the covariant components of the baseline vector as shown schematically in figure 6. The stopped fringes of the incoming wave fronts from the radio source provide a physical realization of the basis 1 -forms which define the contravariant quantities $\widehat{\mathrm{s}}^{1}, \widehat{\mathrm{s}}^{2}, \widehat{\mathrm{s}}^{3}$. In the space-fixed coordinate frame defined by radio sources $\mathrm{s}(1), \mathrm{s}(2), \mathrm{s}(3)$, the contravariant basis vectors or 1 -forms $\widehat{\mathrm{s}}^{1}, \widehat{\mathrm{s}}^{2}, \widehat{\mathrm{s}}^{3}$ have components

$$
\begin{aligned}
& \widehat{\mathrm{s}}^{1}=\left[\begin{array}{lll}
1 & 0 & 0
\end{array}\right] \\
& \widehat{\mathrm{s}}^{2}=\left[\begin{array}{lll}
0 & 1 & 0
\end{array}\right] \\
& \widehat{\mathrm{s}}^{3}=\left[\begin{array}{lll}
0 & 0 & 1
\end{array}\right]
\end{aligned}
$$

by definition. From equation (90), the baseline vector $\vec{\beta}^{\mathrm{k}}$ has representation

$$
\vec{\beta}^{\mathrm{k}}=\mathrm{c} \tau_{1}^{\mathrm{k}} \widehat{\mathbf{s}}^{1}+\mathrm{c} \tau_{2}^{\mathrm{k}} \widehat{\mathrm{s}}^{2}+\mathrm{c} \tau_{3}^{\mathrm{k}} \widehat{\mathbf{s}}^{3}
$$

where the covariant space-fixed components of $\vec{\beta}^{k}$ given by

$$
\begin{aligned}
& \beta_{1}^{\mathrm{k}}=c \tau_{1}^{\mathrm{k}} \\
& \beta_{2}^{\mathrm{k}}=\mathrm{c} \tau_{2}^{\mathrm{k}} \\
& \beta_{3}^{\mathrm{k}}=\mathrm{c} \tau_{3}^{\mathrm{k}}
\end{aligned}
$$

are thus measured directly without reference to source coordinates.

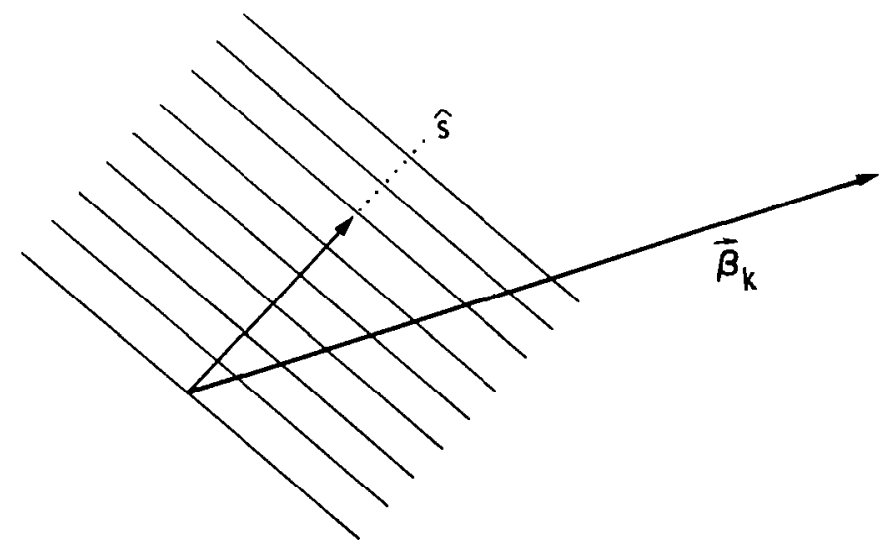

Figure 6. 
It is traditional in geodesy to measure contravariant components of baseline vectors - the infinitesimal displacement vector being defined as the archetypical contravariant vector. To obtain the contravariant space-fixed components $\beta^{\text {ik }}$ of the baseline vector $\vec{\beta}^{k}$ from the covariant space-fixed components $\beta_{i}^{\mathrm{k}}$ given by the delay observable and equations (96). requires knowledge. of the metric tensor $\mathrm{g}_{\mathrm{ij}}$ of the nonorthogonal space-fixed coordinate frame of the quasars $\mathrm{s}(1), \mathrm{s}(2), \mathrm{s}(3)$ shown schematically in figure 7 . The contravariant components $\beta^{\text {ik }}$ are given by

where

$$
\beta^{\mathrm{ik}}=\mathrm{g}^{\mathrm{ij}} \beta_{\mathrm{j}}^{\mathrm{k}}
$$

$$
g^{i j}=g_{i j}^{-1}
$$

and where

$$
\mathrm{g}_{\mathrm{ij}}=\left[\begin{array}{ccc}
1 & \cos \widehat{\mathrm{s}(1) \mathrm{s}(2)} & \cos \widehat{\widehat{s}(1) \mathrm{s}(3)} \\
\cos \widehat{\mathrm{s}(2) \mathrm{s}(1)} & 1 & \cos \widehat{s(2) \mathrm{s}(3)} \\
\cos \widehat{s(3) \mathrm{s}(1)} & \cos \widehat{\mathrm{s}(3) \mathrm{s}(2)} & 1
\end{array}\right]
$$

This procedure does not require knowledge of absolute source coordinates but merely the relative angles or the direction cosines between the quasars $s(1), s(2), s(3)$. This measurement can also be made very accurately by long baseline interferometry.

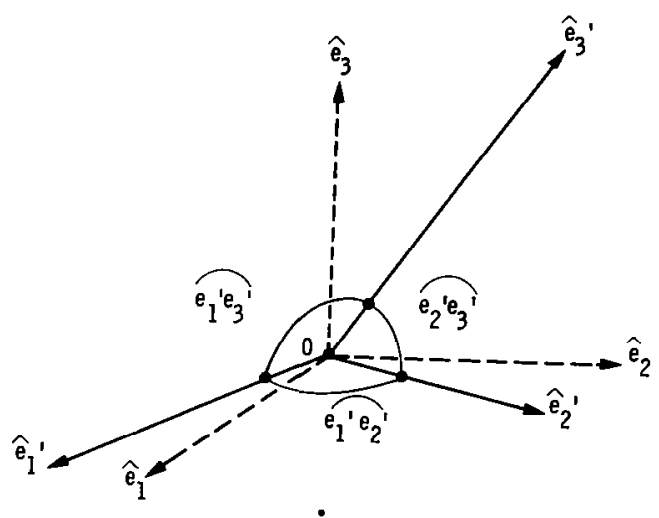

Figure 7.

We now consider the case where observations are made of a radio source $\mathrm{s}(\mathrm{J}), \mathrm{J}=4,5,6 \ldots$ which is not one of the three sources $s(1), s(2), s(3)$ dcfining the fundamental directions in the space-fixed frame. The delay observable on the source $\mathrm{s}(\mathrm{J})$ and baseline $\vec{\beta}^{\mathrm{k}}$ is $\tau^{\mathrm{k}}(\mathrm{J})$ where

$$
\tau^{\mathrm{k}}(\mathrm{J})=\frac{1}{\mathrm{c}} \widehat{\mathrm{s}}(\mathrm{J}) \cdot \beta^{\mathrm{k}}
$$

and where $\widehat{\mathrm{s}}(\mathrm{J})$ is a unit vector in the direction of the radio source $\widehat{\mathrm{s}}(\mathrm{J})$. $\widehat{\mathrm{s}}(\mathrm{J})$ can be written in terms of the funamental contravariant basis vectors $\widehat{\mathrm{s}}^{1}, \widehat{\mathrm{s}}^{2}, \widehat{\mathrm{s}}^{3}$ as 


$$
\widehat{\mathrm{s}}(J)=\mathrm{s}_{1}(J) \widehat{\mathrm{s}}^{1}+\mathrm{s}_{2}(J) \widehat{\mathrm{s}}^{2}+\mathrm{s}_{3}(J) \widehat{\mathrm{s}}^{3}
$$

where

$$
\begin{aligned}
& \mathrm{s}_{1}(\mathrm{~J})=\cos \widehat{s(1) \mathrm{s}(\mathrm{J})} \\
& \mathrm{s}_{2}(\mathrm{~J})=\cos \widehat{\mathrm{s}(2) \mathrm{s}(\mathrm{J})} \\
& \mathrm{s}_{3}(\mathrm{~J})=\cos \widehat{\mathrm{s}(3) \mathrm{s}(\mathrm{J})}
\end{aligned}
$$

Equations (102) indicate that any of the quasar radio sources $\mathrm{s}(\mathrm{J}), \mathrm{J}=4,5,6 \ldots$ can be observed to obtain the space-fixed covariant components of the interferometer baseline providing the direction cosines of the unit vector $\mathrm{s}(\mathrm{J})$ relative to the three chosen fundamental space-fixed directions given by sources $s(1), s(2), s(3)$ are known. The angles $\widehat{s(1) s(J)}, \widehat{s(2) s(J)}, \widehat{s(3) s(J)}$ can be measured accurately hy long baseline interferometers. This suggests that a cataloguing procedure in terms of the direction cosines to three chosen fundamental sources instead of right ascension and declination might be usefully adopted in the near future.

It is possible that the three chosen radio sources $s(1), s(2), s(3)$ will exhibit proper motion relative to the cosmic space-fixed radio frame defined by the ensemble of quasars. Should this be the case it will present no great difficulty to the above procedure. The ensemble of quasars would then be chosen to define the space-fixed frame and to possess a Maxwellian velocity distribution and to exhibit no mean rotation. With this assumption, it would remain only to tie the positions of the chosen sources $s(1), s(2), s(3)$ to the ensemble of quasars by periodic measurements of the angles $\widehat{\mathrm{s}(1) \mathrm{s}(\mathrm{J})} \mathrm{s(2) \textrm {s } ( \mathrm { J } )} \widehat{\mathrm{s}(3) \mathrm{s}(\mathrm{J})}, \mathrm{J}=4,5,6 \ldots \mathrm{N}$ where $\mathrm{N}$ could be of the order of 100 to correctly account for any proper motion they might exhibit.

Finally, it may perhaps be useful in the light of the general theory of relativity to retain and to distinguish between two sets of space-fixed basis vectors. The first set $\widehat{s}^{1}, \widehat{\mathrm{s}}^{2}, \widehat{\mathrm{s}}^{3}$ described above span what could be called a cosmic inertial frame. The second set $\widehat{\mathrm{E}}_{1}, \widehat{\mathrm{E}}_{2}, \widehat{\mathrm{E}}_{3}$ to be defined dynamically by a relativistic ephemeris of the solar system span what could be called a local inertial frame. The theory of general relativity allows for these two inertial frames to be relatively rotating [Weinberg, 1972, pp. $239 \mathrm{ff}]$.

\section{REFERENCES}

Brillouin, L., Tensors in Mechanics and Elasticity, Academic Press, 1964.

Cannon, W. H., "The Classical Analysis of the Response of a Long Baseline Radio Interferometer" Geophys. J. R. Astr. Soc., 53, 503-530, 1978.

Explanatory Supplement to the Astronomical Ephemeris and the American Ephemeris and Nautical Almanac, published by the Nautical Almanac Offices of the United Kingdom and United States of America, 1961. 
Jones, H. E., "Geodetic Ties Between Continents by Means of Radio Telescopes" Canadian Surv., $23,377-388,1969$.

McKenzie, D. P., "Plate Tectonics" in The Nature of the Solid Earth, Chapt. 13, Robertson, E. C., Hays, J. F., Knopoff, L., eds. McGraw Hill, 1972.

Misner, C. W., Thorne, K. S., Wheeler, J. A., Gravitation, Freeman, 1973.

Mueller, I. I., Spherical and Practical Astronomy as Applied to Geodesy, Ungar, 1969.

Munk, W. H., MacDonald, G. J. F., The Rotation of the Earth - A Geophysical Discussion, Cambridge University Press, 1960.

Thomas, J. B., "An Analysis of Long Baseline Radio Interferometry" Deep Space Network Progress Report, J.P.L. Technical Report 32-1526, Vol. VII, 1972.

Thompson, W., "On the Rigidity of the Earth" Phil. Trans. R. Soc. London, 153, 1863.

Weinberg, S., "Gravitation and Cosmology: Principles and Applications of the General Theory of Relativity," Wiley and Sons, 1972.

Woolard, E. W., "The Theory of the Rotation of the Earth around its Centre of Mass," Astronomical Papers Prepared for the Use of the American Ephemeris and Nautical Almanac Vol. XV, Part 1, U.S. Government Printing Office, Washington, D.C. 1953. 


\section{APPENDIX}

\section{The Definition of the Mean Global Strain Tensor $E_{i j}$ and the Mean Global Rotation Tensor $\Lambda_{\mathbf{i j}}$}

The definition of the mean global strain tensor $E_{i j}$ and the mean global rotation tensor $\Lambda_{i j}$ as given in equations (79) and (80)

$$
\begin{aligned}
& E_{i j}=\frac{1}{\sum_{k=1}^{N} k} \sum_{k=1}^{N} k e_{i j}^{k} \\
& \Lambda_{i j}=\frac{1}{\sum_{k=1}^{N} k} \sum_{k=1}^{N} k \Omega_{i j}^{k}
\end{aligned}
$$

can be shown to satisfy two desirable objective criteria. These are discussed below.

\section{$E_{i j}$ - the Mean Global Strain Tensor}

The definition of $E_{i j}$ minimizes, in a weighted least squares sense the net departure between the squared baseline length variations $\overline{\delta \mathrm{s}}{ }^{2}$ which would be predicted assuming a mean global strain $\mathrm{E}_{\mathrm{ij}}$ and the individual squared baseline length variations $\delta \mathrm{s}^{2}$ observed by the long baseline interferometer.

$$
\begin{aligned}
\delta s^{k^{2}} & =e_{i j}^{k} b_{i}^{k} b_{j}^{k} \\
\delta s & k^{2}=E_{i j} b_{i}^{k} b_{j}^{k}
\end{aligned}
$$

Net weighted squared departure between predicted $\overline{\delta \mathrm{s}}^{\mathrm{k}^{2}}$ and observed $\delta \mathrm{s}^{\mathrm{k}^{2}}$ squared baseline length variations is $D^{2}$ where

$$
D^{2}=\sum_{k=1}^{N} w^{k}\left(\delta s^{k}-\delta s^{2}\right)^{2}
$$




$$
\begin{gathered}
D^{2}=\sum_{k=1}^{N} w^{k}\left(e_{i j}^{k} b_{i}^{k} b_{j}^{k}-E_{\ell m} b_{\ell}^{k} b_{m}^{k}\right)^{2} \\
D^{2}=\sum_{k=1}^{N} w^{k}\left[\left(e_{i j}^{k} b_{i}^{k} b_{j}^{k}\right)^{2}-2 e_{i j}^{k} E_{\ell m} b_{i}^{k} b_{j}^{k} b_{\ell m}^{k} b_{m}^{k}+\left(E_{\ell m} b_{\ell}^{k} b_{m}^{k}\right)^{2}\right]
\end{gathered}
$$

A choice of $E_{\ell m}$ which minimizes $D^{2}$ is given by

$$
\frac{\partial D^{2}}{\partial E_{p q}}=0
$$

This gives a condition

$$
\begin{aligned}
& \sum_{k=1}^{N} w^{k}\left(-2 e_{i j}^{k} \frac{\partial E_{\ell m}}{\partial E_{p q}} b_{i j}^{k_{j} k_{j} b_{\ell} b_{m}^{k}}+2\left(E_{i j} b_{i}^{k_{j} b_{j}^{k}}\right) \frac{\partial}{\partial E_{p q}}\left(E_{\ell m} b_{\ell}^{k} b_{m}^{k}\right)\right)=0 \\
& \sum_{k=1}^{N} w^{k}\left(-2 e_{i j}^{k} b_{i}^{k} b_{j}^{k} b_{\ell} b_{m}^{k} \delta_{\ell p} \delta_{m q}+2 E_{i j} b_{i}^{k_{b} k_{j} b_{\ell} b_{m}^{k}} \delta_{\ell p} \delta_{m q}\right)=0 \\
& \sum_{k=1}^{N} w^{k}\left(-2 e_{i j}^{k} b_{i}^{k} b_{j} b_{p}^{k} b_{q}^{k}+2 E_{i j} b_{i}^{k_{j}} k_{j} b_{p} b_{q} k\right)=0 \\
& \sum_{k=1}^{N} w^{k}\left(-2 e_{i j}^{k}+2 E_{i j}\right) b_{i j}^{k} b_{j} k_{p} k_{q} k=0
\end{aligned}
$$

Since this condition should be fulfilled independently of the particular choice of baseline net work we conclude that we shall in general require

$$
\sum_{k=1}^{N} w^{k}\left(-2 e_{i j}^{k}+2 E_{i j}\right)=0
$$


or

$$
E_{i j}=\frac{1}{\sum_{k=1}^{N} w^{k}} \sum_{k=1}^{N} w^{k} e_{i j}^{k}
$$

\section{$\Lambda_{\mathrm{ij}}$ - the Mean Global Rotation Tensor}

The definition of $\Lambda_{\mathrm{ij}}$ minimizes, in a weighted least squares sense, the magnitude of the net departures between the mean global rotation vector $\Lambda_{i}$ and the rotation vectors $\Lambda_{i}^{k}$ of the individual baselines observed by the long baseline interferometer.

$$
\begin{aligned}
& \Lambda_{i}=\varepsilon_{i \ell m} \Lambda_{\ell m}^{k} \\
& \Omega_{i}^{k}=\varepsilon_{i \ell m} s \Omega_{\ell m}^{k}
\end{aligned}
$$

The magnitude of the net weighted squared departure between the mean global rotation vector $\Lambda_{i}$ and the observed rotation vectors $\Omega_{i}^{k}$ of the individual baselines is $\theta^{2}$

$$
\theta^{2}=\sum_{k=1}^{N} w^{k}\left(\Omega_{i}^{k}-\Lambda_{i}\right)\left(\Omega_{i}^{k}-\Lambda_{i}\right)
$$

but since

$$
\Omega_{i}^{k}-\Lambda_{i}=\varepsilon_{i \ell m}\left(\Omega_{\ell m}^{k}-\Lambda_{\ell m}\right)
$$

we have

$$
\theta^{2}=\sum_{k=1}^{N} w^{k} \varepsilon_{i \ell m} \varepsilon_{i p q}\left[\Omega_{\ell m}^{k}-\Lambda_{\ell m}\right]\left[\Omega_{p q}^{k}-\Lambda_{p q}\right]
$$

From the properties of the alternating tensor this can be written

$$
\theta^{2}=\sum_{k=1}^{N} w^{k} \delta_{\ell p} \delta_{m q}\left[\Omega_{\ell_{m}}^{k}-\Lambda_{\ell_{m}}\right]\left[\Omega_{p q}^{k}-\Lambda_{p q}\right]
$$




$$
\begin{gathered}
\theta^{2}=\sum_{k=1}^{N} w^{k}\left[\Omega_{\ell m}^{k}-\Lambda_{\ell m}\right]\left[\Omega_{\ell m}^{k}-\Lambda_{\ell m}\right] \\
\theta^{2}=\sum_{k=1}^{N} w^{k}\left[\Omega_{\ell m}^{k}{ }^{2}-2 \Lambda_{\ell m} \Omega_{\ell m}^{k}+\Lambda_{\ell m}{ }^{2}\right] .
\end{gathered}
$$

A choice of $\Lambda_{\ell m}$ which minimizes $\theta^{2}$ is given by setting

$$
\frac{\partial \theta^{2}}{\partial \Lambda_{\mathrm{pq}}}=0
$$

This leads to the condition

$$
\begin{aligned}
& \sum_{k=1}^{N} w^{k}\left[-2 \frac{\partial \Lambda_{\ell m}}{\partial \Lambda_{p q}} \Omega_{\ell m}^{k}+2 \Lambda_{\ell m} \frac{\partial \Lambda_{\ell m}}{\partial \Lambda_{p q}}\right]=0 \\
& \sum_{k=1}^{N} w^{k}\left[-2 \Omega_{\ell m}^{k} \delta_{\ell p} \delta_{m q}+2 \Lambda_{\ell m} \delta_{\ell p} \delta q\right]=0 \\
& \sum_{k=1}^{N} w^{k}\left[-2 \Omega_{p q}^{k}+2 \Lambda_{p q}\right]=0 .
\end{aligned}
$$

The solution to this equation is simply

$$
\Lambda_{p q}=\sum_{k=1}^{\frac{1}{N}} w_{k}^{k} \sum_{k=1}^{N} w^{k} \Omega_{p q}^{k} .
$$




\title{
CRUSTAL DEFORMATION AT VERY LONG BASELINE INTERFEROMETRY SITES DUE TO SEASONAL AIR-MASS AND GROUND WATER VARIATIONS
}

\author{
A. Stolz ${ }^{1}$ and D. R. Larden ${ }^{2}$ \\ School of Surveying, University of South New Wales
}

\begin{abstract}
Rearrangements of the geographic distribution of air-mass and groundwater load and deform the earth's crust. The seasonal deformation normal to the earth's surface is calculated at stations involved or interested in VLBI geodesy and at hypothetical sites in Australia and Brazil using global atmospheric pressure data, values for groundwater storage, and load Love numbers deduced from current earth models. The annual range of deformation approaches the centimeter-level measuring potential of the very long baseline interferometry (VLBI) technique at Greenbank, Haystack, and the Brazil site. Baselines between stations of the European network and the northeastern part of the United States and between Brazil and Australia could be affected at this level of accuracy.
\end{abstract}

\footnotetext{
1Present address: University FAF at Munich, Astronomical and Physical Geodesy, Werner-Heisenberg-Weg 39, D8014 Neubiberg, West Germany.

2Joint Institute for Laboratory Astrophysics, University of Colorado and National Bureau of Standards, Boulder, CO 80309, U.S.A.
} 


\section{INTRODUCTION}

The new space techniques of laser ranging to artificial satellites and the moon and VLBI have the potential of centimeter-level measurement accuracy. With these systems, the accuracy of determining dynamic earth motions can be comparable to the basic measurement accuracies obtainable (National Academy of Sciences, 1978). It is quite obvious that models for these motions will play an important role in the analysis of the data for positioning to centimeter-level accuracies. In this paper, we make a first attempt at one of the many modelling studies which need to be done. We estimate the crustal "breathing" that occurs when seasonal atmospheric pressure systems move across the globe and when there are seasonal variations in groundwater storage. Results are presented for existing VLBI stations in Europe and the United States and hypothetical sites in Australia and Brazil.

Baseline variations between groups of observatories are determined. The mathematical formulation of the problem, the data used, and the principal assumptions made are briefly reviewed.

\section{METHOD}

The problem of crustal deformation caused by the redistribution of surface masses is conveniently solved by means of the load Love numbers $h_{n}^{\prime}$ and $\ell_{n}^{\prime}$ and the change in potential at the surface arising from the variable load (Munk and MacDonald, 1960). The change in potential may be written as

$$
\Delta \mathrm{U}=\Sigma \Delta \mathrm{U}_{\mathrm{n}}=4 \pi \mathrm{Gr} \sum_{\mathrm{n}} \frac{\mathrm{q}_{\mathrm{n}}}{2 \mathrm{n}+1}
$$

where

$$
q_{n}=\frac{2 n+1}{4 \pi} \int_{s} q(\phi, \lambda ; t) P_{n}(\cos \psi) d S
$$

is the nth degree surface spherical harmonic representation of the variable surface load $q(\theta, \lambda ; t)$ at a particular point on the globe, $G$ is the gravitational constant, $r$ is the earth's radius, $P_{n}(\cos \psi)$ is the Legendre polynomial, and $\psi$ is the angle between radius vector at the computation point and the radius vector at the disturbing load. The corresponding crustal motions are

$$
\mathrm{dr}, \mathrm{rd} \theta, \mathrm{rd} \lambda=\left\{\sum_{\mathrm{n}} \mathrm{h}_{\mathrm{n}}^{\prime} \frac{\Delta \mathrm{U}_{\mathrm{n}}}{\mathrm{g}}, \sum_{\mathrm{n}} \ell_{\mathrm{n}}^{\prime} \frac{1}{\mathrm{~g}} \frac{\partial\left(\Delta \mathrm{U}_{\mathrm{n}}\right)}{\partial \theta}, \sum_{n} \ell_{\mathrm{n}}^{\prime} \frac{1}{\mathrm{~g} \sin \theta} \frac{\partial\left(\Delta \mathrm{U}_{\mathrm{n}}\right)}{\partial \lambda}\right\}
$$

where $\theta$ is colatitude, $\lambda$ is east longitude, and $\mathrm{g}$ is local gravity.

We applied equations (1), (2), and (3) to air-mass and groundwater data. The term groundwater refers to moisture stored on the earth's land surface, and includes snow, variable water storage in the 
form of plant material, and water between the surface and the shallowest position of the watertable. The complete calculations are rather lengthy. Since the load Love numbers $\ell_{n}^{\prime}$ required for the problem are about two orders of the magnitude smaller than their $h_{n}^{\prime}$ counterparts and the radial crustal distortions are already small, we calculated only dr.

\section{DATA}

We used the January, April, July, and October averages of sea-level pressure compiled by Schutz and Gates $(1971,1972,1973,1974)$ which are given at gridpoints spaced every $4^{\circ}$ of latitude and $5^{\circ}$ of longitude over the entire earth. These data need to be adjusted back to station elevation before evaluation of $\Delta U_{n}$. Laplace's formula was used to make the necessary adjustment. The groundwater calculations are based on van Hylckama's (1956) monthly compilations for $10^{\circ} \times 10^{\circ}$ areas. We have taken his January, April, July, and October volumes and divided these by the area of the corresponding compartment to yield surface loads. Data beyond $70^{\circ} \mathrm{S}$ does not appear and have been ignored. Dahlens's (1976) load Love numbers for earth model $1066 \mathrm{~A}$ were preferred for no special reason.

\section{RESULTS}

The calculation for the radial deformations due to atmospheric loading and groundwater have been published separately by Stolz and Larden (1979) and Larden (1980), ${ }^{*}$ respectively, and are depicted in contour form on maps for January, April, July, and October. The crustal motion attributable to the atmosphere approaches significance over parts of Asia, in Greenland, and in Antarctica. It is well below $1 \mathrm{~cm}$ in other parts of the globe. The deformations due to groundwater variations are also small and reach $1 \mathrm{~cm}$ only over the Himalayas and the northern United States. For this study, we have combined the results of Stolz and Larden (1979) and Larden (1980) to produce the amplitude and phase of the annual radial deformation (the annual term of deformation at a particular location is $A$ cos ( $\odot$-phase), where $A$ is the amplitude and $\odot$ is the sun's longitude measured from the beginning of the year) at sites which are actively involved or are interested in VLBI experiments. The horizontal gradients of the deformations over Europe and parts of the United States are small. We have averaged the distortions for these regions (table 1). Thus, Owens Valley and Goldstone become SW U.S. $\left(37^{\circ} \mathrm{N}, 246^{\circ} \mathrm{E}\right)$, Ft. Davis and Richmond become SE U.S. $\left(30^{\circ} \mathrm{N}, 270^{\circ} \mathrm{E}\right)$, Haystack and Greenbank become NE U.S. $\left(45^{\circ} \mathrm{N}, 280^{\circ} \mathrm{E}\right)$, and stations in Spain, England, Holland, Germany, Italy, Sweden, Poland, Finland, and Crimea (U.S.S.R.) become Europe $\left(50^{\circ} \mathrm{N}, 10^{\circ} \mathrm{E}\right)$. The results are plotted in figure 1. The vertical motions at Greenbank, Haystack, and Brazil have an annual range of roughly $1 \mathrm{~cm}$. Since the VLBI technique measures baselines and it is possible that the motions at two sites compound to produce bigger motions, we studied the baselines listed in table 2 . Only the baselines Europe-NE U.S. (a) and Brazil-Australia (c) (an unlikely possibility because the sites are separated by nearly $180^{\circ}$ in longitude) vary by approximately $1 \mathrm{~cm}$ (figure 2 ).

\footnotetext{
*Larden, D. R., Ph.D. Thesis, University of New South Wales, Sydney, N.S.W., Australia, 1980.
} 
Table 1

Radial Deformation

\begin{tabular}{|l|c|c|}
\hline & $\begin{array}{c}\text { Amplitude, } \\
\mathrm{mm}\end{array}$ & $\begin{array}{c}\text { Phase, } \\
\text { deg. }\end{array}$ \\
\hline Europe & 3.1 & $233(\mathrm{a})$ \\
Australia & 1.7 & 19 (b) \\
SW U.S. & 2.4 & $266(\mathrm{c})$ \\
SE U.S. & 1.9 & $260(\mathrm{~d})$ \\
NE U.S. & 5.8 & $243(\mathrm{e})$ \\
Brazil & 4.5 & $282(\mathrm{f})$ \\
\hline
\end{tabular}
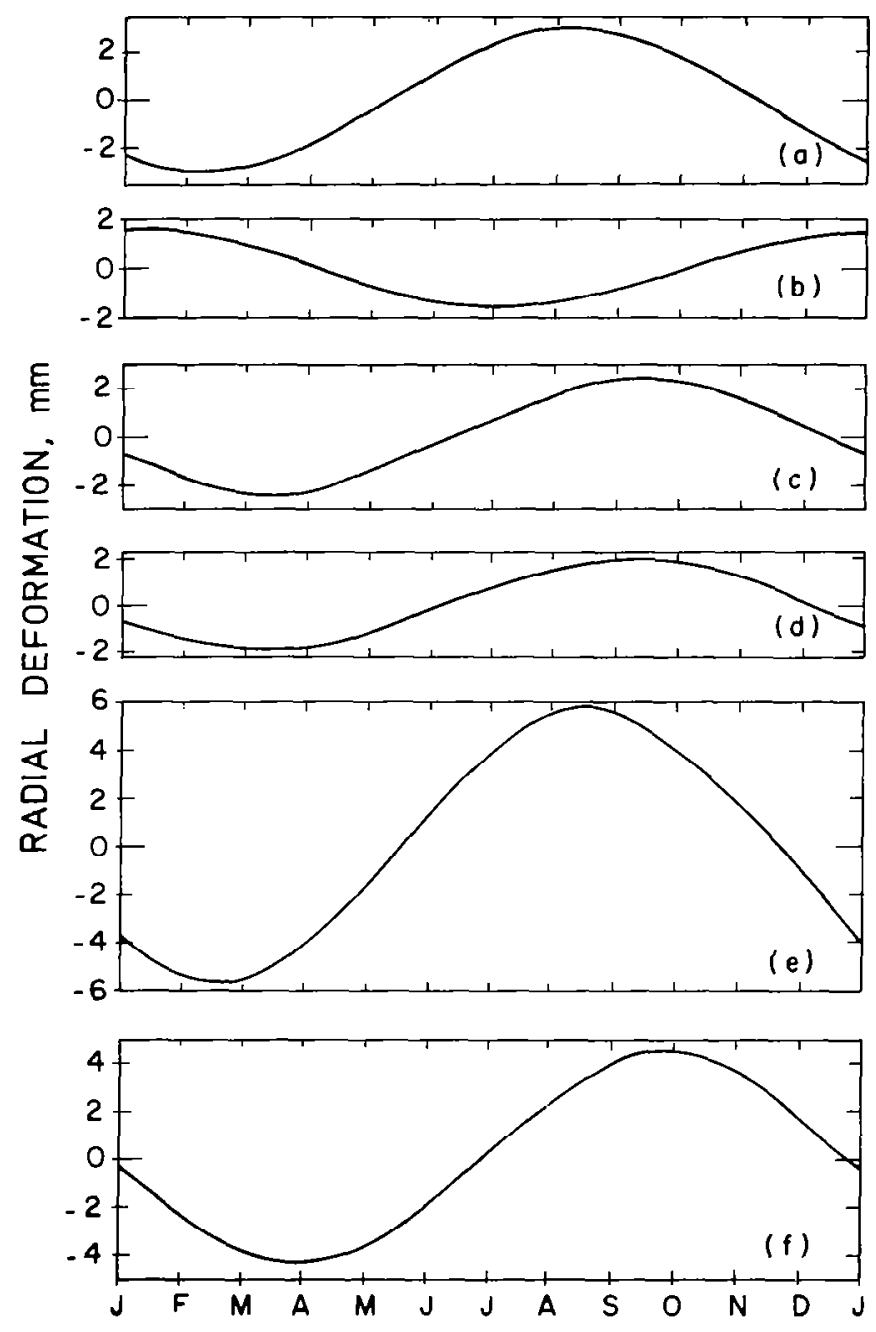

Figure 1. Radial deformations for sites located in: (a) Europe; (b) Australia; (c) SW U.S.; (d) SE U.S.; (e) NE U.S.; and (f) Brazil. 
Table 2

Baseline Variations

\begin{tabular}{|c|c|c|}
\cline { 2 - 3 } \multicolumn{1}{c|}{} & $\begin{array}{c}\text { Amplitude, } \\
\mathrm{mm}\end{array}$ & $\begin{array}{c}\text { Phase, } \\
\text { deg. }\end{array}$ \\
\hline (a) Europe, NE U.S. & 4.2 & 240 \\
NE U.S., SE U.S. & 0.9 & 247 \\
SE U.S., SW U.S. & 0.8 & 264 \\
(b) SE U.S., Brazil & 3.0 & 276 \\
(c) Brazil, Australia & 4.2 & 303 \\
\hline
\end{tabular}

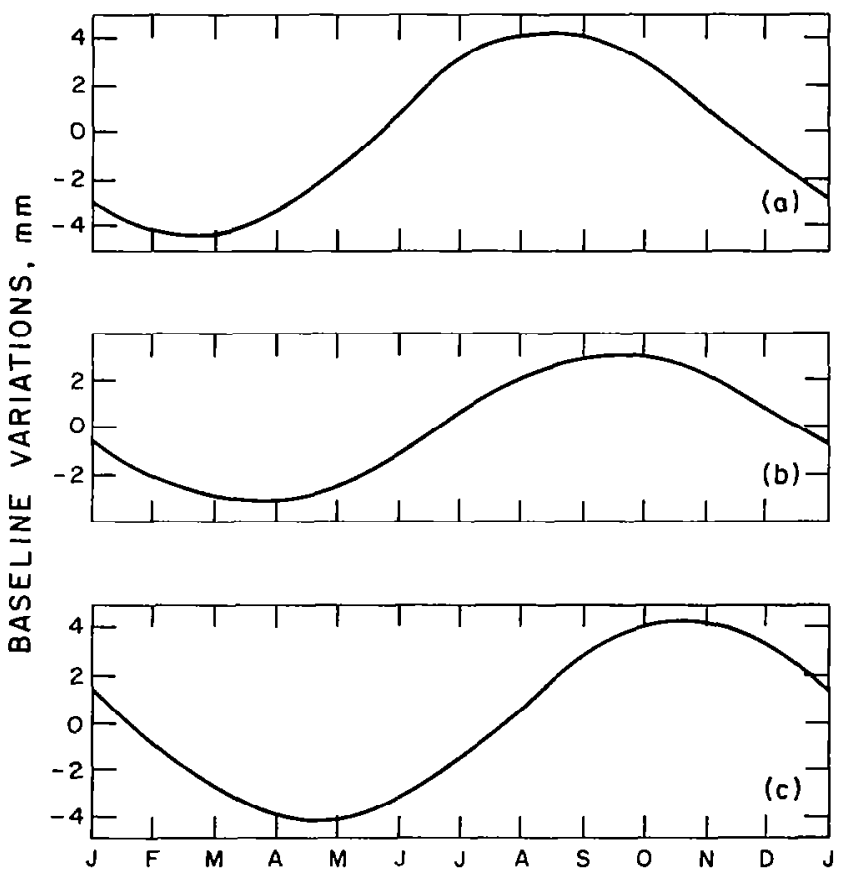

Figure 2. Baseline variations between: (a) Europe and NE U.S., (b) SE U.S. and Brazil; and (c) Brazil and Australia. 


\section{DISCUSSIONS}

When performing the deformation calculations, we need to allow for changes in sea level produced by the response of the oceans to the atmospheric pressure variations and the interchange of water between land and oceans, and atmosphere and oceans. The inverted barometer rule states that for every millibar of atmospheric pressure at a particular place over and above the mean pressure over the entire ocean surface the sea surface is locally depressed by $1 \mathrm{~cm}$. This rule is normally invoked to account for the response of the oceans. This introduces a number of problems. The two main ones are: (1) oceanic mass conservation is implied, which is impossible if we also wish to consider the interchange of water between the constituent parts of the earth; and (2) we end up with a sea surface which is not quite an equipotential. Fortunately, their effect on our results is small (Stolz and Larden, 1979). Data for sea-level variations caused by the exchange of water between land and oceans and atmosphere and oceans are too inaccurate at present to make a reliable estimate of the effect on our results. Larden (1980), op. cit.) has estimated the contribution by assuming that the moisture which is not in the atmosphere or on land during any season is in the oceans. Our results change at most by $1 \mathrm{~mm}$.

Another difficulty, concerning the zero degree load Love numbers, arises from the manner in which we have produced the results presented here. The zero degree load Love numbers vanish if mass is conserved (Rochester and Smylie, 1974), but not if the effect of air mass and groundwater is calculated separately as we have done. Thus, our results contain a zero degree contribution which should not be there. Larden (1980) has considered this problem and finds the results to be in error by roughly 10 percent. This clearly does not affect the conclusion we may draw from the calculations; that is, the general seasonal variations in the continental and intercontinental baselines are small. Accordingly, until considerable improvement is achieved in instrument stabilities and corrections for ionospheric and tropospheric effects, it does not seem necessary to adjust baseline determinations for seasonal variations, and even then, these variations will only be marginally significant.

\section{ACKNOWLEDGMENT}

This paper was written while the first author was the holder of an Alexander von Humboldt Research Fellowship. The second author is supported by a Fulbright Travel Award.

\section{REFERENCES}

Dahlen, F. A., The Passive Influence of the Oceans upon the Rotation of the Earth, Geophys. J. R. Astr. Soc., 46, 363-406, 1976.

Munk, W. H., and G. J. F. MacDonald, The Rotation of the Earth, Cambridge University Press, London, 1960.

National Academy of Sciences, Geodesy: Trends and Prospects, Washington, D.C., 1978. 
Rochester, M. G., and D. E. Smylie, On Changes in the Trace of the Earth's Inertia Tensor, J. Geophys. Res., 32, 4948-4951, 1974.

Schutz, C., and W. Gates, Global Climatic Data for Surface, $800 \mathrm{mb}, 400 \mathrm{mb}$ : January, ARPA Rept. R-915-ARPA, Rand Corporation, Santa Monica, 1971.

Schutz, C. and W. Gates, Global Climatic Data for Surface, $800 \mathrm{mb}, 400 \mathrm{mb}$ : July, ARPA Rept. R-1029-ARPA, Rand Corporation, Santa Monica, 1972.

Schutz, C. and W. Gates, Global Climatic Data for Surface, $800 \mathrm{mb}: 400 \mathrm{mb}$ : April, ARPA Rept. R-1317-ARPA, Rand Corporation, Santa Monica, 1973.

Schutz, C., and W. Gates, Global Climatic Data for Surface, $800 \mathrm{mb}, 400 \mathrm{mb}$ : October, ARPA Rept. R-1425-ARPA, Rand Corporation, Santa Monica 1974.

Stolz, A., and D. R. Larden, Seasonal Displacement and Deformation of the Earth by the Atmosphere, J. Geophys. Res., in press, 1979.

van Hylckama, T. E. A., The Water Balance of the Earth, Drexel Inst. of Technol., Publ. in Climatology, 9, 59-1 10, 1956. 



\title{
GEOPHYSICAL AND ASTRONOMICAL MODELS APPLIED IN THE ANALYSIS OF VERY LONG BASELINE INTERFEROMETRY
}

\author{
Chopo Ma and James W. Ryan \\ Goddard Space Flight Center \\ Bruce R. Schupler \\ Computer Sciences Corporation
}

\begin{abstract}
Very long baseline interferometry (VLBI) presents an opportunity to measure at the centimeter level such geodetic parameters as baseline length and instantaneous pole position. In order to achieve such precision, the geophysical and astronomical models used in data analysis must be as accurate as possible. The Mark-III interactive data analysis system includes a number of refinements beyond conventional practice in modeling precession, nutation, diurnal polar motion, UT1, solid earth tides, relativistic light deflection, and reduction to solar system barycentric coordinates. The algorithms and their effects on the recovered geodetic, geophysical, and astrometric parameters will be discussed.
\end{abstract}




\section{INTRODUCTION}

If the earth were an isolated, rigid, homogeneous, spherical, airless body, the calculation of the VLBI observables, delay and delay rate, would be quite straightforward. In this simple system, the VLBI delay is given by the projection of the baseline, measured in units of light travel time, in the direction of the observed source; i.e., by the dot product of the baseline vector and the source unit vector. The delay rate is the derivative of delay with respect to time. On the idealized earth, the baseline is attached rigidly to the terrestrial coordinate system. Viewed from the celestial reference frame in which the sources are fixed, the components of the baseline vector change as the baseline is carried around by the rotation of the earth; consequently, the VLBI delay has a diurnal sinusoidal signature. Unfortunately for simplicity but perhaps fortunately for scientific interest, the situation of the real earth is more complex and a number of models are required to describe it. The object of most of these models is to compute the position and velocity of the stations and baseline in the celestial reference frame at the epoch of observation. This paper describes the models and algorithms which are used by the East Coast VLBI group and which are implicit in the work described by Robertson (1979) and by Knight (1.979).

It should be pointed out that other models for such phenomena as the propagation medium, antenna geometry, source structure, and clock instability are necessary for correct computation of the VLBI observables. As these effects will be considered in detail by other papers, they will not be discussed further here.

\section{Classes of Models}

As shown in figure 1, the models can be divided schematically into four classes. It should be emphasized that this division is only schematic; the physics underlying different models is of ten closely related. The divisions follow the different modes of mathematical expression used. Models related to the rotation of the entire earth do not affect the baseline length but do affect its orientation and the orientation of the earth with respect to the celestial reference frame. Models related to the displacement of individual stations, such as solid earth tides and ocean loading, affect the baseline length. The model related to displacement of individual sources takes into account the gravitational deflection of the incoming radio signals by the sun. Models related to the orbital motion of the earth will not be discussed further since they are the subject of the paper to be given by Prof. Shapiro (1979). It should be noted, however, that the position and velocity of the earth with respect to the solar system barycenter are computed directly from the Planetary Ephemeris Program (PEP) tape provided by the Massachusetts Institute of Technology (MIT); consequently, no circular approximations appear in aberration or potential.

Figure 2 shows the mathematical form used for each class of model. The models in the first class are implemented as rotation matrices or products of rotation matrices: long period polar motion or wobble (W), diurnal polar motion (D), diurnal spin (S), nutation (N), and precession (P). These transform the components of the baseline from the terrestrial coordinate system to the reference 


\section{ROTATION OF ENTIRE EARTH}

2. DISPLACEMENT OF INDIVIDUAL STATION

3. DISPLACEMENT OF INDIVIDUAL SOURCE

4. ORBITAL MOTION

Figure 1. Classes of models.
1. $\overrightarrow{\mathrm{B}}_{1950}=$ PNSDW $\overrightarrow{\mathrm{B}}_{0}$

2. $\vec{x}=\vec{x}_{0}+\Delta \vec{x}_{E T}\left(+\Delta \vec{x}_{O L}\right)$

3. $\hat{\mathrm{S}}=\hat{\mathrm{S}}_{0}+\Delta \hat{\mathrm{S}}_{\mathrm{GD}}$

Figure 2. Model algorithms.

celestial coordinate system. The models of the second class are implemented as small translations of the station coordinates that vary with time for earth tides (ET) and ocean loading (OL). In figure 2, the parentheses surrounding the second term on the second line indicate that this correction has not yet been used in our analyses. The single model of the third class is implemented as a small rotation of the source unit vector for gravitational deflection (GD).

\section{Rotation Models}

The purpose of the rotation models is to transform from the terrestrial coordinate system, in which the stations are initially located and in which, except for tectonic processes and short period tidal effects, the station coordinates are invariant, to the reference celestial coordinate system in which the VLBI observables are calculated. The origin of the terrestrial system is defined by the adopted coordinates of the intersection of antenna axes of the $37-\mathrm{m}$ telescope at the Haystack Observatory $(x=+1492406.691 \mathrm{~m}, \mathrm{y}=-4457267.330 \mathrm{~m}, \mathrm{z}=+4296882.102 \mathrm{~m})$. These values were derived from the coordinates of the Mars antenna in the Goldstone complex of the Deep Space Network as determined from tracking data and the vector baseline from Goldstone to Haystack as determined from VLBI data. The reference celestial coordinate system is defined by the mean equator and equinox of 1950.0 with the origin at the solar system barycenter. The conceptual basis for the various rotations is that they represent the motion of the earth in space and the motion of the pole on the earth since 1950.0 .

Figure 3 shows the north polar region. The wobble matrix rotates the z-axis successively about the $\mathrm{x}$ - and $\mathrm{y}$-axes from the geographic origin at 0 to the position of the slowly moving pole defined conventionally by optional instruments. Since our analyses have usually been performed considerably later than the VLBI observations were made, we use as a priori values the pole positions distributed by the Bureau International de l'Heure (BIH) in circular D. These initial pole positions are not necessary, and we normally recover polar motion when analyzing more than 1 day's data. The second set of rotations for diurnal polar motion takes the z-axis from the slowly moving pole to the instantaneous pole. We use the seven largest terms in the series for the diurnal pole offset derived by McClure (1973), which has a maximum radius of $60 \mathrm{~cm}$. While the effect is quite small $(<.02$ arcsec), we feel that we have good evidence for it in our data. See C. Ma (1978) for a detailed discussion. 


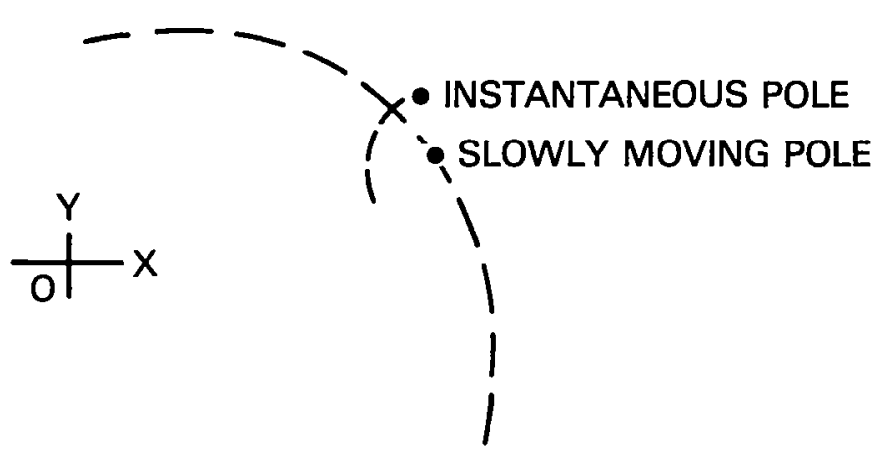

Figure 3. Wobble - long period polar motion diurnal polar motion.

The matrix $\mathrm{S}$ for diurnal spin rotates the coordinate system about the z-axis in order to re-align the $\mathrm{x}$-axis from the terrestrial origin of longitude at the Greenwich meridian to the celestial origin of right ascension at the vernal equinox. The rotation angle is the sum of four terms: (1) a constant sidereal rate from the classical equation of Newcomb given in the Explanatory Supplement to the American Ephemeris and Nautical Almanac (ESAENA), (2) the equation of the equinoxes for the difference between mean and apparent sidereal time, (3) an offset in UT1 for variation in true rotation rate, and (4) four terms suggested by Woolard (1959) for theoretical short period variations in rotation rate. The a priori values for UT1 offset are taken from circular D. As in the case of long period polar motion, the initial value for UT1 offset is not necessary and is normally estimated if more than 1 day is analyzed. The terms suggested by Woolard are included because the smoothing used by the BIH removes short period variations. These terms have fortnightly and monthly periods with magnitudes less than $0.8 \mathrm{msec}$. The algorithm used to compute the Woolard terms was provided by J. G. Williams (1974).*

The geometry for nutation is shown in figure 4. Instead of the commonly used first-order matrix for nutation, we apply an exact product of three rotations. The first rotation is about the $\mathrm{x}$-axis (perpendicular to the flat figure at $T$ ) from the true equator to the mean ecliptic through an angle equal to the true obliquity; i.e., the sum of the mean obliquity, $\epsilon$, and the nutation in obliquity, $\Delta \epsilon$. The second rotation is about the z-axis along the mean ecliptic by an angle equal to the nutation in longitude, $\Delta \psi$. The last rotation is about the $\mathrm{x}$-axis at $\mathrm{M}$ from the mean ecliptic to the mean equator of date by an angle equal to the mean obliquity. The values for the nutations in obliquity and longitude come from the standard Woolard series given in the ESAENA as interpolated from tabulated values on the PEP ephemeris tape. In addition, seven terms suggested by Melchior (1971) to model elastic effects are used. The periods range from 18.6 years to fortnightly with amplitudes up to 0.02 arcsec in obliquity and 0.04 arcsec in longitude. The algorithm using the coefficients from Melchior's table IXB was also provided by J. G. Williams.* 


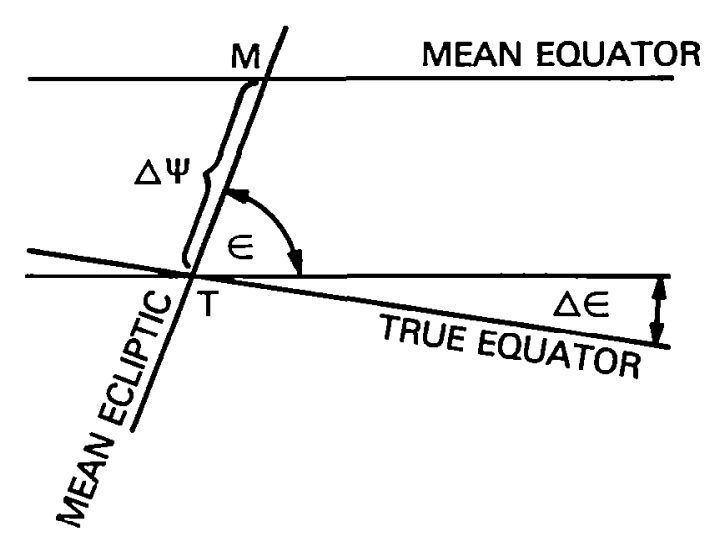

Figure 4. Nutation.

It should be mentioned that the new nutation series to be proposed by the Working Group on Nutation to the IAU in Montreal will affect the models for diurnal polar motion, diurnal rotation, and nutation.

The model for precession follows conventional practice given in the ESAENA with rotations by $z$, $\theta$, and $\zeta$. However, these angles are calculated from the constant of general precession suggested by Fricke $(1967,1971)$, which is 1.10 arcsec/century greater than the standard value. The algorithm allows the precession constant to be recovered, but the short span of VLBI data limits the precision of the estimate. The model and a priori source positions will require changes when epoch 2000.0 coordinates are adopted.

\section{Station Displacement Models}

The tidal potential is calculated exactly using the equation shown in figure 5 . The geocentric station vector is $A$, and the geocentric vector to the moon or sun, derived from the PEP ephemeris tape, is $R$. The distance from the station $P$ to the attracting body $M$ is $p .0$ is the origin of the tertestrial coordinate system. This total tidal potential includes both the second degree harmonic as well as higher degree harmonics, which are smaller by factors of $A / R$. To scale the station displacement from the tidal potential, we use the Love numbers calculated by Dahlen $(1976 ; h=0.609$, $1=0.085$. Because of the geometry of the baselines we have used so far, the effect of solid earth tides on baseline length is only at the level of a few centimeters while the effect on baseline orientation is negligible. The earth tide model has been used to estimate the Love numbers and a tidal lag angle.

The aspect of ocean loading that affects VLBI observations is the displacement of a station as the tide moves masses of water from place to place, causing the crust to move in response. We have 


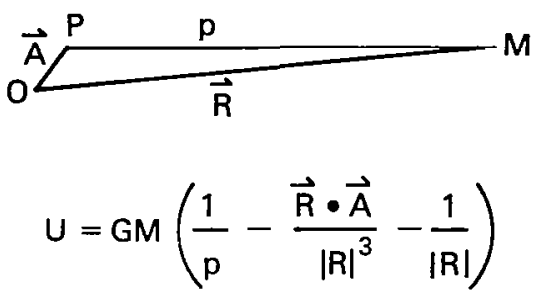

Figure 5. Solid earth tides.

investigated the model of Farrell (1977)* for the M2 and 01 tides. According to the model, the displacement at Haystack is less than $5 \mathrm{~cm}$. Although we have prepared the computer code for implementing the model and scaling other tidal components, we have not applied ocean loading because of uncertaintics as to its usefulness.

\section{Source Displacement Model}

The apparent displacement of a source caused by gravitational deflection of the incoming signal by the sun is modeled using a simple expression given by Shapiro (1967). The expression is applicable at all angles and allows the relativistic parameter gamma to be recovered. The necessary geometry between the source, the sun, and the earth is computed from the PEP ephemeris tape.

\section{Summary}

We have spent considerable effort in examining models, selecting and coding algorithms, and checking the results both internally and with other programs. Further details of these and other models can be found in Robertson (1977), C. Ma (1978), and the documentation of the CALC program in the Mark III data analysis system. Based on work done with older VLBI data, we believe that our geophysical and astronomical models are ready for the better data possible with the Mark III system.

There are several areas in which we expect to improve our models, especially in ocean loading and nutation. We will examine other ocean loading models with different ocean tides and response functions. We will also investigate further the use of tidal gravimeter data from which the displacement might be scaled directly for the period of an experiment. For nutation, we are considering the formulation and series recently computed by Wahr (1979).** In addition, we are working with G. Kaplan of the U.S. Naval Observatory to estimate the coefficients of the nutation series using both VLBI and connected-link interferometer data.

We expect that scientifically interesting results will continue to be forthcoming as the data allow the modcls to improve and as the models permit the data to be better understood.

\footnotetext{
*W. E. Farrell, private communication, 1977.

**J. N. Wahr, Ph.D. thesis, U. of Colorado, 1979.
} 


\section{REFERENCES}

Dahlen, F. A., Geophys. J. R. A. S. 46, 363,1976.

Explanatory Supplement to the Astronomical Ephemeris and the American Ephemeris and Nautical Almanac, HM Stationery Office, 1961.

Fricke, W., Astron. J. 72, 1368, 1967.

Fricke, W., Astron. and Astrophys. 13, 298, 1971.

Knight, C. A., NASA CP Radio Interfer. Tech. for Geod., 1980.

Ma, C., NASA TM 79582, Goddard Space Flight Center, 1978.

McClure, P., Goddard X-592-73-259, Goddard Space Flight Center, 1973.

Melchior, P., Celest. Mech. 4, 190, 1971.

Robertson, D. S., Goddard X-922-77-228, Goddard Space Flight Center, 1977.

Robertson, D. S., et al., NASA CP Radio Interfer. Tech. for Geod., 1980.

Shapiro, I. I., Science 157, 806, 1967.

Shapiro, I. I., et al., NASA CP Radio Interfer. Tech. for Geod., 1980.

Woolard, E, W., Astron. J. 64, 140, 1959. 


\section{RELATIVISTIC FORMULATION OF THE VLBI OBSERVABLE}

S. A. Gourevitch, R. Epstein, and I. I. Shapiro

Massachusetts Institute of Technology

Presented but not submitted. 
$-\cdots$ 
RADIO SOURCE POSITIONS AND ASTRONOMICAL CONSTANTS

T. A. Clark

NASA/Goddard Space Flight Center

Presented but not submitted. 


\title{
VLBI MEASUREMENTS OF RADIO SOURCE POSITIONS AT THE JET PROPULSION LABORATORY \\ G. H. Purcell, Jr., J. L. Fanselow, J. B. Thomas, E. J. Cohen, D. H. Rogstad, O. J. Sovers, L. J. Skjerve, and D. J. Spitzmesser Jet Propulsion Laboratory
}

\begin{abstract}
We present the results of approximately 1300 observations of 67 radio sources. Most of our measurements were made at the stations of the Deep Space Network in California, Spain, and Australia, at wavelengths of 13.1 and $3.6 \mathrm{~cm}$, between 1971 and 1978 . The formal errors in the derived source positions are generally in the neighborhood of 0.01 seconds of arc, and the positions agree fairly well with those published by other groups.
\end{abstract}




\section{INTRODUCTION}

The observational material I am going to discuss this afternoon is identical with what Brooks Thomas discussed this morning in connection with the geophysical parameters - namely baselines, UT1, and polar motion. However, I am going to consider it now from the standpoint of the source positions.

Historically, JPL has been interested in radio source positions as an adjunct to its VLBI measurements of parameters required for spacecraft navigation, in particular, UT1, polar motion, and station locations. When our system becomes fully operational, we expect to make measurements of station locations and polar motion accurate to $30 \mathrm{~cm}$ or better, and UT1 at a comparable level. In order to achieve this goal, we require a source catalog with positions accurate to something like 0. . 01 . Furthermore, we expect to observe at arbitrarily assigned times, with only about 2 hours of data per week on each baseline. Our catalog therefore needs to be fairly large, say a hundred sources; and we want the objects well distributed over the accessible sky.

JPL is also interested in using extragalactic sources more directly for spacecraft navigation by making differential measurements between spacecraft and nearby natural sources. For that reason, we have a special interest in sources near the ecliptic - particularly those parts of the ecliptic where spacecraft are likely to turn up.

Finally, we have recently started a project to test the stability of the celestial reference frame by measuring differential proper motion in a few close pairs of sources.

\section{EQUIPMENT, OBSERVATIONS, AND REDUCTION PROCEDURES}

I am not going to dwell on our equipment and reduction procedures, because Brooks has already discussed those in some detail, and they have been described elsewhere (Purcell et al., 1978); but I would like to remind you of some of the salient points. Our data consist of about 1300 observations of 67 sources made in the course of 30 observing sessions between 1971 and 1974 and in 1977 and 1978. Most of these sessions involve the baselines between the Deep Space Network stations in California and Spain, and California and Australia; but there is also one session between Haystack and Owens Valley. During the earlier years, we observed only at a wavelength of $13.1 \mathrm{~cm}$, and we measured only fringe rates; more recently, we have been using bandwidth synthesis methods to measure delays as well as rates; now, we generally observe simultaneously at 13.1 and $3.6 \mathrm{~cm}$. In several of these sessions, we have combined data at the two frequencies to reduce the corrupting influence of the ionosphere on the observations.

In the final step of the reduction procedure, we fitted all the data simultaneously with an analytic model containing 384 adjustable parameters. Of these parameters, 133 describe the source positions, the others are divided among the clock model (129), the troposphere model (64), UT1 and polar motion (43), and station locations (14). 


\section{RESULTS}

Figure 1 shows the distribution on the sky of our 67 sources, and, on a different scale, the formal errors on the estimated right ascensions and declinations. Very large errors, those greater than $0 . " 03$, have not been plotted. In addition, one source, NRAO 140, was used as the reference point for right ascension and therefore has no horizontal error bar. The figure shows that the sources are, in fact, fairly well distributed over the visible sky, and that the errors in the positions are mostly in the neighborhood of 0."01. You can see that the positions of southern sources - ones that we can observe only on the California-to-Australia baseline - are generally less well determined than those of the northern sources. I attribute this result largely to the fact that these southern sources are visible for only about 4 hours. This circumstance leads to correlations among the errors in some of the parameters, and thus to a general weakening of that part of the solution. Until recently, there were very large errors in the declinations of some sources near the equator that had been observed only on the California-to-Spain baseline, which has a small polar projection. However, we have relieved much of that problem now by adding some observations of these sources on the other baseline.

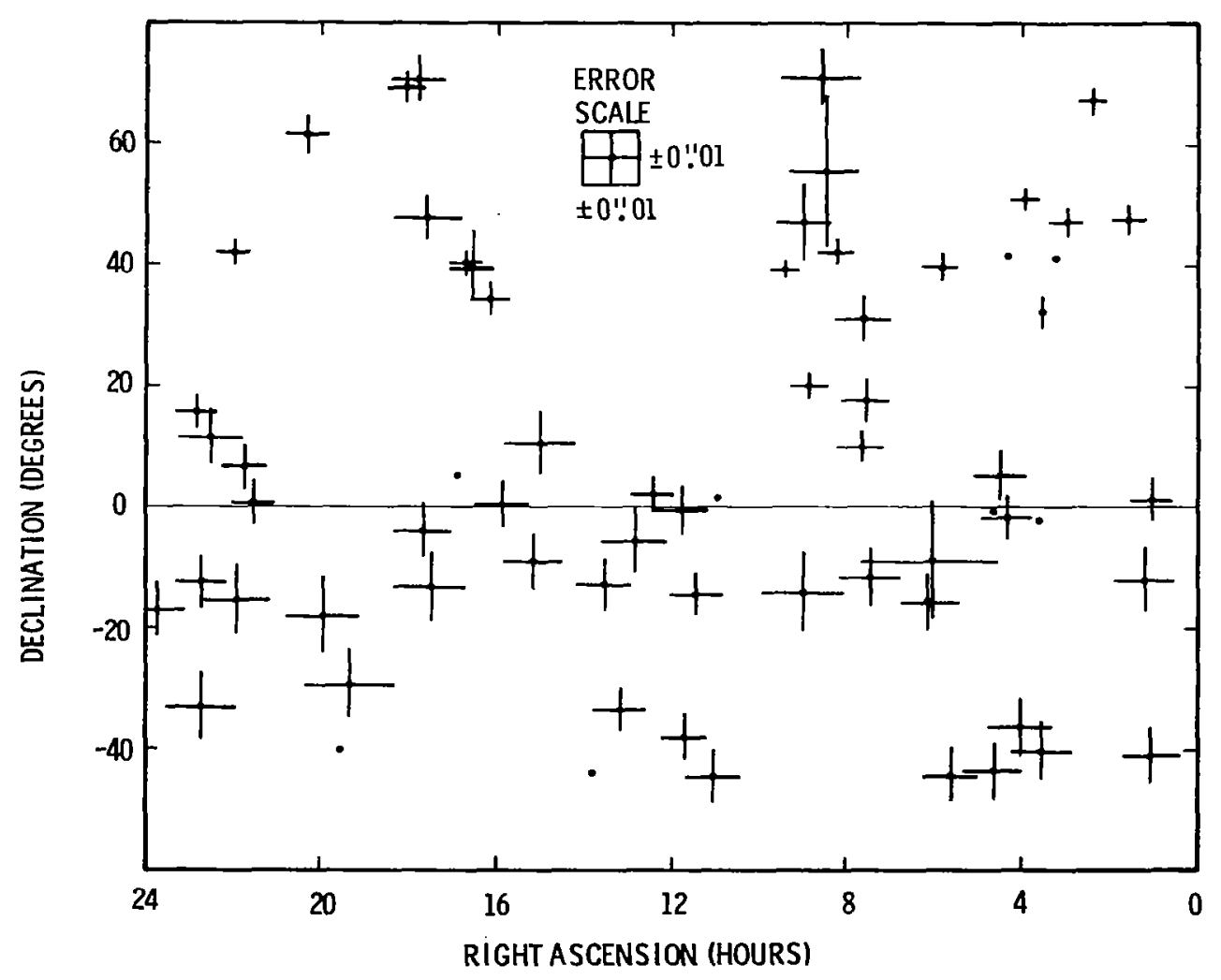

Figure 1. Distribution of sources. 
I would like to emphasize today the progress our group has made in the last year or so. Figures 2 and 3 are histograms that show the distributions of the formal errors in our source positions as they were 9 months ago (the white blocks) and as they are now (the black blocks). Notice that each error bin contains a white column and a black column, with the shorter column always placed in front so that both can be seen. Figure 2 shows that the mean formal error in right ascension has decreased from about 0." 032 (for 52 sources) in September of last year to 0."014 (for 63 sources) now - an improvement of better than a factor of two. The improvement in the declinations is even more dramatic (figure 3), from 0." 030 (for 45 sources) to $0 . " 011$ (for 63 sources).

We are, of course, continuing to compare our results with those of other groups. To this end, we have constructed a composite reference catalog from the lists of Clark et al. (1976), Elsmore and Ryle (1976), and Wade and Johnston (1977). This catalog is a weighted average of the three lists, with slight adjustments in the origin of right ascension of two of them, to produce the best overall agreement. In a few cases of serious discrepancy, we deleted individual values from the average. Finally, we readjusted the origin of right ascension of the composite list to produce the best overall agreement with our own list, and computed the residual differences between the two.

Figure 4 shows the differences between the right ascensions in the reference catalog and those in our list, for 28 sources. I think you can see that the scatter in the points is entirely consistent with the error bars; in fact, chi-square per degree of freedom is 0.99 . However, the agreement in the declinations, shown in figure 5 , is quite poor, owing mainly to a mean difference between the reference list and the JPL list of about $-0 .{ }^{\prime \prime} 02$. We have as yet no satisfactory explanation for this difference.

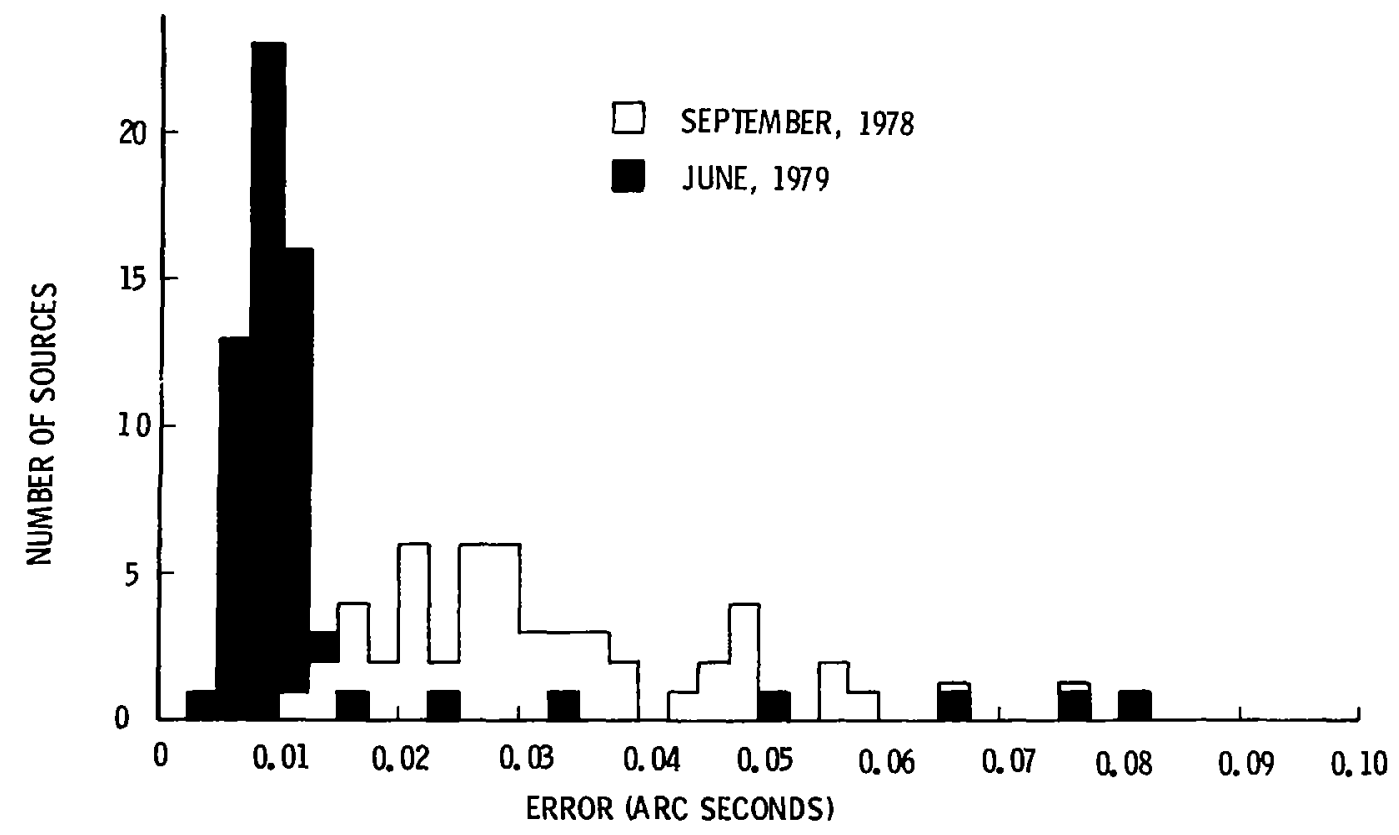

Figure 2. Distribution of errors in right ascension. 


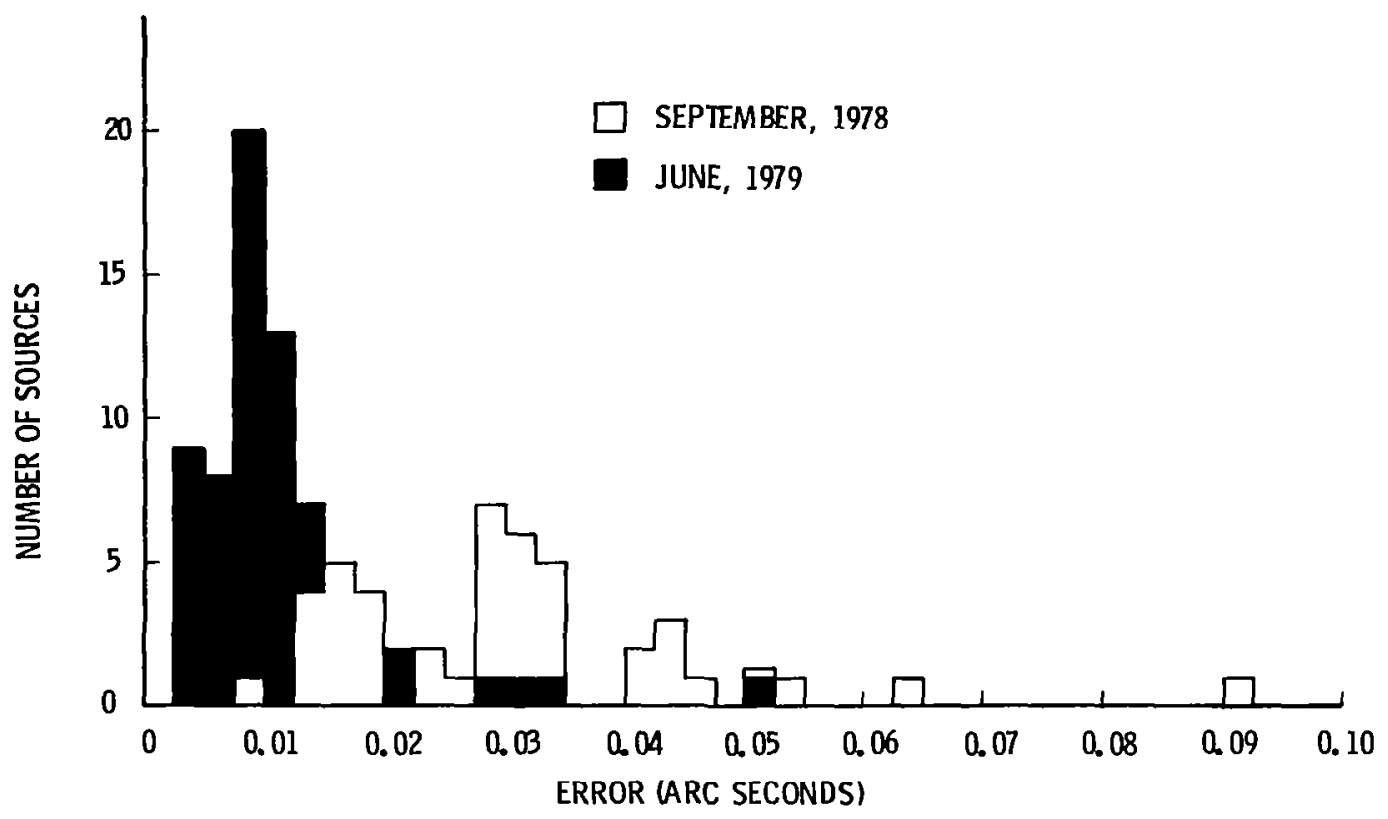

Figure 3. Distribution of errors in declination.

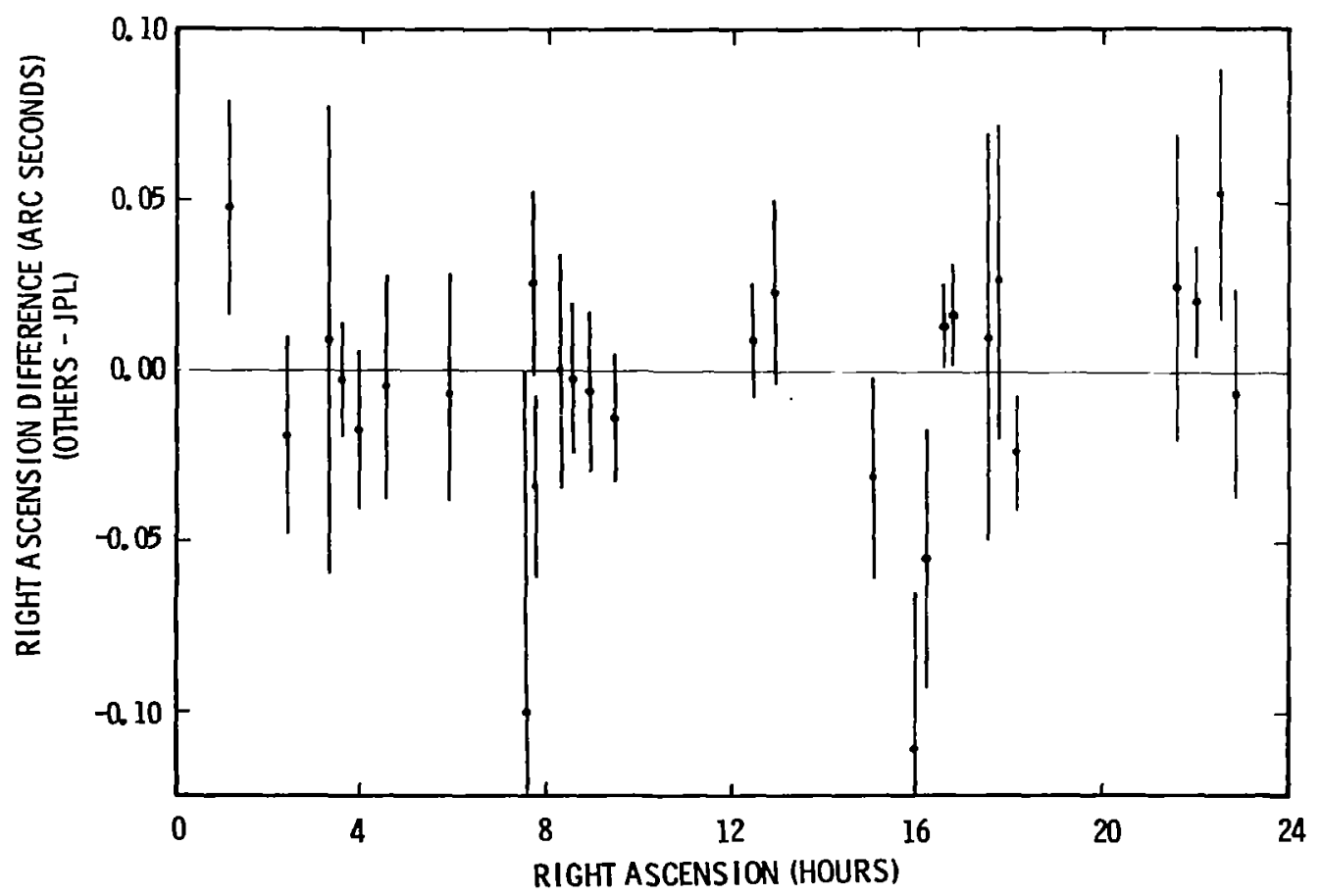

Figure 4. Comparison of right ascensions. 


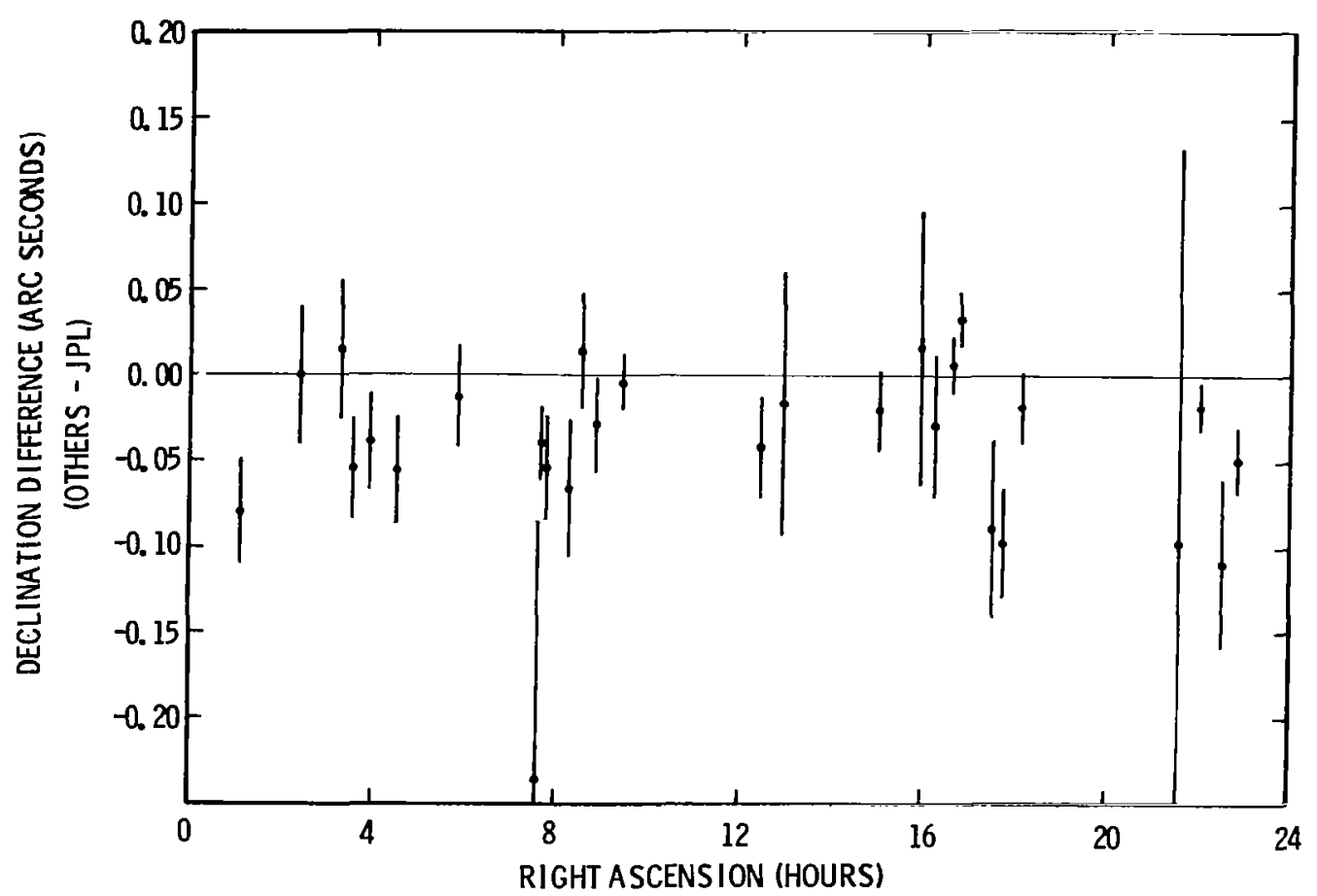

Figure 5. Comparison of declinations.

The general problem of comparing two sets of source positions is an awkward one just now. The fact that different groups are reducing their data using different nutation series and different rates of precession is certain to lead to systematic differences in results at a level of $0 . " 01$, especially when the epochs of the observations differ. Fortunately, it seems likely that we can resolve these differences in the near future.

Table 1 presents the source positions themselves. Note that these positions are barycentric. To obtain positions consistent with normal practice in optical catalogs, you must add the elliptic aberration terms that are provided. Keep in mind also that we reduced the positions to 1950.0 coordinates using the conventional (Woolard) nutation series and Newcomb's value of the precession constant.

\section{FUTURE PLANS}

We have made substantial progress in the past year, and we expect this progress to continue in the near future. Even at our present level of sensitivity, we can easily reach 50 or 60 more sources. We are improving our calibration techniques to account better for fluctuations in instrumental and propagation delays. We are also continuing to improve our delay model in anticipation of accuracies substantially better than $0 . " 01$. 
Table 1

Radio Source Positions

\begin{tabular}{|c|c|c|c|c|c|c|}
\hline \multirow{2}{*}{$\begin{array}{l}\begin{array}{l}\text { SOURCE } \\
\text { NAME }\end{array} \\
\text { P } 0104-408\end{array}$} & $\begin{array}{l}\text { SOURCE POSITION } \\
\text { RIGHT ASCENSION }\end{array}$ & \multicolumn{2}{|c|}{$\begin{array}{cc}\text { (SOLAR SYSTEM BARYCENTER, } \\
\text { ERROR } & \text { DECLINATION }\end{array}$} & \multirow{2}{*}{$\begin{array}{c}\begin{array}{c}1950.0) \\
\text { ERROR }\end{array} \\
0.0106\end{array}$} & \multicolumn{2}{|c|}{$\begin{array}{cc}\text { ELL. ABERRATION } \\
\text { R.A. } & \text { DEC. } \\
\end{array}$} \\
\hline & $01^{h_{04}}{ }^{m_{27}} \cdot 57081$ & 0.00092 & $-40^{\circ} 50^{\prime} 21^{\prime \prime} 3385$ & & 0.00263 & -0.2442 \\
\hline P $0106+01$ & $0106 \quad 04.51419$ & 0.00054 & +01 1901.1607 & 0.0080 & 0.00215 & -0.0207 \\
\hline P 0113-118 & 011343.21450 & 0.00074 & $\begin{array}{lll}-11 & 52 & 04.4395\end{array}$ & 0.0118 & 0.00297 & -0.0977 \\
\hline DA 55 & $0133 \quad 55.09459$ & 0.00066 & $+4736 \quad 12.6398$ & 0.0053 & 0.00724 & 0.2273 \\
\hline DW $0224+67$ & $02 \quad 24 \quad 41.14105$ & 0.00083 & $+67 \quad 0739.4256$ & 0.0049 & 0.02483 & 0.2741 \\
\hline OE 400 & $\begin{array}{lll}03 & 00 & 10.09103\end{array}$ & 0.00060 & $+4704 \quad 33.5013$ & 0.0055 & 0.01868 & 0.1882 \\
\hline 3C 84 & $\begin{array}{lll}03 & 16 & 29.54517\end{array}$ & 0.00593 & +411951.7340 & 0.0310 & 0.01869 & 0.1564 \\
\hline P $0332-403$ & $03 \quad 32 \quad 25.20880$ & 0.00091 & $\begin{array}{llll}-40 & 18 & 23.8807\end{array}$ & 0.0099 & 0.01999 & -0.1861 \\
\hline NRAO 140 & $03 \quad 33 \quad 22.38543$ & $\star$ & $+3208 \quad 36.5561$ & 0.0058 & 0.01809 & 0.1104 \\
\hline CTA 26 & $03 \quad 36 \quad 58.91001$ & 0.01674 & $\begin{array}{lll}-01 & 56 & 16.8612\end{array}$ & 1.1855 & 0.01559 & -0.0370 \\
\hline NRAO 150 & $0355 \quad 45.23306$ & 0.00058 & $+5049 \quad 20.1587$ & 0.0038 & 0.02673 & 0.1598 \\
\hline P $0402-362$ & $\begin{array}{lll}04 & 02 & 02.56611\end{array}$ & 0.00094 & -361311.7154 & 0.0107 & 0.02144 & -0.1545 \\
\hline VRO 41.04 .01 & $04 \quad 2027.96800$ & 0.04666 & +414306.7997 & 590 & 0.02470 & 0.1123 \\
\hline P 0420-01 & $04 \quad 20 \quad 43.51973$ & 0.00059 & $\begin{array}{lll}-01 & 27 & 28.6683\end{array}$ & 0.0085 & 0.01846 & -0.0337 \\
\hline $3 \mathrm{C} 120$ & $04 \quad 30 \quad 31.58204$ & 0.00064 & +051459.6152 & 0.0091 & 0.01908 & -0.0112 \\
\hline P $0438-43$ & $04 \quad 38 \quad 43.14982$ & 0.00092 & $-43 \quad 38 \quad 53.4225$ & 0. & 0.02687 & -0.1437 \\
\hline NRAO 190 & $04 \quad 40 \quad 05.27072$ & 0.00218 & $\begin{array}{lll}-00 & 23 & 20.5738\end{array}$ & 0.0327 & 0.01951 & -0.0298 \\
\hline P $0537-441$ & $\begin{array}{lll}05 & 37 & 21.03832\end{array}$ & 0.00088 & $\begin{array}{llll}-44 & 06 & 44.6030\end{array}$ & 0.0097 & 0.03038 & -0.0891 \\
\hline DA 193 & $\begin{array}{lll}05 & 52 & 01.37726\end{array}$ & 0.00058 & $+3948 \quad 21.9245$ & u. & 0 . & 0.0277 \\
\hline P 0605-08 & $\begin{array}{lll}06 & 05 & 36.00444\end{array}$ & 0.00164 & $\begin{array}{lll}-08 & 34 & 20.2899\end{array}$ & 0.0216 & 0.02271 & -0.0368 \\
\hline P $0607-15$ & $\begin{array}{lll}06 & 07 & 25.95624\end{array}$ & 0.00074 & $\begin{array}{lll}-15 & 42 & 03.2823\end{array}$ & 0.0103 & 0.02336 & -0.0424 \\
\hline P $0727-11$ & $07 \quad 27 \quad 58.07365$ & 0.00074 & $\begin{array}{lll}-11 & 34 & 52.5831\end{array}$ & & & -0.0150 \\
\hline P $0735+17$ & $\begin{array}{lll}07 & 35 & 14.10211\end{array}$ & 0.0 & $\begin{array}{r}+174909.3064 \\
\end{array}$ & & 0.0 & -0.0502 \\
\hline OI 363 & $07 \quad 3800.15161$ & 0.00077 & $+31 \quad 1902.1255$ & 0.0087 & 0.02594 & -0.0655 \\
\hline DW $0742+10$ & $07 \quad 42 \quad 48.44097$ & 0.00056 & $+1018 \quad 32.6957$ & 0.0062 & 0.02241 & -0.0435 \\
\hline OJ 425 & $08 \quad 14 \quad 51.64006$ & 0.00057 & $\begin{array}{lll}+4232 & 07.8557\end{array}$ & 0.1 & 0.02854 & -0.1099 \\
\hline 4C 55.16 & $\begin{array}{lll}08 & 31 & 04.34169\end{array}$ & 0.00147 & $+55 \quad 44 \quad 41.4885$ & 0.0287 & 0.03617 & -0.1428 \\
\hline 4C 71.07 & $\begin{array}{lll}08 & 36 & 21.49599\end{array}$ & 0.00291 & $+7104 \quad 22.6217$ & 0.0107 & 0.06203 & -0.1609 \\
\hline OJ 287 & $08 \quad 5157.22910$ & 0.00048 & +201758.5040 & 0.0046 & 0.02063 & -0.0894 \\
\hline OJ 499 & $\begin{array}{lll}08 & 59 & 39.95510\end{array}$ & 0.00093 & +470256.9833 & 0.0139 & 0.02778 & -0.1586 \\
\hline P $0859-14$ & $\begin{array}{lll}08 & 59 & 54.92583\end{array}$ & 0.00100 & -14 U3 38.8984 & 0.0143 & 0.01950 & 0.0186 \\
\hline
\end{tabular}

* NRAO 140 right ascension reference

The research described in this paper was carried out at the Jet Propulsion Laboratory, California Institute of Technology, under NASA contract NAS7-100.

\section{REFERENCES}

Clark, T. A., Hutton, L. K., Marandino, G. E., Counselman, C. C., Robertson, D. S., Shapiro, I. I., Wittels, J. J., Hinteregger, H. F., Knight, C. A., Rogers, A. E. E., Whitney, A. R., Niell, A. E., Ronnang, B. O., and Rydbeck, O. E. H.; Astron. J., 81, 599, 1976.

Elsmore, B., and Ryle, M.; Monthly Notices Roy. Astron. Soc., 174, 411, 1976. 
Table 1 (continued)

\begin{tabular}{|c|c|c|c|c|c|c|}
\hline \multirow{3}{*}{$\begin{array}{c}\begin{array}{c}\text { SOURCE } \\
\text { NAME }\end{array} \\
4 \mathrm{C} \quad 39.25\end{array}$} & \multirow{2}{*}{$\begin{array}{l}\text { SOURCE POSITION } \\
\text { RIGHT ASCENSION } \\
\end{array}$} & \multirow{2}{*}{\multicolumn{2}{|c|}{$\begin{array}{cr}\text { (SOLAR SYSTEM BARYCENTER, } \\
\text { ERROR } & \text { DECLINATION }\end{array}$}} & \multirow{3}{*}{$\frac{\begin{array}{c}1950.0) \\
\text { ERROR }\end{array}}{0.0037}$} & \multicolumn{2}{|c|}{ ELL. ABERRATION } \\
\hline & & & & & R.A. & DEC. \\
\hline & $09^{h_{2}} 23^{\mathrm{m}_{55}} \mathrm{~s}_{29607}$ & 0.00048 & $+39^{\circ} 15^{\prime} 23^{\prime \prime} .7306$ & & 0.02258 & -0.1607 \\
\hline P $1055+01$ & $1055 \quad 55.29831$ & 0.01438 & $+0150 \quad 04.5763$ & 1.4224 & 0.01039 & -0.0383 \\
\hline P $1104-445$ & $1104 \quad 50.35273$ & 0.00091 & $\begin{array}{llll}-44 & 32 & 53.1988\end{array}$ & 0.0093 & 0.01346 & 0.1971 \\
\hline P $1127-14$ & $1127 \quad 35.65773$ & 0.00061 & $\begin{array}{lll}-14 & 32 & 54.5078\end{array}$ & 0.0082 & 0.00775 & 0.0534 \\
\hline P 1144-379 & $1144 \quad 30.84975$ & 0.00073 & $\begin{array}{lll}-37 & 55 & 30.8424\end{array}$ & 0.0083 & 0.00747 & 0.1804 \\
\hline P $1148-00$ & $\begin{array}{lll}11 & 48 & 10.11818\end{array}$ & 0.00065 & $-00 \quad 07 \quad 13.1473$ & 0.0089 & 0.00554 & -0.0279 \\
\hline $3 C 273$ & $12 \quad 26 \quad 33.24224$ & 0.00052 & $+02 \quad 1943.3448$ & 0.0065 & 0.00178 & -0.0424 \\
\hline $3 \mathrm{C} 279$ & $1253 \quad 35.83076$ & 0.00080 & $\begin{array}{lll}-05 & 31 & 08.0123\end{array}$ & 0.0 & -0.00091 & 0.0044 \\
\hline OP -322 & $\begin{array}{lll}13 & 13 & 20.04317\end{array}$ & 0.00076 & $\begin{array}{lll}-33 & 23 & 09.8640\end{array}$ & 0.0087 & -0.00343 & 0.1627 \\
\hline DW $1335-12$ & $13 \quad 34 \quad 59.80762$ & 0.00005 & $\begin{array}{lll}-12 & 42 & 09.8032\end{array}$ & 0.0091 & -0.00511 & 0.0455 \\
\hline P $1349-439$ & $13 \quad 4950.30438$ & 0.00484 & $\begin{array}{llll}-43 & 57 & 48.3349\end{array}$ & 0.0224 & -0 & 0.2071 \\
\hline OR 103 & $\begin{array}{lll}15 & 02 & 00.17015\end{array}$ & 0.00085 & $+1041 \quad 17.8086$ & 0.0117 & -0.01310 & -0.0804 \\
\hline P $1510-08$ & $\begin{array}{lll}15 & 10 & 08.91346\end{array}$ & 0.00072 & $-08 \quad 54 \quad 47.6550$ & 0.0103 & -0.01369 & 0.0144 \\
\hline DW $1555+00$ & $\begin{array}{lll}15 & 55 & 17.70879\end{array}$ & 0.00061 & to0 $06 \quad 43.5522$ & 0.0087 & -0.01686 & -0.0290 \\
\hline DA 406 & $16 \quad 11 \quad 47.93564$ & 0.00057 & $+34 \quad 20 \quad 19.9820$ & 0.0060 & -0.02170 & -0.1427 \\
\hline NRAO 512 & $16 \quad 38 \quad 48.19450$ & 0.00070 & +395230.2209 & 0.0137 & -0.02534 & -0.1361 \\
\hline $3 \mathrm{C} 345$ & $1641 \quad 17.62954$ & 0.00054 & $+39 \quad 54 \quad 10.9214$ & 0.0046 & -0.02551 & -0.1341 \\
\hline DW $1656+05$ & $\begin{array}{lll}16 & 56 & 05.63938\end{array}$ & 0.00513 & $+05 \quad 1946.8476$ & 0.2463 & -0.02037 & -0.0429 \\
\hline NRAO 530 & $1730 \quad 13.55606$ & 0.00085 & $\begin{array}{lll}-13 & 02 & 45.8376\end{array}$ & 0.0123 & -0.02217 & -0.0033 \\
\hline OT 465 & $\begin{array}{lll}17 & 38 & 36.34191\end{array}$ & 0.00118 & +473928.8555 & 0.0083 & -0.03244 & -0.0908 \\
\hline P 1741-038 & $\begin{array}{lll}17 & 41 & 20.63771\end{array}$ & 0.00067 & $-034848.889 y$ & 0.0100 & $-0.021: 7$ & -0.0223 \\
\hline $1749+\% 01$ & 174903.45582 & 0.00189 & $+70 \quad 06 \quad 39.7027$ & 0.0087 & -0 & -0.0866 \\
\hline $3 C 371$ & $\begin{array}{llll}18 & 07 & 18.61013\end{array}$ & 0.00128 & $+65 \quad 48 \quad 57.1599$ & 0.0050 & -0.06517 & -0.0616 \\
\hline ov -236 & $1921 \quad 42.25904$ & 0.00121 & $\begin{array}{lll}-29 & \therefore 0 & 26.3129\end{array}$ & 0.0132 & -0.02579 & -0.0520 \\
\hline P $1933-400$ & $1933 \quad 51.15233$ & 0.00709 & $-40 \quad 04 \quad 47.4184$ & 0.0518 & -0.02909 & -0.0689 \\
\hline OV-198 & $19 \quad 58 \quad 04.62966$ & 0.00092 & $\begin{array}{lll}-17 & 57 & 16.9055\end{array}$ & 0.0142 & -0.02272 & -0.0604 \\
\hline OW 6.37 & $\begin{array}{lll}20 & 21 & 13.34445\end{array}$ & 0.00104 & +612718.0444 & 0.0071 & -0.04349 & 0.1094 \\
\hline P $21 \approx+004$ & $21 \quad 34 \quad 05.22134$ & 0.00052 & to0 $28 \quad 25.1264$ & 0.0084 & -0 & -0.0267 \\
\hline P $2145 \div 76$ & $21 \quad 45 \quad 36.09253$ & 0.00058 & +064340.9133 & 0.0085 & -0.01614 & 0.0001 \\
\hline $0 X-192$ & $\begin{array}{lll}21 & 55 & 23.25580\end{array}$ & $0.0008 \mathrm{I}$ & $\begin{array}{lll}-15 & 15 & 30.0017\end{array}$ & 0.0130 & -0.01588 & -0.0941 \\
\hline VRO 42.22 .01 & $22 \quad 00 \quad 39.37976$ & 0.00052 & $+4202,08,4480$ & 0.0046 & -0.02010 & 0.1516 \\
\hline CTA 102 & $22^{h} 30^{m} 07^{s} \cdot 81548$ & 0.00079 & $+11^{\circ} 28^{\prime} 22^{\prime \prime} .7831$ & 0.0102 & -0.01286 & 0.0286 \\
\hline $\mathrm{OY}-172.6$ & $22 \quad 43 \quad 39.80282$ & 0.00063 & $-12 \quad 22 \quad 40.2035$ & 0.0097 & $-0.011 / 3$ & -1.0912 \\
\hline P $2245-328$ & 224551.51756 & $0.0009 y$ & $\begin{array}{lll}-32 & 5 \mathrm{i} & 44.2844\end{array}$ & 0.0127 & -0.01342 & -0.1852 \\
\hline $3 C 454.3$ & 225129.52926 & 0.00047 & $\dot{r} 15 \quad 52 \quad 54.2989$ & 0.0061 & -0.01121 & 0.0549 \\
\hline P 2345-16 & 234527.68691 & 0.00068 & -164752.4898 & 0.0102 & -0.00606 & -0.1229 \\
\hline
\end{tabular}

Purcell, G. H., Cohen, E. J., Fanselow, J. L., Rogstad, D. H., Skjerve, L. J., Spitzmesser, D. J., and Thomas, J. B.; in F. V. Prochazka and R. H. Tucker (eds.), IAU Colloquium 48, University Observatory Vienna, Vienna, p. 185, 1978.

Wade, C. M., and Johnston, K. J.; Astron. J., 82, 791, 1977. 


\title{
A VERY LONG BASELINE INTERFEROMETRY SKY SURVEY
}

\author{
R. A. Preston and D. D. Morabito \\ Jet Propulsion Laboratory
}

\section{INTRODUCTION}

The development of new high-accuracy celestial reference frames composed of compact extragalactic radio sources will permit astrometric and geodetic studies of previously unobtainable accuracy to be performed (Shapiro and Knight, 1970; Counselman, 1975). These studies require the use of the observational technique of very long baseline interferometry (VLBI). Previous investigators have utilized only a relatively small set of detected compact sources in initial experimental development of the technique. In this paper, we discuss a systematic VLBI sky survey which has been undertaken to find a suitable set of compact celestial radio sources from which a more complete VLBI reference frame can be constructed. The results of this survey are presently being used to form a VLBI reference frame of about 100 to 200 sources by determining precise relative positions (Purcell et al., 1979).

\section{SELECTION OF CANDIDATE SOURCES}

The survey was conducted by searching known celestial radio sources for compact components by means of VLBI observations. Our baseline lengths were about $7 \times 10^{7}$ RF wavelengths $(\lambda=13.1$ $\mathrm{cm}$ ), so the spatial wavelengths being sampled by the interferometer were generally on the order of a few milliarcseconds. Hence, the radio sources detected by this survey have a measurable portion of their total flux density contained in components that are no more than a few milliarcseconds in angular extent. Into this category of radio sources fall certain quasars, BL Lacertae type objects, and galactic cores.

In order to keep from searching all known celestial radio sources for VLBI components, we have used existing information on radio sources as clues to source size. The following criteria proved useful in determining which sources might have structure confined to the milliarcsecond level:

(1) Spectrum - Power does not fall off with increasing frequency as rapidly as more typical radio sources in the range 1 to $10 \mathrm{GHz}$. An even stronger indicator is if the spectrum is flat, sloped upward, or peaked in this range. Such behavior is typical of the synchrotron self-absorption characteristic of compact sources. Of all the indicators of milliarcsecond

*This paper represents the results of one phase of research carried out at the Jet Propulsion Laboratory, California Institute of Technology, under Contract No. NAS7-100, sponsored by the National Aeronautics and Space Administration. 
structure, spectral properties have proved the most useful. In the absence of any other information about a source, we selected sources whose spectral index between 2.7 and $5.0 \mathrm{GHz}$ was $\gtrsim-0.6\left(\mathrm{~S} \equiv \mathrm{S}_{\mathrm{o}} \mathrm{f}^{a}\right)$.

(2) Variability - Identified as radio or optical variable.

(3) Optical Identification - Identified as extragalactic.

(4) Size Limits - A short baseline interferometer can determine if a source has components smaller than its resolution limit (resolution does not exceed $\sim 1$ arcsecond).

(5) Interplanetary Scintillation - Radio sources that show scintillations when viewed through the solar corona possess components with angular sizes $\lesssim 0.1$ arcsecond.

(6) Detection in High Frequency Surveys - A larger percentage of sources detected in surveys between 1 and $10 \mathrm{GHz}$ are VLBI sources than is the case at lower frequencies.

In general, we chose as candidates only those sources whose total flux densities were $\gtrsim 0.7$ Jansky at our observing frequency $(2.3 \mathrm{GHz})$.

To date, about 1000 candidate sources have been observed, with 665 of these being detected with VLBI.

\section{DATA COLLECTION AND REDUCTION}

The observations were all performed with pairs of antennas within NASA's Deep Space Network (DSN) which were separated by intercontinental distances. In practice, this meant observing on the Goldstone-Australia or Goldstone-Spain baselines. All the observations were performed at 2290 MHz. The data were recorded on the Mark II VLBI recording system, which preserved a $1.8 \mathrm{MHz}$ data bandwid th by digitally sampling at a 4-Mbs rate (Clark, 1973).

Matching tapes from the two antennas were cross-correlated on a special hardware/software computer at the National Radio Astronomy Observatory in Charlottesville, Virginia. Computer manipulation of the output of this correlator yielded the correlation constant, $\rho$, for each observation, or the fraction of bits on the two tapes that were correlated. Correlation constants were then converted into correlated flux densities, $S_{c}$ (i.e., VLBI source strengths) by means of the expression:

$$
\mathrm{S}_{\mathrm{c}}=2.6 \rho \sqrt{\mathrm{T}_{1} \mathrm{~T}_{2}\left(\frac{\mathrm{dS}}{\mathrm{dT}}\right)_{1}\left(\frac{\mathrm{dS}}{\mathrm{dT}}\right)_{2}}
$$


where

$$
\begin{aligned}
& S_{c}=\text { correlated flux density in Jansky } \\
& T_{i}=\text { the measured system temperature at antenna in } K
\end{aligned}
$$

$(\mathrm{dS} / \mathrm{dT})_{\mathrm{i}}=$ the inverse sensitivity of antenna $\mathrm{i}$ in Jansky/K (i.e., how strong does a source have to be in total flux density (Jansky) to raise the system temperature $1 \mathrm{~K}$ ).

Most sources were observed for at least 3 minutes, but, for data processing, the observations were broken into 1 -minute segments. The $5 \sigma$ detection limit for this search for VLBI sources was about 0.1 Jansky. The random uncertainty in detected source strength was about 0.02 Jansky. However, in practice, systematic errors at the 5 or 10 percent level dominate the random contribution for inost sources.

If a priori source positions are in error, the tapes must be correlated over a range of relative tape delay and delay rate offsets in order to detect a VLBI source. Appropriate searches in these parameters were performed so that the sky was completely searched within 0.5 arcminutes of all nominal source positions. Almost all a priori source position errors should be covered by this degree of position searching. In addition, once detections were achieved, the measured delay and delay rate offsets allowed positional errors to be measured to about one arcsecond.

\section{RESULTS}

Figures 1a, 1 b, and 1 c display the general sky distribution of detected VI.BI sources as a function of VLBI source strength. These maps are equal area representations, so that local spatial densities in one area may be compared to local densities in another. Catalogs of source strengths and positions will be published elsewhere.

It should be noted that the strengths of the sources are a function of time, baseline length, and baseline orientation. Although the plots may not always show the true strength of a particular source at a certain epoch and on a certain baseline, they do indicate the general sky densities of sources as a function of strength.

Figure 1a shows the global distribution of all 665 detected sources. The lower limit of correlated flux strength is about 0.1 Jansky. Several things are evident from this plot:

(1) In general, the distribution is dense and rather uniform.

(2) Pairs of DSN antennas cannot commonly view the sky below $\approx-45^{\circ}$ declination. 

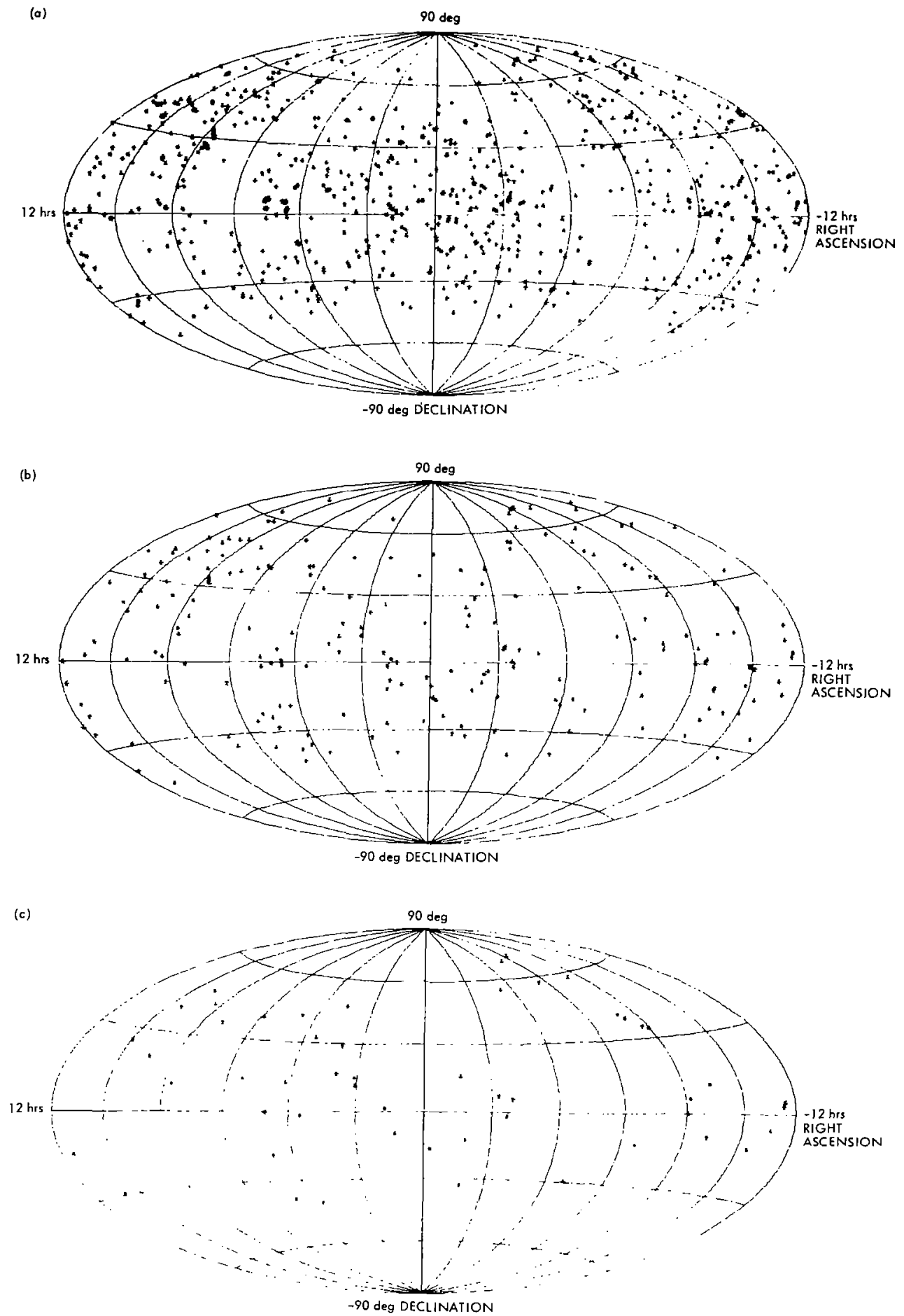

Figure 1. Sky distribution of VLBI sources: (a) 665 sources $\geq 0.1$ Jansky, (b) 210 sources $\geq 0.5$ Jansky, (c) 52 sources $\geq 1.0$ Jansky. 
(3) The north polar area is more sparsely populated. This is directly due to a lack of sufficient high frequency surveys in this region to easily identify candidate sources to observe.

(4) The areas near the plane of the galaxy (not shown) are more sparsely populated. This is particularly evident between $\pm 30^{\circ}$ declination at right ascensions of about -5 and +7 hours. This is due to the fact that high frequency radio surveys often skip the galactic regions and that interstellar charged particles near the galactic plane can cause intrinsically small sources to be scattered to large angular sizes.

We should point out that this is not nearly a complete map of the VLBI sky at the level of 0.1 Jansky. Since one of our criteria for choosing candidate sources was, in general, to keep only those sources with total flux densities greater than 0.7 Jansky, we have missed a large number of very compact sources with lower total flux densities.

Figure $1 \mathrm{~b}$ shows the distribution of 210 sources with correlated flux densities greater than 0.5 Jansky. This is also a rather dense and even distribution, and is a more complete representation of the VLBI sky than was the 0.1 Jansky map.

The DSN Block 1 VLBI System is being developed to calibrate radio metric navigation data from deep space probes by measuring earth rotational position and clock epoch and rate offsets (Fanselow et al., 1978). The sensitivity of this system to VLBI source strength is $0.5 \mathrm{Jansky}$. In order to conscrve antenna time, this system is intended to produce the appropriate calibrations with only 1 or 2 hours of observing on each of two intercontinental baselines. During that short time span, a single pair of DSN antennas will scc only a small scgment of sky in common. In order to properly estimate the desired parameters, there must be a good distribution of sources within this small common area of sky. This must be true at any time of day. Hence, this system requires a source cata$\log$ of about 100 sources. The number of sources we have found with strengths greater than 0.5 Jansky is nicely matched to the number of required sources.

Figure 1c displays the distribution of 52 sources with correlated flux densities greater than 1.0 Jansky. This is also quite a complete representation of the VLBI sky, but the distribution is no longer very dense. Note that less than 10 percent of the total number of detected VLBI sources have correlated flux strengths greater than 1.0 Jansky.

The falloff in number of sources with increasing source strength is shown more clearly in the histogram of figure 2. The number of detected sources at source strengths less than $0.5 \mathrm{Jansky}$ is smaller than the true sky distribution, due to our candidate selection criteria and our approximate sensitivity limit of 0.1 Jansky. The actual number of existing VLBI sources with correlated flux densities greater than 0.1 Jansky is probably several thousand. At the high end of the source strength scale, we see that only six sources are stronger than $2.0 \mathrm{~J}$ ansky and none are stronger than $3.5 \mathrm{Jansky}$. 


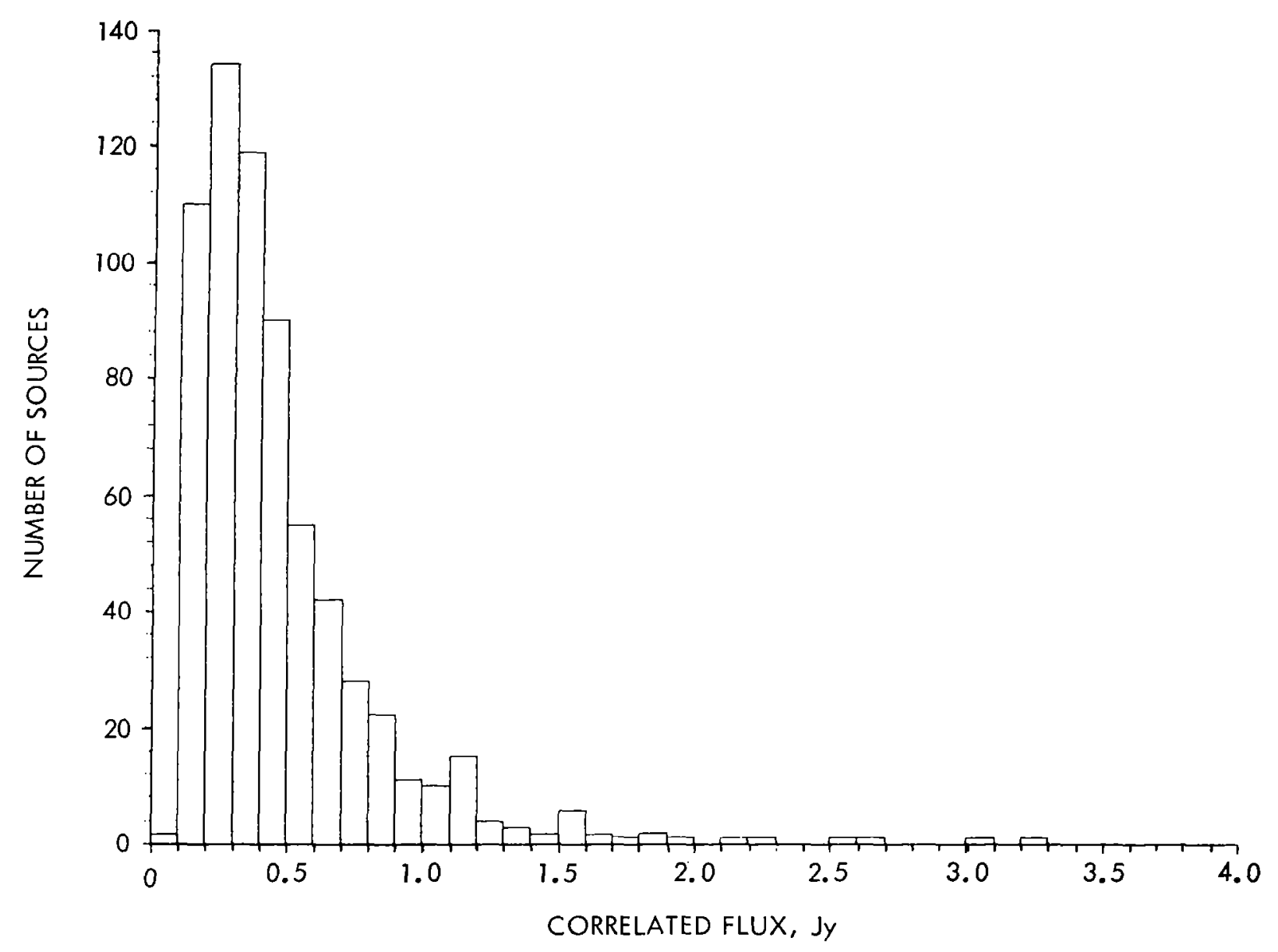

Figure 2. Histogram of number of sources vs correlated flux.

Geodetic VLBI investigations will often involve baselines shorter in length than the intercontinental baselines of this survey. A similar $13 \mathrm{~cm}$ VLBI survey has been completed on the same portion of sky for baselines of about $200 \mathrm{~km}$ length. The results of this survey show that if a given number of sources are required to form a VLBI reference frame, the sensitivity of a $200 \mathrm{~km}$ baseline $13 \mathrm{~cm}$ VLBI system need only be about half that of a similar intercontinental system. This difference is due to the fact that the shorter baseline system is sensitive to sources of larger maximum angular size. Actually, because of the shorter periods of source mutual visibility on the longer baselines, the number of sources necessary to form a reference frame for an intercontinental VLBI system would probably be larger than that for a $200 \mathrm{~km}$ system. Hence, intercontinental systems might have to be several times as sensitive as $200 \mathrm{~km}$ systems. 


\section{REFERENCES}

Clark, B. G.; Proc. of the IEEE, 61, 9, 1242-1248, 1973.

Counselman, C. C., III; Rev. Geophys. Space Phys., 13, 270-271, 1975.

Fanselow, J. L., et al.; Time and the Earth's Rotation, IAU Symp. No. 82, Reidel, 1979.

Purcell, G. H., et al.; NASA CP 2115, Radio Interferometry-Tech. for Geodesy, 1980.

Shapiro, I. I., and Knight, C. A.; Earthquake Displacement Fields and Rotation of the Earth, Reidel, 284-301, 1970. 



\title{
ASTROMETRY USING BASIC MARK II VERY LONG BASELINE INTERFEROMETRY
}

\author{
J. H. Spencer, E. B. Waltman, K. J. Johnston \\ E. O. Hulburt Center for Space Research \\ Naval Research Laboratory \\ D. C. Backer \\ Radio Astronomy Laboratory \\ University of California
}

\begin{abstract}
Two experiments have been performed in April and September 1976 to determine precise positions of radio sources using conventional Mark II very long baseline interferometry (VLBI) techniques. Four stations in the continental United States observed at a wavelength of $18 \mathrm{~cm}$. The recording band width was 2 MIIz. The preliminary results using analyses of fringe rate and delay are discussed and the source positions compared with the results of other measurements.
\end{abstract}


In early 1976, the Naval Research Laboratory (NRL) undertook a program to use the North American Very Long Baseline Network to investigate its astrometric potential. New techniques for determining source positions and baselines were to be investigated. Little was known at that time about the astrometric properties of the Mark Il processor in Charlottesville, Virginia. Objectives included (1) developing a grid of self-consistent baselines that could be used in this and other experiments, (2) testing ideas of how to produce a consistent source catalog of a large number of sources in a short time, and (3) developing a method for increased position accuracy by using phase comparison.

The method of using relative phase between sources previously had been limited to separations of $10^{-2} \mathrm{rad}$ (Wittels, 1975).* If an adequate source catalog could be used as a starting point with wellknown baselines, a low observing frequency might provide a long enough coherence time to switch between distant sources enough times to track the phase and determine lobe ambiguities. In practice, we compared closely-spaced pairs with other closely-spaced pairs. The $2 \mathrm{MHz}$ bandwidth of the Mark II system would be needed to obtain a good signal-to-noise ratio even for extremely short scans. The concept leads to accuracies at the level of absolute phase when relative phase is maintained for a day.

By using an array of baselines, fringe rates would be used to determine initial source positions to $\sim 0$."05 using the longest baselines. Relative phase from the shortest baseline, Maryland Point to Green Bank, would then establish an improved position catalog using its 0." 15 spacing. Then the longest baselines, spacing $\sim 0 . " 01$, would again be used to determine the final catalog, one with errors at the few milliarcsecond level.

Two observing sessions of 48 hours each were used for this program. In April 1976, data were obtained from the Owens Valley and Maryland Point observatories. In September 1976, all baselines between Green Bank, Haystack, Maryland Point, and Owens Valley have produced valuable data. The observations were taken at $18 \mathrm{~cm}$, the lowest standard frequency of the network, to provide a long coherence time. Hydrogen maser frequency standards were used at all sites. The data were correlated on the National Radio Astronomy Observatory (NRAO) processor in Charlottesville and reduced using programs developed at NRL. In this paper, we will discuss the fringe rate source catalog based on our September data set.

Application of basic Mark II techniques, as was done here, could investigate large numbers of survey sources to eliminate from further study those that show too much structure to be of astrometric interest. These techniques should be used at several frequencies to study the frequency dependency of the centroids of the source emission. As has been shown by Perley and Johnston (1979) from their VLA maps, the size and spectral index of 3C371 depends on frequency. At low frequencies, an extended nonthermal component 3 arcseconds northwest of the compact component biases the position found for this source. Measurements of $3 \mathrm{C} 371$ by Elsmore and Ryle (1976) made at $5 \mathrm{GHz}$ 
with a resolution of 3 " and by Wade and Johnston (1977) at $2.7 \mathrm{GHz}$ with a resolution of 0.6 have a discrepancy of $0 \$ 1$ in right ascension. Readhead et al. (1978) discuss systematic changes in structure and position angle with frequency such as found in 3C273 and 3C345. Structural differences such as these can be expected to lead to discrepancies in positions that can be resolved by a more complete study of the spatial and spectral characteristics of radio sources.

Both the delay and fringe rate properties of our data were used to determine source position catalogs and baselines. Because only the $2 \mathrm{MHz}$ bandwidth of the Mark II system was used (no synthetic bandwidth), delay solutions that were precise enough to be of interest could not be established. The period of the $4 \mathrm{MHz}$ clock (250 nanoseconds) limits solutions to about 25 nanoseconds. For a $3500 \mathrm{~km}$ baseline, this is an error of almost $0 . " 5$ in source position or 5 to 8 meters in baseline.

The fringe rate depends on source position, baseline and instrumental clock in the basic form

$$
\phi=\Omega \mathrm{B}_{\mathrm{e}} \cos \mathrm{h} \cos \delta+\mathrm{R}
$$

where $\Omega$ is related to the earth rotation rate, $\mathrm{B}_{\mathrm{e}}$ is the equatorial component of the baseline, $\mathrm{h}$ is the source angle from the meridian of the baseline, $\delta$ is the source declination, and $\mathrm{R}$ is a functional for the oscillator frequency offsets between the independent oscillators at each station. For our data, $\mathrm{R}$ was a constant to within the error of measurement.

It is important to note that in equation (1), a small change in the magnitude of the baseline $B_{e}$ is inseparable from a change of opposite sign in $\cos \delta$. This is because in this analysis $\Omega \mathrm{B}_{\mathrm{e}} \cos \delta$ are each individually time independent, only their product is determined. Outside constraints (such as limiting $\cos \delta$ over the range $0 \leq \cos \delta \leq 1$ ) must be used to factor this measured product. Observing a large number of sources over the sky will establish a source catalog with the same error in cos $\delta$, but will not remove it (Shapiro, 1976). Similarly, a grid of baselines can be established using many stations. Baseline direction vectors and rclativc lengths can be found, but the scale of length cannot be separated from $\cos \delta$ without outside information. Likewise, the zero of right ascension cannot be determined from the observable data alone.

It is difficult for radio astronomers to establish a right ascension for a catalog. Clark et al. (1976) and Wade and Johnston (1977) established their zero point in right ascension by adopting the position of 3C273B determined by Hazard et al. (1971) from lunar occultation timings. Elsmore and Ryle (1976) established their zero point relative to the FK4 position of $\beta$ Persei. Fanslow (1978)* chose the position of NRAO140 as the zero point. The position of NRAO140 was established such that it minimized the difference between the optical and radio positions for those sources in the catalog having optical counterparts. In this paper, $3 \mathrm{C} 273 \mathrm{~B}$ was used to set the zero point of right ascension.

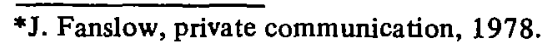


A variety of techniques has been examined to establish the zero of declination. We have tried holding the Owens Valley to Haystack baseline fixed to the value provided by Knight (1979) and Thomas et al. (1979). This value should be good to a few centimeters. Fixing one baseline provides a scale length and establishes the other baselines, thus the source positions.

One could fix a single source position and thus force the others and the baseline scale to follow. This approach was considered but not used because the declination of no one source is known well enough to grant it this favored status.

Instead, the fringe rate catalog of table 1 has its declinations fixed by a statistical average of the 10 best sources that also appear in the catalog of Wade and Johnston (1977). We did not select a weighted mean catalog of all the above mentioned catalogs (see Johnston et al. 1979, and Elsmore 1978), because a systematic error found later in one of the component catalogs could be too hard to remove. The root mean square of the residual of the solution is $0.4 \mathrm{MHz}$ or 0. . 05 . A comparison of the solution obtained by fixing a baseline to the solution of table 1 shows that there are no significant differences to the source catalog.

In table 2, the average differences in position for sources common in the above catalogs versus Wade and Johnston (1977) are displayed. As stated above, our average declination difference is defined to be zero. Also, fringe rate positions must degrade near the equator because the error in declination goes as $1 / \cos \delta$.

The conclusion is that fringe rate techniques and Mark II VLBI can be readily used at a large number of stations to establish baselines to $\sim 1$ meter and source catalogs. Certain errors became known or cancelled because of the grid of baselines, and we were able to get a satisfactory solution using only 48 hours of data. This technique may become important in studying the spatial, spectral, and temporal characteristics of large numbers of sources.

\section{REFERENCES}

Clark, T. A., Hutton, L. K., Marandino, G. E., Counselman, III, C. C., Robertson, D. S., Shapiro, I. I., Wittels, J. J., Hinteregger, H. F., Knight, C. A., Rogers, A. E. E., Whitney, A. R., Niell, A. E., Rönnäng, B. O., and Rydbeck, O. E. H.; Astron. J., 81, 599, 1976.

Elsmore, B. and Ryle, M.; M.N.R.A.S., 174, 111, 1976.

Elsmore, B.; Proc. of IAU Colloquium \#48 “Modem Astrometry,” 1978.

Hazard, C., Sutton, J., Argue, A. N., Kenworthy, C. M., Morrison, L. V., and Murray, C. A.; Nat. Phys. Sci., 233, 89, 1971. 
MOVEMENTS TERRESTRIAL AND CELESTIAL

Table 1

Fringe Rate Catalog

\begin{tabular}{|c|c|c|c|c|c|c|}
\hline Source & \multicolumn{3}{|r|}{$\alpha$} & \multicolumn{3}{|r|}{$\delta$} \\
\hline $0224+671$ & 02 & $24^{m}$ & 41 s. $1695 \pm .0022$ & 67 & 07 & $39: 690 \pm .006$ \\
\hline $3 C 84$ & 03 & 16 & $29.5637 \pm .0005$ & 41 & 19 & $51.883 \pm .005$ \\
\hline NRAOI40 & 03 & 33 & $22.4055 \pm .0008$ & 32 & 08 & $36.618 \pm .011$ \\
\hline $\operatorname{CTA} 26$ & 03 & 36 & $58.9568 \pm .0030$ & -01 & 56 & $15.755 \pm .445$ \\
\hline $3 C 120$ & 04 & 30 & $31.5945 \pm .0018$ & 05 & 14 & $59.180 \pm .089$ \\
\hline OJ 287 & 08 & 51 & $57.2544 \pm .0008$ & 20 & 17 & $58.346 \pm .014$ \\
\hline $\mathrm{DA} 267$ & 09 & 23 & $55.3204 \pm .0010$ & 39 & 15 & $23.549 \pm .007$ \\
\hline $3 C 274$ & 12 & 28 & $17.5750 \pm .0060$ & 12 & 40 & $01.250 \pm .200$ \\
\hline $3 C 273$ & 12 & 36 & 33.2460 & 02 & 19 & $41.667 \pm .380$ \\
\hline $3 C 279$ & 12 & 53 & $35.9278 \pm .0161$ & -05 & 31 & $05.406 \pm .940$ \\
\hline OQ208 & 14 & 04 & $45.6167 \pm .0043$ & 28 & 41 & $29.205 \pm .046$ \\
\hline OQ172 & 14 & 42 & $50.4260 \pm .0130$ & 10 & 11 & $12.000 \pm .300$ \\
\hline $1546+027$ & 15 & 46 & $58.2900 \pm .0080$ & 02 & 46 & $05.300 \pm .400$ \\
\hline $1548+056$ & 15 & 48 & $06.8870 \pm .0110$ & 05 & 36 & $11.800 \pm .300$ \\
\hline $1555+001$ & 15 & 55 & $17.6837 \pm .0054$ & 00 & 05 & $08.544 \pm 24.7$ \\
\hline NRAO512 & 16 & 38 & $48.1733 \pm .0023$ & 39 & 52 & $30.116 \pm .028$ \\
\hline $3 C 345$ & 16 & 41 & $17.6053 \pm .0009$ & 39 & 54 & $10.797 \pm .010$ \\
\hline $3 C 418$ & 20 & 37 & $07.4628 \pm .0022$ & 51 & 08 & $35.727 \pm .010$ \\
\hline $2134+004$ & 21 & 34 & $05.2353 \pm .0075$ & 00 & 28 & $00.795 \pm 3.27$ \\
\hline BL Lac & 22 & 00 & $39.3635 \pm .007$ & 42 & 02 & $08.569 \pm .006$ \\
\hline CTA102 & 22 & 30 & $07.7833 \pm .0091$ & 11 & 28 & $22.531 \pm .155$ \\
\hline $3 C 454.3$ & 22 & 51 & $29.5102 \pm .0023$ & 15 & 52 & $54.150 \pm .040$ \\
\hline
\end{tabular}


Table 2

Weighted Mean Differences. Wade and Johnston (1977) - Other Catalogs

\begin{tabular}{|l|c|c|c|}
\hline \multicolumn{1}{|c|}{ Catalog } & $\begin{array}{c}\text { Sources in } \\
\text { Common }\end{array}$ & $\Delta \alpha$ & $\Delta \delta$ \\
\hline Clark et al. (1976) & 17 & $-0.0001 \pm 0.0006$ & $0.002 \pm 0.009$ \\
Elsmore \& Ryle (1976) & 17 & $-0.0060 \pm 0.0015$ & $0.049 \pm 0.016$ \\
Fanslow (1978) & 10 & $-0.0032 \pm 0.0026$ & $0.012 \pm 0.017$ \\
This paper & 10 & $0.0002 \pm 0.0020$ & $0.000 \pm 0.016$ \\
\hline
\end{tabular}

Johnston, K. J., Spencer, J. H., Kaplan, G. H., Klepczynski, W. J., and McCarthy, D. D.; Proc. of Dec. 1978, U.S.N.O. Symp. on Star Catalogs, 1979.

Knight, C. A., NASA CP 2115, Radio Interferometry Techniques for Geodesy, 1980.

Perley, R. and Johnston, K. J.; submitted to Astron. J., 1979.

Readhead, A. C. S., Cohen, M. H., Pearson, T. J., and Wilkinson, P. N.; Nature, 276, 768, 1978.

Shapiro, I. I.; Methods of Experimental Physics, vol. 12C, N. L. Meeks editor, Academic Press, pp. $264-266,1976$.

Thomas, J. B., Fanslow, J. L., Cohen, E. J., Purcell, G. H., Rogstad, D. H., Sovers, O. J., Skjerve, L. J., and Spitzmesser, D. J.; NASA CP 2115, Radio Interferometry Techniques for Geodesy, 1980 .

Wade, C. M. and Johnston, K. J.; Astron. J., 82, 791, 1977. 


\title{
DIFFERENTIAL RADIO ASTRONOMY OF GALACTIC OBJECTS
}

\author{
D. C. Backer \\ Radio Astronomy Laboratory \\ University of California
}

\section{INTRODUCTION}

In contrast to the very distant quasars and galaxies used for geodetic studies discussed so far, pulsars, radio stars, and other galactic continuum objects have large transverse motions from proper motion, parallax, and orbital effects which can be measured with recently developed microwave interferometers. We (the author in collaboration with R. A. Sramek of the National Radio Astronomy Observatory (NRAO)) established a program in 1974 designed to measure these motions with the 35-km interferometer in Green Bank, West Virginia, at an observing wavelength of $11.1 \mathrm{~cm}$. Our first results $(1976, \underline{\mathrm{AJ}}, \underline{81}, 430)$ demonstrated the sensitivity of this instrument to the proper motions of pulsars: Motions of $0 . " 05 \mathrm{yr}^{-1}$ were detectable for these faint objects with several observing sessions spaced over $1 \frac{1 / 2}{2}$ years. We will discuss our differential astrometry technique and present an improved proper motion and a parallax limit for pulsar $1929+10$ and a limit on the space velocity of the enigmatic object in SgrA.

\section{TECHNIQUE}

Most of you are familiar with the $35-\mathrm{km}$, radio-link interferometer developed by the NRAO and currently operated by the U.S. Naval Observatory (USNO). Three baselines, each with dual polarization, are formed between a remote 45-foot (13.7-meter) telescope and the local 85-foot (25.9-meter) telescopes in Green Bank. Six simultaneous interferometers are thus recorded providing necessary sensitivity and extremely useful redundancy. A small amount of tropospheric phase noise is also eliminated. We observe a sequence of reference (A) - program object - reference (B) - program object - etc., with references located on nearly opposite sides of the program object. A weighted average of reference data will yield the equivalent data of a pseudo-reference source as close as possible to the program object. In principle, three references could be observed and the pseudoreference placed coincident with the program object to remove phase fluctuations which vary linearly over the object/reference region of the sky. However, the sky density of catalogued compact objects, instrumental sensitivity, and temporal variations of the phase fluctuations conspire to limit our observations to two reference sources.

The online phase residuals from Green Bank were first corrected for known errors in baseline, clock, atmosphere, and source position in Charlottesville, and then averaged to form an amplitude and phase for each baseline and polarization for each 5 to 10 minute scan. These data were taken to Berkeley for further analysis containing the following steps: 
(1) position update for errors ascertained after initiating a standard reduction procedure in Charlottesville;

(2) integration over polarization channels and three successive hour-angle scans to obtain unambiguous phase estimates on the faint program object;

(3) removal of lobe ambiguities;

(4) linear interpolation in time of the reference phases to the time of object observations and subtraction of weighted sum of these interpolated values from the object phases;

(5) fitting of the above "first difference" phase for each baseline on each day and each epoch to a pseudo-position offset. The pseudo-position offsets for any program object may be modeled by

$$
\underset{\sim \sim}{\Delta S_{p}}=\underset{\sim}{\Delta S_{0}}+\mu T+\underset{\sim}{\sim}(T)+\text { orbital effects }(T)
$$

where $\underset{\sim}{\Delta S_{0}}$ is a constant and $T$ is the interval of time from initiation of observations. Source structure effects may also enter the model.

\section{$1929+10$ RESULTS}

An example of the effectiveness of this technique is demonstrated in figure 1 for the pulsar $1929+$ 10. The pseudo-position offsets in milliarc seconds are shown during 1974 to 1976 for position axes $(\mathrm{x}, \mathrm{y})$ rotated $40^{\circ}$ from the $(a, \delta)$ axes to coincide with the normal axes of the measurement errors. This results from our 4-hour observation providing limited UV coverage in pa $-50^{\circ}$. The results for each day and baseline are given separately. The agreement of the Y-offset measurements with a proper motion of $0.08 / \mathrm{yr}$ is excellent. This corresponds to a transverse velocity component of $40 \mathrm{~km} / \mathrm{s}$ at a distance of $100 \mathrm{pc}$. The sine wave in figure 1 is the parallactic shift for an object at a distance of $50 \mathrm{pc}$ - clearly $1929+10$ is not closer than $50 \mathrm{pc}$.

In figure 2, we compare absolute positions for $1929+10$ from our program and from timing measurements which effectively employ an interferometer with a baseline of $1 \mathrm{AU}$ but which has a delay resolution of only 10 to $1000 \mu \mathrm{sec}$. The right ascension measurements are in excellent agreement. Our declination disagrees slightly with the timing measurements which disagree with each other in $\mu^{\delta}$.

\section{SgrA}

In 1975, we began observing the compact object in our galactic center, SgrA. If this enigmatic object is at rest in the gravitational barycenter of our galaxy, then observations of SgrA with respect 


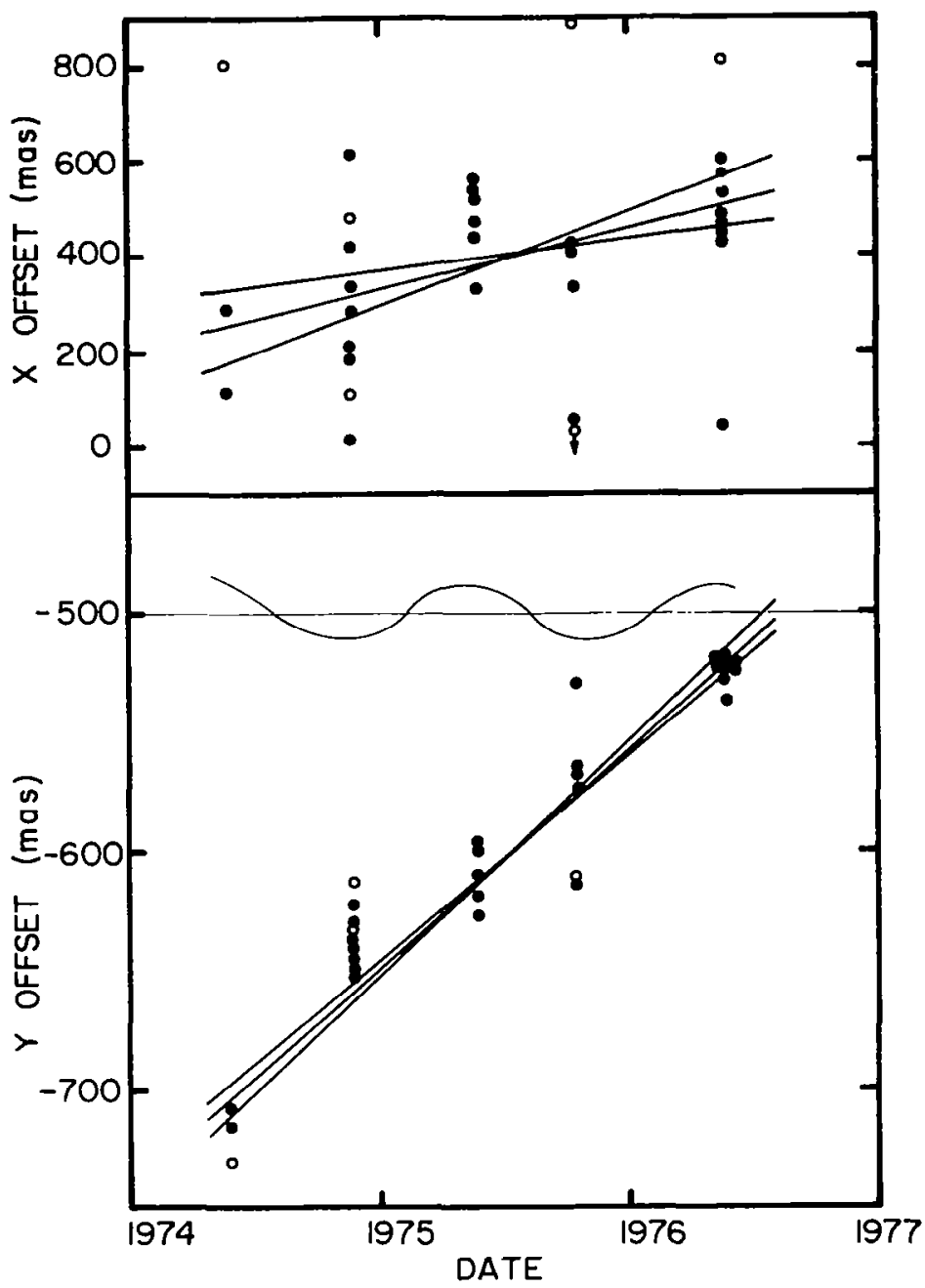

Figure 1. Pseudoposition offsets along axes at position angle of $40^{\circ}$ showing proper motion of pulsar $1929+10$. Offsets are given in milliarc seconds. The sinusoid in the lower section is the expected offset from trigonometric parallax at a distance of $50 \mathrm{pc}$. Open circles designate data with large errors. 


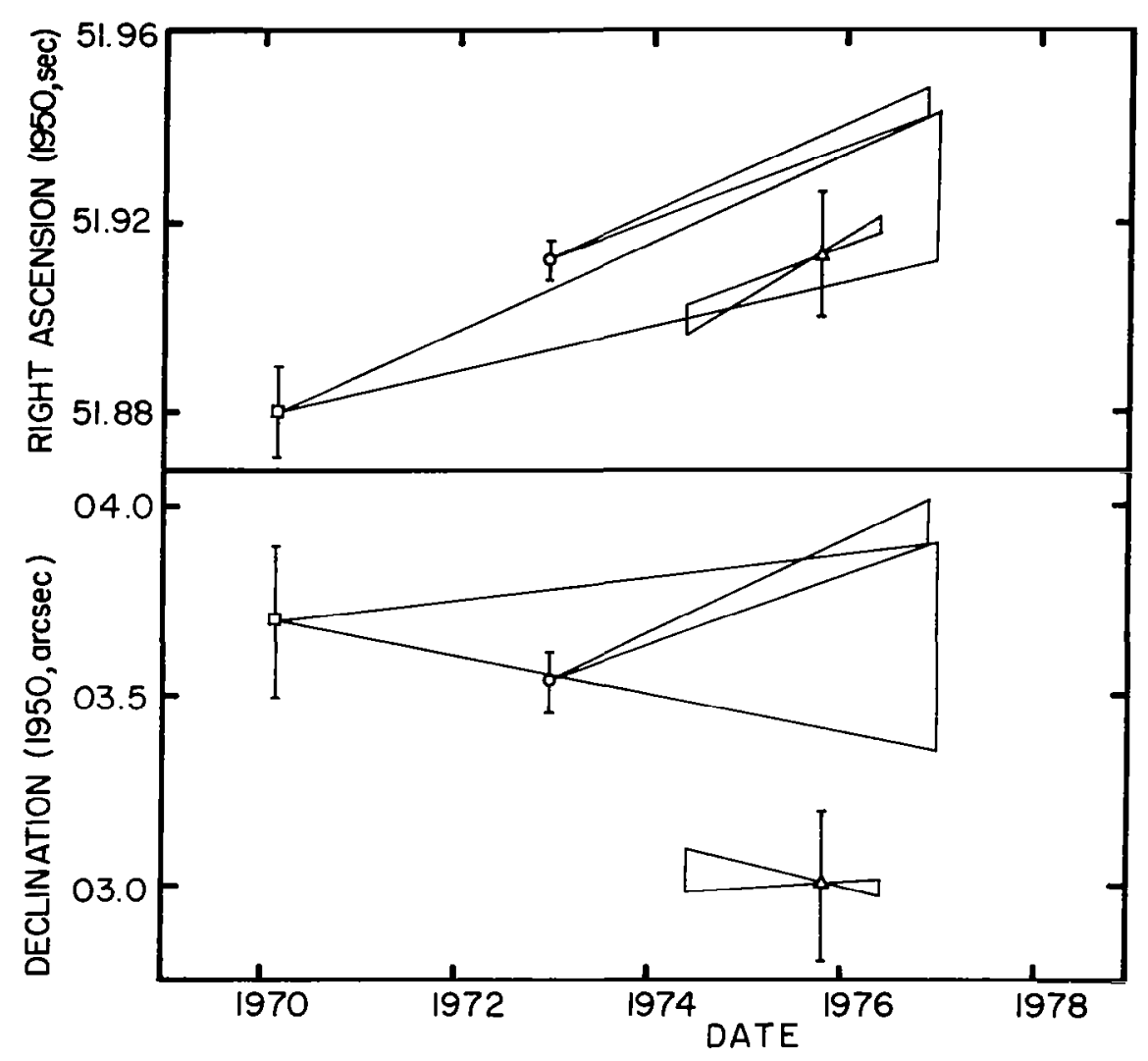

Figure 2. Absolute position and proper motion of $1929+10$ from our interferometer measurements $(\Delta)$, and from timing measurements $(\square=$ Helfand et al. 1979, Ap. J., in press; $O=$ Gullahorn and Rankin 1978, A. J., 83, 1219). The right ascension is $19 \mathrm{~h} 29 \mathrm{~m}$; the declination is $10^{\circ} 53^{\prime}$.

to an extragalactic frame would directly measure the rotation rate of the galaxy. It is likely, however, that SgrA is not at rest - the infrared studies of Lacy and Townes indicate a dynamical center not coincident with this radio object and velocities of order $300 \mathrm{~km} \mathrm{~s}^{-1}$. In this case, we would remove an assumed galactic rotation rate from a SgrA measurement and hope to learn more about the nature of this object.

Figure 3 shows, unfortunately, that measurements with the $35-\mathrm{km}$ instrument using reference sources $2^{\circ}$ and $3^{\circ}$ away can only place an upper limit of $\mu_{\mathrm{y}} \leq 0 . " 01 \mathrm{yr}^{-1}$ (in 2 ycars) at a position angle roughly parallel to the galactic plane. The galactic rotation rate is $0 . " 005 \mathrm{yr}^{-1}$ in this plane. The limit corresponds to a transverse velocity limit of $1000 \mathrm{~km} \mathrm{~s}^{-1}$ at $10 \mathrm{kpc}$ - moderately interesting, but we would like to do 10 times better. 


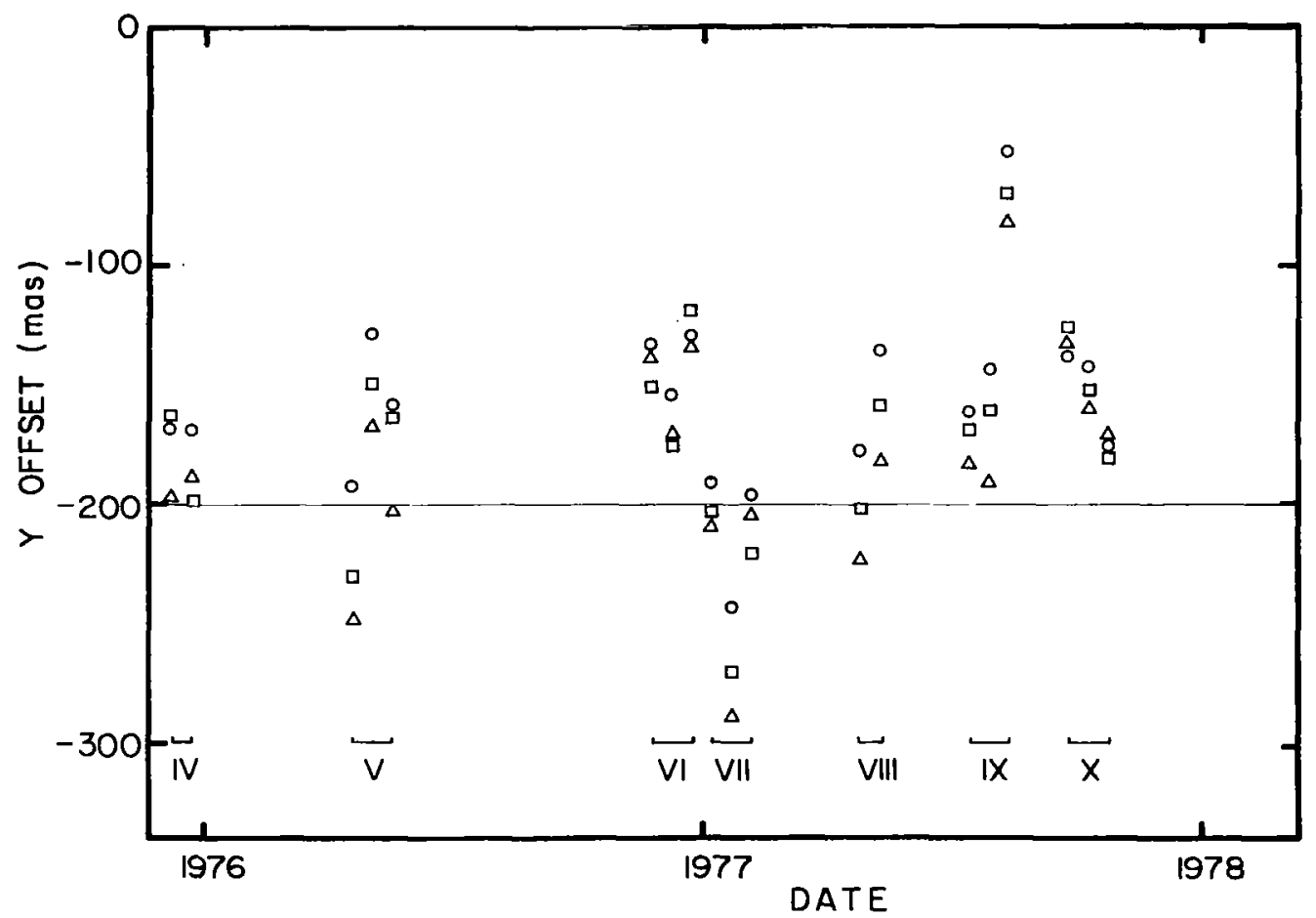

Figure 3. Pseudoposition offsets along an axis nearly parallel to the galactic plane for SgrA. Systematic differences between offsets from the three baselines $(O=14, \square=34$, $\Delta=24$ ) probably result from confusion in the vicinity of SgrA. The measurements were taken at epochs IV through $X$.

We seem to be affected by structure which causes a 0. "030 shift of the 14 baseline offset relative to 24. Tests indicate the shift is not consistent with a first difference lobe error. The shift may well result from structure of, or confusion near, SgrA.* Estimates of interstellar scattering for SgrA would predict an apparent size of 0.300 at $11.1 \mathrm{~cm} \lambda$.

We note that the sun passes near SgrA in December. Estimates of the differential deflection predicted by general relativity give 0.025 - a factor which must be removed before combining these data for formal proper motion limits.

I should emphasize that figure 3 contains pseudo-position offsets for every day of every epoch independent of the often variable weather in Green Bank. This plot then gives an indication of the reliability for absolute astrometry at declinations of $-30^{\circ}$ ( $\sec z \gtrsim 3$ ).

\footnotetext{
* This shift has been identified as the result of comparing $Y$ offsets on slightly different frames of reference for the three baselines. An improved analysis of these observations will be presented elsewhere (Backer and Sramek, 1980, in preparation).
} 
Closer reference sources, a longer time base and, of course, a more southerly site are required to make further progress on this object.

My thanks to Fred Crews and Jim Coe at NRAO for making every epoch a success and to Tom Clark for support while at GSFC. 


\title{
SOURCE STRUCTURE CORRECTIONS TO THE GEODETIC VERY LONG BASELINE INTERFEROMETRY OBSERVABLES
}

\author{
William D. Cotton \\ Department of Earth \& Planetary Sciences \\ Massachusetts Institute of Technology
}

This paper will attempt to address two questions: (1) why does source structure affect very long baseline interferometry (VLBI) observations at all, and (2) how does source structure corrupt the geodetic observables? Then, finally, evidence will be presented which shows that corrections for source structure are possible and necessary for centimeter-level accuracy VLBI measurements.

Why does structure affect VLBI observations? How is it possible to determine this structure? Before considering more complex sources, let us first review the response of an interferometer to a point source. In this case, the received signal can be considered to contain two parts, (1) noise from the source which will be identical at the two antennas (see figure 1), and (2) the receiver noise, etc., which is independent at the two antennas. Thus, when the signals from the two antennas are correlated, the peak of the amplitude of the correlation function is proportional to the ratio of the system power due to the source to the total system power, independent of the observing geometry:

$$
|\rho| \propto \frac{\mathrm{P}_{\text {source }}}{\mathrm{P}_{\text {total }}}
$$

Unfortunately, nature was not generous enough to supply point sources useful for geodetic applications, and we are forced to consider more complex situations.

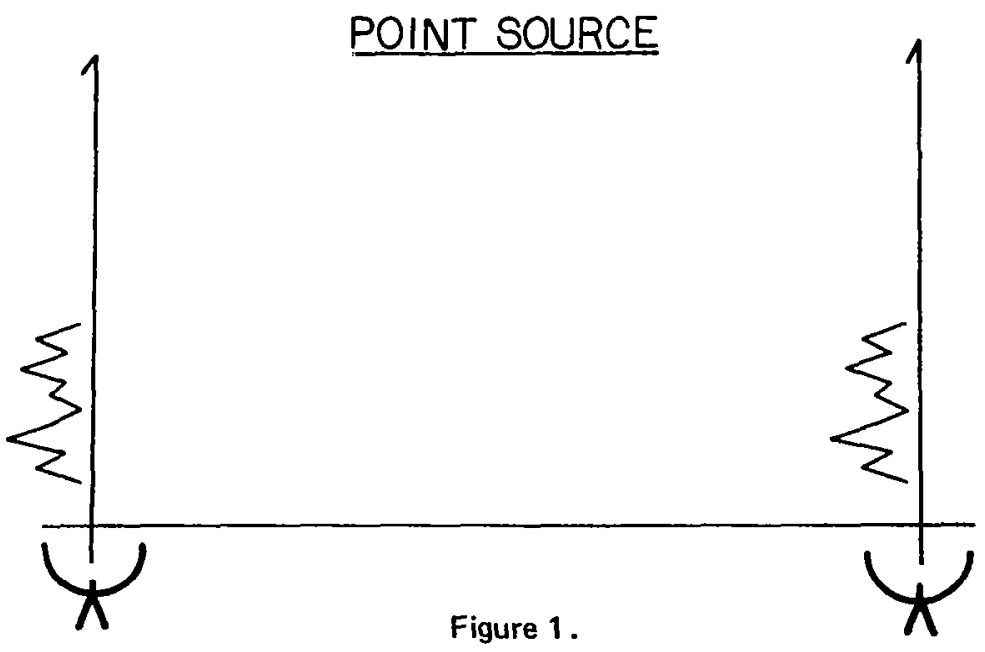


The simplest possible extended source is an equal brightness double point source, which will be sufficient to show the general features of extended structure. In this case, the signal from each component is still identical at the two antennas but with different delays. This is demonstrated in figure 2 on a greatly exaggcrated scale. The delay difference causes the two patterns to interfere, reducing the observed correlation amplitude. In this case, the correlation amplitude is of the form:

$$
|\rho| \propto \frac{\mathrm{P}_{\text {source }}}{\mathrm{P}_{\text {total }}} \cos (2 \pi \nu \Delta \tau)
$$

where $\nu=$ the observing frequency. The delay difference depends on the observing geometry so the observed correlation amplitude will be different on different baselines and vary with time on a given baseline.

\section{EQUAL DOUBLE}

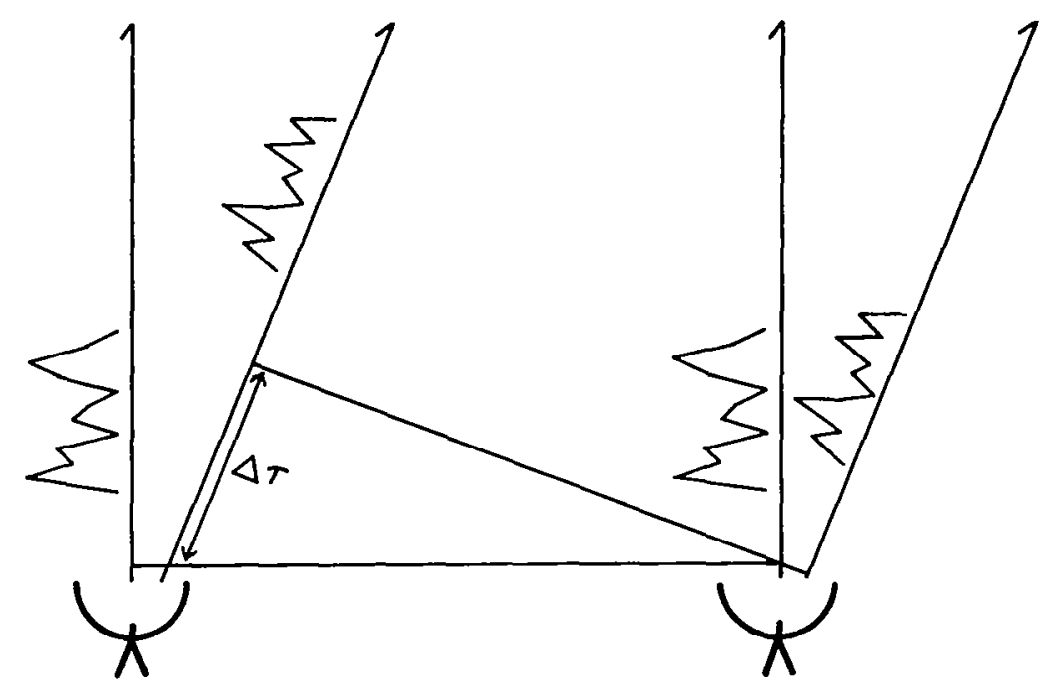

Figure 2.

In the most general case, the observed correlation function is proportional to the so-called visibility function which is the Fourier transform of the source brightness distribution:

$$
\rho \propto \mathrm{V}(\mathrm{u}, \mathrm{v})=\iint \mathrm{B}(\mathrm{x}, \mathrm{y}) \exp [-2 \pi \mathrm{i}(\mathrm{ux}+\mathrm{vy})] \mathrm{dxdy}
$$

where $\mathrm{B}(\mathrm{x}, \mathrm{y})$ is the brightness distribution. Note that the visibility is complex as this point will be relevant to the following discussion. It is possible to deduce the source structure if a sufficient number of observations of the correlation function arc made and properly calibrated. (See Cotton, 1979, Astron. J., 84, 1122.) 
The second question posed at the beginning of the paper was what effect does source structure have on the geodetic observables, group delay, and fringe rate. The group delay is defined as the derivative of fringe phase with respect to angular frequency:

$$
\tau=\frac{\mathrm{d} \phi}{\mathrm{d} \omega}
$$

Since the visibility, or structure phase, can vary arbitrarily rapidly with observing frequency, especially near amplitude minima, the measured group delay can be affected to an arbitrary degree. Extreme cases of this phenomenon are easily detectable because of the very pronounced minimum in the observed correlation amplitude. The structure contribution to the observed group delay is given by the following relationship:

$$
\tau_{s}=\frac{\operatorname{Re}(V) \frac{d \operatorname{Im}(V)}{d \omega}-\operatorname{Im}(V) \frac{d \operatorname{Re}(V)}{d \omega}}{|V|^{2}}
$$

Likewise, the contribution of the source structure to the observed fringe rate is given by:

$$
\dot{\phi}_{s}=\frac{\left[\operatorname{Re}(V) \frac{\partial \operatorname{Im}(V)}{\partial u}-\operatorname{Im}(V) \frac{\partial \operatorname{Re}(V)}{\partial u}\right] \frac{d u}{d t}}{|V|^{2}}+\frac{\left[\operatorname{Re}(V) \frac{\partial \operatorname{Im}(V)}{\partial v}-\operatorname{Im}(V) \frac{\partial \operatorname{Re}(V)}{\partial v}\right] \frac{d v}{d t}}{|V|^{2}} .
$$

Nature has added a further complication; that is, that many sources have time variable structure. This has two effects: (1) the corrections to group delay and fringe rate are time variable and (2) the apparent position of the source can change. To resolve these difficulties, we need to monitor the source structure and to pick, on physical grounds, a feature in the source which is most likely to be at a constant position in the sky.

The bottom line of this paper is, of course, how much effect does source structure really have? To address this question, figure 4 shows the effects due to the structure of 3C 345 on observations on the Goldstone-Sweden baseline in May 1974. The map of 3C 345 used is shown in figure 3. The reference feature for this source is the component on the left which has had a relatively constant brightness and size for the last few years and is probably fixed in the nucleus of the parent object.

In figure 4, the corrections to the delay and rate as well as the visibility amplitude and phase are plotted as a function of Greenwich Sidereal Time. Figure 4 indicates that corrections to individual delay observations can be many centimeters, especially near minima in the visibility amplitude.

In order to avoid giving the impression that the problem is always this severe, figure 5 shows perhaps a more nearly typical case, Haystack-Goldstone, for the same source and epoch in which the maximum corrections are only a few centimeters. Since geodetic measurements involve a number of 


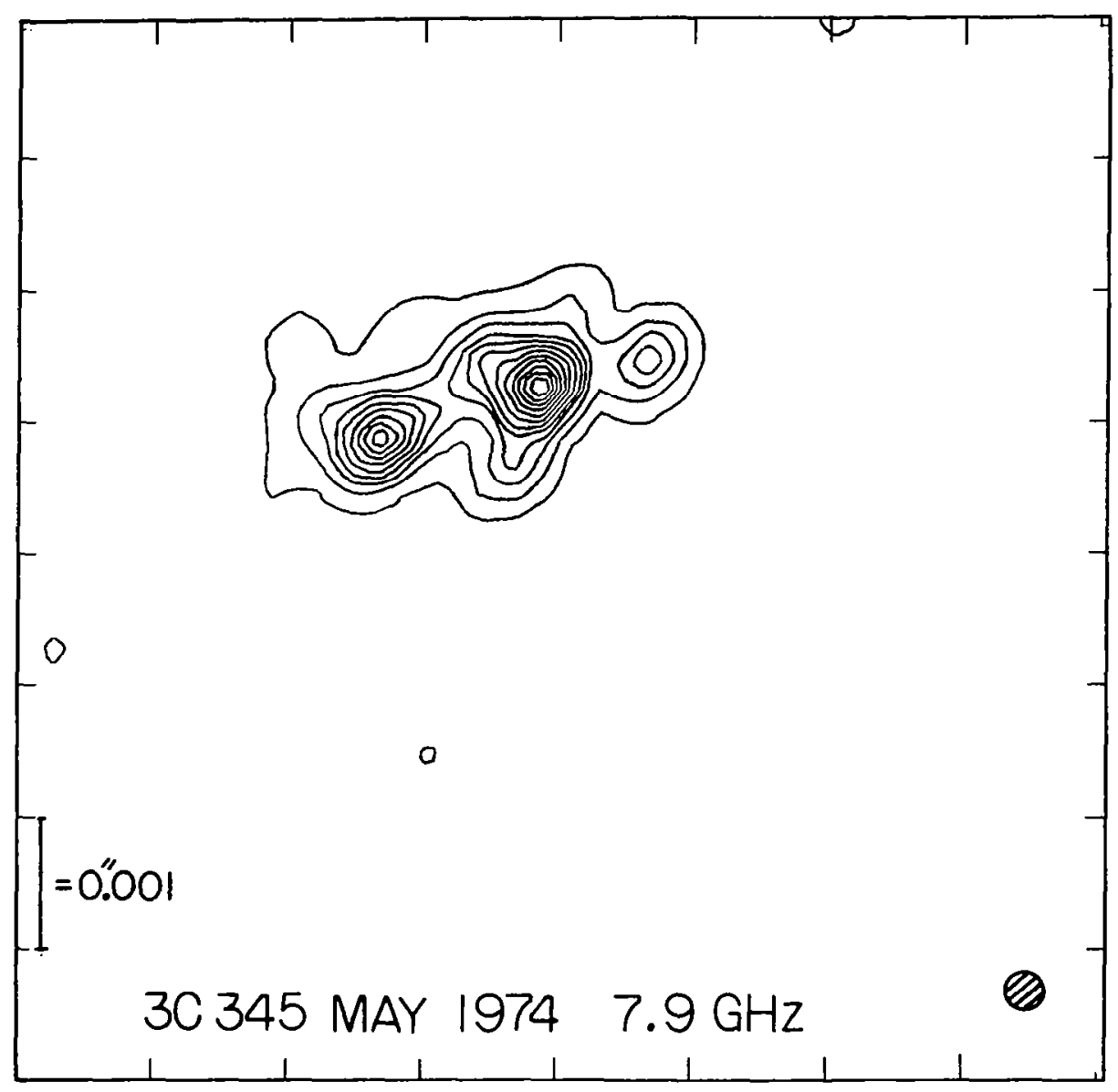

Figure 3 .

sources, the effects of structure on the final results will be somewhat diluted. While we have not performed conclusive tests, we feel that structure has probably not had a serious effect on results to date. However, if the full potential of the Mark III system is to be realized, source structure corrections must be made. 

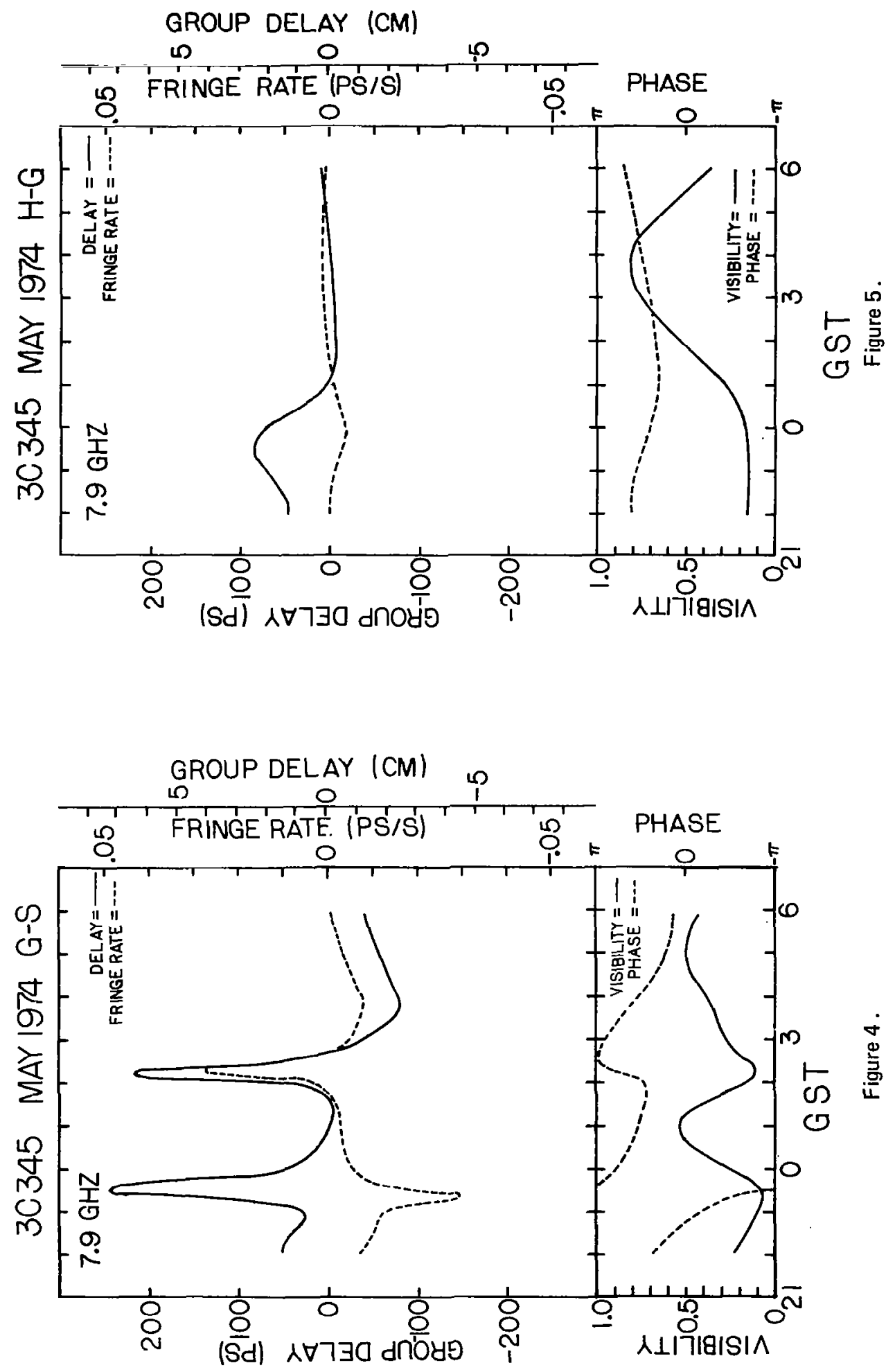
EFFECTS OF SOURCE STRUCTURE ON GEODETIC MEASUREMENTS

\author{
K. Johnson \\ Naval Research Laboratory
}

Presented but not submitted. 
SESSION C

DEGREES KELVIN AND DEGREES OF PHASE 


\title{
HYDROGEN MASER FREQUENCY STANDARD
}

\author{
R. F. C. Vessot \\ Smithsonian Institution \\ Astrophysical Observatory
}

\section{INTRODUCTION}

Since its invention in 1959 by Kleppner, Goldenberg, and Ramsey (1962) and Kleppner et al. (1965), the hydrogen maser has become an indispensable component of very long baseline interferometer systems. During the last decade, the development of the maser and its supporting control and signal procuring equipment has been strongly benefited by two phases of a project to measure the gravitational redshift, the first of which began in 1964 with NASA support and which has continued until the recent successful test of the equivalence principle using a space-borne maser (Vessot and Levine, 1979).

The intent of this paper is to describe the fundamental theoretical limitations of the maser and the systematic processes that cause instability and to describe some aspects of recently designed masers. A design for field use that has evolved from the development of the space-borne maser will be described. The performance of this type of maser is close to theoretical limits imposed by thermal noise. Further developments of smaller masers for space and terrestrial use and our recent work on masers operating at low temperatures will also be discussed.

\section{The Maser Oscillator}

The hydrogen maser is an oscillator whose power comes from the careful release of energy from the hyperfine interaction of the electron and proton of an aggregate of hydrogen atoms. This energy is, of course, familiar to radio astronomers as the $21-\mathrm{cm}$ line of atomic hydrogen. Figure 1 shows a schematic diagram of the maser and the hyperfine levels of atomic hydrogen. In the maser, atoms are obtained by dissociating molecular hydrogen by placing molecular hydrogen in a radio frequency (rf) discharge tube at a pressure of a few tenths of a torr. The outlet of this rf dissociator is usually a set of collimator tubes which produces a fairly narrow, cylindrical beam of neutral atomic hydrogen directed along the axis of a 6-pole state selector magnet that provides a highly inhomogeneous magnetic field in the radial direction (4-pole magnets are used successfully also). The field strength along the axis of the magnet is zero and increases quadratically in magnitude with radius to a level of about 10 kilogauss at the circularly arrayed pole tips of the magnet. Atoms enter the magnet from the source collimator within a conical spread of a few degrees. Those in the $F=1, m_{F}=1$, and $\mathrm{m}_{\mathrm{F}}=0$ states have induced magnetic dipole moments that interact with the magnetic gradient so as to experience a force directed radially inward and are focussed into a storage bulb. Atoms in 

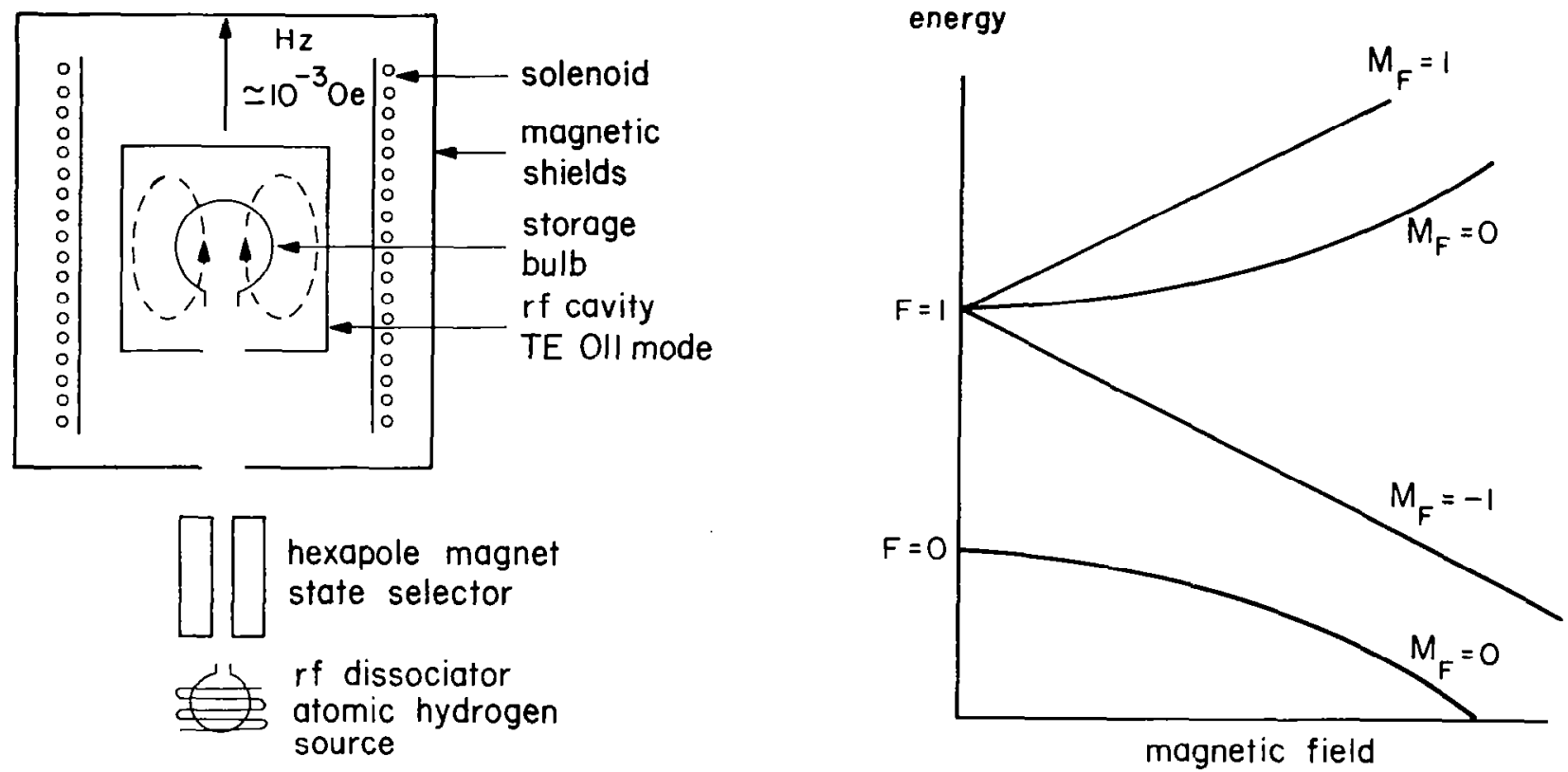

Figure 1. Schematic diagram of atomic hydrogen masers and energy levels of ground-state atomic hydrogen.

the $F=1, m_{F}=1$, and $F=0, m_{F}=0$ states have dipole moments of opposite sign and are directed outward. The field strength and length of the magnet are designed so as to focus the $F=1, \mathrm{~m}_{\mathrm{F}}=0$, and $m_{F}=1$ state atoms into the storage bulb through its collimator. (Note that the lateral dimensions of figure 1 are exaggerated. Typically, the beam diameter is about $3 \mathrm{~mm}$ and the magnet length about $7 \mathrm{~cm}$.)

The storage volume is the heart of the maser. It serves to confine a constantly replenished set of atoms in an in-phase region of microwave rf magnetic field within a $\mathrm{TE}_{111}$ cavity resonator, so that phase coherent stimulated emission of microwave energy from the atoms will result. According to Heisenberg's uncertainty principle of quantum mechanics, the longer the unperturbed interaction time, the narrower the resonance linewidth that can be achieved. Our objective is to store the atoms as unperturbed as possible for as long as possible so as to have as narrow a resonance as possible to define the maser's output frequency. Using kinetic theory, we can design bulbs several centimeters in diameter which, at room temperature, will confine the average hydrogen atom for about 1 second. In principle, we can thus obtain a resonance width of $1 \mathrm{~Hz}$ provided all other perturbations to the hydrogen atom can be controlled. The successful operation of the maser hinges on our providing a coating which reflects the hydrogen atom hundreds of thousands of times without appreciably altering the phase of the precessing, magnetically coupled, electron and proton (let alone allowing the atoms to make unwanted transitions or even to chemically combine with other atoms or recombine with themselves). The currently used coatings are usually made of Teflon $®$ (duPont). 
The energy is released from the state-selected atoms stored in the bulb and delivered to the cavity resonator whose quality factor is sufficiently high that the rf magnetic field from its stored energy will continue to stimulate atoms that follow so as deliver power equal to the combination of cavity dissipation and power coupled to the external receiver system. Under these conditions, the level of oscillation is sustained.

As in all oscillators where two or more resonators are coupled electromagnetically, there is a frequency pulling effect which is proportional to the ratio of the quality factor $Q_{c}$ of the pulling resonator (here the cavity) and the pulled resonator (here the atom, which has a quality factor $\mathrm{Q}_{\ell}$ ). The relationship is:

$$
\Delta \mathrm{f}_{\text {pulling }}=\frac{\mathrm{Q}_{\text {cavity }}}{\mathrm{Q}_{\text {atoms }}} \cdot \Delta \mathrm{f}_{\text {atom } \mathrm{s}}
$$

This is an approximation. The exact relationship also involves the spin exchange frequency shift [4]; however, the "pulling" effect that remains is still proportional to the cavity, offset.

When we look at typical values for masers, we find that $\mathrm{Q}_{\text {cavity }} \sim 30,000$ and $\mathrm{Q}_{\text {atoms }}=\mathrm{Q}_{\ell} \sim 2 \times 10^{9}$, so that to keep the frequency within $\Delta \mathrm{f} / \mathrm{f} \sim 10^{-15}$ the cavity resonance frequency changes must be kept to $\pm 0.1 \mathrm{~Hz}$.

A further condition for stable oscillation is that a low level ( 1 milligauss) uniform magnetic field must be applied in the direction of the magnetic component of the rf field to provide an axis of direction so that the $\Delta \mathrm{M}_{\mathrm{F}}= \pm 0$ transitions, allowed from the selection rules of quantum mechanics, will be selectively stimulated. The transition $F=1, \mathrm{~m}_{\mathrm{F}}=0 \rightarrow \mathrm{F}=0, \mathrm{~m}_{\mathrm{F}}=0$ in hydrogen at low field $(<<550$ Gauss) has second-order frequency dependence with magnetic field given by $\Delta f_{\text {magnetic }}=2,750 \mathrm{H}^{2}$ Gauss. For these so-called "clock" transitions, the frequency sensitivity to change in magnetic field is least at low fields. However, the fields must be maintained constant and uniform enough so that, throughout the storage volume, the moving atoms experience no motioninduced magnetic variations with spectral components that will excite transitions between the $\Delta \mathrm{M}_{\mathrm{F}} \pm 1$ hyperfine sublevels. At weak fields, these transitions follow the relationship $\mathrm{f}_{\mathrm{z}}=1.4 \times 10^{6}$ Gauss $^{-1}$. These transitions are used to measure the average magnetic field in the bulbs by applying from an external coil a transverse rf field whose frequency is scanned until a dip is observed in the maser's oscillation level.

The confinement of the atoms during the storage process also leads to systematic frequency shifts and poses a limitation to the available signal power output. The quality factor associated with the aggregate of stored atoms is given by

$$
\mathrm{Q}_{\ell}=\frac{\omega}{2 \gamma}
$$


where $\omega=2 \pi$ (oscillation frequency) and $\gamma$ is the total transverse relaxation rate of the average atom in the bulb, which has the following major components:

$$
\gamma_{2 \mathrm{~T}}=\gamma_{\text {storage }}+\gamma_{\text {wall }}+\gamma_{\text {spin }}+\gamma_{\text {magnetic }}+\ldots
$$

For a storage bulb of volume $V_{b}$ with a hole of area $A_{e}$ and collimator factor $K$, we have:

$$
\gamma_{2}=\frac{\overline{\mathrm{vA}} \mathrm{e}}{4 \mathrm{KV} \mathrm{v}_{\mathrm{b}}}+\frac{\overline{\mathrm{v} p}}{\lambda}+\overline{\mathrm{v}}_{\mathrm{r}} \sigma_{\mathrm{se}} \cdots
$$

Here,

$$
\begin{aligned}
& \bar{v}=\sqrt{8 \mathrm{kT} / \pi \mathrm{m}} \\
& \mathrm{v}_{\mathrm{r}}=\mathrm{v} / \sqrt{2}=\text { average relative velocity } \\
& V_{b}=4 / 3 \pi R^{3} \text { bulb volume }
\end{aligned}
$$

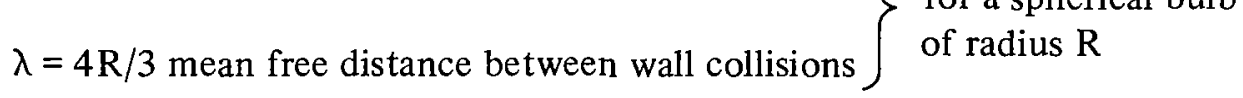

$$
\begin{aligned}
& p=\text { probability of loss per wall collision } \\
& \sigma_{\mathrm{se}}=\text { spin exchange cross section } \\
& \mathrm{n}=\text { density of atoms in bulb }
\end{aligned}
$$

Equation 1 can also be written as follows:

$$
\gamma_{2}=\left(\frac{8 \mathrm{kT}}{\mathrm{m}}\right)^{1 / 2}\left(\frac{3}{16} \cdot \frac{\mathrm{A}_{\mathrm{e}}}{\mathrm{KR}^{3}}+\frac{3}{4} \cdot \frac{\mathrm{P}}{\mathrm{R}}+\frac{\mathrm{n} \sigma_{\mathrm{se}}}{\sqrt{2}}\right)
$$

and we see that the kinetic behavior of the atoms affects three processes:
(1) the rate of emergence from the bulbs,
(2) the rate of wall collision relaxation, and
(3) the rate of loss from spin exchange relaxation. 
These effects limit the storage time and, hence, the $Q$ of the oscillating system. However, the spin exchange relaxation and wall relaxation are also accompanied by a frequency shift and the shift per collision is also a function of temperature. The temperature dependence of the wall relaxation probability depends very much on the type of surface. To complete the picture, we note that the spin exchange frequency shift also depends on the proportion of atoms in the various hyperfine states that enter the bulb.

The key to obtaining stability, then, is to keep the temperature, magnetic fields, and cavity resonance variables under close control. The solution is a question of design, and a considerable amount of engineering has been involved in the development of masers, chiefly in the control of temperature and eliminating the cavity resonator's sensitivity to temperature changes, the control of magnetic fields, the shielding of the atoms from outside field variations, and the control of atomic hydrogen beam flux.

The VLB-10 and -11 series masers built by the Smithsonian Astrophysical Observatory (SAO) have evolved from spacecraft mascr designs wherc ruggedncss, light wcight, small size, and low powcr consumption have been important considerations. All of these have cavity structures made of Cervit R (Owens-Illinois), a very stablc, low thermal expansion, glass-ceramic material that is well isolated mechanically from stress variation induced by barometric changes. Of the 14 masers built, all have large 8-element ion pumps with about 5-year capacity.

Figure 2 shows the lightweight (185 gram) fused silica spherical bulb with its integral collimator tube mounted to the end plate of the all-Cervit cavity. The fact that the dielectric constant of fused silica has a small but significant tempcraturc coefficient makes it important to load the cavity diclectrically as little as possible; hence, the emphasis on a lightweight bulb. The cavity-bulb assembly has a temperature coefficient of resonance frequency $\delta \mathrm{fc} / \delta \mathrm{T} \simeq-300 \mathrm{~Hz} / \mathrm{C}^{\circ}$. The cavity end plates and cylinder interior are coated with silver and, for mechanical stability, their joints are lapped. The assembly is clamped together with about $200 \mathrm{~kg}$ prcload applicd by cannister surrounding the cavity equipped with a constant force Belleville washer to minimize temperature induced stress changes from the cannister. As shown in figure 3, the asscmbly is mounted on a false bottom within a vacuum chamber (shown in the background) so as to avoid the effects of barometric pressure variations. The vacuum chamber is securcd to the base plate with three thin-walled titanium studs and connected by a thin-walled titanium bellows neck tube to the ion pump shown, without its magnets, in figure 4. The neck is equipped with a temperature controlled guard station to which are connected the small diameter coaxial cables leading to the cavity.

Immediately surrounding the cavity vacuum chamber is a nested set of four layers of magnetic shields made of carefully annealed moly-permalloy $\circledast$ (Allegheny Ludlum) which are separated at their ends by glass foam thermal insulation and insulated radially by layers of ethafoam $\circledast$ (Dow Corning). Not shown is a closely fitted aluminum oven cannister that surrounds the second magnetic shield (counting from inside). This oven is divided into two separately controlled zones and operates at $43^{\circ} \mathrm{C}$. The cavity vacuum chamber is temperature controlled in three separate zones at $50^{\circ} \mathrm{C}$. As seen in figure 5, three of the four magnetic shields have torrispherical ends to minimize 


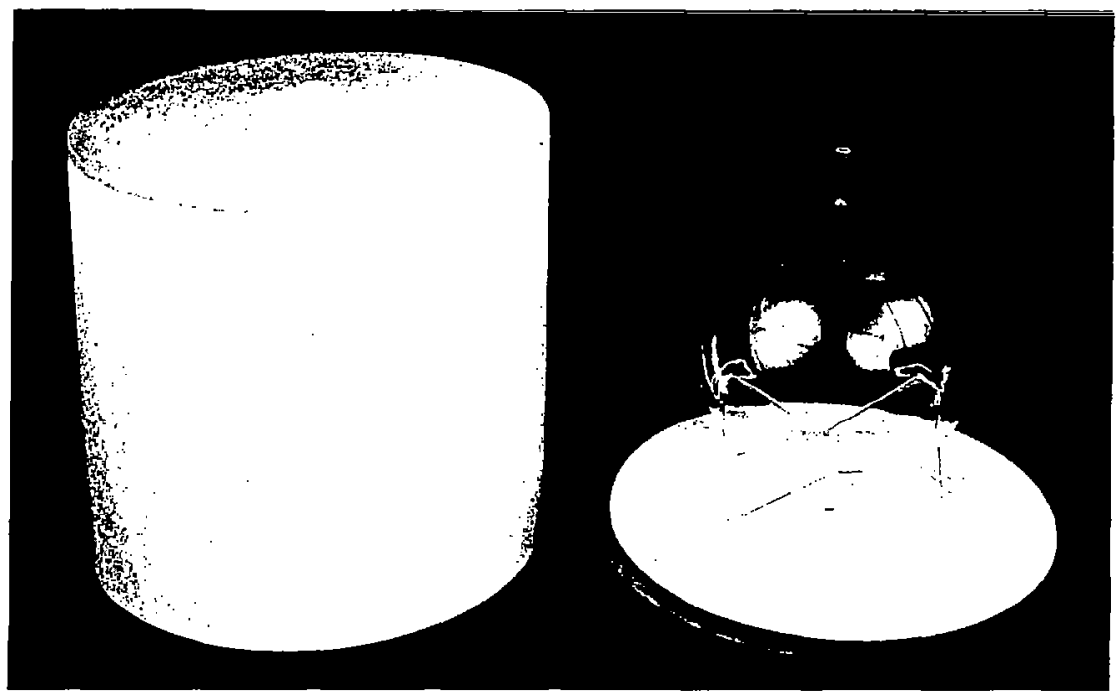

Figure 2. Cervit cavity and fused silica storage bulb used in SAO's VLG-11 series masers.

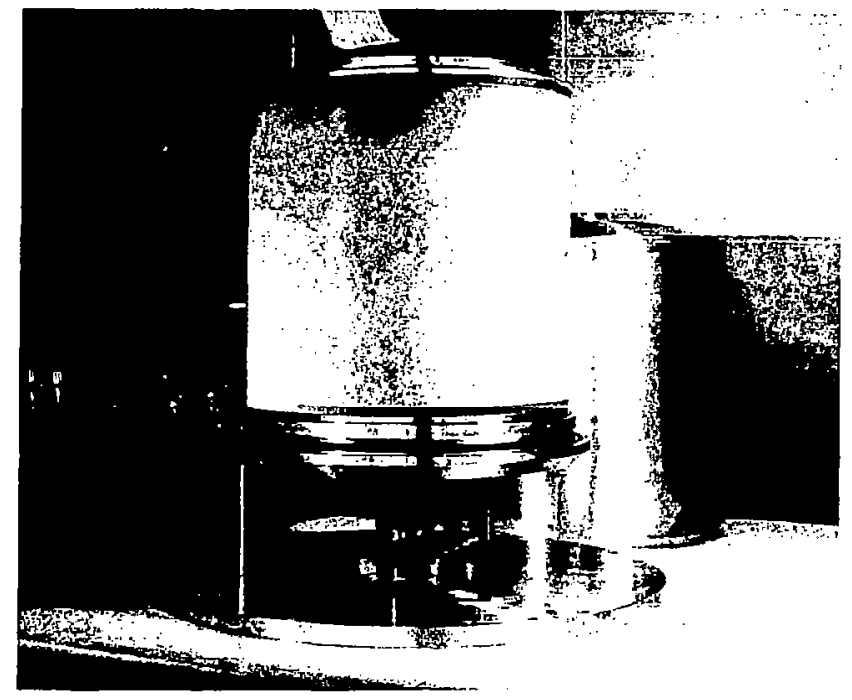

Figure 3. Assembly of Cervit cavity showing false bottom to isolate the cavity from barometric stress changes. Vacuum bell jar is shown in background. 


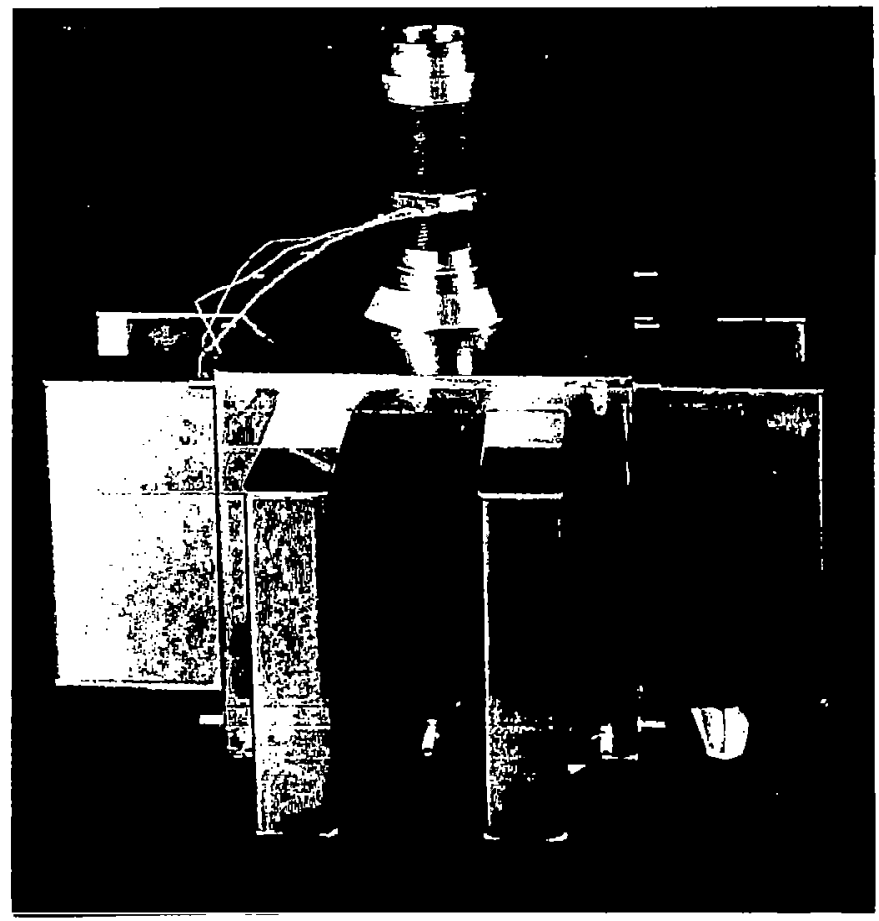

Figure 4. Eight-element Ultek ion pump shown without magnets. Titanium bellows vacuum connection to bell jar base is shown with heat station installed.

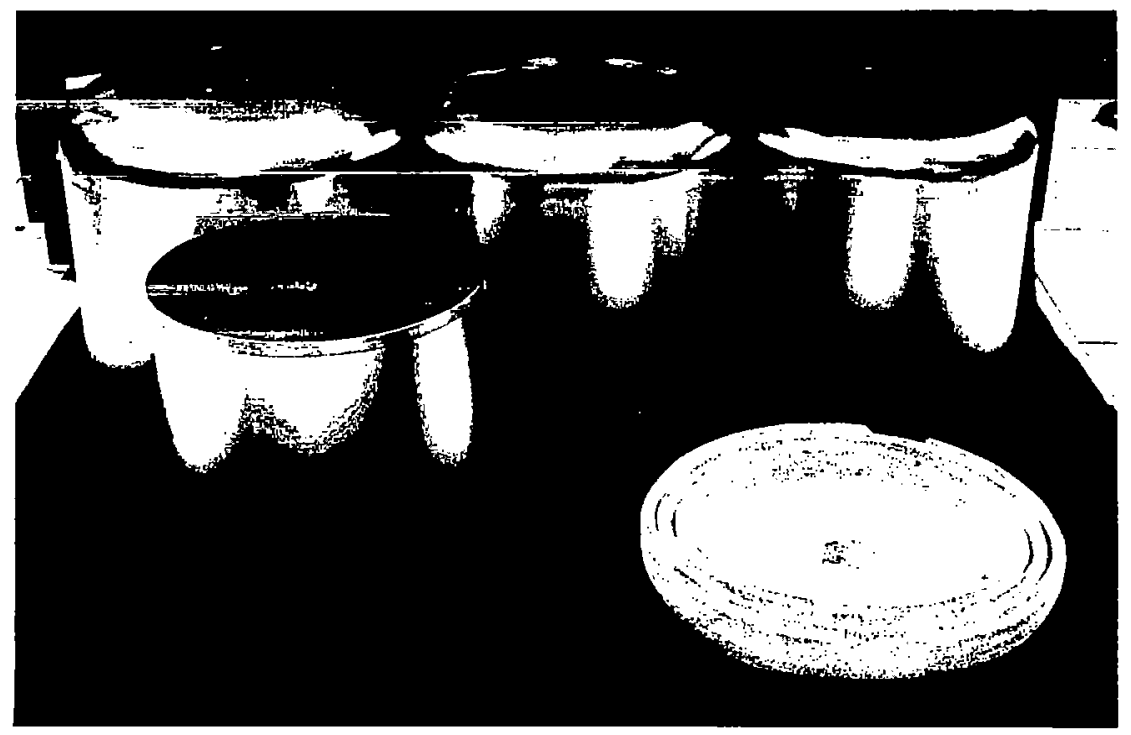

Figure 5. Four-layer magnetic shields showing torrispherical domes on outer shields and foam-glass separators for thermal isolation. 
local mechanical stress and the consequent loss of magnetic permeability. The innermost shield has flat ends and encloses a three-section printed circuit solenoid shown in figure 6. This is symmetrically wound so that the windings proceed forward on the inside and return on the outside and the go-return wires are overlaid as strip lines to minimize stray fields that could spoil the homogeneity of the magnetic field in the bulb.

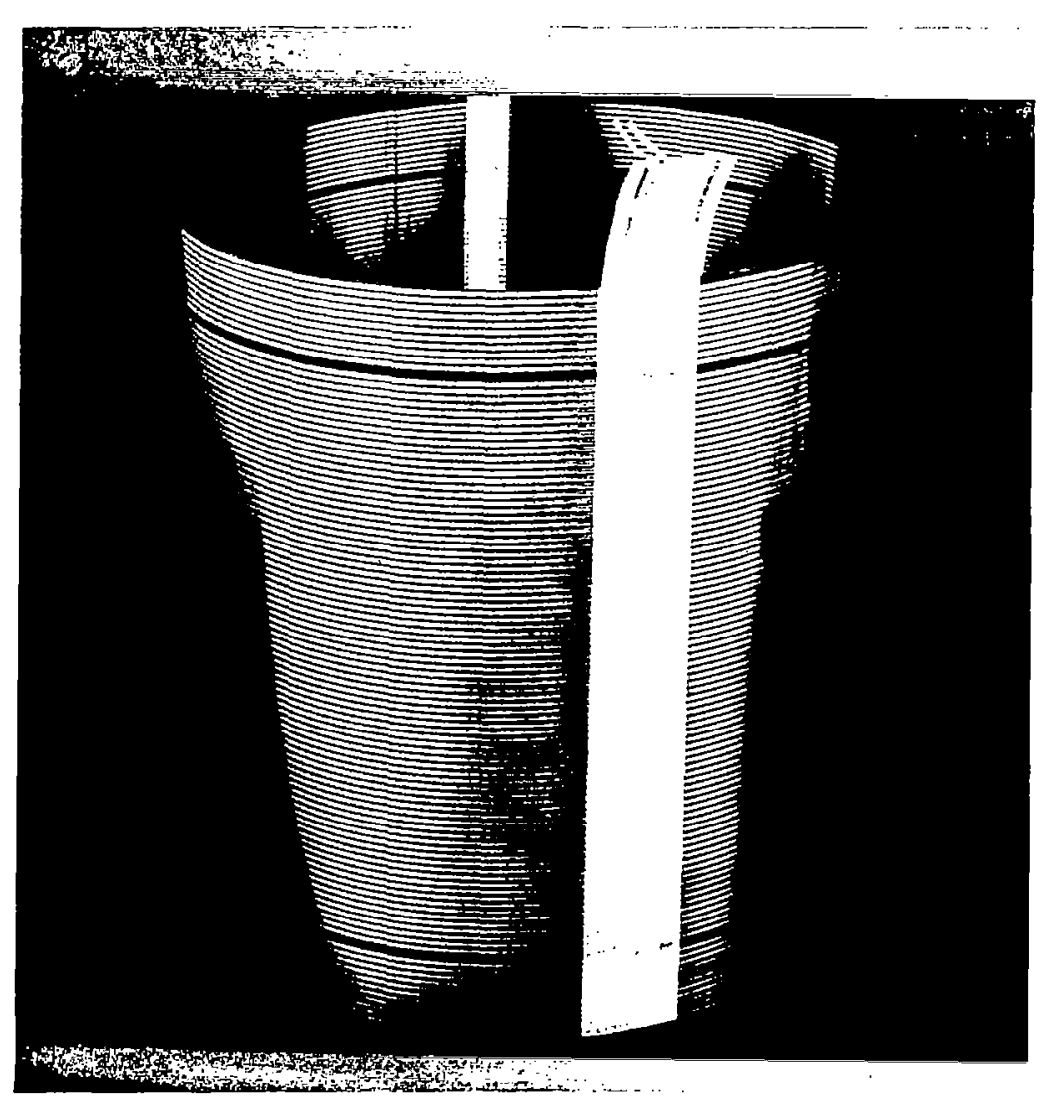

Figure 6. Two-sided printed circuit solenoid. Inner and outer windings in each of three sections form a complete solenoid with go-return wires connected by two-sided strip line to minimize stray magnetic fields.

The three separately-controlled solenoid sections are arranged so that axial second-order magnetic field variations can be compensated without adding spurious fourth order variations.

The completed maser assembly is shown in figure 7 with side panels and electronic box covers removed. The upper cylindrical structure is the magnetic shield surrounding the ion pump; beneath it can be seen the outermost magnetic shield for the cavity assembly. All panels swing out on hinges for servicing, and the electronic circuit boards for the maser control can be unplugged for testing and servicing. The aluminum frame is mounted on casters and the whole system weighs $300 \mathrm{~kg}$ and consumes 120 watts of dc power at 28 volts. 


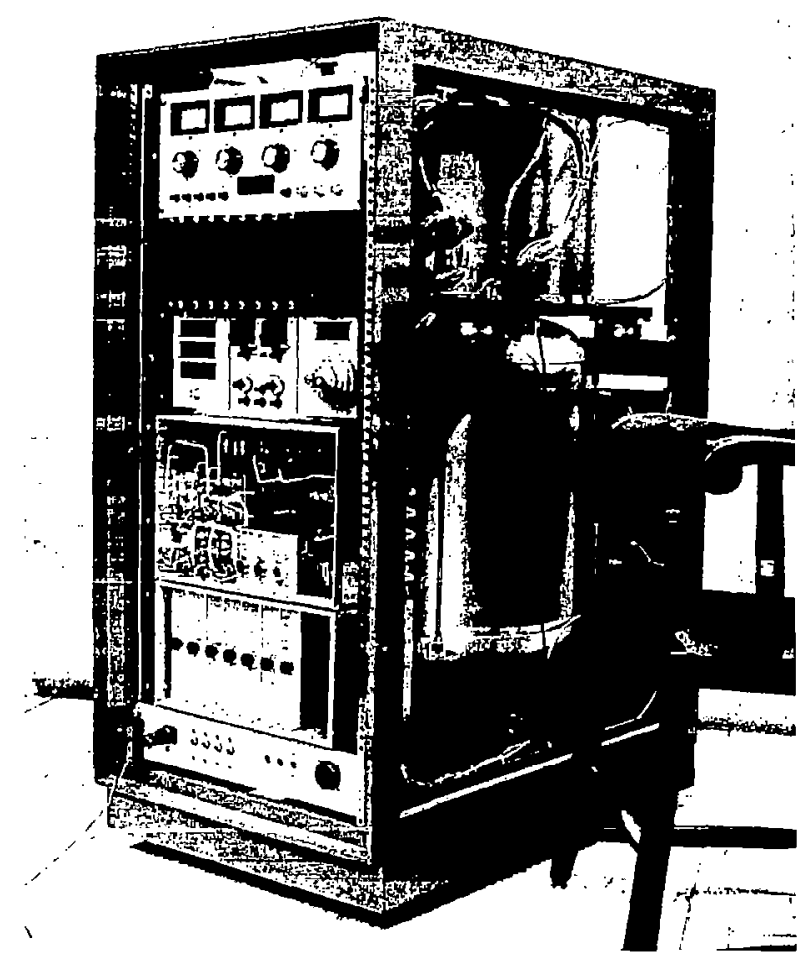

Figure 7. Assembled VLG-11 maser with access panels removed, control and monitor panels hinge out. The eight control modules can be unplugged for testing.

\section{Fundamental Limitations to Maser Stability}

The previous section discussed some aspects of engineering approaches to cope with the systematic frequency shifts in masers that are used in field applications. This section addresses the fundamental thermodynamic limitations of the maser's stability.

There are two reasonably separable sources of fundamental instability in the hydrogen maser. The first is the result of thermal noise, $\mathrm{kT}$, with frequency components lying within the atomic transition linewidth. The effect of this noise is given by the expression

$$
\frac{\Delta \mathrm{f}}{\mathrm{f}}(\tau)=\sigma_{\mathrm{f}}(\tau)=\frac{1}{\mathrm{Q}_{\ell}} \sqrt{\frac{\mathrm{kT}}{\mathrm{P}_{\mathrm{b}} \tau}} \ldots
$$


where $Q_{\ell}$ is the atomic line $Q, P_{b}$ is the power delivered by the atoms, and $T$ is the absolute temperature of the oscillator. The fractional instability $\Delta f / f=\sigma(\tau)$, which is the one-sigma probability of the difference between two adjacent measurements, each of duration $\tau$, is a function of the averaging time interval, $\tau$ (Allen, 1966). For this type of instability, we see that the expectation value of the $\sigma_{\mathrm{f}}$ vs. $\tau$ plot will show a $\tau^{-1 / 2}$ behavior. This expression is fundamental to all oscillators.

However, in the case of hydrogen masers where the signal output is rather weak, typically $10^{-12}$ watts or so, we must contend with another manifestation of thermal noise - that of the noise added to the signal at the input to the receiver system. Here, the instability is described by the expression

$$
\frac{\Delta \mathrm{f}}{\mathrm{f}}(\tau)=\sigma_{\mathrm{a}}(\tau)=\frac{1}{2 \pi \mathrm{f} \tau} \sqrt{\frac{\mathrm{FkTB}}{\mathrm{P}_{0}}} \ldots
$$

where $F$ is the noise figure of the receiver system, $B$ is effective noise bandwidth of the overall system, and $\mathrm{P}_{0}$ is the power delivered to the receiver. We note that a $\sigma$ vs. $\tau$ plot of this type of instability shows a $\tau^{-1}$ behavior. These two processes can be added as incoherent effects, and we obtain

$$
\sigma(\tau)=\left(\sigma_{\mathrm{a}}\right)^{2}+\left(\sigma_{\mathrm{f}}\right)^{1 / 2}=\left\{\frac{\mathrm{kT}}{2}\left[\frac{\mathrm{FB}}{2 \pi^{2} \mathrm{f}^{2} \mathrm{P}_{0} \tau^{2}}+\frac{1}{\left(\mathrm{Q}_{\ell}\right)^{2} \mathrm{P}_{\mathrm{b}} \tau}\right]\right\}^{1 / 2} .
$$

This behavior is illustrated in figure 8 , which shows the performance data from the VLG-11 series masers described earlier. The maser operating parameters are listed in the figure and provide data for predicting the stability which is shown in the dotted line. The data follows the prediction reasonably well up to $\tau \sim 3,600$ seconds, where $\sigma(\tau)=6 \times 10^{-16}$. For $\tau>3,600$ seconds, very slow systematic drifts between the masers, which were likely to have resulted from an air conditioner failure 10 days earlier, made the statistical representation inappropriate.

\section{A Cryogenically Cooled Hydrogen Maser}

From the previous data, it is clear that we are close to the limits imposed by thermodynamics. Increasing the power level to offset the noise by increasing the atomic hydrogen flux intensity can improve the short term stability but does so at the expense of the longer term behavior. The result of increasing the flux is to reduce the atomic line $\mathrm{Q}$ which results from increasing the density $\mathrm{n}$ of the atoms in the bulb and thus, raising the level of spin exchange relaxation (see equations 1 and 2).

In late 1977, experiments by D. Kleppner and co-workers at the Massachusetts Institute of Technology (MIT) showed that atomic hydrogen could be stored in gaseous form at temperatures as low as $4 \mathrm{~K}$ (Crampton et al., 1979). This led to the speculation that the frozen molecular hydrogen that coated the walls of the storage vessel and prevented recombination could also serve as a wall coating to preserve the phase of the oscillating hydrogen atom during collisions. Crampton and his colleagues showed this was not a good wall coating for hydrogen masers (Crampton et al., 1978). 


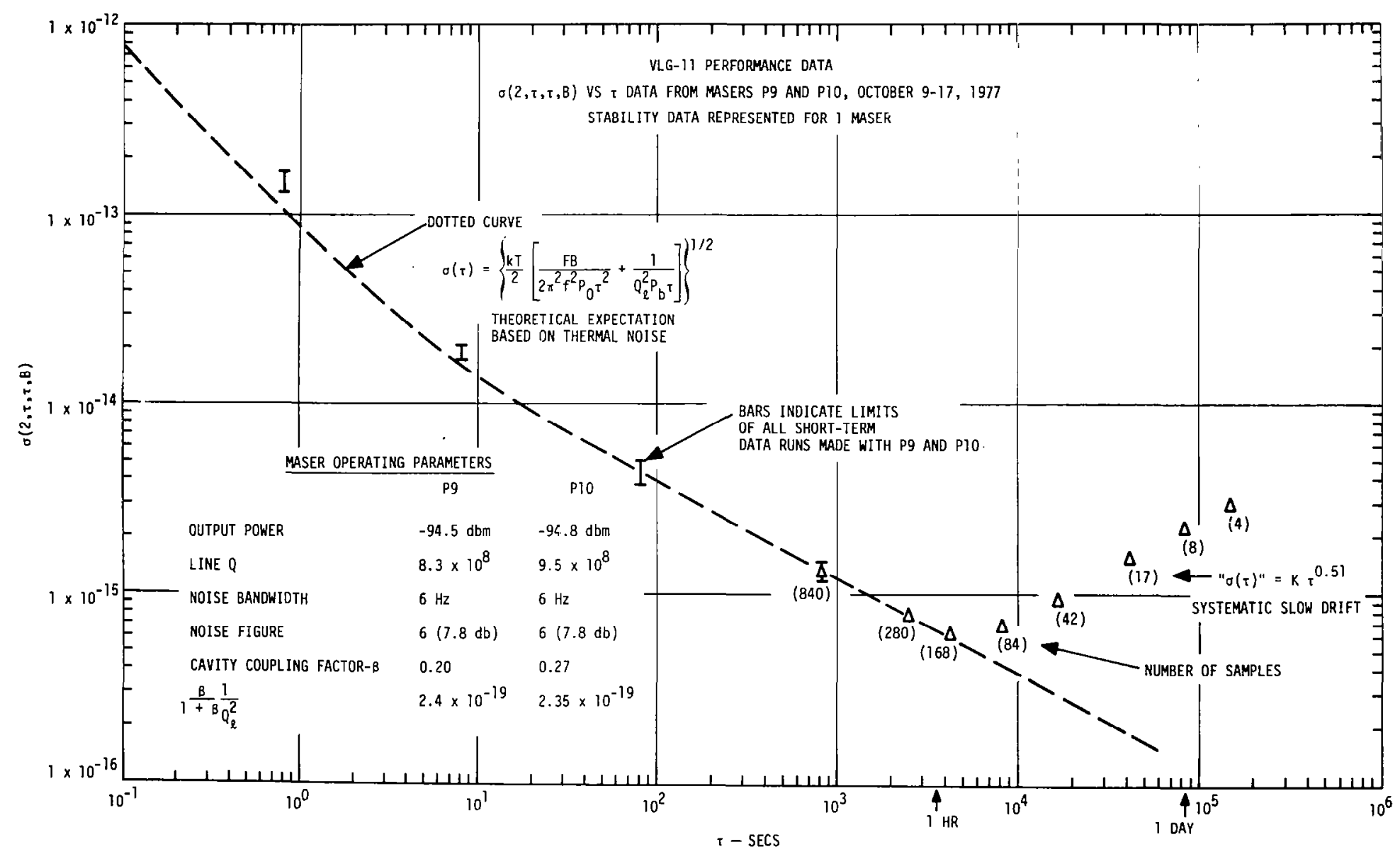

Figure 8. Allan variance plot of performance VLG-11 masers. Dotted lines shows theoretically expected performance. 
During a recent program at SAO to study low temperature operation of a maser, we succeeded in making a maser oscillate at $25 \mathrm{~K}$ by introducing a carbon tetrafluoride into the cavity and freezing it onto the surface (Vessot et al., 1979). This provides a wall coating that consists essentially of tightly bound fluorine atoms. This surface, to the impacting hydrogen atom (at least from the outside), appears quite similar to the Teflon surface normally used at room temperature. We believe that studies at low temperature will gain us a great deal of information about the hydrogen interaction process during collisions and that, from a better understanding of wall materials, we may be able to design a more suitable surface for use at room temperature.

The advantages to be realized from low temperature operation (Vessot et al., 1977), even at $25 \mathrm{~K}$, are obvious if we look at equation 2 , where the effects or thermal noise are considered. In addition, we should remember that the spin exchange cross-section for hydrogen-hydrogen collisions, $\sigma_{\text {se }}$, diminishes very drastically for temperatures below $100 \mathrm{~K}$ (Allison, 1972). At 25K, it is at about 40 percent of its room temperature value; at $10 \mathrm{~K}$, it is about 10 percent. This makes it possible to increase the power level substantially.

From our understanding of the behavior of room temperature masers, we can extrapolate the expected performance at lower temperatures only if we can control the wall relaxation probability, $\mathrm{p}$, in equation 2. Figure 9 shows the relationship of the frequency stability of the SAO VLG-series masers and the expected performance of the maser that operates at $25 \mathrm{~K}$. The projected stability for operation at $4 \mathrm{~K}$ is also shown; however, we still have to demonstrate its operation.

In the near future, we expect to make frequency comparisons between two cold masers, each in its own cryostat, using low noise electronics equipment housed in a separate cryostat to see how well we can realize the expected performance.

\section{Developments Toward Small Size Active and Passive Masers}

Until recently, the size of the hydrogen maser has been chiefly dictated by the cavity resonator, which for the $\mathrm{TE}_{110}$ mode tuned at $1.42 \mathrm{GHz}$ typically has inside dimensions $28 \mathrm{~cm}$ diameter and $28 \mathrm{~cm}$ length. Other cavity structures and modes have been studied (Mattison et al., 1975 and 1976) and tested, including dielectrically loaded structures and "magnetron" structures (Peters, 1978). While the impetus for smaller hydrogen masers mostly results from space applications, their use as portable devices can be foreseen.

The small size of the $\mathrm{TE}_{111}$ mode cavity made it possible to use a 7 " I.D. dewar for the cryogenic maser experiments mentioned earlier. The cavity is shown in figure 10. A comparison of the size, weight, and power of the maser designed for the 1976 redshift experiment and a projected spaceborne maser using the $T E_{111}$ mode cavity is shown in figure 11 . 


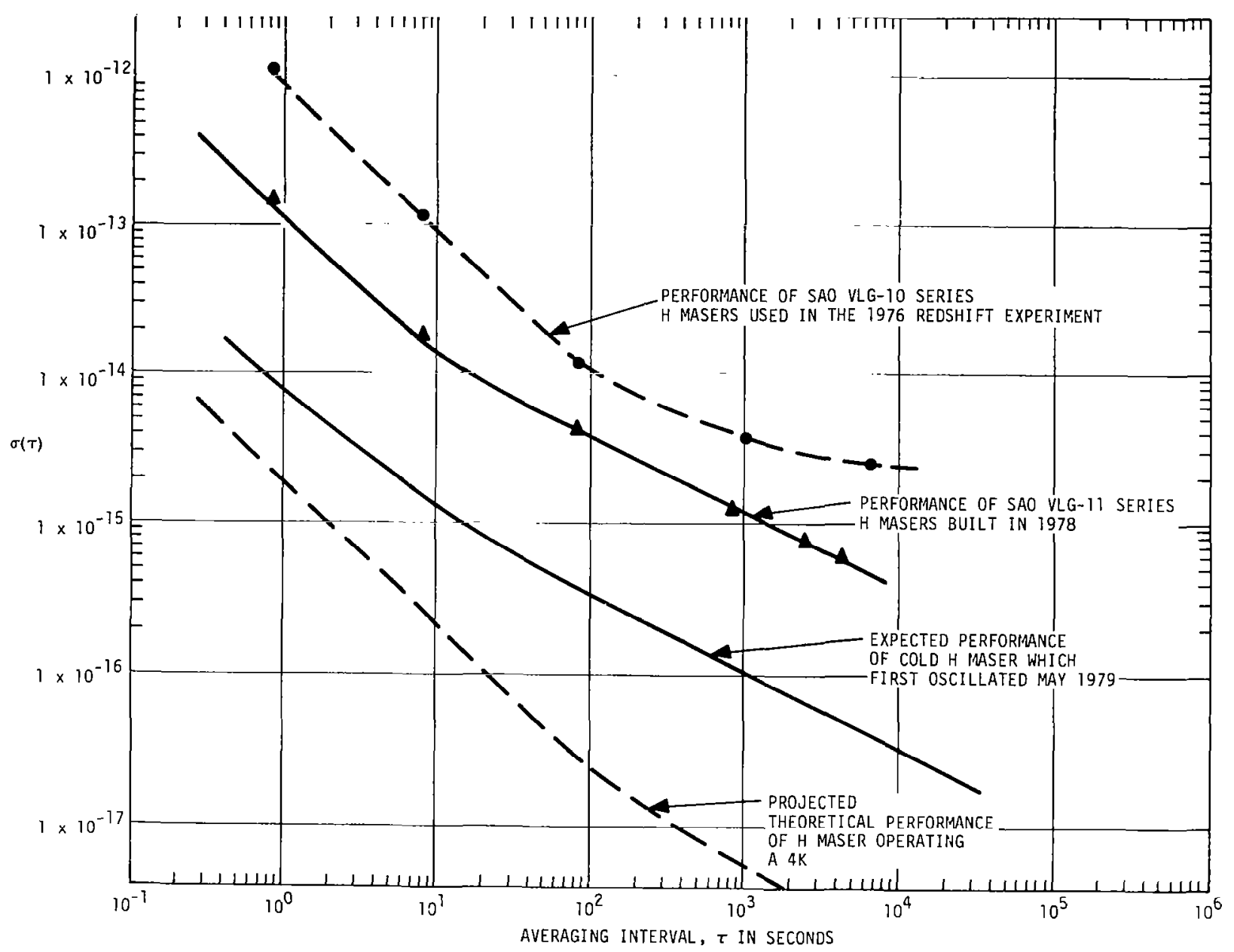

Figure 9. Projected performance of cold hydrogen masers. Lower solid line is predicted for operation at 30K. A maser was operated at 25K in May, 1979 [8] . 


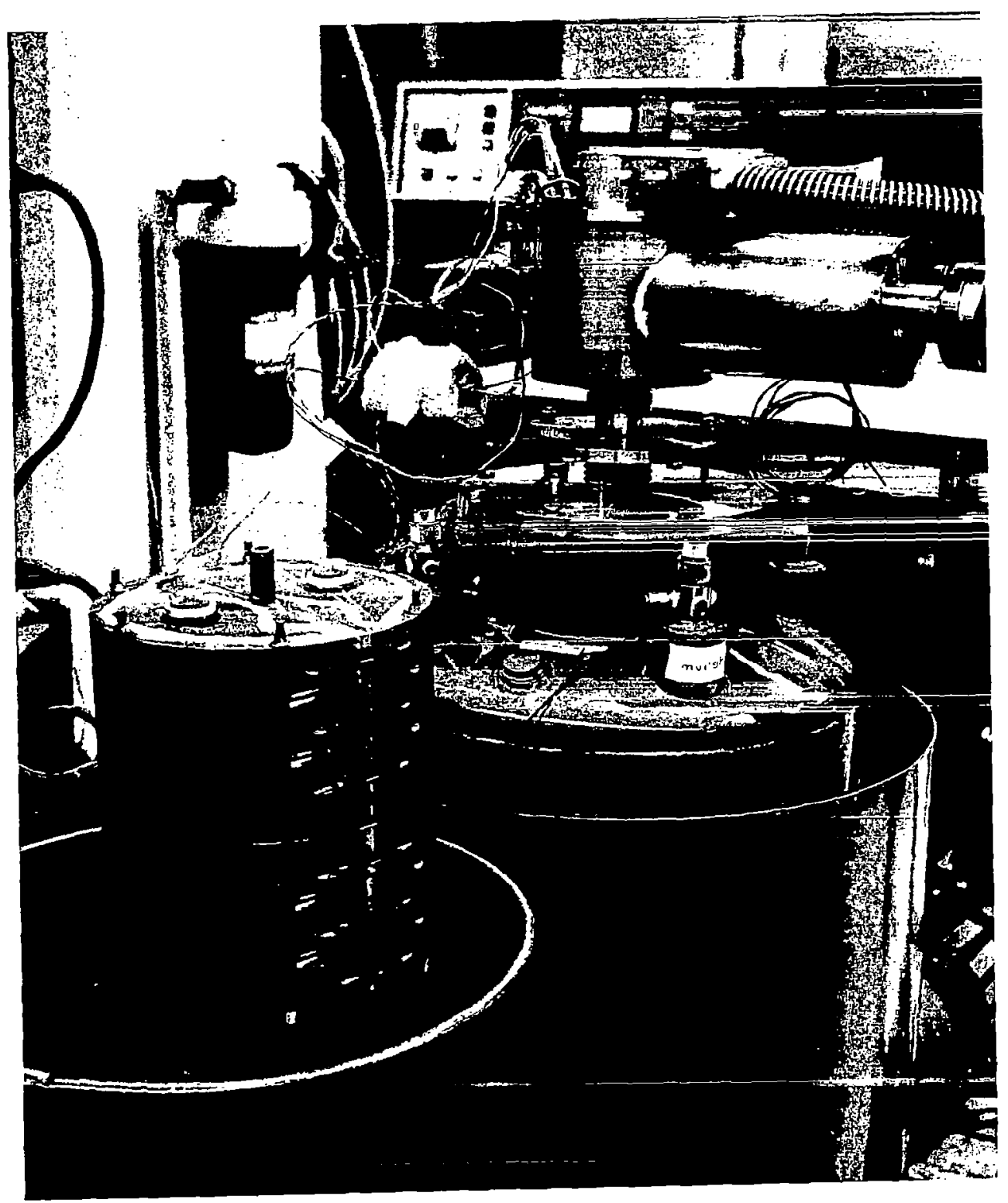

Figure 10. TE-111 mode cavity and cold hydrogen maser dewar system. Cavity is 7 " diameter. 


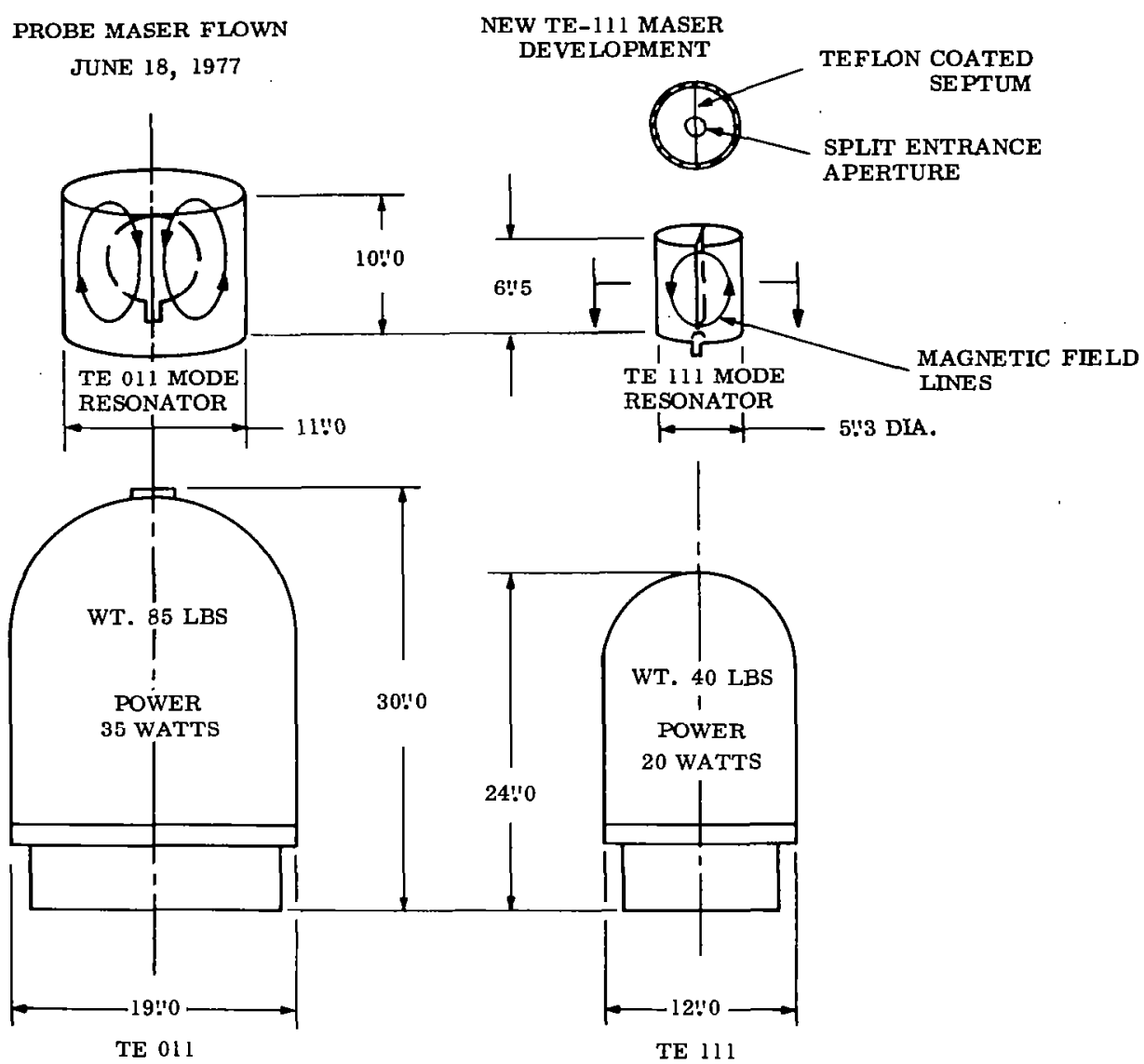

Figure 11. Comparison of size, weight and power of spaceborne masers using TE-111 cavity and mascrs used in 1976 spacecraft test of redshift [3] .

Thus far, we have discussed the behavior of oscillating masers as ultra stable frequency sources. When operated below threshold, the hydrogen maser, operating as an amplifier, can be used to control the frequency of a flywheel oscillator. Since the cavity resonator no longer must have a $\mathrm{Q}$ high enough to maintain oscillation, one can use compact resonators with lower Q. One of these resonators is shown in figure 12. This is an experimental development model (Mattison et al., 1979) leading to a design for space-borne use in the Global Positioning System. The cavity resonator is made of a very thick walled cylinder of sapphire crystal silvered on the outside. The dielectric boundary between the inner region and the outer conductor is chosen to be at the position that optimizes the filling factor of the inner region, which is Teflon coated and serves as the storage volume. Storage times of $0.140 \mathrm{sec}$ have been achieved.

Figure 13 shows the complete assembly, which comprises six zones of thermal control, five separate magnetic trimming coils, and a transverse "zeeman" coil (all-printed circuits), and four stages of magnetic shields. 


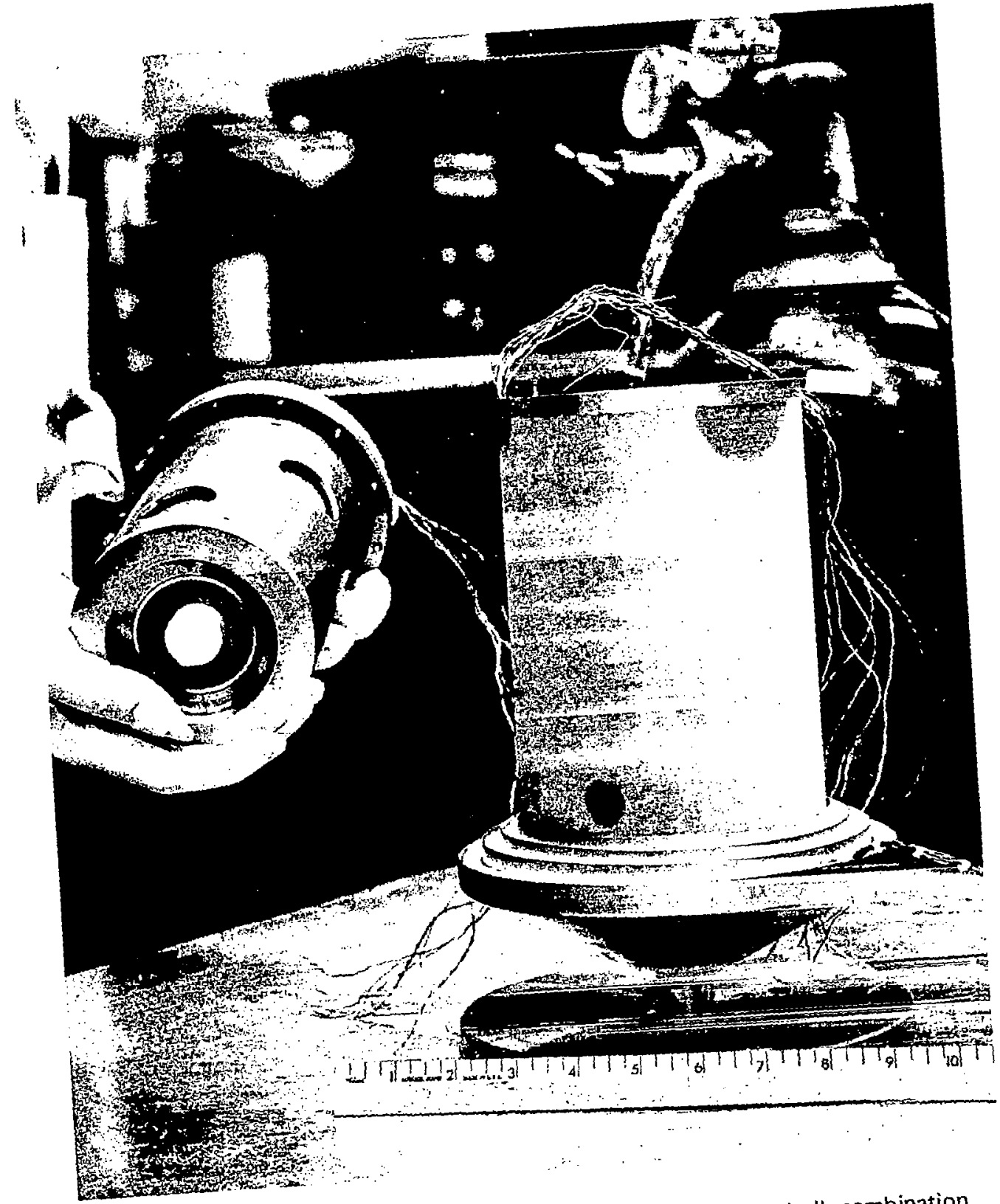

Figure 12. Resonator assembly using thick-walled sapphire cavity-bulb combination for passive hydrogen maser. Vacuum chamber
with four-layer magnetic shiclds and insulation. 


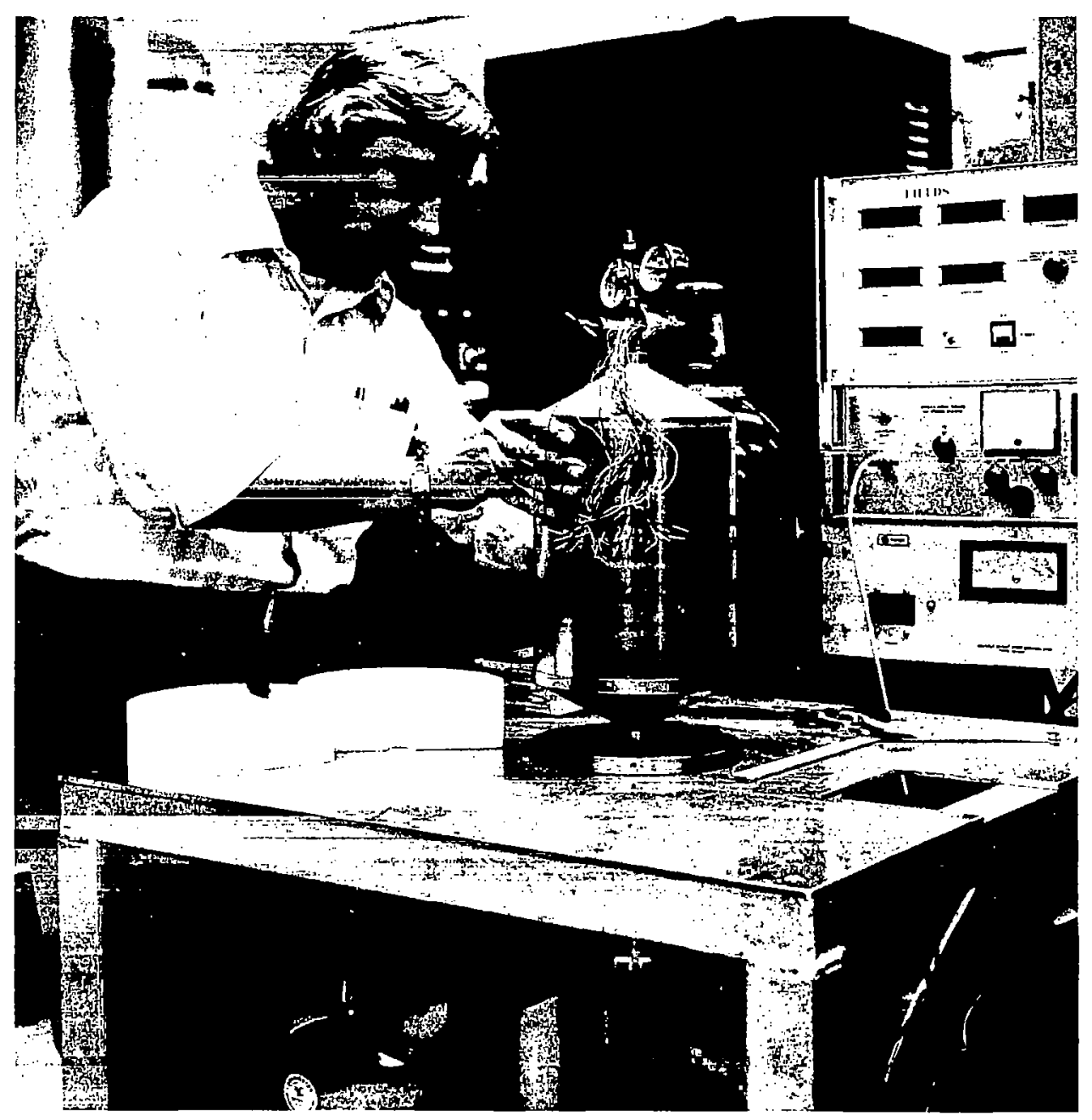

Figure 13. Passive maser cavity-bulb assembly on test stand showing magnetic shields with conical ends.

Even further reduction of size is possible with newly developed magnetron type of resonators which produce a large volume of in-phase rf field in which to store the atoms while occupying relatively little volume.

\section{Conclusion}

It has been 20 years since the maser was invented, and there has been fairly rapid growth in developments for making the maser a practical device. Stability data have steadily improved and now seem to be close to the limits imposed by the effects of thermal noise. Further work to understand the properties of the hydrogen atom as it collides with the storage surfaces is in progress and, in the 
writer's opinion, there is still considerable improvement obtainable in the performance of atomic hydrogen masers as frequency standards.

\section{REFERENCES}

Allan, D. W., Proc. IEEE 54, 221, 1966.

Allison, A. C., Phys. Rev. A. $\underline{5}$, No. 6, 2695, 1972.

Crampton, S. B., Phys. Rev. 132, 2154, 1963.

Crampton, S. B., Greytak, T. J., Kleppner, D., Phillips, W. D., Smith, D. A., and Weinrib, A., Phys. Rev. Lett. 42, No. 16, 1039, 1979.

Crampton, S. B., Phillips, W. D., and Kleppner, D., Bull. Am. Phys. Soc. 23, 86, 1978.

Kleppner, D., Berg, H. C., Crampton, S. B., Ramsey, N. F., Vessot, R. F. C., Peters, H. E., and Vanier, J., Phys. Rev. 138, No. 4A, 972, 1965.

Kleppner, D., Goldenberg, H. M., and Ramsey, N. F., Phys. Rev. 126, 603, 1962.

Mattison, E. M., Blomberg, E. L., Nystrom, G. U., and Vessot, R. F. C., "Design, Construction, and Testing of a Small Passive Hydrogen Maser." Proc. 33rd Annual Symposium on Frequency Control. U.S. Army Electronics Research and Development Command, Monmouth, New Jersey, 30 May - 1 June 1979 - Atlantic City, New Jersey, in press.

Mattison, E. M., Levine, M. W., and Vessot, R. F. C., Proc. 8th Annual Precise Time and Time Interval Planning Meeting, U.S. Naval Research Laboratory, 30 November - 2 December 1976, P. 355, 1976.

Mattison, E. M., Vessot, R. F. C., and Levine, M. W., Proc. 7th Annual Precise Time and Time Interval Planning Meeting. Goddard Space Flight Center, December 2-4, 1975, P. 243, 1976.

Peters, H. E., Proc. 32nd Annual Symposium on Frequency Control. U.S. Army Research and Development Command, Monmouth, New Jersey, 31 May - 2 June 1978 - Atlantic City, New Jersey, P. 469, 1978.

Vessot, R. F. C., Levine, M. W., and Mattison, E. M. Proc. 9th Annual Precise Time and Time Interval Planning Meeting. Goddard Space Flight Center, 29 November - 1 December 1977, P. 549, 1978.

Vessot, R. F. C. and Levine, M. W., General Relativity and Gravitation, 10, No. 3, 181, 1979. 
Vessot, R. F. C., Mattison, E. M., and Blomberg, E. L., "Research with a Cold Hydrogen Maser," Proc. 33rd Annual Symposium on Frequency Control. U.S. Army Electronics Research and Development Command, Monmouth, New Jersey. 30 May - 1 June 1979 - Atlantic City, New Jersey, in press. 



\title{
THE PERFORMANCE OF NASA RESEARCH HYDROGEN MASERS
}

\author{
Victor S. Reinhardt \\ Goddard Space Flight Center \\ Lauren J. Rueger \\ The Johns Hopkins University \\ Applied Physics Laboratory
}

\begin{abstract}
The Johns Hopkins University (JHU), Applied Physics Laboratory (APL), under contract to NASA/ Goddard Space Flight Center, is engineering a new generation of field operable hydrogen masers (NR) based on prior NASA NP and NX designs. These units incorporate improvements in magnetic shielding, lower noise electronics, better thermal control and have a microprocessor for operation, monitoring and diagnostic functions. They are ruggedly built for transportability and ease of service anywhere in the world.
\end{abstract}




\section{INTRODUCTION}

NASA has planned experiments in the coming years that will require reference frequency sources of better than $10^{-14} \Delta \mathrm{f} / \mathrm{f}$ for 1000 second averaging times to be available at remote sites throughout the world. The NR masers are being designed to meet this need and to provide monitoring functions to assure that the reference frequency is valid during experiments. This paper is a status or progress report on the development program.

\section{NR Design Improvements}

The NR masers have major design improvements as compared with past designs. A major breakthrough in terms of field serviceability is field interchangeable vacuum pumps. Hydrogen consumption in the NR masers has been reduced to allow the maser's Vacion pumps to be connected to the maser through a series of $1 \frac{1 / 2}{\text { inch }}(3.81 \mathrm{~cm})$ O.D. vacuum line valves. This enables the vacuum pumps to be replaced in the field without letting the maser up to air. Dry runs have shown that a pump can be replaced and the maser put back in operation in about 2 hours. Only a small fore pump to pump out the replacement Vacion pump is needed to do this.

The NR design has improved magnetic shielding. There are four concentric shells of molypermalloy $0.050^{\prime \prime}(0.13 \mathrm{~cm})$ thick shields spaced about $3 / 4$ inch $(1.9 \mathrm{~cm})$ apart. The Vacion pumps have individual molypermalloy shields, and the source assembly is shielded with the same weight stock.

The thermal time constant of the resonant cavity has been increased about tenfold by mounting it on delrin plastic standoffs and reducing the size of the escape openings of the radiation shields.

The frame and all constant temperature surfaces have been made of heavy gauge aluminum to reduce thermal gradients. The power supplies have been isolated and remotely placed in the rear of the cabinet to minimize local hot spots.

One of the shipping problems with the NP and NX masers was the reluctance of air carriers to take a device with a high-pressure hydrogen gas bottle on board. The NR has eliminated this problem by a low pressure electrolysis system for its hydrogen supply.

The JHU/APL experience in low noise electronics was used to provide new multipliers, I.F. amplifiers, and mixers for the NR design.

The magnetic field currents in the main solenoid and neck coil were put under a part in a million regulations and are now independent of any primary supply voltage variation.

A number of circuits in the NP and NX designs that are in the VCO control loop were not in temperature-controlled compartments. The NR design has placed the following circuits in a new temperature-controlled compartment, the $\mathrm{VCO}$, the autotune reference oscillator, the autotune 
reference oscillator, the autotune multipliers, the $5.7 \mathrm{kHz}$ synthesizer, the $5 \mathrm{MHz}$ isolation buffers, the magnetic current regulator, and the cavity control 18 bit D/A converter.

The $5.7 \mathrm{kHz}$ synthesizer is based on a very reliable design qualified for space service on another APL program. It is a three-stage cascaded divide and mix system that provides a frequency resolution of $10^{-17}$ at the maser frequency.

Recent maser application experiences have indicated the requirement for good buffer isolation. A new buffer design has been provided that realizes $120 \mathrm{~dB}$ of output to input isolation.

A microprocessor, the Intersil IM 6100, provides in software many functions formerly done by discrete logic. A clock system provides two separate one pulse per second outputs that are each adjustable in epoch. An autotuner function automatically tunes the maser from either an internal crystal reference or an external reference oscillator. Sensors are provided to measure voltages, currents, and temperatures, as well as switch positions; these are converted to digital form for recording purposes. The microprocessor can also in terface on any of three RS/232-C communications interfaces.

There has been a high degree of parts and subsystem interchangeability provided. It takes only a few minutes to change any major electronic package.

\section{Maser Block Diagram}

In figure 1 is shown a block diagram of the NR maser. The maser has its own thermal servos, the inner cavity is a thermally tuned aluminum cylinder that is held about $2^{\circ} \mathrm{C}$ above the outer oven temperature. The receiver is in a separately thermal-controlled box adjacent to the top of the maser cavity. It includes $5 \mathrm{MHz}$ to $0.4 \mathrm{MHz} ; 20 \mathrm{MHz}$; and $1400 \mathrm{MHz}$ frequency synthesizers and the 5.7 $\mathrm{kHz}$ phase detector. The new temperature controlled box contains the VCO, buffers, reference oscillator, autotuner multipliers and mixer, the $18 \mathrm{bit} \mathrm{D} / \mathrm{A}$ converter to control the cavity, and the magnetic regulator with controls. The microprocessor is not temperature controlled. It contains the clock, the keyboard, the display of five lines of 20 characters each, the alarm, and the external interface circuits for teletype and recorder. The vacuum electronics, gas pressure controls, and power system are not shown on the diagram.

\section{Hardware Detailed Design}

In figure 2 is shown the basic design of NASA masers. Hydrogen is introduced into the source bulb where it is dissociated and leaves through the collimator. A variable field quadrupole electromagnet selects the atoms of the appropriate energy, and a mechanical beam shutter is used to modulate the beam flux. The quartz storage bulb is elongated in this design and the inner surface coated with Teflon. The resonant cavity has movable end plates for coarse frequency adjustment and is thermally controlled for fine frequency adjustment. The cavity is magnetically shielded and multistage temperature controlled. 


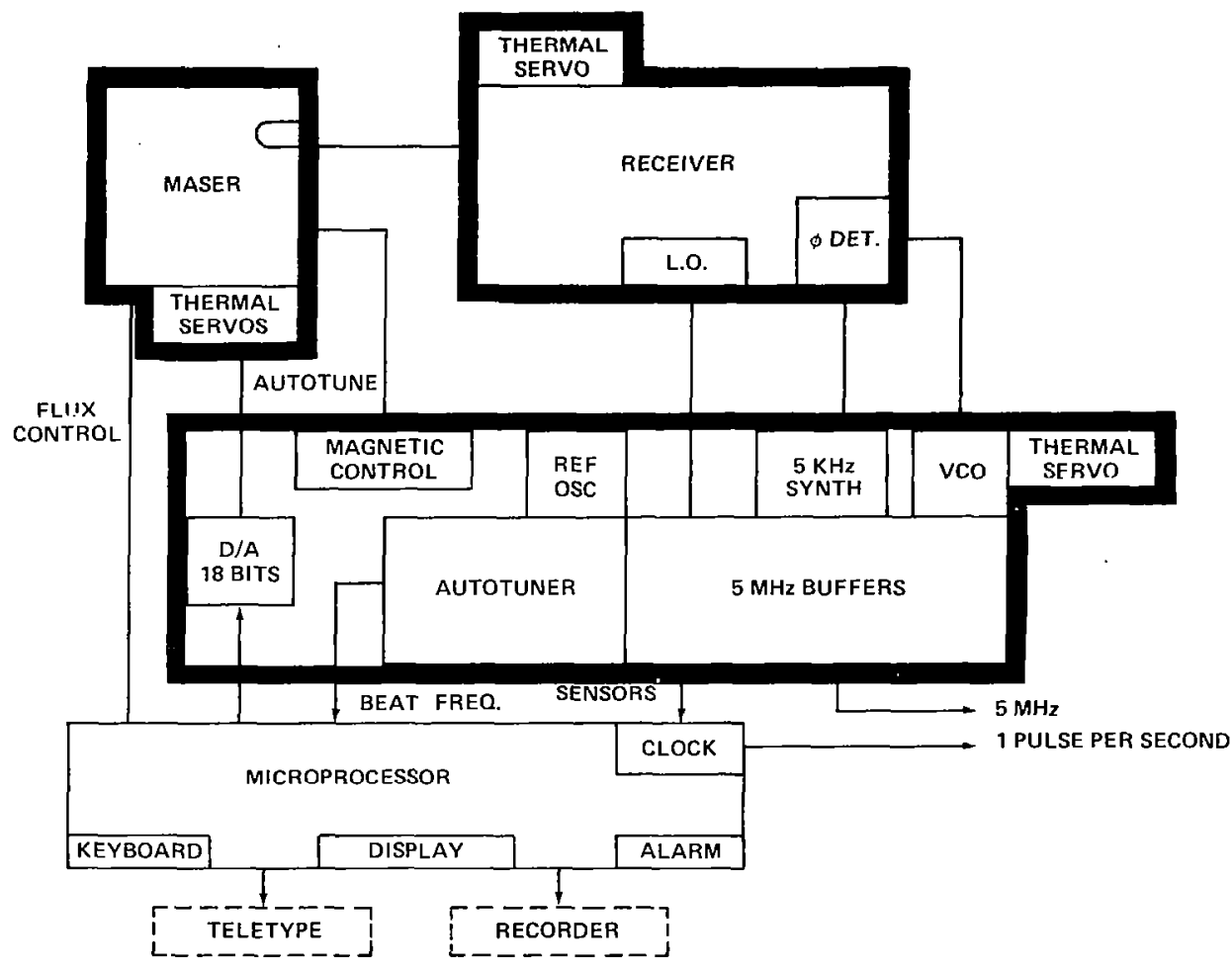

Figure 1. NR maser block diagram.

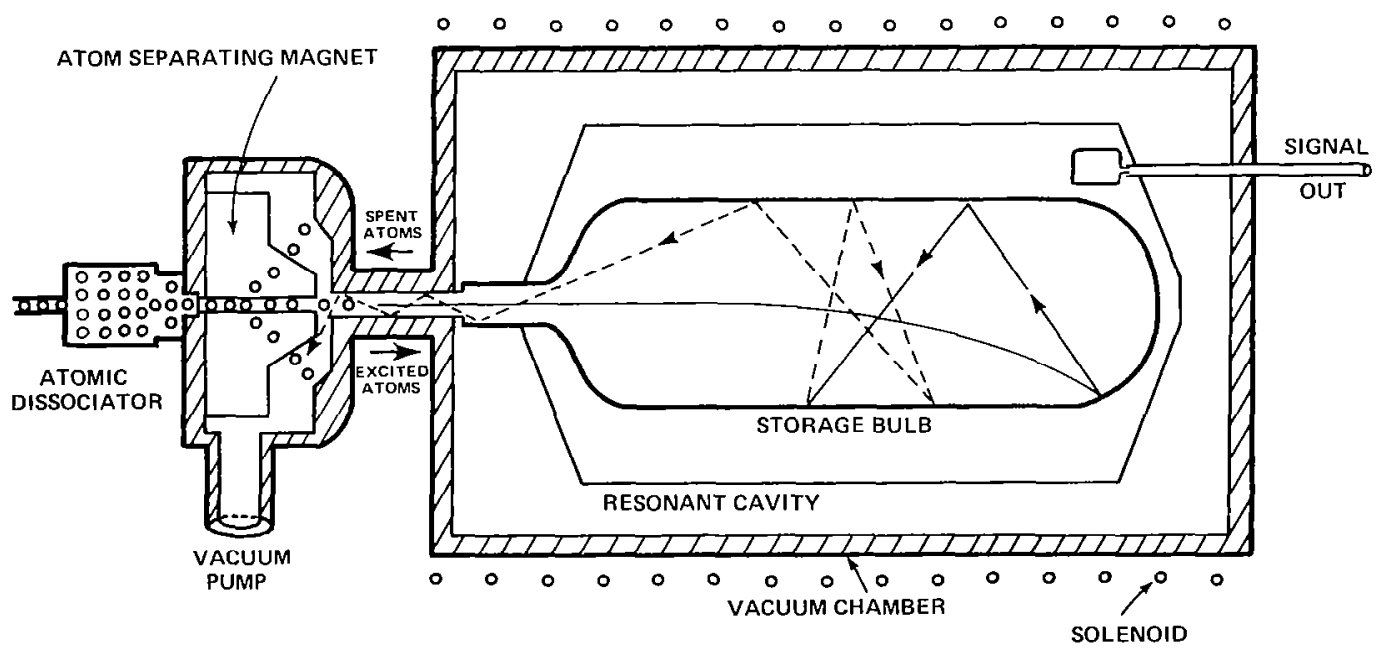

Figure 2. Principal elements of the NASA hydrogen maser. 
In figure 3 is shown the Vacion pumping system of the maser. Notice there are two Vacion pumps. One pumps the storage bulb and source region, and the other pumps the bell jar and microwave cavity region. The dual pumping system is used to reduce contamination of the storage bulb from materials outgassed by the bell jar or cavity. The system is designed so all O-rings exposed to the ambient air pumped by the bell jar system except for one O-ring which seals the source dissociator bulb to the source region.

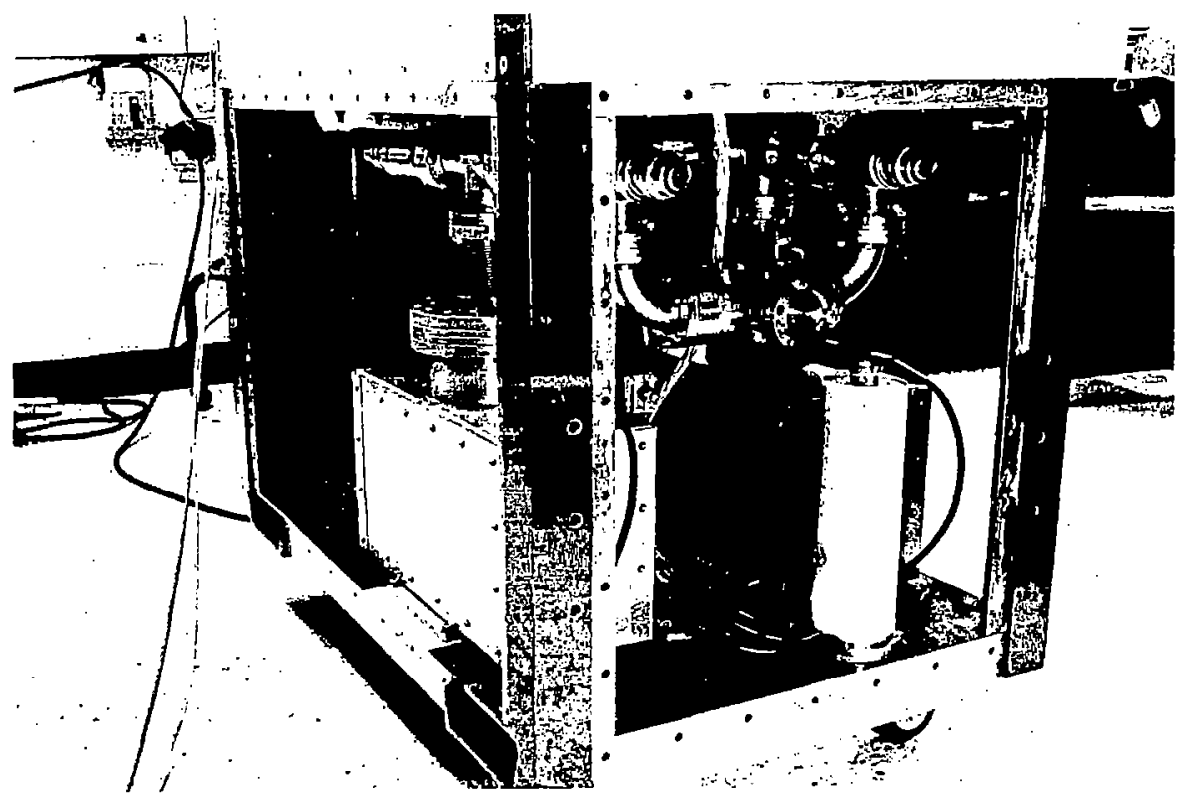

Figure 3. NR maser pumping system.

In figure 4 is shown the new hydrogen generators. Application of 1.5 volts at 2 milliamperes provides hydrogen by electrolysis of a water solution of potassium hydroxide. The negative electrode has a palladium film that provides spectroscopically pure hydrogen flow at room temperature.

In figure 5 is shown the source assembly; the electromagnets, the mechanical shutter, the two-stage vacuum system, one pumping on the vacuum seals, the other pumping on the spent hydrogen. Fiber optics are provided to observe the color and intensity of the discharge in the source bulb. 


\section{RADIO INTERFEROMETRY}

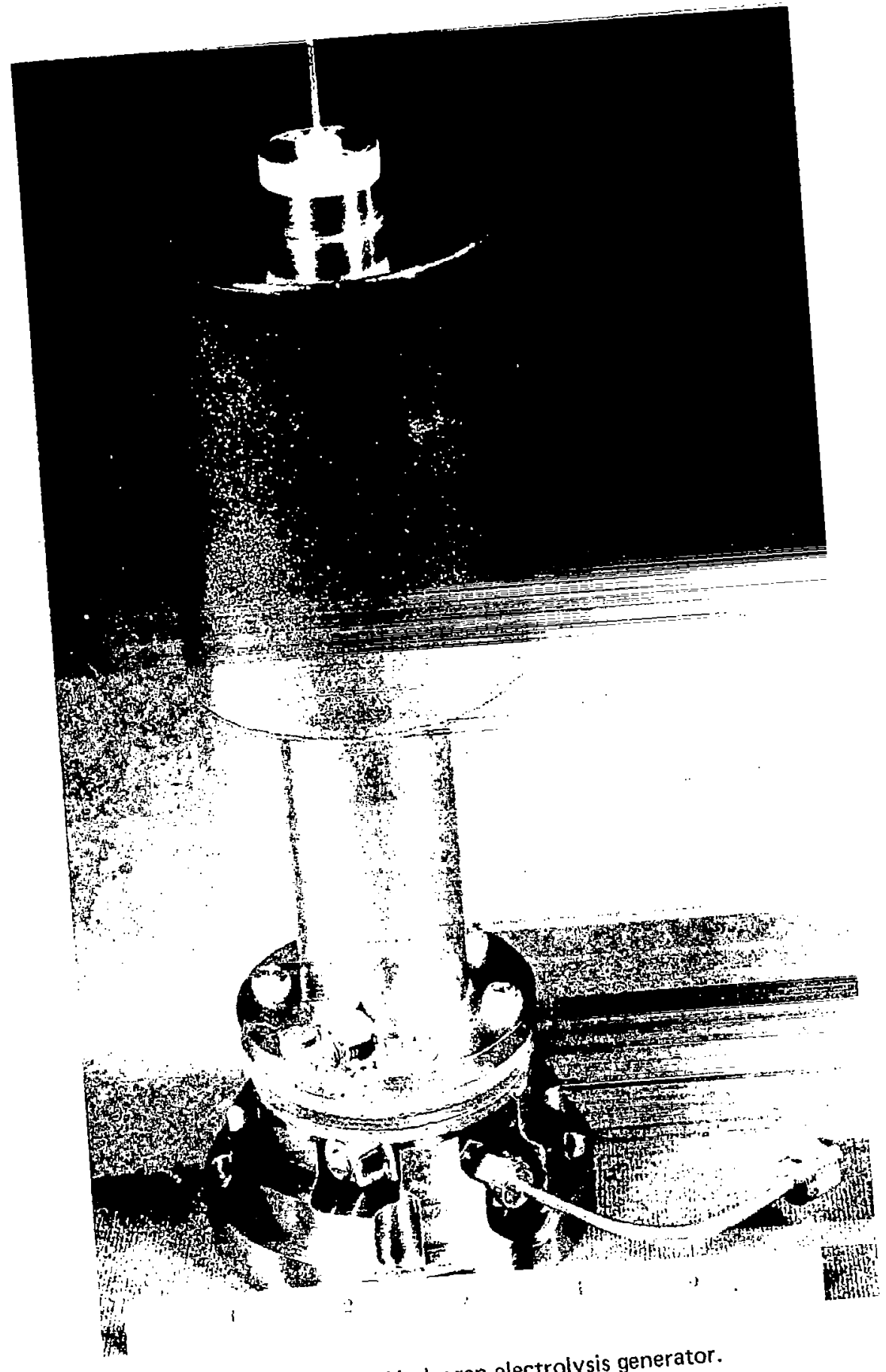

Figure 4. Hydrogen electrolysis generator. 


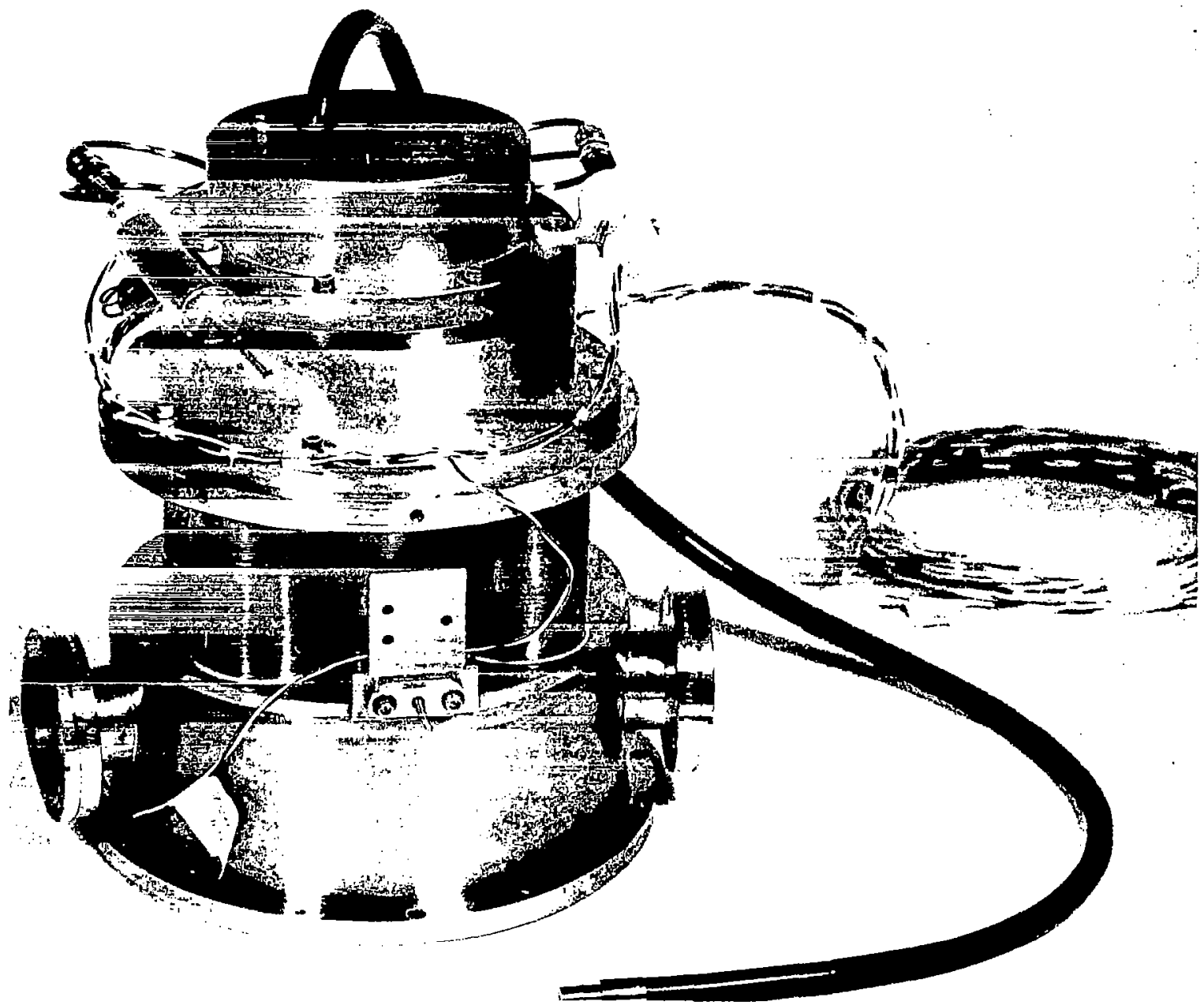

Figure 5. Source assembly.

In figure 6 are shown the various cylinders that make up the physics package. Starting on the left is the primary resonant cavity with heater, Zeeman coil, and thermistors attached by a ceramic cement; two aluminum radiation shields; the vacuum chamber with its heater and thermistor sensors; the solenoid with end trim coils; a magnetic shield, a thermal insulator, a magnetic shield, a thermal insulation; a magnetic shield; and finally, a heavy aluminum constant temperature cylinder.

In figure 7 is shown the base on which these cylinders fit. The lower movable end plate of the resonant cavity can be seen, and the quartz storage bulb is in place.

In figure 8 is shown the front view of the assembled NR maser. All controls except the microprocessor keyboard are enclosed behind doors. The meters indicate vital functions in the maser. 


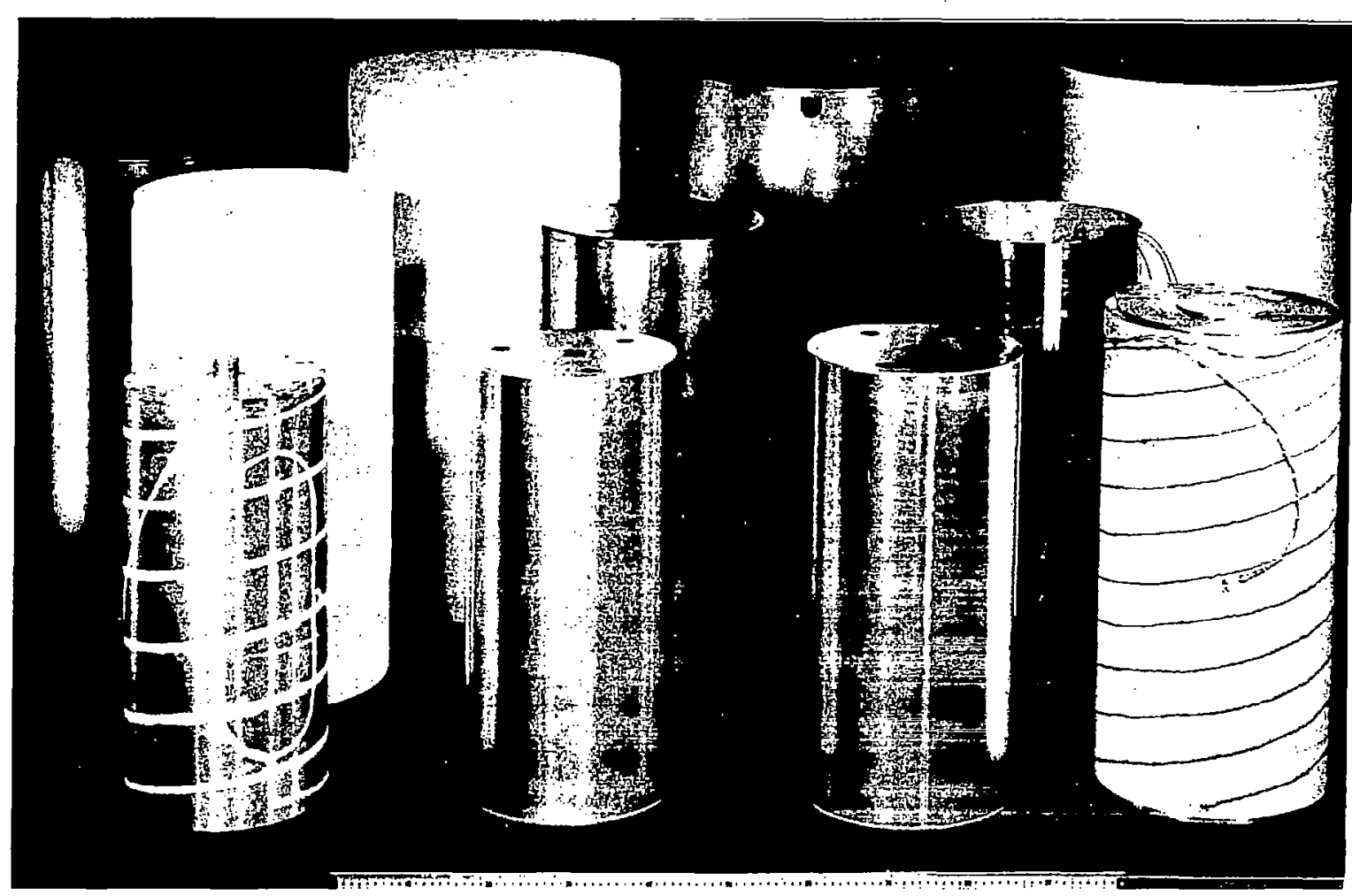

Figure 6. Physics package cylinders.

The lower right meter indicates the I.F. signal level at $5.7 \mathrm{kHz}$. The lower left meter indicates VCO phase lock. The red jewel at the bottom is a fiber optics indication of the source color and intensity.

In figure 9 is shown the maser opened up to indicate the accessibility of the maser for servicing. On the bottom are fork-lift holes for handling the maser on a loading dock. At the rear are stored four 12 -volt batteries for standby power. The vacuum system is made of standard Varian $1 \frac{1}{2}$ inch $(3.81$ $\mathrm{cm}$ ) O.D. vacuum line assemblies. The rear of the microprocessor is visible; the third thermal control box is shown extended on its rails. On the front are the magnetic field controls; on the bottom are the manual synthesizer controls (the synthesizer can also be under microprocessor control). The caster assemblies are for rolling the maser around on the level and are detachable leaving a package about $22^{\prime \prime}(55.8 \mathrm{~cm})$ wide, $38^{\prime \prime}(96.5 \mathrm{~cm})$ deep and $62^{\prime \prime}(156.5 \mathrm{~cm})$ tall. The rear door carries all of the switching regulator supplies: 120 volts $\mathrm{AC}$ to 28 volts $\mathrm{DC} ; 28 \pm 5$ volts $\mathrm{DC}$ to 28 volts DC; 28 volts DC to \pm 18 volts; and 28 volts $\mathrm{DC}$ to +5 volts.

In figure 10 is shown further opening of the microprocessor modules and a panel removed from the third thermal servo box. The Oscilloquartz model B5400 oscillators are visible along the edge, the current regulator circuits are on the hinged lid, the synthesizer boards are visible. 


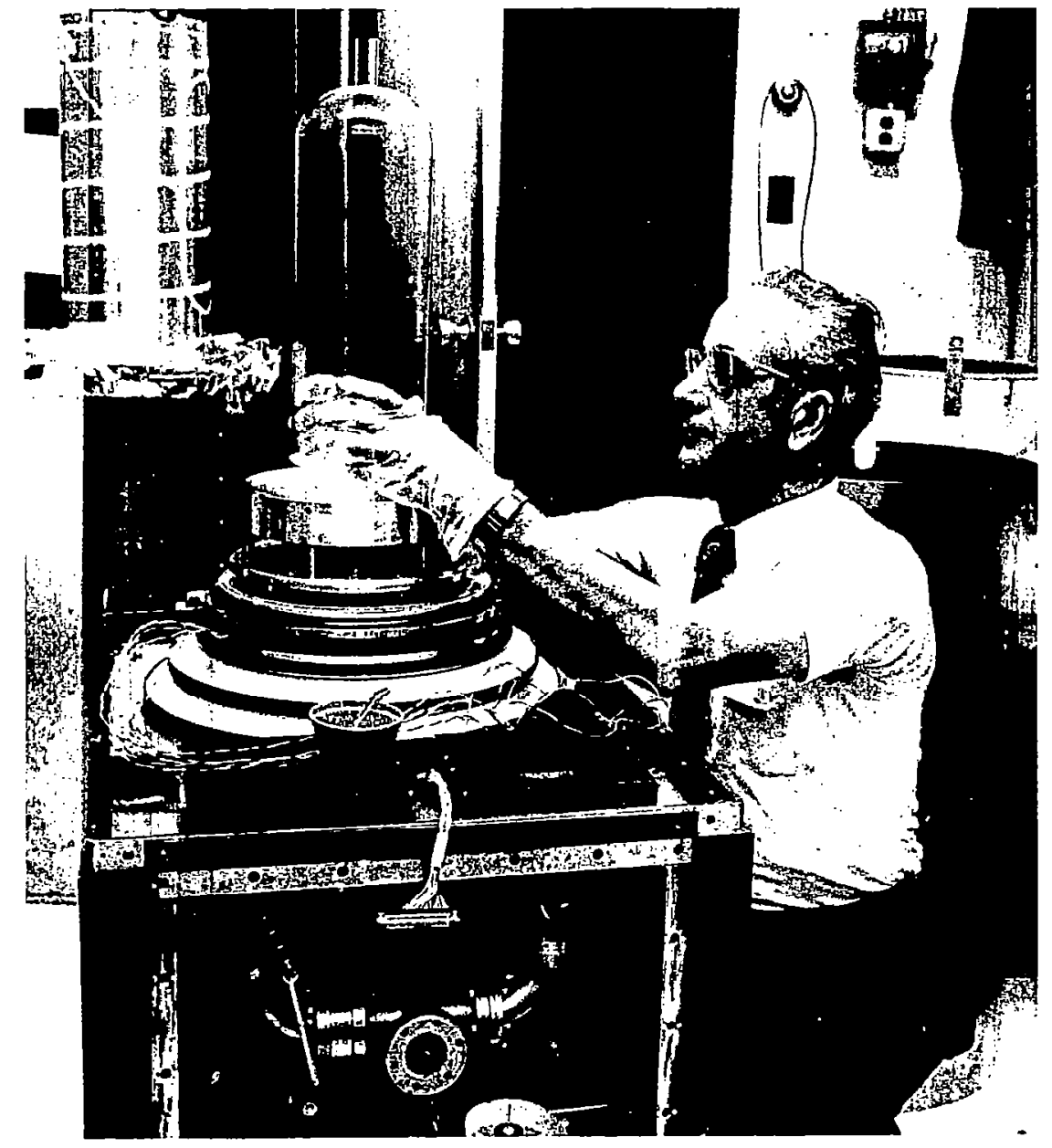

Figure 7. Physics package base with storage bulb.

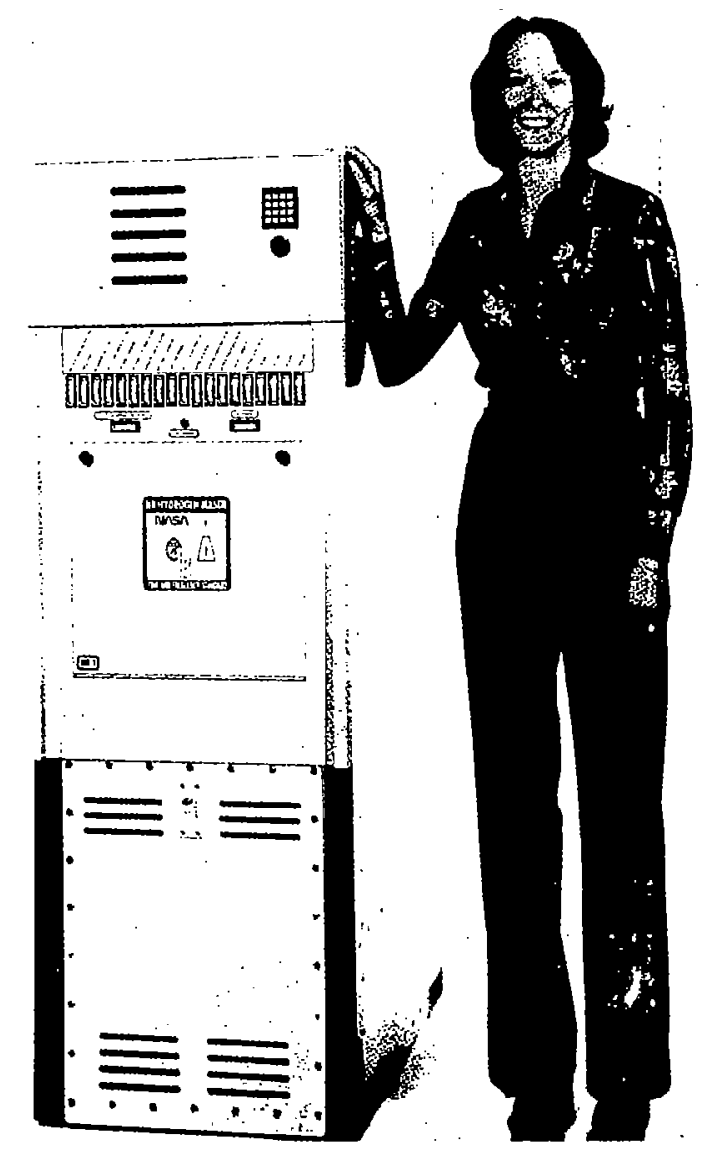

Figure 8. Front view of NR maser. 


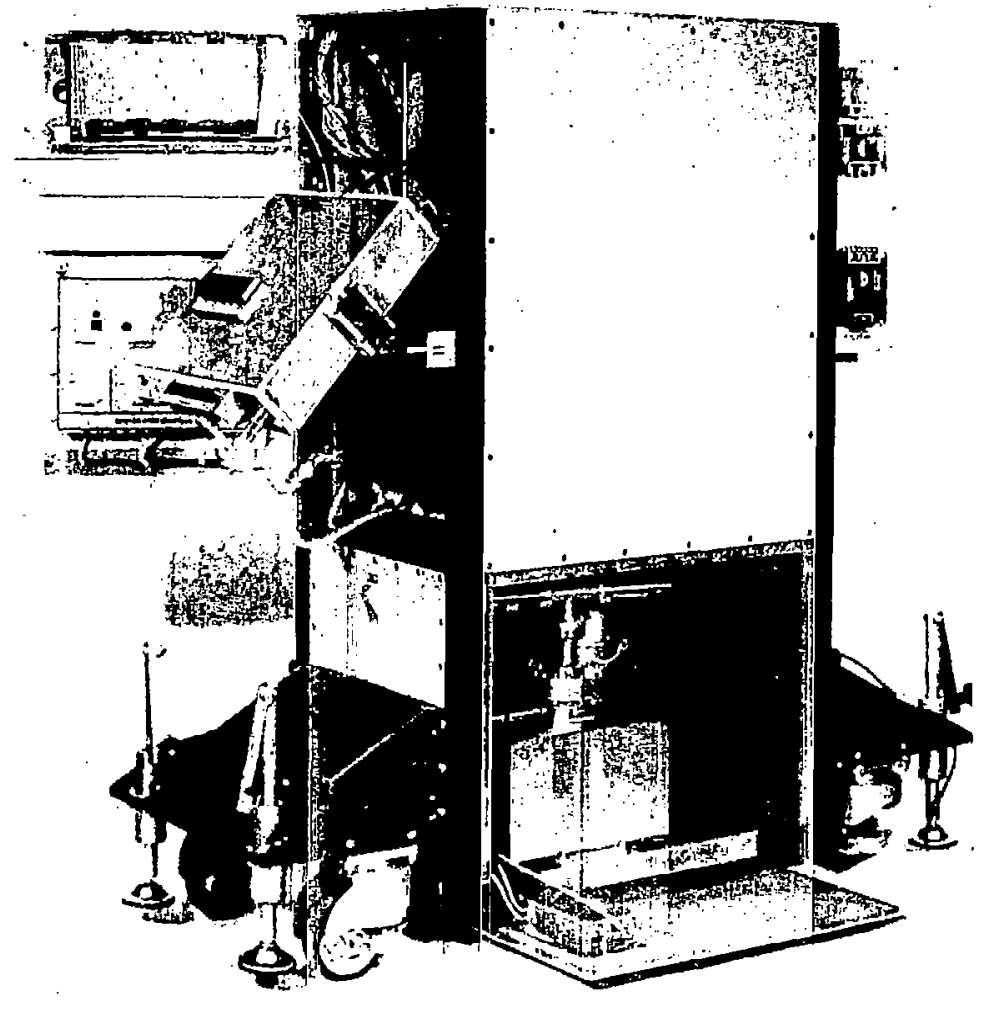

Figure 9. NR maser opened for servicing.

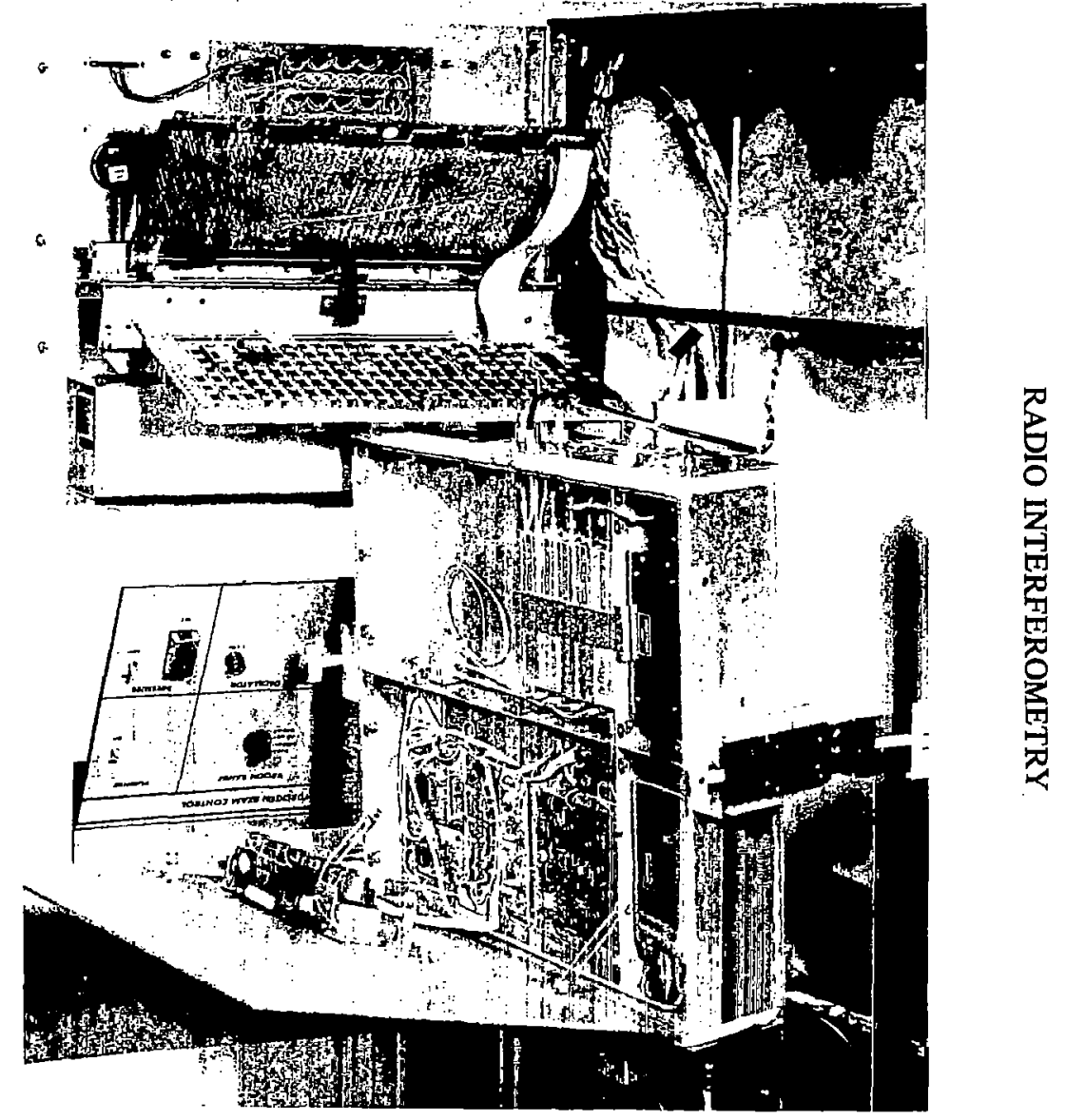

Figure 10. Close-up of NR maser exposed electronics. 
In figure 11 is shown a close-up of a synthesizer board to show how the parts are labeled as in the schematic diagrams, making servicing easy.

In figure 12 is shown the receiver; the multipliers are at the top, the maser signal goes through a low noise preamplifier, a double-balanced image-reject mixer, and the I.F. amplifiers. The overall noise figure is less than $2 \mathrm{~dB}$.

In figure 13 is shown the 200 to $1400 \mathrm{MHz}$ multiplier.

In figure 14 are shown the APL staff working part-time on the maser program who have brought the design to its present status. Clockwise from lower left: C. M. Blackburn, E. E. Mengel, D. W. Stover, P. J. Underwood, A. G. Bates, H. L. Smigocki, L. J. Rueger, L. E. Stillman, and J. R. Norton.

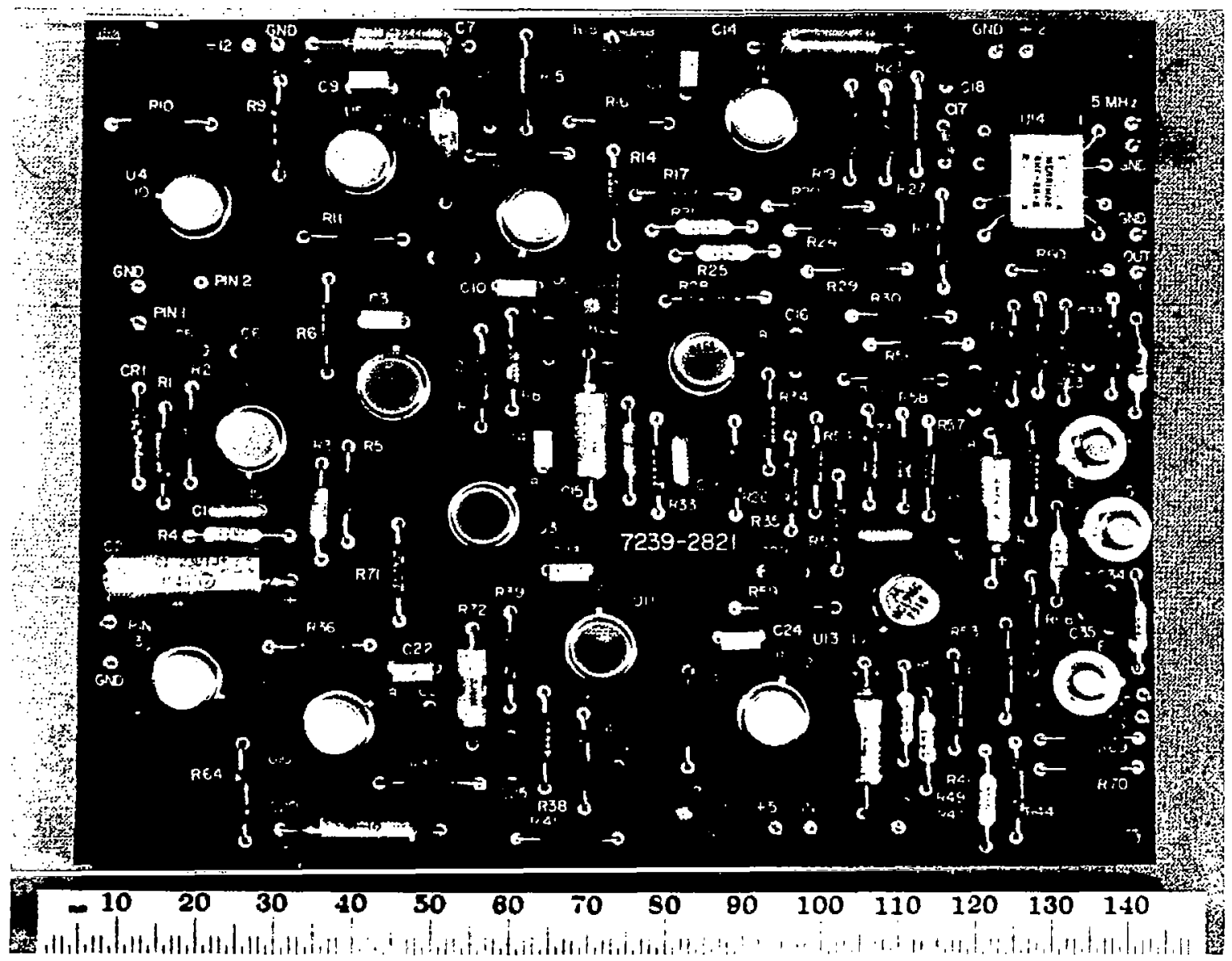

Figure 11. NR maser synthesizer board \#2. 


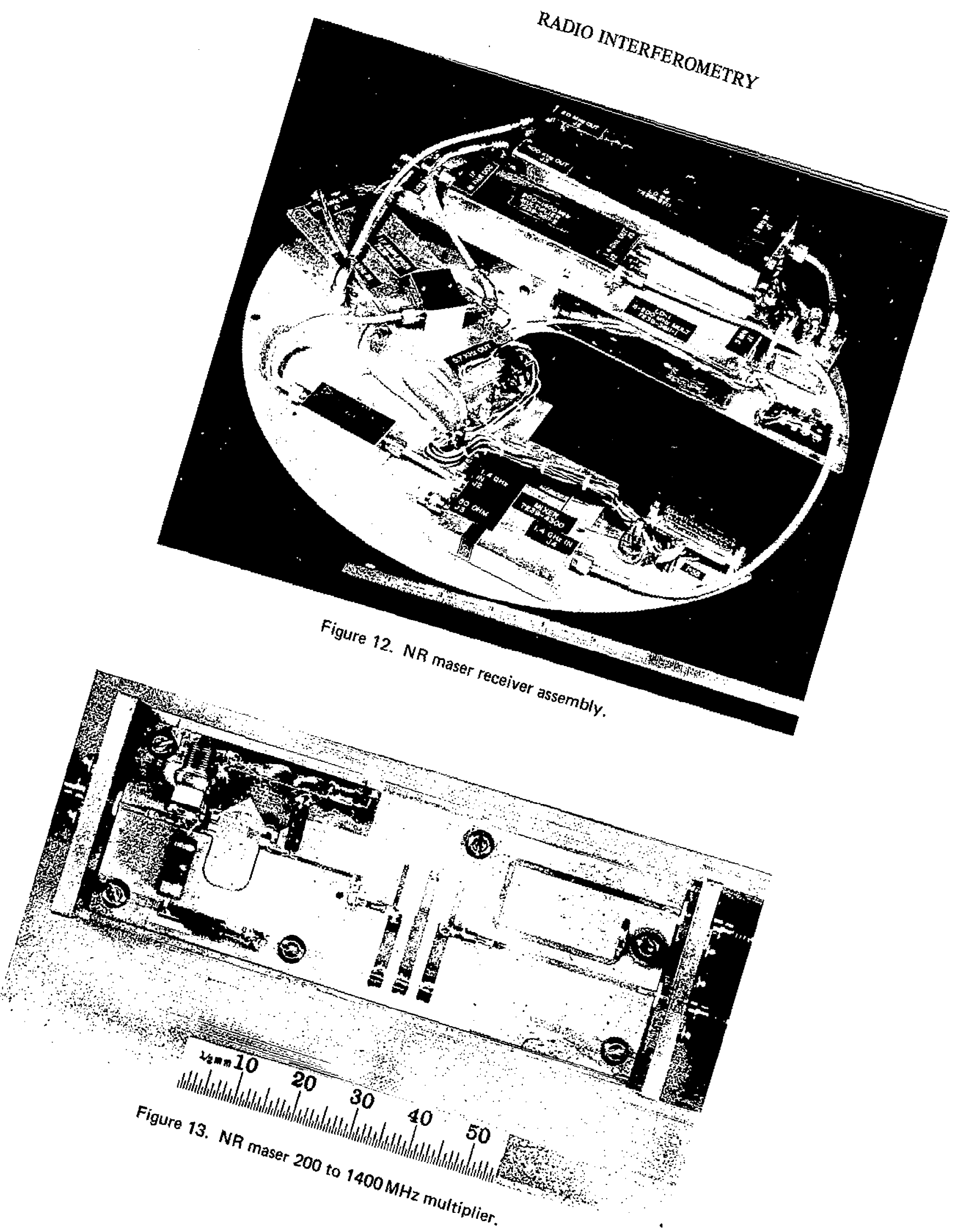




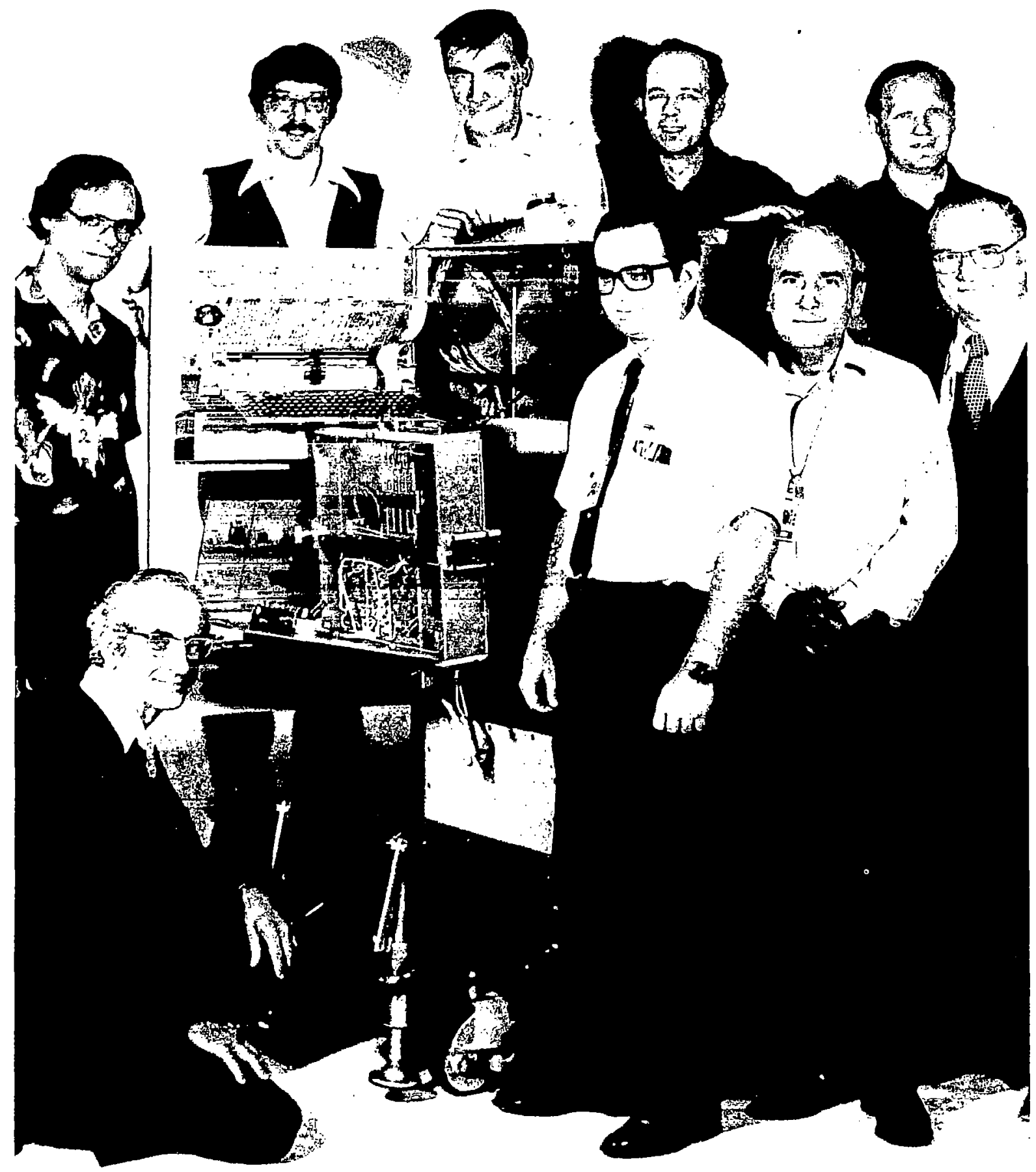

Figure 14. APL staff workers on NR maser. 


\section{Results to Date}

Line Q. Two NR masers have been constructed and are designated NR-1 and NR-2. The line $Q$ has been measured as $2 \times 10^{9}$.

Pressure Sensitivity. The pressure sensitivity of NR-2 has been measured by placing it in an altitude chamber. The average pressure sensitivity was $5 \times 10^{-15}$ per inch $(2.54 \mathrm{~cm})$ of mercury.

Magnetic Sensitivity. The magnetic susceptibilities of the NR masers were measured by placing the masers in a large solenoid and observing changes in the maser frequency output against a reference maser. The standard procedure was to switch the solenoid field between plus and minus 0.5 gauss and to observe the difference in the maser frequency output. The Zeeman frequency for each solenoid setting was also measured to establish the internal magnetic field values and to determine the shielding factor of each maser's magnetic shields. For each measurement set, the coil was switched a few times before taking data to eliminate transient effects due to slight regaussing of a maser's magnetic shiclds by the solenoid field. For the one gauss change at the test maser, the ficld change at the reference maser was only $1.5 \mathrm{mG}$. This corresponds to less than $10^{-15}$ frequency shift in the reference maser.

Figure 15 shows the magnetic susceptibility $(\mathrm{dy} / \mathrm{dH})$ of NR-1 as a function of Zeeman frequency (Z) for the maser main field coil at minus polarity (arbitrary reference). Notice there are two nulls in $\mathrm{dy} / \mathrm{dH}$. One is at $\mathrm{Z}=775 \mathrm{~Hz}$. Further data at higher Zeeman frequencies showed dy/dH becoming increasingly negative until it reached a value of $-4.9 \times 10^{-13} / \mathrm{G}$ at $Z=13750 \mathrm{~Hz}$, the highest $Z$ at which data was taken.

Figure 16 shows the magnetic susceptibility of NR-1 for the maser main field polarity positive. Notice that only one null in $\mathrm{dy} / \mathrm{dH}$ appears at the low value of $\mathrm{Z}$, and that around $\mathrm{Z}=775 \mathrm{~Hz}$, $\mathrm{dy} / \mathrm{dH}$ is about $1 \times 10^{-13} / \mathrm{G}$. Further data shows $\mathrm{dy} / \mathrm{dH}$ becoming more positive at higher $Z$ until a value of $5.8 \times 10^{-13} / \mathrm{G}$ is reached at $Z=13440 \mathrm{~Hz}$, the highest $Z$ at which data were taken. Over the whole range of $Z$ for which data were taken for both signs of the main field, the change in $Z$ $(\mathrm{dZ} / \mathrm{dH})$ remained the same to within $\pm 1 \mathrm{~Hz}$. Its average value was $\mathrm{dZ} / \mathrm{dH}=38.9 \mathrm{~Hz} / \mathrm{G}$ which corresponds to a shield factor (SF) for NR-1 of $3.6 \times 10^{4}$.

Figure 17 shows susceptibility data for NR-2 for its main field positive, notice the zero in dy/dH at $\mathrm{Z}=750 \mathrm{~Hz}$. For NR-2's main field, negative $\mathrm{dy} / \mathrm{dH}-1.8 \times 10^{-15} / \mathrm{G}$ at $\mathrm{Z}=769 \mathrm{~Hz}$. The change in sign of the main field from NR-1 to NR-2 for producing the higher field null in $\mathrm{dy} / \mathrm{dH}$ was caused by inadvertent reversal of the main field coil connections in NR-2. For this data in NR-2,

and

$$
\mathrm{dZ} / \mathrm{dH}=60.5(5) \mathrm{Hz} / \mathrm{G}
$$

$$
\mathrm{SF}=2.3 \times 10^{4}
$$

Notice that the null in $\mathrm{dy} / \mathrm{dH}$ occurs at approximately the same $\mathrm{Z}$ even though there is a large difference in shielding factor between the NR masers. 


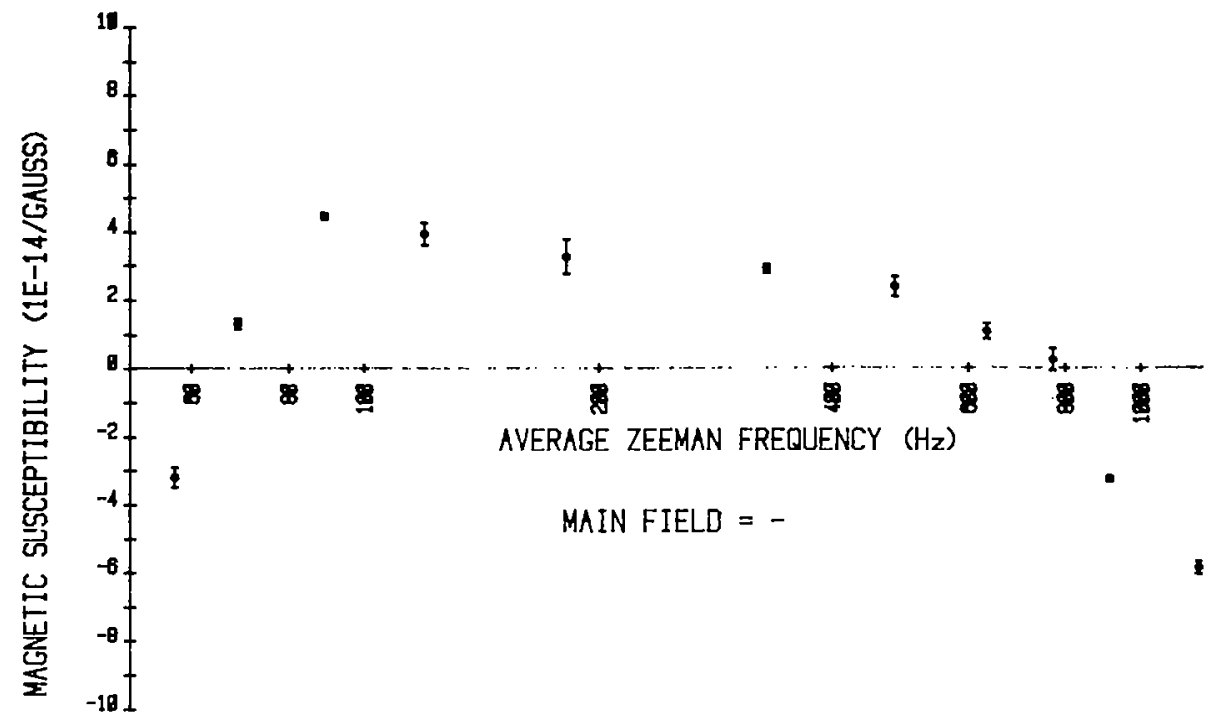

MAGNETIC SUSCEPTIBILITY OF NR-1

FOR 1 GAUSS AMBIENT FIELD CHANGE

Figure 15. Magnetic susceptibility of NR-1 for main field -.

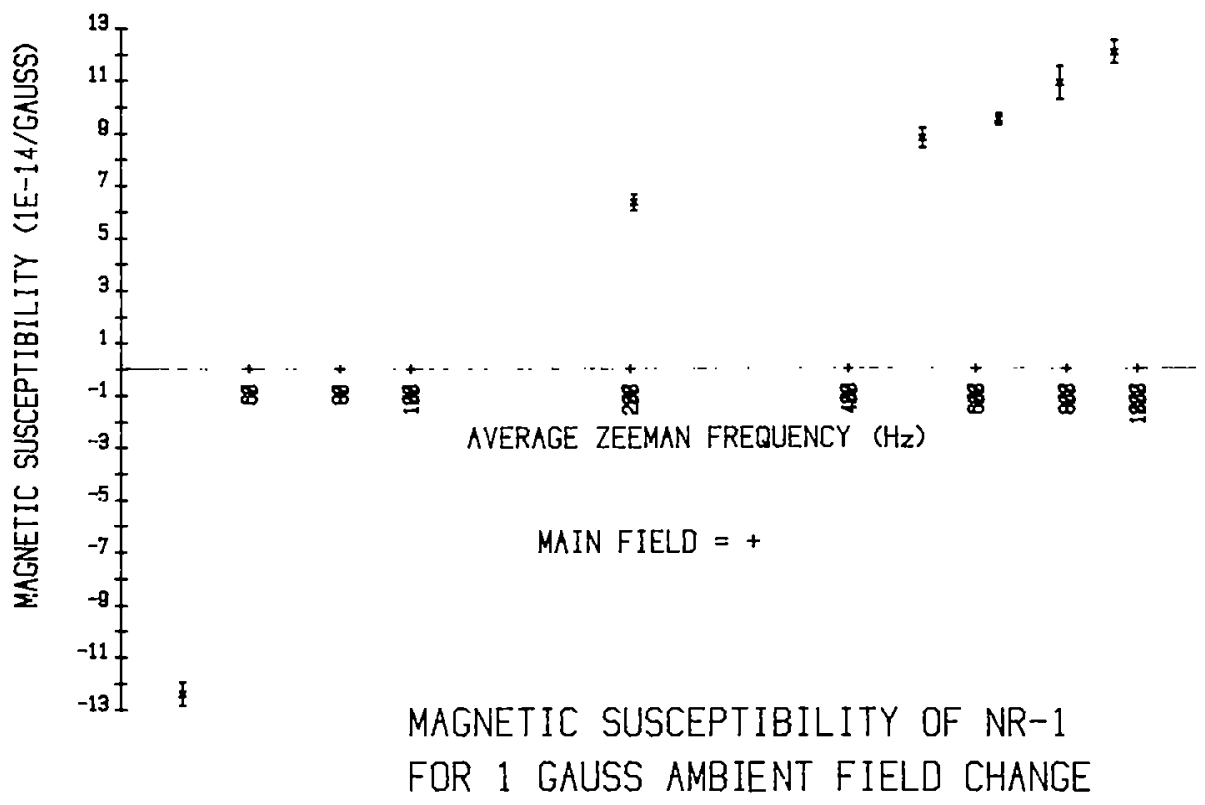

Figure 16. Magnetic susceptibility of NR-1 for main field + . 


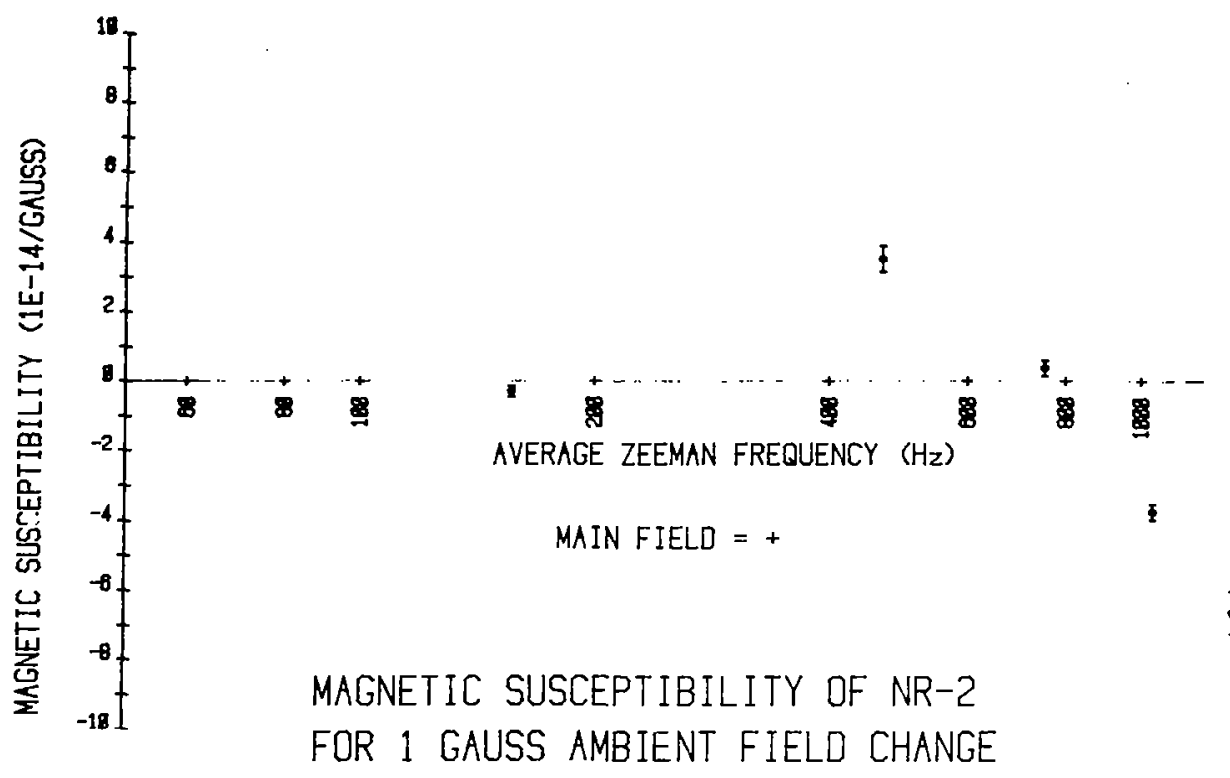

Figure 17. Magnetic susceptibility of NR-2 for main field + .

Figure 18 shows data on the repeatability of the zero susceptibility point at $\mathrm{Z}=752 \mathrm{~Hz}$ of NR-2. NR-2 was chosen because it has the lower shielding factor. For the first five data points, the standard measurement procedure was used. For the next data points, the solenoid (coil) was changed only once and several frequency measurements were made for each solenoid setting. This was done to check for any systematic errors introduced by the standard measurement procedure and to determine the one time frequency shift caused by regaussing of the maser magnetic shields by the solenoid. Notice that when going from a solenoid field both from 0 to $+.5 \mathrm{G}$ and from 0 to $-5 \mathrm{G}$ the maser frequency shifted by $1.5 \times 10^{-14}$. (The data in the chart are normalized to susceptibility units). Using all susceptibility data except that representing the permanent shifts, one obtains:

$$
\begin{aligned}
& \text { mean } \mathrm{dy} / \mathrm{dH}=-0.23 \times 10^{-14} / \mathrm{G} \\
& \text { standard deviation of points }=1.08 \times 10^{-14} / \mathrm{G}
\end{aligned}
$$

Thus, NR-2 would be characterized after degaussing as undergoing a one time permanent $1.5 \times 10^{-14}$ shift from a large magnetic disturbance and then having a susceptibility of about $1 \times 10^{-14} / \mathrm{G}$ for subsequent disturbances.

The data outlined indicates that by finding the zero susceptibility point in each NR maser, one can obtain operational magnetic susceptibilities of $1 \times 10^{-14} / \mathrm{G}$ in these masers. Whether this technique can be applied to hydrogen masers of other designs is another matter since the data presented here may depend on the details of the NR maser design. 


$\begin{array}{lcc} & \begin{array}{c}\text { ZEEMAN FREQUENCY } \\ (\mathrm{Hz})\end{array} & \begin{array}{c}\text { SUSCEPTIBILITY } \\ (1 E-14 / \text { GAUSS })\end{array} \\ & 751.8 & 0.17(6) \\ \text { START: } & 748.2 & 1.58(16) \\ \text { AFTER MILD MAGNETIC SHOCK: } & 752.1 & -0.38(25) \\ \text { AFTER DEGAUSS: } & & \\ \text { AFTER SEVER MAGNETIC SHOCK } & 752.7 & -0.54(68) \\ \text { \& DEGAUSS: } & 752.5 & -1.41(43) \\ \text { AFTER DEGAUSS: } & & \\ \text { AFTER DEGNUSS } & 752.5 & -3.21(74) \\ \text { \& COIL O TO -.5 GAUSS: } & 752.5 & -1.49(77) \\ \text { COIL -.5 TO +.5 GAUSS: } & & \\ \text { AFTER DEGAUSS } & 752.2 & 2.90(43) \\ \text { \& COIL D TO +.5 GAUSS: } & 752.2 & 0.46(46) \\ \text { COIL +.5 TO -.5 GAUSS: } & & \end{array}$

Figure 18. Repeatability of zero susceptibility point of NR-2.

Temperature Sensitivity. The temperature sensitivity of NR-1 was measured. Its value was $4.5 \times 10^{-14}$ per ${ }^{\circ} \mathrm{C}$ with about a one daytime constant. This is consistent with past measurements on the NX masers which showed a temperature sensitivity of $4 \times 10^{-14}$ per ${ }^{\circ} \mathrm{C}$. Because the microprocessor measures the temperature at several points on the maser, this temperature sensitivity can be effectively reduced to zero. Once a maser's temperature coefficient and time constant are measured, the frequency output of the maser can be filtered to extract the predictable temperature shifts. This can be done after the fact by recording temperature changes via the RS-232 interface or in real time by using the RS-232 interface to change the maser synthesizer settings. Though this has not been done yel, such a correction algorithm can be incorporated into the microprocessor programming.

Frequency Stability. Figure 19 shows $\sigma_{y}(\tau)$ of NR-1 versus NX-2 normalized for one maser. The data are shown both for NR-1's autotuner on and off. This data points out another improvement in the NR masers. The phase shifts which occurred in older Goddard Space Flight Center (GSFC) masers when the flux is changed during autotuning has been reduced to less than one picosecond. As seen from the data, this means that the NR masers can be continuously autotuned with virtually no reduction in their short term stability. 


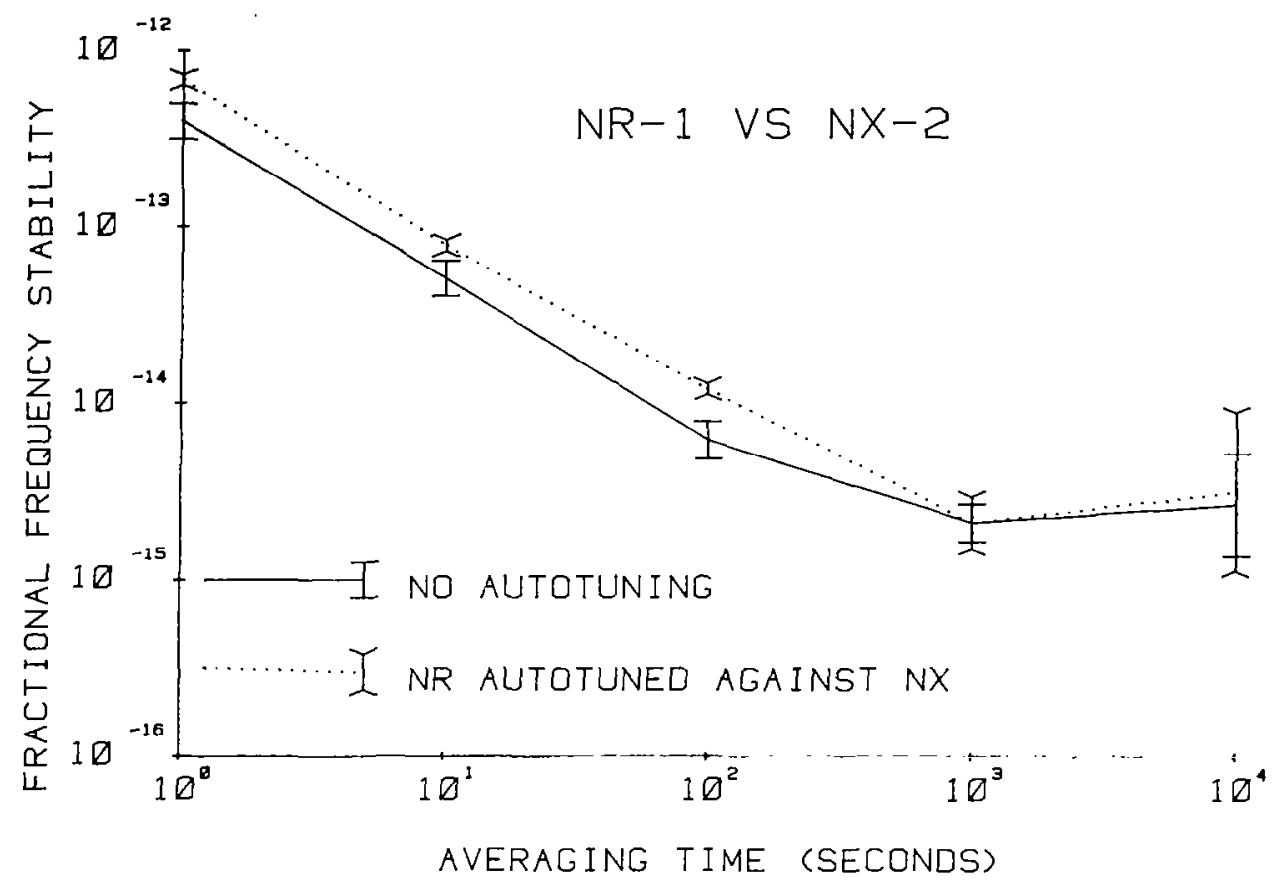

Figure 19. NR-1 stability data. 


\title{
ULTRA-LOW-NOISE MICROWAVE AMPLIFIERS*
}

\author{
Robert C. Clauss and David L. Trowbridge \\ Jet Propulsion Laboratory \\ California Institute of Technology
}

The Jet Propulsion Laboratory (JPL) has been active in the development and use of masers for more than 20 years (Reed et al., 1973). Cavity and traveling-wave masers in open- and closed-cycle helium refrigerators have been designed and built at frequencies between $900 \mathrm{MHz}$ and $42 \mathrm{GHz}$. Noise temperatures of $2.1,3.5,8.5$, and 13 Kelvins have been measured at $2.3,8.4,15$, and $25 \mathrm{GHz}$ respectively. More than 50 field-operational systems have been supplied to the NASA Deep Space Network (DSN); these systems have accumulated over 2 million hours run time. Current maser systems used in the DSN provide $40 \mathrm{MHz}$ bandwidth. $150 \mathrm{MHz}$ bandwidth has recently been demonstrated in the laboratory at $8.4 \mathrm{GHz}$. K-band masers (near $24 \mathrm{GHz}$ ) can provide $500 \mathrm{MHz}$ band width. The combination of a parametric upconverter with a K-band maser can provide a $500 \mathrm{MHz}$ bandwidth at S-band or X-band frequencies with maser-like noise temperatures; a 31/2 Kelvin noise temperature at $2300 \mathrm{MHz}$ has been demonstrated.

The 64-meter antenna at Goldstone, California is shown in figure 1. Five ultra-low-noise travelingwave maser (TWM) systems are in use; two at S-band (2300 MHz) and three at X-band ( $8400 \mathrm{MHz})$. Each maser operates in a closed-cycle helium refrigerator (CCR) at 4.5 Kelvin. Total system temperatures as low as 15 Kelvins have been achieved during spacecraft tracking operations at S-band. Typical X-band operating system temperatures are between 25 and 30 Kelvins.

A $960 \mathrm{MHz}$ cavity maser cooled to 4.2 Kelvin in an open-cycle helium dewar was installed on a 26meter antenna in September 1960. Figure 2 shows a liquid helium transfer at the prime focal point, 80 feet $(27.84 \mathrm{~m})$ above ground. The maser contains a 4-liter liquid helium tank and an 8-liter liquid nitrogen tank. The system will operate for 36 hours between refills on a moving antenna. Figure 3 shows 25 -liter storage containers of liquid helium and liquid nitrogen.

A recently developed printed-circuit traveling-wave maser is shown in figure 4. This X-band TWM is particularly compact; several structures can be used in one super-conducting magnet. The ruby bar (shown just above the scale) has 44 one-half wavelength copper elements along a $8.5 \mathrm{~cm}$ length. A sapphire bar, shown just above the ruby bar, is placed against the copper elements; the ruby, copper, sapphire assembly is placed into the housing so that the sapphire bar contacts the resonant isolator assembly. The isolator assembly contains a continuous strip of polycrystalling yttrium-iron garnet between two alumina bars and is shown located in the housing. A beryllium-copper spring

\footnotetext{
*'This paper presents one phase of research carried out at the Jet Propulsion Laboratory, California Institute of Technology, under contract NAS7-100, sponsored by the National Aeronautics and Space Administration.
} 


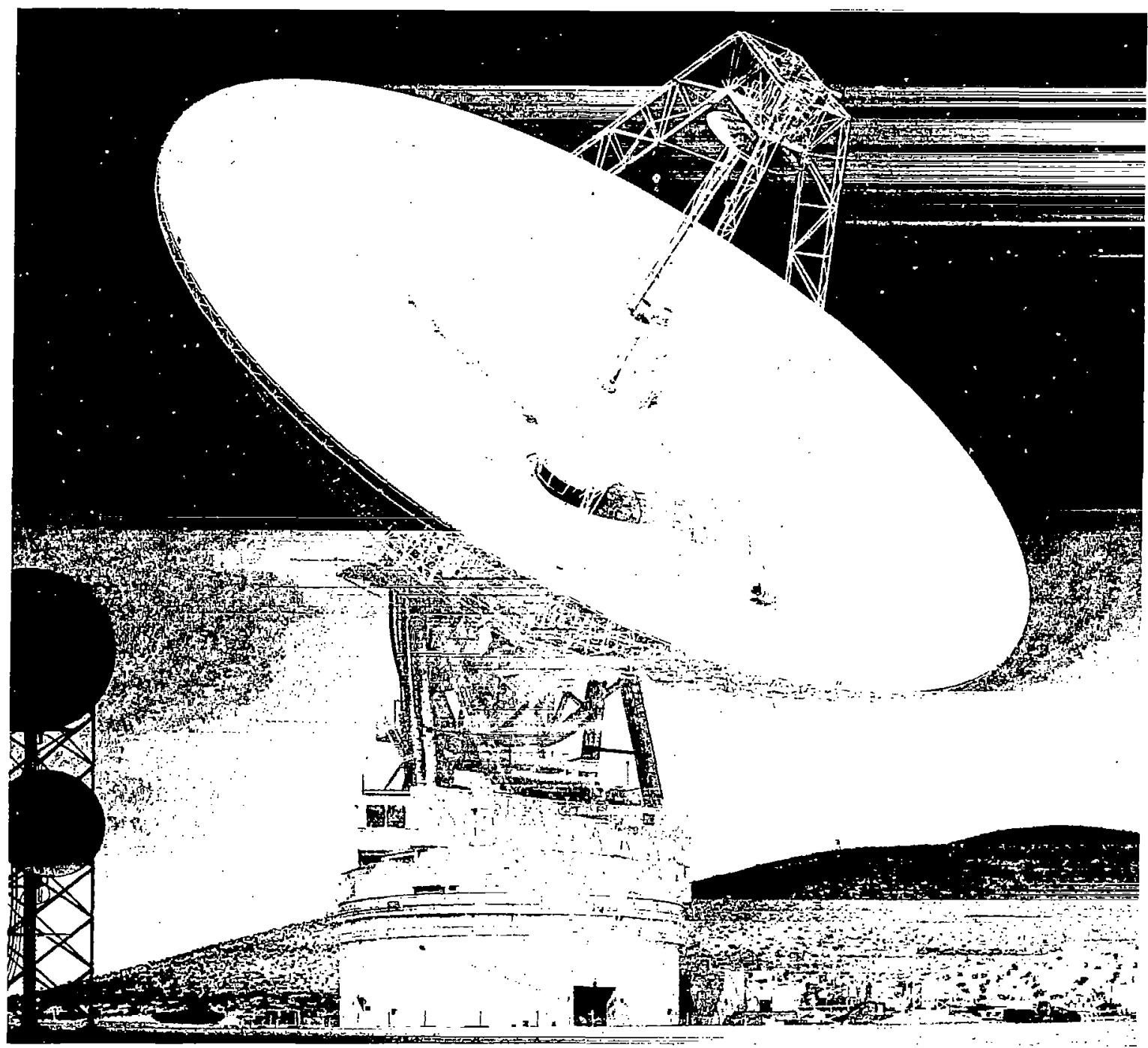

Figure 1. The 64-meter antenna at Goldstone, California.

behind the isolator assembly applies pressure to the assembly so the ruby bar will achieve intimate contact with a copper cover (not shown) to provide a good thermal path to the 4.5 Kelvin station of a closed-cycle refrigerator. Signal input and output connectors (with matching elements) are shown; the one at the left is disassembled to show details. Signals to be amplified travel through the assembly at $1 / 50$ the speed of light. The "slowed-wave" interacts strongly with the ruby and is amplified as it travels through the structure. The ruby is biased with a 5000 gauss magnetic field along the direction of the fingers. A population inversion of the energy levels is achieved by saturating the ruby with pump energy (Siegman, 1964). Pump energy at 19 and $24 \mathrm{GHz}$ enters at the left of the structure, through an alumina filled waveguide. The resonance isolator is properly shaped to operate in the 5000 gauss field required by the ruby for operation at $8400 \mathrm{MHz}$. The isolator highly 


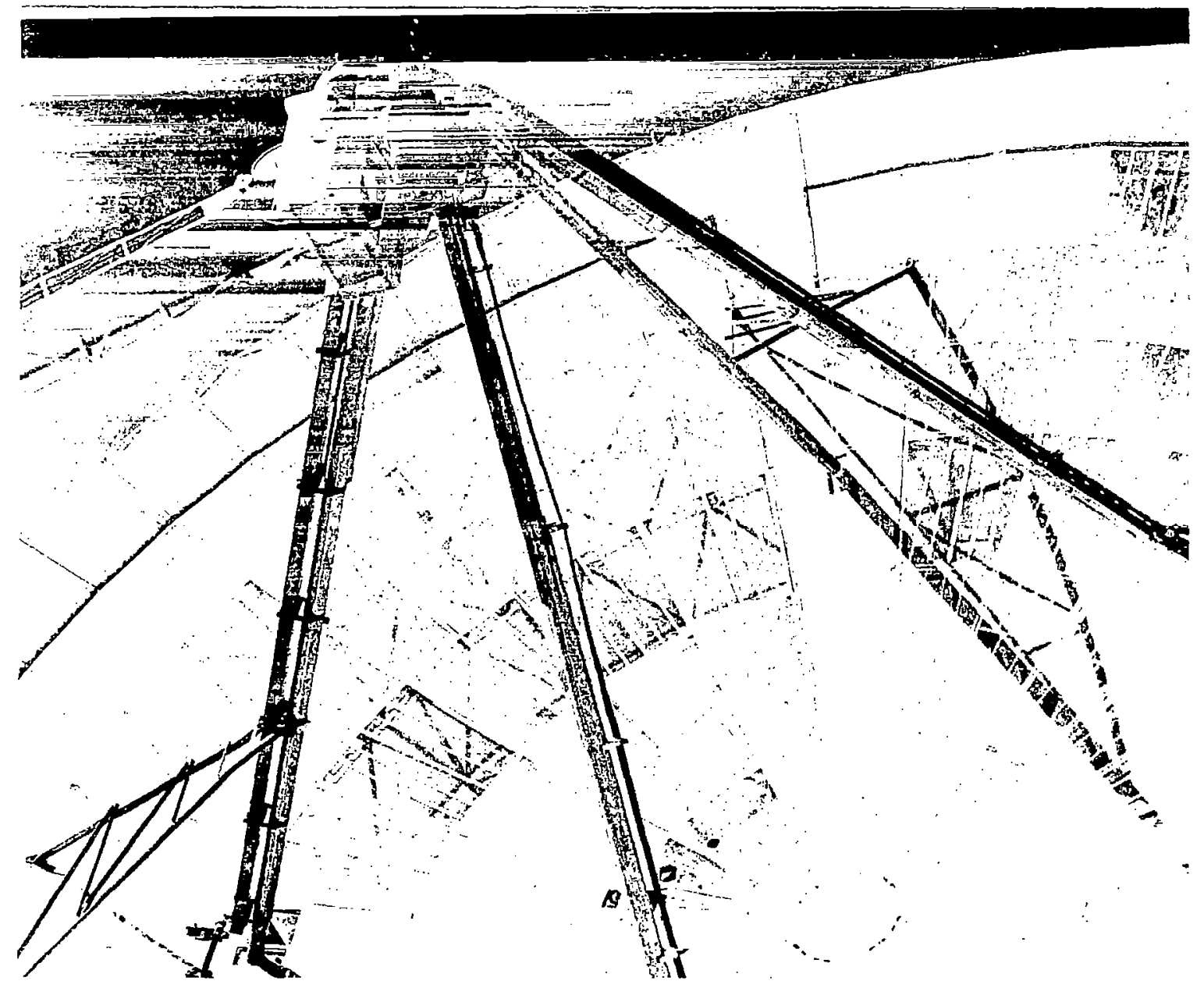

Figure 2. Liquid helium transfer at the apex of a 26-meter antenna.

attenuates signals traveling from output to input, thereby preventing regenerative amplification. The direction of amplification, with proper isolation, is determined by the polarity of the magnetic field and can be reversed by changing the direction of current flow through the 5000 gauss superconducting magnet. Amplifiers of this type can be used to achieve bandwidths of several hundred $\mathrm{MHz}$ with noise temperatures of less than 5 Kelvin.

Figure 5 shows a $2295 \mathrm{MHz}$ maser developed in 1964. The TWM structure was purchased from Airborne Instruments Laboratories and the closed-cycle helium refrigerator was purchased from the Arthur D. Little Corporation. Fourteen systems of this type were installed in the DSN during the 1960's. The TWM is shown connected to a liquid-helium-cooled waveguide termination. The total system noise temperature in this configuration was measured at 15 Kelvins (Clauss et a1., 1964). The equipment racks behind the maser contain: (1) temperature controllers for the permanent 


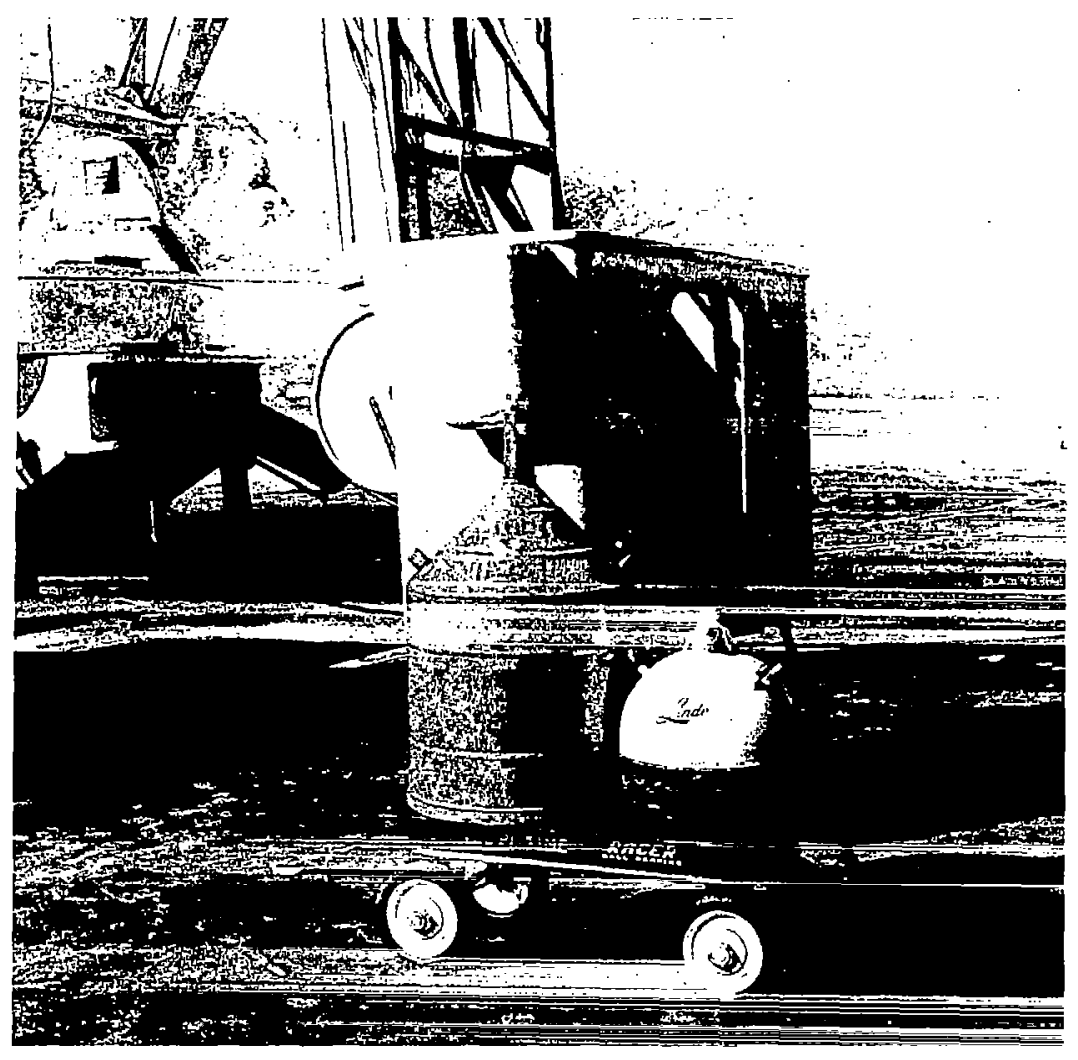

Figure 3. Containers of liquid helium and liquid nitrogen.

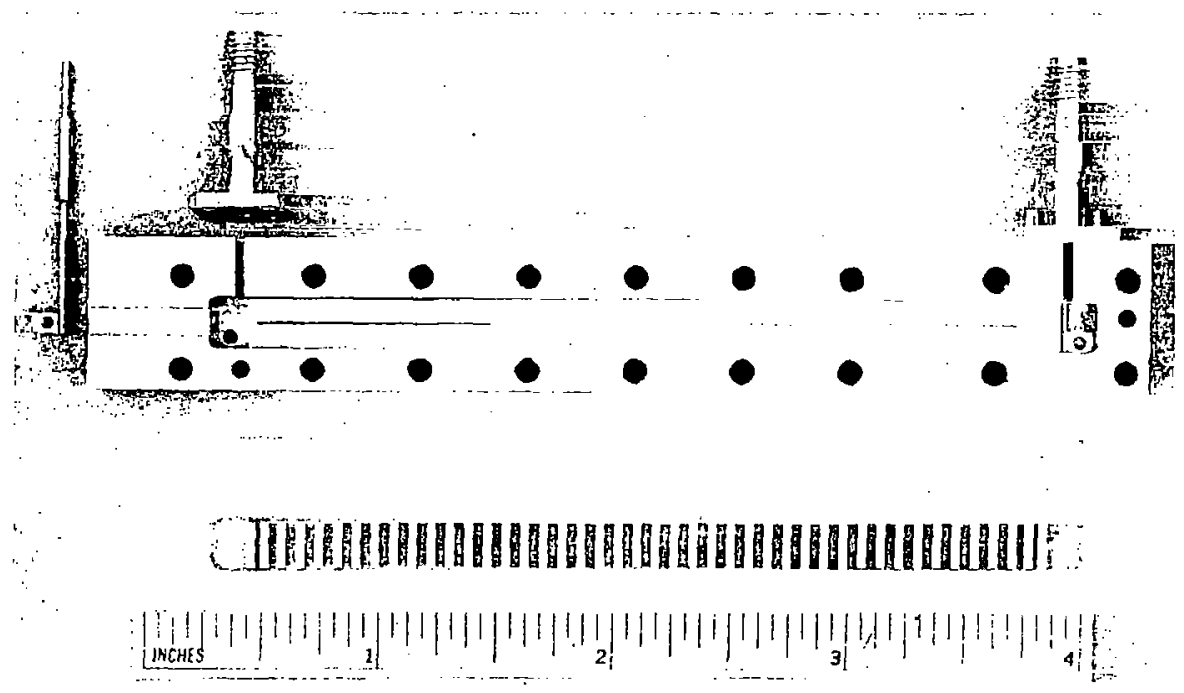

Figure 4. Printed circuit slow-wave comb structure for $\mathbf{X}$-band maser. 


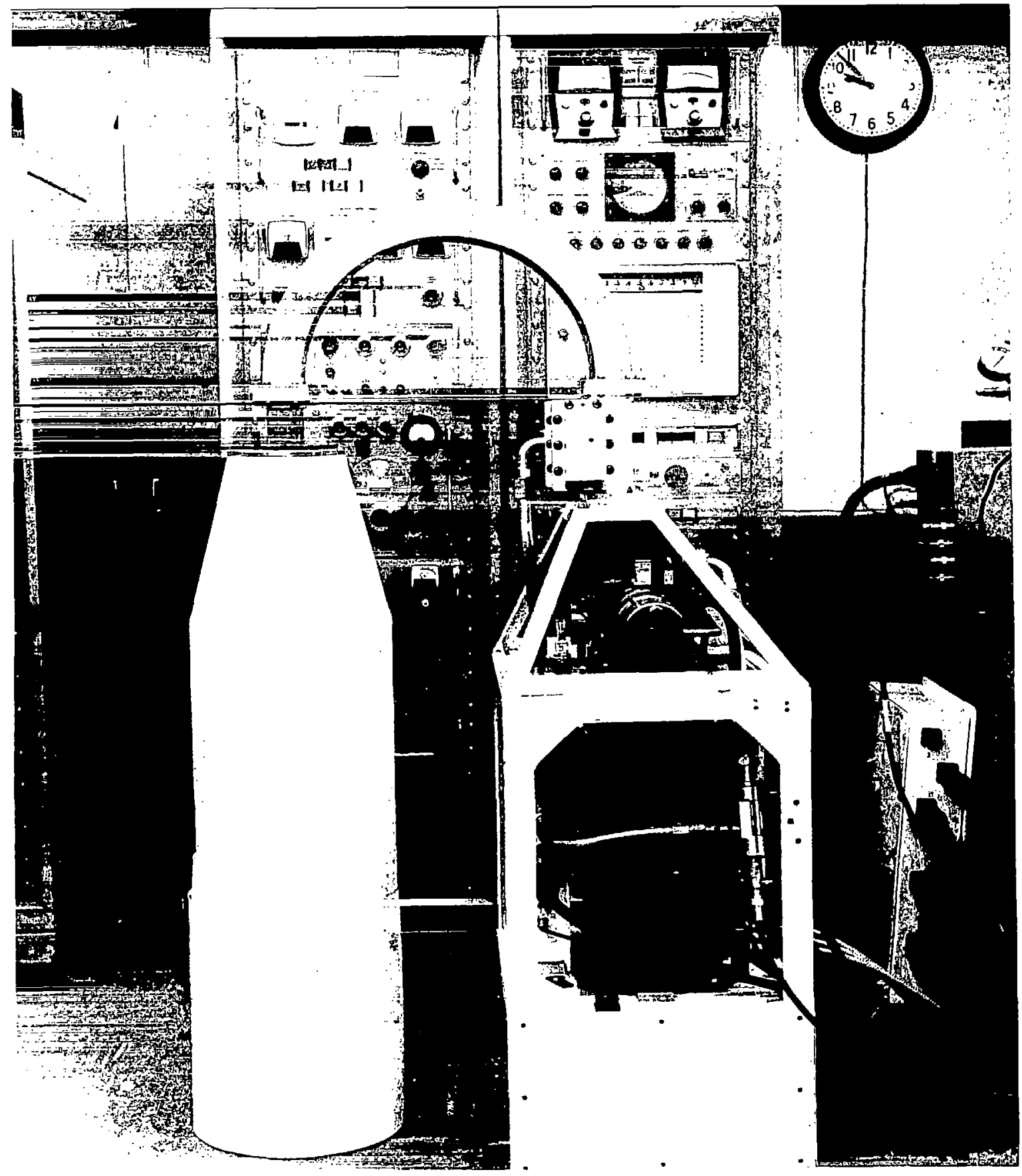

Figure 5. S-band maser and liquid helium-cooled waveguide termination. 
maser magnet, (2) a spectrum analyzer, (3) a strip chart recorder to record stability performance, (4) calibration switch controls, (5) noise source power supplies, (6) detectors with adjustable bandwidth and integration times, (7) refrigeration controls and monitors, (8) a klystron (pump) power supply, (9) the maser magnet trim power supply, and (10) a signal generator used for measuring maser gain. A super-hetrodyne receiver is used to monitor maser performance. A helium compressor used with the cryogenic refrigerator is now shown. Helium gas lines, cables, and junction boxes are also needed. This complete system is typical of a maser installation in the DSN; it is easy to see why each system costs several hundred thousand dollars.

Figure 6 shows an X-band TWM structure typical of those used in the DSN today. A net gain of $45 \mathrm{~dB}$ is achieved, flat within $1 \mathrm{~dB}$, from $8400-8440 \mathrm{MHz}$. The effective input noise temperature is $8 \pm 2$ Kelvins. A TWM like this one is being assembled for the portable 4-meter antenna station for radio interferometry. It will be modified for performance in an open-cycle helium dewar with reduced gain $(\simeq 25 \mathrm{~dB})$ and increased band width $(\simeq 140 \mathrm{MHz})$.

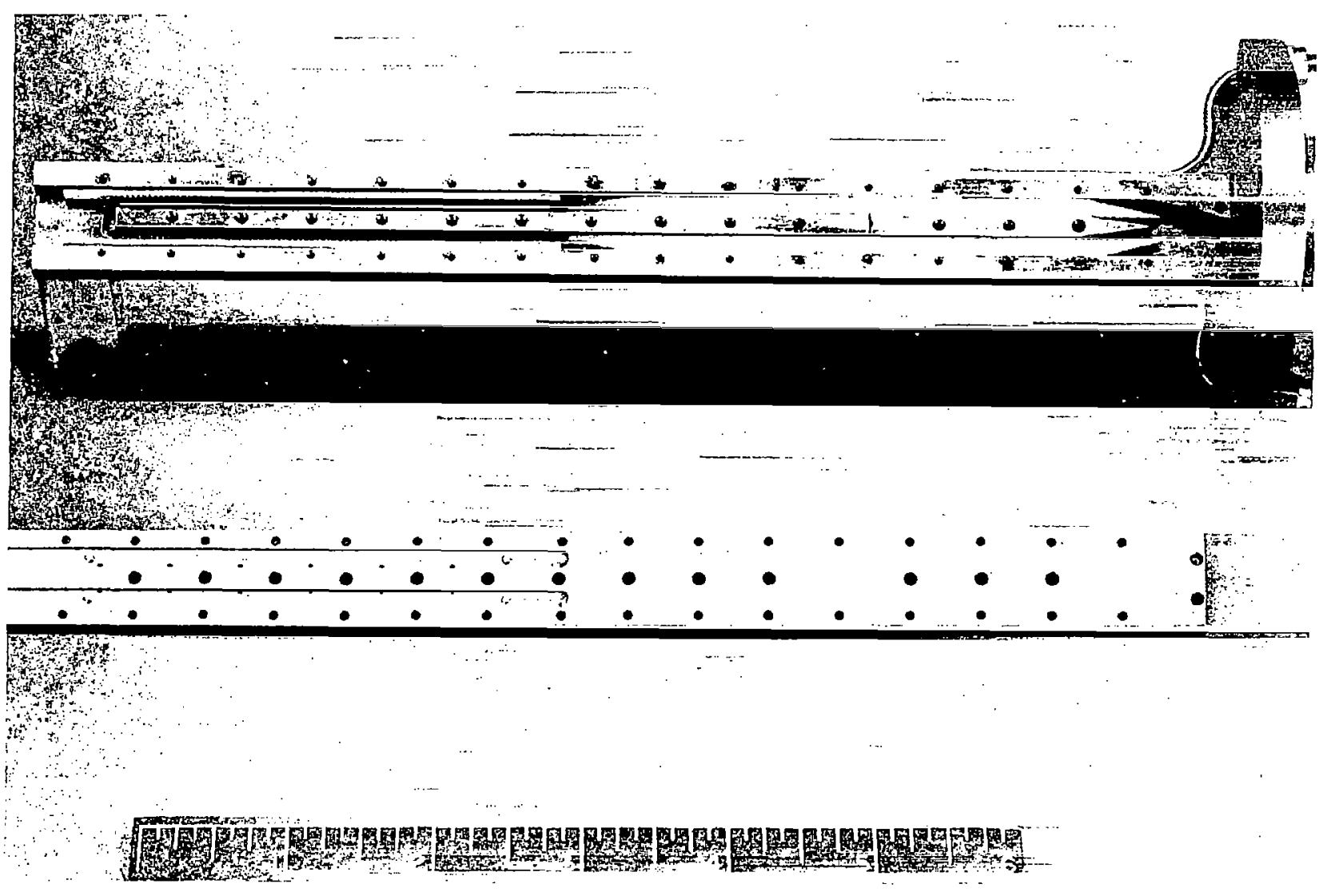

Figure 6. Typical X-band comb-type traveling-wave maser. 
A reflected-wave K-band maser is shown in figure 7. Only one-half of the amplifier is shown so the circulators, matching sections, and ruby-filled waveguides are visible. The unit was developed at JPL under a contract from the National Radio Astronomy Observatory (NRAO). The cooperative effort between JPL and NRAO has produced a wide-band maser design that will find application for both radio astronomy and planetary spacecraft communication (Moore and Clauss, 1979). Signals are directed to and from ruby-filled waveguides by circulators built in reduced height WR-42 waveguide. The round circulator ferrite elements are surrounded by white alumina matching elements. Three circulators are terminated with poly-iron loads; these circulators act as interstage isolators. Each ruby-filled waveguide is connected to a circulator through a multistep matching transformer. The opposite end of the ruby-filled waveguide (not shown) contains a waveguide-beyond-cutoff filter used to introduce pump energy. The signal is amplified as it travels from the circulator to the pump filter. At the filter, it is reflected and travels back towards the circulator, being amplified a second time. The circulator directs the signal to the next stage of amplification. Four stages are used to achieve $30 \mathrm{~dB}$ net gain with $240 \mathrm{MHz}$ bandwidth at the center of the maser tuning range. The tuning range is limited by the circulator bandwidth, in this case, from 18.3 to $26.5 \mathrm{GHz}$. A 10 kilogauss superconducting magnet is used (Cioffi, 1962); improvements in the magnet design at NRAO resulted with $550 \mathrm{MHz}$ maser bandwidth at $30 \mathrm{~dB}$ net gain.

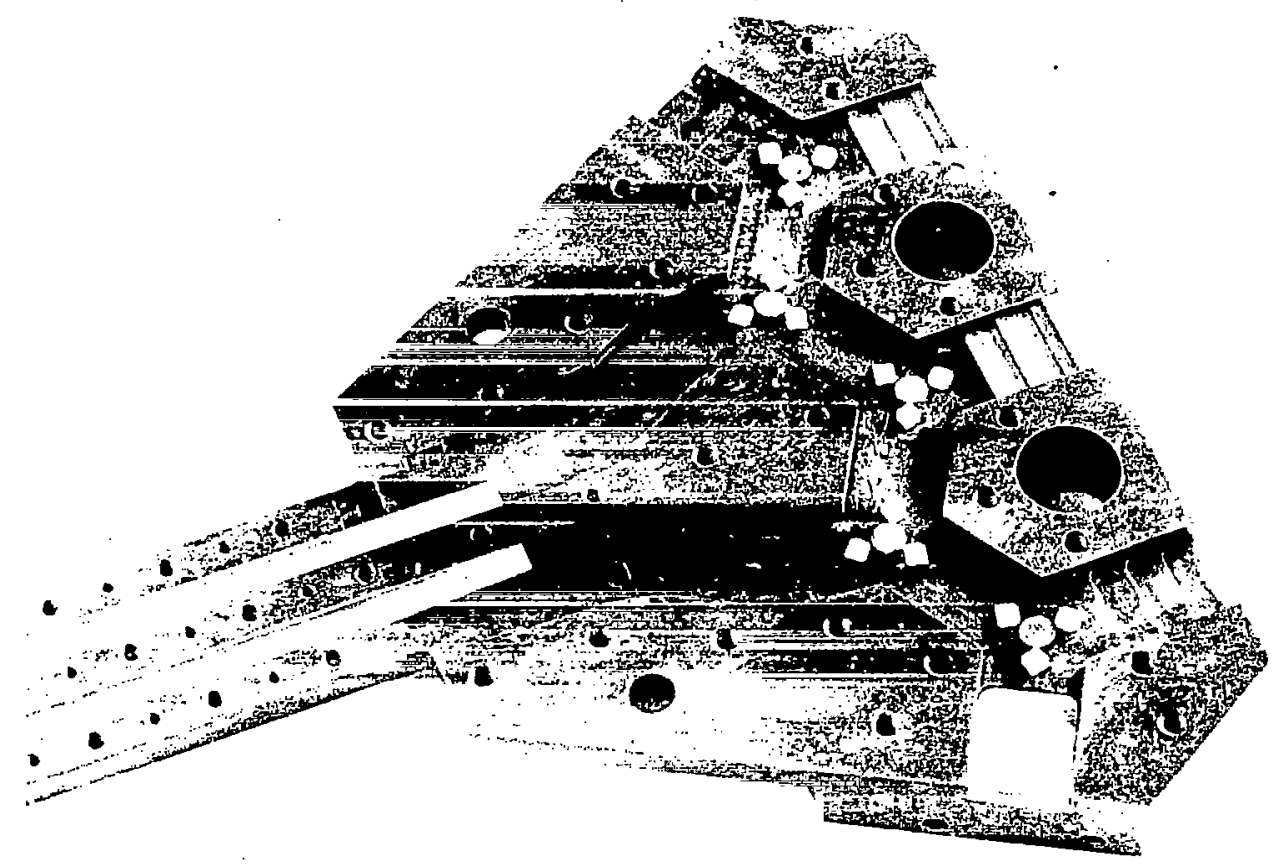

Figure 7. K-band reflected-wave maser structure. 
The maser and magnet operate in a closed-cycle refrigerator at $4.5 \mathrm{Kelvin}$ (Higa and Wiebe, 1967). The package is shown in figure 8. The input waveguide connection is shown at the top. Units of this type have achieved an effective input noise temperature of 10 Kelvins.

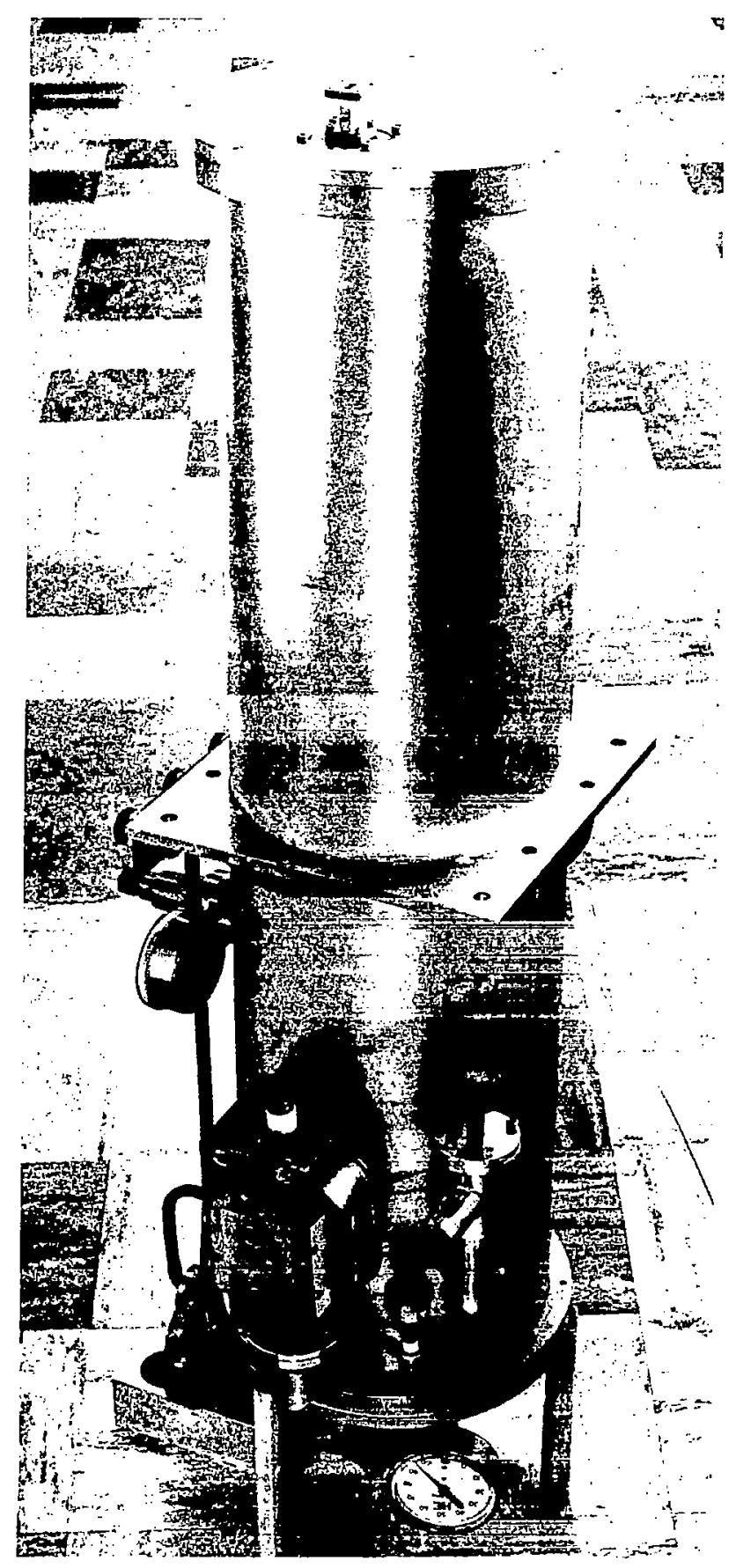

Figure 8. K-band maser package. 
Figure 9 shows the combination of an S-band to K-band up-converter and a K-band maser in a 4.5 Kelvin CCR. The input transmission line center conductor is cooled to 4.5 Kelvin along its entire length. A fused quartz dome provides a vacuum seal in the ambient S-band waveguide. This combination provides maser like noise temperatures with bandwidths up to $500 \mathrm{MHz}$ (Petty et al., 1978).

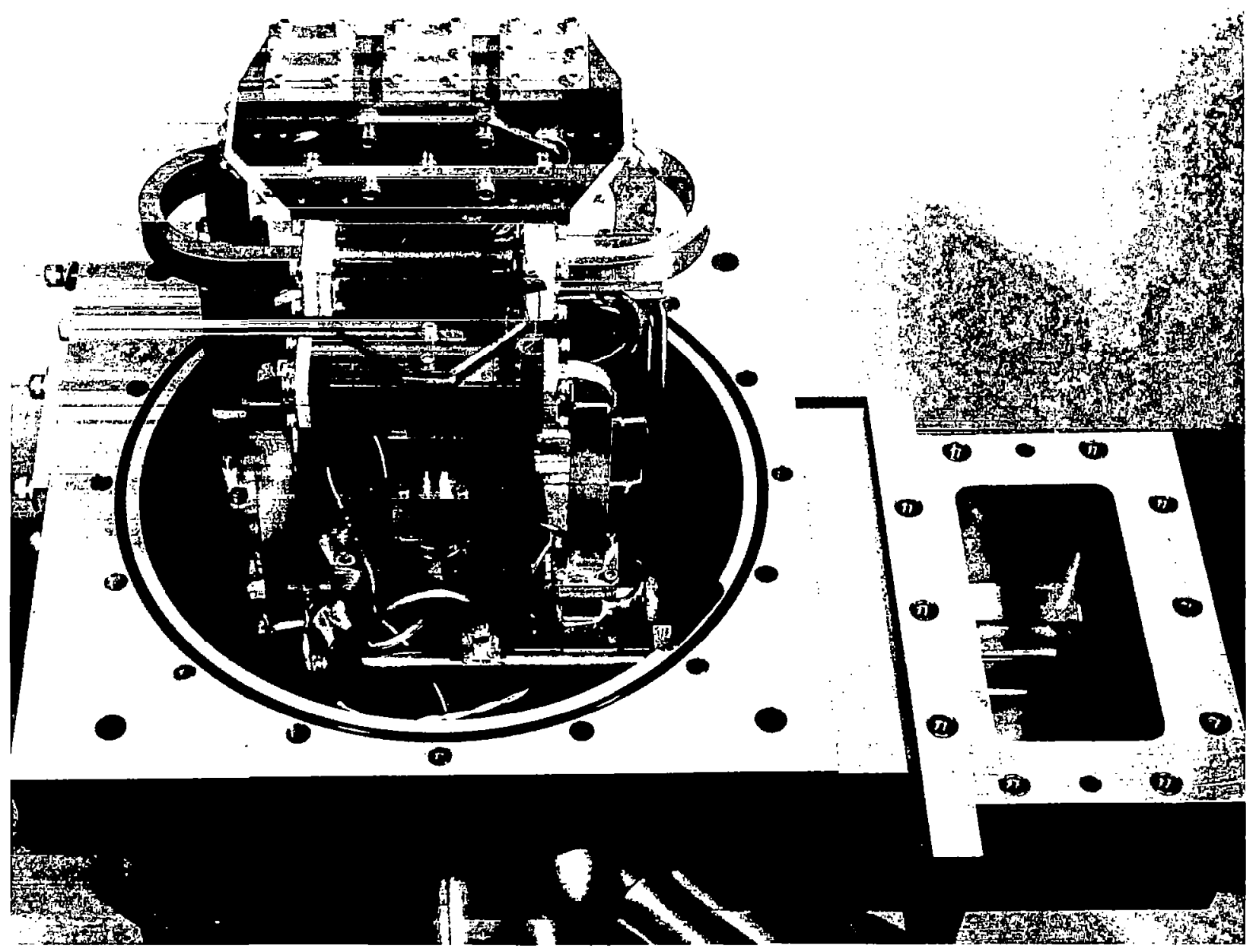

Figure 9. S-band to K-band up-converter with maser and low-loss input line.

Figure 10 shows an S-band maser and feed-horn on the roof of our building at JPL. A well-matched microwave absorber is shown above the horn; it introduces ambient noise into the system. Removal of the absorber allows the 5 Kclvin sky noise to enter the system. Precise mcasurements of the power change are used to determine the total system operating temperature; 8 Kelvins were measured. The maser contributes 2 Kelvin, 1 Kelvin is contributed by the waveguide transducer and feed-horn; the remaining 5 Kelvins are contributed by the atmosphere and cosmic background at 2.3 $\mathrm{GHz}$. 


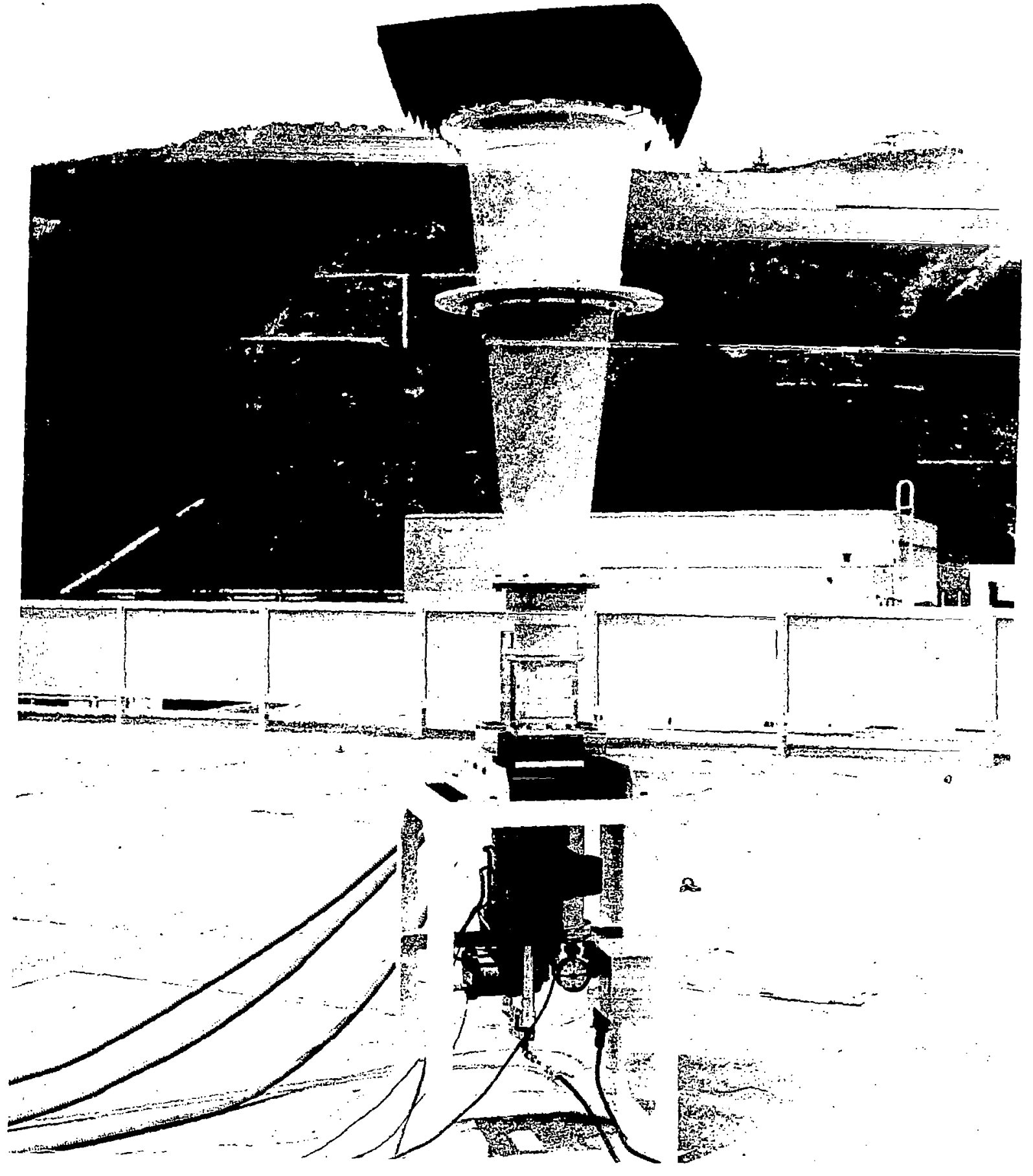

Figure 10. Low-noise S-band maser during noise temperature evaluation. 
A summary of noise temperatures measured since 1960 is shown in figure 11 .

Figure 12 shows maser bandwidths achieved since 1960. Our first cavity maser (at JPL) achieved a bandwidth of $0.7 \mathrm{MHz}$ with $20 \mathrm{~dB}$ net gain. Many other organizations were involved in the development of masers during the late 1950s and the 1960s. Much of their technology was used in JPL maser designs. By the late 1960s most workers discontinued the development of masers; bandwidth limitations together with the high cost and complexity of 4 Kelvin cryogenic systems seemed to indicate a limited future for the ultra-low-noise amplifiers. Satellite communications systems required much greater bandwidth than had been achieved with masers. Increased transmitter power in satellites was used to assure the needed signal to noise ratio at the earth stations without the use of ultralow-noise amplifiers. In contrast, deep space communications did not require extremely wide bandwidth and spacecraft transmitter power was limited. The use of ultra-low-noise receiving systems for deep space communications was a cost effective way to assure the reception of signals, including high quality television pictures, from distant planets. A significant omission from figure 12 and from the presentation of this paper in June was the achievement of a $130 \mathrm{MHz}$ bandwidth with a C-Band maser using rutile. The rutile-meander-line maser was developed by the NASA-Goddard Space Flight Center (Johnson, 1967). Recent cooperative work between JPL and NRAO show that it will be possible to build a ruby maser at $40 \mathrm{GHz}$ with a bandwidth of $1 \mathrm{GHz}$ (Neff et al., to be published).

Figure 13 shows the stability characteristics of a maser using a super-conducting magnet on a moving antenna. Measurements of maser performance on antennas and in the laboratory (Trowbridge, 1975 ) were used to compute the typical values shown.

Figure 14 shows a wide range of costs associated with the construction of masers systems. Development costs are not included; the dollar amounts indicated are for existing designs. Commercially available components are used where possible. Procurement, fabrication, manpower, and overhead costs are included using current (1978-1979) experience at JPL as a guide.

\section{REFERENCES}

M. S. Reid et al., "Low-Noise Microwave Receiving Systems in a Worldwide Network of Large Antennas," Proceedings of the IEEE, Vol. 61, No. 9, pp. 1330-1335, Sept. 1973.

A. E. Siegman, Microwave Solid State Masers, New York: McGraw-Hill, 1964.

R. C. Clauss, W. Higa, C. Stelzried, and E. Wiebe, "Total System Noise Temperature: $15^{\circ} \mathrm{K}$," IEEE Trans. Microwave Theory Tech., Vol. MTT-12, pp. 619-620, Nov. 1964.

C. R. Moore and R. C. Clauss, "A Reflected-Wave Ruby Maser with K-Band Tuning Range and Large Instantaneous Bandwidth: IEEE Trans. Microwave Theory Tech., Vol. MTT-27, No. 3, pp. 249-256, Mar. 1979. 


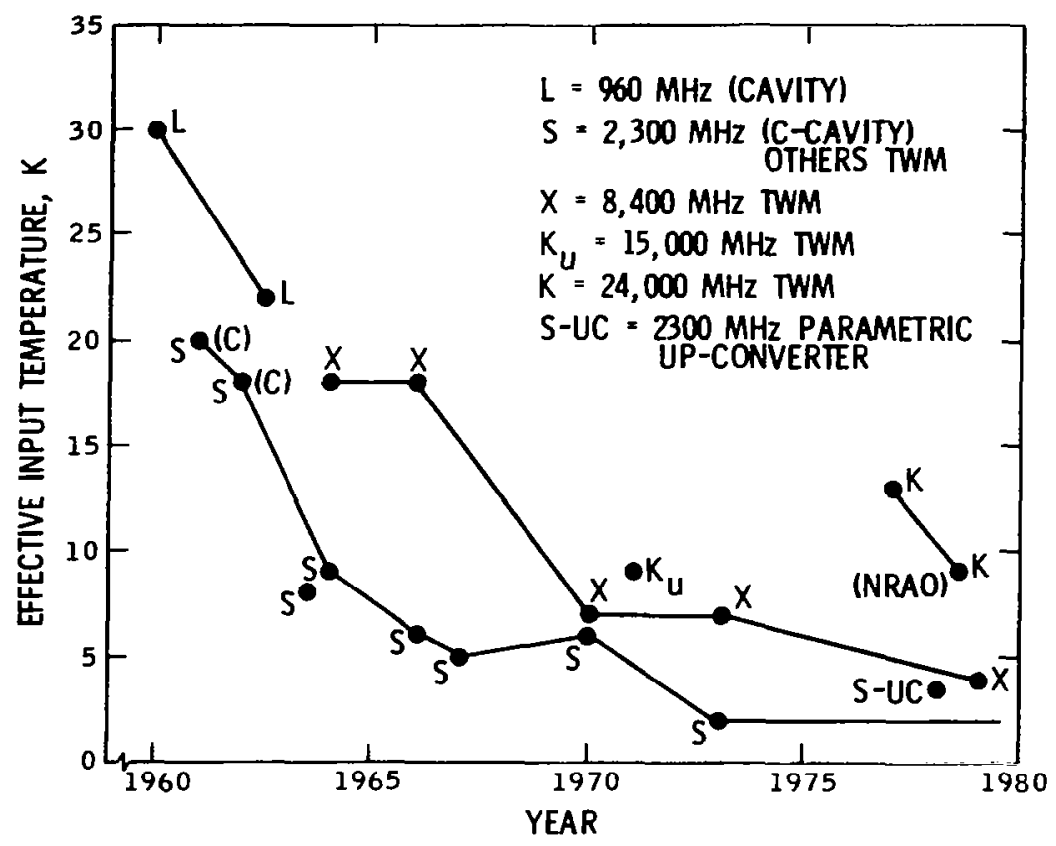

Figure 11. Maser noise temperatures.

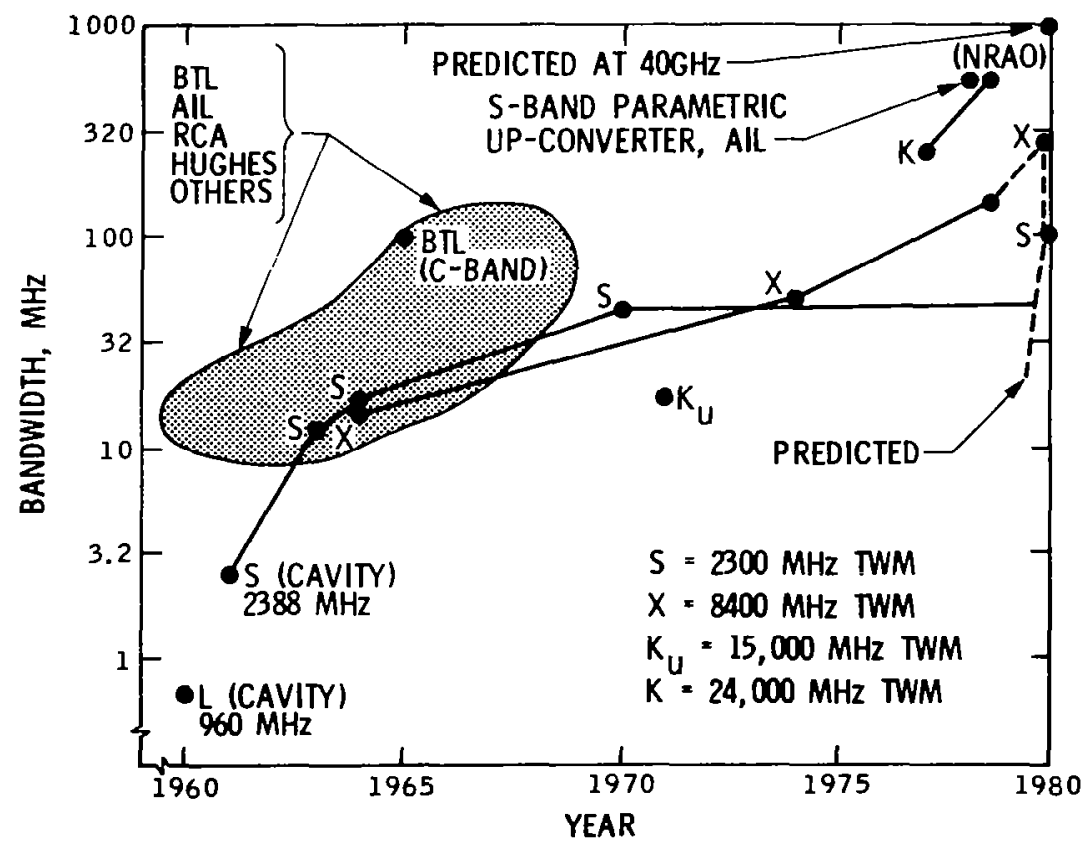

Figure 12. Maser bandwidth. 
- CHANGES in PUMP FREQUJENCY, PUMP AMPLITUDE, AND REFRIGERATION TEMPERATURE ARE THE MAIN CONTRIBUTORS TO INSTABILITY FOR MASERS USING SUPERCONDUCTING MAGNETS.

- USE OF PERMANENT MAGNETS TYPICALLY DEGRADES MASER STABILITY BY A FACTOR OF FIVE.

- RAPID aNTENNa MOTION THAT CHANGES REFRIgERATOR POSITION BETHEEN VERTICAL AND HORIZONTAL CAUSES TEMPORARY GAIN CHANGES ( $\approx 1$ MINUTE) OF UP TO 1/2 DB.

MASER STABILITY VS. TIME

\begin{tabular}{|c|c|c|l|}
\hline TIME INTERVAL & $\begin{array}{c}\text { GAIN } \\
\text { (DB) }\end{array}$ & $\begin{array}{l}\text { PHASE } \\
\text { (DEG.) }\end{array}$ & $\begin{array}{l}\text { DELAY } \\
\text { (NANOSECONDS) }\end{array}$ \\
\hline I SEC & \pm .01 & \pm .1 & \pm .01 \\
$10 \mathrm{SEC}$ & \pm .02 & \pm .2 & \pm .02 \\
100SEC & \pm .05 & \pm 1 & \pm .1 \\
8 HOURS & \pm .2 & \pm 3 & \pm .3 \\
\hline
\end{tabular}

Figure 13. Maser stability characteristics on a moving antenna.

- PARAMETRIC UP-COIVERTER -.-.-.-- \$10,000 TO \$20,000 EA.

. UP-CONVERTER PUI:P --

. MASER AiND S.C. MAGNET ------ \$40,000 T0 \$60,000 EA.

. PUMP PACKAGE - -

- UAVEGUIDES, WINDOWS, HARDWARE --- $\$ 10,000$ TO $\$ 10,000$

. POWER SUPPLIES, CONTROLS ---.--- \$15,000 TO \$50,000

- CABLES, GAS LINES, SUPPORT EQUIP, $\$ 15,000$ TO $\$ 50,000$

- MONITOR AND CAL. EQUIP. - - 0 TO $\$ 50,000$

. CRYOGENICS, DEWAR --.--- \$5,000 TO \$10,000

CLOSED CYCLE (4K) REFRIG. --.-- \$35,000 TO $\$ 70,000$

. DOCLMENTATION AND MANUALS --.---- \$2,000 TO \$50,000

- TOTAL COST FOR MINIMUM SYSTEM ---------\$102,000

- total cost FOR DUAL MASER,

MULTIPLE-UP-CONVERTER SYSTEM $\$ 800,000$

Figure 14. Ultra-low-noise microwave amplifiers cost ranges. 
P. P. Cioffi, "Approach to the Ideal Magnetic Circuit Concept through Superconductivity," J. Appl. Phys., Vol. 33, pp. 875-879, Mar. 1962.

W. H. Higa and E. Wiebe, "A Simplified Approach to Heat Exchanger Construction for Cryogenic Refrigerators," Cryogenic Technology, Vol. 3, pp. 47-48, 50-51, Mar./Apr. 1967.

S. Petty, D. Neff, and D. Norris, "Low-Noise Receivers: S-Band Parametric Upconverter Development." Jet Propulsion Laboratory Deep Space Network Progress Report 42-48, pp. 31-47, Dec. 15, 1978.

C. C. Johnson, "The Maser Experiment," 4th Space Congress: The Challenge of the 1970's, Cocoa Beach, Fla., Canaveral Council of Technical Societies, Apr. 3-6, 1967.

D. Neff and C. R. Moore, "Maser Ruby Inversion Ratios between 27 and $43 \mathrm{GHz}$," to be published.

D. L. Trowbridge, "X-Band Traveling Wave Maser Amplifier," Jet Propulsion Laboratory Deep Space Network Progress Report 42-28, pp. 69-77, Aug. 15, 1975.

J. M. Urech et al., "S-Band Maser Phase Delay Stability Tests," Jet Propulsion Laboratory Deep Space Network Progress Report 42-48, pp. 102-117, Dec. 15, 1978. 


\title{
PHASE AND GROUP DELAY CALIBRATION \\ OF A VERY LONG BASELINE INTERFEROMETER BY EAST COAST VLBI GROUP
}

\author{
A. E. E. Rogers \\ Haystack Observatory
}

\begin{abstract}
Precisely timed pulses injected into the input of each receiver have been used to calibrate the phase and group delay through each interferometer terminal. The short duration (less than 35 picoseconds) pulses are generated at a $1 \mathrm{MHz}$ rate directly from the output of the frequency standard. The pulses are injected into the receiver at a level low enough to produce less than one percent increase in system temperature, yet can be extracted during processing with a high enough signal-to-noise ratio to determine the phase of the calibration "rails" within 1 degree in 1 second of integration. The calibration system also includes precise cable measurement electronics and a pulse echo for clock synchronization.
\end{abstract}




\section{INTRODUCTION}

Very long baseline interferometer (VLBI) systems used for geodetic applications have to be either extremely phase and delay stable or be accurately calibrated. This paper describes a phase and group delay calibration system with millimeter level precision. The calibrator consists of two basic subsystems. The first is a tunnel-diode pulse generator; the second is a cable phase delay measurement subsystem.

\section{THEORY OF OPERATION}

The system utilizes the $5 \mathrm{MHz}$ output from the frequency standard to produce short duration $(<50$ picosecond) calibration pulses at a $1 \mathrm{MHz}$ rate. These pulses are injected into the receiver through an input coupler and are detected by the VLBI processing system. The calibration pulses are generated at a precisely known epoch within the 1 microsecond ambiguity. A component of the $5 \mathrm{MHz}$ signal used to generate the calibration pulses is returned along the same cable and phase compared with the transmitted signal in order to precisely determine the phase delay (with 200 nanosecond ambiguity) in the cable driving the calibration pulse generator. The system block diagram is shown in figure 1. The reflected $5 \mathrm{MHz}$ signal is modulated in order that it may be distinguished from other intermediate reflections. In addition, a component of the calibration pulse itself is returned along the cable and can be viewed on an oscilloscope to resolve the 200 nanosecond ambiguity present in the cable measurement subsystem.

\section{PULSE GENERATOR}

\section{General Description}

The phase calibrator consists of a tunnel-diode pulse generator driven by a $5 \mathrm{MHz}$ square wave. The pulses (both positive and negative) emerge from the tunnel diode and are gated with a diode attenuator so that only one positive pulse per microsecond appears at the output. The block diagram of the pulse generator is shown in figure 2 .

\section{Pulse Duration}

The tunnel-diode switching time $\mathrm{t}$ (at constant current) is given approximately by the following equation for constant a current drive.

$$
\mathrm{t}=(\mathrm{VF}-\mathrm{VP})^{*} \mathrm{CV} /(\mathrm{IP}-\mathrm{IV})
$$

Where $\quad \mathrm{VF}=$ Forward voltage

$$
\mathrm{VP}=\text { Peak point voltage }
$$$$
\text { IP }=\text { Peak point current }
$$

$575 \mathrm{mV}$

$90 \mathrm{mV}$

$10 \mathrm{~mA}$ (increased to $16 \mathrm{~mA}$ in later units) 

IV = Valley point current
$1 \mathrm{~mA}$
$\mathrm{CV}=$ Valley point terminal capacitance $1 \mathrm{pF}$. Thus $\mathrm{t}=$ approx 50 picoseconds. (30 picoseconds in later units)

The condition of constant current is not well maintained as the driving impedance is close to 50 ohms so that the load line intersection point is somewhat below VF. The effect is to reduce the pulse voltage without having much effect on the switching time.

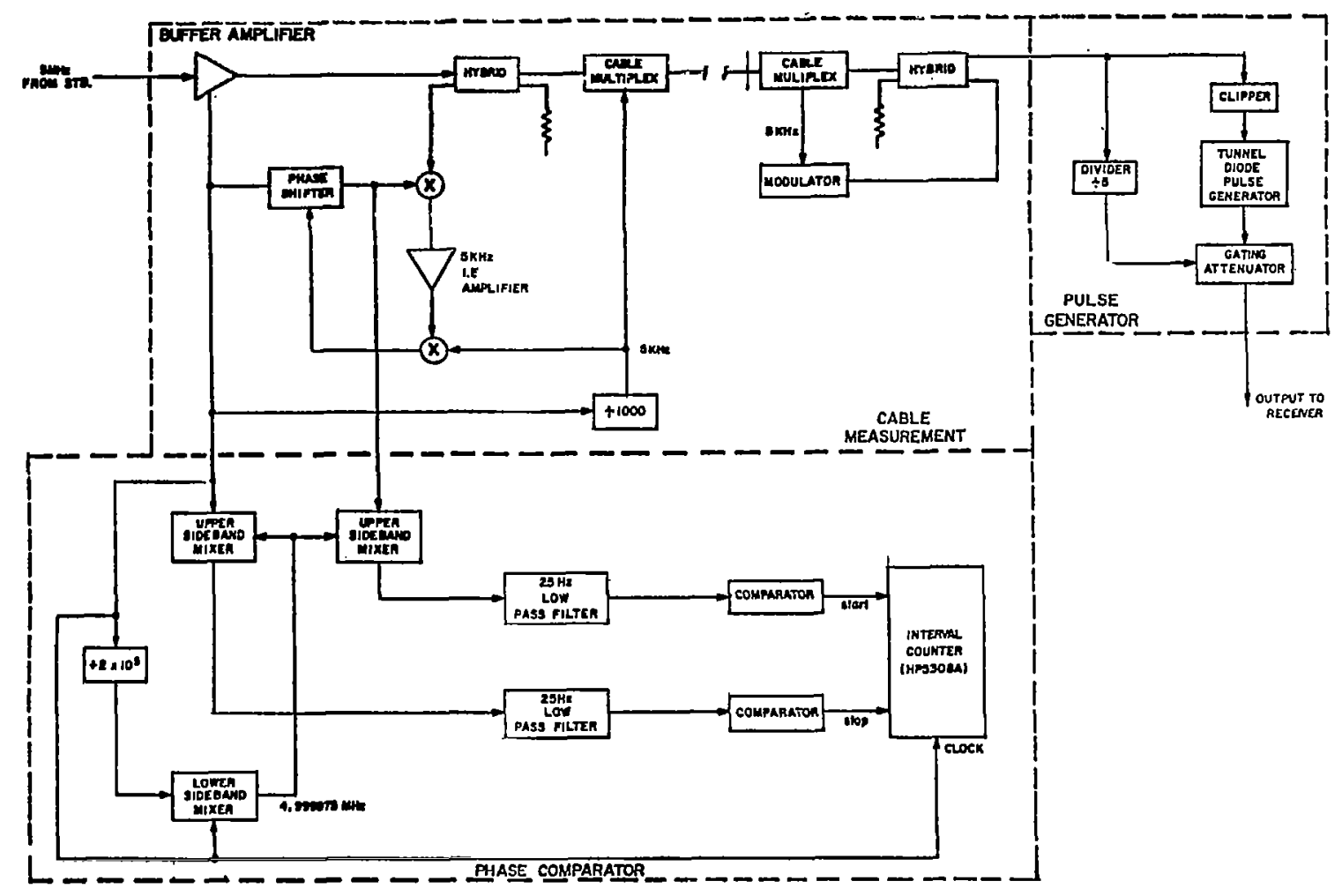

Figure 1. Delay calibration system block diagram.

\section{Output Power}

The output temperature (averaged over several $\mathrm{MHz}$ ) is approximately:

$$
2(\mathrm{VL}-\mathrm{VP})^{2} /\left(\mathrm{RL}^{*}\left(\mathrm{w}^{2} \mathrm{t}^{2} / 12+1\right)^{*} \mathrm{~T}^{*} \mathrm{Kw}^{2}\right) \text { approx } 5 \times 10^{4} \operatorname{deg} \mathrm{K} \text { (at } 10 \mathrm{GHz} \text { ) }
$$

where

$$
\begin{aligned}
\mathrm{w} / 2 \mathrm{pi} & =\text { Center Frequency }(\mathrm{Hz}) \\
\mathrm{T} & =\text { Pulse Repetition Period } \\
\mathrm{RL} & =\text { Load Resistance Seen by Diode } \\
\mathrm{K} & =\text { Boltzmann Constant } \\
\mathrm{VL} & =\text { Load line intersection voltage }
\end{aligned}
$$




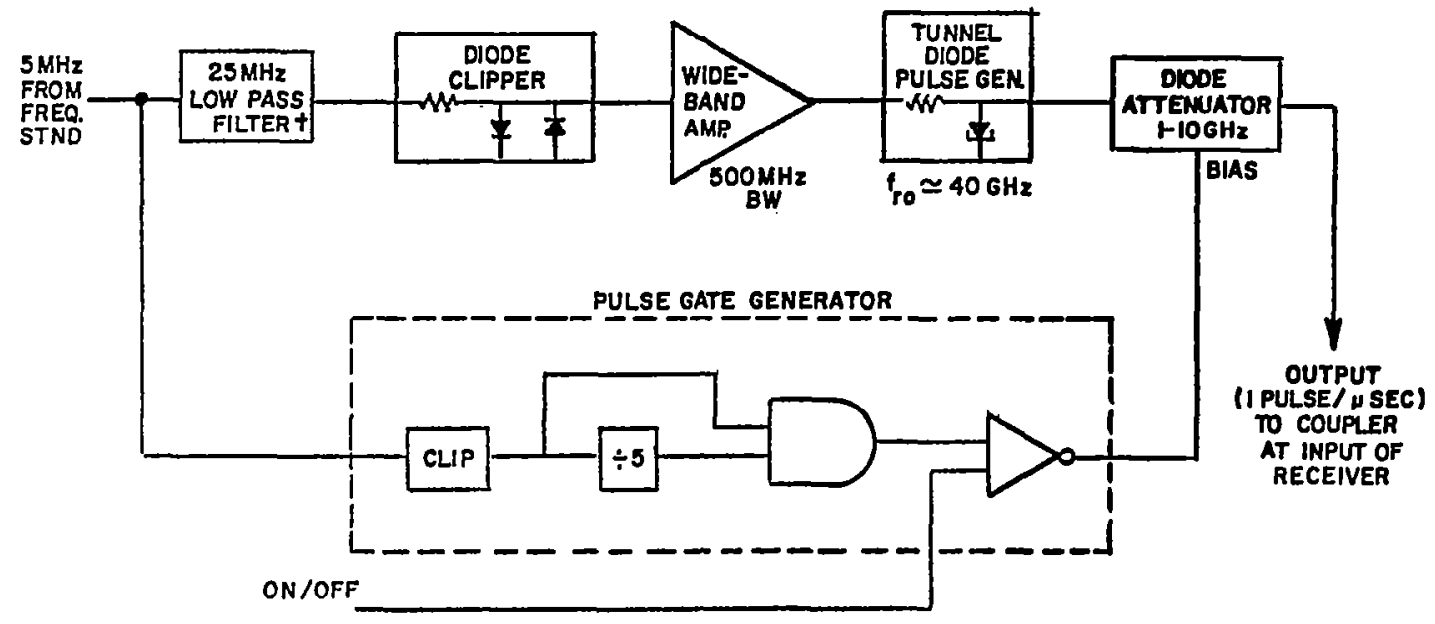

† PART OF CABLE MULTIPLEXER

Figure 2. Delay calibrator pulse generator.

\section{CABLE MEASUREMENT SYSTEM}

\section{General Description}

The delay through a cable changes with temperature (approx $1 \times 10^{*}-4$ per deg $C$ ) and flexure. Pressurized cables also change their electrical length with pressure. The phase delay through a cable also varies in almost the same way as the group delay but with small differences owing to dispersion and multiple-reflection effects.

\section{Theory}

The transmitted signal VT(t) is reflected after modulation by $m(t)$ so that the signal received back at the transmitting end is

$$
V R(t)=m(t) R e(\exp (j w t) \exp (-2 j p)) \quad R e=\text { real part of }
$$

This signal is mixed with a version of the transmitted signal phase shifted by $\mathrm{P}$ and low-pass filtered to form

$$
V D(t)=m(t) \operatorname{Re}(\exp (-2 j p) \exp (j P))
$$

which is mixed with the modulation and low pass filtered again so that

$$
V o(t)=\left\langle m^{2}(t)\right\rangle \cos (2 p-P) .
$$


Vo(t) is integrated and the loop closed on the phase shifter so that

$$
P=2 p+2 \pi(N \pm 1 / 4) \text { where } N \text { is an integer constant }
$$

allowing the cable phase-delay $\mathrm{p}$ to be measured from the measurement of $\mathrm{P}$. A high-resolution phase comparator has been developed for the measurement of $\mathbf{P}$ with picosecond precision.

\section{PICOSECOND PHASE COMPARATOR}

The signal whose phase is to be measured is mixed down from $5 \mathrm{MHz}$ to $25 \mathrm{~Hz}$ using a local oscillator signal of $5 \mathrm{MHz}-25 \mathrm{~Hz}$ which is derived from the reference signal. The mixing operation results in a time expansion of $2 \times 10^{* *} 5$ which is sufficiently large that a digital time interval counter (such as HP5308A counter with D/A converter 5311B) operating at a $5 \mathrm{MHz}$ clock rate can now be used to determine the phase of the $25 \mathrm{~Hz}$ signal with respect to the reference $25 \mathrm{~Hz}$ signal with quantization of only $1.8 \times 10^{* *}-3$ degrees. One count corresponds to 1 picosecond two-way phase delay or 0.5 picosecond one-way delay. Cycle averaging can be used to provide even higher resolution.

Figure 1 shows a block diagram of the comparator. The outputs of the comparator are two $25 \mathrm{~Hz}$ square waves with relative phases equal to the relative phases of the $5 \mathrm{MHz}$ input signals. Single sideband mixing and filters are required to minimize the presence of spurious image signals and harmonics.

\section{MEASUREMENTS OF CALIBRATOR PERFORMANCE}

Output power can be measured by comparing output with that of a noise diode. A typical output power level is $60 \mathrm{Kelvin}$ at $7850 \mathrm{MHz}$ when coupled into the receiver through a $30 \mathrm{~dB}$ coupler. The VLBI processor requires only a relatively weak calibration signal since the processor ex tracts the signal by correlating the signals from each station with a sine and cosine at the frequency of the calibrator rail. With this method, a calibrator signal strength of 1 percent is sufficient for the phase to be determined within 1-degree rms with 1 second coherent integration.

The stability of the calibrator was measured by observing the phase of the frequency rails from the calibrator in the receiver video output. The overall sensitivity of the phase calibrator to temperature changes was measured by cycling the temperature of the calibrator from 30 degrees $C$ to 40 degrees $\mathrm{C}$. In addition, an attempt was made to thermally isolate the individual components within the calibrator and so measure the individual coefficients. The results of these measurements are shown in table 1. The sensitivity of the calibrator to input ac voltage changes was undetectable being less than 1 ps per percentage voltage change. 
Table 1

Temperature Sensitivity of Phase Calibrator

\begin{tabular}{|l|l|}
\hline 25 MHz low pass filter & $+1.0+/-5 \mathrm{ps} / \mathrm{deg} . \mathrm{C}$ \\
Diode clipper & $+10+/-2 \mathrm{ps} / \mathrm{deg} . \mathrm{C}$ \\
Wideband amplifier & $-3+/-1 \mathrm{ps} / \mathrm{deg} . \mathrm{C}^{*}$ \\
Tunnel Diode & $-2+/-1 \mathrm{ps} / \mathrm{deg} . \mathrm{C}^{*}$ \\
Overall sensitivity & $6+/-2 \mathrm{ps} / \mathrm{deg}$. C \\
(measured separately) & \\
\hline
\end{tabular}

*A negative coefficient indicates that an increase in temperature decreases the phase delay through the device.

\section{TESTS OF THE VLBI RECEIVER AND CALIBRATOR}

In order to acquire high quality VLBI data, it is useful to test the performance of each interferometer terminal. An adjustable line in the calibrator cable allows testing of the cable measurement system and can be used to change the phase of the calibrator. Since the frequency of the calibration rail being observed is known, a complete test of the system can be made by comparing the change in cable length from the cable measurement system with that computed from the changc in fringe count observed when the adjustable line is changed. The coherence of the calibrator and receiver local oscillator can be measured from the ratio of power in the calibrator rail to the total power contribution of the calibrator. The fractional coherence is given by

$$
(\mathrm{Ts} / \mathrm{Tcal})^{1 / 2} \text { Acal }
$$

where

$$
\begin{aligned}
\text { Ts } & =\text { system temperature } \\
\text { Tcal } & =\text { effective temperature of the calibrator from total power } \\
\text { Acal } & =\text { measured correlation coefficient of calibrator. }
\end{aligned}
$$

Complete tests of the calibrator performance can be made by injecting a common noise source in to two terminals to form a "zero baseline." In this configuration, receiver local oscillator phases and IF cable lengths can be changed. If the calibration system is working correctly, these changes should produce no changes in the calibrated delay measurements. In addition, the calibrator cable length can be changed, and the observed delay changes compared with the corresponding changes in the cable measurement readout. Figure 3 gives a summary of the calibration technique. 
REASON FOR USE:

TO CALIBRATE DRIFTS IN GROUP AND PHASE DELAY THROUGH:

1) LOW NOISE AMPLIFIERS - WITH TEMPERATURE AND MAGNETIC FIELD 3) LOCAL OSCILLATORS - WITH TEMPERATURE

METHOD:

INJECTION OF SHORT DURATION PULSES $(\approx 35$ PS $(1 \mathrm{CM}))$ INTO RECEIVER AT A RATE OF 1 PULSE/MICROSECOND

DETECTION METHOD:

EXTRACTION OF THE PHASE OF EACH CALIBRATION "RAIL"

IN THE DATA STREAM FROM EACH STATION

SENSITIVITY:

WITY A SIGNAL OF ONLY 1\% (OF TOTAL POWER) PHASE CAN BE MEASURED TO WITHIN 1 DEGREE R.M.S. IN 1 SECOND

PERFORMANCE :

1) PRECISION

1 PS $(0.3 \mathrm{MM})$

2) TEMPERATURE COEFF, 6 PS $/{ }^{\circ} \mathrm{C}$

EXPERIMENTS THAT DEMONSTRATE PERFORMANCE:

1) CALIBRATION SEEN TO IMPROVE RESULTS IN EARLY EXPTS 72-74

2) HAYSTACK-WESTFORD BASELINE REPEATABILITY 5 MM 74-76

3) "Z.ero baseline tests" - show agreement between MEASURED DELAYS, CABLE LENGTH ELECTRONIC READOUT AND LINE STRETCHER MECHANICAL READOUT TO BETTER THAN

4) RESIDUALS OF LONG RASELINE EXPTS EXAMINED WITH AND WITHOUT CALIBRATION CLEARLY SHOW THE EFFECTIVENESS OF THE CALIBRATION SYSTEM

Figure 3. Phase and group delay calibration. 


\title{
USE OF A DIGITAL TONE EXTRACTOR FOR REAL-TIME PHASE ANALYSIS*
}

\author{
Elliott H. Sigman and Gary S. Parks \\ Jet Propulsion Laboratory \\ California Institute of Technology
}

\begin{abstract}
With demands for increased accuracy and reliability for very long baseline interferometry (VLBI) experiments, single station phase calibration techniques are becoming a reality. The presence of phase calibrator tones within the VLBI band-pass make it possible, for the first time, to monitor accurately the health of the entire instrumental data path. The digital tone extractor, designed and built by the Jet Propulsion Laboratory is exactly such a device. This real-time VLBI system monitor is a safeguard against most instrumental breakdowns and operator errors.
\end{abstract}




\section{INTRODUCTION}

A digital tone extractor which monitors the phase integrity of a VLBI recording system in real time has been developed at the Jet Propulsion Laboratory. This digital tone extractor monitors phase calibrator tones injected at the antenna and tracks their phase as a function of time. It is capable of maintaining 0.001 cycle phase accuracy over the course of a VLBI experiment in accordance with the accuracy requirements of centimeter VLBI work. This real-time VLBI system monitor is a safeguard against most instrumental breakdowns and operator errors.

\section{IMPLEMENTATION}

The digital tone extractor interconnects with the VLBI recording system to extract phase calibrator tones from the digital data stream. A typical VLBI system with a digital tone extractor is shown in figure 1 .

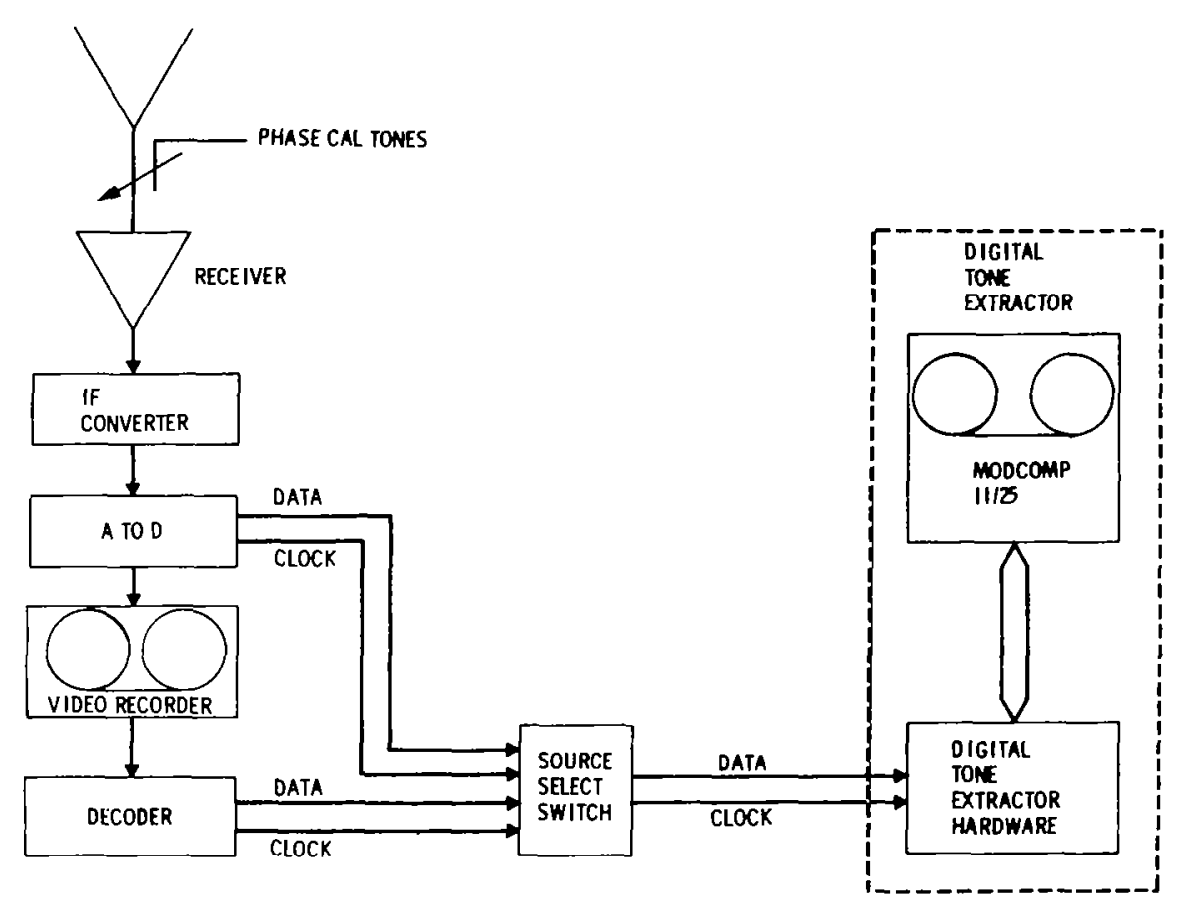

Figure 1. VLBI hardware configuration.

Stable tones from the phase calibrator are injected into the system at the antenna. From the antenna, the signals go through the front end of the receiver and are down-converted to baseband by the IF converter. A one-bit A to D converter digitizes the baseband data, after which it is formatted for recording on the video recorder. 
The digital tone extractor can monitor the digital data stream either before or after it has been recorded on tape as selected by the SOURCE SELECT switch. When monitoring data recorded on tape, a decoder is used which is connected to the read-after-write head of the tape recorder. Along with the digital data, clock signals which are synchronous with the data are sent to the digital tone extractor.

The digital tone extractor synthesizes two tones in phase quadrature at the baseband frequency of a phase calibrator tone. These synthesized tones are cross-correlated with the incoming data and integrated to provide sine and cosine correlations. From these two correlations, the residual phase and amplitude of a phase calibrator tone is obtained.

A block diagram of the digital tone extractor is presented in figure 2. As shown, a programmable digital frequency synthesizer in the digital tone extractor synthesizes a tone at the baseband frequency of a phase calibrator tone. The clock that is brought in with the data is used to synchronize this synthesizer. Quadrature sine and cosine outputs of the synthesizer are cross-correlated with the incoming data, and the results are accumulated in two 32-bit registers. With 32-bit resolution, the hardware is capable of maintaining 0.001 cycle accuracy for up to 1 second of integration time. The integration time is controlled by a precision clock which is externally programmable. The sine and cosine accumulations are periodically sent to a MODCOMP mini-computer through a microprocessor. The phase of the frequency synthesizer is also updated by the MODCOMP. In the MODCOMP's software, 64-bit arithmetic is used which enables the digital tone extractor to maintain $12 \times 10^{-6}$ degree accuracy over a 24 -hour period.

The hardware of the digital tone extractor is controlled by the microprocessor which can store the phase and frequency of the tone synthesizer and the sine and cosine accumulations at any time and retrieve them at a later time. When these numbers are retrieved, the phase of the tone synthesizer can also be updated by the microprocessor. With this capability, the digital tone extractor can monitor time-multiplexed tones such as are encountered in time-multiplexed bandwidth synthesis experiments.

A typical output from a two channel bandwidth synthesis experiment is presented in figure 3 . Each printout represents the results of 15 integrations of 12 seconds duration each. The average residual phase and average magnitude or amplitude are indicated in the last two lines. The slope of a line, which is a least squares fit to the 15 points, is indicated by the "Phase Drift." The scatter from this line is shown by the "RMS Phase Jitter."

During a VLBI experiment, excessive phase drift or rms phase jitter can alert the operator to a malfunction in the VLBI recording system. An alarm could be activated by the digital tone extractor sof tware to warn the operator when these numbers become excessive. 


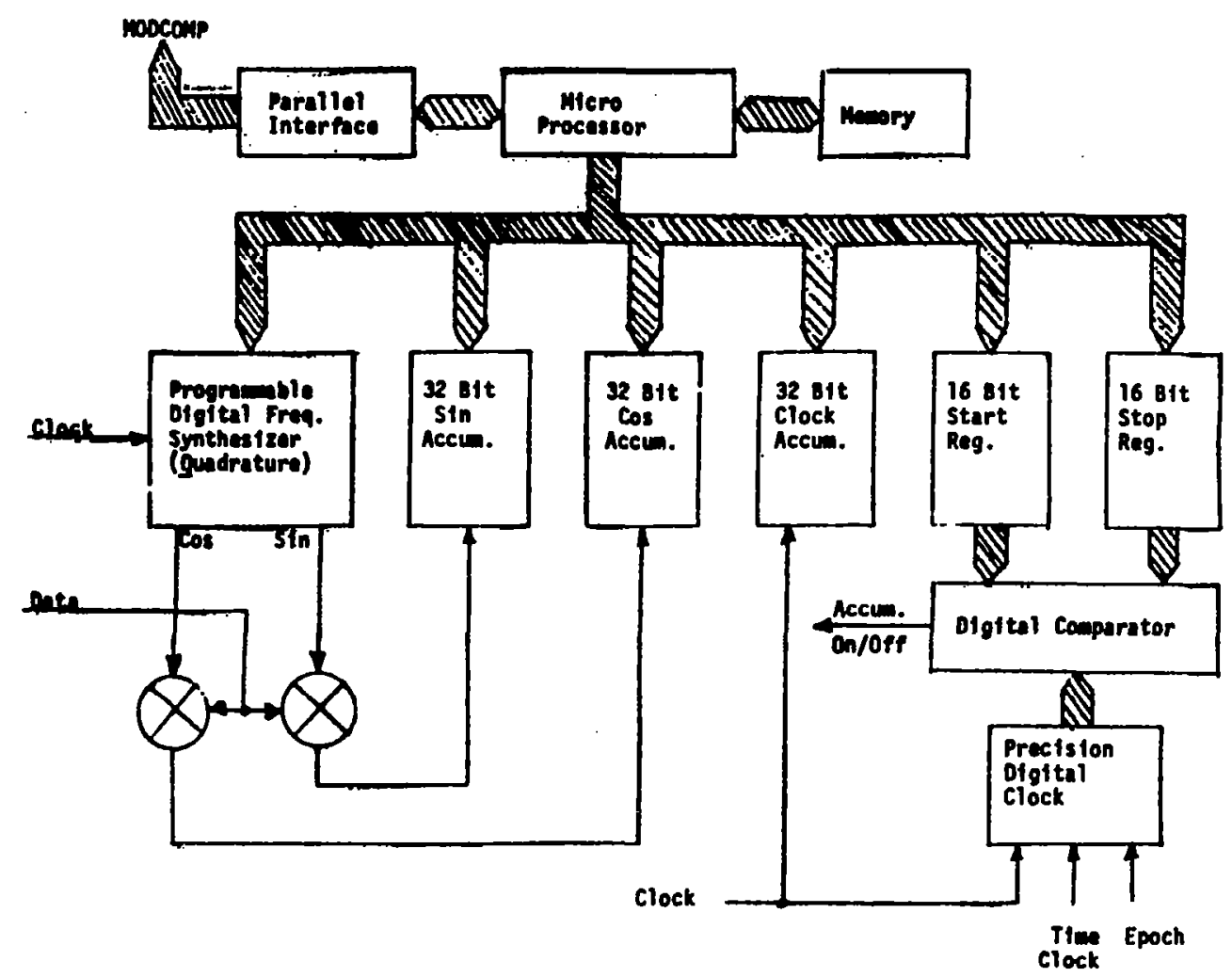

Figure 2. Digital tone extractor.

\section{APPLICATIONS}

The digital tone extractor performs, in real time, the same process on phase calibrator tones as the VLBI correlator. Since the digital tone extractor can monitor what is actually recorded on tape, it can indicate in real time the performance of the entire recording system. Thus, it can warn of an equipment malfunction when it happens and can save an experiment.

The digital tone extractor can also be used as a tool for debugging the VLBI system. It can look at data before and after it is recorded on tape to isolate faults in the recording system. In addition, a frequency synthesizer can be fed into a part of the VLBI system, and the phase can be monitored in order to isolate equipment with phase instabilities.

In addition to VLBI applications, the digital tone extractor has other uses which are of interest at the Jet Propulsion Laboratory. For example, it can be used to track spacecraft tones. The software can be programmed with a polynomial for frequency in order to track the frequency fluctuations of a spacecraft. Alternatively, an iterative software algorithm could be used to make the digital tone extractor follow the spacecraft frequency fluctuations and extract the Doppler shift of the spacecraft. 


\begin{tabular}{|c|c|c|}
\hline \multirow[t]{2}{*}{ BASEBAND TONE NUMBER 1} & CH. 1 & $\begin{array}{l}\text { PHASE DRIFT }=-0.009 \text { DEG } / \text { SEC } 130: 11: 42: 36 \\
\text { RMS PHASE JITTER }=1.096 \text { DEG } \\
\text { AVG PHASE }=152.01 \text { DEG } \\
\text { MAGNITUDE }=0.1354195785\end{array}$ \\
\hline & $\mathrm{CH} .2$ & $\begin{array}{l}\text { PHASE DRIFT }=-0.012 \text { DEG/SEC } 130: 11: 42: 36 \\
\text { RMS PHASE JITTER }=1.192 \text { DEG } \\
\text { AVG PHASE }=-144.132 \text { DEG } \\
\text { MAGNITUDE }=0.0869244525\end{array}$ \\
\hline \multirow[t]{2}{*}{ BASEBAND TONE NUMBER 1} & $\mathrm{CH} . \mathrm{l}$ & $\begin{array}{l}\text { PHASE DRIFT }=0.001 \text { DEG/SEC 130:11:46:36 } \\
\text { RMS PHASE JITTER }=0.662 \text { DEG } \\
\text { AVG PHASE }=151.525 \text { DEG } \\
\text { MAGNITUDE }=0.1355491886\end{array}$ \\
\hline & $\mathrm{CH} .2$ & $\begin{array}{l}\text { PHASE DRIFT }=-0.003 \text { DEG/SEC } 130: 11: 46: 36 \\
\text { RMS PHASE JITTER }=0.884 \text { DEG } \\
\text { AVG PHASE }=-145.476 \text { DEG } \\
\text { MAGNITUDE }=0.0868894043\end{array}$ \\
\hline \multirow[t]{2}{*}{ BASEBAND TONE NUMBER 1} & $\mathrm{CH} . \mathrm{I}$ & $\begin{array}{l}\text { PHASE DRIFT }=0.043 \text { DEG } / \text { SEC } 130: 11: 50: 36 \\
\text { RMS PHASE JITTER }=3.661 \text { DEG } \\
\text { AVG PHASE }=154.289 \text { DEG } \\
\text { MAGNITUDE }=0.1361540671\end{array}$ \\
\hline & $\mathrm{CH} .2$ & $\begin{array}{l}\text { PHASE DRIFT }=0.035 \text { DEG } / \text { SEC } 130: 11: 50: 36 \\
\text { RMS PHASE JITTER }=3.345 \text { DEG } \\
\text { AVG PHASE }=-144.127 \text { DEG } \\
\text { MAGNITUDE }=0.0872003583\end{array}$ \\
\hline
\end{tabular}

Figure 3. Digital tone extractor output.

\section{ACKNOWLEDGMENTS}

The concept of the digital tone extractor was originated by J. L. Fanselow. His enthusiastic support and suggestions have been instrumental in developing this device. 


\title{
ĊONSIDERATIONS IN THE PLACEMENT OF PHASE CALIBRATOR TONES*
}

\author{
L. E. Young \\ Jet Propulsion Laboratory \\ California Institute of Technology
}

\begin{abstract}
In the use of tones to calibrate unwanted instrumental phases for very long baseline interferometry experiments, certain problems exist which are related to the placement of these phase calibrator tones, for example:

1. A bias exists in an analytically generated stopping function used during correlation if its frequency satisfies the following condition; $f=f_{s} m / n$, where $f_{s}=$ sampling frequency, $\mathrm{m}=$ any integer, $\mathrm{n}=$ any odd integer.

2. Due to the quantized representation of sine waves in the stopping function, odd harmonics of the fundamental frequencies are generated. Several mechanisms are available through which these harmonics can cause errors in the residual phase extracted from the recorded tones.
\end{abstract}

3. When multiple tones are injected into a pass band, intermodulation products can occur.

The magnitude of these various effects will be discussed along with strategies designed to avoid them.

\footnotetext{
*This paper represents the results of one phase of research carried out at the Jet Propulsion Laboratory, California Institute of Technology, under Contract No. NAS 7-100, sponsored by the National Aeronautics and Space Administration.
} 
The very long baseline interferometry (VLBI) development group, at the Jet Propulsion Laboratory (JPL), has been investigating the phase calibrator technique for measuring instrumental delays added to radio data at the receiving station. In the JPL procedure, two or more tones are injected into each channel of incoming data. Multiple tones are required for the adequate phase calibration of spacecraft signals, which are received in different localized regions of the passband from day to day. When recorded with quasar signals, multiple tones provide redundancy, as well as a continuous monitor of the passband phase versus frequency response. The availability of multiple tones simplifies the system of data taking and analysis by allowing the bandwidth synthesis process to be done on a single scan basis, without the need for extensive measurements to be taken at the receiving stations. This is felt to be an important benefit of multiple tone phase calibration for achieving accurate results with an operational program of VLBI experiments.

In our experience with phase calibration, we have noticed some systematic sources of phase errors, some of which were not previously understood among the radio-astronomy community, which are the subject of this talk.

Shown in figure 1 are hypothetical spectra of tones in the recorded data and in the stopping function. The residual tone phase is extracted in the correlation process after it is beat to 0 frequency with an analytically generated stopping function. Systematic errors stem from the coincidence of extraneous tones in the data with tones in the stopping function. These accidental coincidences are of two types. For the first type, the interfering tone in the data occurs at or near the frequency of the phase calibrator tone; while, for the second type, the interfering data tone is stopped by one of the harmonics in the stopping function. I will briefly describe the origin of the extra tones in the data and stopping function, with emphasis on the troublesome case of amplitude occurring at 0 frequency in the spectra of both the recorded data and the stopping function. In particular, I will be describing the JPL/CIT 4 megabits per second systems used for recording and correlating data, but the descriptions can be easily generalized to other systems.

The stopping function is generated in three steps as is shown in figure 2. In the first step, an analytical model is produced with the nominal frequency of the phase calibration tone and a known initial phase. However, before this model is compared with the data it is quantized to three levels and sampled at 4 megabits per second. As a result of the quantization, all odd harmonics of the nominal tone frequency are present, and the sampling causes these harmonics to be aliased in to the 2 $\mathrm{MHz}$ passband. The tone stopping functions planned for the JPL/CIT Block I/II correlators have 255 levels, which will practically eliminate any harmonics. The design of these correlators will be discussed by Dr. Rogstad in Thursday afternoon's session.

The recorded data also has extra tones resulting from various sources. The $5 \mathrm{kHz}$ modulation used in the cable compensator causes small side bands at $\pm 5 \mathrm{kHz}$ to the injected tones. There is generally a bit-stream bias present; that is, an excess of either 1 bit or 0 bits in the recorded data. For example, this can be caused by dc level offsets in the sampling electronics. Some power feeds into the data from tones in the opposite sideband. When multiple tones are used, intermodulation products can become important. These intermodulation products are formed when the tones in the data are converted to a two-level bit-stream. (See figure 3.) 


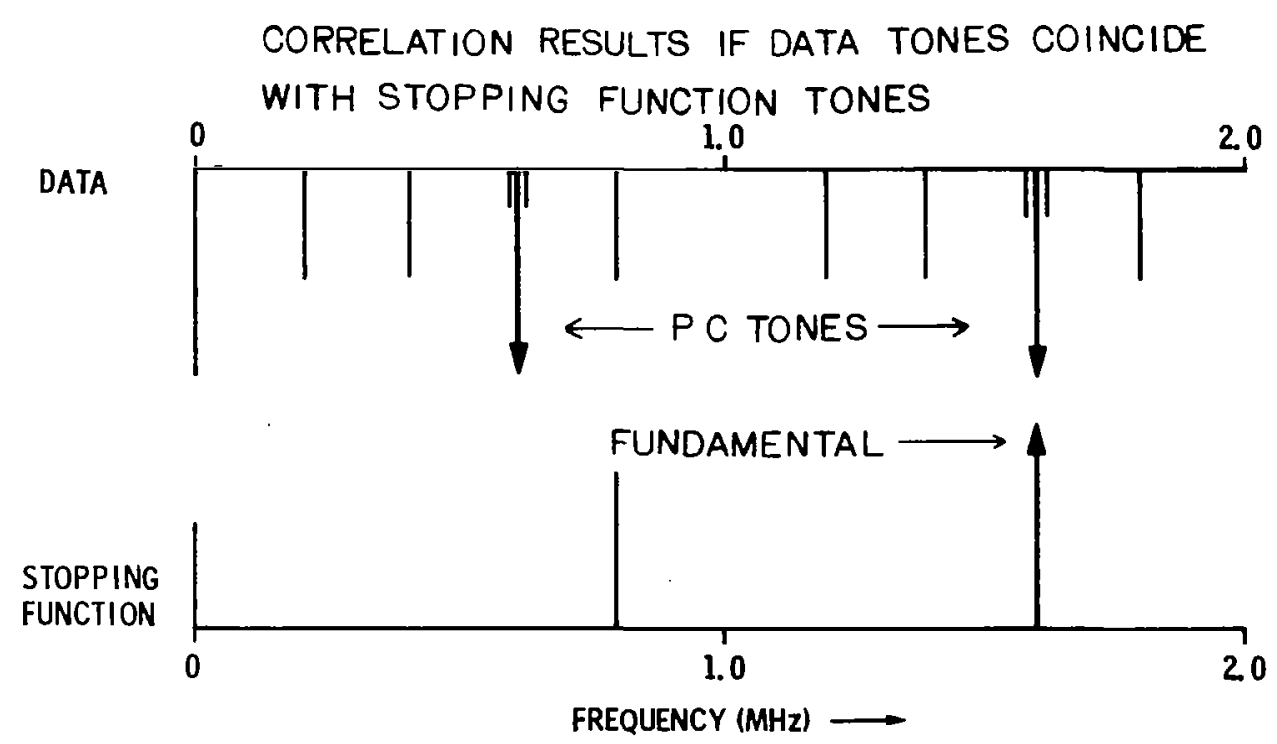

Figure 1. Systematic error sources.

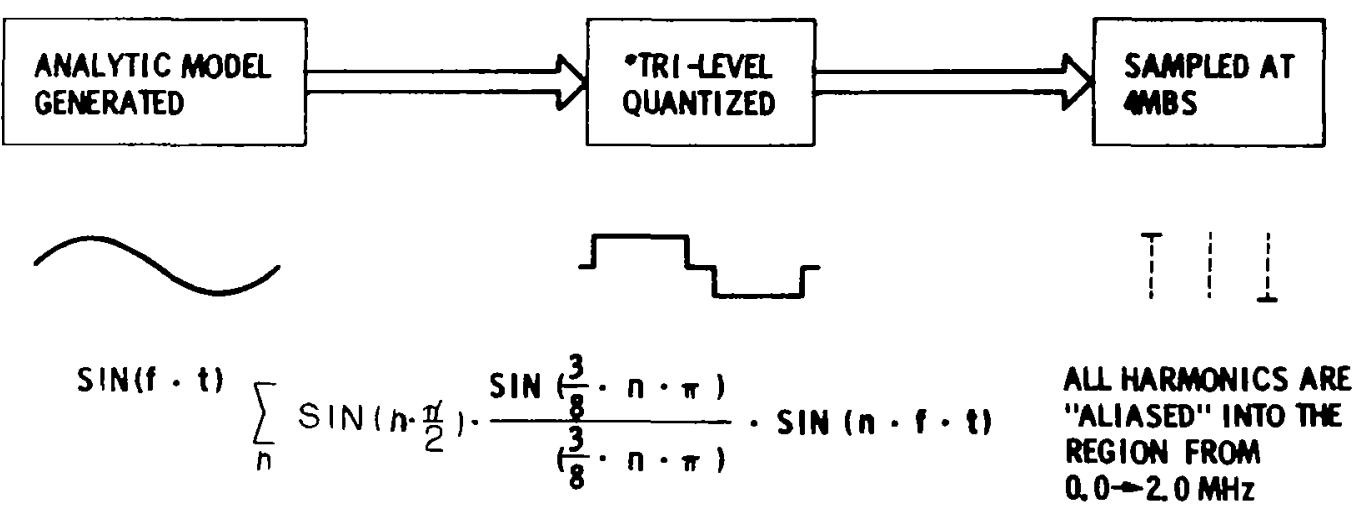

-A 256 LFVEL QUANTIZED STOPPING FUNCTION FOR TONES IS PLANNED FOR THE JPL/CIT BLOCK II CORRELATOR

Figure 2. Stopping function. 
(1) PHASE CALIBRATOR TONES
(2) SIDEBANDS AT $\pm 5 \mathrm{kHZ}$
(3) BIT STREAM BIAS,

-4) INTERMODULATION PRODUCTS

(5) SIDEBAND FEETHROUGH

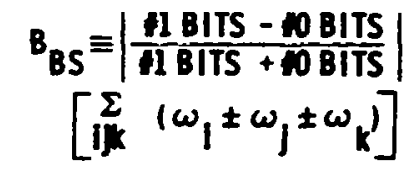

DATA
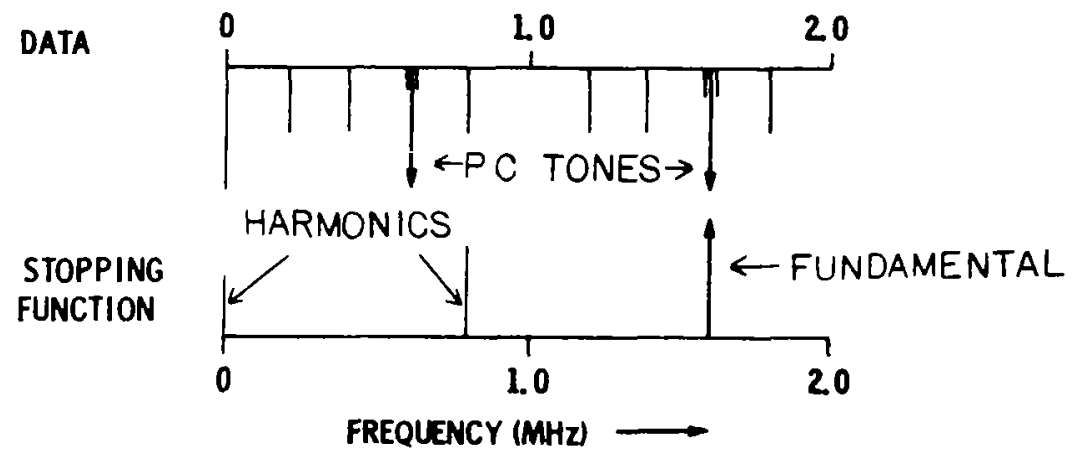

- IMPORTANT ONLY FOR MULTIPLE TONE CASE

Figure 3. Tones present in recorded data.

Because the bit-stream bias is frequently comparable in amplitude to the phase calibrator tones, let us consider the origin of amplitude in the stopping function at 0 frequency. Figure 4 pictures the tri-level quantized stopping function for a tone at $4 / 3 \mathrm{MHz}$. It can be seen that this frequency is commensurate with the sampling rate in such a way that there are exactly 3 bits per cycle, and for the initial phase shown, there are 2 bits at the one level for each bit sampled at the zero level. A stopping function bias $\left(\mathrm{B}_{\mathrm{SF}}\right)$ occurs for some initial phase whenever the stopping function frequency can be expressed as $F=S \cdot m / n$, where $S$ is the sampling rate, $m$ is any integer, and $n$ is any odd integer. This is simply the condition for an odd number of bits per cycle and for an odd harmonic to fall at, or alias to, 0 frequency.

The effect of this bias in the stopping function is that it correlates with the bit-stream bias $\left(B_{B S}\right)$, giving an erroneous addition to the amplitude and phase of the stopped phase calibrator tone.

Figure 5 illustrates how an approximate angular error can be attributed to this erroneous amplitude. Here, the correlator output has been drawn as a vector in the complex plane representing the phase and amplitude of a correlated signal. The resultant vector $\vec{A}$ is seen to be displaced in angle from the phase due to the phase calibrator tone by $\Delta \phi$, where, for $\Delta \phi<<\pi / 2, \Delta \phi \leq \mathrm{TAN}^{-1}$ (erroneous correlated amplitude/P.C. tone correlation amplitude).

Figure 6 contains formulas to express the limits on systematic phase errors mentioned in this talk. These formulas are valid only if the phase error is much less than $\pi / 2$. In short, we have found that some care must be taken to prevent systematic phase errors due to the incorrect placement of phase 
calibrator tones, but, with the use of the formulas presented in figure 6, as well as calculations of $\mathrm{B}_{\mathrm{SF}}$, we have found this to be a straightforward task.

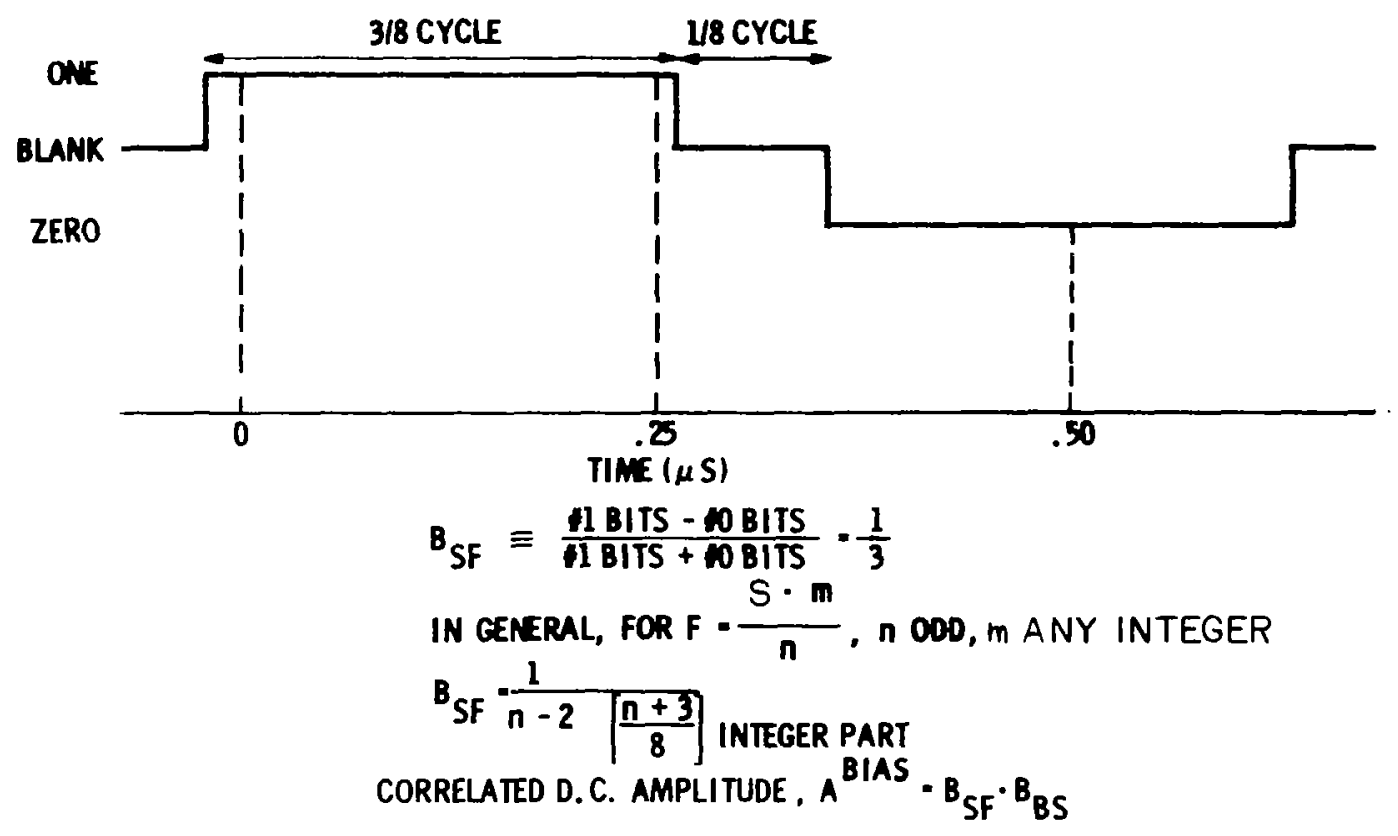

Figure 4. Stopping function for P.C. tone at $F=4 / 3 \mathrm{MHz}$, sampling rate $S=4 \mathrm{MHz}$.

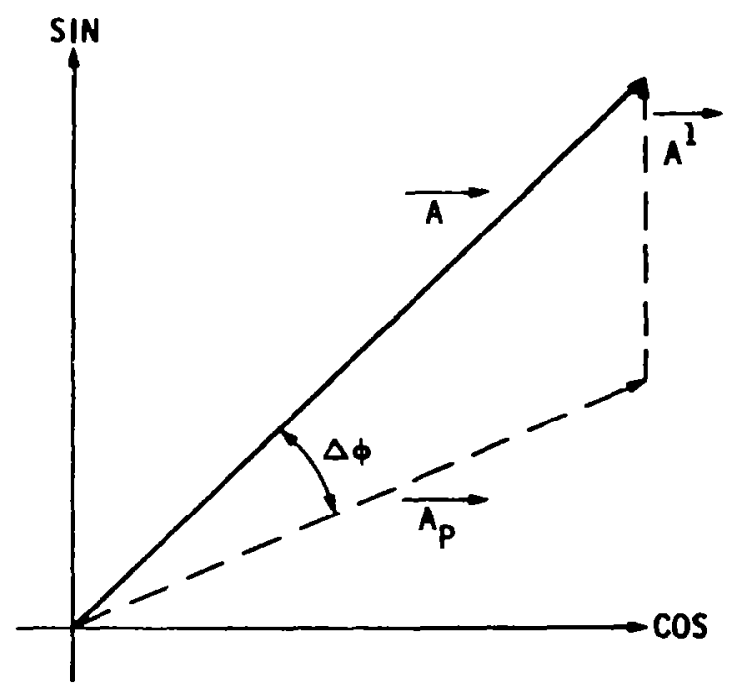

$\vec{A}$ - TOTAL AMPLITUDE AND PHASE IN THE CORRELATOR OUTPIT $\overrightarrow{A_{P}}=$ THE COMPONENT OF $\overrightarrow{A D U E}$ TO THE P.C. TONE

$\overrightarrow{A^{1}}=$ ERROR COMPONENT OF $\vec{A}$ DUE TO ACCIDENTAL CORRELATIONS

$\triangle \phi \leq \operatorname{TAN}^{-1}\left(\frac{A^{1}}{A_{P}}\right.$

Figure 5. Phase error. 
I. D. C. COMPONENTS

$$
\Delta \phi{ }^{B I A S} \leqslant T^{-1}\left[\frac{1.6 \cdot B_{B S} \cdot B_{S F}}{\sqrt{P_{T}^{T}}}\right]
$$

$P_{T}$ IS THE FRACTIONAL PASSBAND POWER IN EACH TONE.

\section{STOPPING FUNCTION HARMONICS}

$$
{ }_{\Delta \phi} \mathrm{SFH}_{(f)} \lesssim \operatorname{TAN}^{-1}\left[\sum_{n} \frac{A_{S F^{(f}}{ }^{\prime}}{A_{S F}(f)} \cdot \frac{A_{D}\left(f_{n}+\Delta f\right)}{\sqrt{2 P_{T}}} \cdot \frac{\operatorname{SIN}\left(\pi \cdot \Delta f \cdot T_{\text {int }}\right)}{\left(\pi \cdot \Delta f \cdot T_{\text {int }}\right)}\right]
$$

$A_{\text {SF }}(f)$ IS THE STOPPING FUNCTION AMPLITUDE AT f, A ${ }^{(f)}$ IS THE

DATA AMPLITUDE AT $f$, AND $\tau_{\text {Int }}$ IS THE CORRELATION INTERVAL.

III. INTERMODULATION PRODUCTS

$\Delta \phi$ INTER $_{(f)} \lesssim \operatorname{TAN}^{-1}\left[\frac{P_{T^{-N_{i n t}}}}{12}\right]$

$N_{\text {int }}$ IS THE NUMBER OF INTERMODULATION PRODUCTS WHICH FALL AT THE FREQUENCY $f$.

Figure 6. Phase error (continued). 


\title{
THE SENSITIVITY OF A VERY LONG BASELINE INTERFEROMETER \\ A. E. E. Rogers and the East Coast VLBI Group \\ Haystack Observatory
}

\begin{abstract}
The theoretical sensitivity of various methods of acquiring and processing interferometer data are compared. It is shown that for a fixed digital recording capacity one bit quantization of single sideband data filtered with a rectangular bandpass and sampled at the Nyquist rate yields the optimum signal-to-noise ratio (SNR). The losses which result from imperfect bandpass, poor image rejection, approximate methods of fringe rotation, "fractional bit correction," and loss of quadrature are discussed. Also discussed is the use of the "complex delay function" as a maximum likelihood fringe estimator.
\end{abstract}




\section{INTRODUCTION}

For interferometric observations of continuum radio sources, the signal-to-noise ratio (SNR) is proportional to the square root of the bandwidth as well as depending linearly on the ratio of the geometric mean of the antenna temperatures to the geometric mean of system temperatures. The constant of proportionality depends on the method of processing. It has been shown (Rogers, 1970) the SNR with the maximum likelihood analog processing is given by:

$$
\mathrm{SNR}=\mathrm{A}(2 \mathrm{BT})^{1 / 2}
$$

where

$$
\begin{aligned}
\mathrm{A} & =\text { Correlation amplitude }=\mathrm{Ta} / \mathrm{Ts} \\
\mathrm{Ta} & =\text { Geometric mean of antenna temperatures (correlated portion) } \\
\mathrm{Ts} & =\text { Geometric mean of system temperatures } \\
\mathrm{B} & =\text { Bandwidth }(\mathrm{Hz}) \\
\mathrm{T} & =\text { Coherent integration time }(\mathrm{sec})
\end{aligned}
$$

The SNR is defined in this equation to be the ratio of the magnitude of the signal vector to the rms of the component of the noise vector normal to the signal vector. Thus in the strong signal case, the rms phase noise (in radians) is (1/SNR). It was also shown (Meeks, 1976) that the best SNR is achieved with single sideband receivers and perfectly rectangular bandpass filters. The magnitude $R$ of the noise vector has a Rayleigh distribution.

$$
P(R)=R\left(e^{-R^{2} / 2}\right)
$$

so that the probability of the noise being falsely interpreted as a signal in a search of $N$ independent channels of delay and delay rate is given by

$$
P E=1-\left(1-e^{-R^{2} / 2}\right)^{N}
$$

which is plotted in figure 1. Thus, an SNR of at least six is required to detect a signal if a large search must be made in delay and rate.

\section{SNR with Digital Data Recording}

If the interferometer data has to be stored or transmitted over a link, then SNR will be limited by the recording or transmission channel capacity. The best SNR is achieved by using the largest possible bandwidth without under-sampling. This condition is achieved by using one bit (two-level sampling of infinitely clipper data) quantization of the data sampled at the Nyquist rate. Over sampling the data produces correlation between samples and increases the noise level while undersampling reduces the signal by aliasing. In this case,

$$
\mathrm{SNR}=(2 / \pi) \mathrm{A}(2 \mathrm{BT})^{1 / 2}
$$




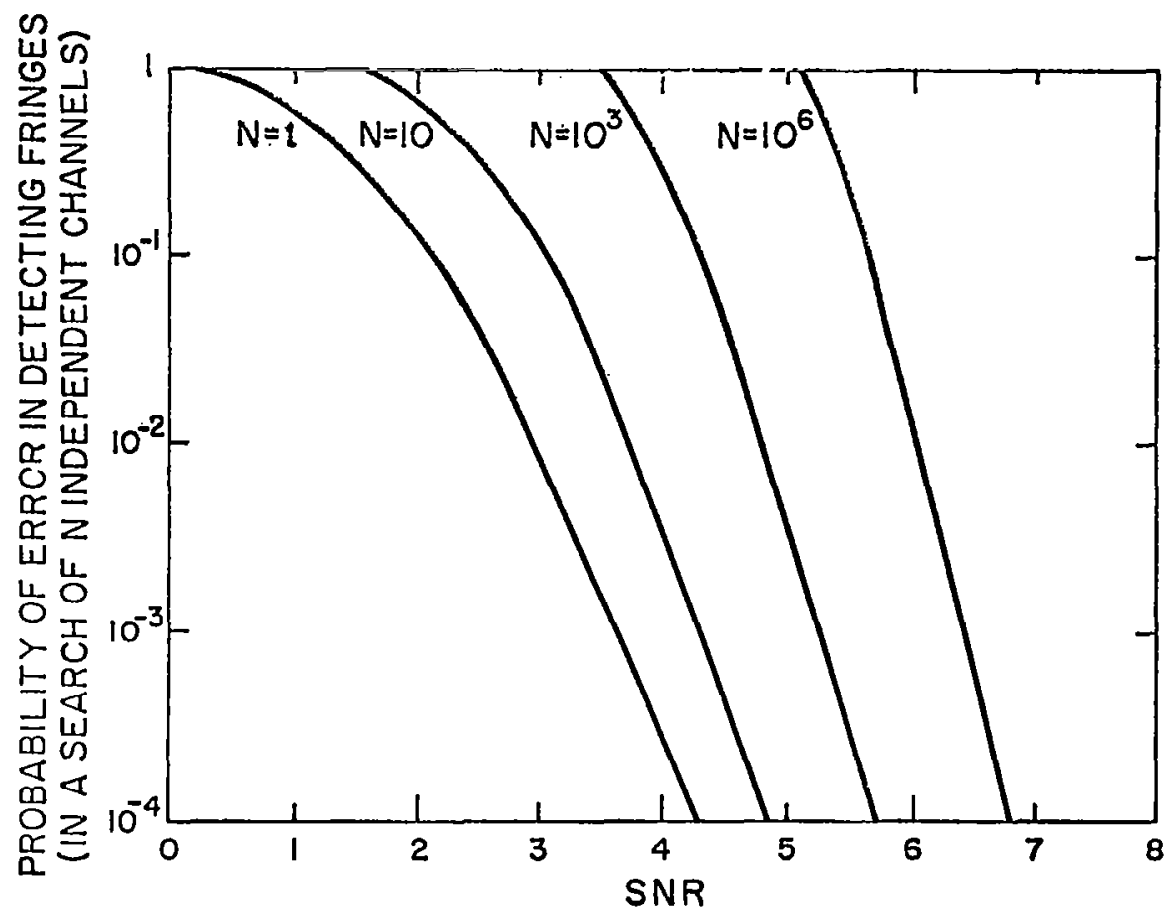

Figure 1.

where $2 \mathrm{BT}=$ the total number of bits processed. The factor $(2 / \pi)$ is the clipping factor which results from the one bit quantization of the signal. The signal is reduced by this factor in applying the Van Vleck correction in going from the cross-correlation $\operatorname{pc}(\tau)$ of the clipped signals to an estimate of the true cross-correlation function $\mathrm{R}(\tau)$ given by

$$
\mathrm{R}(\tau)=\sin ((\pi / 2) \operatorname{pc}(\tau) \approx(\pi / 2) \operatorname{pc}(\tau) \text { when } \operatorname{pc}(\tau)<<1
$$

It should be emphasized that while increasing the number of levels in the quantization and oversampling improve the SNR for the spectral-line interferometry, they result in a loss of SNR in the continuum case. For example, with four-level sampling, the quantization degradation factor is only 1.135 (Bowers and Klingler, 1972) as compared with $(\pi / 2)=1.571$ for the two-level case. The spectral line SNR is thereby increased by 1.384 while the continuum SNR is reduced because the $\sqrt{2}=1.414$ degradation, which results from having to reduce the bandwidth by a factor of two to accommodate the increased number of samples, exceeds the 1.384 gain above. Optimal three-level quantization, however, results in little change in SNR for the continuum and some improvement in the spectral line SNR. 


\section{Loss from Imperfect Bandpass}

There are two loss factors which result from using a bandpass filter which is not perfectly rectangular in shape. The first is due to aliasing or foldover of noise from frequencies above the bandedge $B$, while the second loss factor is due to an increase in the statistical dependence of one sample upon the next. The first loss factor is given by

$$
L_{f}=\int_{0}^{B} B(\omega) d \omega / \int_{0}^{B}(B(\omega)+B(2 B-\omega)) d \omega
$$

where $B(\omega)$ is the bandpass (power response).

When two data streams $x_{i}$ and $y_{i}$ are cross-correlated, the noise correlation $n$ is given by

$$
\mathrm{n}=\frac{1}{\mathrm{~N}} \sum_{\mathrm{i}=1}^{\mathrm{N}} \mathrm{x}_{\mathrm{i}} \mathrm{y}_{\mathrm{i}}
$$

where $\mathrm{N}=$ the number of samples correlated

$$
\mathrm{n}^{2}=\frac{1}{\mathrm{~N}^{2}} \sum_{\mathrm{i}} \sum_{\mathrm{j}} \mathrm{x}_{\mathrm{i}} \mathrm{y}_{\mathrm{i}} \mathrm{x}_{\mathrm{j}} \mathrm{y}_{\mathrm{j}}=\frac{1}{\mathrm{~N}}\left[1+2 \sum_{\tau=1} \mathrm{pc}(\tau)\right]
$$

so that the second loss factor is given by

$$
\mathrm{Lc}=\left[1+2 \sum_{\tau=1}^{\mathrm{N}} \mathrm{pc}(\tau)\right]^{-1 / 2}
$$

Table 1 shows the results of numerical calculations of the loss which results from the use of Butterworth filters. The loss is reduced as the number of poles are increased, and the two loss factors total 3 percent loss for a 7-pole filter. Other filter types may result in lower loss factors but have phase characteristics that are more nonlinear.

\section{Double Sideband}

Double sideband receivers can be used for interferometry, but they result in a SNR loss of $\sqrt{2}$. At first glance, it is difficult to see why there is a reduction in SNR because one can argue that while the noise level is doubled (both sidebands folded on each other), the signal level is also doubled. Actually, the upper and lower sideband signals have opposite fringe rates. Fringes from the two 
Table 1

Butterworth Low Pass Filter Losses due to Foldover and Imperfect Bandpass Shape

\begin{tabular}{|c|c|c|c|c|}
\hline $\begin{array}{c}\text { No. of } \\
\text { poles }\end{array}$ & $\begin{array}{c}\text { Optimum } \\
\text { location of } \\
3 \mathrm{~dB} \text { point in } \\
\text { point in } \\
\text { percent of B }\end{array}$ & $\begin{array}{c}\text { Foldover } \\
\text { loss in } \\
\text { percent }\end{array}$ & $\begin{array}{c}\text { Loss due to } \\
\text { imperfect } \\
\text { bandpass in } \\
\text { percent }\end{array}$ & $\begin{array}{c}\text { Total } \\
\text { loss } \\
\text { in percent }\end{array}$ \\
\hline 2 & 67 & 7 & 5 & 12 \\
3 & 79 & 5 & 3 & 8 \\
4 & 80 & 3 & 3 & 6 \\
5 & 88 & 3 & 2 & 4 \\
6 & 90 & 3 & 1 & 3 \\
7 & 91 & 2 & 0.3 & 2.3 \\
9 & 96 & 1 & 0.5 & 1.5 \\
\hline 10 & 96 & 2 & & \\
\hline
\end{tabular}

sidebands can be obtained separately and then averaged, but this results in a net SNR which is a factor of $\sqrt{2}$ lower than the optimum single sideband case. Imperfect image rejection in a single sideband interferometer results in some SNR loss. The amount of this loss is given by expressions similar to that of equation (6); that is, the loss results from aliased noise. Some reduction in this loss factor can be achieved if the signals from all the images are also processed and averaged together.

\section{Loss of Quadrature}

In order to approach the optimum SNR given by equation (4), the data must be processed in a manner which correctly extracts and coherently adds quadrature components of the interferometer. Complete lack of a quadrature channel degrades the SNR by 2. Incoherent combination of the quadrature channels degrades the SNR by $\sqrt{2}$. Any optimal processing method must completely reject fringe rate images. For example, if an interferometer is observing a radio source, fringes with the opposite fringe rate from an artificial "gedanken" radio source at the same position moving with minus twice the sidereal rate should be completely rejected.

\section{Complex Delay Function as a Maximum Likelihood Estimate}

A complex delay function $\mathrm{D}(\tau)$ is defined as the time-reversed Fourier transform of the crossspectral function $\operatorname{Sxy}(\omega)$ multiplied by a window function which is unity for positive frequency and zero for negative frequency. Alternately, the delay function can be derived by convolving the cross-correlation function with a complex function whose real and imaginary parts are the noiseless 
cross-correlation for a fringe phase of 0 and 90 degrees respectively. The delay function is also the likelihood function which, when maximized, gives the best estimate of delay and delay rate.

\section{Loss from Approximate Methods of Fringe Rotation}

Most VLBI correlators use approximate sine and cosine functions for fringe rotation. The harmonic content in these approximations results in a small loss in signal. The loss is 4 percent for the threelevel approximation used in the Mark II and III correlators.

\section{“Fractional Bit Correction" Loss}

When the delay offset between the data streams being correlated is changed, it results in a frequency dependent phase jump which can be corrected without loss by applying continuous correction to the cross-spectral function (Meeks, 1976). Alternately, the fringe rotation phase can be automatically changed by 90 degrees when the delay offset is changed. This simple procedure results in a continuous phase at midband with 45 degree jumps at each edge of the band. The phase averaged over the band is continuous but the SNR is reduced by about 3.5 percent.

\section{Sensitivity of VLBI}

(1) Analog System

$\mathrm{SNR}=\mathrm{A}(2 \mathrm{BT})^{1 / 2}$

where

$$
\begin{aligned}
\mathrm{A} & =\text { Correlation coefficient }=\mathrm{TA} / \mathrm{Ts} \\
\mathrm{TA} & =\text { Geometric mean of antenna temperatures } \\
\mathrm{Ts} & =\text { Geometric mean of system temperatures } \\
\mathrm{B} & =\text { Band width recorded or transmitted }(\mathrm{Hz}) \\
\mathrm{T} & =\text { Coherent integration time }(\mathrm{sec}) \\
\mathrm{SNR} & \triangleq 1 /(\mathrm{R} . \mathrm{M} . \mathrm{S} . \text { phase noise in radians) } \\
\mathrm{PE} & =1-\left(1-\mathrm{e}^{-\mathrm{SNR}^{2} / 2}\right)^{N}-\text { See graph. }
\end{aligned}
$$

(2) Digital System

SNR $=(2 / \pi) \mathrm{A}(2 \mathrm{BT})^{1 / 2} \quad$ 2-level sampling at Nyquist rate

$2 \mathrm{BT}=$ Total \# bits recorded per station 
\# Levels

2

3

4

2 (oversampled by 2 )
Relative SNR (continuum)

$$
1
$$

1

0.979

0.823
Spectral SNR

1

1.271

1.384

1.164

(3) Loss Factors

(a) Aliased or folded noise from failure of filter to cut-off at bandedge $2 \%$

(b) Imperfect shape factor 6f filter

(c) Approximations in fringe rotation

3-Level done on only one station

3-Level done on both stations

(d) "Fractional bit correction"

Method 1 - continuous correction

Method 2 - "Autocorrection"

(e) Double-Sideband - If used SNR reduced by $\sqrt{2}$

(f) Loss of quadrature - imperfect processing Complete loss

SNR reduced by 2

Partial loss

SNR reduced by $\sqrt{2}$

\section{References}

Rogers, A. E. E., Very Long Baseline Interferometry with Large Effective Bandwidth for PhaseDelay Measurement, Radio Science 5, 1239-1247, 1970.

Bowers, F. K. and Klingler, R. J., Quantization Noise of Correlation Spectrometers, 1972.

Meeks, M. L., Methods of Experimental Physics, Vol. 12C Academic Press, Chapter 5, 1976. 

SESSION D

THE MARK III VLBI SYSTEM 


\title{
MARK III SYSTEM OVERVIEW
}

\author{
T. A. Clark \\ NASA/Goddard Space Flight Center
}

\section{INTRODUCTION}

The "Mark III" VLBI system comprises a complete end-to-end VLBI system optimized for both high accuracy geodesy and radio astronomy. It includes the field station electronics for acquiring and recording the VLBI data, the central correlator preprocessing system and a data base/interactiveanalysis computer system.

In the past, the earlier Mark I and Mark II VLBI systems were conceived as tape recorders. With these recording systems in hand, the VLBI community started to figure out what to do with them. With Mark III, we started with the requirements of the geodetic and astronomy job, developed the instrumentation to do the job. For that reason, the Mark III is a total system; it is much more than just a tape recorder.

\section{Mark III Data Flow}

The entire data flow in the Mark III system from schedule generation through field data acquisition to the analytical production of geodetic and astronomical results is shown in figure 1 . One of the key features of the system is the data base concept used to tie together all of the data operations from the beginning to the end. All data is put into the data base so that it is available for later operations and archiving.

The first step in the process of conducting an experiment is to plan the detailed schedule of operations at the VLBI field stations. The program SKED generates the schedules. These schedules are sent to each field station on floppy disks for the station computer. This computer controls the field operations and logs the system status and correlative data during the data acquisition operation. The Mark III wideband VLBI data tapes recorded in the operation are sent to the Mark III correlator, and the station logs (typically on floppy disks) are DE-LOGged into the data base. The paper by Vandenberg, et al. ${ }^{1}$ describes these operations in detail.

The schedule, logs, and miscellaneous data in the data base are used to generate the control instructions for the correlator operations which performs the cross-correlation of the VLBI station tapes and the production of the delay and rate observations. Next a string of operations is performed to process the data. The details of the correlator hardware and software are discussed by Whitney 2 and Nesman. ${ }^{3}$ 


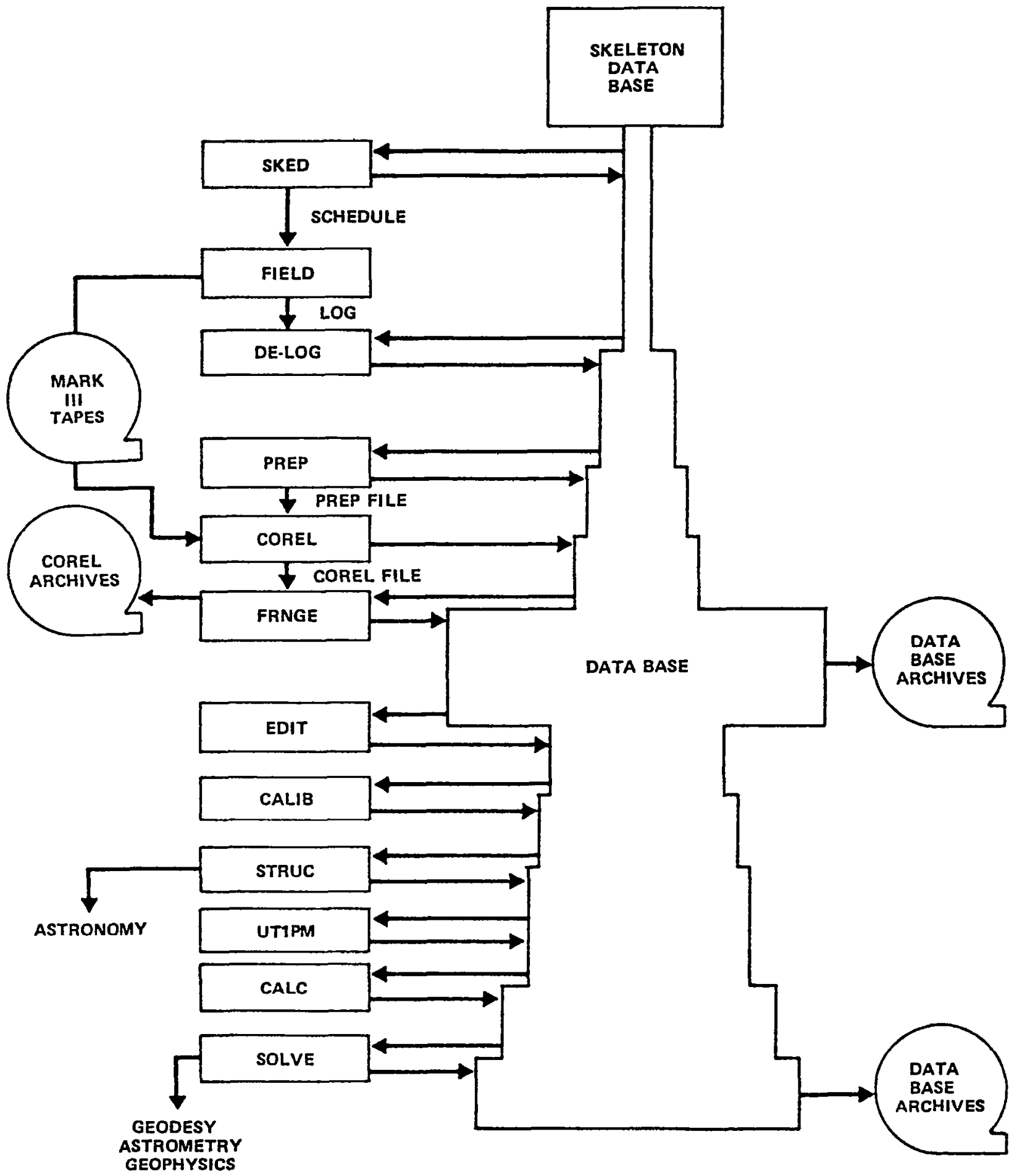

Figure 1. Mark III VLBI data flow. 
The edit operation uses data tests and station log information to edit out bad data. Next the propagation and system calibrations are applied, based on station logs of supporting data and calibration data extracted from the VLBI data in the correlator operation. Then corrections are made for the fact that the extra galactic radio sources are not point sources, but have some structure. The STRUC analysis modules can also be used in the analysis of VLBI data to determine the source structure, which is of considerable interest to the astronomy community. Similarly, UT1 and Polar Motion can be put in as a priori values or they can be determined from the analysis of the VLBI data. In doing a geodetic determination, it is necessary to CALCulate a large number of a priori theoretical values of what the observed parameters were expected to be. The details of these models were presented earlier by Ma, et al. ${ }^{4}$ In the final step, we SOLVE for the geodetic and astrometric parameters. 5

One of the things which we have found from our experience with the earlier VLBI systems was that it is necessary to force a degree of discipline on yourselves to maintain the integrity of the data, maintain the quality of data, and to keep records of all the things that have happened to the data in the processing operations. This Mark III Data Base Handler System preserves the integrity of the observations so that information is not lost and can be recovered at any future time.

Figure 1 shows the flow of data and information through the system. The boxes shown are actually the names of computer programs that do the particular jobs. A computer is used for all of these operations. We have removed repetitive manual operations and rely on very heavy computer involvement. Thus, the information is really flowing through a computer system, and there is a very heavy computer structure. The details of the Mark III Data Base Handler System are presented by Ryan, et al. 6

\section{Mark III VLBI Field Station}

A diagram of the Mark III VLBI field station is shown in figure 2. The key features of the system are as follows:

a. The Mark III receiver has very wideband front ends $(400 \mathrm{MHz}$ centered at $8.4 \mathrm{GHz}, 100 \mathrm{MHz}$ centered at $2.25 \mathrm{GHz})$ for high delay resolution, uses dual frequencies $(8.4 \mathrm{GHz}, 2.25 \mathrm{GHz})$ for extraction of the ionospheric delay, and has continuous system calibration for high accuracy. The details of the receiving system and the continuous calibration method are given by Rogers. ${ }^{7}, 8$

b. The Mark III Data Acquisition Terminal is a very wide bandwidth $(112 \mathrm{Mb} / \mathrm{s})$ data system for a significantly improved signal-to-noise ratio. This is especially important for applications utilizing small antennas or weak sources. This terminal contains a computer for automated control and monitoring of the VLBI station in order to facilitate easy field operations. The details of the Mark III Data Acquisition Terminal are given by Rogers, ${ }^{7}$, Vandenberg, 1 , Hinteregger, 9 and Levine. 10 


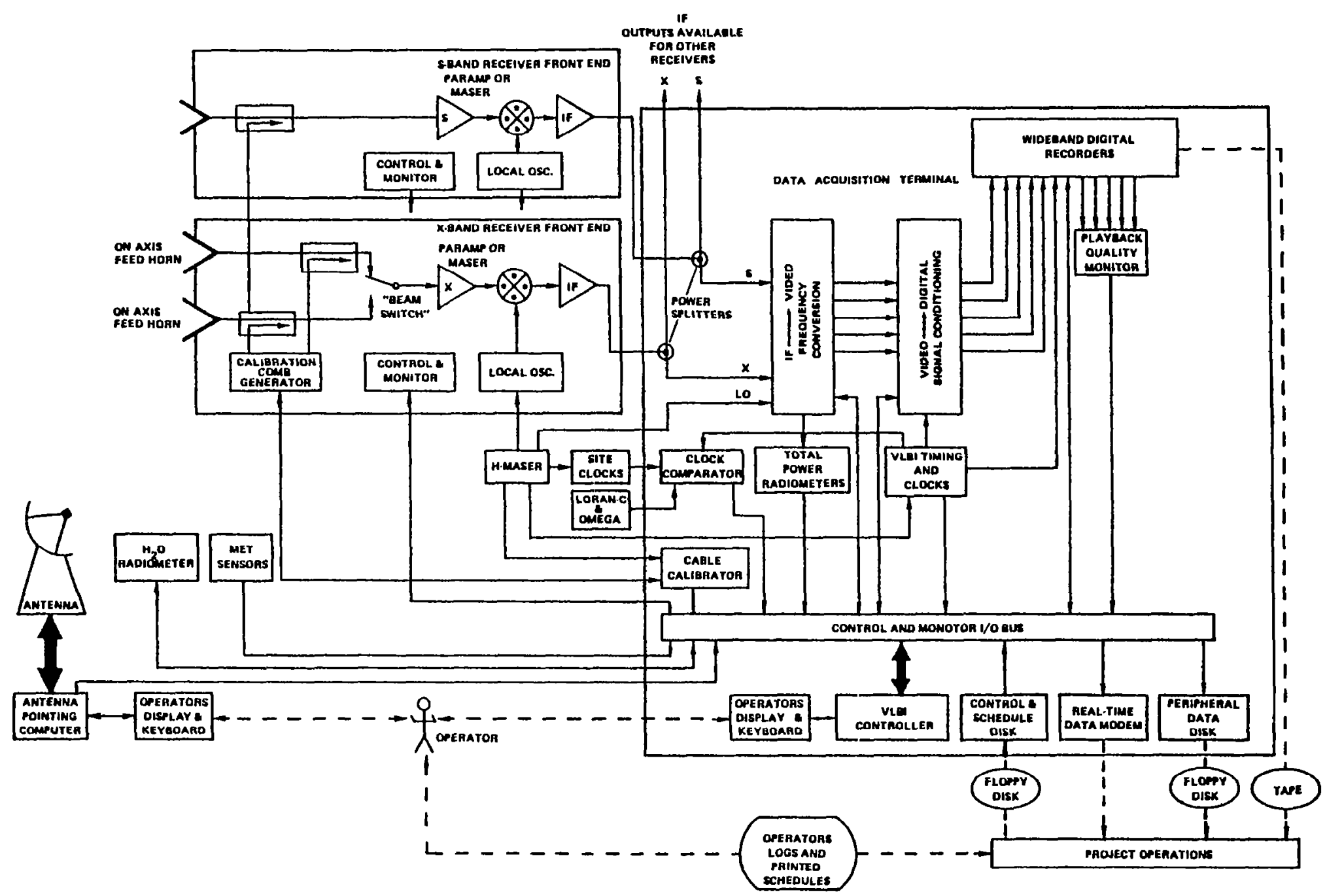

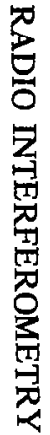

Figure 2. Mark III VLBI field station configuration. 
c. A very stable hydrogen maser frequency standard for all frequencies and timing signals in the Mark III ficld station. Both the NASA NR hydrogen masers, described by Rcinhardt, 11 and the SAO VLG masers, described by Vessot, ${ }^{12}$ have been used in the Mark III VLBI field stations. They provide a frcquency stability of a few parts in $10^{15}$ for a 100 -second pcriod.

d. Tropospheric propagation delay calibrations are determined from measurements at the station with meteorological sensors and microwave water vapor radiometers. The "dry" component of the tropospheric delay is deduced from the meteorological data, and the "wet" component of delay is deduced from water vapor radiometer data. A detailed description of these tropospheric calibration subsystems is given by Resch. 13

The Mark III VLBI system for the field stations has been developed with stand-alone subsystems to enable the use of the Mark III system in all of the configurations of VLBI stations to be employed by the Crustal Dynamics Project. The three categories of configurations to be used by the Project are:

- Fixed VLBI stations with Mark III VLBI capability permanently located at the station.

- Transportable VLBI stations where a transportable Mark III terminal is brought in and connected to a fixed antenna for a short term VLBI measurement.

- Mobile VLBI stations with the entire VLBI station, including the antenna, on wheels for moving from site to site.

The use of the mobile and transportable stations enables the project to make measurements from a large number of sites with relatively few sets of equipment. The fixed stations form a long-term reference network for tying together the measurements with the mobile and transportable stations.

The status, as of June 1979, of the Mark III VLBI systems/stations is that the development is completed and systems are operating at Haystack Observatory, Massachusetts, and National Radio Astronomy Observatory, West Virginia. Additional Mark III systems are in fabrication for fixed stations at the Owens Valley Radio Observatory, California, the Onsala Space Observatory, Sweden, and the Harvard Radio Astronomy Station, Texas. The first transportable Mark III system is in fabrication and will be deployed first to the Max Planck Institute for Radio Astronomy, West Germany. Similarly, the first Mark III system for a mobile VLBI station is in fabrication and will be installed in the ARIES mobile station (Neill, et al. ${ }^{14}$ ). 


\section{REFERENCES}

1. Vandenberg, N., T. Clark, L. Foster, A. Whitney, and G. Lamps, "Automation of the Mark III Field System," Proceedings of Radio Interferometry Techniques for Geodesy Conference, June 1979 , in press.

2. Whitney, A., "Mark III Correlator Hardware and Software," Proceedings of Radio Interferometry Techniques for Geodesy Conference, June 1979, in press.

3. Nesman, E., "Correlator Computer Interface and Module Implementation - Mark III Processor," Proceedings of Radio Interferometry Techniques for Geodesy Conference, June 1979, in press.

4. Ma, C., "Geophysical and Astronomical Models Applied in the Analysis of VLBI Data," Proceedings of the Radio Interferometry Techniques for Geodesy Conference, June 1979, in press.

5. Ryan, J. W. and C. Ma., "The Mark III Interactive VLBI Data Analysis System," Proceedings of Radio Interferometry for Geodesy Conference, June 1979, in press.

6. Ryan, J. W., C. Ma, and B. Schupler, “The Mark III Data Base Handler," Proceedings of Radio Interferometry Techniques for Geodesy Conference, June 1979, in press.

7. Rogers, A., "Microwaves to Megabits," Proceedings of Radio Interferometry for Geodesy Conference, June 1979, in press.

8. Rogers, A., "Phase and Group Delay Calibration of a Very Long Baseline Interferometer," Proceedings of Radio Interferometry for Geodesy Conference, June 1979, in press.

9. Hinteregger, H., "The Mark III Wideband Digital Recorder in Perspective," Proceedings of Radio Interferometry for Geodesy Conference, June 1979, in press.

10. Levine, J. and A. Whitney, "Mark III Real-Time Fringe-Detection System," Proceedings of Radio Interferometry for Geodesy Conference, June 1979, in press.

11. Reinhardt, V. and L. Rueger, "The Performance of the NASA Research Hydrogen Masers," Proceedings of Radio Interferometry for Geodesy Conference, June 1979, in press.

12. Vessot, R., "Hydrogen Maser Frequency Standards," Proceedings of Radio Interferometry for Geodesy Conference, June 1979, in press.

13. Resch, G., "Mark III VLBI System - Tropospheric Calibration Subsystems," Proceedings of Radio Interferometry for Geodesy Conference, June 1979, in press.

14. Neill, A., T. Claflin, T. Lockhart, P. MacDoran, D. Morobito, K. Ong, and G. Resch, "Geodetic Measurcments with a Mobile VLBI Systcm," Proccedings of Radio Interferometry for Geodesy Conference, June 1979 , in press. 


\title{
AUTOMATION OF THE MARK III FIELD SYSTEM
}

\author{
N. R. Vandenberg \\ NASA/Goddard Space Flight Center and Phoenix Corporation
}

T. A. Clark and L. N. Foster

NASA/Goddard Space Flight Center

A. R. Whitney

NEROC/Haystack Observatory

\author{
G. Lampe \\ Ideas, Inc.
}

\begin{abstract}
The many capabilities of the Mark III acquisition system have been designed for fully automatic operation. A system comprising both software and hardware components has been developed which will enable hands-off operation, except for the mounting and dismounting of data tapes, for an entire Mark III experiment. Under automatic control, the acquisition system can easily follow a complicated schedule which includes changing operating configurations and recording multiple observations on a tape without waste.

The operation of the field system begins with the scheduling of observations. An interactive program, SKED, has been developed which provides displays of mutual visibility, automatic calculation of telescope slewing times, and the ability to list and edit the schedule. The output of SKED is a schedule file containing commands in the Standard Notation for Astronomy Procedures (SNAP) language which the field system uses for controlling events during the experiment. The most important features of SNAP include sophisticated time-sequencing of events, automatic logging of all commands and responses, and the ability to define often-used sequences of commands as procedures.

The heart of the field system is a control program, BOSS, running in an HP 1000 mini-computer. BOSS reads the SNAP commands from a schedule file and interprets them in terms of commands and requests to devices. Interactive command input is possible through the operator's display terminal. Communication with all of the Mark III electronics modules is done via a small general purpose interface board (a microprocessor-based ASCII transceiver) which has been installed in each module. Additional devices are controlled and monitored using the IEEE 488 General Purpose Interface Bus.
\end{abstract}




\section{INTRODUCTION}

The many capabilities of the Mark III Data Acquisition System were designed to be completely automatically controlled. Except for the mounting and dismounting of tapes, an entire Mark III experiment can be conducted as a fully automated, hands-off operation. This paper describes the parts of the Mark III system which implement the automation concept. These parts include the scheduling program, the SNAP language, the field system control programs, and the interface boards between the control computer and the electronics modules. As used here, the term "field system" includes the control computer hardware and software as well as the interfaces to the data acquisition system.

\section{SKED - INTERACTIVE SCHEDULING PROGRAM}

Logically, the first operation of an experiment using the Mark III system is the scheduling of observations. A program called SKED has been developed which enables the user interactively to make a detailed very long baseline interferometry (VLBI) observing schedule. First, the user selects the sources and stations to be used in the experiment. For an individual observation, the program automatically calculates the telescope slewing time taking into account slew rates and cable wrap for the selected stations. Slewing times are calculated iteratively to allow for possible significant motion of the source during the time the telescope is moving. Then, a display is available of the sources which are currently mutually visible and the slewing times to each one so the user can select the next source to be observed. As the schedule of observations is being built up in this manner, any portion of it can be listed, edited, and then automatically shifted in time to readjust the run start times around the observations which were inserted or deleted. A Mark III "configuration" is associated with each observation so that different combinations of bandwidth, LO frequencies, and tape recorder modes can be scheduled. One of the outputs of SKED is a data base with information on the planned observations; during processing, the data base will be augmented by the correlated data.

\section{SNAP CONTROL LANGUAGE}

The primary output of SKED is a "schedule file" which will be sent to each telescope participating in the experiment. The method of distribution would normally be to mail a floppy disk with the schedule file; however, if time does not permit this, the file could be transferred by telephone. Since the schedule file is a "human readable" ASCII file, it can be edited before or during an experiment, either at the station or via telephone.

The schedule file contains commands in the Standard Notation for Astronomical Procedures (SNAP) language. These commands are used to control data acquisition during the experiment by sequencing and timing events based on the scheduled observations. Figure 1 shows a sample schedule which illustrates the basic features of SNAP. Using SNAP, events which are to happen at a particular time can be pre-scheduled. For example, the command which starts the tape recording, "ST," could be issued such that it would be executed at a specific time with the SNAP command "ST@120300". If 
only the command "ST" were issued, it would be executed at once. The pre-scheduling feature of SNAP is not intended for an entire observing schedule but is useful for setting up a few events in advance of their execution time. Another feature of SNAP is the ability to schedule events periodically; devices can be monitored by scheduling a request for data every so often. For example, the command "WX@12H,15M" would schedule a request for weather data at 12:00 UT and every 15 minutes thereafter until the end of the experiment or until cancelled. Sequences of commands which are to be often used can be defined as procedures in SNAP and then simply invoked by name. The commands which set up a particular Mark III configuration for several observations might be defined as a procedure; e.g., CON1 in the sample schedule is used to set up the tape drive for 120 ips in the forward direction, enable record track groups 1 and 2, and set up the formatter for mode B and $4 \mathrm{Mbit}$ sample rate. Finally, SNAP has features for schedule flow control. The "f" command followed by a time is used to halt the processing of schedule file entries until the specified time.

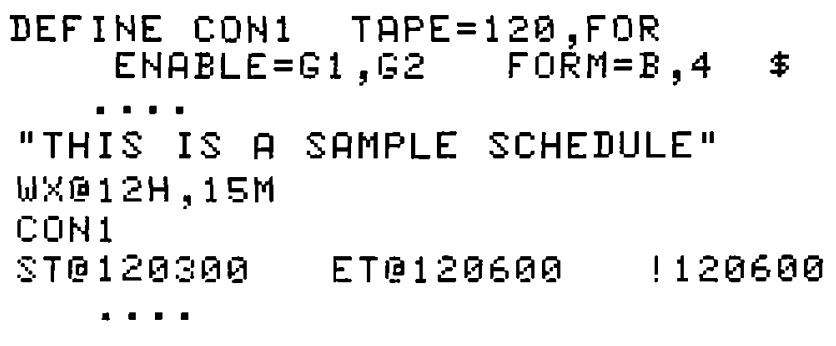

Figure 1. Sample SNAP schedule.

An important feature of SNAP is that all commands, responses to commands, and operator comments are tagged with the time and their source and logged in a disk file. All events during the experiment are thus recorded and available in a specified machine-readable format for complete accountability and automatic delogging after the experiment.

\section{FIELD SYSTEM PROGRAMS}

The field system's main control program is called BOSS. BOSS was written to implement the specifications of SNAP, and in addition some system-dependent features were implemented which were found to be desirable. Figure 2 is a block diagram of the field system programs. BOSS reads SNAP commands from the schedule file and also accepts interactive input from the operator via OPRIN. The SNAP commands are first checked for proper syntax, and if no errors are found, a table is consulted which holds information on the way to interpret this command. BOSS itself interprets commands which deal with the time flow of the schedule, procedure definition, and other control functions. BOSS schedules the external program QUIKR (QUIcK Response) for commands which must be interpreted in terms of parameters to be sent to modules, requests for monitor information from modules, and various testing and display functions. QUIKR parses the parameters in the commands sent to it, formats ASCII messages for electronics modules, and decodes module responses into user-readable responses. QUIKR is organized into segments (or overlays), one for each module 
or set of related commands. The utility programs MATCN, IBCON, and ANTCN handle communications protocol and control of the ASCII transceivers (described below), HP interface bus devices, and the antenna pointing, respectively. The program OPRIN accepts operator input, displays commands and responses as they are processed by BOSS, and schedules various interactive programs for the operator. Finally, MONIT displays current status information.

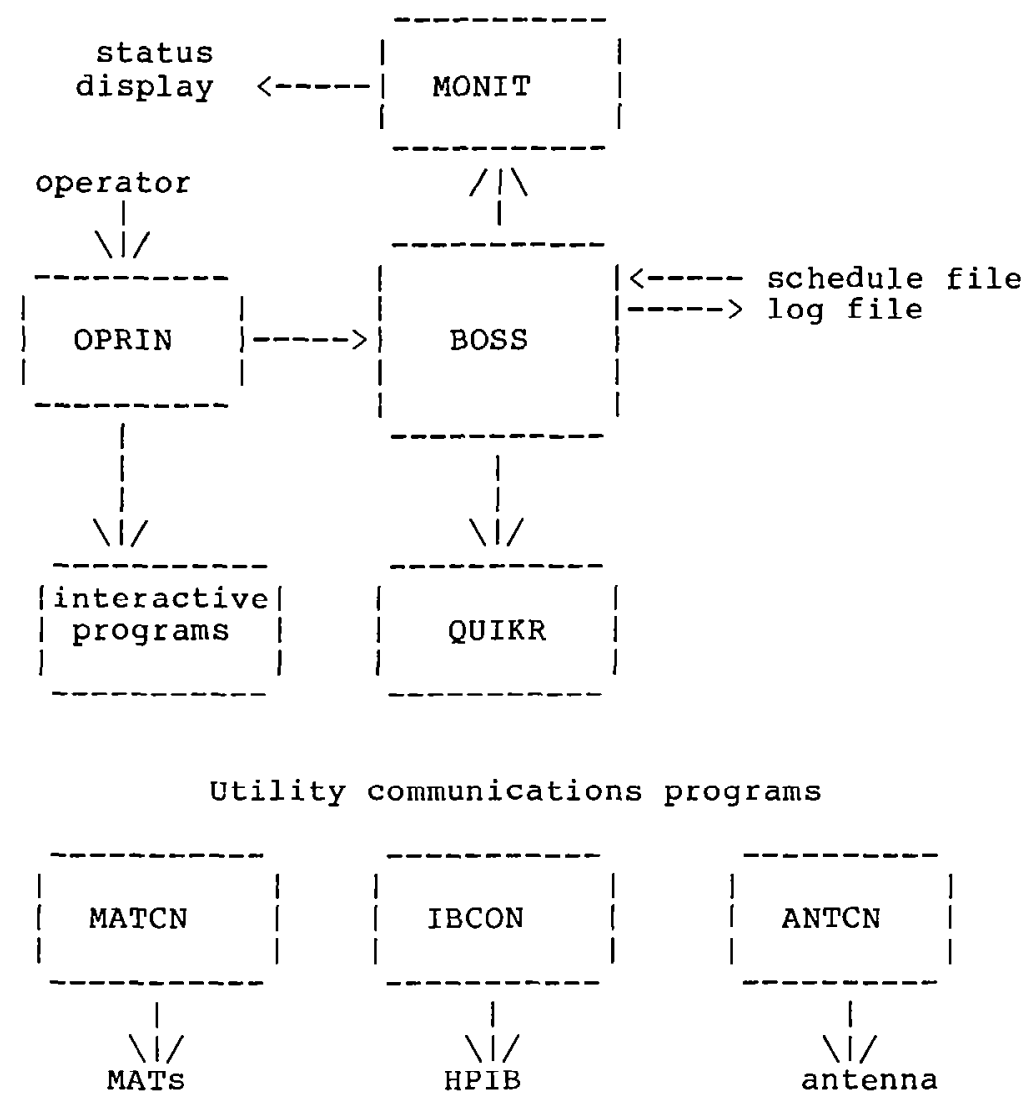

Figure 2. Field system programs.

\section{FIELD SYSTEM COMPUTER HARDWARE}

The hardware which implements the field system control functions comprises a Hewlett-Packard (HP) 1000 series mini-computer which includes in its minimum configuration $64 \mathrm{~K}$ words of memory, a CRT terminal, two high-density ( $0.5 \mathrm{M}$ bytes) floppy disk drives, and assorted I/O cards. Additional memory and/or a hard disk would enable more of the field system software features to be supported. It appears that one floppy disk will be adequate to hold the schedule and log files for approximately 1 day of observing. With the floppy disks, HP's RTE-M operating system is used in which all programs are memory-resident and segments are swapped in from disk. This system will be available at Owens Valley and Green Bank. With a hard disk, HP's RTE-IV operating system is 
used in which all programs and segments are swappable. The field systems at Fort Davis, Haystack, and the ARIES trailer will have this system. In both operating systems, $64 \mathrm{~K}$ words of memory address space are available at any given time, of which the operating system itself uses approximately half.

\section{INTERFACES BETWEEN COMPUTER AND ELECTRONICS}

There are two interfaces between the control computer and the electronics hardware of the Mark III Data Acquisition System. One uses the IEEE-488 General Purpose Interface Bus; commercial devices such as counters and switches are controlled using this interface. The other interface is to the Mark III electronics modules; this interface is accomplished with a small general-purpose board: an 8085 microprocessor-based ASCII transceiver or MAT. There is one MAT per module, and all are daisy-chained together on a "party line." Each MAT has an address between 00 and FF, and when that address is detected in the data stream, the MAT accepts the ASCII characters which follow until a different address appears. The MAT accepts serial teletype-like data from the control computer and presents parallel data and control signals to its module. The MAT interface board has $4 \mathrm{~K}$ bytes of ROM, 768 bytes of RAM, and 72 I/O pins. Of this capacity, the interface functions use somewhat less than $2 \mathrm{~K}$ bytes of ROM, 256 bytes of RAM, and $8 \mathrm{I} / \mathrm{O}$ pins. By using the additional capacity in the microprocessor on each board, it is intended to augment the capabilities of some of the boards in order to remove the burden of simple control and testing functions from the main computer. Among the functions to be implemented is assistance with the acquisition of correlative information such as radiometry data during the experiment. 



\title{
MICROWAVES TO MEGABITS
}

\author{
A. E. E. Rogers \\ Haystack Observatory \\ and \\ East Coast VLBI Group
}

\begin{abstract}
The Mark III system has 14 IF to video converters, each with built-in synthesized local oscillators which have a range of 100 to $500 \mathrm{MHz}$ covered in $10 \mathrm{kHz}$ steps. In the normal widebandcontinuum mode, all 14 upper and all 14 lower sideband video outputs (each with $2 \mathrm{MHz}$ bandwidth) are recorded with a total data rate of $112 \mathrm{Mbits} / \mathrm{sec}$.

In the NASA geodetic observations, two IF bands are simultaneously recorded from a dual band $(\mathrm{S} / \mathrm{X})$ receiver.
\end{abstract}




\section{INTRODUCTION}

In the Mark III configuration for geodetic observations, a dual frequency wideband receiver is used with the Mark III terminal for acquisition and recording of the very long baseline interferometer (VLBI) signals at the observing station.

\section{RECEIVER}

The dual receiver was designed and built for use at the prime focus of antennas with $f / D \simeq 0.4$. The first unit was built for use at the Owens Valley Radio Observatory's 130' (45.24 m) antenna. The receiver is designed to operate simultaneously at $13 \mathrm{~cm}$ (S-band) and $3.6 \mathrm{~cm}$ (X-band). The receiver is packaged along with feeds into a complete front-end box. Table 1 lists the characteristics of the receiver.

Table 1

Summary of Receiver Characteristics

\section{FREQUENCIES}

S-band LO

X-band LO

LO Reference

S-band IF

$\mathrm{X}$-band IF
$2020 \mathrm{MHz}$

$8080 \mathrm{MHz}$

$5 \mathrm{MHz}$

200-550 MHz ( $3 \mathrm{~dB}$ bandwidth)

$100-550 \mathrm{MHz}$ ( $3 \mathrm{~dB}$ bandwidth)

Approximate $3 \mathrm{~dB}$ S-band frequency range (including feed and paramps) $-2220-2320 \mathrm{MHz}$

Approximate $3 \mathrm{~dB}$ X-band range $-8180-8600 \mathrm{MHz}$

NOISE TEMPERATURE

S-band system temperature

$\mathrm{X}$-band system temperature
$80 \mathrm{~K}$ measured on OVRO 130' (45.24 m)

$160 \mathrm{~K}$ measured on OVRO 130' (45.24 m)

\section{FEEDS}

On axis $\mathrm{S} / \mathrm{X}$ dual frequency feed: Polarization - RCP on antenna (feed is LCP)

Off axis X-band feed: Polarization - LCP on antenna (feed is RCP) 
The X-band low noise amplifiers are two separately-packaged parametric amplifiers. Each stage has a noise temperature of about $120 \mathrm{~K}$ and a gain of $13 \pm 1 \mathrm{~dB}$. The paramps are fixed tuned to cover 8.05 to $8.55 \mathrm{GHz}$ with less than $\pm 1 \mathrm{~dB}$ variation over this band.

The X-band channel has a latching circulator in front of the paramps to allow for "Dicke" switching between the on-axis $\mathrm{S} / \mathrm{X}$ feed and an off-axis $\mathrm{X}$-band feed.

The S-band low noise amplifier is a two-stage parametric amplifier. The paramp operates at about $14 \mathrm{~dB}$ per stage, has a $50 \mathrm{~K}$ noise temperature and covers a frequency range in excess of 2.2 to $2.3 \mathrm{GHz}$.

Both S and X channels have a coupler and four-way divider for injection of noise sources and delay calibration signals.

The receiver local oscillators are derived from $5 \mathrm{MHz}$ using a phase-locked oscillator. The S-band oscillator is phase-locked using a harmonic mixing scheme with $80 \mathrm{MHz}$ reference and $20 \mathrm{MHz}$ IF. By successive doubling and filtering, 20 and $80 \mathrm{MHz}$ are derived from $5 \mathrm{MHz}$. The phase locked oscillator is used directly as the S-band LO and is multiplied by four to provide the X-band LO. The oscillator can be locked every $40 \mathrm{MHz}$ within its limited tuning range. The lock point at 2020 $\mathrm{MHz}$ was chosen as the best choice for the frequency range covered by the low noise amplifiers.

The receivers have broadband $(5-500 \mathrm{MHz}) \mathrm{IF}$ amplifiers. A total of $71 \mathrm{~dB}$ gain is provided in $\mathrm{S}$ and $\mathrm{X}$ channels respectively $-40 \mathrm{~dB}$ of this gain is built into the mixer preamplifiers.

The IF output level is about $-10 \mathrm{dBm}$ for both $\mathrm{S}$ and $\mathrm{X}$ channels. However, owing to the rapid increase of cable attenuation with frequency above $100 \mathrm{MHz}$ the X-band IF is corrected on the ground using a cable compensation filter. The compensation filter has a loss of $20 \mathrm{~dB}$ at $200 \mathrm{MHz}$ and $8 \mathrm{~dB}$ at $450 \mathrm{MHz}$. The S-band IF is not compensated owing to its relatively small bandwidth.

\section{MARK III TERMINAL}

In the VLBI configuration used for high accuracy geodesy, the two receiver IF output signals are sent to the inputs of the Mark III terminal. This terminal converts the signals to 28 baseband channels, digitizes the channel signals, formats the digital data, and records the data on magnetic tape.

The IF output from the receiver is connected to an IF distribution module which amplifies and splits the IF power to all frequency converters modules. The distributor is dual channel to allow for the IF's from two radiometers or from two polarizations, etc. In addition, each channel is split into two subchannels, one covering the range 96 to $224 \mathrm{MHz}$ and the other covering the range 216 to $504 \mathrm{MHz}$. This split is required to reduce the loss in signal-to-noise ratio that results from the odd harmonic response of the IF-to-video converters which contain no preselection filters. The 14 converters can be connected to either channel and subchannel via a cable patch. 
Included in the distributor are two wideband square law detectors which can be used for total power monitoring. When used in conjunction with the front end, the detector outputs can be processed by the built-in integrator and microprocessor controlled ASCII transceiver to compute the system temperature and to do radiometry.

The Mark III terminal has 14 video converters each with built-in synthesized local oscillators which have a range of 100 to $500 \mathrm{MHz}$ covered in $10 \mathrm{kHz}$ steps. In the normal wideband-continuum mode of 14 upper and all 14 lower sideband vidco outputs arc used, one going to each recorder channel. Other modes are ones in which fewer tracks are recorded simultaneously if fewer bands are needed. In these modes, all the physical tracks on the tape are recorded after many forward and reverse passes of the tape.

The video converter converts a window in the IF input to video. The conversion is a frequency translation from IF to video using the "phasing" method of single sideband conversion. Both upper and lower sidebands are preserved, low pass filtered, and amplified. The analog video signals are clipped, sampled, and formatted in the same way for each recorder track so that each track can be decoded independently. The Mark III serial data format shown in figure 1 contains complete time of day, auxiliary data, cyclic-redundancy check bits and parity check bits along with the sampled video data bits.

The Mark III terminal uses instrumentation tape recorders presently outfitted with 28-track heads; each track is used to record a $2-\mathrm{MHz}$ bandwidth at $4 \mathrm{MB} / \mathrm{s}$ with a longitudinal density of 33,000 bits per inch so that the total recorded bandwidth is $56 \mathrm{MHz}$, as opposed to the $2 \mathrm{MHz}$ obtainable with Mark II. The system is quite flexible in allowing slower record speeds, and the corresponding narrower bandwidths, for operations that do not require the full bandwidth.

Table 2 summarizes the characteristics of the Mark III terminal.

The Mark III terminal is contained in four equipment racks. Two racks contain the two tape recorder systems, each consisting of a recorder transport, record head drivers, recorded controller and read-after-write/playback electronics. The third rack contains the IF distributor, video converters, formatter and decoder. The fourth rack contains the system control computer and the operators console. Every module in the Mark III can be locally controlled from its front panel or remotely controlled by means of a two-way serial communication system. Thus, a Mark III terminal can be operated manually or can be placed entirely under computer control except for the manual tape changes. When used under computer control, it is very easy for the terminal to follow complicated schedules including starting and stopping the tape and changing mode. It is relatively easy for the user to tailor the mode of operation of the terminal to the observing program requirements in order to make best use of the capabilities of the Mark III and to minimize the amount of tape required for an experiment. 


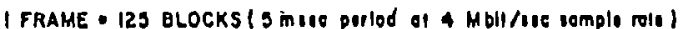

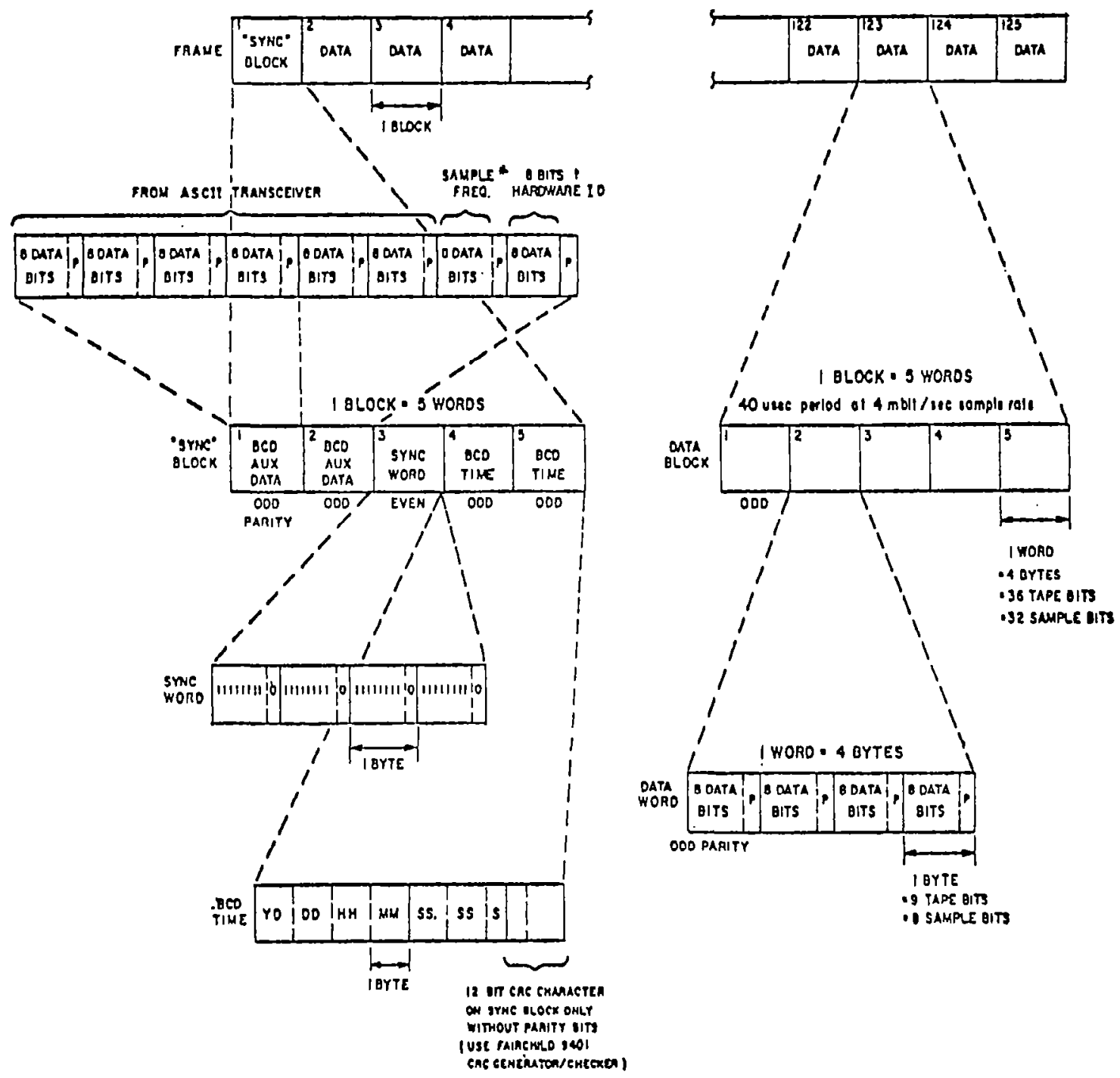

- SAMPLE fatoueney coot

Oo. Mmit/ios

or 4

062

of 0.5

$\begin{array}{ll}03 & 0.29 \\ 02 & 0.129\end{array}$

- hapowate do code siove have AT LEAST ONE DIT SET TO ZERO

Figure 1. Mark III tape format (single track). 
Table 2

Mark III Terminal Characteristics

Recorder Type: Instrumentation

Tape: 1 inch Ampex 797 or equivalent (Ampex 795 on GSA), $9200 \mathrm{ft}$. on glass reels (Corning \#690449)

Number of Tracks: 28 according to EIA standard

Track Width: 25 milli-inches or 635 micrometers

Longitudinal Density: 33,000 bits/inch on each track

Record Code: NRZM ("1" = flux change "0" = no flux change)

Format: see figure 1

Standard Sample Rate: $8,4,2,1,0.5,0.25,0.125 \mathrm{MHz}$

Video Low Pass Filter Bandwidths (10 dB bandwidth) 4,2,1,0.5,0.25,0.125 MHz

Filter Type: 7-pole Butterworth with $3 \mathrm{~dB}$ point at $0.45 \mathrm{x}$ sample rate. In the systems presently being manufactured, 4 converters are fitted with all filters in the upper sideband channel while all the remaining channels are initially outfitted with $2 \mathrm{MHz}$ filters. An external $62.5 \mathrm{kHz}$ filter has to be used for the $0.125 \mathrm{MHz}$ sample rate.

Number of IF to video converters: 14 (each produces separate upper and lower sideband outputs)

Converter LO range: $100-500 \mathrm{MHz}$ selectable in $10 \mathrm{kHz}$ steps.

Number of IF channels: 2 (each converter can be connected to either IF via patch panel)

Operating modes: Wideband -28 tracks recorded in one pass remaining 14 .

Continuum - 14 tracks recorded per pass only seven converters used.

Multiline -4 tracks recorded in each pass the upper sideband outputs from four converters.

Spectral - 1 track recorded per pass-Mark II compatible mode.

Control Computer: Hewlett Packard 1000 


\section{MARK III SYSTEM SENSITIVITY}

The new Mark III acquisition terminal has been developed to make, initially, a more than five-fold improvement (compared with the Mark II system) in the sensitivity of VLBI for astrophysical, astrometric, and geophysical applications. When this is used with the Mark III wideband receiver, the total system has very high delay resolution even when small antennas are used. As an example, table 3 lists the performance characteristics when the Mark III system is used with 4-meter and 18meter antennas.

Table 3

Source $=4 \mathrm{C} 39.25=10 \mathrm{~J}$
Ant. $1=$ Westford $=18 \mathrm{~m}=50 \%$ eff.
Ant. $2=$ Transportable $=4 \mathrm{~m}=60 \%$ eff.
Ts $1=$ Ts $2=160 \mathrm{~K}$
Ta $1=0.45 \mathrm{~K}$
Ta2 $=0.03 \mathrm{~K}$
Correlation Amplitude $=0.12 \%$
Integration time $=500 \mathrm{sec}$.
Recorded bandwidth $=56 \mathrm{MHz}$
Signal to noise ratio $=114$
RMS spanned bandwidth $=100 \mathrm{MHz}$
$\Delta \mathrm{t}$ (group delay) RMS $=14 \mathrm{PS}$ or $4 \mathrm{millimeters}$

\section{MARK III TERMINAL SCHEDULES}

The first Mark III terminals are being built for use in the NASA Crustal Dynamics Project for high accuracy geodetic measurements with both fixed and mobile VLBI stations. The systems are in stand-alone, modular form so that they can be easily transportable to any antenna. Table 4 lists the current schedule for completion of the first nine Mark III terminals. 
RADIO INTERFEROMETRY

Table 4

\begin{tabular}{|c|c|c|c|}
\hline Mark III Terminal & ID Number & Expected Completion Date & Sponsor \\
\hline Haystack & Mk-III-0 & Complete & NASA \\
OVRO & Mk-III-1 & July 1979 & NASA \\
Onsala & Mk-III-2 & September 1979 & USAF/NSF \\
Transportable & Mk-III-3 & September 1979 & USGS \\
Ft. Davis & Mk-III-4 & January 1980 & NASA \\
ARIES & Mk-III-5 & July 1980 & NASA \\
Bonn & Mk-III-6 & July 1980 & MPI \\
NRAO 140 & Mk-III-64 & Complete & NSF \\
NRAO VLA & Mk-III-65 & 1980 & NSF \\
\hline
\end{tabular}




\title{
THE MARK III WIDEBAND DIGITAL RECORDER IN PERSPECTIVE
}

\author{
Hans F. Hinteregger \\ Haystack Observatory \\ VLBI Development Group
}

\begin{abstract}
The tape recorder now used for the Mark III data acquisition and processing system is compared with earlier very long baseline interferometry (VLBI) recorders. At least a quadrupling of track density on tape is anticipated within 2 years to improve tape logistics in high duty cycle VLBI network operations. Wideband $33-1 / 3 \mathrm{kbpi}$ digital channel characteristics of instrumentation recorders and of a modern video cassette recorder are illustrated. Factors which influenced selection of the three major commercial components (transport, heads, and tape) are discussed. A brief functional description and the reasons for development by Haystack Observatory of efficient signal electronics and by NRAO of necessary auxiliary control electronics are given. The design and operation of a digital bit synchronizer, invented and implemented by the author, is illustrated as an example of the high degree of simplicity achieved.
\end{abstract}




\section{INTRODUCTION}

\section{Past}

By way of historical perspective, the physical tape recording formats of the first three generations of very long baseline interferometry (VLBI) systems are shown in figure 1. Mark I, used from 1967 to 1978 , with $150 \mathrm{ips}, 800$ bpi computer drives which consumed a $1 / 2^{\prime \prime} \times 2400^{\prime}$ tape in 3 minutes, recorded a single $0.72 \times 10^{6}$ bit per sec (bps) channel. MarkII, introduced in 1971 and still in use, employing slightly modified helical scan video recorders (originally the Ampex VR660 with 2 "-wide tape and later the IVC825 with $1^{\prime \prime}$-wide tape), raised the data rate to $4 \times 10^{6}$ bps and the record time to at least 1 hour.

\section{Present}

The recorder for Mark III was chosen primarily to increase recordable bandwidth by a very large factor so as to increase the sensitivity of any interferometer using it by the square root of that factor. The basic technology of the 28-channcl wideband instrumentation recorder was selected. A $2 \mathrm{MHz}$ analog channel bandwidth at 120 ips is standard and implies the 33,333 bpi digital recording capability. This high code density is in fact standard for Mark III; at its nominal speed of 135 ips, each channel operates at a data rate of $4 \times 10^{6}$ bps which corresponds to an NRZM-with-parityformatted bit stream of $4.5 \times 10^{6}$ bps. A Mark III recorder can therefore be regarded as 28 Mark II recorders in parallel; for any given interferometer, source, and coherent integration time, Mark III is $\sqrt{28}=5.3$ times as sensitive as Mark II and 12.5 times as sensitive as Mark I.

Running at 135 ips and recording a total data rate of $112 \times 10^{6}$ bps with all channels simultaneously, the Mark recorder consumes the $9000^{\prime}$ working length of a standard instrumentation tape in 800 seconds. At $0.8 \times 10^{9}$ data bits per cubic inch, the volume density of information of a Mark III recording is 130 times that of Mark I, but only 1.5 times that of Mark II. This is in spite of the fact that the 33,333 bpi in-track code density of Mark III is 6 times greater than that of Mark II.

The recorder has demonstrated reliable operation at double bandwidth and speed (224 Mbps @ 270 ips). This double bandwidth capability will be attractive to support only when the volume density of recording is improved so as to maintain constant or reduce the machine's total appetite for tape.

\section{Future}

To appreciate the importance of developing a further substantial increase in the "bits-per-pound-oftape" figure-of-merit of tape and recorder, consider the fact that the yearly cost of shipping tape cross-country for one field site recording $112 \mathrm{Mbps} 30$ percent of the time would be at least $\$ 80 \mathrm{~K}$ for the current 28 track-per-inch system. When high duty cycle VLBI operations become a reality (and since tape is rapidly and continually recycled), tape shipping costs will dominate operational 
THE MARK III VLBI SYSTEM
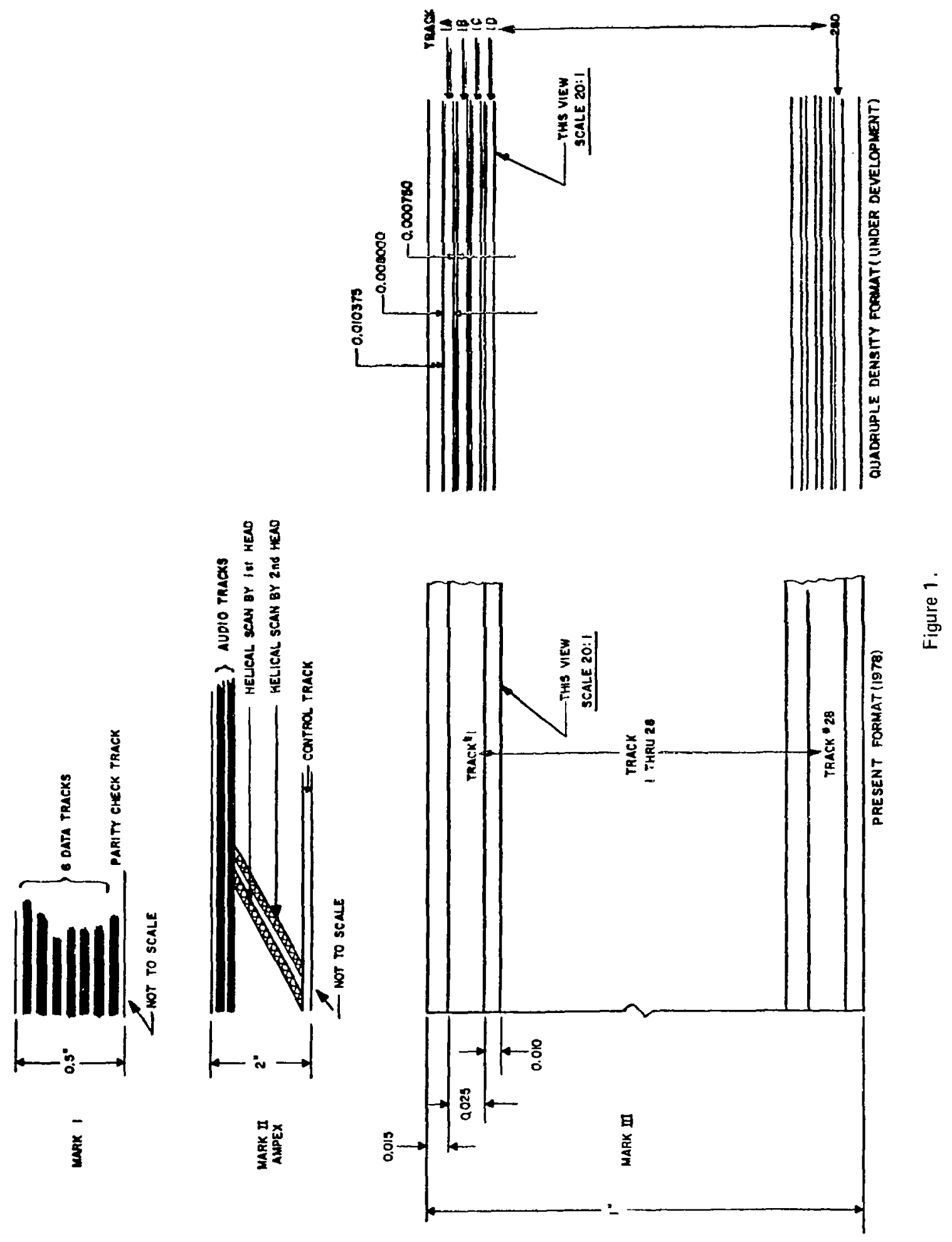
expenses! For this reason, and because important aspects of a 112 track-per-inch system have already been tested, the quadruple track-density format shown under development in figure 1 is thought to be the least density upgrade to which the Mark III recorder will be subjected within the next 2 years. Note that track density on tape is built up in successive passes, not by requiring higher code density or more heads and signal electronics in parallel. Such a narrow-track, multi-pass recorder upgrade will correct the major deficiency of commercial wideband recording technology a failure to capitalize on the known ability to use very much narrower tracks. Such an upgrade will also be intentionally "transparent" to the rest of the Mark III system; only the head assemblies will be changed. Technical requirements are (1) narrow-track (but not necessarily more dense) head stacks with improved head edge placement tolerances and (2) a presumably simple mechanism for accurately positioning (indexing) the stack(s) for each of the multiple passes.

\section{HIGH DENSITY TAPE CHANNELS}

Figure 2 shows the wavelength response of a typical wideband instrumentation recorder channel and also, by way of comparison with the state-of-the-art in "bits-per-pound-of-tape," that of a remarkable consumer product, the VHS video cassette recorder. The VHS curve is derived from data provided to me by Dr. Alan Yen (University of Toronto).

Note that the wideband instrumentation channel response is already 12 to $18 \mathrm{~dB}$ down from its peak at its defined edge; i.e., at a wavelength of $1.5 \mathrm{~m}$. Although this channel has a luxuriously high signal-to-noise ratio (SNR) even at bandedge, it is clearly foolish to attempt to increase bits-per-unitarea significantly by going to yet shorter wavelengths because (1) SNR is dropping more than $18 \mathrm{~dB}$ per octave at bandedge, and (2) proper equalization rapidly becomes much more complicated. On the other hand, when track width is halved, only a $3 \mathrm{~dB}$ loss in SNR should be incurred as long as head or tape (and not amplifier) noise dominates.

Note, in spite of its 30-times-higher tape track density, the VHS bandedge SNR is only about $6 \mathrm{~dB}$ below that of the instrumentation channel. When tape response differences are taken into account, this is in remarkably good agreement with the theoretical expectation. The SNR is still more than adequate, though, due to tape defects, error rates will probably be higher than SNR alone would lead one to expect. A comforting aspect of VLBI in principle and the Mark III system implementation in practice is its great immunity to high error rates. Though error rates are normally less than 1 in $10^{6}$, data with error rates as high as 1 in $10^{2}$ can easily be processed without any important degradation of results.

VHS recorders could probably provide a good "double bandwidth" Mark III equivalent digital channel. It is tempting to envision combining the wide total bandwidth and bulk tape packaging advantages of the multi-channel instrumentation recorder with the very high track density and mass production head/tape technology advantages of the VHS recorder. A simple yet extremely accurate method of stacking and mounting standard VHS heads for use in an instrumentation transport needs to be found to make this idea practical. 


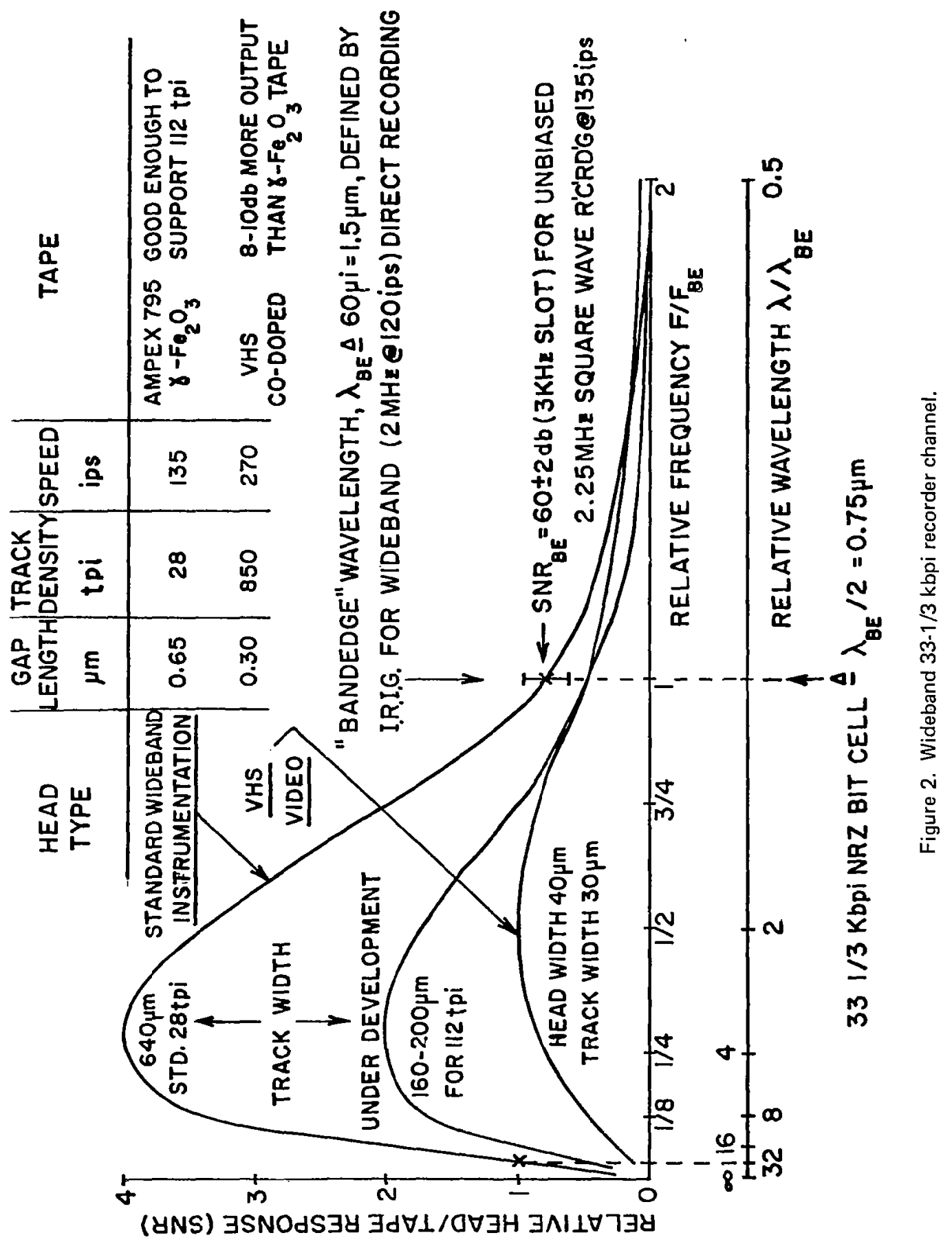




\section{MAJOR COMMERCIAL COMPONENTS}

The Mark III recorder is the result partially of careful selection of three major commercial components (transport, heads, and tape) and partially of the in-house development of simplified signal electronics well-suited to exclusively high-density digital operation.

\section{Transport}

The Honeywell Model 96 was chosen for several reasons:

(1) It is the most mechanically simple machine available; all parts are interchangeable and none require individual adjustment or alignment.

(2) The tape tension required for consistent operation is light and uncritical; the 8-ounce nominal tension is about half that used by most other instrumentation transports. The transport can therefore reliably handle the thinner tapes we hope to use in the future.

(3) Good head-tape contact is maintained consistently even at the maximum speed of 360 ips without increased tension. This was evidenced by recording a $33-1 / 3 \mathrm{kbpi}$ pseudorandom test signal at that speed and reproducing it at 135 ips without a noticeable increase in error rate and also by a loss of at most $1.5 \mathrm{~dB}$ in bandedge SNR compared to a $135 \mathrm{ips}$ recording. The transport is therefore proven for double speed, $270 \mathrm{ips}$, operation.

(4) The tracking consistency for any given piece of tape - the repeatability of the distance between guiding plates and the tape edges where they cross the heads - is superb, about $\pm 3 \mu \mathrm{m}$. It is in fact good enough to support very much narrower track widths, down to $1 \mathrm{mil}(25 \mu \mathrm{m})$ at least, without the complications of a track-following servo.

\section{Heads}

Wideband heads of all-ferrite construction were chosen. Honeywell's standard product, made from "gap bars" manufactured by Matsushita (which, incidentally, also manufactures VHS heads), was selected because it exhibited the reliability and consistency expected from this construction. There are basically two reasons for choosing all-ferrite heads:

(1) Ferrite heads are the most long-lived heads available. They are guaranteed for 3000 hours of wear and may last much longer. Hard-metal-tipped heads by comparison will almost certainly wear out in about 1000 hours.

(2) Because of the monolithic-head-tip geometry of the all-ferrite head, response consistency is much better than for hard-metal-tipped heads. There are two important aspects of this consistency: 
(a) head-to-head response variations, which are typically only $\pm 1 \mathrm{~dB}$ bandedge for ferrite (as opposed to $\pm 4 \mathrm{~dB}$ for hard-metal-tipped heads), and

(b) variations in the shape of the wavelength response as the gap wears down, which are negligible for ferrite but may involve as much as a $10 \mathrm{~dB}$ change in peak-to-bandedge ratio for a hard-metal-tipped head.

The response consistency of ferrite heads made possible the design and implementation of totally adjustment-free signal electronics, without which we could not really consider the wideband digital recorder much more than a laboratory curiosity and certainly not the constant operational workhorse needed for the job.

\section{Tape}

The preferred instrumentation tape selected for Mark III is Ampex 795. Its width is 1 "; the standard length is $9200^{\prime}$; and the tape is packed on a $14^{\prime \prime}$ diameter reel. This tape is the only one currently available under GSA contract; 3M890 is comparable in quality and price.

The Ampex 790 series tapes have an improved, more-consistent short wavelength response compared to earlier $\gamma-\mathrm{Fe}_{2} \mathrm{O}_{3}$ instrumentation tapes such as the Ampex 780 series or $3 \mathrm{M} 888$. The new tapes are also less abrasive and relatively more free of dropouts. The VLBI system is immune to dropouts so there is no need for the specially screened and more expensive tapes advertised for "PCM" use. Thinner versions of modern tapes are desirable, but have yet to be evaluated; double length, $18,400^{\prime}$, on a $15^{\prime \prime}$ reel, would be a nice new standard.

Current $\gamma-\mathrm{Fe}_{2} \mathrm{O}_{3}$ tapes have sufficient output to support a quadrupling of track density ( $\sim 8 \mathrm{mil}$ track width) and still maintain error rates below 1 in $10^{6}$. A much greater track width reduction (for instance, direct use of VHS heads) would require a concomitant switch to a new high output (and higher coercivity) tape. $\mathrm{CrO}_{2}$ (Sony V-16), Co-doped (3M479, Fuji H621, VHS or $\beta$ type slit to $1^{\prime \prime}$ ), or even Fe-particle (3M Metafine) should be considered. VHS/ $\beta$ type is attractive as it is already standard state-of-the-art and thin enough for the double-length reel, but a commercial source willing to supply it slit to 1 " needs to be found.

Iron particle tape, incidentally, has so much output that it should support at least a fourfold reduction in track width, down to 0.3 mil of even the VHS format, which would result in a volume bit density of about $1.6 \times 10^{11}$ bits per cubic inch. This potential volume density is 200 times that of the present Mark III; the future of magnetic tape recording seems, indeed, assured!

\section{RECORDER ELECTRONICS}

Simplified signal electronics were developed at Haystack by the author and a controller was designed at NRAO by Benno Rayhrer, as part of an informal cooperation in the Mark III system design effort. 


\section{The Controller}

(1) Working with a Mark III ASCII transceiver submodule, allows the recorder to be remotely controlled and monitored, like every other module in the system, via a single RS-232 interface.

(2) Exercises the basic tape motion controls.

(3) Controls and displays which channels are recording.

(4) Contains and controls the use of a test set for recorder self-test or spot checking recorder performance.

(5) Controls several multiplexers (signal path switches) in the signal electronics including recorder bypass, track, and equalizer selectors.

(6) Supplies a programmable, timer-controlled synthesized reference frequency to the transport for speed control and initial tape synchronization in processing.

(7) For final lock-up in "tape" mode, in order to maintain fine data synchronization for processing, supplies a properly scaled "tape" signal to the transport which is derived from the bit synchronizer clock of any track in playback.

(8) Supplies a reference frequency at 21 times the a priori fonnatted bit rate for a bank of 32 digital bit synchronizers, and

(9) Controls the "footage" display on the transport directly as a general purpose display for footage, speed, error rate, etc.

The controller includes an 8085 microprocessor and resides on a 7.5"x16.2" (six-section) standard wire-wrap panel with plenty of spare real estate for as yet vaguely defined future functions such as head positioning electronics.

\section{Signal Electronics}

Three kinds of signal electronics modules have been designed for the recorder. All three use standard wire-wrap panels. The Signal Electronics modules are:

(1) Head Driver (HD) module, a small (single-section, 7.5" $\times 2.7^{\prime \prime}$ ) panel which is the entire record head interface for all 28 tracks.

(2) Analog Reproduce (AR) modules, two (two-section $7.5^{\prime \prime} \times 5.4$ ") panels each of which handles one 14-track reproduce head stack, with

(a) 14 preamplifiers, equalizers, post amplifiers and transition detectors (comparators) for parallel singlc-spced (135 ips) playback and 
(b) a pair of independent (potentially redundant) track selectors and dual 6-speed sets of equalizers, equalizer selectors, and comparators.

(3) Input/Output (IO) module, a six-section, 7.5" $\times 16.2^{\prime \prime}$ panel with two identical (odd and even) halves, each of which for input:

(a) receives 14 formatted bit streams and a "tape bit clock" from the Mark III formatter;

(b) substitutes test signals from the controller when put in TEST mode;

(c) passes either sct of signals, reclocked, to the head driver modulc;

(d) passes through individual track record enable signals from the controller;

(e) provides track selectors for BYPASS mode, in which the system can be tested exclusive of the head-tape interfaces by allowing any signal going to head driver module to replace the corresponding track-selected signal coming from an analog reproduce module;

and for output:

(a) receives the 16 (14 parallel and 2 track-and-speed-selected) clipped signals from one analog reproduce module;

(b) causes these parallel inputs or another pair of inputs, called group-commons, to be routed respectively to each of two groups of eight digital bit synchronizers in COMMON mode;

(c) independently selects each group-common input from one of four possibilities: the two odd and two even track-selected channels (This feature allows the distribution of a single channel to up to 32 processor correlator modules which can be operated with mutually offset delays so as to simply accommodate spectral-line VLBI requirements.);

(d) outputs signal-and-clock from 16 digital bit synchronizers to the Mark III processor;

(e) selects any one of these output signal-and-clock pairs to be sent back both to the controller's test receiver and "tape" signal divider and to the field system's dual channel decoder.

\section{RECORDER INTEGRATION}

The decision to undertake in-house development of recorder electronics and to include recorder integration in our plan to facilitate replication of the Mark III system was made in 1975, after evaluation of then available commercial equipment showed that the basic wideband high-density digital recorder was not (and still has not been produced as) a standard, reliable, general-purpose product. 
Unfortunately, the industry approach has been to offer custom add-ons to adapt "direct" analog recorder channels to high-density digital recording; this approach leads inevitably to

(1) very much more complicated, and hence intrinsically less reliable, electronics than needed;

(2) the use of relatively unproven custom circuit designs; and

(3) high cost to the user - typically $\$ 150 \mathrm{~K}$ for a custom quasi-Mark III equivalent recorder partly because too few of these systems are ever made sufficiently alike, so that development costs are never amortized.

The Mark III recorder, by way of a somewhat unfair comparison because it is made available on a non-profit basis and includes no hidden development burden, costs only $\$ 36 \mathrm{~K}$ to replicate - only $\$ 11 \mathrm{~K}$ of which is for production of recorder electronics and recorder integration and more than two-thirds of which goes into the direct purchase of transport $(\$ 12 \mathrm{~K})$ and heads $(\$ 13 \mathrm{~K})$.

Simplification was the major technical goal of the recorder electronics development. More than a ten-fold reduction in parts count, without loss of performance, was achieved compared to the least complex custom industry electronics we know of.

There are no potentiometers or other adjustable components in the signal electronics. This came about because of our early determination that channel characteristics were in fact sufficiently invariant (if the selected major commercial components are used) and because of an explicit effort made to eliminate them in our designs. Standard analog recorders, by comparison, have literally hundreds of pots. The mere thought of maintenance and accountability for set-up of such machines was sufficiently horrific that the ability to hold to the no pots design rule for the Mark III recorder was viewed as salvation.

\section{DIGITAL BIT SYNCHRONIZER}

As a specific example of the simplified circuitry developed, a diagram of the basic bit synchronizer used is shown in figure 3. This invention of the author uses only 2-1/2 IC's in the basic form shown. The circuit is completely digital and will capture any bit rate up to $650 / 21 \simeq 31 \mathrm{MHz}$ provided the reference frequency is within $\pm[100 /(21$ times maximum transitionless run $)]$ percent of 21 times the actual bit rate. The principle of operation is illustrated in the figure. Transitionless bit cells (NRZM zeros) cause the reference to be alternately divided by 10 and 11 so that the clock output frequency is the reference $\div 21$; early transitions (occurring in the first half of the clock cycle) cause the next half cycle to be shortened $1 / 21$ cycle; late transitions (in the second half of the clock cycle) cause the next half cycle to be similarly lengthened. The maximum phase tracking rate is, therefore, one cycle in 21 signal transitions. These bit synchronizers are particularly useful in the multi-channel recorder where all channels run at nominally the same rate, because only one common reference needs to be supplied to all synchronizers. Their completely digital nature implies that densely packaged units will not suffer from mutual interference. The performance of these digital bit synchronizers has proven equal to the best commercial analog units we have tried in the recorder application. 


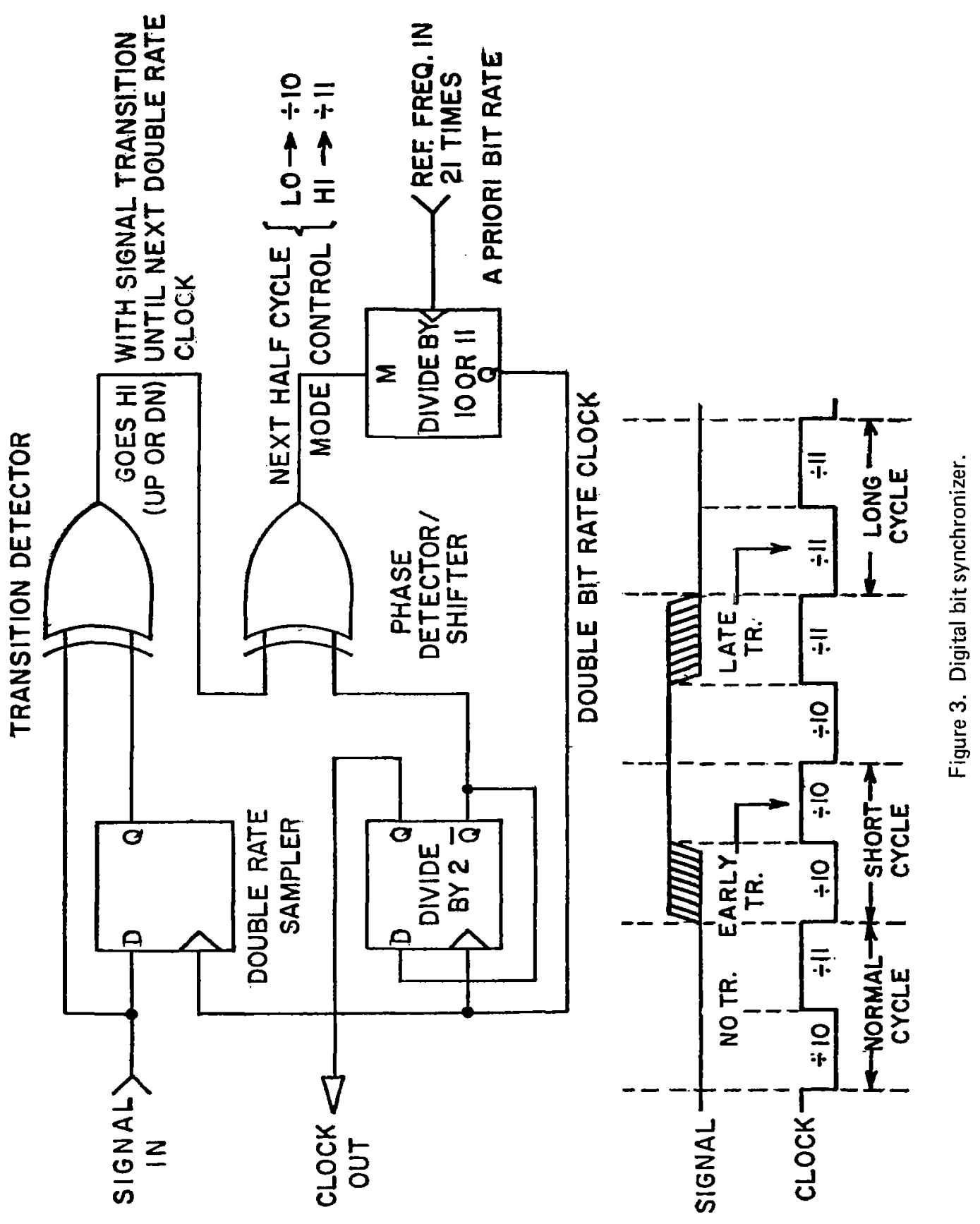




\section{CONCLUSION}

Simplified signal as well as supplementary control electronics are added to the selected commercial transport and head in order to complete a basic but, in our view for the first time, fully-engineered, multi-channel, wideband high-density digital recorder. Though designed specifically to fill the operational needs of the Mark III system, the integrated recorder is nevertheless a relatively efficient general purpose wideband digital mass storage device. As such, because of its simplicity, reliability, and low cost, the Mark III recorder should be attractive in other applications as well. The Mark III recorder's as yet untapped potential to support much higher track density remains to be exploited within the frame work of the Mark III system and promises to give it a long lease on life. 


\title{
MARK III CORRELATOR HARDWARE AND SOFTWARE
}

\author{
Alan R. Whitney \\ NEROC Haystack Observatory
}

\begin{abstract}
The Mark III correlator system is based on a modular philosophy in a manner similar to the Mark III data acquisition system. Each "correlator module" independently processes the data from one track pair. Therefore, 28 modules are necessary to complete a full one-baseline processor and 84 modules for a full 3-baseline processor. Each correlator module has two interfaces: (1) data and clock from each of the two tracks to be correlated and (2) Computer Automated Measurement and Control (CAMAC) dataway interface to the computer. The processor is organized around the IEEE "CAMAC" standard architecture, housing 15 correlator modules in each of 6 "crates." This allows one-pass processing of a full 3-baseline 28-track observation or a 6-baseline (4 station) 14-track observation. The correlator architecture allows easy expansion for up to 8 stations. The computer system is an HP1000 system utilizing a 16-bit minicomputer with disc and tape peripherals. The processing software is also organized in a modular fashion with many independent but cooperative programs controlling the operation of the Mark III processor. Processing time through the correlator is normally real time or faster, with graphics displays providing real-time monitor and control of the processing operation.
\end{abstract}




\section{DESIGN PHILOSOPHY}

In keeping with the philosophy of the Mark III data acquisition terminal, the Mark III correlator has also been designed as a highly modular system. In fact, the correlator system is modular to the extent that the entire correlator is made up of identical modules. Each correlator module is responsible for cross-correlating the data from one track-pair, so that 28 modules are required to process a single 28-track single-baseline observation.

\section{BASIC CORRELATOR MODULE CHARACTERISTICS}

Figure 1 shows a simplified view of the input/output interfaces of the correlator module. Input data to the module is made up entirely of the data and clock signals from each of the two tracks to be correlated. Approximately once every 2 seconds the host computer provides processing parameters to the module and receives the processed data from the previous approximately 2 seconds. As explained in the paper presented by Ed Nesman, this computer interface was selected to conform to the CAMAC standard.

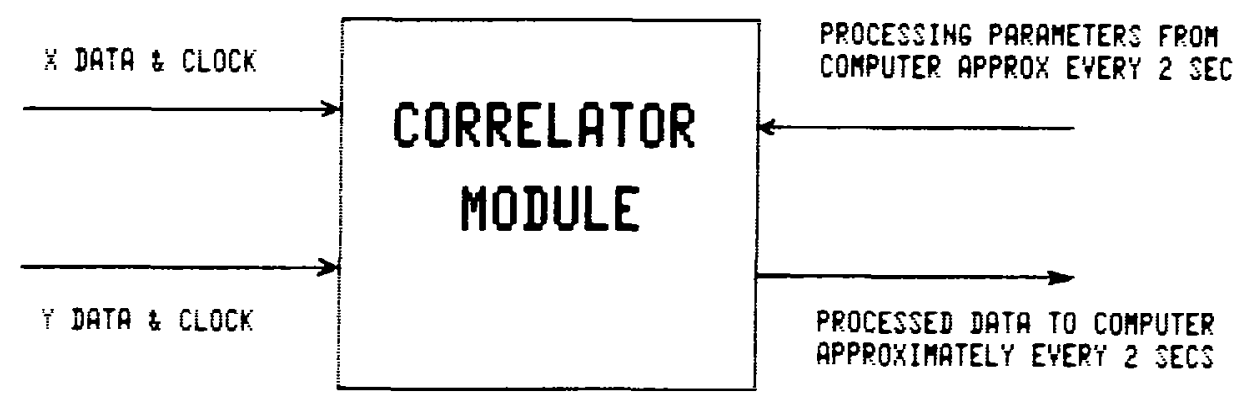

Figure 1. Correlator module input/output.

Figure 2 lists the basic characteristics of the correlator module. As indicated in figure 1, the interface of the recorded data is very simple, consisting only of the data and clock signals from each of the two data streams to be cross-correlated. The X-clock signal is used as a basic clock within the correlator module, so that normal processing will take place regardless of the data rate from the tape recorders; in other words, no control signals indicating data rate to the module are necessary. The reproduce circuitry within the tape recorders is designed in such a manner that the clock signals will always "flywheel" even in the absence of data, so that processing will continue normally even through deep data dropouts.

The cross-correlator section of the module performs processing for 8 complex lags during normal processing or 16 real lags for auto-correlation processing. The accumulation registers are 23 bits wide, allowing the accumulation of slightly more than 2 seconds of data at the 4 Mbps sample rate. 


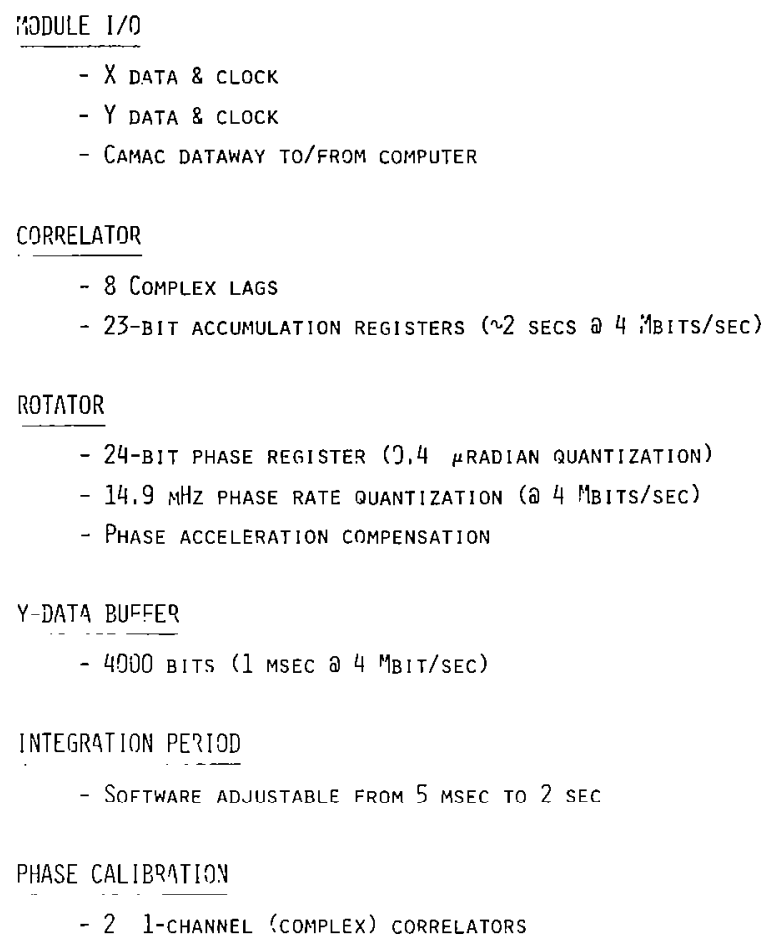

Figure 2. Mark III correlator module characteristics.

Fringe rotation is accomplished through the use of a 24-bit phase register, thus achieving 0.4 microradian resolution. A three-level rotation scheme is used similar to that used by the Mark I correlation system to optimize the signal-to-noise ratio of the processed data. The phase-rate-resolution of the rotator is selectable in powers of two under software control and is normally set to be $14.9 \mathrm{MHz}$ at the $4 \mathrm{Mbps}$ sample rate. The selection of rotator phase-rate resolution must be traded against the maximum fringe-rate that can be processed; selecting the resolution to be $14.9 \mathrm{MHz}$ for the $4 \mathrm{Mbps}$ sample rate limits the maximum rotation speed to approximately $250 \mathrm{kHz}$. Higher fringe rates may be processed by decreasing the phase-rate resolution at the possible expense of shortening the basic correlator integration period due to degraded phase-tracking of the rotator. Also included in the fringe-rotator section of the correlator module is a simple phase-acceleration compensation mechanism which is designed to be sufficient to compensate for any expected fringe-phase acceleration during the normal correlator integration period.

A 4000-bit addressable buffer is provided in the path of the Y-data and Y-clock signal streams so that the $\mathrm{X}$ and $\mathrm{Y}$ tape drives need be synchronized only so well as to maintain the a priori bit delay within this 4000 -bit window. Synchronization of the tape drives to this level is a relatively easy task.

The basic integration period of the correlator module is software-controllable from 1 frame to 512 frames, or equivalently, from $5 \mathrm{msec}$ to 2.56 seconds (tape-time) at $4 \mathrm{Mbps}$ sample rate. For most normal geodetic processing, an integration period of 1 to 2 seconds is used. This provides a search 
range of 1.0 to $0.5 \mathrm{~Hz}$, respectively, which is quite adequate for all normal processing. Since the computer must service the correlator module once each integration period, the load on the host computer varies approximately inversely as the integration period. As the integration period shortens, a point is reached where the number of modules that may be operating simultaneously must be reduced or tape-playback speed reduced in order that the computer may keep up.

Not indicated in figure 2 is the availability of a "pulsar" processing mode, which may be used for special applications. In this mode, a start bit number and stop bit number may be specified within an integration period so that only those bits within the start-stop window are processed. This mode is primarily designed for processing of pulsar data, but may be used to process any pulse-type data or for other special-purpose processing.

Phase-calibration (phase-cal) processing is also provided within the correlator module. Separate processors are used for the $\mathrm{X}$ and $\mathrm{Y}$ data streams, although the frequency of the phase-calibration rail must be the same for both $\mathrm{X}$ and $\mathrm{Y}$. A much-simplified rotation scheme is used for phase-cal processing compared to the rotator described above. Rotator phase-rates are constrained to be such that the phase-cal signal must contain an integral number of sample bits per quarter-phase-cal-period. This condition is met with the phase-cal signals that are normally used. Also, only a two-level rotation scheme is used, since signal-to-noise of the phase-calibration signal is not normally of concern. By imposing these conditions and restraints, the circuitry used for phase-cal processing is considerably simplified compared to that of the main lobe rotator and cross-correlator.

Internal testing of the correlator module is possible through the use of an on-board quasi-random signal generator. Partial testing is possible off-line with no computer servicing necessary. Complete testing using the internal test generator is possible when a host computer is available.

\section{SIGNAL FLOW THROUGH CORRELATOR MODULE}

Figure 3 shows a block diagram of the Mark III correlator module. Both $\mathrm{X}$ and $\mathrm{Y}$ data streams are first decoded by their respective DECODER's. The $\mathrm{X}$ and $\mathrm{Y}$ data streams may be independently selected from any one of eight data streams which may be applied to the module (not shown in figure 3). The decoder has the responsibility of synchronizing itself to the data stream (by use of the sync words embedded in the data streams), extracting the time and auxiliary-data fields, checking the time/aux-data field for errors (using the embedded 12-bit CRCC check character), stripping parity bits and counting parity errors. The decoded X-data is then passed to the X PHASE-CAL DETECTOR and the X-DATA ROTATION-BLANKING. Rotation is controlled by the FRINGE RATE GENERATOR, which generates a three-level quadrature rotation signal according to the parameters received from the host computer. The quadrature-rotation signals from the rate generator are processed by the MODE SELECTOR before actually being applied to the X-data. The mode selector may apply an additional $+/-90 * n$ degree phase-shift in conjunction with a shift in the $\mathrm{X}-\mathrm{Y}$ bit delay to accomplish "automatic fractional-bit-error correction" - more will be said about this later. After rotation, the X-data stream is applied to the CORRELATOR for cross-correlation with Y-data. 


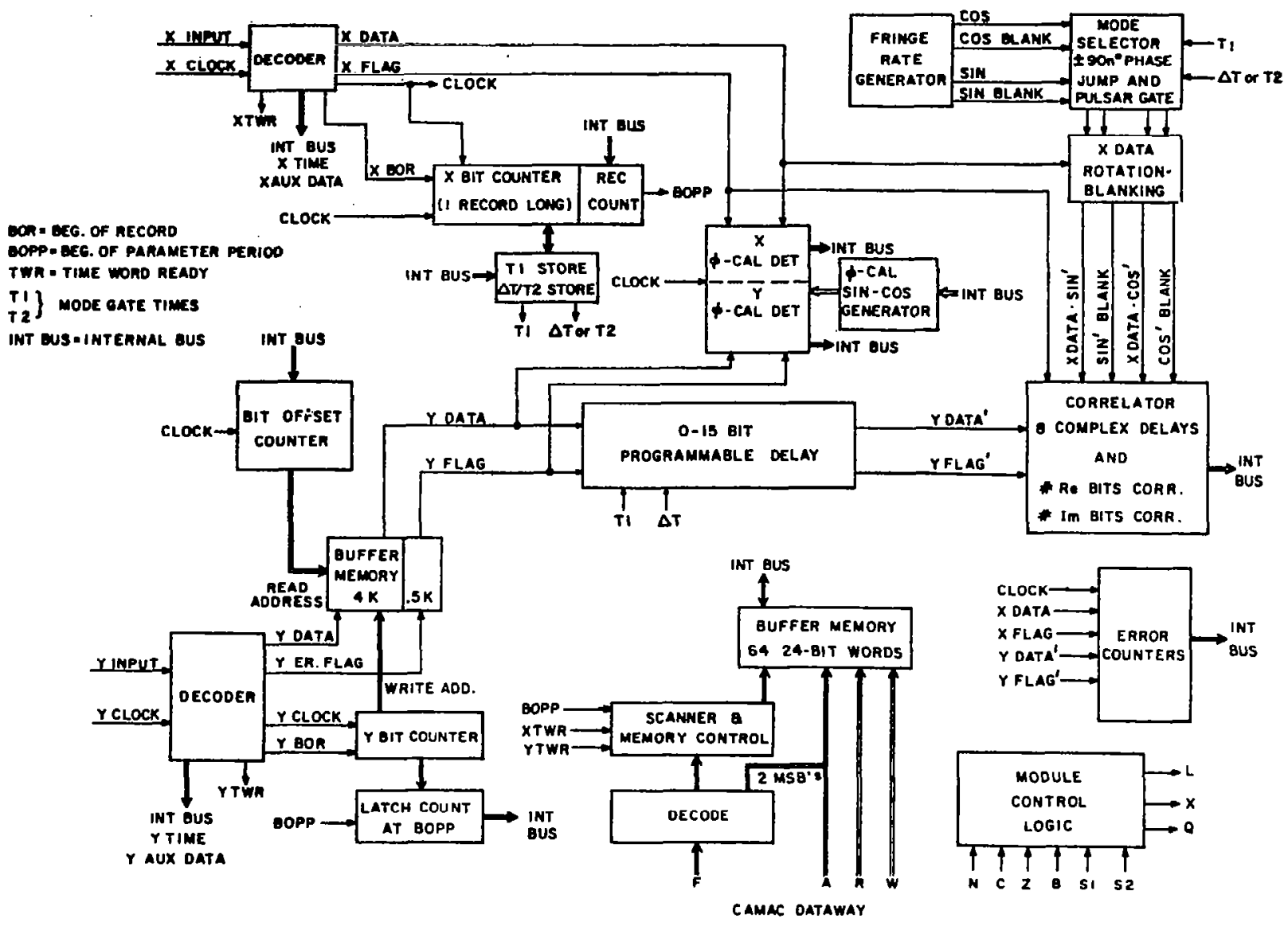

Figure 3. Mark III processor internal block diagram.

After emerging from the Y-decoder, the Y-data stream and accompanying flags are routed to a BUFFER MEMORY capable of storing 4000 bits of data. Data is removed from this buffer memory according to a BIT OFFSET COUNTER which has been set according to the a priori parameters received from the host computer, so that the proper Y-data bits are made available to the correlator. The output from the buffer memory is applied directly to the Y-PHASE-CAL DETECTOR for phase-cal processing, and to a 0-15 BIT PROGRAMMABLE DELAY preceding actual correlation with the rotated X-data. This programmable delay is used to shift the Y-data stream one-bit at a time to the X-data stream as the a priori delay changes within an integration period. Up to 15 such bit shifts may be made within a single integration period, according to the parameters provided by the host computer. Each time such a bit shift takes place, the MODE SELECTOR imposes an additional +/-90 phase shift to the signals emerging from the FRINGE RATE GENERATOR. Through the use of this "automatic fractional-bit error correction" technique, correlation may be carried through many changes in the $X$-to- $Y$ bit delay so long as the model contained in the FRINGE RATE GENERATOR is sufficiently accurate to track the fringe phase. 
The CORRELATOR section of the module does the actual correlation between the rotated X-data stream and the unrotated Y-data stream. Eight complex lags are normally processed, although the correlator may also be configured under software control to do auto-correlation processing of either the $\mathrm{X}$ or $\mathrm{Y}$ data stream.

The determination of tape-recorder synchronization is made by latching the $\mathrm{Y}$ bit count (within a frame) at the beginning of the first $X$ frame of an integration period. Integration period boundaries are constrained to start at X-data frame boundaries, so that with knowledge of the Y-data stream time and LATCH COUNT at the beginning of an integration period, the tape-recorder synchronization may be computed and the necessary commands sent to the tape transports.

All communications with the host computer are "double-buffered" so that computer servicing of the correlator module is completely asynchronous with respect to the $\mathrm{X}$ and $\mathrm{Y}$ data streams except that servicing must take place once each integration period in order to update processing parameters and extract processed data. Each correlator module communicates individually with the host computer. During a normal computer service, approximately 21 bytes are sent computer-to-module and 114 bytes module-to-computer.

\section{CORRELATOR SYSTEM CONFIGURATION}

The Mark III correlator system as currently built is designed for one-pass processing of 3-station/ 28-track data or 4-station/14-track data. Ninety correlator modules are housed in a single rack of six CAMAC crates, fifteen modules/crate. Each of the six crates is identical and receives an identical set of data signals from the four tape transports distributed as follows:

Module 1 - Receives signals from both tracks \#1 and \#2 from each of the four tape drives (recall that each module may select $\mathrm{X}$ and $\mathrm{Y}$ from among eight inputs to the module).

Module 2 - Receives signals from both tracks \#3 and \#4 from each of the four tape drives.

Module 14 - Receives signals from both tracks \#27 and \#28 from each of the four tape drives.

Module 15 - This module is dubbed a "floating module" whose input may be selected to be from any track of any of the four tape drives. Track selection is actually made within the control electronics of the tape drive. Tape drive selection is made at the input to the correlator module itself.

Figure 4 shows the resulting correlator configurations for processing of typical 3 and 4 station data. For the case of 3-baseline/28-track data, each crate processes either the odd or even numbered tracks from one of the three baselines. The floating modules may be assigned arbitrarily for either 
redundancy checking or for special processing. In the 4-station/14-track case, each crate is assigned to one of the six basclines and may process all even or all odd tracks, which is compatible with the standard 14-track mode of data recording. The floating modules may again be assigned as desired.

3 BASELINES/28 TRACKS

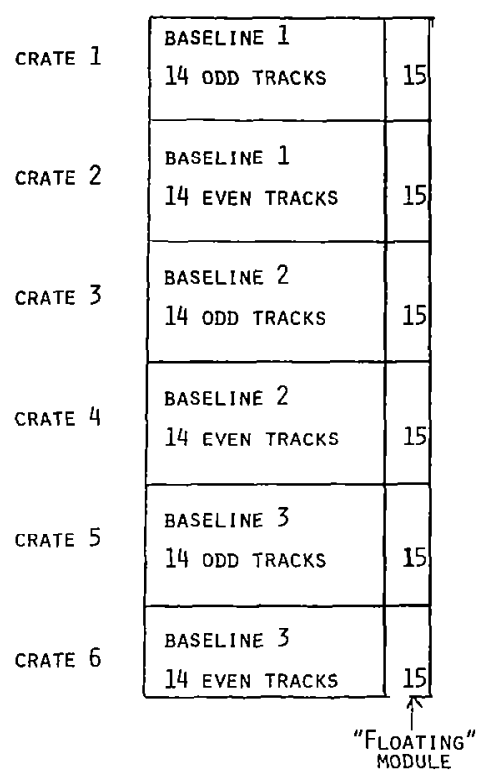

6 BASELINES/14 TRACKS

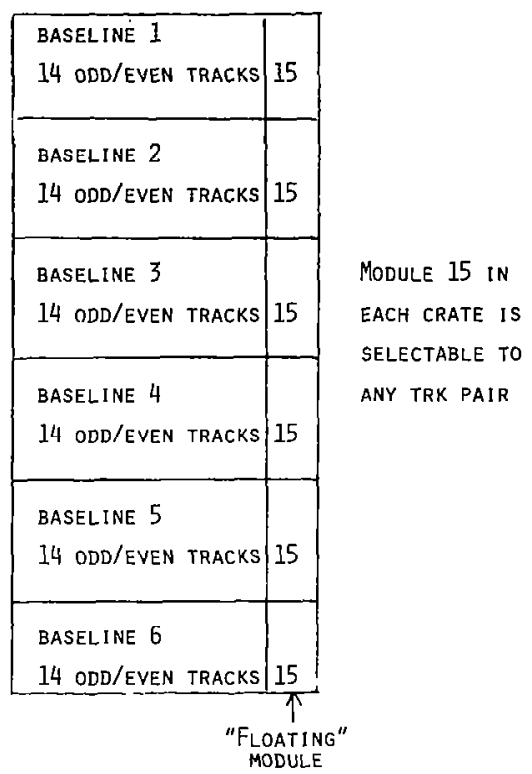

Figure 4. Correlator configuration for $3 \& 4$ stations.

Due to the modularity of the correlator system, it is easily expandable in several ways. With the addition of an identical 6-crate correlator system, 4-station/28-track data may be processed in single-pass. These additional 6 crates may be handled either by the existing host computer or by an additional host computer, if necessary. Because the data interface to the tape transports is very simple, expansion of the system to a large number of stations is a straightforward matter. Correlator modules and host computers may be added to bring the system to any capability desired. Connecting a group of HP1000 computer systems into a multicomputer net for handling a large correlator system is straightforward and cost effective. High-speed data devices such as discs are shared among CPU's, so that all data from a particular processing pass will end up properly concatenated ready for further processing.

\section{SPECTRAL-LINE PROCESSING CAPABILITY}

Although the Mark III processor design is primarily oriented towards the processing of continuum data, provisions have also been made for processing of spectral-line data where many lags are desired. This is done by staggering the a priori delays between modules to process many lags. Using 90 correlator modules to process a single-track, single-baseline spectral-line observation, 60 modules may 
be used to do cross-correlation (480 complex delays) and 15 modules each for $\mathrm{X}$ and $\mathrm{Y}$ autocorrelation (240 real delays). Similarly, a single-track 3-baseline observation may be processed using 22 modules on each baseline for cross-correlation processing (192 complex delays) and 6 modules for autocorrelation processing (96 real delays).

\section{CORRELATOR COMPUTER SUPPORT}

The Mark III correlator system is supported by an HP1000 computer system utilizing a Model $2117 \mathrm{~F}$ CPU. The HP1000 is a 16-bit minicomputer system of modest size, presently incorporating 256 kbytes of semi-conductor memory and $135 \mathrm{Mbytes}$ of high-speed disc storage. All computations and control for the Mark III correlator system are done using this computer. The interface to the correlator hardware is provided by a commercially-available CAMAC branch-driver. Interface to the tape transports is through a standard ASCII RS-232 interface to ASCII Transceivers in the tape transports. No special-purpose or in-house-designed interfaces have been used in the correlator system.

The HP1000 computer system is supported by a standard RTE-IV system supplied by HewlettPackard (HP). This system supports a full multiprogramming environment, allowing many concurrent processing tasks. Full 64-bit precision arithmetic is supported in hardware for all critical calculations and use of a microprogrammed FFT in fringe-search operations make processing speed quite high (competitive with Digital Equipment Corporation VAX for FFT's and common scientific functions).

The computer system is expandable in a modular fashion to meet the needs of a growing processor system. Multiple CPU's may be connected in a variety of configurations, sharing peripherals as desired. Such easy modular expandability is in keeping with the modular expandability of the processor itself.

\section{CORRELATOR SOFTWARE SUPPORT}

The correlator support software is also highly modular. Figure 5 shows a rough outline of data flow through the processor system. The correlator itself is supported by a program called COREL which is really a battery of approximately 10 interacting programs each controlling certain aspects of the processing. Setup for processing a particular observation is done completely through simple ASCII data files which may be generated manually by using the on-line editor or through software which generates the setup files automatically according to an actual observing schedule. All a priori calculations for processor operation are done in real time while the correlator is operating. An on-line graphics display provides for real-time monitoring and control of the processing operation. All raw correlator output data is output to disc to await fringe-search processing. 


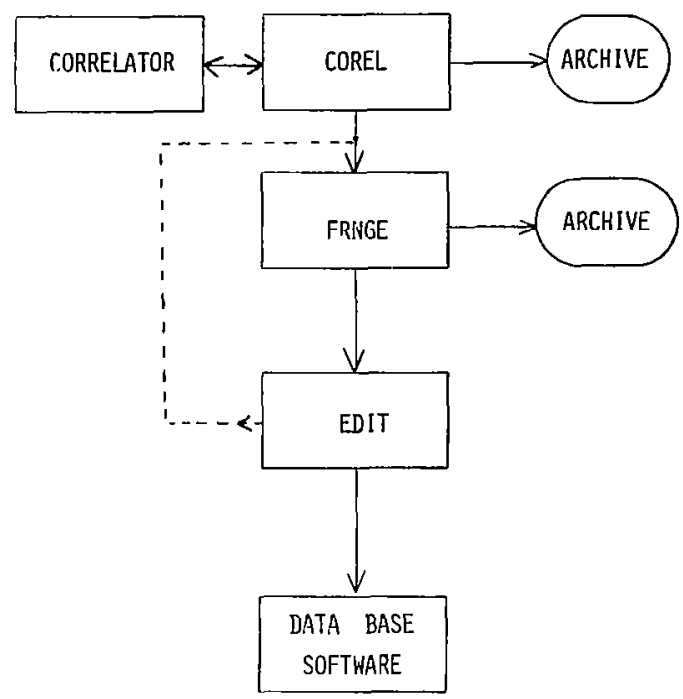

Figure 5. Mark III correlator software flow.

Fringe-search processing is done by program FRNGE, which collects all of the data from a particular observation, one baseline at a time, and does processing to estimate correlation amplitude, group delay, phase-delay rate, fringe phase, etc. The results of the fringe search are again stored to disc to await possible editing with program EDIT. EDIT allows viewing of the FRNGE output as a trouble-shooting aid; after processing by EDIT to remove bad data, etc., FRNGE may be run again. Finally, the edited (if necessary) output of FRNGE is transferred into the DATA BASE to await further processing for geodetic, astrometric, and astronomical parameters. It is important to emphasize that all of these programs may be run in a hands-on interactive mode when necessary or desirable to process troublesome data or do special processing in an efficient manner.

All data is archived to permanent mag tape libraries at two stages in the correlation processing. The raw output of COREL containing all raw correlation coefficients and a prioris is archived so that this data may be retrieved and FRNGE re-run if necessary or desirable. Also, all FRNGE output is archived in a form which EDIT may retrieve and review. This output includes correlation amplitude and phase on short time scales (typically 1 to 2 seconds) for each observation.

During normal geodetic correlation processing of 3-station/28-track data, the computer is occupied 25 to 50 percent of the time with real-time processor-related tasks. The remaining time may be used in any manner desired without interfering with the correlation processing. Normally this "leftover" time is used for fringe-searching on a run which has just completed correlation. 


\title{
CORRELATOR COMPUTER INTERFACE AND MODULE IMPLEMENTATION- MARK III PROCESSOR
}

\author{
Edwin F. Nesman \\ NEROC Haystack Observatory
}

\section{INTRODUCTION}

This paper briefly describes two hardware aspects of the Mark III Processor treated in the previous paper by Alan Whitney. The first area concerns the choice of interface to the controlling minicomputer and the second area concerns the implementation of the correlator module.

\section{COMPUTER INTERFACE}

At the outset of the hardware design associated with the Mark III Processor, it became obvious that one of the major functions which would have to be designed was a large computer-to-correlator module $\mathrm{I} / \mathrm{O}$ bus or module selector to facilitate communications with the 90 independent correlator modules. Another outstanding design task identified was the mechanical packaging of the correlator circuitry. Our investigation of CAMAC (Computer Automated Measurement and Control)IEEE Std 583-1975, convinced us that both of these functions were available to us without the burden of engineering through the use of the standard. Another benefit was that CAMAC provides independence from the host computer, which in our case had not yet been specified. The connecting link to the host computer, of course, must be computer-specific; this link, the branch driver, is available for most popular minicomputers. In our case, an HP1000 minicomputer was selected; although a branch-driver of American manufacture was not available, a unit of European manufacture was.

As with most human endeavors, perfection does not exist or in any event last very long. We have deviated from the CAMAC standard in our processor development in two areas. The first area is power. We require +5 volts at 80 amperes per crate complement of 15 correlator modules. Consequently, we have converted the +6 -volt bus to +5 -volt use and in addition have defined two adjacent buses for +5 -volt use in order to increase current-carrying capacity into each module. The second area involves the physical size of the correlator modules and the crates into which they are plugged. Our final correlator circuit design is comprised of 340 elements, mostly dual in-line package integrated circuits. In order to accommodate this population, our module is 3.5 inches taller, 5.3 inches deeper, and 1.5 times as wide as a standard single-width CAMAC module. In each of the six crates required for our system, we accept 15 modules in the first 22.5 slots. We mate with the standard 
CAMAC dataway (backplane) by means of an auxiliary connector edge board offset by one-half a module width in alternate modules. Procurement of the special CAMAC hardware did not require generation of engineering drawings and the costs were very reasonable.

\section{MODULE IMPLEMENTATION}

The circuit design of the Mark III correlator module represents a conservative approach in that no custom LSI was undertaken; it was felt that the risks in project time and funding were not warranted cven though a reduction in number of circuit elements might have resulted. The majority of the circuits used are low-power Schottky TTL types, with the balance being NMOS RAM's, TTL RAM's, and several PROM's used as sequence controllers. Power consumption is about 26 watts per module.

As mentioned previously, the component count had reached 340 at the end of the design phase. A normal single-width CAMAC module holds in the order of 80 to 90 IC's using printed circuitry as an interconnection media. Multiple CAMAC modules and a single "large" CAMAC module were considered as possible packaging forms for the correlator. The "large" CAMAC module approach was chosen because of the difficulty in partitioning the correlator with minimum interconnections, the fabrication economy of a single large planar assembly, and the desire to minimize the number of modules (thereby staying within the hardware capacity of a single CAMAC branch which requires only two HP I/O slots). Wrapped-wire interconnections were used in order to allow easy modifications during the development phase and were also utilized in the production phase to avoid the high costs and risks associated with multi-layer printed-circuit boards. Another benefit of the wrappedwire approach is increased component density; our "large" CAMAC module holds four times the IC's in three times the volume of a printed-circuit single-width module.

Our experience in the production and checkout of correlator modules has been positive. The bulk of the fabrication is accomplished by contract machine wrapping, leaving component population of the panel as the major in-house task, along with checkout. Checkout of each module is averaging four hours. Approximately half the modules work when plugged in; the balance have some combination of wiring or population problem.

The cost of the materials and services for each module is under $\$ 1000$, with the allocation roughly equal among the basic wrapped-wire panel, wiring services, and integrated circuits. 


\title{
MARK III VLBI SYSTEM - TROPOSPHERIC CALIBRATION SUBSYSTEMS*
}

\author{
G. M. Resch \\ Jet Propulsion Laboratory
}

\begin{abstract}
Tropospheric delay calibrations are implemented in the Mark III system with two subsystems. Estimates of the "dry" component of tropospheric delay are provided by accurate barometric data from a subsystem of surface meteorological sensors (SMS). An estimate of the "wet" component of tropospheric delay is provided by a water vapor radiometer (WVR). Both subsystems interface directly to the ASCII Transceiver bus of the Mark III system and are operated by the control computer. Seven WVR's are under construction and will be used at various radio observatories both as Mark III subsystems and as general observatory calibration tools. These instruments are designed to operate in proximity to a radio telescope and can be commanded to point along the line-of-sight to a radio source. They should provide a delay estimate that is accurate to the $\pm 2 \mathrm{~cm}$ level.
\end{abstract}

\footnotetext{
*This paper represents the results of one phase of research carried out at the Jet Propulsion Laboratory, California Institute of Technology, under NASA Contract No. 7-100, sponsored by the National Aeronautics and Space Administration.
} 
Any VLBI system that presumes centimeter level accuracy must provide for good tropospheric calibration. In the Mark III data acquisition system, this will be handled by two important subsystems. The first consists of a collection of surface meteorological sensors (SMS). Initally, this package will include an accurate barometer to provide an estimatc of the "dry" delay component, a temperature sensor, and a humidity measuring device. The later two measurements could provide an estimate of the "wet" delay component based on a surface model if necessary. Later, we may add a device to estimate cloud cover and a solar hygrometer. Responsibility for this package rests with the group at Goddard Space Flight Center. The second tropospheric calibration subsystem consists of a water vapor radiometer (WVR) that can estimate the "wet" delay component along the line-of-sight of the associated radio telescope. Seven of the WVR's are being constructed at the Jet Propulsion Laboratory (JPL).

The functional description of the WVR is shown in figure 1 . Our goal is to provide a $\pm 2 \mathrm{~cm}$ delay calibration for all elevations above $20^{\circ}$ under most atmospheric conditions. When it is raining, the assumptions on which the algorithms are based will be violated and the instruments will not operate properly. Similarly, a film of liquid $\mathrm{H}_{2} \mathrm{O}$ on the cover of the horns (e.g., dew) introduces large errors. More work is needed to define the range of operating conditions and to provide some way to remove the accumulation of liquid $\mathrm{H}_{2} \mathrm{O}$.

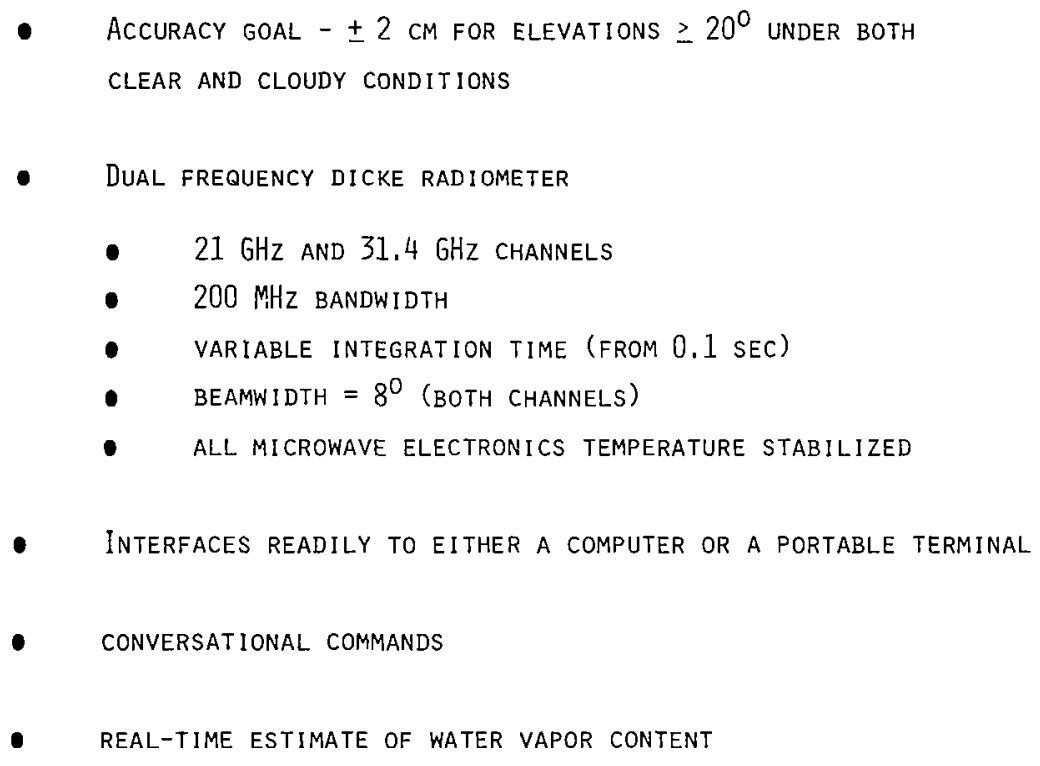

Figure 1. WVR functional description.

The instrument consists of two microwave channels, one centered at $21 \mathrm{GHz}$ and the other at $31.4 \mathrm{GHz}$, to provide an on/off line measurement. Each channel operates double sideband with a total bandwidth of $200 \mathrm{MHz}$. The effective system temperature in each channel is approximately $1100^{\circ} \mathrm{K}$. The microwave module sits on a simple azimuth/elevation positioner and can be pointed to within $1^{\circ}$ of a specified position. However, the elevation readout is accurate to $\pm 0.3^{\circ}$. The microwave module contains waveguide loads at ambient temperature and at $100^{\circ} \mathrm{C}$ in order to provide internal calibration. 
Both the microwave and the positions modules are interfaced to a local control panel intended primarily as an aid to troubleshooting. The primary interface to the user will be via a simple serial RS232 line from the microprocessor. However, should a malfunction be suspected, the operator can go to the local control panel and, in principle, determine which module is misbehaving. The microprocessor consists of a single board microcomputer and an analog $\mathrm{I} / \mathrm{O}$ interface board on which there are 32 multiplexed analog inputs to a 12-bit A/D converter and two 12-bit D/A converters. The control program will reside in ROM (Read Only Memory) and a small amount of RAM (Random Access Memory) will provide space for a data buffer. Note however that the WVR does not provide a capability for storing large amounts of data - that is the responsibility of the user.

Earlier today, Tom Clark described the approach taken to control all of the functions of the Mark III data acquisition system. A microprocessor will control each module and all activity will be coordinated by a central minicomputer. Both the SMS package and the WVR were designed to operate as modules in this system. Since the electrical interface chosen is particularly simple and relatively "universal," the SMS and WVR could also serve as general observatory support instruments. Any microcomputer with an RS232 interface or, for that matter, a portable terminal could be used to control and acquire data from these subsystems.

From independent tests performed during the last several years, we convinced ourselves that the WVR really can measure water vapor. About a year ago, it was realized that what was needed was a clear-cut demonstration that the WVR could improve the quality of VLBI data. To this end, we have concentrated on building seven instruments that will be provided to various installations. Figure 2 shows our construction schedule. The first unit was built from parts cannibalized from the engineering model of a spacecraft radiometer. It was delivered to Project ARIES in early May and is now being tested. Units R2 and R3 were built using microwave components from Sense Systems Inc. (Temple City, California). The hardware has been completed and awaits testing and calibration. Unit R3, the Haystack WVR, was delivered only yesterday; Unit R2 for the Owens Valley Radio Observatory has been completed but is still at JPL. Units R4 through R7 are being constructed from new components and are in various stages of assembly. All hardware will be completed and delivered by October 1979. The design, assembly and fabrication of all WVR hardware has been the responsibility of the Microwave Radiometry Group at JPL under the able direction of Mr. Noboru Yamane.

The software that will reside in the WVR microprocessor which will provide the control and communication features is still in the definition stage. Version 2 of this software is supplied with the WVR that you will see on display. We expect to go through several more versions of this software during the next year as the specifications are refined by feedback from the users of these instruments. More importantly, we expect that these instruments will contribute to increasingly accurate VLBI results during the next year. 


\section{RADIO INTERFEROMETRY}

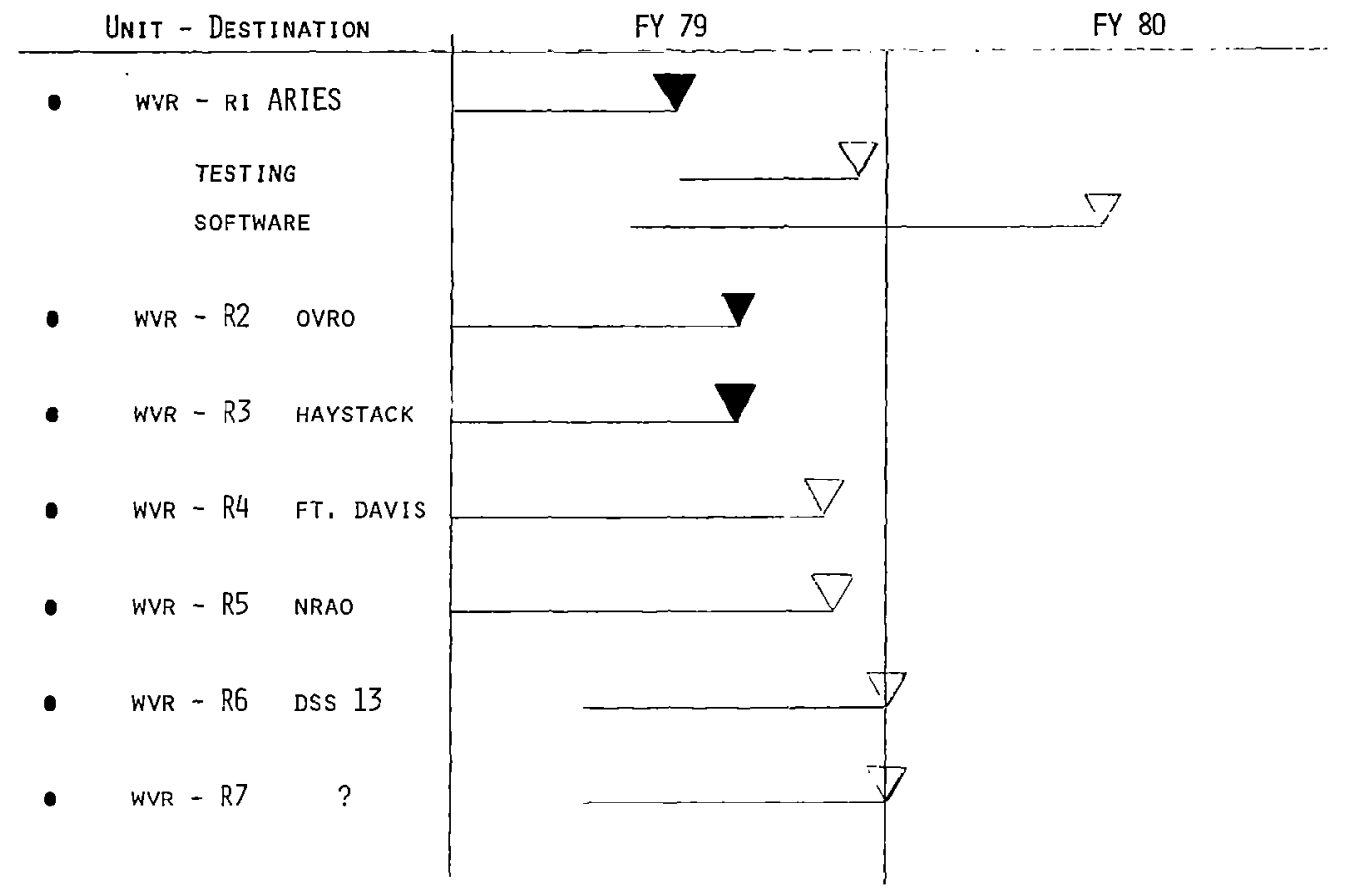

Figure 2. WVR construction. 


\title{
MARK III REAL-TIME FRINGE-DETECTION SYSTEM
}

\author{
J. I. Levine and A. R. Whitney
}

NEROC Haystack Observatory

\begin{abstract}
A RAM memory built into the Mark III decoder module allows the capture of 1 Megabit of data. Data may be collected either in real time or from a pre-recorded tape. Once collected, the data may be retrieved using a standard EIA serial data link. The data may be transmitted to a remote computer for cross-correlation processing with similar data from other stations to verify fringes in "real time." The data may also be analyzed by a local computer to verify phase-calibration, bandpass, format, etc., during a Mark III observing session.
\end{abstract}




\section{INTRODUCTION}

A well-known difficulty in conducting very long baseline interferometry (VLBI) experiments is real-time verification of proper data taking. Much money and effort has been spent in rushing newly recorded tapes back to a processing center to verify that everything is in order. With the recent development of high-density, low-cost semi-conductor memories, it has become practical and economical to capture a small segment of VLBI data in local memory and transmit that data over ordinary telephone lines to a remote processing facility where it may be cross-correlated with similar data from other sites for fringe verification. The Mark III Data Buffer has been designed for this purpose.

\section{HARDWARE IMPLEMENTATION}

The Data Buffer system is a 1-Megabit memory which is physically mounted within the Mark III Dual-Decoder module and accepts data from the " $\mathrm{A}$ " section of the decoder for storage. Data may be collected in real time in either "bypass" mode or read-after-write, or after-the-fact by playing back a pre-recorded tape.

Operation of the Data Buffer typically is as follows: Once armed by the host computer, the buffer waits for a sync signal from the decoder (normally on the 10-sec mark). When this sync signal is detected, all following data is stored to the capacity of the memory. Data is acquired in a single, continuous 1-Megabit window but is blocked into 4-kilobit segments for transmission to the host.

The data stored is exactly that which would have been written onto a Mark III tape and includes all header, synchronization, and parity bits. The data buffer contents, therefore, represent a "slice" of Mark III tape, 1 track wide and 1 Megabit long.

All functions of the data buffer may be controlled through a host computer. The data source and operating modes are reported to the host computer in a status byte sent with each transmission. Identification codes, segment number, and a binary checksum are also reported to the host on each transmission. Re-transmission of any segment can be requested by the host in case of difficulty.

An auxiliary data source may be selected by a rear-panel toggle switch and a set of 3 BNC jacks. This might be used, for example, to capture Mark II data in a similar manner.

A set of 13 memory test patterns can be loaded and verified by the host in order to detect malfunctioning circuits. The expected positions of the Mark III header information from frame to frame may also be exploited for error detection purposes.

While each data buffer is wired for a full megabit of memory, capacity may be reduced in 0.25 Megabit increments for economy. Also, the design will directly accommodate 64-kbit memory chips when they become economical, expanding the memory capacity to 4 Megabits. 


\section{SOFTWARE SUPPORT}

A full set of software-support programs has been developed to retrieve and process the data collected by the Mark III Data Buffer. The data stored within the data buffer may be accessed by either a local or remote computer. Transmission to a local computer may be done at 9600 baud, requiring only a few minutes to transmit a full buffer. The local computer can examine the data for proper format and process it for phase-cal signals and bandpass. Data transmission to a remote computer is normally done using ordinary dial-up telephone lines at 1200 baud. At these data rates, approximately 15 to 20 minutes is needed to transmit a full 1-Megabit buffer. Data rates up to approximately 4800 baud can be supported through the use of more sophisticated modem equipment. Cross-correlation and phase-calibration processing is done in software in a manner exactly analogous to the Mark III Processor and is displayed in real-time as data is received from the data buffer. Software integration periods may be shortened to any desired value to search for the possible presence of phase-modulation of local oscillators at power-line frequencies, a problem that has occasionally plagued VLBI experiments.

Figure 1 displays the results of "real-time" Mark III fringes on 3C84 at X-band obtained between Haystack and Green Bank during an experiment on August 4, 1979. Figure 1a displays the fringerate spectrum; note that there is no evidence of local-oscillator phase-modulation at power-line frequencies. Figure $1 \mathrm{~b}$ displays the correlation amplitude and residual-fringe-phase versus time, one point for each 5 -msec frame. Also displayed in figure $1 \mathrm{~b}$ is the phase-calibrator phase for each station. 

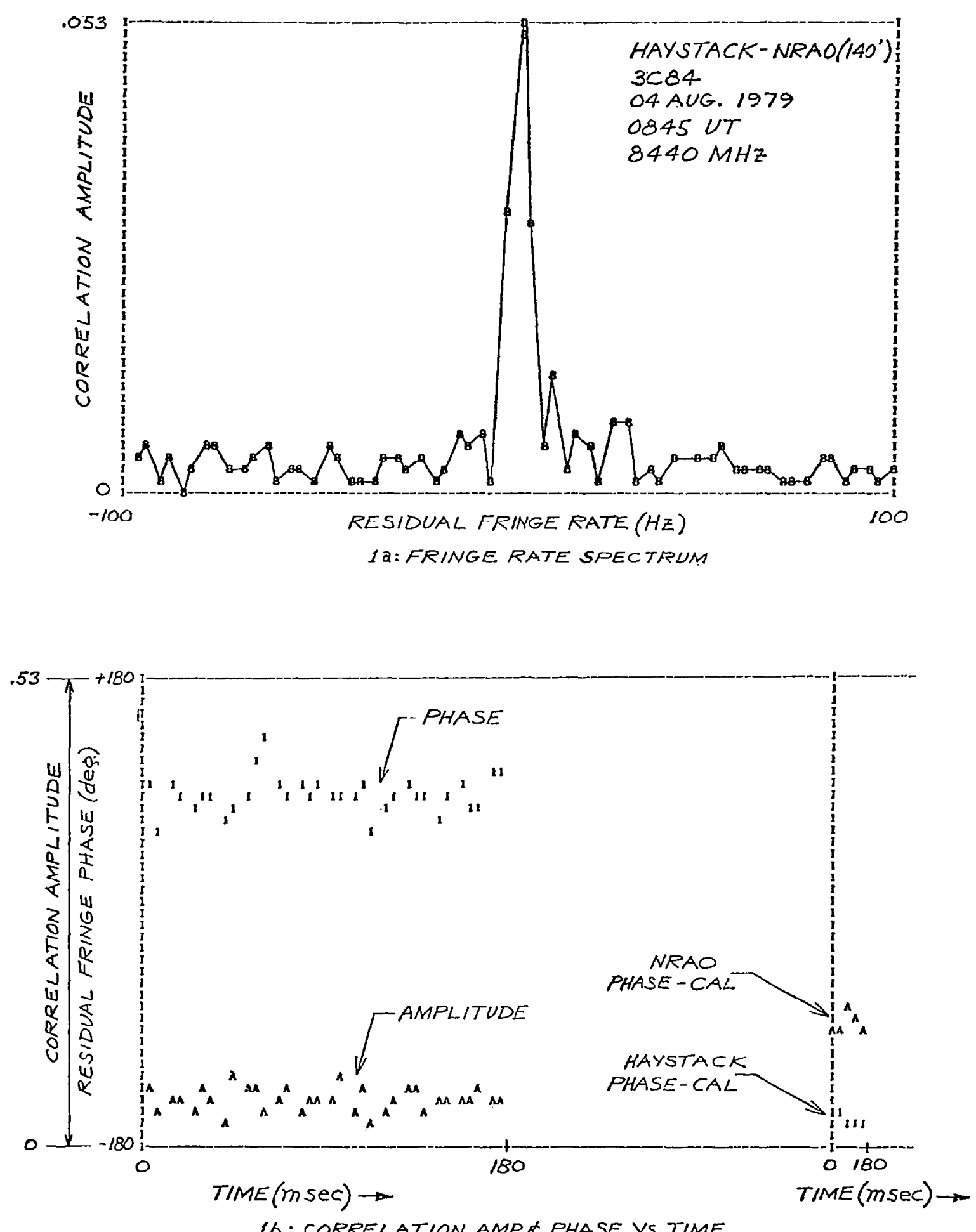

Figure 1. "Real-time" Mark III fringes. 


\title{
THE MARK III DATA BASE HANDLER
}

\author{
James W. Ryan and Chopo Ma \\ NASA Goddard Space Flight Center
}

\author{
Bruce R. Schupler
}

Computer Sciences Corporation

\begin{abstract}
As a part of the overall commitment to build the Mark III very long baseline interferometry (VLBI) system, the decision was made to build an integrated software system which would span all aspects of VLBI computer activities from scheduling of the experiments to production of the ultimate geophysical and astrometric parameters. It was decided that underlying this system would be a sophisticated data base handler which would act to tie the programs together. The commercially available data base systems were investigated and found unsuited to our needs. An entirely new data base handler was developed by our group; it was written in Fortran and is currently implemented on the Hewlett-Packard $21 \mathrm{MX}$ and the IBM 360/91. The system addresses six major problem areas which plague scientific data analysis: the data base handler should (1) provide for an easily specified method of data interchange among programs, (2) provide for a high level of data integrity, (3) accommodate changing requirements, (4) promote program accountability, (5) provide a single source of program constants, and (6) provide a central point for data archiving.
\end{abstract}


One of the major areas in which the Mark III VLBI system has been significantly upgraded over the Mark I system is the area of information flow and data handling. The following discussion presents the goals we set for ourselves in developing an information system for the Mark III and how well we achieved those goals.

\section{DATA HANDLING PROBLEMS}

VLBI data processing is quite complex and involves no fewer than four major software systems and numerous small programs between the acquisition of data and the generation of geophysical results. In the Mark I system, the interchange of data among the programs was handled using formatted cards or card-image tape files. In some cases, the output of one program was not compatible with the input of the next and required an intermediate step to transform the data. The complications inherent in this arrangement prompted the first attribute which our new data handling system would have: it should provide a common, easily specified means of data interchange among all the programs of the VLBI software system.

VLBI observations which can be used for astrometric measurements will be of interest for some time. It is not inconceivable that observations made now will be of interest 100 years hence; astrometric measurements made several hundred years ago are still reprocessed in present studies. Some of the astronomical phenomena we observe have periods of many thousand years and there is no substitute for a long time base. Moreover, the very complexity of VLBI processing requires the storing of many types of correlative data for every astrometric observation. These requirements necessitated the second attribute of our new data handling system: it should provide for an extremely high degree of data integrity. Not only should the system maintain without error the numeric values of the data, but it should also provide a scheme for retaining the identity of the data elements.

In our formatted card data handling system, addition of new data elements was quite difficult. Usually, an attempt was made to put the new data in some unused columns in the existing format. When the unused columns were exhausted, a partial solution was to replace old data which were no longer of interest. Ultimately, the interpretation of special flags was required to allow proper decoding of the information on data cards. Backward compatibility was often impossible, and each time a new data element was added many of the programs required changes. Experience with these problems defined the third attribute of our new data system: it should easily accommodate changing data requirements. It should be possible to add new data elements without destroying backward compatibility and without requiring program modification simply because the new data exist.

The Mark I data handling system had no provision for tracking the status of programs which produced the data stored in the system. At times, results could not be reproduced because it was impossible to know what versions of the programs generated them. To avoid this difficulty, the fourth attribute of the new data system was specified: it should provide a simple method for programs to add data in to the system to $\log$ their status. 
Another feature of the old system was that each program contained its own set of program constants internally; e.g., the speed of light and the locations of the observatories. It was nearly impossible to trace what constants had been used to generate a given result. Moreover, when a new value of a constant was adopted each program using that constant needed modification. The system was cumbersome and prone to errors. Consequently, the fifth attribute was suggested: the system should maintain a core of important constants which all programs would be required to use.

Finally, the old system provided no uniform method for archiving the data. Various parts of the data were stored on cards or in files maintained by individuals. The cards and files were archived by the individuals in whatever way they chose or perhaps not archived at all. This proliferation and absence of control gave rise to the final attribute of our new system: it should consist of a single set of well-defined files where all data of long-term interest reside and should have a well-defined, nearly automatic scheme to archive these files.

\section{DATA BASE HANDLER SELECTION RATIONALE}

The six areas summarized in figure 1 provided a set of specifications for the data handling scheme we desired. The first conclusion we drew from reviewing these specifications was that we needed a data base structure and a handler to manipulate it. In the formatted card scheme, individual users read from and wrote into the data files directly; there was no interface between the users and the data, hence no control. Putting a program between the users and the data immediately generated two further requirements: compatibility with all computers which predictably could be used for VLBI data processing and ability to automatically transfer data created on one of the machines to any other. At the time the goals were specified, a UNIVAC 1108, a CDC 3300, or IBM 360/91, and a Hewlett-Packard $21 \mathrm{MX}$ were used in VLBI processing. The common features of these machines and their operating systems dictated that the data base handler must operate on any machine which supported Fortran and sequential files. No such system existed. The only data base available on the $21 \mathrm{MX}$ was Hewlett-Packard's IMAGE system, which was not transferrable to the other computers. Thus, we were left with no option but to write our own system.

\section{DATA INTERCHANGE BETWEEN PROGRAMS}

2. DATA INTEGRITY

3. ACCOMMODATION OF CHANGING REQUIREMENTS

4. PROGRAM ACCOUNTABILITY

5. PROGRAM CONSTANTS

6. DATA ARCHIVING

Figure 1. Data management problems. 
The second conclusion was that we did not need the full hierarchical and associative structure of a complete data base system. VLBI data are largely organized in a sequential manner based on time of observation. Data not associated with an observation time are minimal; e.g., the catalog of VLBI observatories. The absence of a requirement for tree structures, daisy chains, and other data associations greatly simplified our task.

\section{STRUCTURE OF THE VLBI DATA BASE HANDLER}

The easiest way to explain how the VLBI data base handler achieves its goals is to describe it in some detail. In fact, this explanation will be considerably more detailed than is necessary to use the system and will contain details which are unknown to most of our users.

The system consists of two distinct parts: one, a set of files existing on disk packs and tapes; and two, a set of utility subroutines which allow users to access the information in these files. Users never directly read or write the files and need not know the details of how the data are formatted in the files. To the user, the storage medium is "format free." A user does need to know something about the sequencing of his data in the files but nothing about data in which he has no interest.

Figure 2 shows how a file is structured. Each data base file consists of four major subsets of records:

1. The file begins with the identification records. These consist of seven records of fixed format which contain information such as the data base name, update version number, creation date, etc.

2. Next are an indefinite number of sets of history records. Each time the data base file is updated the user must supply one or more lines of text explaining who he is and what he is doing. These lines are then incorporated into a new set of history records which is added to the output file after the existing history records. The new history records also contain the datc/time the update was created, the machinc type and facility on which the update was made, and the version of the data base handler in use.

3. The third subset consists of the table of contents records. The VLBI data base handler supports up to 99 different types of data records. Consequently there can be as many as 99 tables of contents. Each type of data record that exists in a file has a corresponding table of contents. The existence of different data record types allows users to segregate data for convenience in processing and efficient use of storage capacity.

4. The final subset of records are the data records which contain the user information. Up to the physical limits of the storage medium, i.e., the size of the largest disk file, there can be any number of occurrences of each type of data record with the different types of data records interleaved in any fashion. 


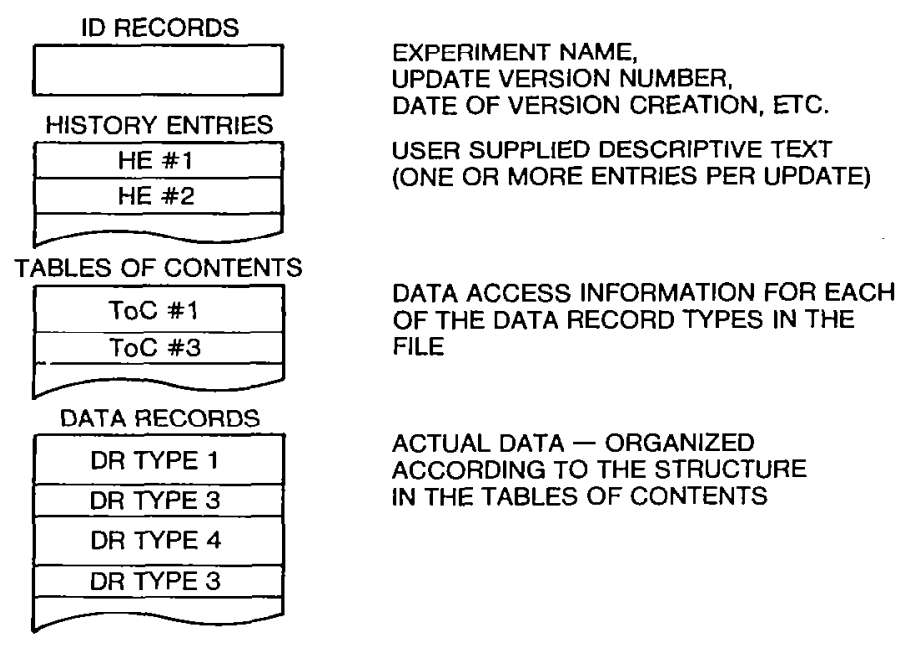

Figure 2. File structure.

\section{TABLE OF CONTENTS ORGANIZATION}

The information in the data records is organized into arrays which are accessed by means of 8character ASCII codes called LCODE's. The correspondence between the LCODE passed by the user and the actual position of the data in the data record is defined in the table of contents. Figure 3 shows the structure of a typical table of contents. It is a matrix of ASCII and integer data in which each row of the matrix contains information pertaining to one array in the data record. A row contains the 8-character LCODE, the version number of the update when the array was inserted or last changed, three dimensions which define the structure of the array, and 32 characters of userprovided text description which identify the array contents. Three types of arrays can be defined real numbers, integers, and ASCII characters. When the user requests an array he specifies the type desired. The data base handler finds the actual physical location for a particular array in the data record by locating the correct row in the table of contents and using the array types and dimensions of all the preceding arrays in the table of contents.

The version number and test descriptions in the table of contents are features which are essential to achieving two of the goals set for the system. The version number allows the user to know precisely when an array was entered into the system or last modified. The text descriptions play a key part in maintaining the identity of the data elements.

In the example shown in figure 3, each of the three types of arrays is shown. "VLIGHT" is the 8character code for an array containing the real number which is the speed of light in meters/second. Note that an array containing a single number has dimensions $(1,1,1)$. "NUMBSTAR," which also has dimensions $(1,1,1)$, is an integer which specifies the number of radio sources in the source cata$\log$ of the data base file. "STRNAMES," which has dimensions $(4,6,1)$, is list of 6 star names, each of which is 8 characters ( 4 words) long. It is part of the radio source catalog. 


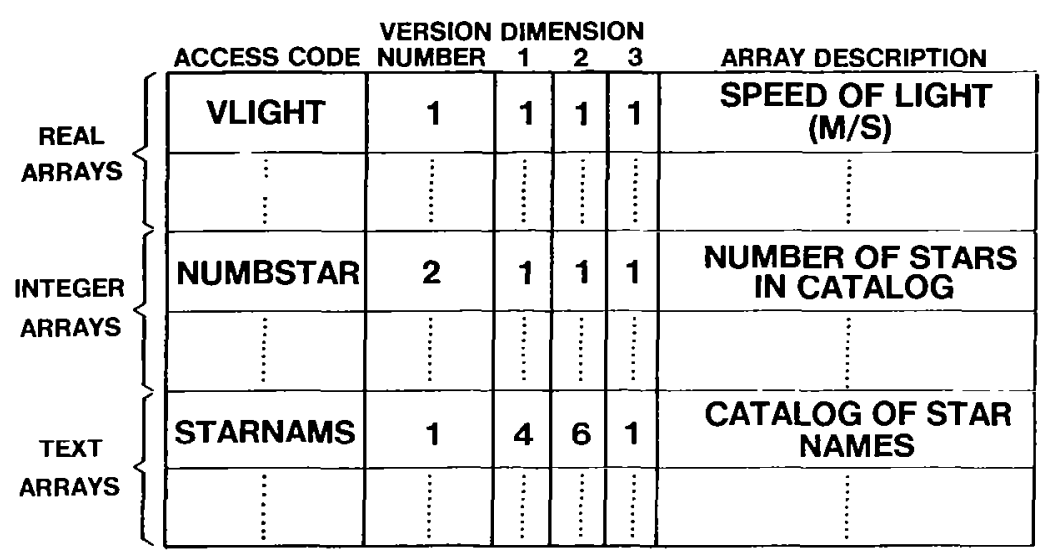

Figure 3. Organization of a table of contents.

It should be emphasized that new tables of contents may be introduced and existing tables of contents may be modified at any update of a data base file. It is not necessary or desirable to define all information and allocate all space at file creation.

\section{DATE BASE HANDLER USER INTERFACE}

Users interface with the data base handler by calling the necessary subroutines from their programs. Figure 4 is a list of the various utilities and a short description of their functions. The list is generally in the order of use.

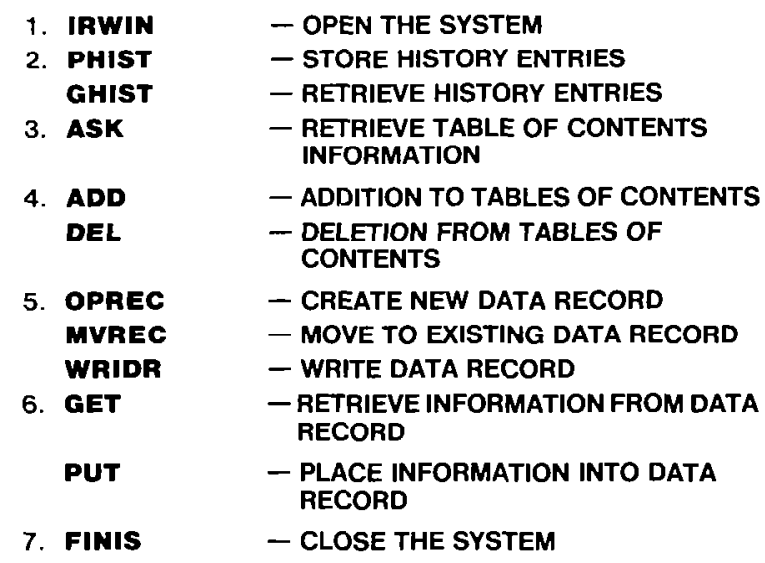

Figure 4. Data base user interface.

The system operates in three modes: (1) a "read-only" mode which is by far the most common mode used and in which the user only extracts information from the data base files, (2) an "update" mode in which the user adds new data or extracts and modifies existing data, and (3) a "create" mode in which an entirely new data base is created. In our application, the create mode is rarely used. 
The user initializes the data base handler by calling IRWIN. In the call, he specifies the name of the input data base file, the mode (i.e., read-only, update, or create), and the unit number of a terminal or printer where error messages can be sent. IRWIN returns a flag indicating whether the system has successfully opened the file and is ready to proceed. Most other data base utilities also return a flag showing whether the desired function has been properly executed.

The next calls deal with history entries. GHIST returns to the user the history information from the input file. PHIST stores new history records in the output file. In create and update modes, the user must provide at least one history entry; otherwise, the system will not allow the user to continue forming the output file.

At this point in the update and create modes, the user must deal with the tables of contents through the routines ADDA, ADDI, ADDR, DELA, DELI, and DELR. The three ADD routines allow the user to tell the system to provide array space for text, integer, or real arrays, respectively. In the call to the ADD routines, the user specifies the data record type, the access code (LCODE) for the array, the dimensions, and array text description. If the data record type does not yet exist, the necessary table of contents is created. If an array already exists in the input file and the user wishes to modify some of the data stored in that array, the array must be added through one of the ADD routines as if it did not exist. This requirement insures that the version counter correctly tracks the array's last modification. The DEL routines allow the user to delete arrays which exist in the input file. Once an array specification has been deleted from the table of contents, all occurrences of that array in the data records are automatically deleted from the output file. Through the ASK utility, the user can also query the system about the tables of contents of the input file. The user can ask whether an array corresponding to a specific LCODE exists in the file and what its dimensions and text description are.

In order to store data in or retrieve data from a data record, the user must make that record active in the system; i.e., bring the record to a buffer in memory. A record can be made active in one of two ways: by calling the utility MVREC, which activates a record, either by type or sequence, through reading it from the input file; or by calling OPREC, which creates an empty record. Once a record is active, the actual data accesses can occur. Arrays are retrieved and stored by using the utilities GETA, GETI, GETR, PUTA, PUTI, and PUTR. The three GET routines retieve text, integer, and real arrays, respectively; the three PUT routines store arrays in an identical fashion. In the calls to the PUT and GET routines, the user specifies the desired array by passing the appropriate LCODE and a buffer into which the data are returned in the case of a GET and out of which the data are passed in the case of a PUT. The user must also pass the array dimensions in order to insure a correct linkage between the user program and the data base handler system. The dimensions passed to the GET routines can be smaller than those specified for the array in the table of contents; the system will only return as much as specified by the passed dimensions.

In the update and create modes, the user must specifically dispose of the active record when he has finished by calling WRIDR or DELDR. WRIDR causes the active record to be written to the output file. DELDR simply deletes it. By using DELDR, records which exist in the input file but are unwanted in the output file can be eliminated. 
After calling WRIDR or DELDR, the user can make another record active by calling MVREC or OPREC. In a typical application, most of the interaction with the data base system involves a large loop beginning with a call to MVREC or OPREC to make a record active, several calls to GET and PUT for actual data access, and ending with a call to WRIDR to output the active record. In this fashion, the user can move through the records of an entire data base file.

When the user is finished with a data base file, he terminates the system by calling the utility FINIS. In the update mode, if the user has moved through only a portion of the input file when FINIS is called, the remainder of the input file is then copied into the output file. This copying is provided as the default to insure that data are not inadvertently lost in updating.

In general, when the data base handler detects some unexpected condition, it sets an error flag and returns control to the user. Such an error condition occurs, for example, when the user attempts to GET an array using an LCODE which does not exist in the table of contents. However, there are abnormal conditions which the data base handler detects which can only occur if some internal condition is completely incorrect or if the user has attempted to misuse the system. A typical example of the latter is an attempt to store data using one of the PUT routines after initially specifying readonly mode in calling IRWIN. In these cases, the system writes a error message in plain English on the device specified in the call to IRWIN, closes the files, and terminates the user program.

\section{SUMMARY - PROBLEMS AND THEIR SOLUTIONS}

Figure 5 summarizes the six problems which our data handling system was to alleviate and the solutions to these problems. In review, the requirements were:

1. The system should provide a common, easily specified means of data interchange among all the programs of the VLBI software system. This goal has been accomplished. If two users wish to pass data through a data base file (one generating the data, the other using them), they need only agree on the data record type in which the data are to be stored, the array type and dimensions for holding them, and an 8-character LCODE to access them. Once the data are in the system, any user can access them.

2. The system should provide for data integrity; it should not only preserve the numeric values of the data but also retain the identity of the data elements. The system satisfies this requirement in at least two ways. Associated with each array in a data base file is a 32-character text description. These descriptions if used conscientiously clearly define the data elements. In addition, the history records give each user an opportunity to make a permanent $\log$ in the output file of precisely what he was doing when he updated the data base file.

3. The system should accommodate changing requirements. It automatically preserves backward compatibility and does not require programs to be modified simply because new data have been added. With the data base handler, users are isolated from data files and need not know precisely what data are in them or how the files are formatted. New data can be added and a user who is not interested in those data need never know they exist. 


\begin{tabular}{|c|c|}
\hline 1. DATA INTERCHANGE & $\begin{array}{l}\text { THEDATA BASE FILES PROVIDE A COMMON, EASILY } \\
\text { SPECIFIABLE LINK BETWEEN PROGRAMS. USED ON } \\
\text { MANY MACHINES. }\end{array}$ \\
\hline 2. DATA INTEGRITY & $\begin{array}{l}\text { USER NEVER DIRECTLY WRITES DATA FILES. DATA } \\
\text { SET MODIFICATIONS CAUSES A NEW FILE TO BE } \\
\text { CREATED AND THE OLD FILE IS PRESERVED. }\end{array}$ \\
\hline $\begin{array}{l}\text { 3. ACCOMMODATION OF } \\
\text { CHANGING REOUIRE- } \\
\text { MENTS }\end{array}$ & $\begin{array}{l}\text { USERS CAN ADD NEW DATA WITH NO IMPACT ON } \\
\text { ON EXISTING DATA. DATA RECORD AND FILE SIZE } \\
\text { EXTENSIBILITY LIMITED ONLY BY DISK CAPACITY. }\end{array}$ \\
\hline $\begin{array}{l}\text { 4. PROGRAM } \\
\text { ACCOUNTABILITY }\end{array}$ & $\begin{array}{l}\text { USERS MUST ADD HISTORY RECOROS WHEN } \\
\text { UPDATING. PROGRAMS CAN AUTOMATICALLY ADD } \\
\text { TEXT INFORMATION ON PROGRAM VERSION } \\
\text { NUMBER, LAST COMPILATION DATE }\end{array}$ \\
\hline $\begin{array}{l}\text { 5. PROGRAM } \\
\text { CONSTANTS }\end{array}$ & $\begin{array}{l}\text { ALL CONSTANTS CAN BE STORED IN THE DATA } \\
\text { BASEWHEN CREATED AND ALL PROGRAMS CANBE } \\
\text { REQUIRED TO GET CONSTANTS FROM THE DATA } \\
\text { BASE. }\end{array}$ \\
\hline 6. DATA ARCHIVING & $\begin{array}{l}\text { WELL DEFINED INTERNAL FORMAT; DATA } \\
\text { DESCRIPTIONS CONTAINED IN TABLES OF } \\
\text { CONTENTS. ALL DATARESIDES IN A SINGLE SET OF } \\
\text { WELL CONTROLLED FILES WHICH CAN BE } \\
\text { ARCHIVFD ON TAPE IN A NFARI.Y AIJTOMATIC } \\
\text { FASHION. }\end{array}$ \\
\hline
\end{tabular}

Figure 5. Problems and solutions.

4. The system should provide a simple method for programs to log their status. In our application, all data base files contain a data record of type 1. Each file contains only one data record of this type; it is called the "header record" and is the first data record in the file. This record contains all information which is needed on a once-per-file basis; e.g., the core of important constants and the radio source and VLBI observatory catalogs. The various programs of our system store text messages in ASCII arrays in the header record to log their status. These messages contain information such as the load module number of the program and the versions and compilation dates of all the major subprograms. By looking at these messages, we can know what versions of the programs created the output data, and by maintaining copies of obsolete program versions, we can reproduce old results if desired.

5. The system should maintain a core of important constants which programs would be required to use. In our application, all data base files are ultimately derived by updating a single existing file, the skeleton data base file which contains nothing more than the core of important constants. Since all other files result from some sequence of updates to the skeleton file, they retain the core of constants.

6. The system should provide a well-defined, nearly automatic method of data archiving. In this system, all data of permanent interest reside in data base files; therefore, the problem of individuals being responsible for archiving the data they generate is eliminated. There is simply one common pool of data. Since the system obviously produces many files - one might even say it proliferates files - a data base file cataloging system has been instituted. Conceptually, it sits above everything which has been described earlier. The catalog logs the existence of every data base file created, its relationship to other data base files, the disk device on which it resides, and whether it has been archived. One individual is responsible for backing up the files on archive tapes and clearing the disks of files which are no longer in active use. The disk-to-tape and tape-to-disk routines are functions in the catalog system and are used for archiving and restoring and for data transmission between machines. 


\section{DIFFICULTIES WITH THE USE OF THE VLBI DATA BASE HANDLER}

The data base handler which has been implemented meets all the original specifications. However, the system is not without drawbacks. The following is a list of the principal difficulties:

1. Data access requires much more computer time than in the formatted card scheme. On the IBM 360/91, there is no problem since the time involved is negligible with either scheme. On the HP $21 \mathrm{MX}$, where most processing is done interactively and where increased time means some individual must sit waiting at a terminal, the problem does exist. In a typical minicomputer application, a user accessing nearly all the information in a 200-observation data base file may wait 3 minutes; whereas with formatted cards, he might wait 20 to 30 seconds. A faster CPU would reduce the delay.

2. Formatted cards are ideally suited for sorting. In fact, sorting was one of the reasons why computer cards were invented. Data base files are not easily sorted, and we do not have general purpose data base sorting routines. We have written one data base sorting program to do a very specific kind of sorting, and with minor modifications, it could be made to do other types of sorting.

3. Cards formatted identically can easily be concatenated, usually by simply stacking two card decks one behind the other. Concatenation of data base files is not so simple. Because of the organization of the header record it is not possible to write a single program to merge two arbitrary VLBI data base files. In order to merge two files, one must, in general, make use of the actual sense of the information contained in the files. No mechanical scheme simply involving manipulations of data record types, access codes, and dimensions will work. To date, we have skirted this problem by merging the information from separate data base files in the user programs at the time the information is processed. We are, however, developing a program for concatenating a limited class of data base files.

\section{CONCLUSION}

In summary, we are quite satisfied with the performance of our data base handler as applied to our VLBI problem. It has some small drawbacks, but we have been able to work around them, and it is infinitely better than the system of formatted cards which it replaced. In fact, had we continued using formatted cards, it is likely that our entire software system would have collapsed around us. 


\title{
MARK III INTERACTIVE DATA ANALYSIS SYSTEM
}

\author{
James W. Ryan and Chopo Ma \\ NASA/Goddard Space Flight Center
}

Bruce R. Schupler

Computer Sciences Corporation

\begin{abstract}
The Mark III very long baseline interferometry (VLBI) software system is an integrated set of programs covering all aspects of VLBI computer activity from schedule generation through field data acquisition to the production of "publication ready" parameter values. The interactive data analysis system is a major subset of this system and consists of two major and a number of small programs. These programs provide for the scientific analysis of the observed values of delay and delay rate generated by the VLBI data reduction programs and produce the geophysical and astrometric parameters which are among the ultimate products of VLBI. The two major programs are CALC and SOLVE. CALC generates the theoretical values of VLBI delay and delay rate as well as partial derivatives based on a priori values of the geophysical and astrometric parameters. SOLVE is a least squares parameter estimation program which yields the geophysical and astrometric parameters using the observed values provided by the data processing system and theoretical values and partial derivatives provided by CALC. SOLVE is a highly interactive program in which the user selects the exact form of the recovered parameters and the data to be accepted into the solution.
\end{abstract}




\section{INTRODUCTION}

In the Mark III very long baseline interferometry (VLBI) system, complete processing of data from schedule generation through production of geophysical results involves a series of large and sophisticated programs. Figure 1 outlines this flow. The data processing can be thought of as consisting of three distinct elements: acquisition, reduction, and analysis. The programs SKED and FIELD constitute the acquisition element; these programs are used to schedule the experiments and to control the acquisition system while the raw data are being recorded. The programs DE-LOG, PREP, COREL, FRNGE, and EDIT constitute the reduction element; this includes all processing between the cross-correlation of the Mark III tapes and the production of the delay and rate observations. The programs CALIB, STRUC, ASTRO, CALC, and SOLV constitute the analysis element; included here is all data processing required to recover the geophysical and astrometric parameters from the delay and delay rate observations. This paper will be limited to a discussion of the analysis element.

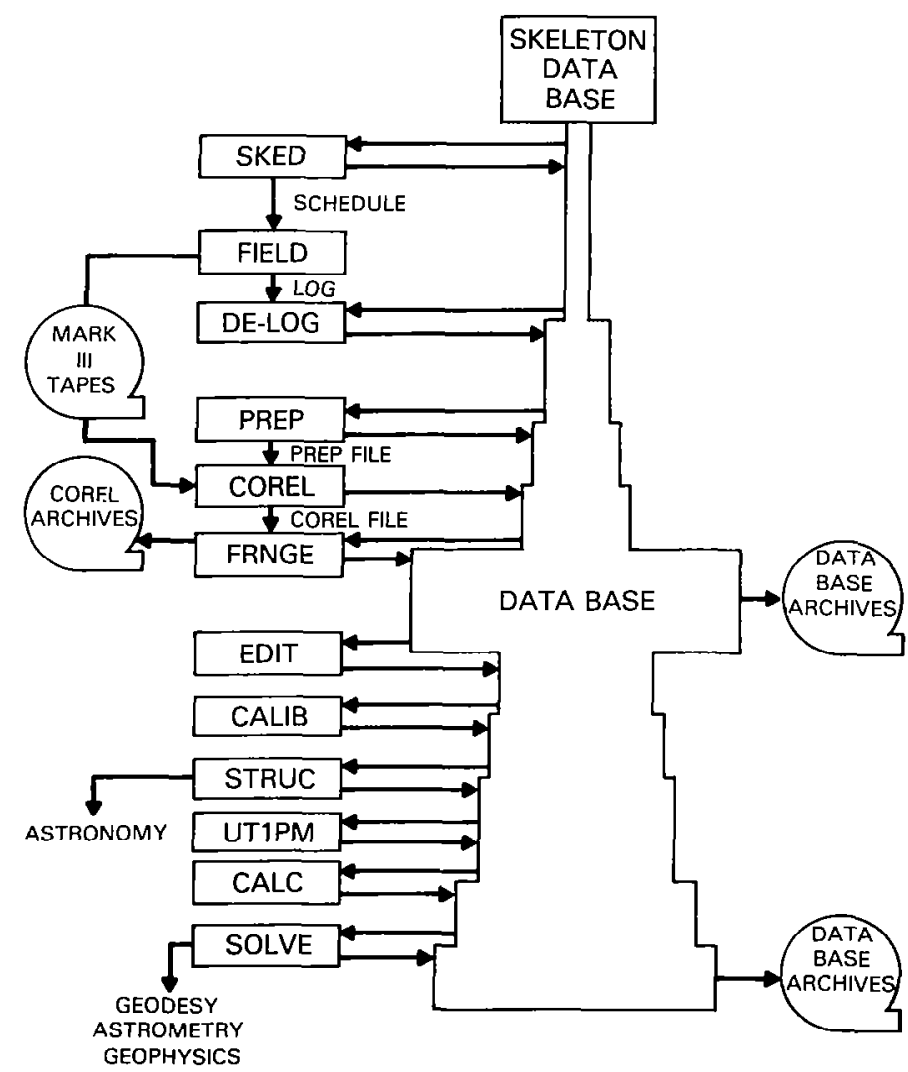

Figure 1.

\section{INTERACTIVE ANALYSIS SYSTEM DESIGN CONSIDERATIONS}

In 1975 when NASA elected to pursue the development of the Mark III VLBI system, the decision was made to develop an entirely new analysis system as well as hardware and data reduction systems. At that time, a program called VLBI3 was used to analyze VLBI data. It was a large batch 
mode program which could be run on the IBM 360 computers at Goddard and the Massachusetts Institute of Technology (MIT). Based on experience with VLBI3, a series of overall design considerations was set. Figure 2 highlights those considerations, and the following summarizes them.

\section{HIGH LEVEL OF DATA INTEGRITY AND ACCESSIBILITY}

2. HIGHLY RESPONSIVE SYSTEM

3. PROCESS AN UNLIMITED NUMBER OF POINTS AND RECOVER AN UNLIMITED NUMBER OF PARAMETERS

\section{BUILT WITH STATE-OF-THE-ART MODELS}

5. SIMPLE TO USE

6. WELL WRITTEN, DOCUMENTED, AND CONTROLLED

7. INEXPENSIVE TO USE

Figure 2. VLBI analysis system design considerations.

1. The system should provide for an extremely high level of data integrity. It should be easy to access any data set, and any data of permanent value should be saved automatically.

2. The system should be responsive. It should be possible to carry out simple analysis tasks in a matter of minutes and more complex tasks in minutes to hours.

3. Subject only to the physical limitations of the computer on which the system would operate, it should be possible to combine an unlimited number of data points and recover an unlimited number of parameters.

4. The system should contain state-of-the-art models for all phenomena which affect VLBI data.

5. The system should be simple to use. System operation should be straightforward and not require knowledge of the internal workings of the programs. With only a few hours of "hands on" experience it should be possible for a person who is familiar with VLBI analysis procedures to operate the system competently. The system should present the user with a default set of analysis specifications which in most cases would require only a small number of modifications to generate the desired result. The system should attempt to protect the user from blunders and should log with the output all analysis specifications set by the user.

6. The configuration of the system should be well controlled. It should be possible by reviewing the output of the system to know exactly what versions of the software generated the output. Moreover, the software should be completely documented and written with modern concepts of good code. 
7. The system should be so inexpensive to operate that the cost of the computer time required to carry out data analysis would never be a consideration in deciding whether or not to perform the analysis.

\section{RESPONSE TO THE DESIGN CONSIDERATIONS}

In response to the first design consideration, the Mark III Data Base System was developed. The system consists of a set of files and a software system for accessing the files. In addition, there is a catalog system which allows the user to find any file quickly, which tracks the relationships among files, and which provides for nearly automatic archiving of files. The data system is covered in detail in the appendix to this paper.

Once the decision was made to develop a formal data system the second design consideration, system responsiveness, quickly surfaces as the driving consideration. The existing analysis program, VLBI3, was unresponsive because it was run as a batch job on a large computer system and because it calculated from first considerations all theoretical and partial derivatives for the data being processed even though that data may have been analyzed many times in the past. For complex analysis tasks, VLBI3 required amounts of computer time which were often available only at night or on weekends. Based on this experience, the decision was made that the new system should be an interactive system; that is, that the analyst using a terminal would set up the run via a dialog with the program. The program would carry out the estimation and return the result to the user's terminal or printer in at most a few minutes of elapsed time. This could not be accomplished using the large computer systems available at Goddard; only through the use of a dedicated minicomputer could an interactive system be achieved.

The decision to use a minicomputer, which has much slower execution speed than the large batch machines, forced the decision to divide the analysis into a number of smaller, separable tasks. In the main, the tasks were divided into two groups: those which are carried out only once or infrequently for a given data set, and those which must be carried out every time a new analysis is attempted. The programs CALIB, STRUC, and UT1PM were specified as small, "stand alone" minicomputer programs. Their functions are to add to a data base calibration data, source structure corrections, and UT1 and polar motion tables respectively. At least in principle, the information they store is to be entered only once for each data base. The function of computing the theoretical observations and partial derivatives and the function of doing the least squares estimation, both of which were done in VLBI3, were assigned to two programs: CALC and SOLVE, respectively. Computing the theoretical observations and partial derivatives is a typical example of a function which must be carried out only infrequently. Since in our application, the solutions need not be iterated, the only time the computation of the theoreticals and partials would be redone would be if the a priori constants were changed or if a model were improved. The least squares processing obviously depends on the detailed specifications of the analysis to be accomplished. Separating these functions proved to be the key decision in achieving adequate system responsiveness. With the system as it exists today, new data can be processed through the entire chain of analysis programs in less than 24 hours and old data can be reprocessed through SOLVE in a matter of minutes. 
Achieving the second design consideration, the ability to process a large number of data points and to recover a large number of parameters, proved to be exclusively a function of SOLVE. It was implemented on a Hewlett-Packard (HP) 21MX minicomputer, which has rather severe constraints on program size (programs may not exceed 32K 16-bit words). Because of this limitation, it was not possible to maintain large matrices in core, and if a standard least squares algorithm had been implemented, fewer than 100 parameters could have been recovered. In order to circumvent this limitation, a algorithm called arc parameter elimination was implemented. The parameters to be recovered are divided into two classes: arc and global parameters. Arc parameters are those which affect only a limited, usually time-bound, subset of the data. These include clock polynomials, tropospheric refraction scale factors, and values of UT1 and polar motion. Global parameters are those which affect a broad cross-section of the data; these include the site and source coordinates, the precession constant, and earth tide parameters. Using the arc parameter elimination technique, data are added to the solution one experiment at a time, and at each step, the arc parameters are algebraically eliminated. Only a matrix containing the global parameters is carried forward. Once all the data have been added, the global matrix is inverted. The values of the parameters which result are identical to those which would have resulted from an inversion of a matrix containing the global parameters and all the arc parameters. With a modest amount of additional effort, the arc parameters can also be recovered. We have already produced a solution based on 8171 data points containing 535 recovered parameters of which 485 were arc dependent and 50 were global, and this solution scarcely tested the limits of the system.

The third design consideration, that state-of-the-art models should be used, involves nearly all of the analysis programs but is of special significance for CALC. Currently CALIB is little more than a prototype of the program which will ultimately calibrate the effects of cable delay and tropospheric refraction. The experiments which have been conducted to date have not produced on an operational basis the data needed to carry out these calibrations. Once the water vapor radiometers become operational, CALIB will be brought up to the state-of-the-art. STRUC is now no more than a program specification, and even the source structure models which would drive this program have not been tested to see if they would improve the analysis of delay and delay rate data. The quality of the data acquired to date has been such that source structure corrections have not been needed, but it is expected that the Mark III system will produce data which will require source structure corrections if the full potential of the data is to be achieved. CALC, the program which computes theoreticals and partials, has been fully operational for almost 2 years. CALC may well be the most complete program in existence for modeling VLBI delay and delay rate, and with the exceptions of ocean loading and ionospheric refraction, contains models for every phenomenon which significantly affects VLBI observations. The models in CALC were the subject of Chopo Ma's paper "Geophysical and Astronomical Models Applied in the Analysis of VLBI Data" already presented at this conference. SOLVE does not contain models for VLBI observations.

The fourth consideration, that the programs should be simple to use, is one that we have worked with great vigor to satisfy. UT1PM is an interactive program which operates on the HP $21 \mathrm{MX}$ and stores UT1 and polar motion information in data bases. It prompts the user in plain English at all decision points and is so foolproof that it could be run with no more instruction than how to set 
the program running. CALIB and STRUC are not yet operational, but they will be written with the same standards for simple use as UT1PM. CALC is maintained by a single individual in our group, but the operation of CALC is so simple that anyone of our group who desired a CALC run may generate independently. Anyone modestly familiar with VLBI processing could be taught to run CALC with no more than 30 minutes of instruction. Simplicity of use has had its greatest impact on the design of SOLVE. As stated above, SOLVE takes the actual and theoretical observations and partial derivatives placed in the data base by other programs and performs a least squares estimation to recover geophysical, astrometric, and other parameters. In order to carry out this function, data bases must be selected, individual data points must be accepted or rejected, and the configurations of clock polynomials, tropospheric refractions scale factors, and numerous other parameters must be specified. To accomplish this, SOLVE presents the user with a series of plain text menus which contain the various options which may be selected. After consulting the selected data bases, the program sets up a default configuration for all parameters which may be selected. To make a specific run, the user usually selects only a small number of options in the menus. The program contains numerous cross-checking procedures to help insure that the user does not blunder. For example, if more than one data base is accessed, the program checks to insure that the a priori constants used to generate the theoretical observations are identical. If the user attempts to solve for a parameter for which the data has no sensitivity, SOLVE detects that condition, informs the user, and generates the solution with that parameter eliminated. Since SOLVE presents the analyst with so many options for combining the data and selecting parameters, and since this power could generate many improperly specified solutions, considerable further effort will be spent placing more sophisticated cross-checking procedures into SOLVE.

The fifth design consideration is that of software configuration control and software coding and documentation standards. UT1PM and CALC have been the most successful in meeting this design consideration. Both programs were completed early in the project and have been stable for almost 2 years. All operational CALC processing has been accomplished with four thoroughly documented benchmark systems. Morcover, the old systems have bcen maintaincd so that any CALC run can be recreated. Both programs have been written with explicit coding and documentation standards. The two guiding principles have been "keep it simple stupid" and "machines are cheap and people are expensive." In any conflict between coding efficiency and coding clarity, clarity has always been chosen. We have not been as successful with configuration control for SOLVE, but that reflects more than anything else the nature of the SOLVE's job. It has been and still is the program which limits the complexity of the data analysis our system can achieve. There is relentless pressure to bring the latest improvements in SOLVE on line quickly. In the fall of 1979, a new version of SOLVE with significantly upgraded capabilities will become operational, and SOLVE should become more stable.

The final design consideration is that the cost of computer time to carry out the analysis should be negligible. This has been by far the easiest design consideration to achieve. When compared with the cost of reducing data such as satellite orbit measurements or planetary ranging data, the cost of analyzing VLBI data has always been small. Even so, at MIT, analysis with VLBI3 was often hampered by the availability of computer funds. In our current system, most of the work is carried out 
on the dedicated minicomputer, and no incremental cost is incurred in making a run. The total computer cost for the operation and maintenance of CALC on the IBM 360-91 at Goddard is less than $\$ 3000$ per year, an insignificant cost in the overall project budget.

\section{SUMMARY}

Since 1975, our group has expended almost 15 man-years in the development of the Mark III VLBI analysis system. We have by no means completed our task, but we can already carry out analysis tasks which only a few years ago could only be dreamed of. It is our hope and expectation that the analysis system will always measure up to the quality of the data produced by the Mark III data acquisition system. 


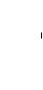


SESSION E

WAVES OF THE FUTURE AND OTHER EMISSIONS 


\title{
WATER VAPOR AS AN ERROR SOURCE IN MICROWAVE GEODETIC SYSTEMS: BACKGROUND AND SURVEY OF CALIBRATION TECIINIQUES*
}

\author{
E. S. Claflin and G. M. Resch
}

Jet Propulsion Laboratory

\begin{abstract}
At microwave frequencies, the delay imposed by tropospheric water vapor becomes a limiting error source for high accuracy geodetic systems. The mapping of tropospheric induced errors into "solved-for" parameters depends upon baseline length and observing strategy. Simulation analysis (and experience) indicates that in some cases, errors in estimating tropospheric delay can be magnified in their effect on baseline components. We have surveyed the various techniques by which tropospheric water can be estimated or measured with particular consideration to their possible use as a calibration technique in support of VLBI experiments. The method of remote sensing using a microwave radiometer seems to be the most cost effective way to provide an accurate estimate of water vapor delay.
\end{abstract}

\footnotetext{
*The research described in this paper was carried out at the Jet Propulsion Laboratory, California Institute of Technology, under NASA Contract NAS7-100, sponsored by the National Aeronautics and Space Administration.
} 


$$
\begin{aligned}
& \text { Refractivity - } \quad N=(n-1) \times 10^{6} \\
& =\frac{77.6 \mathrm{P}}{\mathrm{T}}+1722 \frac{\rho_{\mathrm{V}}}{\mathrm{T}} \\
& \text { "Electrical" Length }-\quad L=\int_{0}^{\ell} n d s \\
& \text { Path Delay - } \Delta \mathrm{L}=\int_{0}^{\ell}(\mathrm{n}-1) \mathrm{ds}=\mathrm{L}-\ell \\
& \text { Water Vapor Path Delay }-\Delta \mathrm{L}_{\mathrm{V}}=\mathrm{k} \int\left(\frac{\rho_{\mathrm{v}}}{\mathrm{T}}\right) \mathrm{ds} \\
& \text { Precipitable Water Vapor }-\quad \mathrm{V}=\frac{1}{\rho_{\mathrm{w}}} \int \rho_{\mathrm{v}} \mathrm{ds} \\
& \text { Ratio - } \Delta \mathrm{L}_{\mathrm{v}} / \mathrm{V} \approx 6
\end{aligned}
$$

Figure 1. Refractivity and path delay.

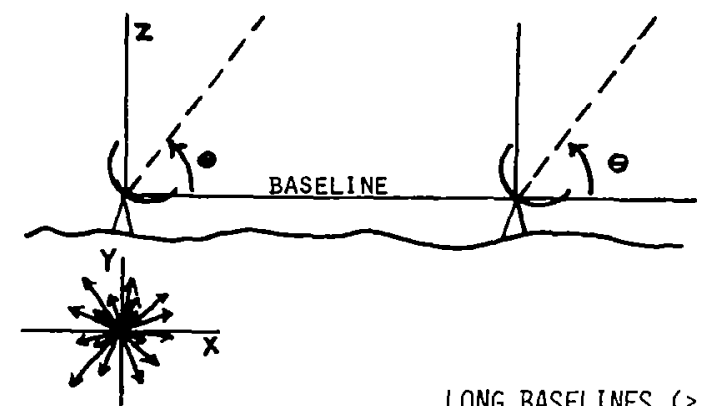

LONG BASELINES ( $>1000 \mathrm{KM})$

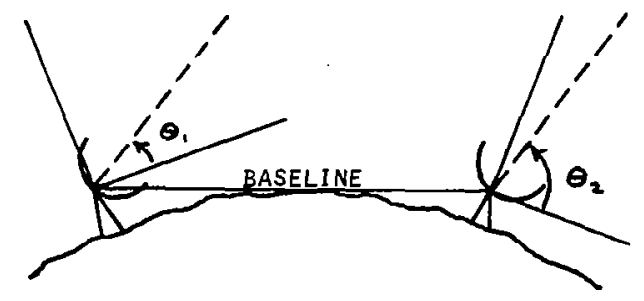

- Local verticals nearly parallel,

- hater vapor error affects only LOCAL VERTICAL COORDINATE,

- Strong common mode rejection,

- hater vapor delay cannot be solved for.

Figure 2.

- Local verticals not parallel.

- hater vapor error enters all COMPONENTS OF BASELINE,

- No COMmon mode ReJection.

- hater vapor delays can be solved for. 


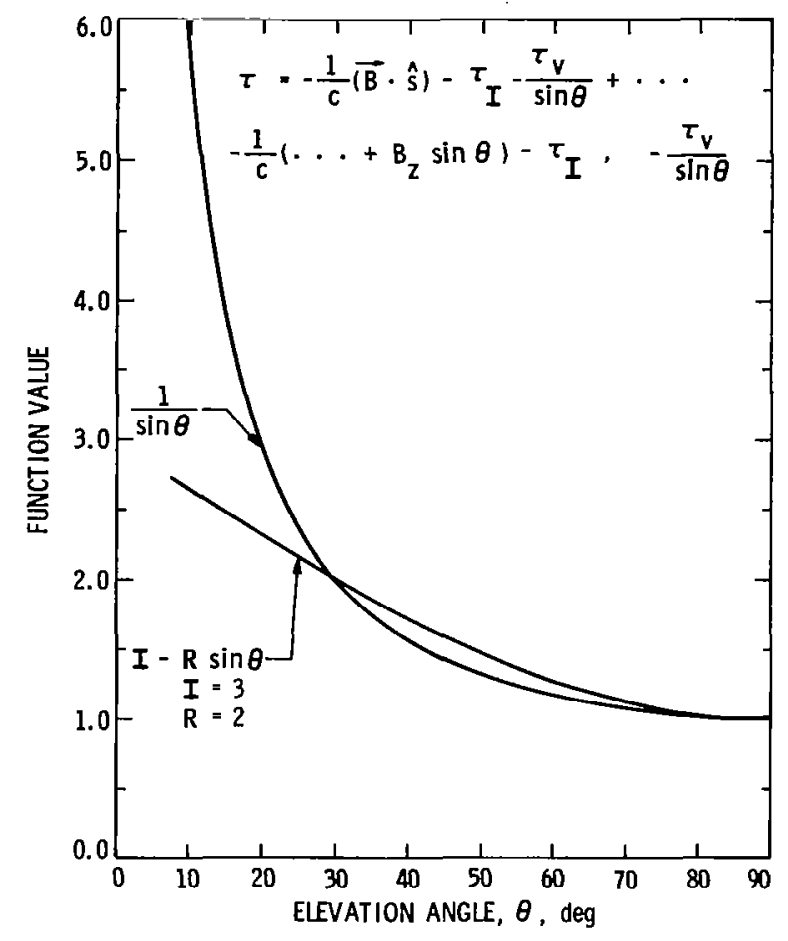

Figure 3. Similar functions: $(\operatorname{Sin} \theta)^{-1}$ and $(1-R \operatorname{Sin} \theta)$.

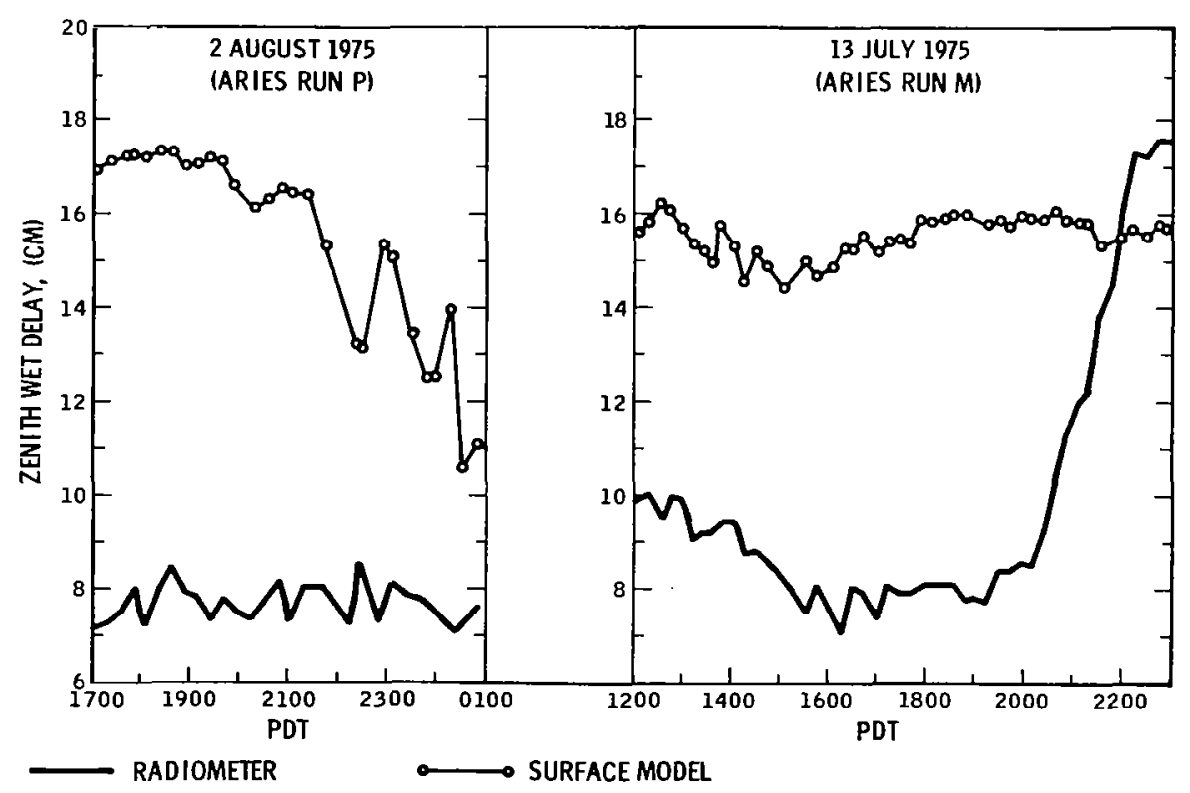

Figure 4. Zenith path delay during ARIES experiments as predicted by radiometer and a surface model. 
I. IN SITU MEASUREMENTS
A. RADIOSONDES
B. AIRCRAFT
C. DRONES

II. TRANSMISSION MEASUREMENTS
A. SOLAR HYGROMETERS
B. $22 \mathrm{GHZ}$ SATELLITE TRANSMITTER
c. OPTICAL/MICROWAVE TRANSMITTER

III. REMOTE SENSING
A, LIDAR
B. INFRARED WVR
C. MICROWAVE WVR

Figure 5. Water vapor measurement techniques.

PART I. IN SITU MEASUREMENTS

- Radiosondes - $\$ 50$ K capital investment, $\$ 100$ per launch, RECEIVING EQUIPMENT CAN BE AUTOMATED. Provides measurement at "Zenith". ACCURACY DIFFICULT TO SPECIFY. OPERATES DAY OR NIGHT AND DURING RAINFALL,

- Instrumented - Expensive $\$ 1600$ per day, AIRCRAFT $\}$ OPERATIONALLIY INCONVENIENT.

FLIGHTS RESTRICTED IN OVERCAST CONDITIONS. POTENTIAL CALIBRATION ACCURACY $-1 \mathrm{cM}$.

- Instrumented) - \$65 K CApital investment (Radiosonde receiving equipment). DRONE $\quad$ OPERATIONALLY INCONVENiENT.

NOT SUITABLE FOR AUTOMATION.

SAFETY HAZARD.

POTENTIAL ACCURACY BETTER THAN SURFACE MODEL.

Figure 6. Water vapor measurement techniques. 
PART II. TRANSMISSION MEASUREMENTS

- Solar Hygrometer - Inexpensive $\leq 12 \mathrm{~K}$, can be automated. ONLY OPERATES IN SOLAR DIRECTION, INOPERATIVE UNDER CLOUDS OR AT NIGHT. ACCURACY PROBABLY BETTER THAN 3 CM AT ZENITH DEPENDS ON SOLAR ELEVATION ANGLE.

$\left.\begin{array}{l}22 \mathrm{GHz} \text { Satellite } \\ \text { Transmitter }\end{array}\right\}$ VERY EXPENSIVE, ELECTROMAGNETIC POLLUTION. ONLY OPERATES IN DIRECTION OF SATELLITE. POOR ACCURACY POTENTIAL.

- Optical/Microwave $\}^{-}$Direct measure of water vapor delay. TRANSMITTER $\}$ EXPENSIVE OPERATIONAL.LY. NEEdS REPACKAGING FOR USE IN AERIAL PLATFORM. OPERATES DAY/NIGHT, BUT NOT UNDER CLQUDS. VERY HIGH POTENTIAL ACCURACY $\sim 0.3 \mathrm{cM}$,

Figure 7. Water vapor measurement techniques.

PART III. Remote Sensing

- lidar (laser Radar) - Equipment investment - \$l00 K. BULKY PACKAGING NOT SUITABLE FOR MOBILE OPERATIONS. GOOD SPATIAL AND TEMPORAL RESOLUTION OF VAPOR PROFILE. OPERATES DAY OR NIGHT, NOT UNDER CLOUDS. ACCURACY POTENTIAL BETTER THAN $1 \mathrm{~cm}$.

- Infrared hater Vapor $\}$ - Opacity too high. RADIOMETER (WVR)

- Microwave water Vapor $\xi$ Cost \$85K.

RADIOMETER (WVR) $\}$ COMPACT PACKAGE CAN BE AUTOMATED, OPERATES DAY OR NIGHT, NOT DURING RAINFALL. POTENTIAL ACCURACY $\sim 1 \mathrm{CM}$ LINE-OF-SIGHT,

Figure 8. Water vapor measurement techniques. 


\title{
THE ESTIMATION OF THE PROPAGATION DELAY THROUGH THE TROPOSPHERE FROM MICROWAVE RADIOMETER DATA
}

\author{
J. M. Moran and B. R. Rosen \\ Harvard-Smithsonian Center for Astrophysics
}

\begin{abstract}
Uncertainty in the estimate of the microwave propagation delay through the troposphere is a principal limiting factor to the accuracy of the technique of very long baseline interferometry (VLBI). This uncertainty is due primarily to tropospheric water vapor, the total amount and vertical distribution of which is variable. Because water vapor both delays and attenuates microwave signals, the propagation delay, or wet path length, can be estimated from the microwave brightness temperature near the $22.235 \mathrm{GHz}$ transition of water vapor.
\end{abstract}

We analyzed the data from a total of 240 radiosonde launches taken simultaneously in 1974 at Chatham, Massachusetts; Albany, New York; and Portland, Maine. Estimates of brightness temperature at 19 and $22 \mathrm{GHz}$ and wet path length were made from these data. The wet path length in the zenith direction could be estimated from the surface water vapor density to an accuracy of $5 \mathrm{~cm}$ for the summer data and $2 \mathrm{~cm}$ for winter data. Using the brightness temperatures, the wet path could be estimated to an accuracy of $0.3 \mathrm{~cm}$.

Two dual-frequency radiometers constructed by the National Radio Astronomy Observatory (NRAO) were refurbished in order to test these techniques. These radiometers were capable of measuring the difference in the brightness temperature at $30^{\circ}$ elevation angle and at the zenith to an accuracy of about $1^{\circ} \mathrm{K}$. In August 1975, 45 radiosondes were launched from Haystack Observatory over an 11-day period. Brightness temperature measurements were made simultaneously at 19 and $22 \mathrm{GHz}$ with the NRAO radiometers. The rms error for the estimation of wet path length from surface meteorological parameters was $3.2 \mathrm{~cm}$, and from the radiometer brightness temperatures, $1.5 \mathrm{~cm}$. 


\section{INTRODUCTION}

The neutral atmosphere retards and attenuates propagating electromagnetic waves. The retardation limits the accuracy to which very long baseline interferometry can be used to measure radio source positions and baseline vectors between the antennas. Atmospheric water vapor can contribute up to about $40 \mathrm{~cm}$ of excess propagation path length in the zenith dircction at microwave frequencies. The exact amount cannot be predicted accurately from ground level meteorological variables since the water vapor is not well mixed in the atmosphere. However, since the index of refraction and absorption coefficient are functions of the water vapor density, the brightness temperature due to the self-emission, a weighted integral of the absorption coefficient, is related to the path length, the integral of the index of refraction. Hence, ground-based radiometric measurements can be used to estimate the excess phase path. Early evaluations of the effectiveness of this technique were made by Waters (1967) and Shaper, Staelin, and Waters (1970).

The vertical profiles of temperature and water vapor density are routinely obtained from radiosondes launched by the National Weather Service (NWS). From these data, the index of refraction can be calculated and the path delay estimated. The microwave absorption coefficient can also be calculated and the brightness temperature estimated from the equation of radiative transfer. We completed a theoretical study based on radiosondes launched at three locations in New England in order to determine how well the path delay could be estimated from: (1) surface meteorological data, (2) radiosonde data from a remote station, and (3) microwave radiometry. We also conducted an 11-day experiment during which we compared actual radiometry data from two microwave radiometers with values of brightness temperatures and path length derived from radiosonde data. Our study was focused on establishing how well various techniques work. We used the method of linear regression analysis and examined the residuals. Substantial progress has been made recently by $\mathrm{Wu}$ (1979) and Claflin, Wu, and Resch (1978) on establishing useful a priori prediction algorithms. A full report of our work is available in Moran and Penfield (1976).

\section{BACKGROUND PHYSICS}

The excess propagation path length is given by

$$
L=10^{-6} \int_{0}^{\infty} \mathrm{N}(\mathrm{h}) \mathrm{dh}
$$

where $\mathrm{N}(\mathrm{h})$ is the refractivity of the air as a function of height. The refractivity of moist air is described by the Smith-Weintraub equation (Bean and Dutton, 1966),

$$
\mathrm{N}=\frac{77.6}{\mathrm{~T}}\left(\mathrm{P}+\frac{4810 \mathrm{e}}{\mathrm{T}}\right),
$$

where 


$$
\begin{aligned}
& \mathrm{T}=\text { temperature }\left({ }^{\circ} \mathrm{K}\right) \\
& \mathrm{P}=\text { total pressure }(\mathrm{mb}) \\
& \mathrm{e}=\text { partial pressure of water vapor }(\mathrm{mb})
\end{aligned}
$$

The first term in equation (2) arises from the displacement polarizations of all the air constituents including about a 1-percent contribution from water vapor at the surface. It is called the "dry term." The second term in equation (2) is due to the dipole moment of the water vapor molecule and is called the "wet term."

The refractivity of air is essentially independent of frequency from 0 to $30 \mathrm{GHz}$. The dispersive component of the index of refraction associated with the $22.235 \mathrm{GHz}$ transition of water vapor has a refractivity which is less than 0.02 (Liebe, 1969).

Using the ideal gas law, the dry and wet components of the refractivity, $N_{D}$ and $N_{v}$, can be written as

$$
\begin{gathered}
\mathrm{N}_{\mathrm{D}}=\frac{77.6 \mathrm{P}}{\mathrm{T}}=2.70 \times 10^{4} \rho_{\mathrm{D}} \\
\mathrm{N}_{\mathrm{v}}=3.73 \times 10^{5} \frac{\mathrm{e}}{\mathrm{T}^{2}}=1720 \frac{\rho_{\mathrm{v}}}{\mathrm{T}}
\end{gathered}
$$

where $\rho_{\mathrm{D}}$ and $\rho_{\mathrm{V}}$ are the densities of dry air and water vapor in grams per cubic meter. At the surface, $N_{D}$ typically varies between 250 and 300; while in New England, $N_{V}$ varies from between about 10 and 100 .

The dry gas obeys the equation of hydrostatic equilibrium which leads immediately to the result that the excess dry path is

$$
\mathrm{L}_{\mathrm{D}}=10^{6} \int \mathrm{N}_{\mathrm{D}} \mathrm{dh}=\mathrm{AP}_{\mathrm{O}}
$$

where $P_{0}$ is the total pressure at the surface, $A=77.6 \frac{\mathrm{R}}{\mathrm{gm}}=0.2276 \mathrm{~cm} \mathrm{mb}^{-1}, \mathrm{R}$ is the universal gas constant, $\mathrm{m}$ is the molecular weight of dry air, and $\mathrm{g}$ is the surface gravity constant. Hence, the value of $L_{D}$ at sea level at the standard pressure of $1013 \mathrm{mb}$ is $231 \mathrm{~cm}$ at $45^{\circ}$ latitude. $L_{D}$ can therefore be estimated to an accuracy of less than $1 \mathrm{~cm}$ provided the pressure is measured to an accuracy of a few millibars since departures from hydrostatic equilibrium are small (Hopfield, 1971).

The partial pressure and density of water vapor are related by the ideal gas law, so that

$$
\rho_{\mathrm{v}}=\frac{217 \mathrm{e}}{\mathrm{T}} \mathrm{gm}^{-3} \text {. }
$$


The wet path length is therefore

$$
\mathrm{L}_{\mathrm{v}}=1720 \int_{0}^{\infty} \frac{\rho_{\mathrm{v}}(\mathrm{h})}{\mathrm{T}(\mathrm{h})} \mathrm{dh} .
$$

The assumption that $\rho_{\mathrm{v}}$ is an exponential function with a scale height of $2.2 \mathrm{~km}$ and $\mathrm{T}$ is constant at $290^{\circ} \mathrm{K}$ leads to the approximate formulas

$$
\mathrm{L}_{\mathrm{v}} \approx 1.3 \rho_{\mathrm{v}}
$$

and

$$
\mathrm{L}_{\mathrm{v}} \approx 1.0 \mathrm{e} \text {. }
$$

More exact analysis for prediction of path length from surface meteorological data are given by Saastamoinen (1973).

The brightness temperature at the surface of the earth, derived from the equation of radiative transfer, can be written as

$$
\mathrm{T}_{\mathrm{B}}(\nu)=\mathrm{T}_{\mathrm{c}} \mathrm{e}^{-\tau \nu}+\int_{0}^{\infty} \mathrm{T}(\ell) \alpha(\nu, \ell) \mathrm{e}^{-\tau^{\prime}(\nu, \ell)} \mathrm{d} \ell,
$$

where

$$
\tau_{\nu}^{\prime}=\int_{0}^{\ell} \alpha(\nu, \ell) \mathrm{d} \ell
$$

and

$$
\tau_{\nu}=\int_{0}^{\infty} \alpha(\nu, \ell) \mathrm{d} \ell
$$

and where $\ell$ is the distance along the ray path from the observer, $\alpha(\nu, \ell)$ is the absorption coefficient, $T_{c}$ is the brightness temperature of any extraterrestial radiation source, $\tau_{\nu}$ is the total atmospheric opacity along the ray path, and $\tau_{\nu}$ is the opacity between the point of emission and the observer.

The principal contribution to the microwave absorption coefficient are water vapor and oxygen. The opacity due to oxygen at $22 \mathrm{GHz}$ is about 0.013 nepers; therefore, the brightness temperature contribution is only about $4^{\circ} \mathrm{K}$. This contribution changes very little with time. The absorption coefficient for oxygen, taken from Meeks and Lilley (1960) has been included in our calculations. The absorption coefficient due to the water vapor for the $6_{16}-5_{23}$ transition having a rest frequency, $\nu_{0}$, of $22.23508 \mathrm{GHz}$ is given by Staelin (1966) as 


$$
\begin{aligned}
\alpha(\nu, \ell)= & 3.24 \times 10^{-4} \mathrm{e}^{-664 / \mathrm{T}} \frac{\nu^{2} \mathrm{P} \rho_{\mathrm{v}}}{\mathrm{T}^{3.125}}\left(1+0.0147 \frac{\rho_{\nu} \mathrm{T}}{\mathrm{P}}\right) \\
\cdot & {\left[\frac{1}{\left(\nu-\nu_{\mathrm{o}}\right)^{2}+\Delta \nu^{2}}+\frac{1}{\left(\nu+\nu_{\mathrm{o}}\right)^{2}+\Delta \nu^{2}}\right] } \\
& +2.55 \times 10^{-8} \rho_{\mathrm{v}} \nu^{2} \frac{\Delta \nu}{\mathrm{T}^{3 / 2}} \mathrm{~cm}^{-1}
\end{aligned}
$$

where

$$
\Delta \nu=2.58 \times 10^{-3}\left(1+0.0147 \frac{\rho_{\mathrm{v}} \mathrm{T}}{\mathrm{P}}\right) \frac{\mathrm{P}}{(\mathrm{T} / 318)^{0.625}}
$$

and $\nu$ is the frequency in $\mathrm{GHz}$.

The brightness temperature at center line, for the case where $\tau_{\nu} \ll 1$, is therefore

$$
\mathrm{T}_{\mathrm{B}} \propto \int_{0}^{\infty} \frac{\rho_{\mathrm{V}}}{\mathrm{PT} \cdot 875} \mathrm{e}^{-644 / \mathrm{T}} \mathrm{dh}
$$

whereas

$$
\mathrm{L}_{\mathrm{v}} \propto \int_{0}^{\infty} \frac{\rho_{\mathrm{v}}}{\mathrm{T}} \mathrm{dh} .
$$

To a first approximation, we find that $\mathrm{T}_{\mathrm{B}}\left(\mathrm{H}_{2} \mathrm{O}, \nu=22 \mathrm{GHz}\right)\left({ }^{\circ} \mathrm{K}\right) \sim 2.1 \mathrm{~L}_{\mathrm{v}}(\mathrm{cm})$. P decreases by 10 percent per kilometer and $\mathrm{T}$ by 2 percent per kilometer so that a given amount of water vapor contributes more heavily at higher altitudes to the brightness temperature than to the wet path length although saturation tends to reduce the discrepancy. Frequencies can be chosen to maximize the correlation between path length and brightness temperature (Wu, 1979).

\section{RADIOSONDE STUDIES}

Radiosondes are launched routinely at 11 and 23 hours UT from Portland, Maine; Chatham, Massachusetts; and Albany, New York by the National Weather Service. We obtained data for 50 occasions during which simultaneous launches were made at the three stations in July and August 1974 and for 30 occasions in January and February 1974. The data of the significant reporting points were used in our analysis. The geopotential height was calculated from the pressure and temperature (Hess, 1959), and the water vapor density was calculated from the dew point depression and temperature. The absorption coefficient was calculated as a function of height from $\rho_{\mathrm{v}}, \mathrm{P}$, and $\mathrm{T}$. 
The wet path and the brightness temperatures were calculated from equations (7), (10), (13), and (14) using the trapezoidal rule for integration. The mean and $r \mathrm{rs}$ of $L_{v}$ were $17 \pm 7 \mathrm{~cm}$ and $5 \pm 2 \mathrm{~cm}$ for summer and winter.

The calculated values of wet path length and brightness temperature were compared in various ways with a simple least-mean-square analysis program. Some of the results are shown in table 1 . To see how well the wet path length could be predicted from the surface density of water vapor, $\rho_{0}$, the data were fit to the equation

$$
\mathrm{L}_{\mathrm{v}}=\mathrm{A}_{1} \rho_{\mathrm{o}}+\mathrm{A}_{2} \text {. }
$$

The rms deviations in path length were typically $5 \mathrm{~cm}$ for the summer data and $2 \mathrm{~cm}$ for the winter data. Hence, surface data only offers a slight improvement in the estimate of $\mathrm{L}_{\mathrm{V}}$ compared to predictions from the climatic mean.

Tablc 1

rms Error in Predicting Wet Path Length from Various Parameters

\begin{tabular}{|l|c|c|}
\hline \multicolumn{1}{|c|}{ Predictor } & $\begin{array}{c}\text { summer } \\
\sigma \\
(\mathrm{cm})\end{array}$ & $\begin{array}{c}\text { winter } \\
\sigma \\
(\mathrm{cm})\end{array}$ \\
\hline Seasonal mean (1) & 6.9 & 3.1 \\
Seasonal mean (2) & 4.4 & 2.2 \\
Surface meteorology (1) & 5.1 & 2.1 \\
Surface meteorology (2) & 3.7 & 2.0 \\
Surface meteorology (3) & 3.9 & 2.4 \\
Remote station radiosonde (1) & 5.0 & 1.5 \\
$\mathrm{~T}_{22}$ and $\mathrm{T}_{19}(1)$ & 0.3 & 0.2 \\
$\mathrm{~T}_{22}(1)$ & 0.4 & 0.4 \\
\hline
\end{tabular}

NOTES: (1) Portland-Chatham-Albany data from this study.

(2) Huntington, W. Va. data from Schaper et al. (1970).

(3) Nlbany, N.Y. data from Crane (1976). 
The hypothesis that the wet path at one station might be useful in predicting the wet path at another was examined. Wet path data from the station $i$ was fit to the wet path data from the station $\mathrm{j}$ with the equation

$$
L_{v_{i}}=A_{1} L_{v_{j}}+A_{2} \quad i \neq j .
$$

The mean of the rms deviations were $5 \mathrm{~cm}$ for the summer data and $1.5 \mathrm{~cm}$ for the winter. The wet path length is therefore fairly well decorrelated over a distance of $200 \mathrm{~km}$. Hence, the remote profiles are no better than local measurements of surface meteorology for the prediction of the wet path length.

The correlation between brightness temperatures at 22 and $19 \mathrm{GHz}, \mathrm{T}_{22}$ and $\mathrm{T}_{19}$, and wet path length were calculated by fitting the data to the equations

$$
\mathrm{L}_{\mathrm{v}}=\mathrm{A}_{1} \mathrm{~T}_{22}
$$

and

$$
L_{v}=A_{1} T_{22}+A_{2} T_{19}+A_{3} .
$$

The rms deviations for the two-temperature fit for the summer and winter data were $0.3 \mathrm{~cm}$ and 0.2 $\mathrm{cm}$, showing that wet path length and brightness temperature are well correlated.

\section{RADIOMETERS}

The two radiometers in our study were built at the National Radio Astronomy Observatory in 1968. Their characteristics are summarized in table 2 . In order to measure the antenna temperatures at two frequencies, 19 and $22 \mathrm{GHz}$, each radiometer was configured as a dual-Dicke-switched radiometer which switched between the antenna and an ambient load at $50 \mathrm{~Hz}$ and between frequencies at $2 \mathrm{~Hz}$. Although the radiometers were very stable, drifting less than $0.3^{\circ} \mathrm{K}(\mathrm{rms})$ over many hours, absolute calibration was difficult. The accuracy of the noise tube calibration was measured to be about 1.5 percent. Hence, the absolute calibration with respect to the $295^{\circ} \mathrm{K}$ load was about $3.5^{\circ} \mathrm{K}$. To avoid the need for absolute measurements, observations were made at different elevation angles. The observable used was the brightness temperature at $30^{\circ}$ elevation angle minus that at the zenith. For a temperature difference of about $30^{\circ} \mathrm{K}$, the calibration error was about $0.5^{\circ} \mathrm{K}$. The synchronous detectors suffered a peculiar problem that increased the rms accuracy of the temperature measurements by $1.5^{\circ} \mathrm{K}$. This was, therefore, the dominant contribution to the total measurement error of about $1.6^{\circ} \mathrm{K}$.

\section{THE AUGUST 1975 RADIOSONDE EXPERIMENT}

In August 1975, an experiment was performed at the Haystack Observatory in Westford, Massachusetts, to determine the accuracy of using data from the NRAO radiometers to predict the wet path length. A direct comparison of the radiosonde and radiometer data was made at that time. 
Table 2

Radiometer Characteristics

\begin{tabular}{|l|l|}
\hline Source & NRAO (constructed 1968) \\
Antenna & $92 \mathrm{~cm}$ paraboloid \\
Beamwidth (FWHM) & 61 arc minutes \\
Peak sidelobe & $-22 \mathrm{db}$ \\
Pointing accuracy & $\pm 0.5^{\circ}$ (potientometer encoders) \\
Receiver & Superhetrodyne, mixer first stage \\
Receiver Temperature & $1500^{\circ} \mathrm{K}$ \\
Bandwidth & $300 \mathrm{MHz}$ \\
$\Delta \mathrm{T}$ (rms) (4 sec) & $0.1^{\circ} \mathrm{K}$ \\
$\Delta \mathrm{T}$ (rms) (5 hours) & $0.2^{\circ} \mathrm{K}$ \\
Noise tube accuracy & $1.5 \%$ \\
Reference load temperature & $294^{\circ} \mathrm{K}$ \\
Tuning range & $18-24 \mathrm{GHz}$ \\
Dicke switching & $4^{\circ} \mathrm{K}$ \\
Observation frequencies & $0.9^{\circ} \mathrm{K}$ \\
Absolute calibration & $19.0,22.2 \mathrm{GHz}$ \\
\hline
\end{tabular}


The radiosondes were launched by NWS at the NASA Wallops Island Facility. The radiosondes contained carbon elements for sensing relative humidity (Brousaides and Morrissey, 1974). Forty-five radiosondes were launched over an 11-day period from August 14 to 25, 1975. The basic data were a series of measurements of pressure, temperature, and relative humidity from the radiosondes and the tracking angles of the telemetry antenna. NWS supplied us with reduced profile data consisting of pressure, temperature, water vapor density, wind speed and direction, and other quantities derived from them, at intervals of 100 feet (after interpolation) from the surface to an altitude of 30,000 feet. Typically, the radiosondes drifted eastward from Haystack and were at an elevation angle of $30^{\circ}$ at $1 \mathrm{~km}$ altitude. The total pressure, partial pressure of water vapor, and temperature at the surface are plotted in figure 1 and show that during the period of the experiment, two weak frontal disturbances passed. The mean profile of water vapor density is shown in figure 2 . The profile has, approximately, an exponential dependence with a scale height of $2.2 \mathrm{~km}$, although the high altitude part decreases more slowly than an exponential. Individual profiles deviated greatly from exponential dependence.
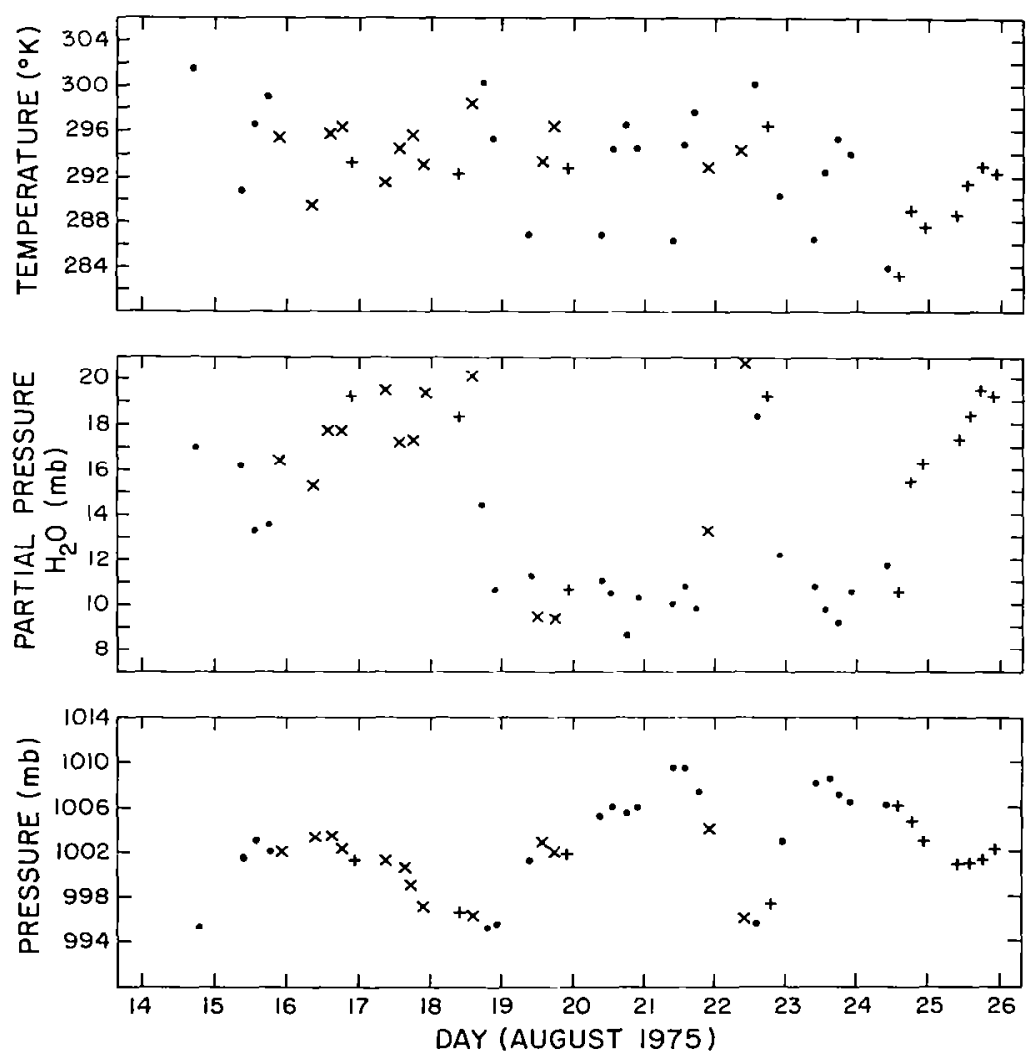

Figure 1. The temperature, partial pressure of water vapor, and total pressure measured at the surface before each radiosonde launch in August 1975. The symbols denote different cloud cover conditions: $(\cdot) \leq 2 / 10$ cover or thin cirrus (21 launches); $(x)>2 / 10$ and $\leq 6 / 10$ cover (13 launches); $(+)>6 / 10$ cover ( 11 launches). 


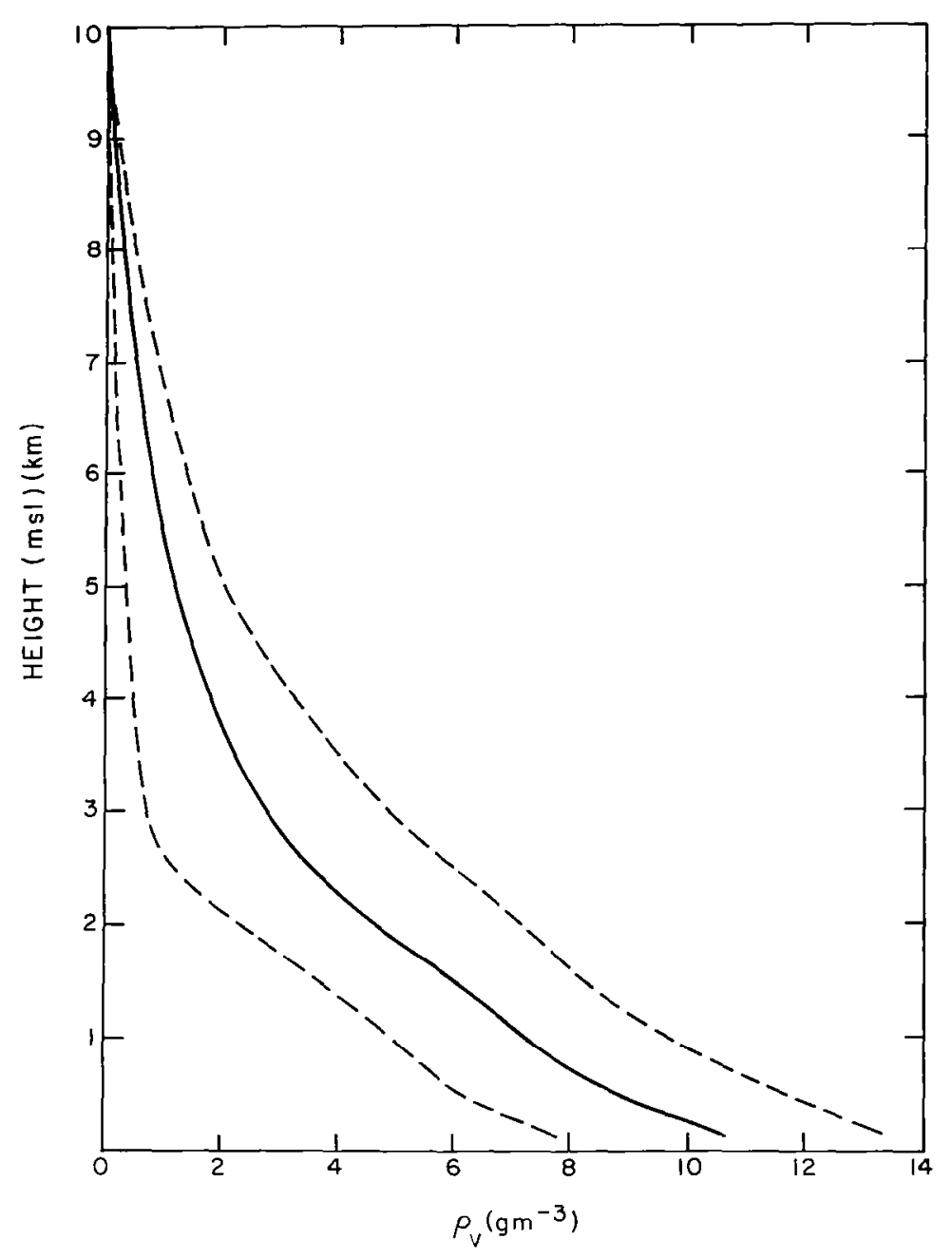

Figure 2. The mean profile of the water vapor from the data of 45 radiosonde launches at Haystack Observatory in August 1975. The dotted lines denote plus and minus one standard deviation.

During each balloon flight, which lasted about 30 minutes, six tipping scans were made at different azimuthal angles. The values of brightness temperature, $\mathrm{T}\left(30^{\circ}\right)-\mathrm{T}$ (zenith), were averaged for each balloon flight. The set of 45 brightness temperatures for each frequency and radiometer were compared to those calculated from the radiosonde data (as described in section II) in order to calibrate the radiometers. The temperature data sets (i.e., temperatures at two frequencies from the two radiometers and the radiosonde estimates) were fit to the equation

$$
T_{i}=A_{1} T_{j}+A_{2} \quad i \neq j .
$$

The values of $\mathrm{A}_{2}$ were typically less than $1^{\circ} \mathrm{K}$. The rms deviation was $1.2^{\circ} \mathrm{K}$ in the comparison of data from radiometers 1 and 2 , implying the radiometer measurements were accurate to $0.9^{\circ} \mathrm{K}$ 
(i.e., $1.2 \% \sqrt{2}$ ). Most of this error was caused by the problem with synchronous detector. The expected radiometer error was $1.6 / \sqrt{6}$ or $0.7^{\circ} \mathrm{K}$. The rms deviation in the fit of radiometer temperatures to radiosonde predicted temperatures was typically $2.5^{\circ} \mathrm{K}$. This suggests that most of the experimental error was in the radiosonde data, or due to the effects of clouds.

Extensive correlation analysis was performed between radiometer temperatures and the wet path and theoretical temperatures estimated from radiosonde data. A plot of the wet path versus brightness temperature at $22 \mathrm{GHz}$ is shown in figure 3. The path length predicted by radiometer 1 from equation (20) is plotted versus the path length estimated from the radiosonde data in figure 4.

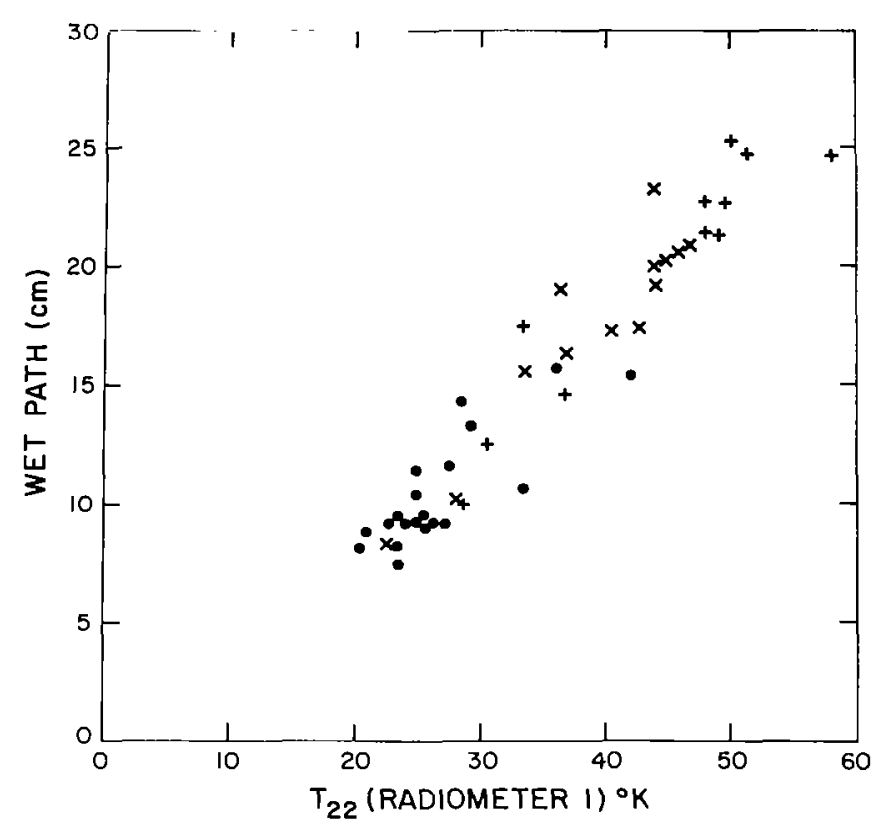

Figure 3. The wet path lengths for the vertical direction calculated from radiosonde profiles versus the brightness temperature ( $\mathrm{T}\left(30^{\circ}\right)-\mathrm{T}$ (zenith)) at $22.2 \mathrm{GHz}$ measured by one of the NRAO radiometers. The symbols, denoting different cloud conditions, are defined in the caption to figure 1. The non-zero intercept is due largely to oxygen emission.

\section{CONCLUSIONS}

The residuals from various regression analyses for the August 1975 experiment are listed in table 3 . We reached the following conclusions:

1. The mean value of the path lengths estimated from radiosonde data for the 45 launches was $14.8 \mathrm{~cm}$ with an rms deviation of $5.6 \mathrm{~cm}$. For the 21 radiosondes launched in clear or nearly clear skies, the mean was $10.3 \pm 2.5 \mathrm{~cm}$. 


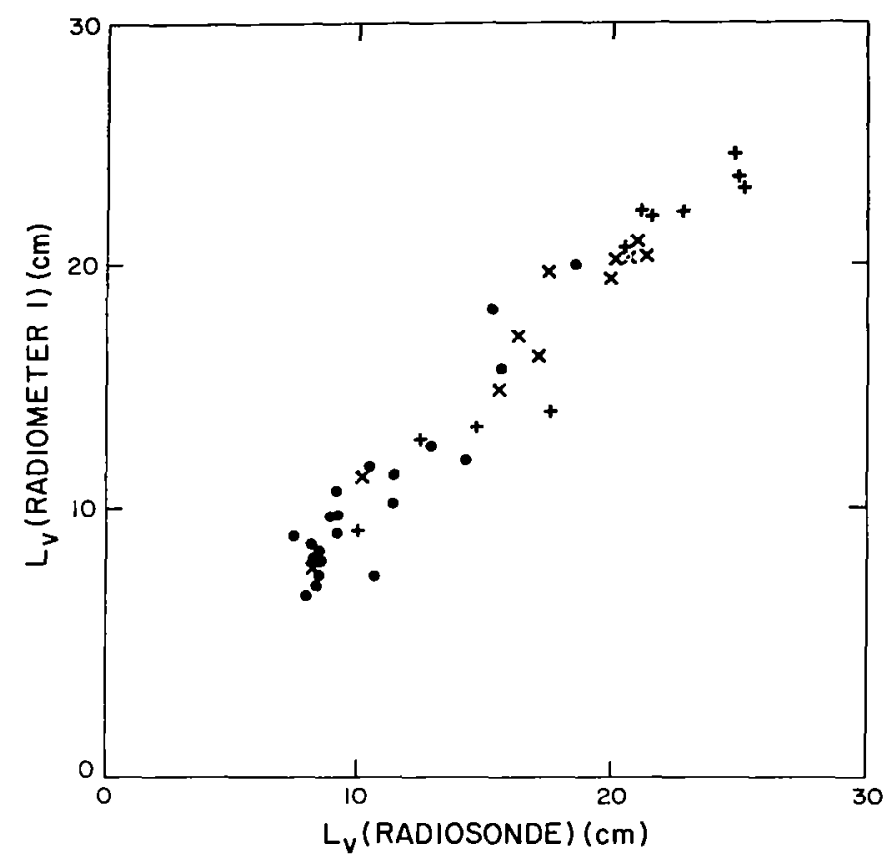

Figure 4. The wet path length, estimated from the formula $L_{v}=T_{1} T_{22}+A_{2} T_{19}+A_{3}$ with temperatures from radiometer 1 versus the wet path lengths derived from radiosonde data. The coefficients $A_{1}, A_{2}$, and $A_{3}$ were derived by fitting the brightness temperatures from the radiometers to the wet path length estimated from the radiosonde data.

2. Prediction of the wet path length from the surface partial pressure of $\mathrm{H}_{2} \mathrm{O}$ had an $\mathrm{rms}$ deviation of $3.2 \mathrm{~cm}$, an improvement over the rms deviation about the mean.

3. Prediction of the wet path length at Haystack from those estimated for Portland, Albany, and Chatham gave no better results than prediction from local surface meteorology.

4. The rms deviation in wet path length estimated from radiometer data was $1.5 \mathrm{~cm}$.

5. The rms deviation in path length predicted from brightness temperatures computed from radiosonde data was only $0.4 \mathrm{~cm}$. This may represent the ultimate accuracy of the technique without adding higher-order regression coefficients or correcting for the effects saturation caused by large microwave opacity.

The results from this experiment were consistent with the results from our previous analysis of radiosondes launched at Albany, Portland, and Chatham. The residuals for the winter data were, of course, much smaller. 
Table 3

Results of Regression Analysis on August 1975 Data

\begin{tabular}{|l|c|c|c|}
\hline \multicolumn{2}{|c|}{ RMS Residuals in Wet Path Length } \\
\hline \multicolumn{1}{|c|}{ Predictor } & \multicolumn{1}{|c|}{ Fit(3) } & All data(1) & Best Weather data (2) \\
\hline Mean & $\mathrm{L}_{\mathrm{v}}=\mathrm{A}_{1} \mathrm{e}+\mathrm{A}_{2}$ & $5.6 \mathrm{~cm}$ & $2.5 \mathrm{~cm}$ \\
Surface partial pressure & $\mathrm{L}_{\mathrm{v}}=\mathrm{A}_{1} \mathrm{~L}_{\mathrm{v}_{1}}+\mathrm{A}_{2} \mathrm{~L}_{\mathrm{v}_{2}}+\mathrm{A}_{3} \mathrm{~L}_{\mathrm{v}_{3}}$ & 3.0 & 1.5 \\
$\begin{array}{l}\text { Path length from radio- } \\
\text { sondes at Portland, } \\
\begin{array}{l}\text { Albany, Chatham } \\
\begin{array}{l}\text { Brightness temper- } \\
\text { atures from NRAO } \\
\text { radiometers }\end{array}\end{array} \\
\begin{array}{l}\text { Brightness temper- } \\
\text { atures estimated from } \\
\text { radiosonde data }\end{array}\end{array} \mathrm{L}_{\mathrm{v}}=\mathrm{A}_{1} \mathrm{~T}_{22}+\mathrm{A}_{2} \mathrm{~T}_{19}+\mathrm{A}_{3}$ & 1.5 & \\
\hline
\end{tabular}

NOTES: (1) 45 data points.

(2) 22 data points for which cloud cover was $\leq 0.2$.

(3) $\mathrm{L}_{\mathrm{v}}$ estimated from radiosonde data.

The stability of the regression coefficients derived presents a problem requiring some study. Wu (1979) has shown that coefficients can be found which are insensitive to climatic conditions if correct pairs of frequencies are chosen. Wu requires that the coefficients satisfy the relation $\left(A_{1} / A_{2}\right)=$ $-\left(\nu_{2} / \nu_{1}\right)^{2}$ so that the brightness temperature of clouds, which is proportional to $\nu^{2}$, does not effect the path length estimate. With this constraint, 19 and $22 \mathrm{GHz}$ are not a good frequency pair. A good way to include the radiometer data in the analysis of VLBI data may be to add the observed brightness temperatures to the data base of measured interferometer observables and estimate the regression coefficients for each site in the general data analysis. In this way the stability of the coefficients under different climatic conditions is less important.

This work was supported under NASA contract NAS5-20975. We thank Hays Penfield and Joe Hayes for construction and operating support, and I. I. Shapiro, C. C. Counselman, and C. A. Knight for helpful discussions. 


\section{REFERENCES}

Bean, B. R., and Dutton, E. J. 1966, Radio Meteorology, U.S. Gov’t. Printing Office.

Brousaides, F. J., and Morrisey, J. F. 1974, Residual Temperature-Induced Humidity Errors in the National Weather Service Radiosonde. AFCRL Report TR-74-0111.

Claflin, E. S., Wu, S. C., Resch, G. M. 1978, DSN Progress Report 42-48, 22.

Crane, R. K. 1976, Refraction Effects in the Neutral Atmosphere in Methods of Experimental Physics ed. by M. L. Meeks, Academic Press, New York, Vol. 12B, p.. 186.

Hess, S. L. 1959, Introduction to Theoretical Meteorology, Holt Co., New York

Hopfield, H. S. 1971, Radio Science 6, 357.

Liebe, H. 1969, IEEE Trans. Ant. Propag. AP-17, 621.

Meeks, M. L., and Lilley, A. E. 1963, J. Geophys. Res., 68, 1683.

Moran, J. M., and Penfield, H. 1976, NASA Contract Report NAS5-20975.

Saastamoinen, J. 1972, AGU Geophysical Monograph 15, The Use of Artificial Satellites for Geodesy (ed: S. Henriksen, A. Mancini, B. Chovitz) p. 247.

Shaper, L. W., Staelin, D. H., and Waters, J. W. 1970, P.I.E.E.E., 58, 272.

Staelin, D. H. 1966, J. Geophys. Res., 71, 2875.

Waters, J. W. 1967, NRAO VLA Scientific Memorandum No. 8.

Waters, J. W. 1976, Absorption and Emission by Atmospheric Gases in Methods of Experimental Physics, ed. by M. L. Meeks, Academic Press, New York, Vol. 12B, p. 142.

Wu, S. C. 1979, I.E.E.E. Trans. Ant. and Prop. AP-27, 233. 


\title{
MICROWAVE RADIOMETRY AS A TOOL TO CALIBRATE TROPOSPHERIC WATER-VAPOR DELAY*
}

\author{
G. M. Resch and E. S. Claflin \\ Jet Propulsion Laboratory
}

\begin{abstract}
The spectrum of atmospheric emission contains an emission line due to the water-vapor molecule at a frequency of $22.2 \mathrm{GHz}$. The strength of the line is proportional to the amount of water vapor along the line-of-sight. In principle, measurement of this line strength provides an independent estimate of water-vapor induced delay that can then be used to correct the interferometrically observed group delay. We have used borrowed radiometers for the past 5 years to refine our understanding of this technique to measure water vapor. Four separate field tests have been completed in which we have compared the radiometer to other techniques that measure water vapor. We have attempted to schedule these experiments at times and locations that would test the validity of our assumptions relating radiometer output (i.e., brightness temperature) to integrated water vapor content. We believe that we can estimate water-vapor induced delay with an accuracy of $\pm 2 \mathrm{~cm}$ for elevation angles above $17^{\circ}$. We are currently constructing seven water vapor radiometers for use at very long baseline interferometry (VLBI) stations. These new instruments are being designed to be compatible with the Mark III data acquisition system. We believe that the technique is ultimately capable of a $1-\mathrm{cm}$ accuracy, and further tests are planned to investigate this possibility. In addition, we plan a re-design and re-packaging of the radiometer with the goal of reducing the total cost.
\end{abstract}

\footnotetext{
*This paper presents the results of one phase of research carried out at the Jet Propulsion Laboratory, California Institute of Technology, under Contract No. NAS 7-100, sponsored by the National Aeronautics and Space Administration.
} 
In the previous paper by Claflin and Resch, you heard how water vapor introduces an error in VLBI measurements. The most cost effective method for calibrating the water-vapor induced errors appears to be the use of microwave radiometry to estimate the total amount of water vapor along the line-of-sight by measuring the strength of the water-vapor emission line at $22.2 \mathrm{GHz}$. Figure 1, taken from Waters (1977), shows the spectrum of the atmosphere for two conditions. The lower curve is the brightness temperature versus frequency for an atmosphere with no water vapor. The upper curve is calculated for an atmosphere containing $2 \mathrm{gm} / \mathrm{cm}^{2}$ of precipitable water vapor. This corresponds to a delay of $12.2 \mathrm{~cm}$ at the zenith - a fairly high zenith delay. Our new radiometers operate at $21 \mathrm{GHz}$ on the shoulder of the emission line and at $31 \mathrm{GHz}$, which is well off the wing of the line. The brightness temperatures that we typically measure are in the range $20^{\circ}$ to $100^{\circ} \mathrm{K}$. The $21 \mathrm{GHz}$ brightness temperature is proportional to the total amount of water vapor in the beam plus the background emission. The $31 \mathrm{GHz}$ channel is used to subtract out the background and is particularly useful for removing the effect of clouds.

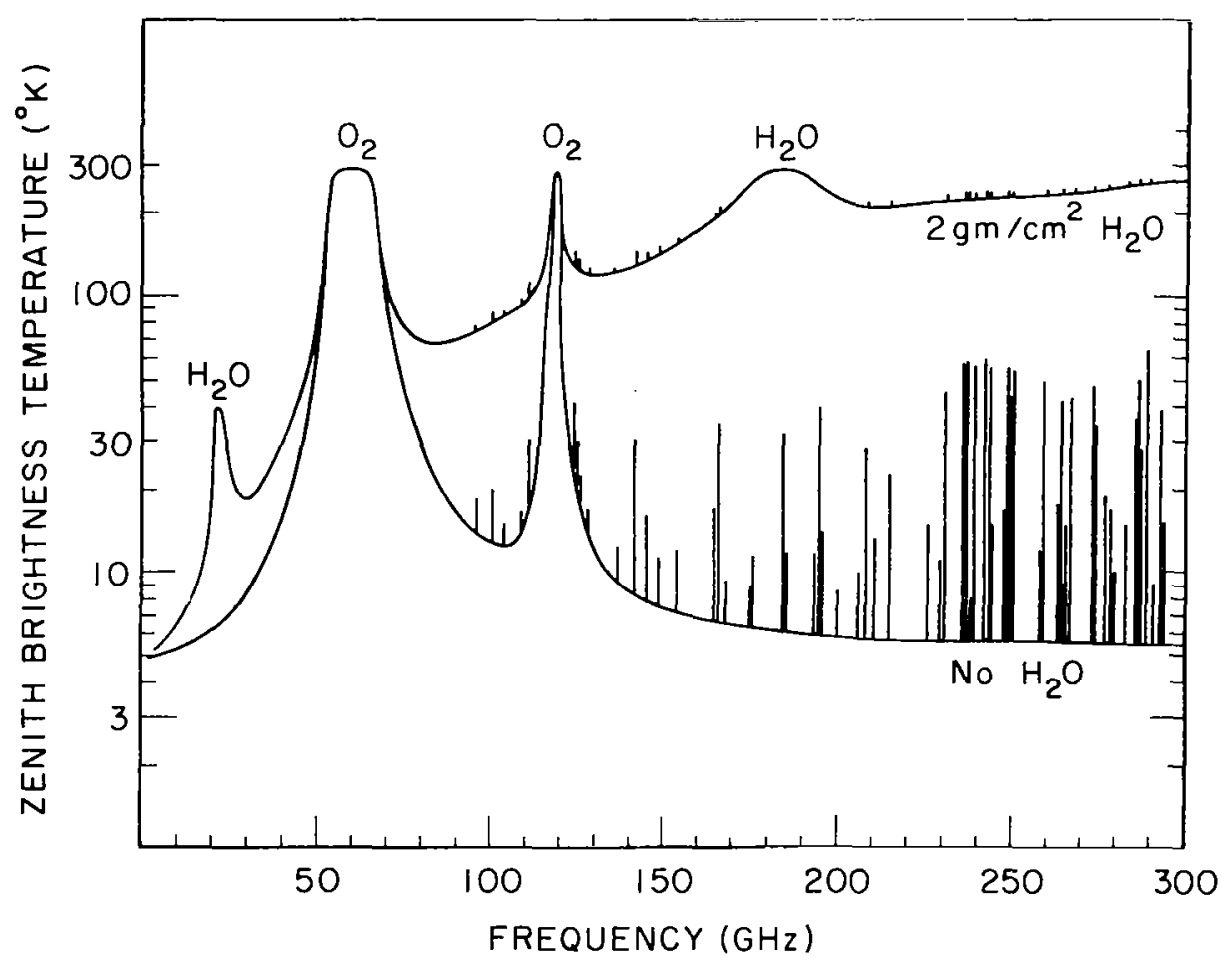

Figure 1.

The water-vapor delay correction can be related to the brightness temperature by a very simple linear relation as shown in figure 2. The quantities $\mathrm{T}_{\mathrm{C}}^{\prime}, \mathrm{A}_{0}, \mathrm{~A}_{1}$, and $\mathrm{A}_{2}$ in this expression are actually functions of surface temperature, pressure, and relative humidity as explained by $\mathrm{Claflin}, \mathrm{Wu}$, and Resch (1978). We have determined the constant terms in these functions from a regression analysis against radiosonde and instrumented aircraft measurements at some particular site. We believe that we know the functional dependence on temperature, pressure, and relative humidity well enough to 
allow us to transport the radiometer to other geographical locations or climates and still retain the instrumental calibration. The quantities $T^{\prime}(22 \mathrm{GHz})$ and $T^{\prime}(31 \mathrm{GHz})$ are the brightness temperatures that have been corrected for opacity and adjusted to an absolute temperature scale.

$$
\begin{aligned}
& \Delta L_{V}=-\left(A_{0} M-T_{C}^{\prime}\right)+A_{1} T^{\prime}(22 \mathrm{GHz})+A_{2} T^{\prime}(31 \mathrm{GHz}) \\
& T_{C}=\text { COSMIC BLACKBODY BACKGROUND TEMPERATURE }=2.8^{0} \mathrm{~K} \\
& M=\text { AIR MASS }
\end{aligned}
$$

Figure 2. WVR path delay algorithm.

When we initially contemplated the use of this technique to support VLBI measurements, we noted two important difficulties: (1) it seemed to require an instrument well calibrated on the absolute temperature scale - this is difficult and expensive, and (2) the measurement of antenna temperatures in the $20^{\circ}$ to $100^{\circ} \mathrm{K}$ range implied a cold internal calibration load would be necessary - this is also operationally difficult and expensive. Both difficulties were eased by using a calibration technique suggested by Water (1975).* This technique is illustrated in figure 3 . The instrument is tipped from the zenith (air mass $=1$ ) to different elevations (higher air mass) and the observed brightness temperature is plotted versus the air mass as shown by the dotted line. In general, this line implies a negative brightness temperature at zero air mass - a nonphysical result. We assume that this nonphysical result is due to two causes: (1) nonlinearity of the tipping curve (i.e., opacity effects), and (2) lack of proper instrumental calibration. We then perform a simple iteration calculation to estimate the opacity and instrumental correction term that would cause the tipping curve to have the proper intercept at zero air mass; i.e., $2.8^{\circ} \mathrm{K}$, the cosmic blackbody background temperature. By using this tipping curve calibration method, we have found that we can forego the cold internal calibration load in favor of a hot load that is much more convenient to operate.

In order to determine the constants that appear in the equation relating brightness temperature to delay, we have used the radiometers in several calibration sessions as shown in figure 4 . We have used two different radiometers and compared them against radiosondes, instrumented aircraft, LIDAR (laser radar), and a microwave refractometer mounted in an aircraft. Some typical results are shown in figure 5. Here, we plot the delay determined by the water vapor radiometer (WVR) along the vertical axis and the delay determined by an instrumented aircraft along the horizontal axis. The aircraft flight pattern was an approximation to the line-of-sight at elevation angles of $10^{\circ}$, $20^{\circ}$, and $30^{\circ}$, and the zenith. The WVR could not tip down to elevation angles of $10^{\circ}$ so we had to extrapolate from data taken at $17^{\circ}$. This data shows a relatively wide range of path delay. Typical of all our data, the RMS of the fit is a bit better than $2 \mathrm{~cm}$. This is one way to demonstrate the WVR - compare it with an independent method of measuring water vapor. Another way, one that probably means more to VLBI practitioners, is to demonstrate that VLBI data improves with WVR calibrations.

*I. W. Waters, private communication, 1975. 


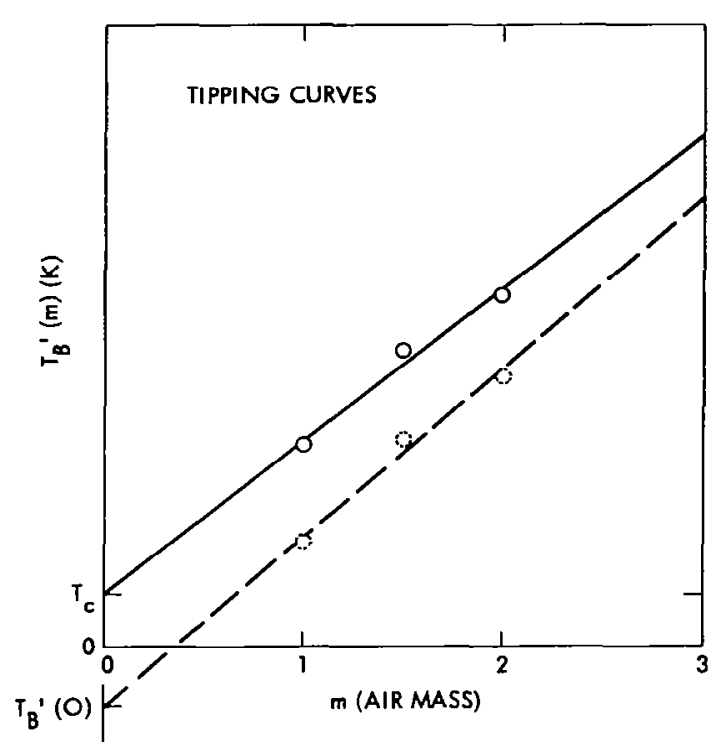

Figure 3 .
- Mar 1974

el Monte, Ca.

- Mar 1975

El Monte, Ca.

- February 1976

Pt, Magu, Ca.

- JUNe 1978

HAMPTON, $V_{A}$.
SMILE vs. RADIOSONDE

$\left.\begin{array}{l}\text { Smile } \\ \text { Nems }\end{array}\right\}$ vs. $\left\{\begin{array}{l}\text { Radiosonde } \\ \text { Aircraft }\end{array}\right.$

SMile vs. $\left\{\begin{array}{l}\text { RAdIOSONDE } \\ \text { AIRCRAFT }\end{array}\right.$

Nems vs. $\left\{\begin{array}{l}\text { Lidar } \\ \text { Refractometer }\end{array}\right.$

Figure 4. WVR calibration sessions.

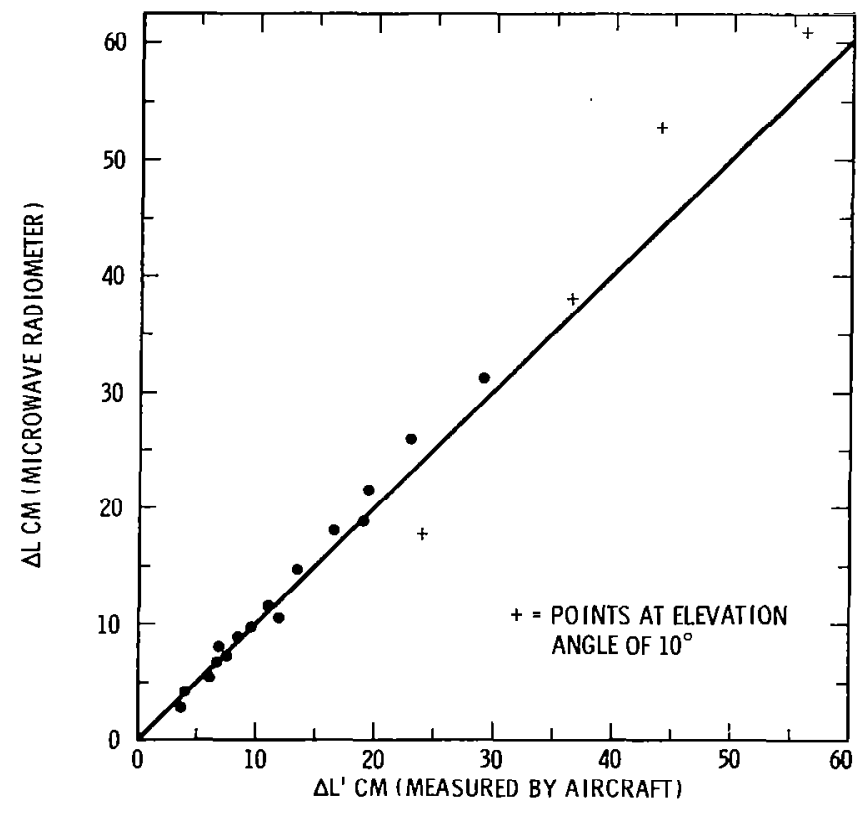

Figure 5. Excess path delay due to water vapor as measured by a microwave radiometer and an instrumented aircraft.

Figure 6 is the first suggestion that the WVR does indeed positively contribute to a VLBI result. We have plotted the results from three experiments that were performed in June 1977 between OVRO and the 9-m ARIES antenna that was then located near the Golden Gate Bridge in San Francisco. The coordinate system chosen was local to the 9-m antenna. The reference point was arbitrary but 
located near the intersection of antenna axis. The data was reduced using two different types of water vapor calibrations. The unfilled squares indicate a solution in which the tropospheric water vapor was estimated from surface meteorological measurements. The filled circles indicate a solution in which the tropospheric water vapor was estimated by using the WVR. The WVR did not observe along the line-of-sight to each radio source, but merely estimated the zenith water-vapor path delay. To the best of my knowledgc, this was the first set of experiments in which WVR's were used at both ends of the baseline.

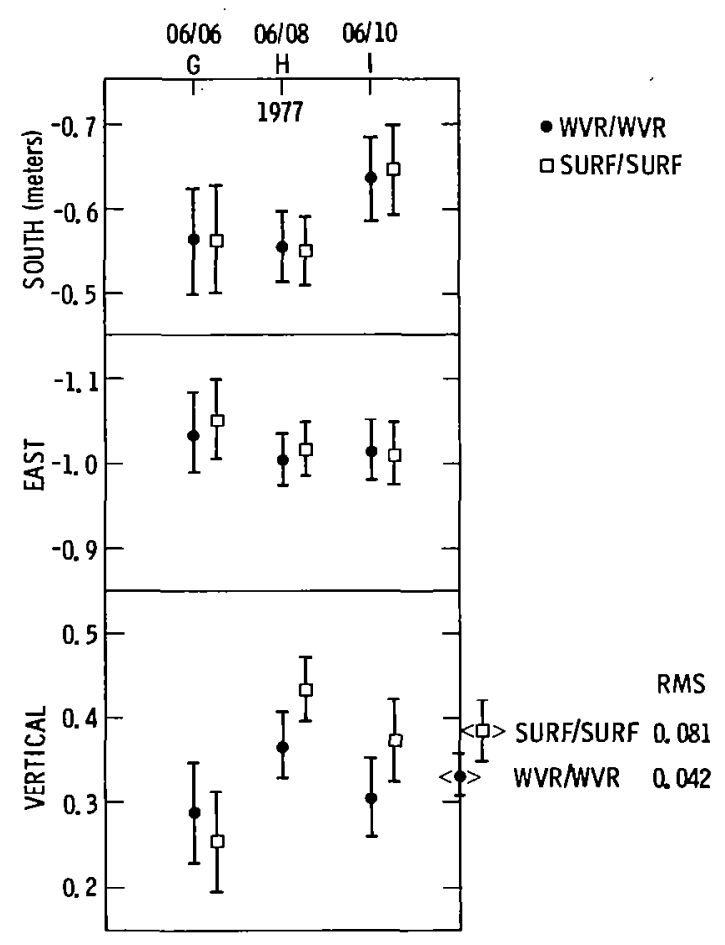

Figure 6. Troposphere calibration comparison OVRO/San Francisco.

In the earlier paper by Claflin and Resch, it was pointed out that propagation medium errors have little effect on the determination of local horizontal coordinates. As you can see in figure 6, the North-South and East-West baseline components are not affected by the choice of tropospheric calibration. However, as we would expect from the previous discussion, the scatter in the local vertical component does improve with the WVR calibration. If we can attach any significance to the RMS of three data points then the "RMS" improves by almost a factor of two by using the WVR calibration - at least the scatter in the data did not get worse. Obviously, the case for the WVR must be established by many more data points. During the past year our main objective has been to complete seven WVR's so that this data can be collected on a variety of baselines. 
Our "guess-estimate" of the error budget for a two-channel WVR is shown in figure 7. The bar on the far left is due to system noise and the cross-hatched area indicates that this error can normally be reduced by averaging. The bar labeled instrumental corrections indicates that the technique of using tipping curves to determine the instrumental constants is itself noisy. The technique error is due to the approximations that we make in deriving our simple algorithm. These errors are roughly proportional to the total amount of water vapor. Also, when we calibrate the instrument by comparing it to an independent method of measuring water vapor, this independent method usually is subject to errors that are roughly proportional to total water vapor content. The root-sum-square of these assumed independent error sources is shown by the bar on the far right. At low values of path delay, the error budget is dominated by system noise. At larger values of path delay, the error is more like a percentage value of the path delay. For the range of typical delay values likely to be encountered in a VLBI experiment, we feel the WVR crrors will be no larger than $2 \mathrm{~cm}$.

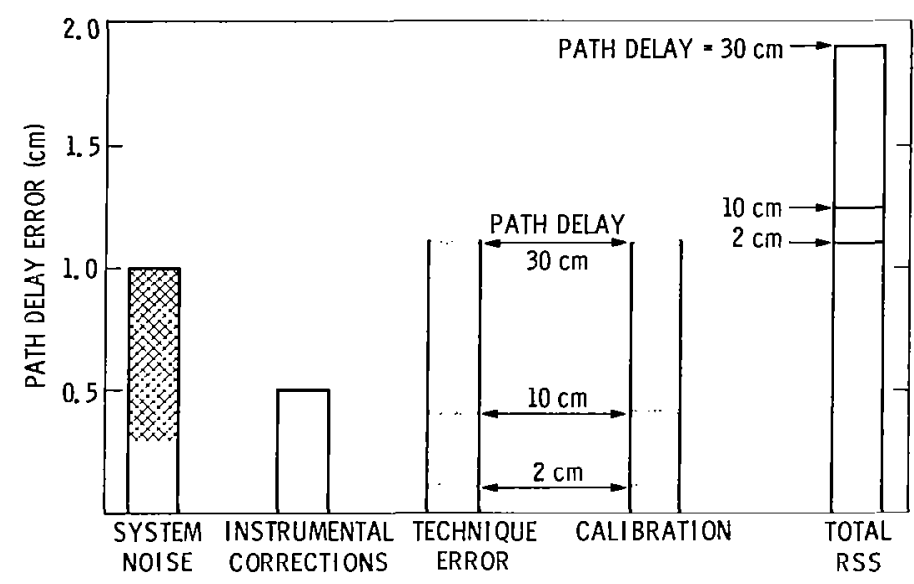

Figure 7. Two-channel WVR error budget.

Even with a 2-cm calibration accuracy, water vapor is likely to be a major error source to the VLBI systems that evolve during the next decade. Simulations of WVR performance suggest that the instrument may be capable of $1-\mathrm{cm}$ accuracy. Figure 8 summarizes the research and development tasks that we feel need work. The priority task is to develop an independent calibration method that is intrinsically more accurate than the WVR. A good possibility is a method that utilizes an optical/microwave device. The basic principal of the device has been described by Thompson (1971). It utilizes a microwave signal to modulate a laser and then transmits both the optical and microwave signals. The optical signal suffers a group delay that is, to first order, proportional to the dry component. The microwave signal is retarded by both dry and wet component. An observer who detected the two signals some distance away would note a phase difference that is proportional to the total amount of water vapor along the propagation path. If we can mount either the transmitter or receiver end of this device in an aircraft and fly it at 20,000 feet (above 98 percent of the tropospheric water vapor), it would provide a subcentimeter calibration of the WVR and practically eliminate a large contributor to the overall WVR error budget. 


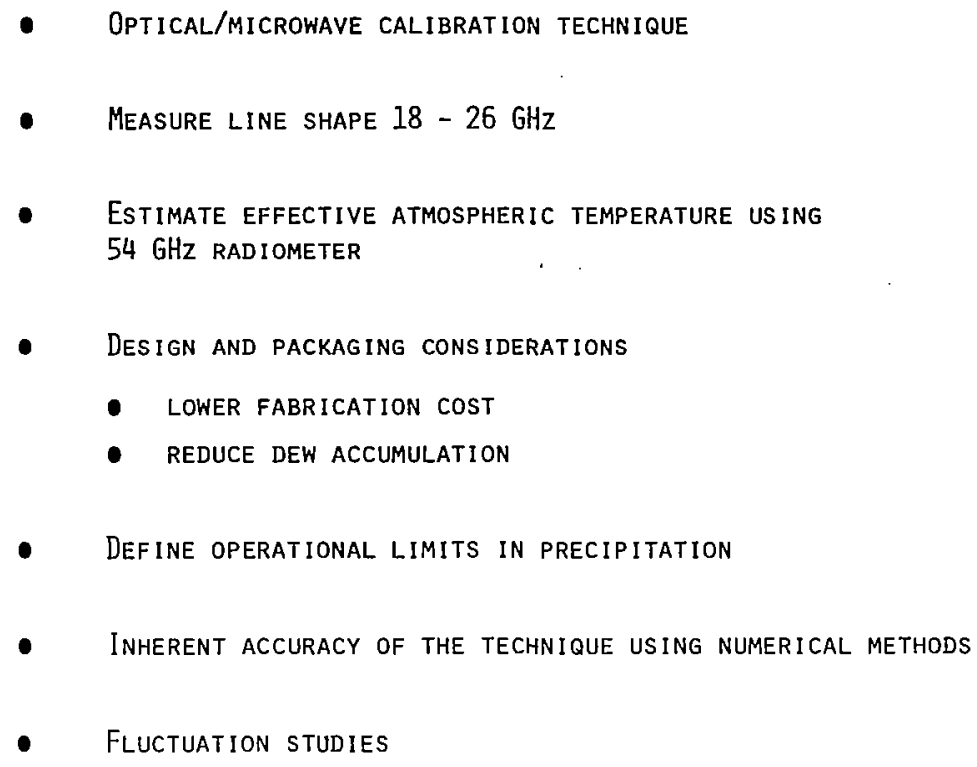

Figure 8. WVR R\&D tasks.

\begin{abstract}
Algorithm error could be reduced by building a new type radiometer that was frequency agile and could measure the line shape. The addition of a $54 \mathrm{GHz}$ channel would also be useful to estimate the effective atmospheric temperature which would improve the accuracy of the algorithm. There are some design and packaging features that could be improved. It may be possible to use a single frequency agile channel which would appreciably lower the fabrication costs. With the present package design, we have trouble often in the early morning hours with dew accumulation. Liquid droplets form on the covering of the horn antennas and give large brightness temperature errors. We will have to experiment with different methods to remove this dew accumulation. The algorithm that relates the brightness temperature to path delay is not valid in heavy precipitation. We need to do more work in order to properly define the operational limits of the technique.
\end{abstract}

We are continuing our work on the inherent accuracy of the remote sensing technique using simulation methods. Our preliminary conclusion is that the WVR should be capable of 1-cm delay accuracy under most conditions. Now that we have several dedicated radiometers we will pay much more attention to fluctuation studies. This is important for several reasons. First, we must be able to separate instrumental and real atmospheric fluctuations. Second, we must examine our assumption that the spatial average of water vapor over the large solid angle of the WVR antenna is equivalent to the longer temporal average over the duration of a VLBI observation. Finally, we must investigate the homogeneity of tropospheric water. This will tell us how to construct an observing strategy with the WVR in both time and space coordinates. The latter point is particularly relevant to a SERIES type network where the spacing between points is a few tens of kilometers. If we do not need a WVR at each of these points, there would be a big cost impact in the initial cost per station. 


\section{REFERENCES}

Claflin, E. S., Wu, S. C., and Resch, G. M., DSN Prog. Rept., 42-48, $28-29$ (Sept. 78)

Waters, J. W., Methods of Experimental Physics, 12-B, 142-176 (1976)

Thompson, Jr., M. C., Rem. Sens. Environ., 2, $37-40$ (1971) 


\title{
PHASE VERSUS DELAY IN GEODETIC VLBI
}

\author{
James Campbell \\ Geodetic Institute \\ University of Bonn
}

\begin{abstract}
For high precise baseline and source position determinations with VLBI, large bandwidth techniques are required to achieve the necessary delay resolution of $\leqslant 0.1 \mathrm{~ns}$. In local interferometry, the interferometric phase observable offers an equally high resolution in spite of the much smaller bandwidths used. In the present paper, the conditions for the applicability of the phase observable in very long baseline interferometry (VLBI) are discussed and examples of adequate observation programs are given. These considerations apply in particular to the Mark II system, which is already in operation at many observatories.
\end{abstract}




\section{DELAY AND PHASE OBSERVATIONS}

In radio interferometry the travelling time $\tau$ of a wavefront between the antennas at two sites is measured by maximizing the cross-correlation function of the two signal streams received at the two antennas. Simultaneously, the phase and the rate of the interference fringes are determined in the processor as they vary with the Earth's rotation. During correlation, the data stream from one station is delayed quasi-continuously in such a way that the changing geometric delay $\tau_{\mathrm{s}}$ is almost completely compensated for. This gives rise to a rather low residual fringe frequency the phase of which slowly varies on the scale of a few turns per minute. In order to be able to observe those fringes which have maximum amplitude, the correlation is carried out simultaneously in a number of delay channels separated by

$$
\Delta \tau=\frac{1}{2 \mathrm{~B}_{\mathrm{o}}}
$$

where $B_{0}$ is the effective bandwidth of the processed signal streams. The output of the correlator is usually described by the complex cross correlation functions, which translates the response of an interferometer system to a point source*:

$$
\mathrm{R}\left(\mathrm{t}, \tau_{\mathrm{i}}\right)=\mathrm{W}_{\mathrm{SNR}} \cdot \mathrm{D}\left(\tau-\tau_{\mathrm{i}}\right) \cdot \mathrm{e}^{-\mathrm{j} \phi_{\mathrm{f}}\left(\mathrm{t}, \tau_{\mathrm{i}}\right)}
$$

where

$\mathrm{W}_{\mathrm{SNR}}=\mathrm{a}$ factor depending essentially on the signal-to-noise ratio (SNR) of the interferometer system observing a given source,

$\mathrm{t}=$ observing time (i.e., UTC),

$\tau=$ actual delay including all instrumental and atmospheric effects,

$\tau_{i}=$ discrete time delays as computed for the different delay channels,

$$
\begin{gathered}
\mathrm{D}\left(\tau-\tau_{\mathrm{i}}\right)=\frac{\sin \pi \mathrm{B}_{\mathrm{o}}\left(\tau-\tau_{\mathrm{i}}\right)}{\pi \mathrm{B}_{\mathrm{o}}\left(\tau-\tau_{\mathrm{i}}\right)} \\
\mathrm{D}=\text { delay resolution function, }
\end{gathered}
$$

$\phi_{\mathrm{f}}\left(\mathrm{t}, \tau_{\mathrm{i}}\right)=\underline{\text { residual fringe phase (including all instrumental and atmospheric effects) }}$

and

$$
\phi_{\mathrm{f}}=\omega_{\mathrm{f}} \cdot t
$$

where $\omega_{\mathrm{f}}=$ residual fringe frequency.

(For more details the reader is referred to Thomas, 1972 or Moran, 1976.)

For the discussion that follows, it is important to note that the interferometric observables used in geodetic VLBI are all contained in equation (2), namely the group delay $\tau(\mathrm{t})$, the phase $\phi_{\mathrm{f}}(\mathrm{t})$, and the fringe frequency $\omega_{\mathrm{f}}(t)$.

*The source structure is expressed by an extra amplitude and phase term which is omitted here for simplicity. 
The group delay is estimated from the delay resolution function (2a), which becomes a maximum for $\tau_{\mathrm{i}}=\tau$. The accuracy with which the exact fraction of $\tau_{\mathrm{i}}$ can be determined, depends mainly on the halfwidth of the delay function, which is given by

$$
\Delta \tau_{\mathrm{h}}=\frac{1}{\mathrm{~B}_{\mathrm{o}}} \text {. }
$$

There are different possible methods of delay estimation* (i.e., Whitney et al., 1976) all of which yield a precision $\sigma_{\tau}$ of roughly 1 percent of $\Delta \tau_{\mathrm{h}}$, depending on the SNR and the available integration time per observation. The following table is meant to give an overall idea on the delay estimation performance of different VLBI systems.

Table 1

\begin{tabular}{|l|c|c|c|c|}
\hline \multicolumn{1}{|c|}{ System } & $\mathrm{B}_{\mathrm{O}}[\mathrm{MHz}]$ & $\Delta \tau_{\mathrm{h}}[\mathrm{ns}]$ & $\sigma_{\tau}[\mathrm{ns}]$ & $c \cdot \sigma_{\tau}[\mathrm{cm}]$ \\
\hline Canadian analog. & 4 & 250 & 2.5 & 75 \\
\hline Mk I c 1 channel & 0.36 & 2,778 & 28 & 840 \\
Mk I synth. 8 channels & $23^{* *}$ & 43 & 0.5 & 15 \\
\hline Mk II 1 channel & 2 & 500 & 5 & 150 \\
\hline Mk III 28 channels & $56^{* *}$ & 18 & 0.2 & 6 \\
\hline Sat-Link (concept.) & 100 & 10 & 0.1 & 3 \\
\hline
\end{tabular}

**high-degree of flexibility (Mk III: $0.2-300 \mathrm{MHz}$ )

The instrumental and atmospheric errors are discussed in connection with the phase observable.

The residual fringe phase and the fringe rate are obtained from the sine and cosine parts of the cross-correlation function (last term in equation (2)). Due to the close relationship of $\sigma_{\mathrm{f}}$ and $\omega_{\mathrm{f}}$ (equation (2b)), these observables are determined simultaneously, either from an ordinary sine wave adjustment or using the fourier transform into the frequency domain, where the maximum of $S$ $\left(\omega_{\mathrm{fi}}\right)$ is estimated. After convolution with $\mathrm{e}^{-\mathrm{j} \omega_{\mathrm{f}} \mathrm{t}}$, the phase for a given instant $\mathrm{t}$ can be computed from the real and imaginary components at lag zero. These methods, which allow to establish the function $\phi(t)$ over a certain interval of time (usually the duration of an uninterrupted source scan), are often referred to as "phase tracking" methods (Thomas, 1972). The accuracy of phase tracking, which is inherent to the system, only weakly depends on system bandwidth through SNR considerations. So in the case of observations on strong sources, no marked differences in accuracy can be found comparing the different VLBI systems. The tracked phase can be converted into a so-called phase delay by using

$$
\tau_{\phi}(\mathrm{t})=\frac{1}{2 \pi \nu_{0}} \phi(\mathrm{t})+\phi_{0}
$$

*Rigorous delay estimation is done iteratively together with the fringe rate estimation: $D=D\left(\tau, \omega_{\mathrm{f}}\right)$. 
where $\nu_{0}=$ center of observing frequency band, and

$\phi_{\mathrm{O}}=$ constant phase zero term, accounting for the unknown number of turns at the start of each source scan.

The phase delay accuracy, which is equivalent to the phase accuracy, is composed of the small phase tracking crror plus the much larger instrumental and atmosphcric phase errors listed below:

- clock stability (phase variations of the local oscillators)

- instrumental delay (electronics, cables + mechanical structure)

- atmospheric delay (ionosphere; troposphere, dry and wet)

- resolved sources add phase variations due to their structure.

Typical phase tracking errors on strong sources are about one-tenth of a turn or smaller; this translates into phase delay errors of some tens of picoseconds only, corresponding to the observing frequency used. The overall phase variations due to the above mentioned effects, however, amount to about $0.1 \mathrm{~ns}$ (if hydrogen masers are used as frequency standards). It is difficult to give a representative number here because of the wide variability of atmospheric and instrumental conditions. It may be possible in the near future to reduce this number by a factor of two or three using satellite LO links, instrumental delay calibration, dual-frequency receiving systems and water vapor radiometry. But even in the less favourable case of $\sigma_{\tau_{\phi}}=0.1 \mathrm{~ns}$, the phase delay accuracy of any narrow band system is equivalent to a group delay precision of a wide bandwidth system with $B_{0}=100$ $\mathrm{MHz}$.

What can be done to take advantage of the phase observables for meaningful geodetic work, will be shown in the next paragraphs.

\section{GEODETIC BASELINE DETERMINATIONS USING PHASE OBSERVATIONS}

There are several ways in which phase observations are made following the purpose of the experiment.

\section{Method A: Uninterrupted Scans for Mapping the Structure of Compact Radio Sources}

In most of these experiments the telescopes are aimed at the same source for many hours, with the exception of a few short calibration scans. During each of these scans, the phase can be tracked without ambiguity problems, even at higher frequencies, as long as interference fringes are seen.

For a unique baseline solution with this kind of data, the following conditions have to be met:

1. The polar component ( $z=$ component) of the baseline has to be kept fixed.

2. At the start of each source scan, a new phase zero unknown (which is equal to a clock offset unknown) has to be introduccd. 
3. The duration of the sources scans should not be shorter than 3 to 4 hours. An optimum is reached if the hour angle range covers $180^{\circ} \cong 12$ hours).

These conditions can be deduced from the observation equations, where the baseline terms expressed in cylindrical coordinates are

$$
\begin{array}{ll}
\mathrm{db}_{1}=\frac{1}{c} \sin \delta & \text { (polar component) } \\
\mathrm{db}_{2}=\frac{1}{c} \cos \delta \cosh & \text { (equatorial component) } \\
\mathrm{d} \lambda_{\mathrm{b}}=\frac{\mathrm{b}_{2}}{\mathrm{c}} \cos \delta \sinh & \text { (longitude of baseline vector) }
\end{array}
$$

with $\delta=$ declination and $\mathrm{h}=$ hour angle of the observed source.

An example of a baseline solution with $13 \mathrm{~cm}$ data on an intercontinnetal baseline is shown in figure 1. These data are the first that could be used with a program completed only very recently. In spite of the poor quality of these data (rubidium clocks were used at both stations), a comparison with the delay solution of the same experiment demonstrates the substantial gain in accuracy:

$$
\begin{array}{ll}
\text { delay solution: } & \mathrm{db}_{1}=47.52 \pm 1.62 \mathrm{~m} \\
\sigma_{\tau}= \pm 9.5 \mathrm{~ns} & \mathrm{db}_{2}=16.00 \pm 2.86 \mathrm{~m} \\
& \mathrm{~d} \lambda_{\mathrm{b}}=4: 99 \pm 0 .: 32 \\
\text { phase delay solution: } & \mathrm{db} b_{1}=--- \\
\sigma_{\tau_{\phi}}= \pm 0.20 \mathrm{~ns} & \mathrm{db}=19.32 \pm 0.24 \mathrm{~m} \\
& \mathrm{~d} \lambda_{\mathrm{b}}=4:{ }^{\prime} 4 \pm 0 .: 03
\end{array}
$$

Another example is shown in figure 2, where phase data on the $832 \mathrm{~km}$ baseline Effelsberg-Onsala at $1.3 \mathrm{~cm}(22.2 \mathrm{GHz})$ were converted into phase delays. Due to the small ambiguity spacing at this high frequency, it proved to be difficult to connect the phases across data gaps larger than 5 minutes (coherence time of the interferometer).

The phase delay solution with scan on $3 \mathrm{C} 273$ and $3 \mathrm{C} 84$ gave the following results:

$$
\begin{array}{ll}
\sigma_{\tau_{\phi}}= \pm 0.15 \mathrm{~ns} & \mathrm{db}_{2}=0.15 \pm 0.054 \mathrm{~m} \\
& \mathrm{~d} \lambda_{\mathrm{b}}=3: 36 \pm 0,008
\end{array}
$$

The drawback of method A, however, is twofold: the baseline solution is incomplete and only a few sources can be observed during one experiment. Moreover, very severe bounds have to be placed on the systematic effects due to the instrumentation and the atmosphere because there is a high sensitivity of these solutions to any non-linear effects in the phase delay observables. 


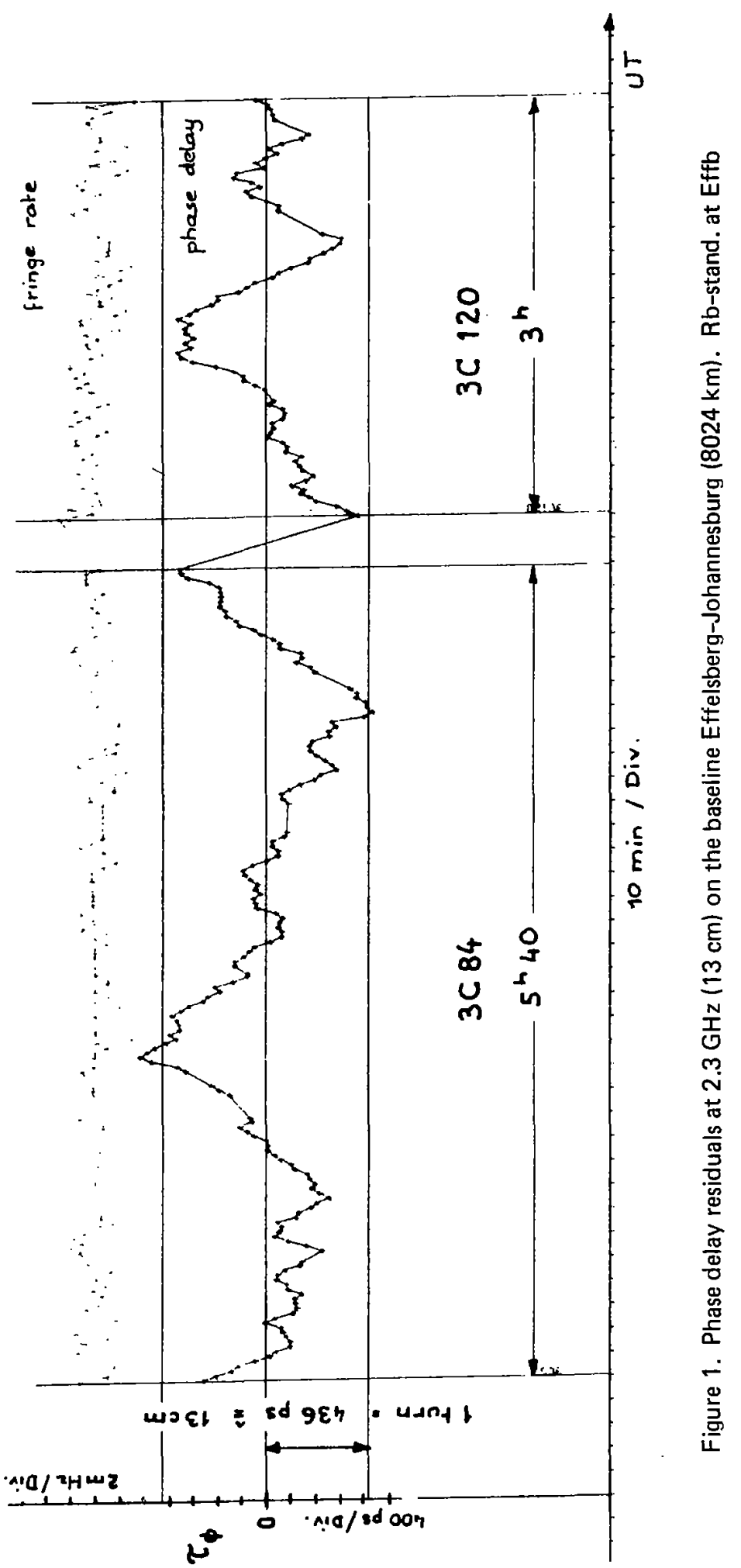




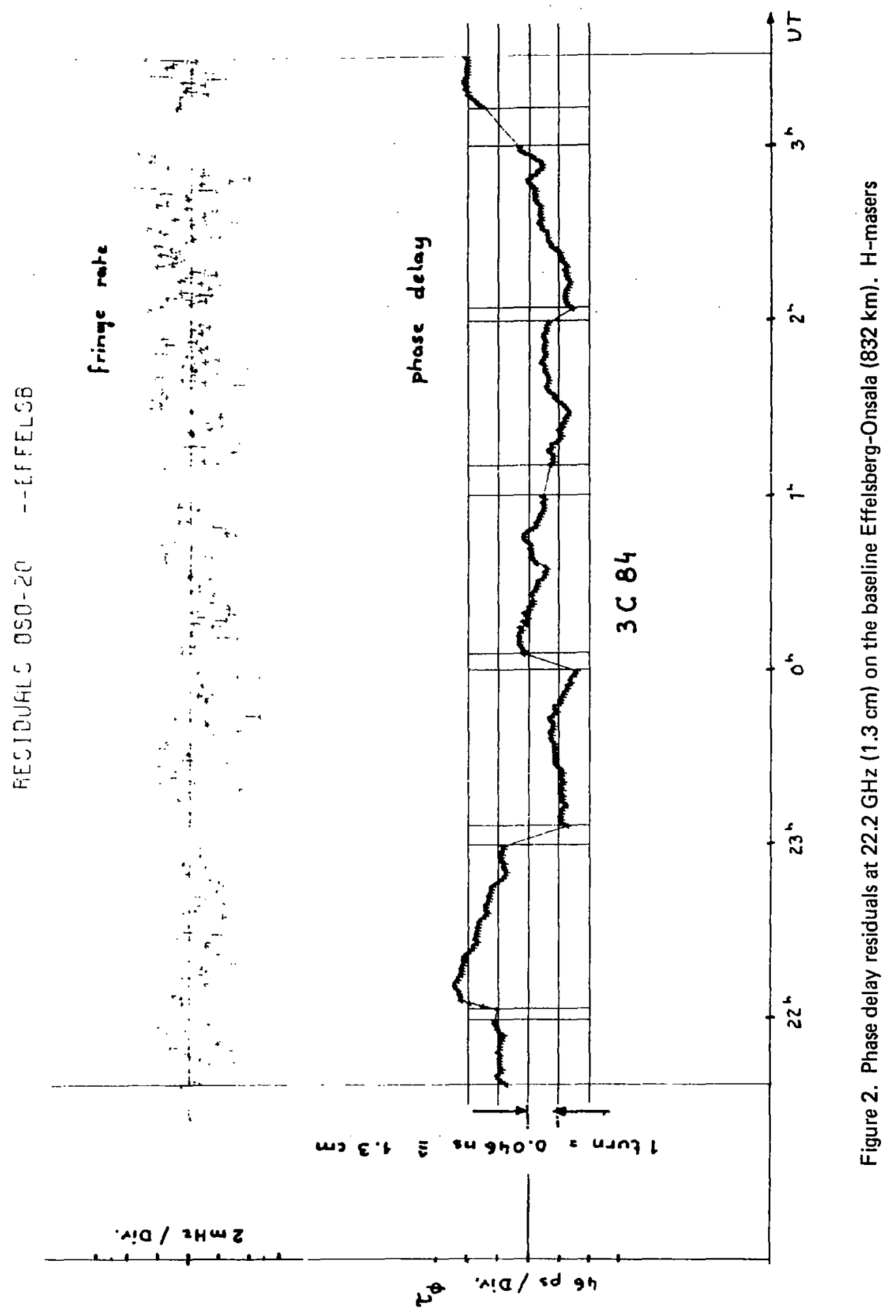


Method B: Interrupted Scans on Source Pairs (Source Switching Between Pairs) for Accurate Determination of Relative Source Positions*

In this case, conditions for a unique baseline solution are:

1. Polar baseline component is fixed.

2. A phase zero unknown for each observed source has to be introduced.

3. Same duration minimum as method $\mathbf{A}$, the optimum being again 12 hours.

4. The length of the individual scans (including telescope drive time) should not exceed a limit set by the possibility to interpolate the phase change up to the correct number of turns between two consecutive scans on the same source. This condition is satisfied if the integrated phase error is substantially smaller than $180^{\circ}$. Remaining phase ambiguities within the scans pertaining to one source can be removed in an iterative process. A portion of a plot still containing some ambiguity errors is shown in figure 3.

The number of sources that can be observed in this scheme is larger by a factor of two, which increases the stability of the solution. Some comparative computations based on a constant observational error of $\sigma_{\tau_{\phi}}=0.1 \mathrm{~ns}$ are listed in the following table:

Table 2

Baseline Effelsberg - Green Bank (6 $335 \mathrm{~km})$

\begin{tabular}{|c|c|c|c|c|}
\hline \multirow{2}{*}{$\begin{array}{l}\text { Source } \\
\text { Pair }\end{array}$} & \multirow{2}{*}{$\begin{array}{l}\text { Decl. } \\
{\left[\begin{array}{l}0 \\
0\end{array}\right]}\end{array}$} & \multirow{2}{*}{$\begin{array}{c}\text { Total Observation } \\
\text { Time }\end{array}$} & \multicolumn{2}{|c|}{$\begin{array}{c}\text { Baseline Component } \\
\text { Accuracy }\end{array}$} \\
\hline & & & $\mathrm{b}_{2}$ & $\lambda_{b}$ \\
\hline $\begin{array}{l}\text { VRO } \\
\text { 3C } 371\end{array}$ & $\begin{array}{l}42^{\circ} \\
70^{\circ}\end{array}$ & $\sim 7 \mathrm{~h}$ & $\pm 8 \mathrm{~cm}$ & $\pm 0: 0^{\prime} 015$ \\
\hline $\begin{array}{l}\text { CTA } 102 \\
\text { 3C } 371\end{array}$ & $\begin{array}{l}11^{\circ} \\
70^{\circ}\end{array}$ & $\sim 7 \mathrm{~h}$ & $\pm 10 \mathrm{~cm}$ & $\pm 0: 0.17$ \\
\hline $\begin{array}{l}\text { VRO } \\
\text { CTA } 102\end{array}$ & $\begin{array}{l}42^{\circ} \\
11^{\circ}\end{array}$ & $\sim 7 \mathrm{~h}$ & $\pm 13 \mathrm{~cm}$ & $\pm 0: 004$ \\
\hline
\end{tabular}

(duration of individual source scans: $24 \mathrm{~min}$ ) 


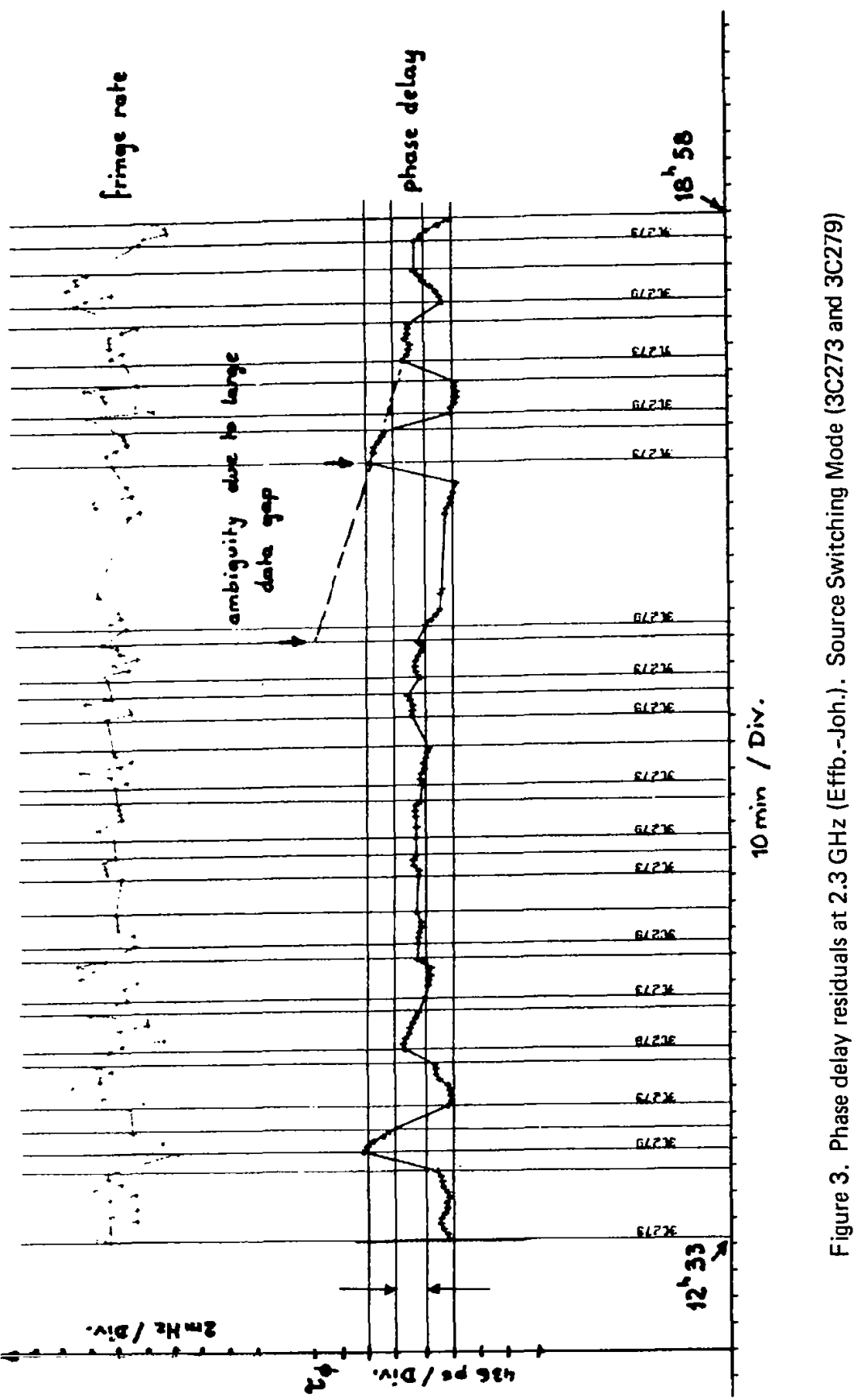




\section{Method C: Interrupted Scans on Many Different Sources}

The sampling of sources all over the commonly visible portion of the sky would, of course, provide the most stable least squares solution, a fact which can best be demonstrated by comparing the correlation matrices of methods $\mathrm{A}, \mathrm{B}$ and $\mathrm{C}$ : unless the observation period is extended over 12 hours or more, the solutions of methods A and B tend to show strong correlations ( 0.9$)$ between some of the estimated parameters. It is interesting, however, that the equatorial baseline projection $b_{2}$ in all cases correlates only very weakly with the other parameters.

The realization of method $\mathrm{C}$ becomes increasingly difficult with an increasing number of sources involved in the switching scheme. This is easily understood because the gaps between scans on the same source widen considerably, preventing correct phase interpolation. In this case, more sophisticated methods of ambiguity elimination are required.

\section{AMBIGUITY ELIMINATION}

In order to obtain a complete baseline solution including the z-component, the remaining phase ambiguities between scans to different sources must be resolved. In Rogers et al., 1978 an interesting method of phase ambiguity elimination is described, which relies on a preliminary wide band delay solution. At the relatively high observing frequency of $7.85 \mathrm{GHz}$, one phase turn corresponds to an ambiguity spacing of $3.8 \mathrm{~cm}$ or $0.13 \mathrm{~ns}$. This was roughly equivalent to the uncertainty of the adjusted group delay observations from the preliminary solution. Now these adjusted delay observations were converted to reference phases which were then subtracted from the observed phases. After suppression of clock drift effects, the phase differences were treated in a special function

$$
f(\vec{b})=\sum_{i} \cos \Delta \phi_{i} .
$$

If $\mathrm{f}(\overrightarrow{\mathrm{b}})$ is maximized by varying the baseline solution parameters, all the different $\Delta \phi_{\mathrm{i}}$ tend to crop around zero phase. The values obtained for $\vec{b}$ in this way are used to remove the ambiguities from the original phase observations, which are then converted to phase delays and processed in the final solution. The accuracy gain proved to be considerable: the postfit rms of the phase delay residuals was down to about $10 \mathrm{ps}$ compared to some $200 \mathrm{ps}^{* *}$ with the group delay solution.

From the above experiences, which were obtained with the Mark I system on a very short baseline $(1.24 \mathrm{~km})$, we may draw one important conclusion (which is independent of baseline length): it is possible to resolve phase ambiguities if a preliminary solution with a delay phase accuracy of about one turn (or even somewhat more) can be achieved. It is obvious that the requirements for the preliminary delay solution will become less stringent with decreasing frequency. However, a lower

\footnotetext{
*This sum was weighted if the observations are not equally spaced.

**The synthesized bandwidth was $\sim 100 \mathrm{MHz}$.
} 
limit of the observing frequency is set by the ionospheric path length variations, which increase with $1 / \nu_{0}{ }^{2}$. Experiments are planned by our group to test the available frequencies at the lower end of the $\mathrm{GHz}$-scale.

With reference to the limited Mark II system's performance, the following configuration appears to hold promise without showing much complexity: A basic observing frequency could be chosen at $1.66 \mathrm{GHz}(18 \mathrm{~cm})$ with an ambiguity spacing of $0.6 \mathrm{~ns}$. In order to resolve this ambiguity, a bandwidth of about $10 \mathrm{MHz}$ would have to be synthesized. This could be achieved with four channels spread over the band (see figure 4). In order to reduce SNR losses, two recording sets are used in parallel, each switching rapidly between two channels. The resulting delay ambiguity of about 300 ns can be solved with the coarse delay of each of the channels, while the fine delay itself will have a halfwidth of $\pm 100 \mathrm{~ns}$. The accuracy of the preliminary delay solution of this configuration should be about 0.8 ns with good SNR, which is in agreement with the above mentioned requirements.

The phase delay accuracy of the proposed system will be limited by the same effects as considered in paragraph 1 , this amounts to a few $\mathrm{cm}$ or about $0.1 \mathrm{~ns}$ on intercontinental baselines.

The proposed Mark II phase delay system could be equivalent to an economy version of the Mark III system with the advantage of being at a lower cost level. On the other hand, if used in conjunction with the highly flexible Mark III system, the proposed scheme should result in a substantial reduction of tape consumption because only two channels would have to be recorded simultaneously (factor of 14).

The $38 \mathrm{MHz}$ Mark II system (bandwid th synthesis), as it is used at present by the JPL-Caltech group, should be particularly suitable for phase delay analysis with ambiguity elimination.

\section{CONCLUSIONS}

There is no doubt that with wide band group delay techniques the optimal geodetic baseline results can be achieved, but it is also clear that these techniques, due to their high costs and complexity, will not be in reach of many observatories.

The test computations with real and simulated data presented here are meant to demonstrate that meaningful geodetic work can be done with the existing Mark II technology if the phase observable is used. In spite of the incomplete baseline solution (i.e., the z-component cannot be determined), it is possible, for example, to measure Earth tides and continental drift on east-west baselines if the changes in the equatorial baseline component are considered. Furthermore, the longitude of the baseline vector can be used for the determination of UT1 variations.

An additional advantage of the proposed phase observation scheme resides in the fact that source structure analysis is possible using the closure phase method on the same data. Thus, the structure is monitored simultaneously and yet independently of the baseline solution. However, due to the 


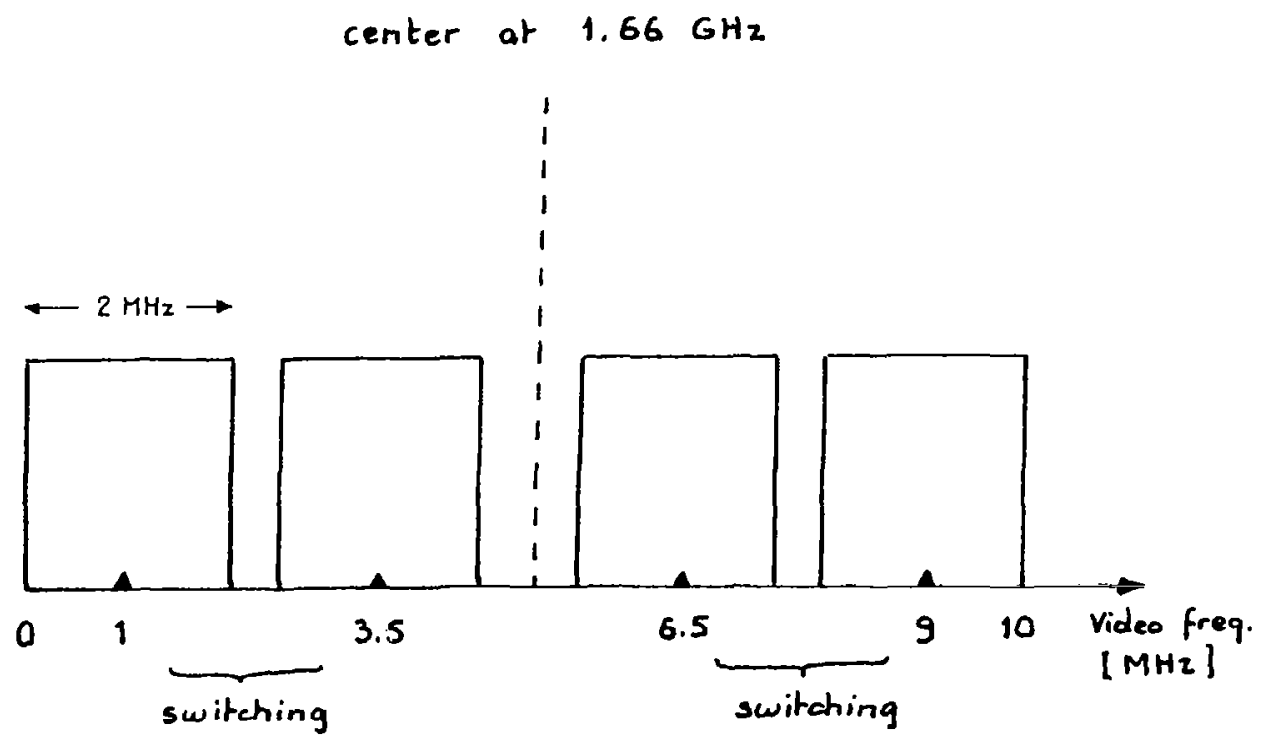

Mk II ree. A Mk II rec. B

a) $10 \mathrm{MHz}$ synthesis

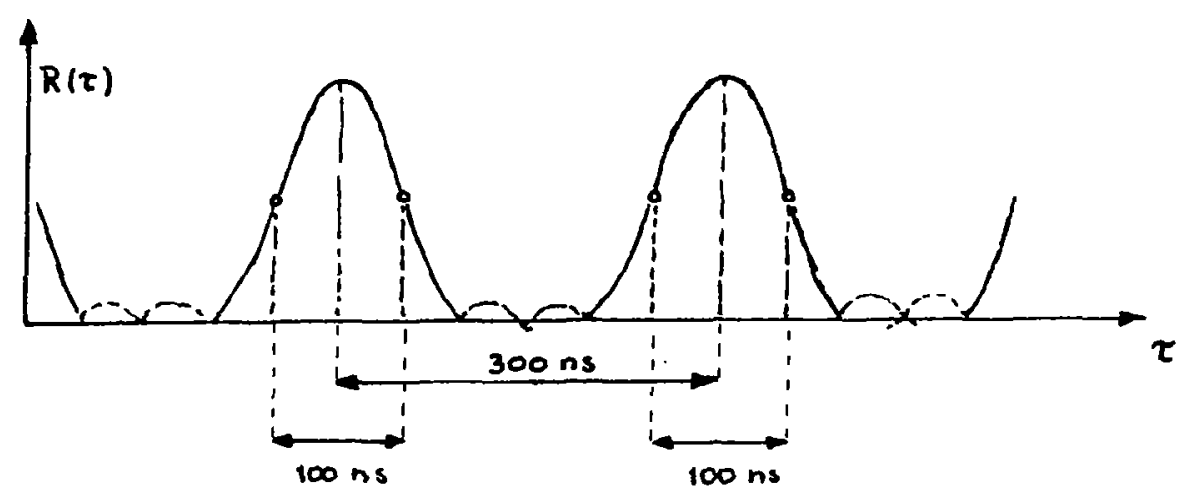

b) fine delay function

Figure 4. System characteristics for ambiguity elimination at $18 \mathrm{~cm}$. 
sensitivity of the pure phase solutions to instrumental and atmospheric effects, the full benefit of the high resolution of the phase observable can only be obtained if these effects are monitored with great care.

As an alternative, a system based on Mark II technology is outlined, which is capable of (a posteriori) resolving the phase ambiguities in order to achieve a complete baseline solution. With this system, it is possible to optimize observing strategies to obtain well conditioned baseline solutions that are less sensitive to long term instrumental and atmospheric effects.

\section{REFERENCES}

Moran, J. M.: Very Long Baseline Interferometric Observations and Data Reduction. Methods of Experimental Physics, Vol. 12, Part C, Radio Observations, Academic Press, New York, 1976.

Rogers, A. E. E. et al.: Geodesy by Radio Interferometry: Determination of a 1.24-km Baseline Vector with $\sim 5 \mathrm{~mm}$ Repeatability. J. Geophys. Res., Vol. 83, p. 325, 1978.

Shapiro, I. I., Wittels, J. J., et al.: Submilliarcsecond Astrometry via VLBI: I Relative Position of the Radio Sources 3C345 and NRAO 512. Preprint submitted to The Astronomical Journal April 1979.

Thomas, J. B.: An Analysis of Long Baseline Radio Interferometry. The Deep Space Network Progress Report, Technical Report 32-1526, Vol. VII, VIII, XVI, Jet Propulsion Laboratory, Pasadena, Calif., 1972.

Whitney, A. R. et al.: A Very-Long-Baseline Interferometer System for Geodetic Applications. Radio Science, Vol. 11, p. 421-432, 1976. 


\title{
PHASE DELAY ASTROMETRY AND ITS GEODETIC APPLICATIONS
}

\author{
J. J. Wittels \\ Massachusetts Institute of Technology
}

The goal of our experiments was to exploit the accuracy inherent in the fringe phase in the determination of source positions.* That accuracy can be utilized by applying differencing techniques in order to circumvent such systematic effects as clock drift and delay due to the propagation media. The best cancellation of systematic effects could be attained by simultaneous observation of two or more sources with the same interferometer. However, having only one antenna at each end of the baseline, we chose to observe alternately two closely spaced sources, allowing a short cycle time and hence good cancellation of systematic effects by the differencing of the measured fringe phases.

The nonsimultaneous nature of our measurements created two difficulties. First, for the best accuracy, the arbitrary number of phase rotations between sequential observations of the same source had to be removed; or at a minimum, no rotations were to remain between sequential measurements of the phase difference between the two sources. Second, we had to assess the effects of systematic errors from the less than ideal cancellation of nonsimultaneous observations of the two sources.

We conducted four experiments between October 1971 and May 1974, each time, cycling between observations of the quasars 3C 345 and NRAO 512, which are only one-half degree apart. Typically, the integration time on each source was about 100 seconds, the cycle time about 4 or 5 minutes, and the full span of the observations 3 to 5 hours. All four experiments were conducted at $3.8 \mathrm{~cm}$ using the Haystack "MA" 37-meter antenna and the Goldstone CA 64-meter NASA antenna; the May 1974 experiment also used the NRAO "WV" 43-meter antenna. All data were recorded and correlated with the Mark I system.

The removal of the $2 \pi n$ phase ambiguities - a process termed phase connection - was acheived for each source separately by manipulating a residual fringe phase, $\varphi^{\mathrm{r}}$, calculated for each observation of a source from the observed total phase, $\varphi^{t}$, by the formula:

$$
\varphi^{\mathrm{r}}=\varphi^{\mathrm{t}}-\varphi^{\mathrm{g}}-\int_{\mathrm{t}_{\mathrm{o}}}^{\mathrm{t}} \dot{\varphi}^{\mathrm{r} \text { poly }}(\mathrm{t}) \mathrm{dt}
$$

where $\varphi^{g}$ is the phase from a simple geometric model, and $\dot{\varphi}^{\text {poly }}$ is the residual fringe rate calculated from a four or five term polynomial fit to the entire ensemble of residual fringe rates of that source during that experiment. The residual fringe rates are those found by subtracting, for each observation of the source, the fringe rate calculated from the same simple geometric model from the total measured fringe rate.

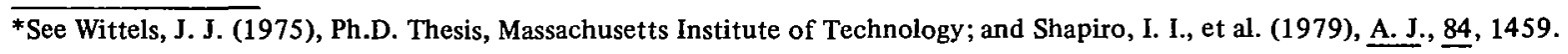


The ambiguities were then removed by a simple computer algorithm that considered pairs of adjacent observations, and, finally, were checked visually. The definitive test of proper phase connection emerges only after parameterized modeling of the differenced phases (This will be discussed later.). Figure 1 shows schematically the desired results of proper phase connection. Clearly, the noisier the individual measurements of phase, the less reliable the phase connection.

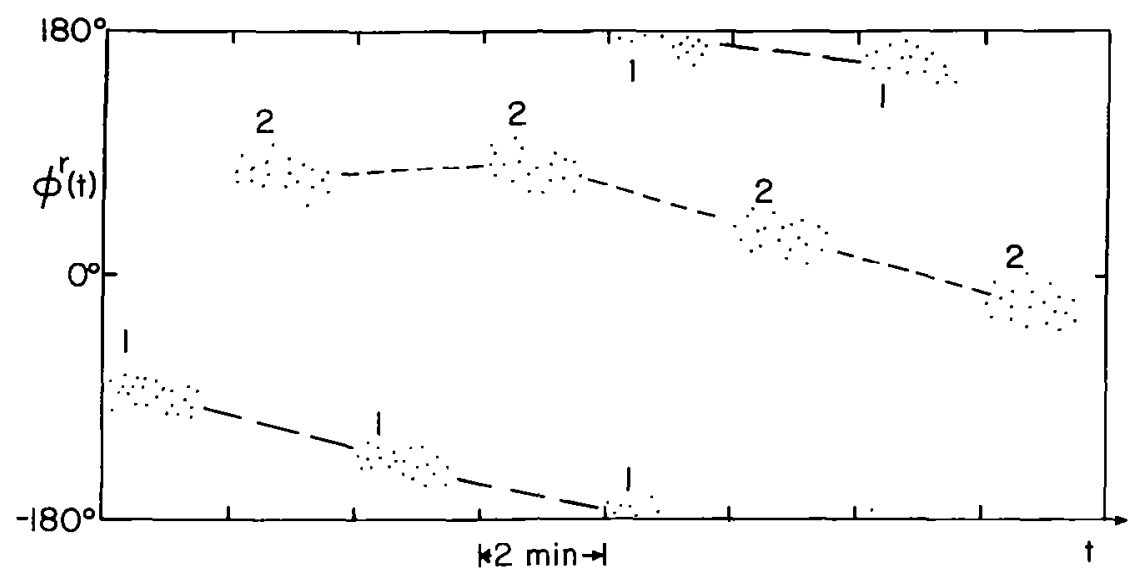

Figure 1.

After removing the ambiguities, the total phase for each observation of each source is reconstructed from the residual phases and the model phases. However, before the phases from the two sources are differenced, one other noncancelling contribution to the total phase should be removed: that due to the structure of the observed source. For noncylindrically symmetric sources, any structure resolvable by the interferometer contributes to the total measured fringe phase. Hence, the best accuracy can only be achieved by observing the source structure and removing its contribution.

For our experiments, NRAO 512 could be considered unresolved. However, 3C 345, as we are now all aware, had extended and time varying structure, which was only inadequately sampled, especially during the first three single-interferometer experiments. Using a simple two-component model for the structure of $3 \mathrm{C} 345$, we corrected the measured phases by removing the structure phase with respect to the model's center of brightness. Hence, the separation determined by subsequent analyses is that between NRAO 512 and the center of brightness of the two components in the model of $3 \mathrm{C}$ 345. The source 3C 345 was sufficiently resolved that the structure phase calculated from the model reached a value of about a third of a phase rotation.

It must be emphasized here that the realization of centimeter accuracy in baseline determinations or of tenth milliarcsecond or better accuracy in relative source positions using fringe phase observations is crucially dependent on knowledge of the structure of the sources. Also, since a very large fraction of observed compact radio sources are time-dependent, the structure must necessarily be observed at the same epoch as the geodetic and astrometric results. 
Having removed the ambiguities and source structure, for each experiment, we differenced each measured fringe phase of one source with the following measured fringe phase of the other source. The ensemble of differenced phases for each experiment was then subjected to a least squares analysis using a comprehensive parameterized model.* The only parameters varied were the right ascension and declination separations of the sources and a constant delay offset, referred to as a constant clock; the parameters for the antenna locations, atmospheric models, earth tides, UT 1 , etc. were kept fixed. Examination of the post-fit residuals was the final determining test of proper phase connection: When all ambiguities had been properly removed, the post-fit residuals would scatter approximately randomly between about \pm 20 psec, where one full rotation comprised 127 psec. If a phase rotation was inserted between observations, such as in a gap in the October 1971 experiment, the post-fit residuals became highly systematic and spanned most of a full rotation, clearly pointing to an error in phase connection.

In testing the sensitivity of the results from the least squares analyses to changes in the values of the fixed parameters and other effects, we found that the greatest change in the solution values resulted from reversing the order of the two sources in the differencing of the phases. Clearly, this sensitivity resulted from the imperfect cancellation of clock drifts and/or propagation media effects due primarily to nonsimultaneous observations. The problem could be best treated by using both the sum and difference of the phases in the solution. However, due to a computer program limitation, we combined instead the differenced variable with the measurcd phases from only the stronger source 3 C 345 . The set of 3C 345 phases alone primarily determined a four or five term polynomial "clock", while the differenced phases determined the right ascension and declination separations of the two sources. The constant clock offset was now fixed at the integral number of phase rotations closest to the value determined for this clock by the earlier solution, in which it had been varied.

The values determined for the right ascension and declination separation, $\Delta \alpha$ and $\Delta \delta$, respectively, from each of the four experiments combine to give a weighted mean of:

$$
\begin{aligned}
& \Delta \alpha_{\mathrm{M}}=2^{\mathrm{m}} 29^{\mathrm{s}} .436680 \pm 0.000008 \\
& \Delta \delta_{\mathrm{M}}=1^{\prime} \quad 40^{\prime} \cdot 72630 \pm 0,00020
\end{aligned}
$$

for the difference in the position of NRAO 512 from that of 3C 345. The distribution of the four values about this mean is shown in table 1 , along with the root-mean-square of the post-fit residuals for both the differenced and the undifferenced observations. Also shown are the comparable values obtained from the group delay data for the two experiments in which wide band synthesis** was used.

\footnotetext{
*See Robertson, D. S. (1975) Ph.D. Thesis, Massachusetts Institute of Technology.

**Whitney, A. R., et al. (1976), Radio Science, $11,421$.
} 
Table 1

\begin{tabular}{|c|c|c|c|c|c|c|}
\hline \multirow{2}{*}{$\begin{array}{c}\text { Experiment } \\
\text { Date }\end{array}$} & \multicolumn{2}{|c|}{$\begin{array}{c}\Delta \alpha-\Delta \alpha_{\mathrm{M}} \\
\left(\mathrm{x} 10^{6} \mathrm{sec}\right)\end{array}$} & \multicolumn{2}{c|}{$\begin{array}{c}\Delta \delta-\Delta \delta_{\mathrm{M}} \\
\left(\mathrm{x} 10^{5} \text { arcsec) }\right.\end{array}$} & \multicolumn{2}{c|}{ Post-fit RMS } \\
\cline { 2 - 6 } & Group & Phase & Group & Phase & $\begin{array}{c}\text { Difference } \\
\text { (psec) }\end{array}$ & $\begin{array}{c}\text { Single } \\
\text { (psec) }\end{array}$ \\
\hline \multirow{2}{*}{$10 / 71$} & & $5 \pm 4$ & & $5 \pm 11$ & 12 & 15 \\
$05 / 72$ & $-40 \pm 80$ & $4 \pm 8$ & $50 \pm 40$ & $-7 \pm 9$ & 19 & 36 \\
$07 / 72$ & & $28 \pm 4$ & & $-14 \pm 19$ & 13 & 18 \\
$05 / 74$ & $150 \pm 430$ & $-45 \pm 5$ & $180 \pm 260$ & $12 \pm 15$ & 14 & 18 \\
\hline
\end{tabular}

The values in table 1 show that the distribution in $\Delta \delta$ is consistent with the formal errors for the individual experiments and for the mean. However, the formal errors for $\Delta \alpha$ are too small compared with the actual scatter. The likely explanation for this discrepancy underlines the importance of simultaneous measurements of source structure: 3C 345 is extended primarily in the right ascension direction. The structure of this source is only poorly determined for the epochs of the first three experiments yet structural scale changes during the $2 \frac{1}{2}$ years spanned by the experiments are of the same order as the scatter in $\Delta \alpha$. Taking the conservative view of errors, we estimate our accuracy as $0^{\prime \prime} .0003$ in both coordinates. A conservative limit on the relative proper motion of the two sources is 0 "'00025 \pm 0 ". $00020 /$ year along a position angle of $220^{\circ} \pm 60^{\circ}$.

In conclusion, the accuracy of the differenced phase technique could be maximized by the following:

1. Full sky coverage for each experiment (11 hours in the case of these two sources with these interferometers) would significantly decouple the determination of $\Delta \alpha$ and $\Delta \delta$.

2. Simultaneous source mapping would keep the measured separations consistently defined. Of course, the reverse application to understanding of the motions within the variable radio sources is also enticing.

3. Good models and values for earth tides, UT1, polar motion, the atmosphere, etc.

4. Simultaneous observations and, or at least, more stability in clocks and receivers, short cycle times, and cooperative propagation media. Fortunately, indications are that we are presently more limited by the equipment than the uncontrollable whims of the propagation media. 


\title{
SATELLITE EMISSION RADIO INTERFEROMETRIC EARTH SURVEYING (SERIES)
}

\author{
P. F. MacDoran \\ Jet Propulsion Laboratory \\ California Institute of Technology
}

\begin{abstract}
The satellites of the Global Positioning System (GPS) offer an important new geodetic resource making possible a highly accurate portable radio geodetic system. A concept called Satellite Emission Interferometric Earth Surveying (SERIES) makes use of GPS radio transmissions without any satellite modifications. By employing the techniques of very long baseline interferometry (VLBI) and its calibration methods, 0.5 to $3 \mathrm{~cm}$ three-dimensional baseline accuracy can be achieved over distances of 2 to $200 \mathrm{~km}$ respectively, with only a few hours of on-site data acquisition. The use of quasar referenced ARIES Mobile VLBI to establish a sparse fundamental control grid will provide a basis for making SERIES GPS measurements traceable to the time-invariant quasar directions. Using four SERIES stations deployed at previously established ARIES sites, allows the GPS satellites apparent positions to be determined. These apparent positions then serve as calibrations for roving SERIES stations at unknown locations to determine their positions in a manner traceable to the quasars. Because this proposed radio interferometric configuration accomplishes its signal detection by crosscorrelation, there is no dependence upon knowledge of the GPS transmitted waveform which might be encrypted. Since GPS radio signal strengths are $10^{5}$ stronger than quasar signals, a great reduction in telecommunications sophistication is possible which will result in an order-of-magnitude-less cost for a SERIES GPS station compared to a quasar based mobile VLBI system. The virtual all-weather capability of SERIES offers cost-effective geodetic monitoring with applications to crustal dynamics and earthquake research.
\end{abstract}

This paper presents the results of one phase of research carried out at the Jet Propulsion Laboratory, California Institute of Technology, under Contract No. NAS 7-100, sponsored by the National Aeronautics and Space Administration. 


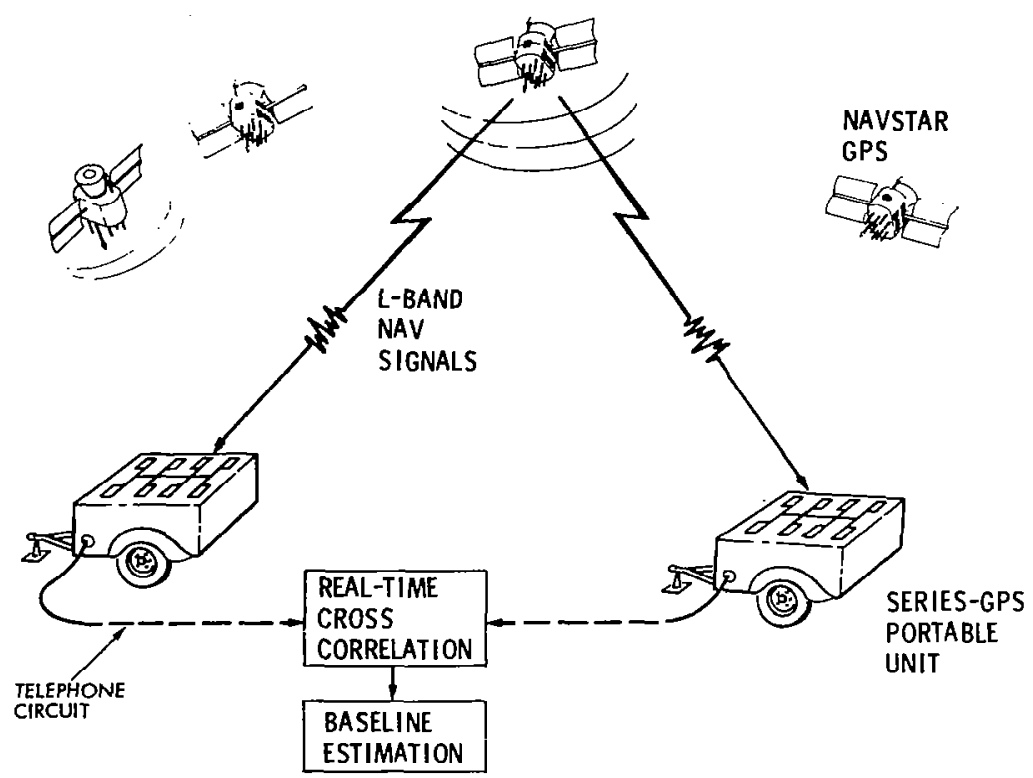

Figure 1. SERIES-GPS.

GOALS: TO EXPIOIT EXISTING SATELIITE RADIO EMISSIONS, ESPECIALLY THOSE OF THE GLOBAL POSITIONING SYSTEM (GPS), AS A RESOURCE FOR COST-EFFECTIVE HIGH ACCURACY GEODETIC MEASUREMENTS WITH EMPHASIS ON GEODYNAMICS APPLICATIONS.

OBJECTIVES:TO DEVELOP AND DEMONSTRATE A SYSTEM CAPABLE OF SUB-DECIMETER THREE DIMENSIONAL ACCURACY ON BASELINES OF SEVERAL HUNDRED KILOMETERS WITH CHARACTERISTICS OF:

- LOW COST PER POINT MEASURED (<\$2K)

- LOW COST field EQUIPMENT $(\leq \$ 100 \mathrm{~K})$

- SHORT TIME ON-SITE (<2 HOURS)

- real-time operations OPTION

- all-weather operations

- unatTENDED station OpERATIONS OPTION

- USE OF gPS EMISSIONS WITHOUT KNOWLEDGE OF CODED WAVEFORMS

- gPS AND GEODETIC POSITIONS TRACEABLE TO TIME-INVARIANT QUASAR DIRECTIONS

Figure 2. Satellite Emission Radio Interferometric Earth Surveying (SERIES). 


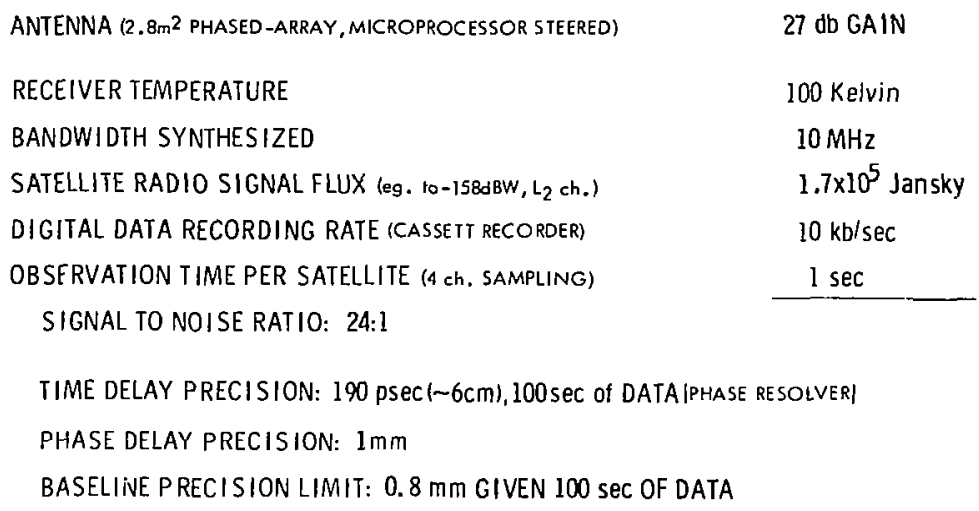

Figure 3. SERIES-GPS system configuration.

- pHASE dELAY PRECISION PER SATELLITE OBSERVATION

- TIME AND FREQUENCY $\left(\Delta \mathrm{f} / \mathrm{f}=2 \times 10^{-12}, \tau=4 \mathrm{sec}\right.$. $)$

- TROPOSPHERE

- DRY (SURFACE METEOROLOGY)

$1.0 \mathrm{~cm}$

- WET (LOW-COST WATER VAPOR RADIOMETFR)

- IONOSPHERE (RESIDUAL FROM DUAL L-BAND CALIBRATION)

- SATELLITE Positional unCERTAINTIES

(ARIES NET OF $2 \mathrm{~cm}$ ACCURACY AT $200 \mathrm{KM}$ SPACING. GPS SATELLITE RANGE TO $1 \mathrm{KM}$ ACCURACY)

$1.0 \mathrm{~cm}$

$1.0 \mathrm{~cm}$
B A SELINE EQUIVALENT

$0.3 \mathrm{~cm}$

$0.3 \mathrm{~cm}$

$$
\begin{aligned}
& \text { ROOT SUM SQUARE } \\
& \text { PER 4 SECONDS: } \\
& 1 \mathrm{~min} \text {. AVERAGE: }
\end{aligned}
$$

Figure 4. Randon error sources for SERIES-GPS system ( $\leqslant 100 \mathrm{~km}$ baselines). 
- SATELLITE POSITIONAL UNCERTAINTIES:

(ARIES NET OF $2 \mathrm{~cm}$ ACCURACY AT $200 \mathrm{KM}$ SPACING; GPS SATELLITE RANGE TO 1 KM ACCURACY)

- $\operatorname{TIME}$ AND FREQUENCY $\left(\triangle \mathrm{l} / \mathrm{f}=6 \times 10^{-11}, \tau=16 \mathrm{sec}\right)$

(INCOHERENT AVERAGING OF $60,16 \mathrm{seC}$ DATA BLOCKS)

- TROPOSPHERE

- DRY (SURFACE METEOROLOGY)

- WET (LOW-COST WATER VAPOR RADIOMETRY)

$1.5 \mathrm{~cm}$

- IONOS PHERE (RESIDUAL FROM DUAL L-BAND CALIB)

$1.0 \mathrm{~cm}$

ROOT SUM SQIJARF: $2.3 \mathrm{~cm}$

COMBINED RANDOM AND SYSTEMATIC ERRORS:

$2.4 \mathrm{~cm}$

Figure 5. Systematic error sources for SERIES-GPS system ( $\leqslant 100 \mathrm{~km}$ baselines).

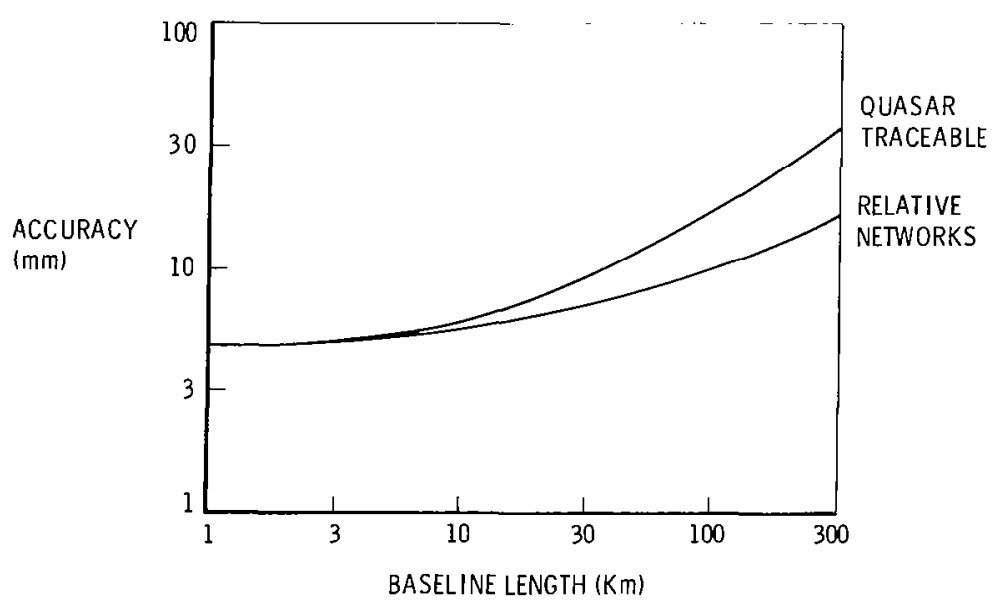

Figure 6. SERIES-GPS accuracy estimates. 


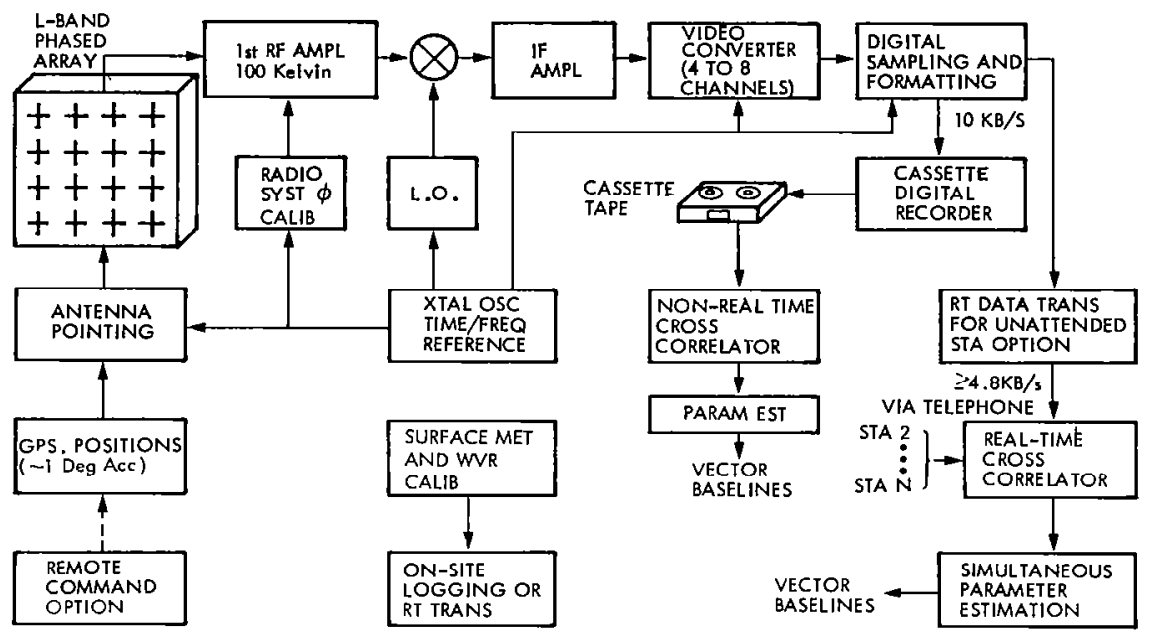

Figure 7. SERIES field station conceptual design.

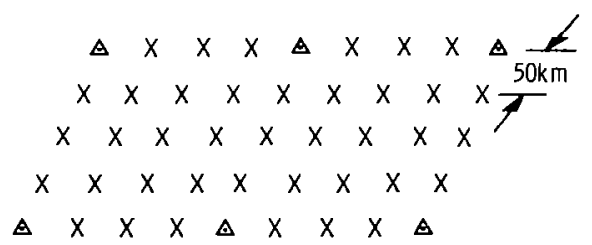

$\triangle \quad$ ARIES DERIVED FUNDAMENTAL CONTROL GRID

$X$ UNKNOWN SITES TO BE MEASURED

OBSERVING STRATEGY

1. FOUR SERIES STATIONS OCCUPY ARIES (A) SITES AND PROVIDE GPS SATELLITE POSITION CALIBRATIONS

2. FOUR ROVING SERIES STATIONS OCCUPY UNKNOWN $(X)$ SIIES

Figure 8. SERIES network densification with quasar traceability. 


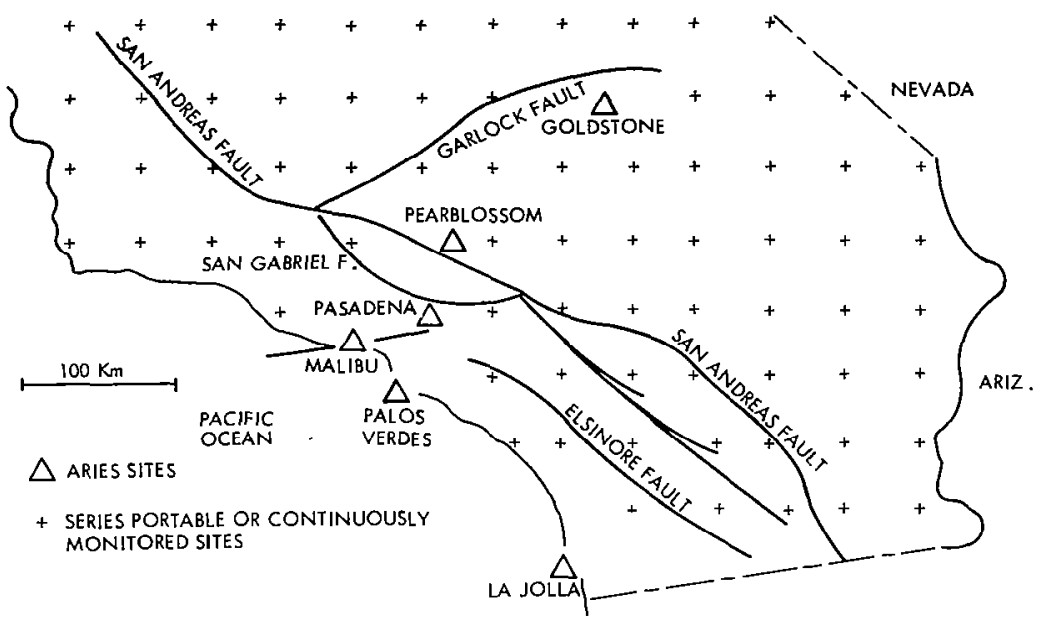

Figure 9. SERIES-GPS monitoring strategy for southern California.

- GeOdetIC NETWORK DENSIFICATION (20 to $100 \mathrm{~km}$ SPACINGS)

- RAPID MULTI-STATION DEPLOYMENT INTO ZONES OF EARTHQUAKE PRECURSORS

- UNATTENDED STATION GEODETIC MONITORING

- EARTHQUAKE PORECAST REgIONS

- kNOWN hazardoUs areas (i.e., VOLCANOES, NUCLEAR WASTE DISPOSAL SITES)

- gPS satellite angular positions Relative to quasar tRaceable aries GEODETIC POSITIONS

- MEASUREMENT PRECISION AND TEMPORAL RESOLUTION IN A REgIME BETWEEN CONVENTIONAL GEODESY AND SEISMOLOGY

- OCEANOGRAPHY - OPEN OCEAN TIDES, GEODETIC CONTROL AND TRENCH MOVEMENT via BOTTOM SONAR TRANSPONDERS

Figure 10. Applications SERIES-GPS.

- RF CYCLE AMBIGUITY RESOLUTION

- dual l-band ionospheric calibration and high precision delay MEASUREMENTS

- saturation efFects in data system due to strong gPs signals

- ASSUMPTION OF ARIES CONTROL FOR SERIES OPERATIONS - hOW OFTEN MUST ARIES REMEASUREMENTS BE DONE TO HOLD $2 \mathrm{~cm}$ ACCURACY AT $200 \mathrm{~km}$ SPACING?

Figure 11. Challenges SERIES-GPS. 


\title{
BACKPACK VLBI TERMINAL WITH SUBCENTIMETER CAPABILITY
}

\author{
C. C. Counselman III and I. I. Shapiro \\ Massachusetts Institute of Technology \\ R. L. Greenspan and D. B. Cox, Jr. \\ Charles Stark Draper Laboratory Inc.
}

The measurement of short vector baselines with subcentimeter repeatability and accuracy using radio interferometric observations of quasars has already been demonstrated (reference 1). This paper describes our plans to achieve comparable performance using inexpensive, backpack portable equipment that processes less than one second of data per baseline redetermination. Figure 1 summarizes some of these objectives.

Our approach exploits the full measurement accuracy inherent in the precise radio signals that will be broadcast by each satellite in the NAVSTAR Global Positioning System (GPS) (reference 2). Figure 2 illustrates the measurement concept. The user equipment at each end of the unknown baseline receives signals from the same set of four or more GPS satellites. The equipment consists of a simple antenna, a GPS receiver, a microprocessor unit and a recording unit (reference 3 ). "Real-time" baseline determination can be accomplished by linking the microprocessor units with a communication channel that can transmit at the rate of about 1 kilobit per baseline redetermination.

Each GPS receiver measures its range to each satellite by means of the wide-band pseudo-noise P-Code modulation that is impressed on the GPS radio frequency carriers which are at $1.226 \mathrm{GHz}$ and $1.57542 \mathrm{GHz}$ (reference 4). These measurements will typically have a precision of about 0.5 meters, which is approximately 2.5 wavelengths at a frequency of $1.57542 \mathrm{GHz}$. The primary use of these relatively coarse measurements in the proposed program is to assist in resolving ambiguities of the more precise carrier-phase measurements that will also be made (see below).

- To develop backpack portable equipment to measure vector BASELINES FROM - 1 KM TO - 100 KM IN LENGTH WITH SUBCENTIMETER TO FEW CENTIMETER ACCURACY.

- To develop equipment that is

- simple in concept and implementation

- reliàble in unattended operation

- inexpensive - less than $\$ \mathbf{\$ 1 5 , 0 0 0}$ per unit

Figure 1. Objectives. 


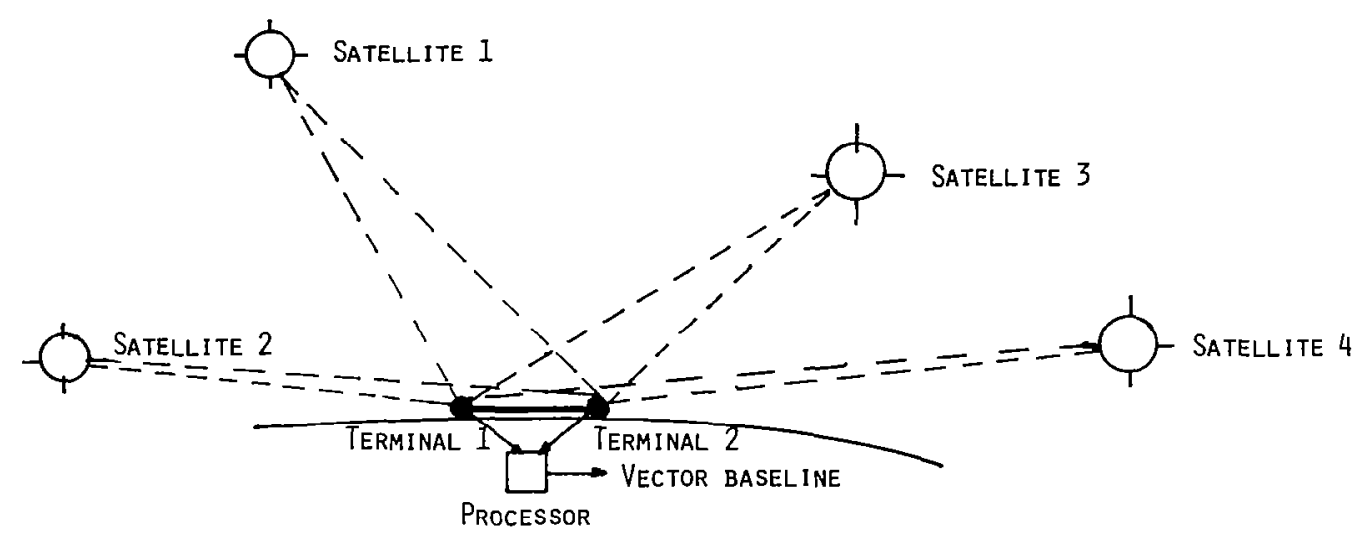

INSTRUMENTATION

- Wideband omnidirectional antenNa to Receive $1.6 \mathrm{GHz}$ and $1.2 \mathrm{GHz}$ GPS transmissions

- Simultaneous reception from all satellites

- Microprocessor-controllej system for data collection and formatting

- Data storage and/or low-volume data link to microprocessor-based data analyzer

Figure 2. GPS measurement concept.

The same knowledge of the pseudo-noise modulation on the transmitted GPS signal that enables the receiver to make coarse range measurements also enables that modulation to be stripped from the received signal, lcaving a sinusoidal component that is modulated only by a low speed (50 bit/second) biphase encoded data signal. This data modulated carrier is tracked in current GPS receivers by a "Costas" phase detector, which uses a phase doubling nonlinearity to remove the effect of the biphase modulation (reference 5). The output of the loop is an unmodulated tone. The phase tracking error will be approximately $8^{\circ}$ in typical noise environments; this value corresponds to approximately $4 \mathrm{~mm}$ at the GPS carrier frequencies (reference 6). The measurement is ambiguous by multiples of half the wavelength (approxinately $10 \mathrm{~cm}$ ) because the Costas loop output does not distinguish between input phases that differ by $180^{\circ}$. However, by using knowledge of the data signal format, one can regain the "lost" factor of two and obtain whole-wavelength $(20 \mathrm{~cm})$ spacing of the ambiguities.

The carrier phases obtained from the signals from four or more satellites (see figure 3 ) at each end of a baseline yield an extremely precise, albeit ambiguous, determination of the vector baseline (reference 3). One primary objective of the current plan is to assess the adequacy and flexibility of several alternatives for resolution of the ambiguities with simple equipment. Two leading approaches are:

1. Collect data from satellite observations over several hours during which the satellite geometry changes significantly. If the number of potential ambiguities from observations of each satellite is small, then the set of ambiguity values, one value for each satellite, that corresponds to the weighted least squares fit to the data will usually be the correct one. 


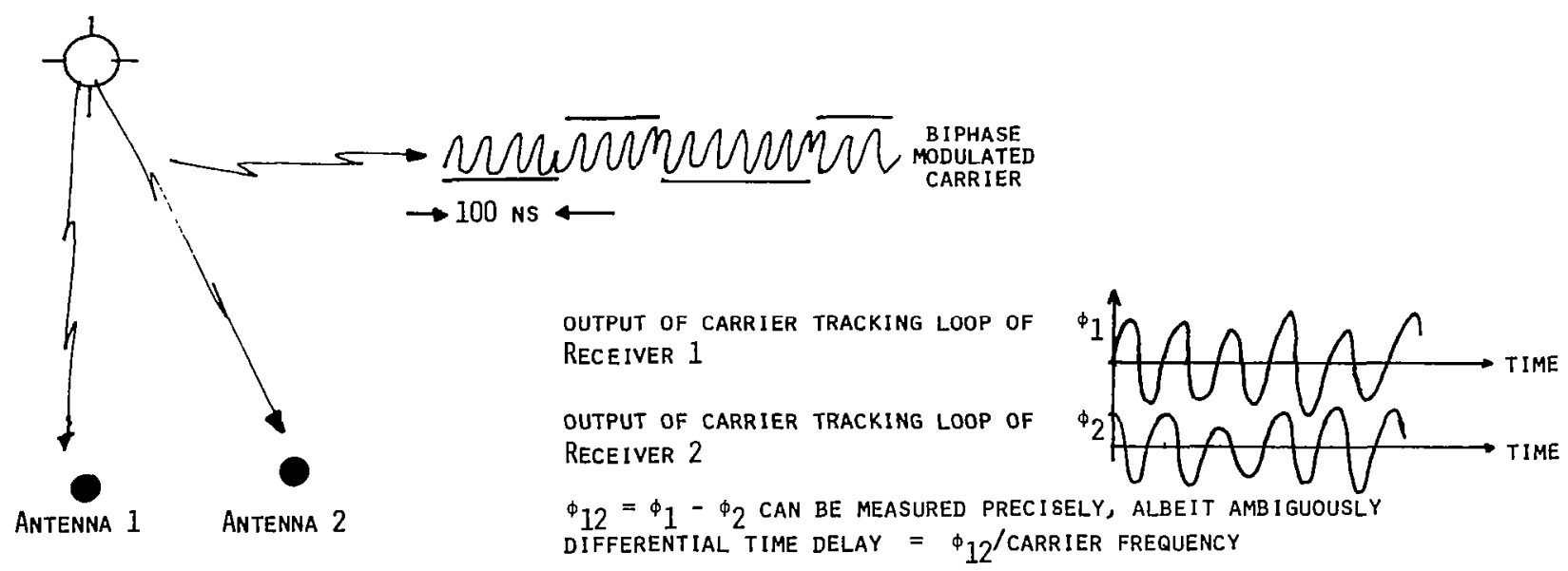

Figure 3. GPS signal processing concept ("reconstructed carrier").

This technique is described in reference 1 . We note that all data used to resolve ambiguities are usable also for accurate determination of the baseline vector, after the ambiguities are resolved.

2. Measure the phase difference between the two GPS carriers received at each end of the baseline from each satellite to within a few degrees (note that both GPS carriers are synthesized coherently from the same oscillator). The corresponding phase-delay ambiguities will be light-time equivalents of integral multiples of the $0.43 \mathrm{~m}$ half-wavelength for the difference frequency ( $349 \mathrm{MHz}$ ). This approach is similar to the use of multiple tones in OMEGA to resolve "lane" ambiguities (reference 7). Used in conjunction with the coarse range measurement, the phase from both GPS carriers will allow a reduction in the initial ambiguities of the phase delay estimates. As the baseline length increases, the effectiveness of this technique will decrease because of ionospheric effects.

As the first phase in the development of the intended system, we plan to conduct an experimental program using commercially supplied GPS receivers. One or more standard baselines defined by receiving antennas will be established and surveyed. Each leg will be less than 100 meters in length. GPS signals received by the antennas will be processed to make a series of baseline determinations under a wide range of environmental conditions. The objectives of this experiment are:

1. to ascertain whether the accuracy of the baseline determination meets our goal (see figure 4);

2. to assess different techniques for ambiguity resolution; and

3. to determine whether the present GPS signal structure will allow the development of inexpensive and effective ground equipment. 
1) Exploitation of KNown "pseudorandom" signal structure (RECONSTRUCTED CARRIER APPROACH) YIELDS MORE THAN $60 \mathrm{DB}$ GAIN,

2) SATEllite transmitted POHER and signal processing gain YIELD A MEASUREMENT SNR EXCEEDING 35 DB PER HZ, EVEN USING A MODEST RECESVER, $T_{N}=430^{\circ} \mathrm{K}$ (NOISE FIGURE $=4.0 \mathrm{DB}$ ),

3) GPS BROADCASTS ACCURATE EPHEMERIS DATA.

4) GPS SUPPORTS CLOCK SYNCHRONIZATION TO 10'S OF NS SO THAT INHERENT EPHEMERIS ACCURACY CAN BE EASILY EXPLOITED.

Figure 4. Why size, weight and complexity objectives are feasible.

\section{REFERENCES}

Rogers, A. E. E. et al., Geodesy by Radio Interferometry: Determination of a $1.24 \mathrm{~km}$ Base Line Vector with $\sim 5-\mathrm{mm}$ Repeatability, Journal of Geophysical Research, Vol. 83, No. 1, 10 January 1978, pp. 325-334.

Milliken, R. J., and C. J. Zollar, Principles of Operation of NAVSTAR and System Characteristics, Navigation, Vol. 25, No. 2, Summer 1978, pp. 95-106.

Counselman, C. C. III, and I. I. Shapiro, Miniature Interferometer Terminals for Earth Surveying, Bulletin Géodésique, Vol. 53, No. 2, August 1979, pp. 139-163.

Borel, M. J. et al., Phase 1 GPS User Equipment, Navigation, Vol. 25, No. 2, Summer 1978, pp. 179-194.

Viterbi, A. J., Principles of Coherent Communication, Section 10.4, pp. 286-293, McGraw-Hill, New York, 1966.

Martin, E.. H., GPS User Equipment Error Models, Navigation, Vol. 25, No. 2, Summer 1978, pp. 201-216.

Pierce, Omega, IEEE Transactions on Aerospace and Electronic Systems, Vol. AES-1, No. 3, December 1965, pp. 206-215. 
Action

USE DETERMINISTIC PROPERTIES OF GPS SIGNALS

HIGH MEASUREMENT SNR

OMNIDIRECTIONAL ANTENNA

SIMULTANEOUS OBSERVATION AND PARALLEL CHANNEL RECEIVER AND iIIGH MEASUREMENT SNR

SHORT MEASUREMENT INTERVALS

DIRECT CARRIER PHASE MEASUREMENTS
CONSEQUENCE

HIGH MEASUREMENT SNR

CAN USE OMNIDIRECTIONAL ANTENNA; INEXPENSIVE RECEIVER

No BEAM STEERING SimULTANEOUS OBSERVATION OF ALL SATELLITES

CARRIER PHASE MEASUREMENTS IN ABOUT 1 SECOND AND BASELINE UPDATE EVERY SECOND IN STEADY STATE*

QUARTZ CRYSTAL CLOCKS

SIMPLE NUMERICAL PROCESSING IN STEADY STATE, BASED ON SOLUTION OF LINEAR EQUATIONS

\footnotetext{
"STEADY STATE = AMBIGUITY REMOVED
}

Figure 5. Properties of GPS-based system .

- Ambiguity resolution reguired at start and occasionally THEREAFTER:

- I.E., Whenever there is a "Long" enough interval BETWEEN BASELINE MEASUREMENTS THAT THE BASELINE MAY CHANGE SIGNIFICANTLY

- Resolve ambiguities by applying concepts tested in HAYSTACK-WESTFORD EXPERIMENTS AND AUGMENTED BY USE OF TWO FREQUENCY BANDS FOR IONOSPHERE REDUCTION

- USE CODE MODULATION (GROUP DELAY) FOR COARSE, BUT UNAMBIGUOUS BASELINE DETERMINATION

TIME REQUIRED DEPENDS ON EXTENT OF GROUND CLUTTER (MULTIPATH)

- USE CARRIER PHASES FOR REFINED BASEL INE DETERMINATION

Figure 6. Ambiguity resolution. 


\section{QBJectives}

- Assess ambiguity resolution techniques,

- Demonstrate accuracy of baseline determination,

Figure 7. Experimental program.

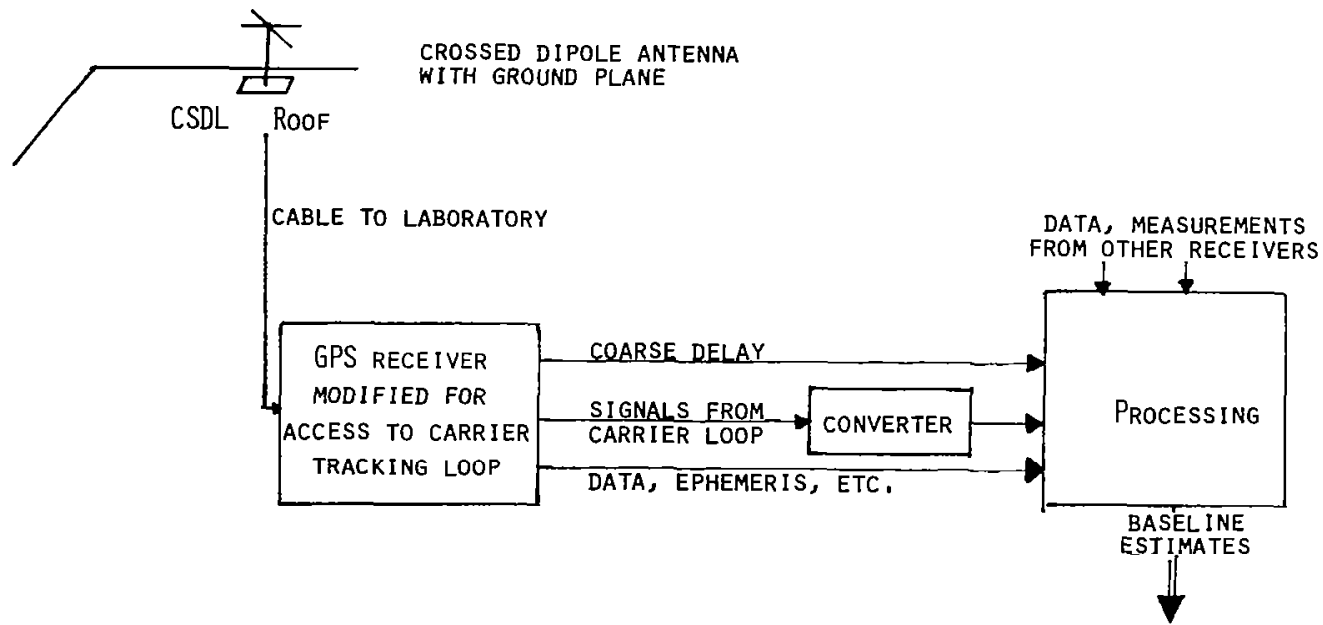

Figure 8. Experimental configuration.

\section{FeATURES}

- Very Short Baseline - < 100 meters (RESULTS CAN BE ACCURATELY CHECKED BY INDEPENDENT TECHNIQUES, IONOSPHERIC REMOVAL NOT TESTED.)

- gPS 4-Channel Receivers

- Crossed Dipole Antennas with Groundplane

- Precise Phase Measurements on Reconstructed Carrier

- Low Data Volume for Each Baseline Redetermination (LESS THAN 1 K BIT)

Figure 9. Experimental program. 


\section{SESSION F}

ADHERENCE AND COHERENCE IN NETWORKS AND PLANS 



\title{
THE EUROPEAN VLBI NETWORK
}

\author{
R. T. Schilizzi \\ Netherlands Foundation for Radio Astronomy
}

\begin{abstract}
A brief review is given of the present capabilities of the European very long baseline interferometry (VLBI) network, including the range of baseline parameters, sensitivities, and recording and other equipment available. Plans for upgrading the recording facilities and the use of geostationary satellites for signal transfer and clock synchronisation are discussed.
\end{abstract}




\section{INTRODUCTION}

This paper presents information concerning the growing VLBI activities in Europe and some of the plans under discussion for future development. The highly mobile VLBI systems discussed elsewhere in these Proceedings are not considered in this paper.

\section{VLBI FACILITIES IN EUROPE}

The locations of the radio observatories are shown in figure 1. All but the Italian and Polish observatories have successfully taken part in observations. The Polish station is expected to be on the air by the end of 1979; the Italian, by 1981-1982. The Italian VLBI plans are far-reaching: at least one, maybe two, antennas of very large array (VLA) design, modern cooled receivers, and the latest in VLBI equipment. Their stations will be a valuable southern addition to the network. Other potential sites also exist, such as the EISCAT antennas in northern Scandinavia and former space tracking antennas in Germany.

The telescopes available for VLBI and some parameters of the European network are shown in tables 1(a) and 1(b). Table 1(a) shows the range of receivers available, the system temperatures, and the telescope diameters. There are a number of large antennas in Europe with moderately good system

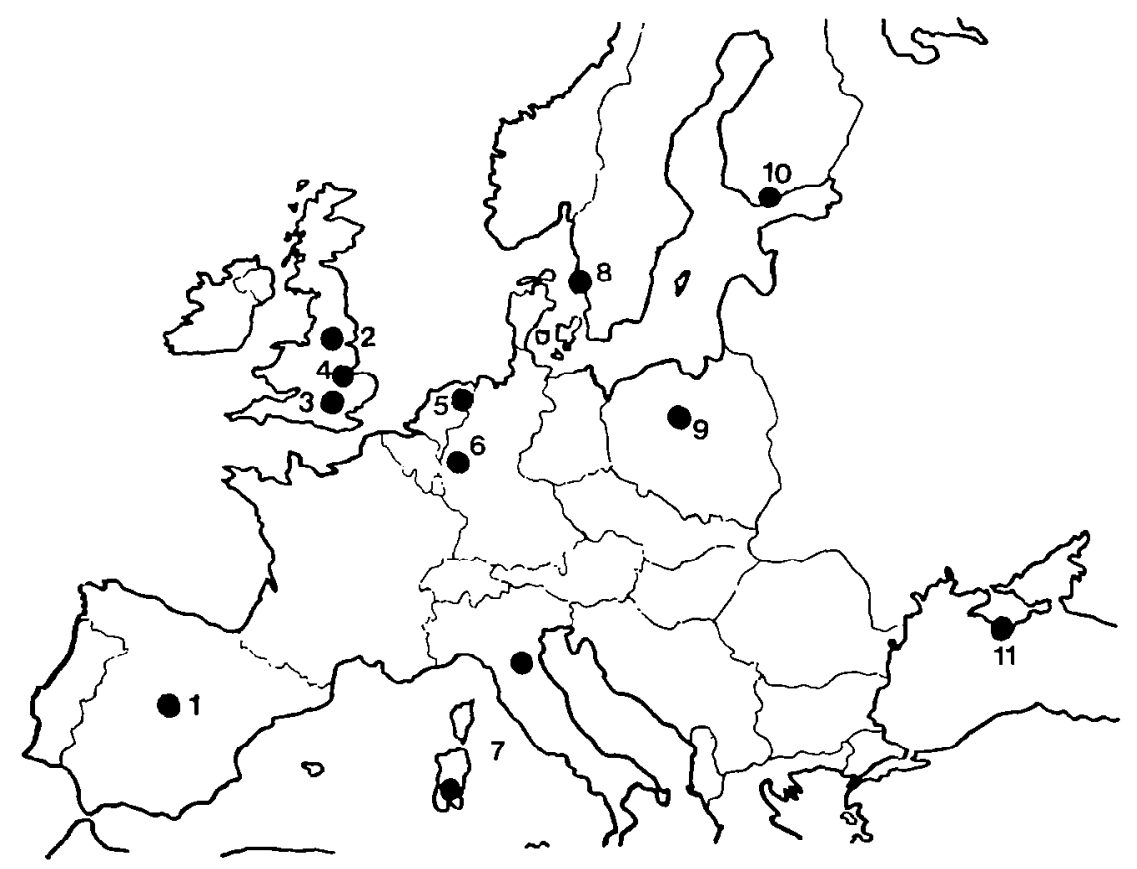

Figure 1. European VLBI observatories: 1. Madrid, 2. Jodrell Bank, 3. Chilbolton, 4. Cambridge, 5. Westerbork/Dwingeloo, 6. Effelsberg, 7. Bologna/Sardinia, 8. Onsala, 9. Torun, 10. Helsinki, 11. Crimea . 
Table 1(a)

Observatories in Europe,

Telescope Diameters and System Temperatures

\begin{tabular}{|c|c|c|c|c|c|c|c|}
\hline \multirow{2}{*}{$\begin{array}{l}\text { Country/ } \\
\text { Observatory }\end{array}$} & \multirow{2}{*}{$\begin{array}{l}\text { Antenna } \\
\text { Diam. (m) }\end{array}$} & \multicolumn{6}{|c|}{ Observing Wavelength $(\mathrm{cm})$} \\
\hline & & 1.3 & 2.8 & 6 & 11 & 18 & 21 \\
\hline \multicolumn{8}{|l|}{ U.K. } \\
\hline Jodrell Bank & $76,26,25$ & 500 & & 60 & 100 & 60 & 60 \\
\hline Chilbolton & 25 & 1300 & 180 & 100 & & & \\
\hline Cambridge & 32 (equiv.) & & & 90 & 75 & & \\
\hline \multicolumn{8}{|l|}{ Netherlands } \\
\hline Westerbork & 93 (equiv.) & & $450^{*}$ & 80 & & & 95 \\
\hline Dwingeloo & 25 & & 480 & 60 & & 37 & 42 \\
\hline \multicolumn{8}{|l|}{ Germany } \\
\hline Effelsberg & 100 & 200 & 75 & 80 & 100 & 60 & 100 \\
\hline \multicolumn{8}{|l|}{ Sweden } \\
\hline Onsala & 26,20 & 100 & & 45 & & 25 & \\
\hline \multicolumn{8}{|l|}{ Finland } \\
\hline Helsinki & 15 & & & (200) & & & \\
\hline \multicolumn{8}{|l|}{ U.S.S.R. } \\
\hline Crimea & 22 & 100 & 100 & 200 & & 250 & \\
\hline \multicolumn{8}{|l|}{ Italy } \\
\hline Bologna & 25 & & & & & & \\
\hline $\begin{array}{l}\text { Sardinia/ } \\
\text { Sicily }\end{array}$ & $(25)$ & & & & & & \\
\hline Poland & & & & & & & \\
\hline Torun & 15 & & & & & & \\
\hline
\end{tabular}

*1 antenna

Note: The DSN station at Madrid (Spain) is also available for VLBI observations. 
Table 1(b)

Range of Sensitivities ( $2 \mathrm{MHz}$ bandwidth, 1 minute integration) and Range of Baseline Lengths, for Interferometers in Europe

\begin{tabular}{|c|c|c|}
\hline $\begin{array}{c}\text { Observing } \\
\text { Wavelength }\end{array}$ & $\begin{array}{c}\text { Baseline lengths } \\
\left(10^{6} \lambda\right)\end{array}$ & $\begin{array}{c}\text { Sensitivities } \\
(\mathrm{mJy})^{*}\end{array}$ \\
\hline $1.3 \mathrm{~cm}$ & $26-212$ & $210-2670$ \\
2.8 & $9-89$ & $80-1030$ \\
6 & $4-46$ & $14-410$ \\
11 & $1.8-6.3$ & $24-150$ \\
18 & $1.4-15$ & $25-360$ \\
21 & $1.2-3.3$ & $16-120$ \\
\hline
\end{tabular}

${ }^{*} 1 \mathrm{mJy}=10^{-29} \mathrm{Watt} / \mathrm{m}^{2} \mathrm{~Hz}$

temperatures, and a number of smaller antennas with quite low system temperatures. In interferometer configurations, this leads to high sensitivity - or alternatively, short integration times per source per hour angle. Moreover, a great number of sources are available for observations with high sensitivity systems.

The only dual-frequency S-X band receiver in Europe is at Onsala.

Table 1(b) further illustrates the range of interferometer sensitivities, assuming a 2-MHz recorded bandwidth and a 1-minute integration. Also shown are the range of baseline lengths at each wavelength; the baselines in kilometers range from about $200 \mathrm{~km}$ to about $3000 \mathrm{~km}$.

All the observatories, with two or three exceptions, have the Mark II recording system based on Ampex or IVC recordcrs, and two obscrvatories are experimenting with vidco cassettc recorder. Hydrogen maser oscillators are not as widespread as in the United States; Onsala, Crimea, and Effelsberg are so endowed. The other stations have $\mathrm{Rb}$ standards.

Correlation and further processing of the data is carried out, for the most part, using the threestation Mark II processor at the Max Planck Institut für Radioastronomie in Bonn, Germany.

\section{PLANS FOR FUTURE VLBI DEVELOPMENT IN EUROPE}

\section{Introduction}

European plans for VLBI at the fixed observatories can be summarized in the following way. There is a need for a VLBI system which fully exploits the technique for geodesy, astronomy, and astrometry, and permits smooth, full-time observations with up to 10 radio telescopes. A smoothly 
operating system, in practice, is one in which all segments are matched; correlator, number of telescopes, computer system, observing time, correlating time.

The means of achieving these aims lie partly in the technical regime and partly in the political regime. On the technical side, there are three areas in which decisions have to be made. The first is the question of how to transfer the huge quantity of bits from remote sites to a central site for correlation. The second is how best to maintain the overall phase coherence of the system. The third is what form the data processor should take.

Included in these considerations are the different requirements of geodesy, astronomy, and astrometry for speed of data throughput, oscillator stability, and so on. From the geodetic point of view, we need only concern ourselves with global crustal dynamics and UT1/polar motion studies; the highly mobile systems discussed elsewhere in these Proceedings appear capable of catering for the needs of local crustal studies, independently of the fixed observatories.

\section{Data Transfer}

On this question, the decision to be made is whether the Mark III system or its successors are the best means of achieving a wide bandwidth capability, or if a satellite-linked system offers significant advantages. For the short and medium term, the decision to utilize the Mark III system is clear. A number of observatories in Europe are committing themselves in mind, if not yet on paper, to Mark III recording systems; Onsala and Effelsberg have orders on paper. For the longer term, though the question of satellite-linked systems needs to be considered. Two studies commissioned by the European Space Agency (ESA) have carried out a detailed investigation of the requirements for such a system ESA Doc. DP PS(78)15,1978; Phase A Study (in preparation).

Why consider the satellite-linked option at all? The primary reason is its operational "simplicity" compared with a tape-based system. This is particularly true for astronomical applications where wide bandwid th observations can be expected for a considerable fraction of the time. The contrasting prospect of a 10 station tape-based system producing 40 tapes an hour is daunting. Moreover, the cost of transporting the tapes to the central processor and return is considerable, in Europe ranging up to $\$ 250 \mathrm{~K}$ per year per observatory.

The real-time aspect of a satellite-linked system is secondary, allowing a check on operation of the system and its stability as the observations are progressing. A quick turnaround on the results is also a possibility which would allow changes in observing strategy if something interesting turned up.

From the geodetic point of view, a fast throughput would be useful, but not critical, for global crustal studies since observations are envisaged only on an intermittent basis. A tape-based system is adequate for this work. Polar motion and UT1 studies are envisaged as quasi-continuous ( 8 hours daily for the Polar-motion Analysis by Radio Interferometric Surveying (Polaris) project, see paper 
by W. E. Carter, these Proceedings) and would benefit from a rapid flow of bits through the correlator. A channcl in the satellite-linked system dedicated to UT 1/polar motion data transfer would probably have an advantage over a tape-based system. On the other hand, astronomers have a vested interest in obtaining geodetic and astrometric information on a regular basis since this provides the basic calibration of the interferometer system for astronomical work. It is likely that, independent of geodesists' needs, there will be a regular monitoring of the vector baseline and source position sets.

\section{Local Oscillator Stability and the Use of the Phase Observable}

Experience over the past few years has shown that present generation hydrogen maser oscillators deliver sufficient stability for geodetic purposes. They are also expensive items. Europe is sparsely populated with masers and heavily populated with observatories in countries with small budgets for radio astronomy. It is not clear whether a wide distribution of hydrogen masers is possible.

An alternative, possibly cheaper (for each observatory), scheme is to link remote oscillators of $\mathrm{Rb}$ quality via geostationary satcllite, thus making VLBI a form of connected element interferometry. The Canadian-United States efforts and Canadian plans in this regard are described in papers by Waltman et al. and Cannon et al. (these Proceedings). Design work is also going on in Europe at the Dwingeloo Radio Observatory in the Netherlands on a local oscillator link scheme via satellite. This is scheduled for its first test in January 1980 using the Orbital Test Satellite. The estimated cost of the ground station plus associated electronics required at each laboratory is estimated to be a factor 5 or 6 cheaper than a hydrogen maser. If this is successful, it will be an attractive solution to the problem of local oscillator stability.

It is anticipated that the use of the phase observable will grow in the coming years both for geodesy and astrometry (e.g., J. Campbell, these Proceedings) and for astronomy. A full use of phase for geodesy and astrometry requires that the a priori knowledge of the vector baselines, the source positions, the troposphere and ionosphere, be sufficiently good to resolve the inherent $2 \pi$ ambiguities. For well-established baseline and source position sets, narrow bandwidth observations utilizing phase should be sufficient for "fine-tuning" the baselines and position and for monitoring small scale changes in them.

\section{The Data Processor}

Ideally, the data processor should be designed to take inputs from either tapes or satellite links. Compatibility with Mark III could be assured by making the basic clock rate of the processor a multiple of $2 \mathrm{MHz}$, and providing parallel-to-serial interfaces for the 28 channel Mark III data before it entered the processor. For a satellite-linked system, the number of bit streams able to be correlated must be identical or greater than the number of stations; there is no opportunity for reprocessing. Design considerations for a wide bandwid th correlator are being evaluated at present. 


\section{Organizational Questions}

Observatories must commit themselves to VLBI operations for a substantial fraction of the time and to support the operations with the required funding.

If a satellite-linked system for either clock synchronization or signal transfer, or both, shows substantial advantages in capability or efficiency over an independent station approach, then ways of ensuring the success of the project need to be found.

There are encouraging signs in Europe on at least the first, and perhaps also the second, of these items. 



\title{
NASA GEODETIC APPLICATIONS OF THE MARK III VLBI SYSTEM
}

\author{
Robert Coates
}

NASA Goddard Space Flight Center

\begin{abstract}
For the past 5 years, NASA has been developing the Mark III Very Long Baseline Interferometer (VLBI) System for very high accuracy geodetic applications, such as measurements of the contemporary tectonic plate motions, large-scale plate stabilities, regional fault motions, Earth rotation changes, and polar motion. Initial deployments at fixed observatories in United States have established an initial reference grid for determinations of stability of the North American Plate and for measurements of polar motion and UT 1. Cooperating stations with new Mark III systems at Onsala, Sweden, and Effelsberg, Germany, observing with the U.S. stations, are to measure the relative plate motion between the North American and the Eurasian Plates. NASA plans to involve additional cooperating institutions throughout the world to develop a global VLBI network for plate motion determinations. NASA is developing mobile VLBI systems utilizing the Mark III for measurements of regional fault motions and crustal distortions. Initial deployments will be in the fault regions in western United States. Discussions have been initiated with foreign organizations regarding cooperative programs for measurement of fault motions in other active regions of the world.
\end{abstract}




\section{INTRODUCTION}

In the 1960's, NASA started supporting the development of the two space techniques, very long baseline interferometry (VLBI) and space laser ranging, for geodetic applications. NASA's interest was in the development of space technology that would permit the measurement of the dynamic motions of the Earth's crust that are believed to be the causes of earthquakes. The global distribution of large earthquakes shows that the earthquakes are most prevalent along the edges of the major tectonic plates. The relative motions between these plates causes severe distortions of the boundaries between the plates. This results in earthquakes. Thus, it is felt that the measurement of the relative plate motions and the crustal distortions along the boundaries of the plates will contribute significantly to a much better understanding of the processes which lead to earthquakes. NASA has concentrated on the development of the VLBI and space laser ranging because these two space techniques offer the potential for making measurements over very long distances with accuracies of better than a part in $10^{8}$. The measurements with these techniques can be made over water and rough terrain. Thus, they seem to be ideal techniques for the measurement of plate motion, regional deformation, and other dynamic motions of the earth.

\section{CRUSTAL DYNAMICS PROJECT}

A coordinated federal program for the application of this space technology to crustal dynamics and earthquake research has been formulated.* The participating agencies are the National Aeronautics and Space Administration (NASA), National Ocean and Atmospheric Administration (NOAA), the United States Geological Survey (USGS), the National Science Foundation, and the Department of Defense (DOD). All of these agencies have a role in earth dynamics and are interested in applying VLBI and lasers to their particular area of activity. NASA has been leading the technology development, and is the focal point for the initial phase of implementation of the VLBI and lasers for crustal dynamics. The NOAA National Geodetic Survey (NGS) is initialing a program to implement an operational VLBI system for polar motion and UT1 measurements. In addition, they plan to implement mobile VLBI for very high accuracy geodetic surveys in North America. The United States Geological Survey and the National Science Foundation have prime responsibility for earthquake research investigations. Their interest in utilizing VLBI is to obtain new and significant measurements and interpretations of the earthquake phenomena. The Department of Defense has a responsibility for high accuracy geodetic survey and mapping, as well as operational UT and polar motion determination.

The success of the NASA developments of VLBI and space laser ranging has prompted NASA to establish a Crustal Dynamics Project for the application of these space techniques to the study of the earth-dynamics motions as related to earthquakes. Both the Goddard Space Flight Center and the Jet Propulsion Laboratory are involved in the project. Goddard is responsible for the management

\footnotetext{
*National Aeronautics and Space Administration, National Oceanic and Atmospheric Administration, U.S. Geological Survey, National Science Foundation, and Defense Mapping Agency, "The Coordinated Federal Program for the Application of Space Technology to Crustal Dynamics and Earthquake Research," November 1978.
} 
of the project, the science coordination and management, the VLBI technology developments, the implementation and operations of fixed VLBI observatory stations, and the laser ranging development and operation. The Jet Propulsion Laboratory is responsible for the mobile VLBI development and operations, plus related Deep Space Network (DSN) activities. In addition to NASA, the Crustal Dynamics Project involves many close cooperative efforts from many other institutions. As an example, the development of the Mark III VLBI for geodetic applications has had the participation of the Haystack Observatory, the Massachusetts Institute of Technology, the National Radio Astronomy Observatory, the Smithsonian Astrophysical Observatory (SAO), the National Geodetic Survey, the Owens Valley Radio Observatory, The Harvard College Observatory, and the Chalmers Institute.

The scientific objectives of the Crustal Dyanmics Project are to improve our knowledge and understanding of:

- The regional deformation and strain accumulation related to large earthquakes in the plate boundary region of the western United States.

- Contemporary relative plate tectonic motions of the North American, Pacific, South American, Eurasian, and Australian Plates.

- The internal deformation of continental and oceanic lithospheric plates with particular emphasis on North America and the Pacific.

- The rotational dynamics of the Earth and their possible correlation to earthquakes, plate motions, and other geophysical phenomena.

- Motions and deformation occurring in regions of high earthquake activity.

In order to achieve these objectives, a rather extensive measurement program utilizing both the VLBI and space laser ranging will be required. Frequent high accuracy measurements of baselines between many stations in active areas near the plate boundaries are required for the determination of the regional deformation and strain accumulation in these active regions. Regular measurements of baselines between a global set of stations on the different plates are required for the determination of the relative tectonic plate motions. Repeated measurements of baselines between several stations on the same plate are needed for the measurement of the internal deformation of the plates. The determination of polar motion and Earth-rotation variations requires daily measurement of PM and UT1 with a global set of stations in stable locations.

An integral part of the Crustal Dynamics Project is the test and validation of the capabilities of the space systems for making high accuracy geodetic measurements. As an example, one of the test experiments to be conducted later on this year is a five-station intercomparison between the VLBI and space laser ranging. Figure 1 shows the location of the five VLBI stations and the five laser stations. The systems are co-located at Westford, Massachusetts; Ft. Davis, Texas; Owens Valley, California; and Goldstone, California. In this intercomparison both the Mark III VLBI systems* and

*T. A. Clark, "Mark III System Overview," Proceedings of Radio Interferometry Techniques for Geodesy Conference, June 1979, NASA CP 2115, 1980. 


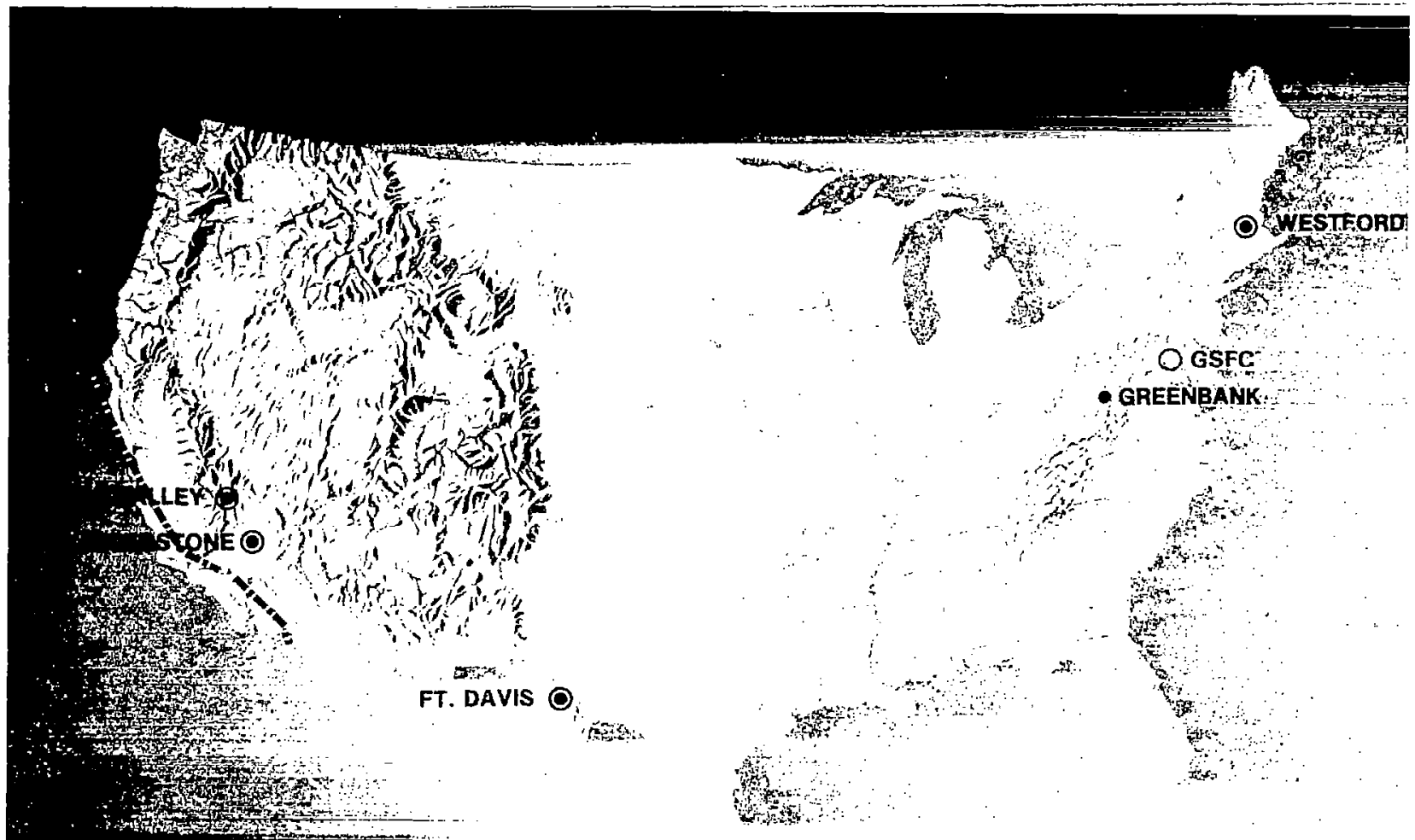

Figure 1. Map of the station locations for the 1979 intercomparison of satellite laser ranging and VLBI geodetic measurements.

the space laser systems will make a series of measurements for the determination of the baselines between the stations. The VLBI and laser results will then be intercompared in order to determine if there are any systematic effects apparent from these measurements. These baselines are expected to intercompare at the 5 centimeter level.

These intercomparison stations are planned to be used continuously by one technique or the other throughout the life of the project in the role of permanent base stations to be used in the determination of regional crustal deformation, internal deformation of the North American plate, and tectonic plate motions relative to the North American plate. It should be noted that the Westford site, the Ft. Davis site, and a new site in Richmond, Florida, make up the NGS Polar-motion Analysis by Radio Interferometric Surveying (Polaris) network* for operational polar motion and UT1 determination.

Figure 2 is a map of the different tectonic regions of the United States and locations of recommended sites for VLBI and laser measurements for the determination of the regional deformation in

*W. E. Carter, "Project POLARIS," Proceedings of Radio Interferometry Techniques for Geodesy Conference, June 1979, NASA CP 2115, 1980. 


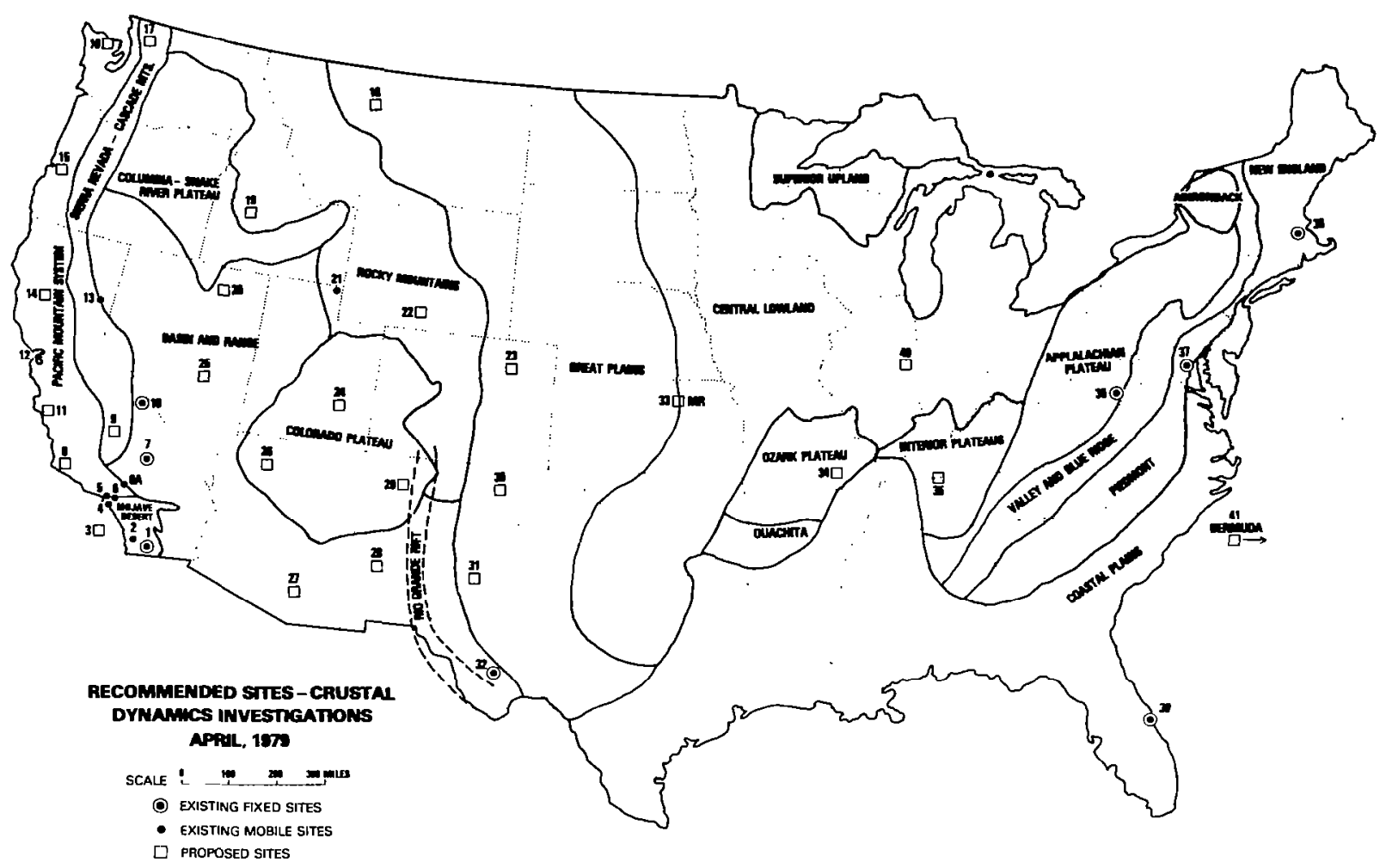

Figure 2. Possible locations of sites for measurements of the regional crustal deformation in the western United States and the internal deformation of the North American plate.

the western United States and the internal deformation of the North American plate. At the present time, this recommendation is a "strawman" proposal by a group of NASA scientists. It is expected that sites similar to these will be firmed up in the next few months through discussions with the scientific community and other government agencies. The final deployment will probably be similar to figure 2. This map shows several fixed and mobile sites that are already in operation, plus many new locations to be established for mobile systems or transportable systems. A total of 41 locations are identified, with the majority of locations in the seismically active western half of the United States.

Figure 3 shows the present deployment of space laser ranging stations around the globe. This includes NASA stations, SAO stations, European Range Observations to Satellites (EROS) stations, and lunar laser ranging stations. This distribution provides several stations on the North American plate, South American plate, the Pacific plate, the Australian plate, and the Eurasian plate for measurements of global plate motions and polar motion/UT 1 . 


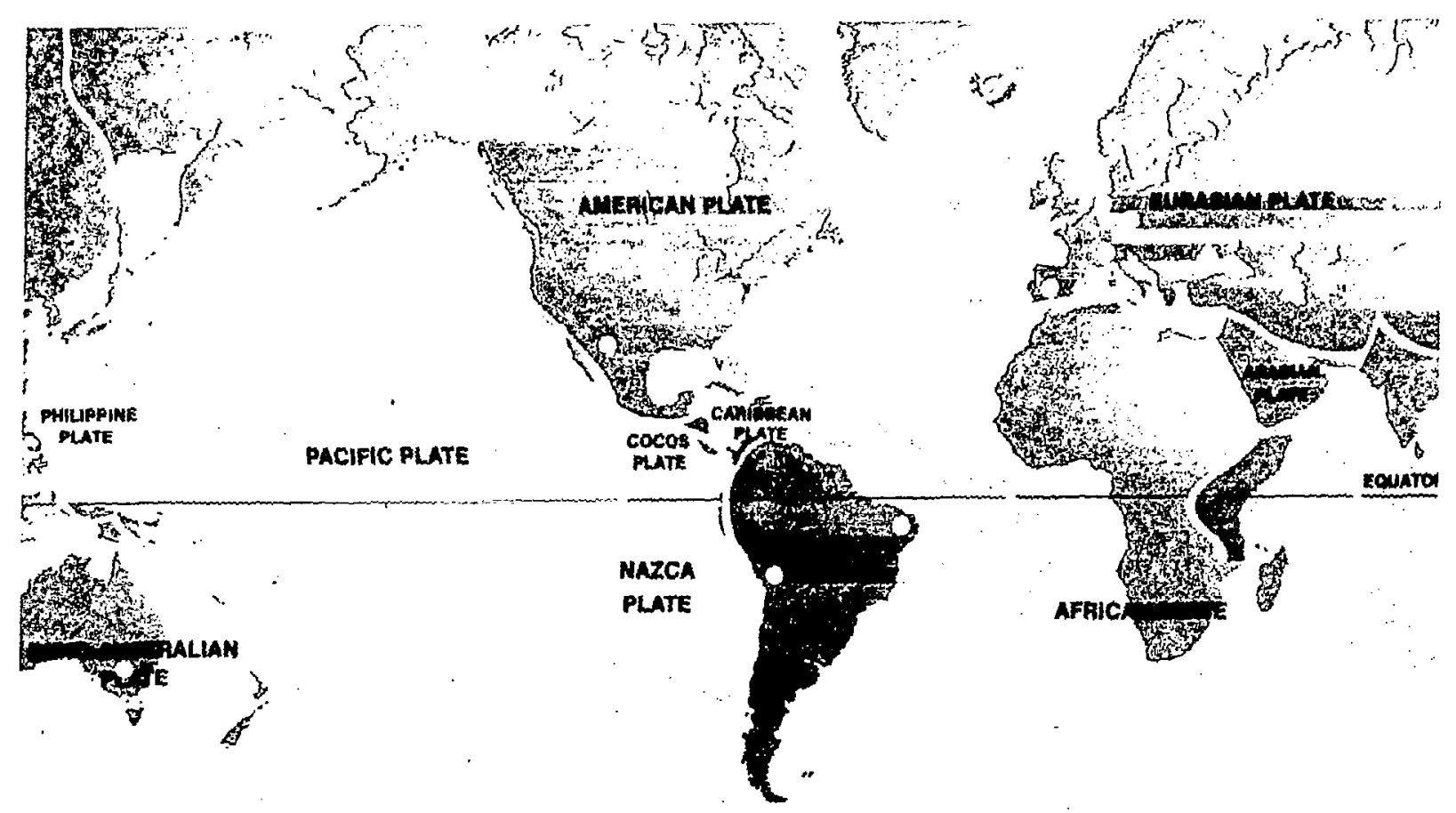

ANTARCTIC PLATE
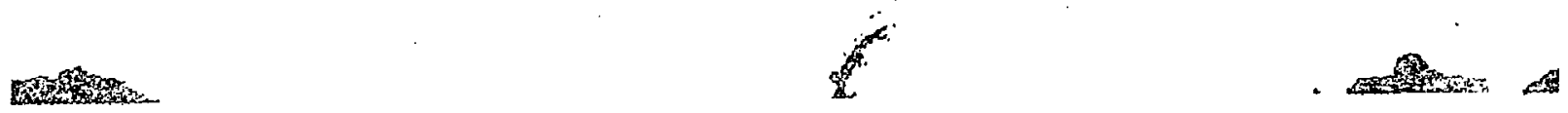

Figure 3. 1979 deployment locations of space laser ranging systems.

In figure 4, the solid dots are the locations of fixed VLBI stations in 1979 which will be used for geodetic measurements. This shows the five VLBI base stations in North America that were discussed above, the DSN stations in Australia and Spain, and the European stations at Onsala, Sweden, and Effelsberg, Germany. This initial deployment will begin the measurements of the plate motions between the three respective plates, the internal defomations of the North American and Eurasian plates, and polar motion/UT1.

The development of geodetic VLBI capability at the two European stations has been accomplished through cooperative joint efforts of several United States and European agencies and institutions. The host institutions are the Chalmers Institute of Technology for the Onsala station and the MaxPlanck-Institut für Radioastronomie for the Effelsberg station. The Mark III VLBI systems are being assembled from subsystems funded by the NASA Crustal Dynamics Project, the host institutions the U,S. Geological Survey and the U.S. Air Force. The Crustal Dynamics Project VLBI team from Goddard Space Flight Center, Haystack Observatory, and Massachusetts Institute of Technology responsible for the implementation of the Mark III unique equipment.

\footnotetext{
tho intent of the Crustal Dynamics Project to encourage organizations throughout the worlc - :itias to make a global joint effort possible for the measurements of $\mathrm{F}$
} 


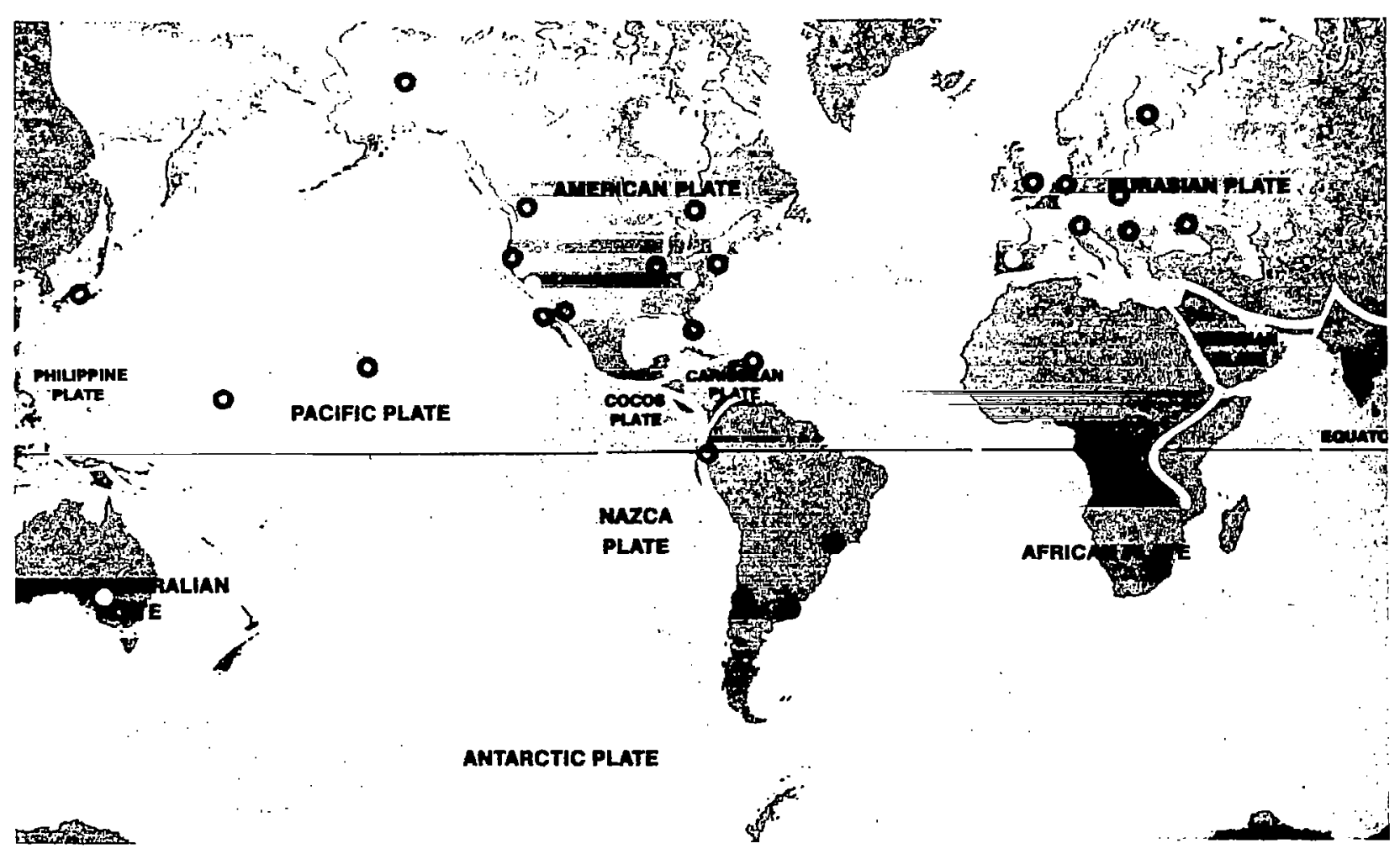

Figure 4. 1979 locations of VLBI fixed stations (solid dots), and locations of a few of the radio astronomy or space tracking antenna facilities (open circles) which could be considered for future geodetic VLBI applications.

motions, crustal motions, and polar motion/UT1. To illustrate the tremendous potential that exists for global VLBI participation, the open circles in figure 4 are a few of the existing radio astronomy or space tracking facilities which could be considered for geodetic VLBI applications. In the development of the Mark III VLBI system, NASA has designed the configuration to be self-contained so that it can be easily moved into an antenna facility for geodetic or astronomical observing sessions and then moved to another facility for other observations. This configuration provides the flexibility to make use of the many existing antenna facilities around the world.

Besides the seismically active region in the western United States discussed earlier, there are many other regions of high earthquake activity along plate boundaries throughout the world. Some of these tectonically important areas are indicated in figure 5. The deployment of mobile VLBI systems in these active regions in a manner similar to that illustrated in figure 2 will provide important information about the deformation in these regions. By monitoring these deformations and other seismic and geodetic measurements, geophysical models of the region can be developed that will provide insight into the reasons for the occurrence of earthquakes in the region. Through extensive cooperation with other organizations and countries, NASA would like to stimulate the development 


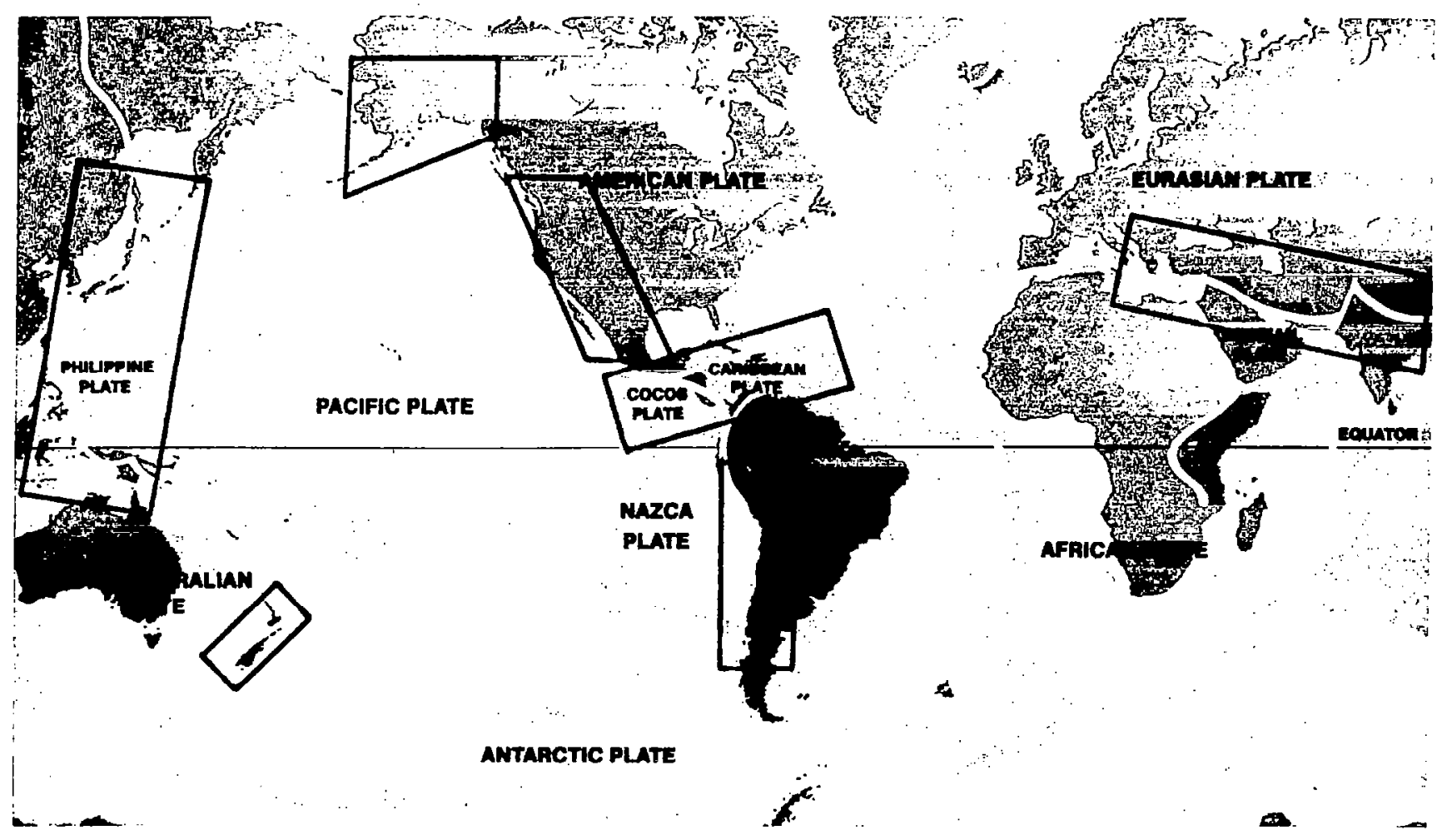

Figure 5. Tectonically important areas of high earthquake activity.

of regional measurement plans with mobile systems in these seismically active areas. This will enhance the scientific understanding of earthquake processes as related to the dynamics of the Earth.

\section{SUMMARY}

The NASA Crustal Dynamics Project is an effort to develop and demonstrate the capabilities of the VLBI and laser ranging techniques for unique high accuracy geodetic measurements which have the potential for significant scientific measurements of the dynamics of the Earth. The thrust of the project is to implement minimal networks to demonstrate the capability and to work with user organizations all over the world to encourage them to build up their own capabilities for carrying on these very significant measurements.

\section{REFERENCES}

Carter, W. E., "Project POLARIS," Proceedings of Radio Interferometry Techniques for Geodesy Conference, June 1979, NASA CP 2115, 1980. 
Clark, T. A., "Mark-III System Overview," Proceedings of Radio Interferometry Techniques for Geodesy Conference, June 1979, NASA CP 2115, 1980.

National Aeronautics and Space Administration, National Oceanic and Atmospheric Administration, U.S. Geological Survey, National Science Foundation, and Defense Mapping Agency, "The Coordinated Federal Program for the Application of Space Technology to Crustal Dynamics and Earthquake Research," November 1978. 


\title{
ON THE VLBI-SATELLITE LASER RANGING "IRON TRIANGLE" INTERCOMPARISON EXPERIMENT
}

\author{
Yehuda Bock, Ivan I. Mueller, Erricos Pavlis \\ Department of Geodetic Science \\ The Ohio State University
}

\begin{abstract}
A very long baseline interferometry (VLBI)-laser intercomparison experiment is planned for the "Iron Triangle" consisting of the stations at Haystack, Owens Valley, and Ft. Davis. The improvements in the variances of the estimable parameters resulting from the addition of the stations at the National Radio Astronomy Observatory (NRAO), Green Bank (Greenbelt, in the laser case), and Goldstone is examined by means of a least squares covariance analysis assuming only random observational errors and disregarding systematic effects. (The latter are clearly aided by additional baseline observations.)
\end{abstract}

The usefulness of a substitute station at Richmond, Florida, is also examined with the idea of improving the accuracy of polar motion. Although this station is not in operation, it would be possible to use a portable laser and VLBI antenna at this site during the intercomparison experiment.

In the case of VLBI, a covariance analysis is performed on multi-baseline configurations. The parameters examined include baseline related quantities, quasar declinations, quasar right ascension differences, and Earth rotation parameters including polar motion. The variances of these parameters are calculated and compared among the various station configurations.

Laser range observations to LAGEOS are simulated, and the variances of the recovered baselines are examined. For the purpose of establishing a lower bound on the achievable baseline standard deviations independent of dynamical errors and assumptions, the laser observations were also analyzed in the geometric mode.

Although the absolute numbers recovered for the variances are not meaningful in themselves, their relative improvements within each of the two systems are instructive in planning for the intercomparison experiments. 


\section{INTRODUCTION}

In anticipation of the upcoming VLBI-laser intercomparison experiments scheduled for 1979-1980, simulations were performed to determine the suitability of the proposed station locations. The criterion was a comparison among the possible station configurations of the standard deviations of baseline and Earth rotation parameters estimated from a least squares covariance analysis. Only the relative magnitudes of the standard deviations were addressed in the analysis. Thus, only random errors were assumed and no provision was made for systematic effects.

The "Iron Triangle," consisting of the stations at Westford (Haystack), Massachusetts, Owens Valley, California, and Ft. Davis, Texas, was regarded as the basic structure of the proposed network with options to incorporate either the Goldstone, California, or Green Bank, West Virginia, station or both. In addition, it was decided to include the Richmond, Florida, station in the analysis since it offered more North-South separation and therefore could strengthen the geometry of the network especially in the recovery of Earth rotation parameters. Although the station is not in operation as yet (it is part of the proposed Polar-motion Analysis by Radio Interferometric Surveying (Polaris) triangle), it would be possible to use a portable antenna at the site if its addition was found to be worthwhile. The effect of adding the more precise STALAS laser at Greenbelt was also considered.

The VLBI and laser simulations were done independently. Obviously, no absolute comparison of the numerical results is possible.

\section{MATHEMATICAL MODEL}

\section{VLBI}

The mathematical model for the time delay "observable" can be written as the inner product of the baseline vector in an Earth-fixed system, and the quasar unit vector rotated from an inertial system into the Earth-fixed frame. The observable, denoted by d, is the product of the time delay and the speed of light, and can be expressed in a simplified manner, suitable for this type of analysis, as follows:

$$
\mathrm{d}=-\left[\Delta \mathrm{X}_{\mathrm{i}} \Delta \mathrm{Y}_{\mathrm{i}} \Delta \mathrm{Z}_{\mathrm{j}}\right]\left[\begin{array}{rrr}
1 & 0 & \xi_{\mathrm{j}} \\
0 & 1 & -\eta_{\mathrm{j}} \\
-\xi_{\mathrm{j}} & \eta_{\mathrm{j}} & 1
\end{array}\right]\left[\begin{array}{ccc}
\cos \theta & \sin \theta & 0 \\
-\sin \theta & \cos \theta & 0 \\
0 & 0 & 1
\end{array}\right]\left[\begin{array}{ll}
\cos \delta_{\mathrm{k}} & \cos \alpha_{\mathrm{k}} \\
\cos \delta_{\mathrm{k}} & \sin \alpha_{\mathrm{k}} \\
\sin \delta_{\mathrm{k}} &
\end{array}\right]
$$

where

$$
\Delta \mathrm{X}_{\mathrm{i}}, \Delta \mathrm{Y}_{\mathrm{i}}, \Delta \mathrm{Z}_{\mathrm{i}} \quad \text { are the coordinate differences of the } \mathrm{i}^{\text {th }} \text { baseline in an Earth-fixed system }
$$$$
\alpha_{\mathrm{k}}, \delta_{\mathrm{k}}
$$$$
\text { are the true right ascension and declination of the } \mathrm{k}^{\text {th }} \text { quasar, respectively }
$$ 
$\theta$

$\begin{array}{ll}\text { Eq. E. } & \text { is the equation of the equinoxes } \\ \text { AI } & \text { atomic time } \\ \text { TA1 } & \text { international atomic time } \\ \text { UTC } & \text { coordinated universal time } \\ \text { UT1 } & \text { observed universal time corrected for polar motion } \\ \text { W }_{d} & \text { conversion factor from universal to sidereal time } \\ \theta_{0} & \text { GAST at initial epoch }\end{array}$

$\xi_{\mathrm{j}}, \eta_{\mathrm{j}}$ is the Greenwich Apparent Sidereal Time (GAST)

$$
\begin{aligned}
\theta= & \theta_{0}+\mathrm{W}_{\mathrm{d}}[\mathrm{TAI}-(\mathrm{TAI}-\mathrm{UT} 1)] \\
= & \theta_{\mathrm{o}}+\mathrm{W}_{\mathrm{d}}[\mathrm{TAI}-(\mathrm{TAI}-\mathrm{A} 1)-(\mathrm{A} 1-\mathrm{UTC}) \\
& \left.-(\mathrm{UTC}-\mathrm{UT} 1)_{\mathrm{BIH}}+\kappa+\dot{\kappa} \mathrm{t}\right]+ \text { Eq. } \mathrm{E} .
\end{aligned}
$$

the components of polar motion that relate the true celestial pole to the terrestrial pole

The estimable parameters whose standard deviations were estimated in these simulations were the following:

A. Quasar parameters

1. Quasar declinations: $\delta_{\mathrm{k}}$

2. Quasar right ascension differences: $\alpha_{k}-\alpha_{o}$ where $\alpha_{o}$ is the adopted right ascension of a low declination quasar, defining the origin of the right ascensions.

B. Earth rotation parameters

1. Polar motion component differences: $\xi_{j}-\xi_{1}, \eta_{j}-\eta_{1}$. The interval of observations (24 hours) was divided into four steps of 6 hours duration. The average values of the first step $\xi_{1}, \eta_{1}$ are adopted; e.g., they can be taken from other sources (Bureau International de l'Heure (BIH)). The remaining steps were 6-hour averages referred to these initial values. For the purpose of our simulations, $\xi_{1}=\eta_{1}=0$.

2. Earth rotation variation parameters: $\dot{\kappa}$ and $\kappa_{j}-\kappa_{1}$, where $\kappa$ and $\dot{\kappa}$ are the first two terms of a polynomial that models the variations in the Earth rotation rate as given by TAI UT1 in the previous expansion for GAST. The $\dot{k}$ rate parameter was taken as constant over the period of observations. The $\kappa_{j}-\kappa_{1}$ earth rotation variation difference parameter was also represented by a step function. The third component of Earth rotation was defined by adopting a value for the first step, $\kappa_{1}$. The remaining steps were 6-hour averages referred to the fixed initial value. The $\kappa_{1}$ value can be obtained from the BIH, but for the purposes of our simulation $\kappa_{1}=0$. 
C. Baseline related parameters

1. $\tau_{\mathrm{i}}, \epsilon_{\mathrm{i}}, \sigma_{\mathrm{i}}$. The baseline components $\Delta \mathrm{X}_{\mathrm{i}}, \Delta \mathrm{Y}_{\mathrm{i}}, \Delta \mathrm{Z}_{\mathrm{i}}$ are nonestimable quantities being affected by errors in $\alpha_{0}, \kappa_{1}, \xi_{1}, \eta_{1}$ described above. The differential relationships between these parameters are as follows;

$$
\begin{aligned}
& \mathrm{d} \tau_{\mathrm{i}}=\mathrm{d} \Delta \mathrm{X}_{\mathrm{i}}+\Delta \mathrm{Y}_{\mathrm{i}}\left(\mathrm{d} a_{\mathrm{o}}-\mathrm{d} \kappa_{1}\right)-\Delta \mathrm{Z}_{\mathrm{i}} \mathrm{d} \xi_{1} \\
& \mathrm{~d} \epsilon_{\mathrm{i}}=\mathrm{d} \Delta \mathrm{Y}_{\mathrm{i}}-\Delta \mathrm{X}_{\mathrm{i}}\left(\mathrm{d} a_{\mathrm{o}}-\mathrm{d} \kappa_{1}\right)+\Delta \mathrm{Z}_{\mathrm{i}} \mathrm{d} \eta_{1} \\
& \mathrm{~d} \sigma_{\mathrm{i}}=\mathrm{d} \Delta \mathrm{Z}_{\mathrm{i}}+\Delta \mathrm{X}_{\mathrm{i}} \mathrm{d} \xi_{1}-\Delta \mathrm{Y}_{\mathrm{i}} \mathrm{d} \eta_{1}
\end{aligned}
$$

The subscript $\mathrm{i}$ refers to the $\mathrm{i}^{\mathrm{t} h}$ baseline.

2. Baseline distances, $\ell_{i}$. These are estimable parameters.

This completes the list of estimable parameters whose total number is given by:

$$
\begin{aligned}
& 3 i+(3(j-1)+1)+(2 k-1)=3 i+3 j+2 k-3 \\
& i=\text { number of baselines } \\
& j=\text { number of steps } \\
& k=\text { number of quasars }
\end{aligned}
$$

A least squares covariance analysis was used to estimate the standard deviations of the parameters. These are obtained from the diagonal elements of the inverted normal matrix (the variancccovariance matrix) which is independent of observations. Its values depend on the geometry, the observation schedule, and the anticipated observational noisc.

The normal matrix $\mathrm{N}$ is derived from the well-known formula

$$
\mathrm{N}=\mathrm{A}^{\mathrm{T}} \mathrm{PA}
$$

where A represents the partial derivative matrix, $A^{T}$ its transpose, and $P$ the weight matrix of observables, in this case the time delays. When observing simultaneously from more than two stations, for instance on a triangle, the time delay measurements are correlated and this must be included in the off-diagonal elements of the variance-covariance matrix of the observables, $\Sigma_{L_{b}}$, where

$$
\mathrm{P}=\sigma_{\mathrm{o}}^{2} \Sigma_{\mathrm{L}_{\mathrm{b}}}^{-1}
$$

$\sigma_{\mathrm{o}}^{2}$ being the a priori variance of unit weight. On a triangle (and similarly on any closed figure), when observing simultaneously, only two sets of time delays (out of a possible three) should be used. The actual observations are registered on tapes located at the three sites. The time delay is a quasi-observable being derived by cross-correlating the tapes at a later time. Denoting the time delay between stations $i, j$ as $\tau_{i j}$, it follows from the mathematical model that $\tau_{12}+\tau_{23}+\tau_{31}=0$. Therefore, after choosing any two combinations of time delays, the third combination will be linearly dependent on the other two and thus does not provide new information. Naturally, for any choice of two tapes, the estimated standard deviations of parameters should be identical. However, 
if the correlations between the time delay "observables" are not included in the $\Sigma_{L_{b}}$ matrix, this will not be the case. The weight matrix of observables is diagonal in this case, and the result is three sets of estimated standard deviations instead of one.

Using our simplified model and assuming that all time delays are "observed" with equal noise, it is easy to construct the $\Sigma_{\mathrm{L}_{\mathrm{b}}}$ matrix. In triangle $1-2-3$, the time delays can be written as

$$
\begin{aligned}
\tau_{12} & =t_{2}-t_{1} \\
\tau_{23} & =t_{3}-t_{2} \\
\tau_{31} & =t_{1}-t_{3}
\end{aligned}
$$

the differences in the arrival times of a given segment of a wavefront at the two antennas. An error propagation is done using the first two time delays from above to determine $\Sigma_{\mathbf{L}_{\mathrm{b}}}$.

The complete variance-covariance matrix $\Sigma_{L_{b}}$ is composed of $2 \times 2$ full blocks along the main diagonal, and zeros elsewhere, for each set of observations at a particular epoch, in the following manner:

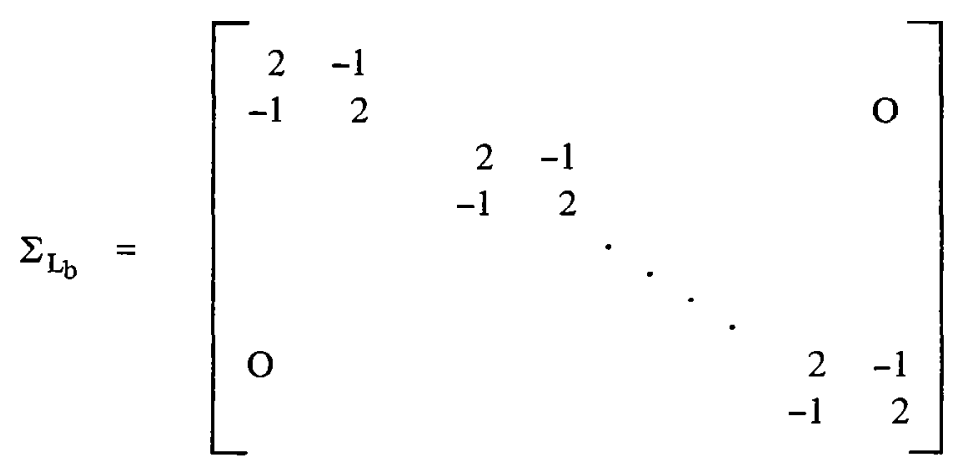

This matrix is inverted and scaled according to the assumed observational noise to arrive at the appropriate $\mathrm{P}$ matrix.

In the "real world," the $\Sigma_{L_{b}}$ matrix will be more difficult to derive, but our tests show that unless the true correlations are known, the results may be very misleading.

\section{LASER}

In this simple simulation study, the only quantities which were allowed to adjust were the station positions $\bar{U}$, the initial satellite state-vector $\left[\bar{X}_{0} \vdots \dot{X}_{0}\right]^{T}$, and the coordinates of the pole $\xi, \eta$. The brief presentation of the mathematical model that follows is 'given in an Earth-fixed coordinate system neglecting the effects of nutation and precession. In compact form, the error equation is as follows:

$$
\left(\rho_{\mathrm{o}}-\rho_{\mathrm{c}}\right)_{\mathrm{i}}=\sum_{\mathrm{j}} \frac{\partial \rho_{\mathrm{c}_{\mathbf{i}}}}{\partial \mathrm{P}_{\mathrm{j}}} \mathrm{dP} \mathrm{P}_{\mathrm{j}}-\mathrm{v}_{\mathrm{i}}
$$


where $\rho_{\mathrm{o}_{\mathrm{i}}}$ denotes the $\mathrm{i}^{\text {th }}$ observed range, $\rho_{\mathrm{c}_{\mathrm{i}}}$ the corresponding prediction based on an approximate set of parameters $P_{j}$. Based on the above general equation and the aforementioned set of parameters, the linearized observation equation is the following:

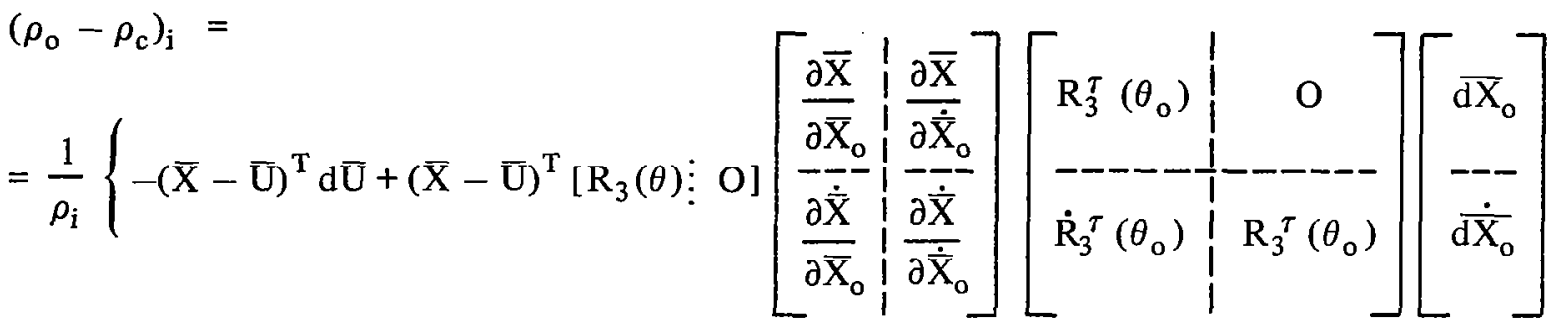

$$
\begin{aligned}
& \left.+\left[\mathrm{X}_{1} \mathrm{U}_{3}-\mathrm{X}_{3} \mathrm{U}_{1} \vdots \mathrm{X}_{3} \mathrm{U}_{2}-\mathrm{X}_{2} \mathrm{U}_{3}\right]\left[\frac{\mathrm{d} \xi}{\mathrm{d} \eta}\right]\right\}-\mathrm{v}_{\mathrm{i}}
\end{aligned}
$$

In the above, the angle $\theta$ denotes the GAST at the epoch of observation and $\theta_{0}$ refers to the initial epoch; $v_{i}$ denotes the random error in the observation. The results presented here are based on a fixed polar motion model; $\xi$ and $\eta$ were not considered as parameters.

\section{SIMULATION PROCEDURE}

\section{VLBI}

The selection of a quasar observation schedule was guided by two considerations:

- that a quasar be observable simultaneously (maximum zenith distance of $80^{\circ}$ ) from all stations at a chosen epoch of observation.

- that the final quasar schedule, over the 24-hour period of the simulations, be evenly distributed in right ascensions and declinations in order to achieve a strong geometry and to provide good recovery for low and high quasar declination-dependent parameters. Since the geometry of the experiments shifts by about only 4 minutes every 24 hours, it was decided that a day of observations would adequately encompass the entire geometry of the problem.

In order to simulate real observing conditions, it was decided to observe a quasar every 10 minutes simultaneously from all stations involved in a particular experiment. Although a typical time delay "observation" requires 3 to 5 minutes, a longer period was taken in order to allow time for antenna slewing and switching of tapes. The observational noise was assumed to be 0.1 nanosecond $(3 \mathrm{~cm})$.

\section{LASER}

For the laser experiments, the observables were simulated ranges to the satellite LAGEOS with a nominal noise level of $10 \mathrm{~cm}$. The observational period was 7 days during which 22 passes were 
co-observed by all stations. The simulations were performed in two modes: in the dynamic mode involving a short arc solution, and in the geometric mode run for the purpose of recovering standard deviations independent of orbital effects and assumptions. It is recognized that the inherent restriction of having simultaneous observations from at least four distant stations makes this mode impractical. The laser results presented are based on the directly estimable baseline distances as obtained from the short-arc solutions.

\section{RESULTS AND CONCLUSIONS}

The various experiments were compared on the basis of the estimated standard deviations of baseline (VLBI and laser) and Earth rotation parameters (VLBI only). However, since the baseline components depend to a certain extent on the coordinate system definition as described earlier, the directly estimable baseline lengths were used as a basis for comparison. As of this time, we have not completed our simulations estimating the recovery of pole coordinates from laser observations.

\section{VLBI}

The VLBI results using the assumed correlations are summarized in table 1 . Experiment 1 using the tapes from the Iron Triangle configuration is the basis of comparison. In experiments $2-4$, the effects of the addition of either Green Bank, Goldstone, or Richmond are examined. In experiment 5 , the effect of adding both Green Bank and Goldstone to the basic configuration is listed. The columns headed by IMPRV give the improvement in the estimated standard deviations of an experiment relative to experiment 1 .

Experiments 2 and 3 show improvements on the order of 5 to 10 percent over experiment 1 . However, the addition of the Richmond station results in improvements of about 25 to 35 percent. Especially apparent is the improvement in the $\Delta \eta$ parameter, because of the significant North-South separation of the Westford and Richmond stations. The closeness of the Green Bank and Goldstone stations to the Iron Triangle configuration implies that the effect of these stations is the same as would be expected from increasing the number of observations in the Iron Triangle itself. Obviously, there is a limit to the number of observations over a given interval of time that can be made especially for the earth rotation parameters which are time dependent. In any case, these stations do not substantially improve the recovery of the parameters of interest. In fact, as seen in experiment 5, the addition of both Goldstone and Green Bank has less effect on the results than the addition of Richmond alone in experiment 4. Thus, it can be concluded that only Richmond would significantly improve the basic configuration, as expected. Naturally, another station providing a similarly favorable geometry such as in Alaska could provide similarly improved results.

\section{LASER}

Table 2 summarizes the laser experiments and gives the improvement of average relative precisions of the three baselines of the Iron Triangle (L1). In each column (L2 - L6) the results of adding stations to the Iron Triangle are listed. The numbers in parentheses are the total number of ranges 


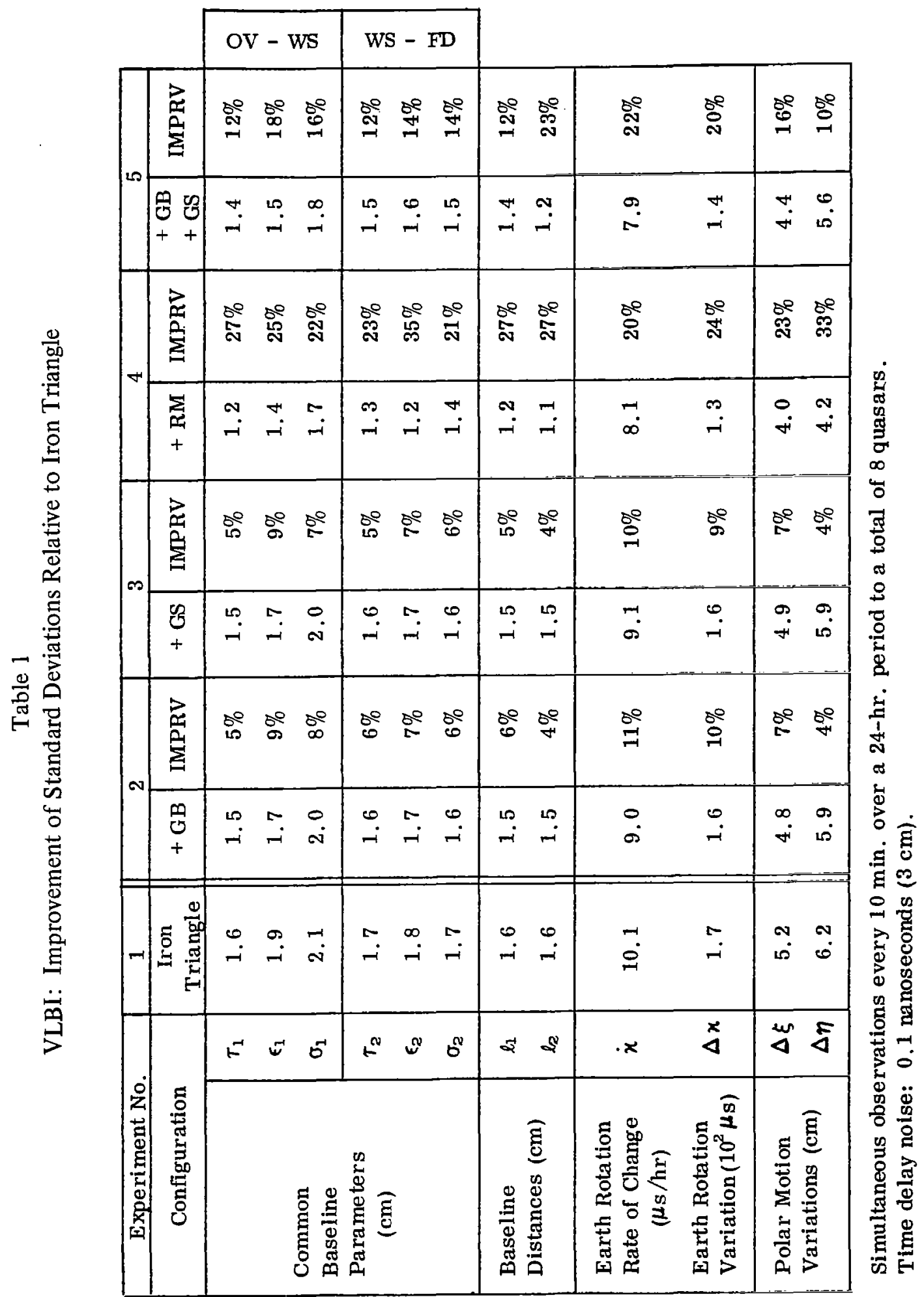


Table 2

LASER: Improvement of Average Relative Precisions

\begin{tabular}{|c|c|c|c|c|c|c|}
\begin{tabular}{|c|} 
Standard of \\
Comparison \\
$\begin{array}{c}\text { Iron Triangle: } \\
\text { L1* } \\
(3969)\end{array}$
\end{tabular} & $\begin{array}{c}\text { L2 } \\
\text { GB, GS, RM } \\
(7843)\end{array}$ & $\begin{array}{c}\text { L3 } \\
\text { GB, GS } \\
(6522)\end{array}$ & $\begin{array}{c}\text { LA } \\
\text { GB } \\
(5337)\end{array}$ & $\begin{array}{c}\text { GB (3 cm) } \\
(5337)\end{array}$ & $\begin{array}{c}\text { L5 } \\
\text { RM } \\
(5290)\end{array}$ & $\begin{array}{c}\text { G6 } \\
(5154)\end{array}$ \\
\hline $\begin{array}{c}\text { Total } \\
\text { Improvement \% } \\
\text { Over L1 }\end{array}$ & 35.6 & 21.6 & 13.0 & 44.6 & 25.9 & 11.6 \\
$\begin{array}{c}\text { Improvement \% } \\
\text { Due to Increase } \\
\text { of Observations }\end{array}$ & 28.9 & 22.0 & 13.8 & 13.8 & 13.4 & 12.3 \\
\hline $\begin{array}{c}\text { Improvement \% } \\
\text { Due to Network } \\
\text { Extension }\end{array}$ & 6.7 & -0.4 & -0.8 & 30.8 & 12.6 & -0.7 \\
\hline
\end{tabular}

* L1 average baseline precision: $1.7 \times 10^{-8}$

for each experiment. The first row shows the percent improvement for each solution due to the combined effect of adding more stations and, therefore, at the same time, increasing the number of observations. The second row gives the percent increase due simply to the increase in the number of observations. Note that in the VLBI experiments only the equivalent of the first row was presented since in that case the numbers in the second row would be less meaningful. The difference of the first two rows in the laser table, depicted in the last row, indicates the net improvement due to network geometry only.

As can be seen from experiment L4A, the addition of the high quality laser at Greenbelt provides the most dramatic improvement, about 31 percent, even though Greenbelt is not part of the Iron Triangle. This is due to the improvement of the LAGEOS orbit from the Greenbelt STALAS observations.

The addition of the proposed Richmond station in experiment L5 gives the best improvement, about 13 percent, when all stations have a laser of the same precision such as MOBLAS. In fact, the addition of all three stations to the Iron Triangle in experiment L 2 gives poorer results than the addition of Richmond alone, or a similarly located station. This is due to the fact that Green Bank and Goldstone introduce six new unknowns to the adjustment which are not compensated for by improved geometry.

As can be seen by the negative percentages in experiments L3, L4, and L6, the addition of Greenbelt with the MOBLAS precision, or Goldstone, or both provides no improvement. Although not presented here, the results from the geometric mode solutions lead to identical conclusions. 


\section{SUMMARY}

Examining the results, we suggest that the STALAS laser at Greenbelt definitely be part of the intercomparison experiments as it is already planned, or of any other experiment. The Richmond site or a similarly suitable one should be considered as a useful addition to the Iron Triangle. From the point of view of random errors, the station at Goldstone or the VLBI at Green Bank is not considered particularly useful. At this time, the study on the effect of systematic errors on both VLBI and laser is not complete. In some cases, these systematic errors may be of great importance in deciding on a particular station configuration as opposed to purely geometric considerations. The elimination of systematic errors is likely to be aided by redundant baseline observations. Other factors may also be considered, such as antenna parameters. Finally, we would like to stress the importance of working as much as possible with estimable parameters such as baseline distances and including the VLBI observation correlations between simultaneously "observed" time delays in a multi-baseline configuration. Neglecting these correlations results in a different set of estimated standard deviations from each possible baseline combination. These estimates are generally overly optimistic compared to the unique set obtained using proper correlations.

\section{ACKNOWLEDGMENTS}

The research leading to this paper was supported by NASA/Goddard Space Flight Center Grant No. NSG 5265.

We would like to thank Irwin Shapiro for enlightening discussions regarding the VLBI data analysis.

\section{REFERENCES}

Arnold, K. (1974), "Geodetic Aspects of Laser Distance Measurements to the Moon and Radio Interference Measurements to Quasars," Gerlands Beitr. Geophysik, 84/4, pp. 249-269.

Cappellari, J. O., C. E. Velez, and A. J. Fuchs, eds. (1976). Mathematical Theory of the Goddard Trajectory Determination System, X-582-76-77, April, Goddard Space Flight Center, Greenbelt, Md.

Dermanis, A. (1977). "Design of Experiment for Farth Rotation and Baseline Parameter Determination from Very Long Baseline Interferometry," Ohio State Univ., Dept. of Geodetic Science Rept. No. 245.

Kaula, W. M. (1966). Theory of Satellite Geodesy, Blaisdell Publ. Co., Waltham, Mass.

Ma, Chopo. (1978). Very Long Baseline Interferometry Applied to Polar Motion, Relativity and Geodesy. PhD thesis, Dept. of Physics and Astronomy, Univ. of Maryland, College Park. 
Martin, T. V. et al. (1976). GEODYN System Description, Vol. I, Part 1, Wolf Research and Development Group, Riverdale, Md.

Robertson, D. S. (1975). Geodetic and Astrometric Measurements with Very-Long Baseline Interferometry. PhD thesis, Dept. of Earth \& Planetary Sciences, Massachusetts Inst. of Technology, Cambridge.

Whitney, A. R. (1974). Precision Geodesy and Astrometry via Very Long Baseline Interferometry. PhD thesis, Dept. of Earth \& Planetary Sciences, Massachusetts Inst. of Technology, Cambridge. 



\title{
THE DEEP SPACE NETWORK CAPABILITIES FOR RADIO INTERFEROMETRY
}

\author{
J. L. Fanselow \\ Jet Propulsion Laboratory \\ California Institute of Technology
}

\begin{abstract}
The Deep Space Network (DSN) consists of very high performance radio tracking stations located in Spain, California, and Australia, together with a ground communications and command facility. As part of the support for deep space tracking, a constantly improving capability for very long baseline interferometry (VLBI) exists at these stations. This paper will describe the current VLBI capability of the DSN and outline the planned capability.
\end{abstract}




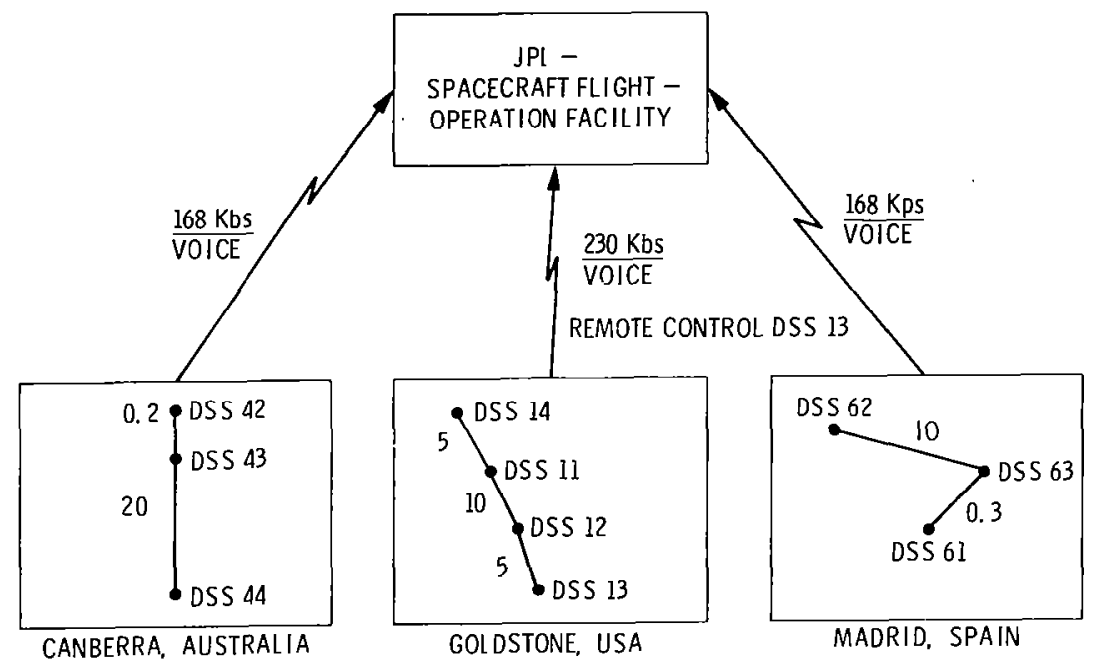

Figure 1. DSN operations facilities.

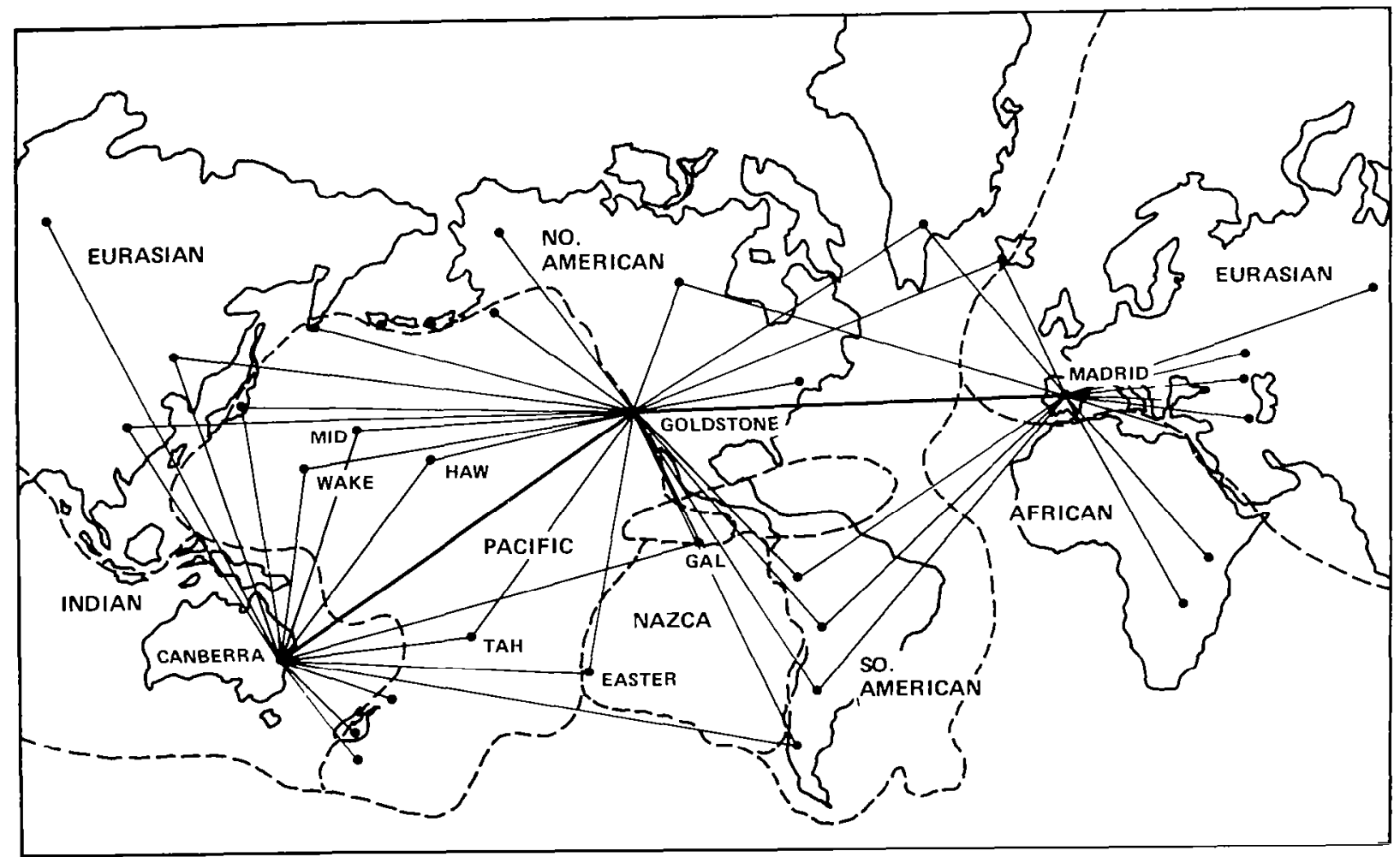

Figure 2. ARIES/64 m DSN global deployment. 
- VLBI DEVELOPMENT

- DEMONSTRATE ACCURACY LIMITS OF VLBI $11-5 \mathrm{~cm}$ ACCURACY IN VECTOR BASELINE MEASUREMENTS IN MID 1980's)

- OPERATIONAL VLBI FOR DSN CALIBRATION (DECEMBER, 1979)

- UTI, POLAR MOTION WITH 30-50 cm ACCURACY

- CloCK EPOCH OFFSET ACCURACY: 10 NSEC

- $\triangle$ F/F OFFSET ACCURACY: $\leqslant 3 \times 10^{-13}$

- WEEKLY DBSERVATIONS DSS 14-43, 14-63 BASELINES

- 24 HOUR DATA THROUGHPUT

- VLBI FOR SPACECRAFT NAVIGATION

- DEVELOP 0.'0I (50 NRAD) GLOBAL SPACECRAFT NAVIGATION INPUT FOR GALILEO ( 1981)

- DEVELOP 0!:001 (5 NRAD) LOCAL SPACECRAFT NAVIGATION INPUT FOR VOIR $(\sim 1984)$

Figure 3. DSN VLBI programs.

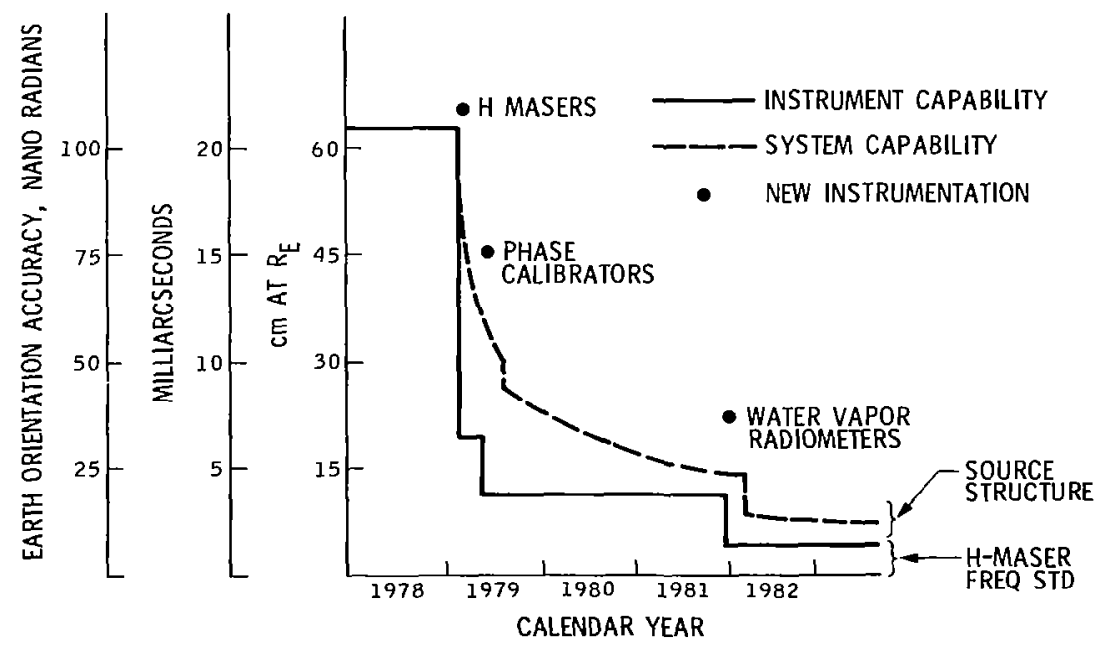

Figure 4. "Best efforts" capability for determining Earth orientation with 12 hours of VLBI data on each of 2 baselines: DSS 14-43, DSS 14-63. 


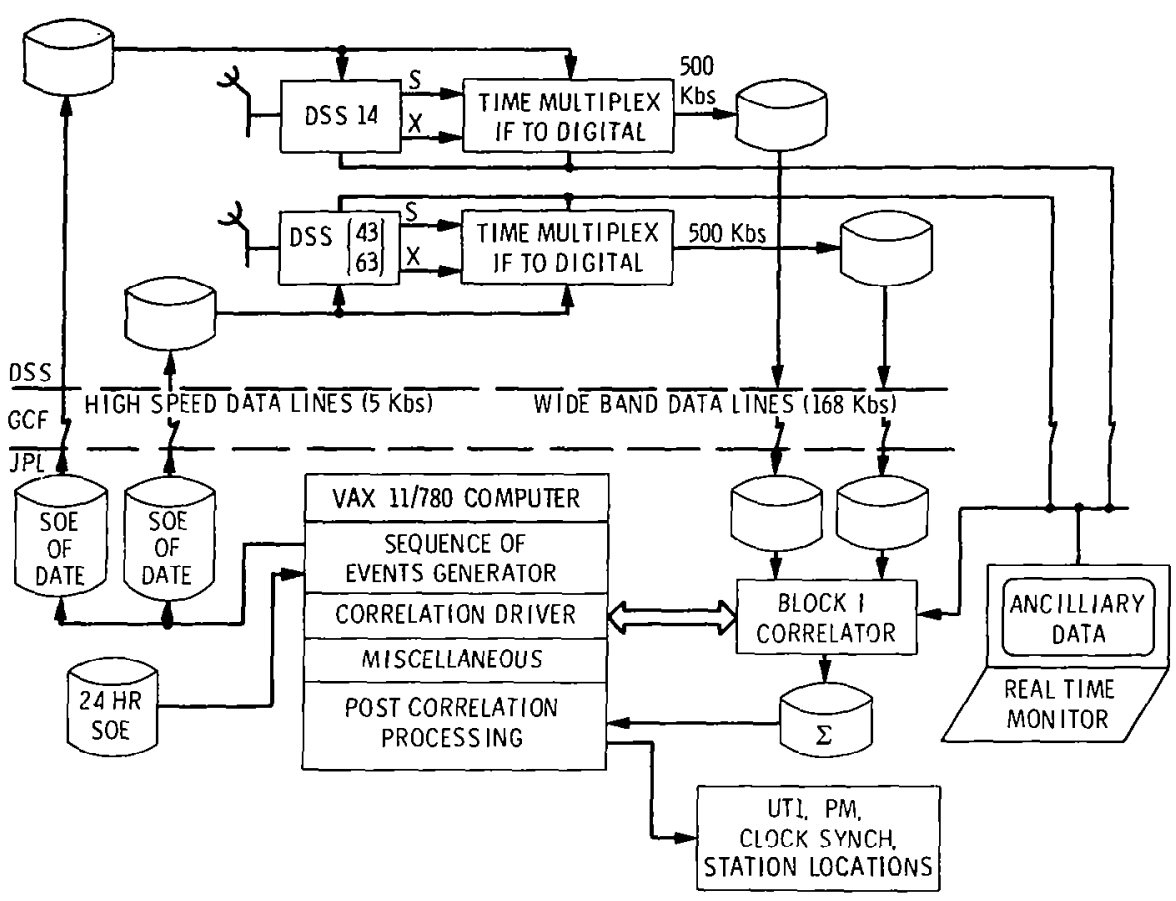

Figure 5. DSN configuration for operational VLBI . 


\title{
THE JET PROPULSION LABORATORY/ CALIFORNIA INSTITUTE OF TECHNOLOGY BLOCK II VLBI PROCESSOR
}

\author{
D. H. Rogstad \\ Jet Propulsion Laboratory \\ California Institute of Technology
}

\begin{abstract}
The very long baseline interferometry (VLBI) groups at the Jet Propulsion Laboratory (JPL) and the California Institute of Technology are jointly constructing a multistation, multi-channel crosscorrelation processor using the $250 \mathrm{kbit}$ to 4 Mbit recording format developed for the Goddard/ Haystack Mark III VLBI system. The design is modular, using bit-sliced micro-processors to perform the routine calculations for phase and delay on a per station basis, as well as for fractional bit-shift correction and Fourier transformation of the correlation coefficients on a per baseline basis. Plans call for completion in 1981 of a three station 28 channel processor that will easily expand to 10 stations, 28 channels.
\end{abstract}




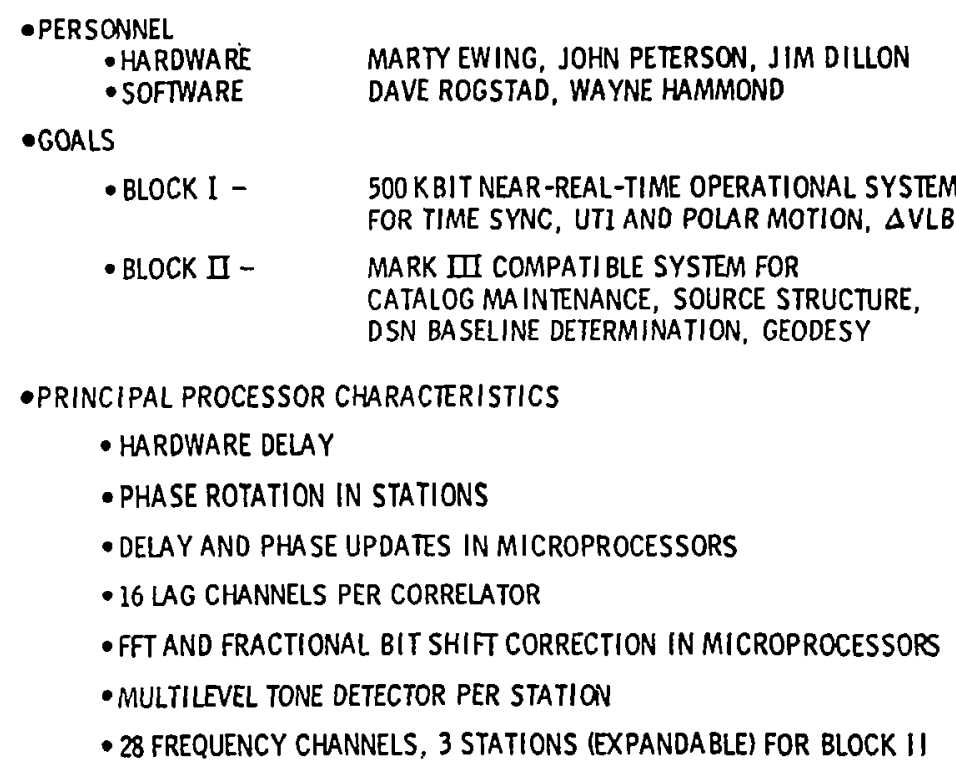

Figure 1. Block I/II VLBI processors.

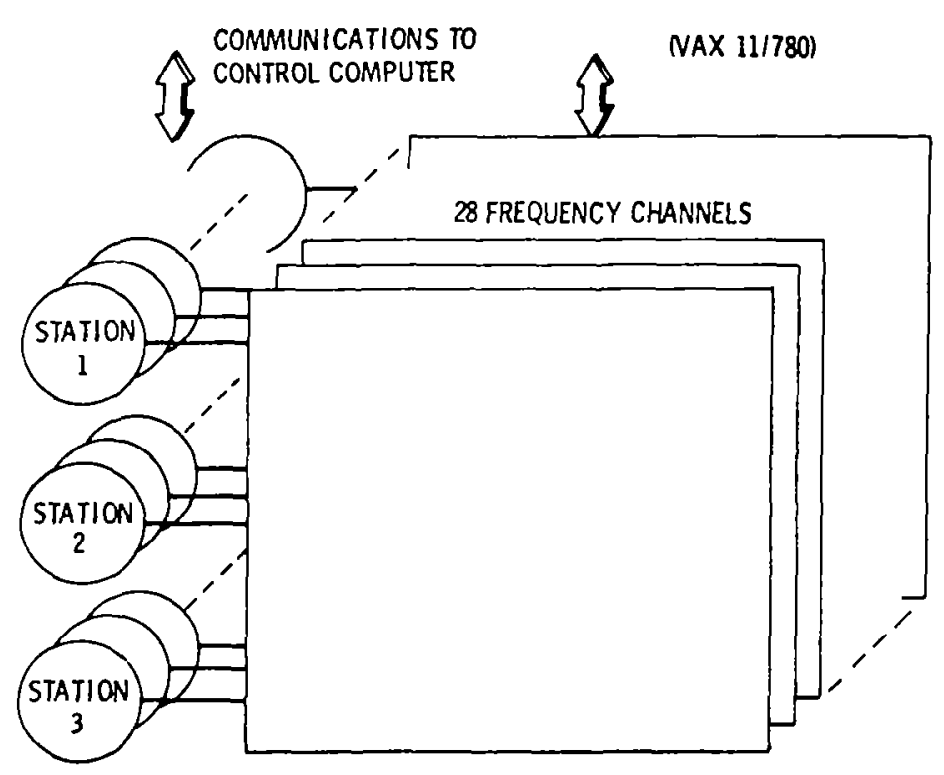

Figure 2. Schematic diagram of Block II VLBI processor. 


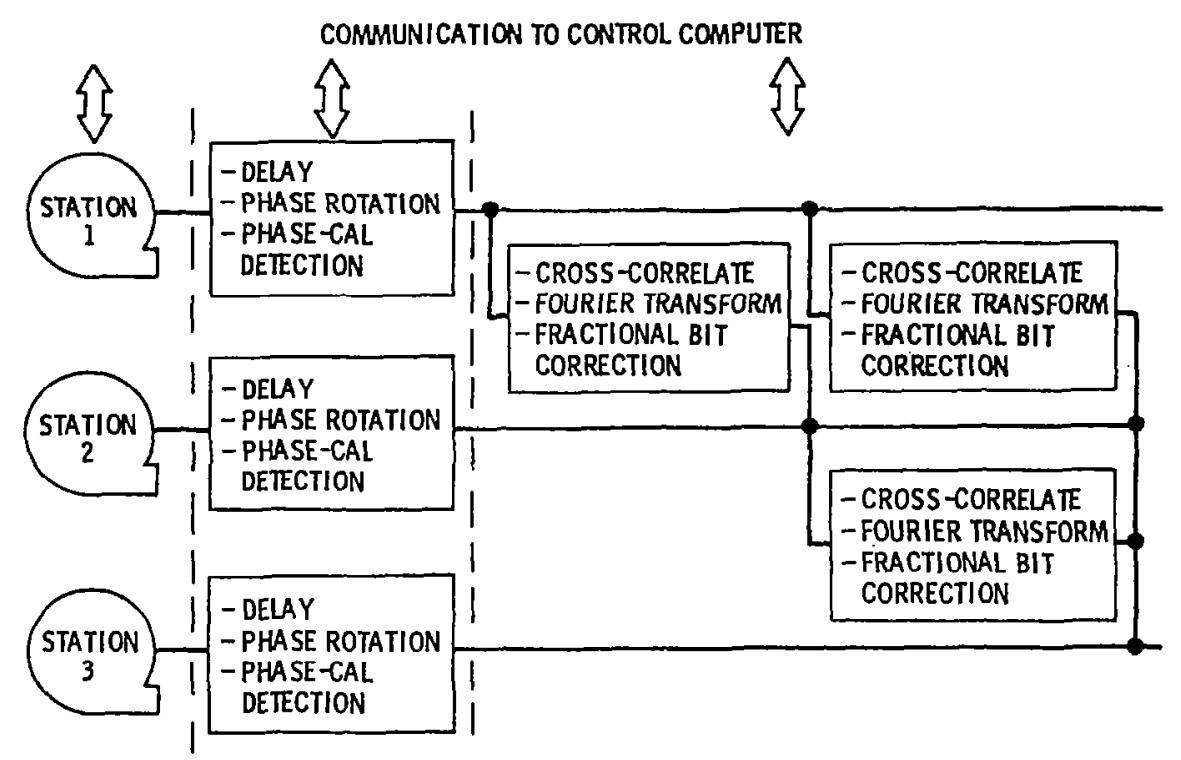

Figure 3. Block diagram of Block II VLBI processor single frequency channel.

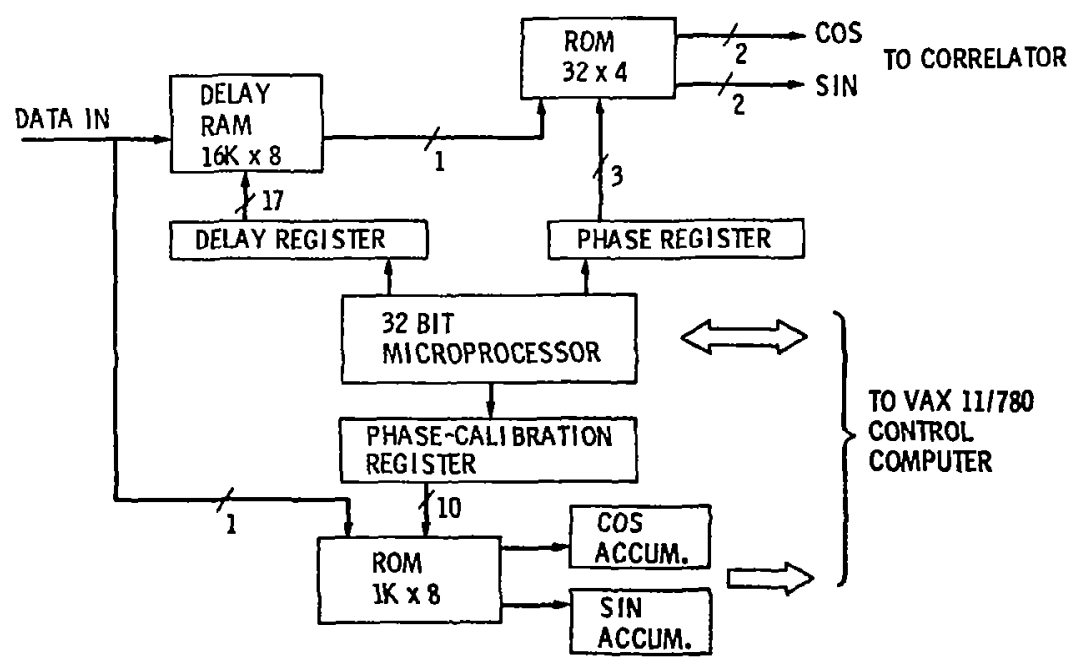

Figure 4. Schematic diagram of station module. 


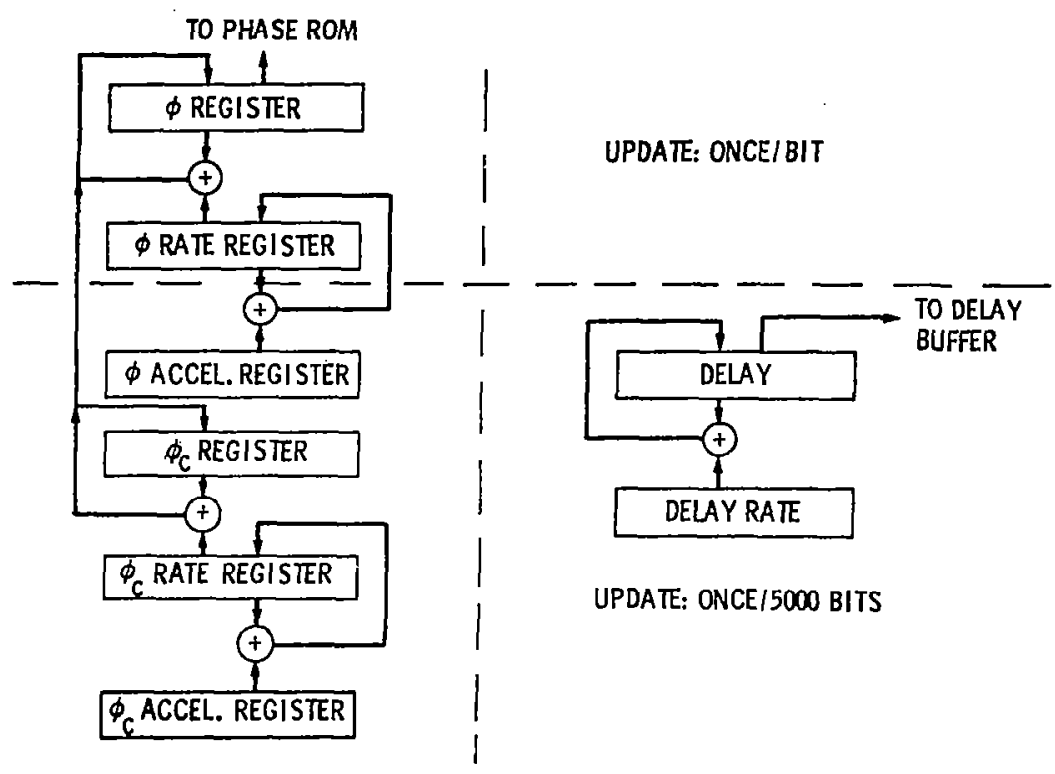

Figure 5. Station module update algorithms.

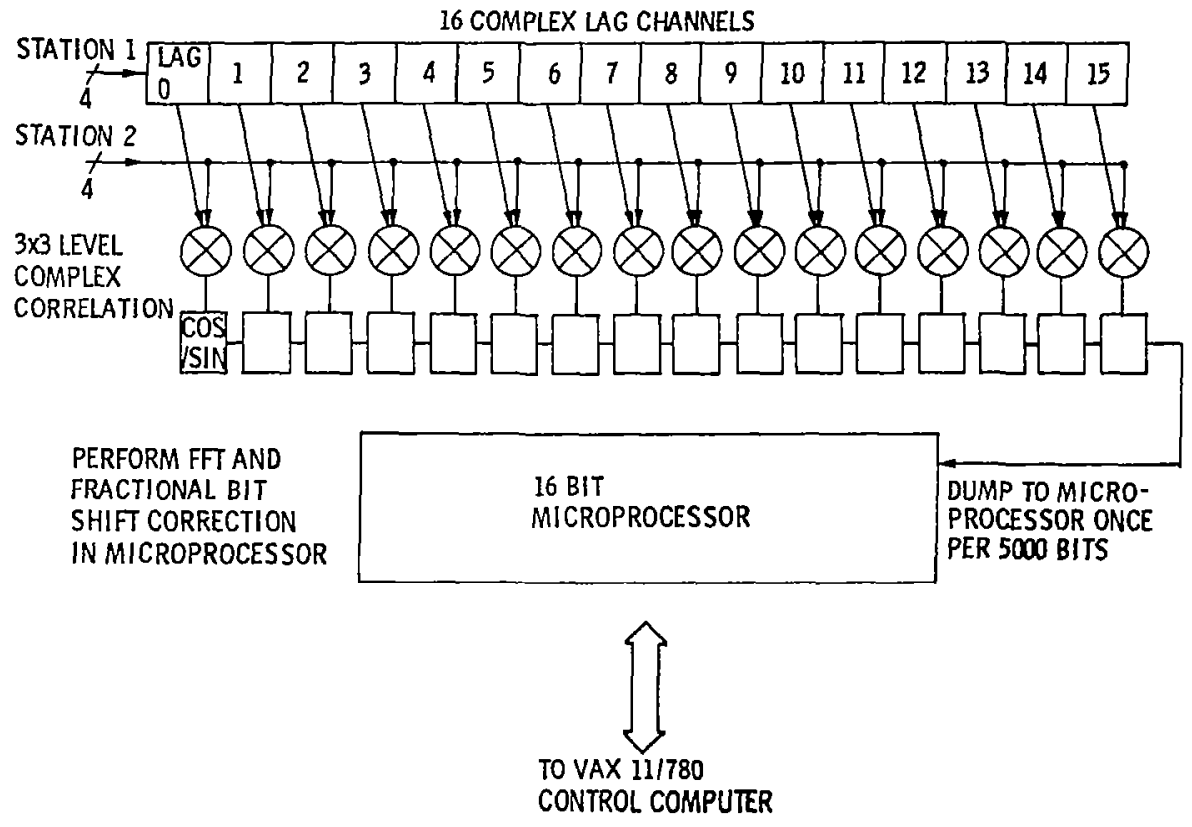

Figure 6. Schematic diagram of correlator module. 


\title{
PROJECT POLARIS: A STATUS REPORT
}

\author{
W. E. Carter \\ National Oceanic and Atmospheric Administration \\ National Ocean Survey, National Geodetic Survey
}

\begin{abstract}
Under project Polaris, NOAA's National Ocean Survey/National Geodetic Survey (NOS/NGS), in close cooperation with the National Aeronautics and Space Administration (NASA), is establishing a network of three observatories that will utilize interferometric observations of extragalactic radio sources to regularly monitor polar motion and UT 1 .

The sites will be: The Harvard Radio Astronomy Station (HRAS), near Ft. Davis, Texas; the Westford Observatory, near Boston, Massachusetts; and the U.S. Naval Observatory Time Service substation, near Richmond, Florida. Present activities are focused primarily on the upgrading and equipping of the HRAS facilities, which are expected to be in limited operation by the end of 1979 . Present planning is for the Polaris network to become fully operational about 1983.
\end{abstract}




\section{INTRODUCTION}

Polaris is an acronym formed from POLar-motion Analysis by Radio Interferometric Surveying. Under project Polaris, the National Ocean Survey/National Geodetic Survey (NOS/NGS), in close cooperation with the National Aeronautics and Space Administration (NASA), is establishing a network of three observatories that will utilize radio interferometric observations of extragalactic radio sources to regularly monitor polar motion and UT 1 .

The project was proposed by the author in 1977 to meet the newly developing demands for higher resolution and accuracy polar motion and Earth rotation data to support modern geodynamic studies. The basis for the selection of radio interferometry, rather than lunar or artificial satellite laser ranging or Doppler satellite tracking, has been reviewed in Carter and Strange (1979) and Carter (1979).

As NOS/NGS developed the Polaris project plan more fully and began to explore various options for its implementation, it became apparent that close cooperation between NOS/NGS and NASA would be beneficial to both agencies. The NASA mission to develop and demonstrate applications of space-related technology and to transfer that technology to operational agencies for application to their mission meshed well with the NOS/NGS Polaris plans. A joint work group was formed. The membership quickly increased to include six organizations: the National Oceanic and Atmospheric Administration (NOAA), NASA, U.S. Naval Observatory, Defense Mapping Agency, National Science Foundation, and the U.S. Geological Survey. They produced a document entitled "Interagency Coordination Plan for Development and Applications of Astronomic Radio Interferometry for Geophysical Sciences" (1978). This document sets out the roles, missions, and plans of the participating agencies.

\section{THE NETWORK}

A minimum of three stations is required to monitor both components of polar motion and UT1 by radio interferometry. Network design criteria include: costs of establishing and operating the network; geometrical strength and stability of candidate networks; potential for multiple users of the facilities; bureaucratic and political appeal.

The sites selected for the Polaris network are (see figure 1):

1. The Harvard Radio Astronomy Station (HRAS) located near Ft. Davis, Texas.

2. The Westford Observatory located in the Haystack Radio Observatory complex near Boston, Massachusetts.

3. The U.S. Naval Observatory Time Service Substation at Richmond, Florida.

The cost of establishing the network will be kept relatively low by using the existing facilities at HRAS and Westford. These facilities can be upgraded to meet the Polaris performance specifications without the necessity of establishing new sites. The cost of maintaining and operating the 


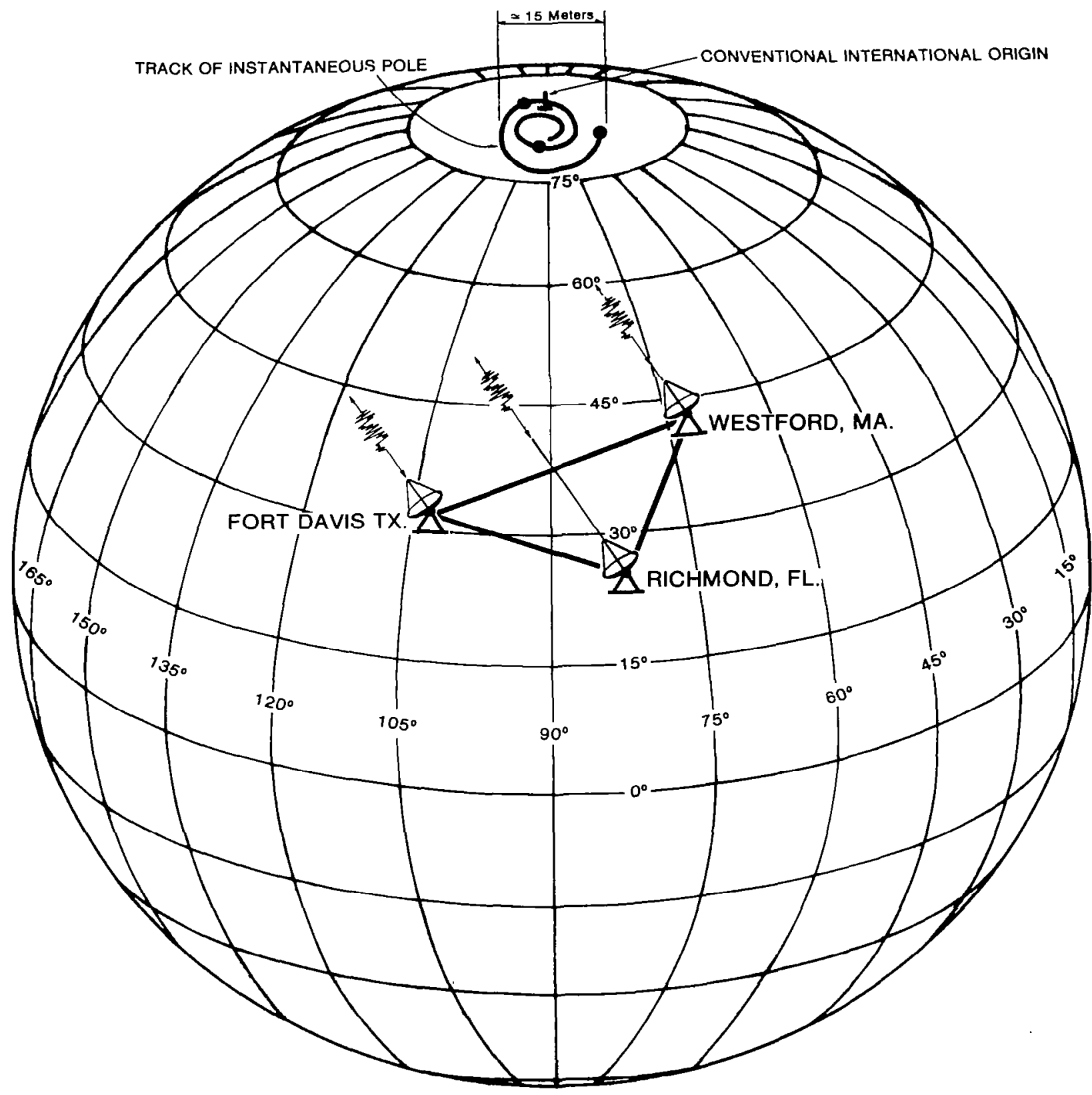

POLARIS

POLar - motion Analysis by Radio Interferometric Surveying

Figure 1. 
network will be minimized by "time sharing" the facilities and the operating personnel. Costly duplication of facilities to meet the overlapping requirements of multiple agencies will be eliminated.

According to computer simulations the HRAS-Westford-Richmond network will be capable of determining both components of polar motion to $\pm 10 \mathrm{~cm}$ and UT 1 to \pm 0.1 millisecond in an observational period of about 8 hours. Better geometrical configurations can be postulated, but the predicted advantages in performance are offset by increased costs and a greater likelihood of degradation resulting from local, regional, and plate instabilities. The three selected sites are all located in reasonably quiet seismic zones away from the edges of the North American Plate.

NASA already had plans to use HRAS in their research activities. One of the important missions of the U.S. Naval Observatory is the determination and transfer of time. That work will benefit from the development of the Richmond Polaris facility.

\section{PRESENT STATUS OF EACH STATION}

The NOS/NGS has negotiated a long tern user agreement with Harvard University to use the HRAS facilities. NOS/NGS, NASA, and Harvard Observatory have jointly formulated plans to upgrade the 27-meter prime focus, equatorially mounted telescope to meet the Polaris performance specifications. Engineers from the three organizations are cooperating in this effort. A Mark III very long base line interferometry (VLBI) Data Acquisition System is under construction. Initial test observations at HRAS are scheduled for the last quarter of 1979 into early 1980.

The NOS/NGS and the U.S. Air Force have reached broad agreement on the long term use of the Westford facilities. The Northeast Radio Observatory Corporation has submitted a detailed plan to upgrade the 17-meter, Cassegrain focus, altitude-azimuth mounted telescope. Work should begin on the long-lead time items during this fiscal year. Present plans indicate that the remaining items will be completed during FY 80 , and that the site will be ready for initial testing by early 1981 . The site will still be heavily dependent upon joint usage of certain Haystack Observatory facilities, such as the hydrogen maser frequency standard, until additional funds can be allocated.

The Carnegie Institute of Washington has agreed to donate a 17-meter, prime focus, equatorial mounted telescope to NOS/NGS. This instrument, now located in Rockville, Maryland, will be relocated in Richmond, Florida. Dismantling of the telescope should be completed this summer, and we hope to re-erect it in Richmond during 1980. The telescope is essentially a smaller aperture version of the HRAS telescope and will require many of the same upgrades. We plan to complete the Richmond station in 1981 and begin full-scale testing of the Polaris network in 1982. 


\section{DATA PROCESSING AND ANALYSIS}

During the testing and initial operational stages of the project, the Haystack Mark III correlator should have adequate capacity to handle the Polaris observational tapes with a reasonable lag time. NOS/NGS recently purchased an F model Hewlett Packard 21 MX mini-computer to replace the older slower machine that has been limiting the performance of the correlator. The new machine will allow the tapes from three stations to be processed through the correlator in about the same span of time as the observational period. Higher capacity disk storage was also added to the correlator.

NASA Goddard Space Flight Center (GSFC) has been developing a mini-computer based data processing and analyzing system for this project. The central processing unit (CPU) is a Hewlett Packard 21 MX Computer (Carter et al., 1978). NOS/NGS personnel have been assisting in developing a new software package and have used the GSFC facilities to analyze Mark I data (Robertson et al., 1978; Robertson et al., 1979). The NOS/NGS expects to have its own processing system operational at NOAA Headquarters in Rockville, Maryland, by the end of 1980.

\section{CONCLUDING REMARKS}

The predicted performance of the Polaris system represents an improvement of a full order of magnitude over our present operational systems. The goals are well within our technological capabilities. Providing that the necessary resources are obtained, as detailed in this paper, we believe that full operational status could be attained within the 1982-83 timeframe.

\section{REFERENCES}

Carter, W. E. and Strange, W. E., 1979: The National Geodetic Survey Project POLARIS. In: C. A. Whitten, R. Green, and B. K. Meade (Editors). Recent Crustal Movements 1977. Tectonophysics, 52:39-46.

Carter, W. E., 1979: Modern Methods for the Determination of Polar Motion and UT 1. Proceedings of the Tenth Annual Precise Time and Time Interval (PTTI) Applications and Planning Meeting, Washington, D.C., November 1978. NASA Technical Memorandum 80250, Goddard Space Flight Center, Greenbelt, Maryland 20771.

Robertson, D. S., Carter, W. E., Corey, B. E., Cotton, W. C., Counselman, C. C., Shapiro, I. I., Whittels, J. J., Hinteregger, H. F., Knight, C. A., Rogers, A. E. E., Whitney, A. R., Ryan, J. W., Clark, T. A., Gater, R. J., Ma, C., and Moran, J. M., 1978: Recent Results of Radio Interferometric Determinations of a Transcontinental Baseline, Polar Motion and Earth Rotation. IAU Symposium No. 82, Cadiz, Spain. In: McCarthy, D. D., and J. D. H. Pilkington (Editors), Time and the Earth's Rotation. 
Robertson, D. S., Clark, T. A., Coates, R. J., Ma, C., Ryan, J. W., Corey, B. E., Counselman, C. C., King, R. W., Shapiro, I. I., Hinteregger, H. F., Knight, C. A., Rogers, A. E. E., Whitney, A. R., Pigg, J. C., and Schupler, B. R., 1979: Polar Motion and UT1 Comparisons of VLBI, Lunar Laser, Satellite Laser, Satellite Doppler, and Conventional Astrometric Determinations; Presented at MIT and NEROC Haystack Observatory Conference on Radio Interferometry Techniques for Geodesy, June 18-22, 1979; Boston. 


\title{
PLANS FOR PHASE COHERENT LONG BASELINE INTERFEROMETRY FOR GEOPHYSICAL APPLICATIONS USING THE ANIK-B COMMUNICATIONS SATELLITE
}

\author{
W. H. Cannon and W. T. Petrachenko \\ York University \\ Canada \\ J. L. Yen \\ University of Toronto \\ Canada \\ J. A. Galt \\ Dominion Radio Astrophysical Observatory \\ Canada \\ W. B. Waltman and S. H. Knowles \\ Naval Research Laboratory \\ J. Popelar \\ Department of Energy, Mines and Resources \\ Canada
}

\begin{abstract}
A pilot project to establish an operational phase stable very long baseline interferometer (VLBI) for geophysical studies is described. The technique of phase transfer between distant oscillators has been demonstrated in an experiment using the Hermes satellite. Based on the results obtained, a phase link employing two-way transmissions of multiple pilot tones via standard satellite communications channels is designed. The project will employ the Anik-B satellite to provide a phase link between ARO in Ontario, Dominion Radio Astrophysical Observatory (DRAO) in Penticton, and Naval Research Laboratory (NRL) in Maryland Point. VLBI observations of a selection of strong compact radio sources will be carried out at two frequencies using the Canadian analog VLBI recording system. By correcting the observed fringe phase using the satellite link phase, a major source of uncertainty in VLBI geophysical measurements can be removed.
\end{abstract}

The instrument will be used to monitor Earth's rotation, the nutation of the Earth's axis, and other geodynamical observations. One objective of the project is to evaluate the feasibility of a dedicated phase coherent geophysical VLBI for UT 1 monitoring to replace the PZT's. 


\section{INTRODUCTION}

The possibility of phase coherent long baseline interferometry was pointed out by Yen et al. [1977] following a series of experiments in which the Hermes communications satellite was used to provide a data link between antennas in Canada and the United States to operate a long baseline radio interferometer in "real time". Following the success of this effort, an application by J. L. Yen and W.H. Cannon to the Federal Department of Communications, Ottawa, Canada, was approved for the use of the Anik-B communications satellite for purposes of constructing a phase coherent long baseline interferometer for geophysical applications. The Anik-B communications satellite was launched on December 9, 1978, and is owned and operated by Telesat Canada. The satellite's $12 / 14 \mathrm{GHz}$ transponder capacity has been leased from Telesat Canada by the Federal Department of Communications and has been made available, free of charge, by the Department of Communications to qualified users.

The initial objectives of this research program are to investigate the potential of domestic telecommunications satellites for conducting phase coherent long baseline interferometry and to test the capability of a phase coherent long baseline interferometer for monitoring short term variations in the Earth's rotation rate and other geophysical studies.

In carrying out these studies, the Anik-B satellite will be used to establish a phase link between the following observatories:

1. ARO, Algonquin Park, Ontario, Canada

2. DRAO, Penticton, B. C., Canada

3. NRL, Maryland Point, Maryland, U.S.A.

The astronomical receivers at each observatory will be dual frequency, $11 \mathrm{~cm}$ and $21 \mathrm{~cm}$, systems using cavity backed spiral feeds. The astronomical data will be recorded using the Canadian analog format on video cassette tape recorders with a $3.5 \mathrm{MHz}$ instantaneous bandwidth at $-3 \mathrm{~dB}$. The long baseline interferometry recording terminals used at each observatory as well as the correlator facility used to obtain the interferometer fringes will be provided by the Radio Astronomy Section of the Herzberg Institute for Astrophysics, Ottawa, Canada. Some general characteristics of this interferometer are summarized below in tables 1 and 2.

Table 1

\begin{tabular}{|l|c|c|c|}
\hline SITE & $\begin{array}{c}\text { ASTRONOMICAL } \\
\text { ANTENNA } \\
\text { DIAMETER }\end{array}$ & $\begin{array}{c}\text { PHASE LINK } \\
\text { ANTENNA } \\
\text { DIAMETER }\end{array}$ & $\begin{array}{c}\text { PHASE LINK } \\
\text { TRANSMITTER }\end{array}$ \\
\hline ARO & 46 meters & 10 meters & 20 watt TWTA \\
DRAO & 26 meters & 4.5 meters & 20 watt TWTA \\
NRL & 26 meters & 26 meters & 20 watt TWTA \\
\hline
\end{tabular}


Table 2

\begin{tabular}{|c|c|c|c|}
\hline $\begin{array}{c}\text { INTERFEROMETER } \\
\text { PARAMETER }\end{array}$ & $\underset{\text { DFAO-ARO }}{\longrightarrow}$ & $\overrightarrow{\mathrm{DRAO}-\mathrm{NRL}}$ & $\overrightarrow{\text { ARO-NRI }}$ \\
\hline $\mathrm{x}$ & $2970 \mathrm{~km}$ & $3160 \mathrm{~km}$ & $190 \mathrm{~km}$ \\
\hline $\mathbf{Y}$ & $-720 \mathrm{~km}$ & $-1260 \mathrm{~km}$ & $-540 \mathrm{~km}$ \\
\hline $\mathbf{z}$ & $-250 \mathrm{~km}$ & $-880 \mathrm{~km}$ & $-620 \mathrm{~km}$ \\
\hline TOTAL, LENGTH & $3060 \mathrm{~km}$ & $3510 \mathrm{~km}$ & $840 \mathrm{~km}$ \\
\hline EQUATORIAL LENGTH & $3050 \mathrm{~km}$ & $3400 \mathrm{~km}$ & $570 \mathrm{~km}$ \\
\hline EAST IONGITUDE & $-13^{\circ}$ & $-22^{\circ}$ & $-71^{\circ}$ \\
\hline DECLINATION & $-5^{\circ}$. & $-14^{\circ}$ & $-48^{\circ}$ \\
\hline $\begin{array}{l}\text { MIN. FRINGE } \\
\text { SPACING } 11 \mathrm{~cm}\end{array}$ & $7: 4 \times 10^{-3}$ & $6: 4 \times 10^{-3}$ & $27: 0 \times 10^{-3}$ \\
\hline $\begin{array}{l}\text { MIN. FRINGE } \\
\text { SPACING } 21 \mathrm{~cm}\end{array}$ & $14: 1 \times 10^{-3}$ & $12: 2 \times 10^{-3}$ & $51: 5 \times 10^{-3}$ \\
\hline
\end{tabular}

The entries in table 2 have been rounded off and are therefore only approximate values.

\section{THE OPERATION OF A SATELLITE PHASE LINK}

\section{General Description}

Radio interferometry ideally requires local oscillator signals at each antenna site whose phases remain coherent over extended, ideally infinite, intervals of time. In connected element interferometry, this property is easily achieved by distributing local oscillator signals via cable or microwave links from a central source. In long baseline interferometry (LBI) the separation between the antennas generally precludes the generation of phase coherent local oscillator signals by such means, and local oscillator phase coherence at each antenna site is traditionally achieved by phase locking the local oscillator, independently at each site, to separate frequency standards whose absolute phase stability is known to be very high.

Since the phase observable is extremely sensitive to geodetic and geophysical phenomena considerable effort has been devoted to improving the intrinsic (i.e., uncorrupted by atmospheric propagation effects) phase stability of long baseline interferometers. Part of this effort has been directed towards the development of frequency standards, particularly hydrogen masers, of extraordinarily high absolute phase stability [Vessot et al., 1977]. However, such devices are becoming both expensive and bulky and will require operation in very carefully controlled environments to achieve their maximum potential [Vessot et al., 1977]. 
While absolute phase stability over extended periods is essential for many applications such as the Doppler tracking of spacecraft, it is inessential for long baseline interferometry applications which carry with them the somewhat weaker requirement of phase coherence or relative phase stability over extended periods. The satellite phase link is a technical development which is intended to exploit the opportunity presented by the somewhat relaxed requirements of the interferometer applications. There appear to be no fundamental physical impediments preventing satellite phase links using inexpensive and robust rubidium frequency standards or even high quality crystal oscillators from achieving long baseline interferometer operation with intrinsic phase stability comparable to that achievable using super stable hydrogen masers.

The operation of the Anik-B satellite phase link is illustrated in figures 1 and 2. Figure 1 illustrates the recording procedure; figure 2, the playback procedure. In the Anik-B experimental program, the recording and playback operations will remain distinct. However, in an operational system, they would be combined by sending the recorded signal from one antenna to the other, whereupon the mixing shown in figure 2 would be performed, thereby achieving real-time operation of the phase link.

In the present configuration of the Anik-B phase link, the phase of the astronomical local oscillators $\phi_{1}$ and $\phi_{2}$ at antenna sites \#1 and \#2, respectively, are used, along with four phase-locked local

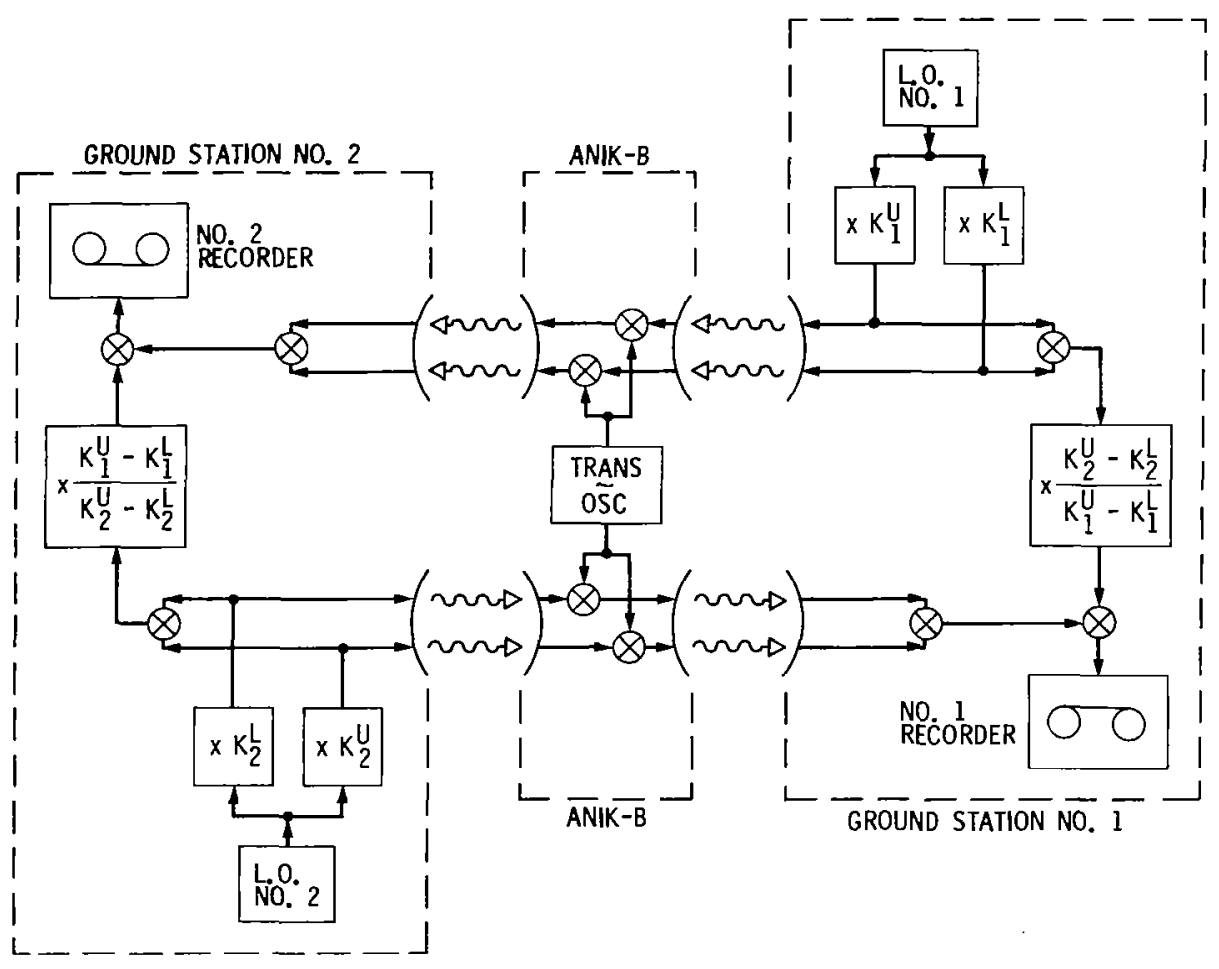

Figure 1. 


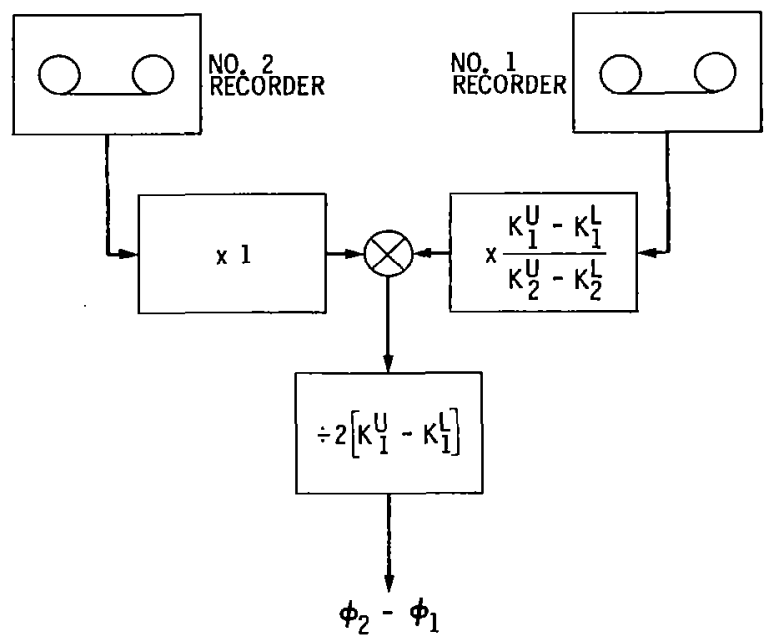

Figure 2.

oscillators to generate two pairs of signals. The high (upper) frequency pair, denoted by a superscript $\mathrm{U}$, and low (lower) frequency pair, denoted by a superscript $\mathrm{L}$, are separated by $60 \mathrm{MHz}$ in each case. These signals with phase $\mathrm{K}_{1}^{\mathrm{U}} \phi_{1}, \mathrm{~K}_{1}^{\mathrm{L}} \phi_{1}$ at site \#1 and $\mathrm{K}_{2}^{\mathrm{U}} \phi_{2}, \mathrm{~K}_{2}^{\mathrm{L}} \phi_{2}$ at site \#2, where $\mathrm{K}_{1}^{\mathrm{U}}, \mathrm{K}_{1}^{\mathrm{L}}$ and $\mathrm{K}_{2}^{\mathrm{U}}, \mathrm{K}_{2}^{\mathrm{L}}$ are known rational numbers with $\mathrm{K}_{1}^{\mathrm{U}}>\mathrm{K}_{1}^{\mathrm{L}}$ and $\mathrm{K}_{2}^{\mathrm{U}}>\mathrm{K}_{2}^{\mathrm{L}}$, are transmitted to Anik-B via the $14 \mathrm{GHz}$ up-link channel. The phases of the signals arriving at Anik-B from site \#1 are $\mathrm{K}_{1}^{\mathrm{U}}\left[\phi_{1}-\phi_{\mathrm{G} 1}-\phi_{\mathrm{M} 1}\right], \mathrm{K}_{1}^{\mathrm{L}}\left[\phi_{1}-\phi_{\mathrm{G} 1}-\phi_{\mathrm{M} 1}\right]$ and from site $\# 2$ are $\mathrm{K}_{2}^{\mathrm{U}}\left[\phi_{2}-\phi_{\mathrm{G} 2}-\phi_{\mathrm{M} 2}\right]$, $\mathrm{K}_{2}^{\mathrm{L}}\left[\phi_{2}-\phi_{\mathrm{G} 2}-\phi_{\mathrm{M} 2}\right]$, where $\phi_{\mathrm{G} 1}, \phi_{\mathrm{G} 2}$ refer to the geometric phase delays and $\phi_{\mathrm{M} 1}, \phi_{\mathrm{M} 2}$ refer to the propagation medium phase delays at the local oscillator frequency between Anik-B and the antenna sites \#1 and \#2, respectively.

In transitting the satellite, the translation oscillator on board Anik-B converts the up-link signal at $14 \mathrm{GHz}$ to a down-link signal at $12 \mathrm{GHz}$, subtracting the phase of the onboard translation oscillator $\phi_{\mathrm{T}}$. The phases $\phi_{21}^{\mathrm{U}}, \phi_{21}^{\mathrm{L}}$ of the signal arriving at site \#1 from site \#2 are therefore:

$$
\begin{aligned}
& \phi_{21}^{\mathrm{U}}=\mathrm{K}_{2}^{\mathrm{U}}\left[\phi_{2}-\phi_{\mathrm{G} 2}-\phi_{\mathrm{M} 2}\right]-\mathrm{K}_{2}^{\mathrm{U}^{\prime}}\left[\phi_{\mathrm{G} 1}+\phi_{\mathrm{M} 1}\right]-\phi_{\mathrm{T}} \\
& \left.\phi_{21}^{\mathrm{L}}=\mathrm{K}_{2}^{\mathrm{L}}\left[\phi_{2}-\phi_{\mathrm{G} 2}-\right] \mathrm{M} 2\right]-\mathrm{K}_{2}^{\mathrm{L}}\left[\phi_{\mathrm{G} 1}+\phi_{\mathrm{M} 1}\right]-\phi_{\mathrm{T}},
\end{aligned}
$$

and the phases $\phi_{12}^{\mathrm{U}}, \phi_{12}^{\mathrm{L}}$ of the signal arriving at site \#2 from site \#1 are therefore:

$$
\begin{aligned}
& \phi_{12}^{\mathrm{U}}=\mathrm{K}_{1}^{\mathrm{U}}\left[\phi_{1}-\phi_{\mathrm{G} 1}-\phi_{\mathrm{M} 1}\right]-\mathrm{K}_{2}^{\mathrm{U}^{\prime}}\left[\phi_{\mathrm{G} 2}+\phi_{\mathrm{M} 2}\right]-\phi_{\mathrm{T}} \\
& \phi_{12}^{\mathrm{L}}=\mathrm{K}_{1}^{\mathrm{L}}\left[\phi_{1}-\phi_{\mathrm{G} 1}-\phi_{\mathrm{M} 1}\right]-\mathrm{K}_{1}^{\mathrm{U}^{\prime}}\left[\phi_{\mathrm{G} 2}+\phi_{\mathrm{M} 2}\right]-\phi_{\mathrm{T}},
\end{aligned}
$$


where the factors $\mathrm{K}_{1}^{\mathrm{U}^{\prime}}, \mathrm{K}_{1}^{\mathrm{L}^{\prime}}$ and $\mathrm{K}_{2}^{\mathrm{U}^{\prime}}, \mathrm{K}_{2}^{\mathrm{L}^{\prime}}$ are introduced to account for the fact that the up-link and down-link frequencies are different.

The three stages of mixing which occur at each ground station as shown in figure 1 produce recorded signals with phases $\Phi_{1}$ and $\Phi_{2}$ at stations \#1 and \#2, respectively, where

$$
\begin{aligned}
& \Phi_{1}=\left[K_{2}^{\mathrm{U}}-K_{2}^{\mathrm{L}}\right]\left[\phi_{2}-\phi_{\mathrm{G} 2}-\phi_{\mathrm{M} 2}\right]-\left[\mathrm{K}_{2}^{\mathrm{U}^{\prime}}-\mathrm{K}_{2}^{\mathrm{L}^{\prime}}\right]\left[\phi_{\mathrm{G} 1}+\phi_{\mathrm{M} 1}\right]-\left[\mathrm{K}_{2}^{\mathrm{U}}-\mathrm{K}_{2}^{\mathrm{L}}\right] \phi_{1} \\
& \Phi_{2}=\left[\mathrm{K}_{1}^{\mathrm{U}}-\mathrm{K}_{1}^{\mathrm{L}}\right]\left[\phi_{1}-\phi_{\mathrm{G} 1}-\phi_{\mathrm{M} 1}\right]-\left[\mathrm{K}_{1}^{\mathrm{U}^{\prime}}-\mathrm{K}_{1}^{\mathrm{L}^{\prime}}\right]\left[\phi_{\mathrm{G} 2}+\phi_{\mathrm{M} 2}\right]-\left[\mathrm{K}_{1}^{\mathrm{U}}-\mathrm{K}_{1}^{\mathrm{L}}\right] \phi_{2} .
\end{aligned}
$$

Now $\mathrm{K}_{1}^{\mathrm{U}^{\prime}}-\mathrm{K}_{1}^{\mathrm{L}^{\prime}}=\mathrm{K}_{1}^{\mathrm{U}}-\mathrm{K}_{1}^{\mathrm{L}}$ and $\mathrm{K}_{2}^{\mathrm{U}^{\prime}}-\mathrm{K}_{2}^{\mathrm{L}^{\prime}}=\mathrm{K}_{2}^{\mathrm{U}}-\mathrm{K}_{2}^{\mathrm{L}}$, since the frequency separation on the uplink and down-link are identical. Hence, the recorded phases at each antenna site are:

$$
\begin{aligned}
& \Phi_{1}=\left[\mathrm{K}_{2}^{\mathrm{U}}-\mathrm{K}_{2}^{\mathrm{L}}\right]\left[\phi_{2}-\phi_{1}-\phi_{\mathrm{G}}-\phi_{\mathrm{M}}\right] \\
& \Phi_{2}=\left[\mathrm{K}_{1}^{\mathrm{U}}-\mathrm{K}_{1}^{\mathrm{L}}\right]\left[\phi_{1}-\phi_{2}-\phi_{\mathrm{G}}-\phi_{\mathrm{M}}\right]
\end{aligned}
$$

where

$$
\begin{aligned}
& \phi_{\mathrm{G}}=\phi_{\mathrm{G} 1}+\phi_{\mathrm{G} 2} \\
& \phi_{\mathrm{M}}=\phi_{\mathrm{M} 1}+\phi_{\mathrm{M} 2} .
\end{aligned}
$$

Following this, it is clear that the playback procedure shown in figure 2 produces the local oscillator phase $\phi_{2}-\phi_{1}$.

\section{Timing Requirements}

The timing requirements for the data manipulation in a satellite phase link of the Anik-B type can be estimated from the expressions for the recorded signals $\Phi_{1}$ and $\Phi_{2}$. The recorded signals $\Phi_{1}$ and $\Phi_{2}$ are seen to depend on the sum of the phase difference $\phi_{2}-\phi_{1}$ and the phases $\phi_{\mathrm{G}}$ and $\phi_{\mathrm{M}}$ all of which, in this treatment, have been referenced to the local oscillator frequency $\omega_{0}$.

For local oscillators phase locked to even relatively poor frequency standards with frequency instabilities as large as $10^{-10}$, the phase rate $\left|\frac{d}{d t}\left(\phi_{2}-\phi_{1}\right)\right|$ is of the order of $10^{-10} \omega_{\mathrm{o}}$ radians $\sec ^{-1}$. For an observing frequency of $3 \mathrm{GHz}\left(\omega_{\mathrm{o}}=6 \pi \times 10^{9}\right)$, it follows that $\left|\frac{\mathrm{d}}{\mathrm{dt}}\left(\phi_{2}-\phi_{1}\right)\right| \cong 2$ radians $\mathrm{sec}^{-1}$ and a timing error of $\epsilon$ seconds introduces an error in the measurement of the order of $2 \epsilon$ radians.

The phase rate $\frac{d \phi G}{d t}$ is a Doppler shift due to the motion of the satellite relative to the ground stations, changing the total geometric path length of the phase link. Yen [1977] has shown that for a satellite in a geosynchronous orbit inclined at $0.2^{\circ}$ to the equator that 


$$
0.2586 \mathrm{sec}<\frac{\phi_{\mathrm{G}}}{\omega_{\mathrm{o}}}<0.2588 \mathrm{sec}
$$

and that

$$
\frac{1}{\omega_{\mathrm{o}}}\left|\frac{\mathrm{d} \phi_{\mathrm{G}}}{\mathrm{dt}}\right|<5 \times 10^{-9} .
$$

It follows that the phase rate $\left|\mathrm{d} \phi_{\mathrm{G}} / \mathrm{dt}\right|$ is the order of $5 \times 10^{-9} \omega_{\mathrm{o}}$ radians $\mathrm{sec}^{-1}$. For an observing frequency of $3 \mathrm{GHz}\left(\omega_{\mathrm{o}}=6 \pi \times 10^{9}\right),\left|\mathrm{d} \phi_{\mathrm{G}} / \mathrm{dt}\right| \cong 10^{2}$ radians sec $^{-1}$ and a timing of $\epsilon$ seconds introduces an error in the measurement of the order of $10^{2} \epsilon$ radians.

The phase rate $\left|\mathrm{d} \phi_{\mathrm{M}} / \mathrm{dt}\right|$ is due to temporal variations in the propagation medium along the path of the phase link and will be due to temporal variations in the tropospheric refractive index and the ionospheric integrated electron density. Rcasonable upper limits can be placed on $\mathrm{d} \phi_{\mathrm{M}} / \mathrm{dt}$ by considering the magnitude of the tropospheric and ionospheric phase delays and the typical time scales of their variations.

$\phi_{\mathrm{M}}$ can be decomposed into a tropospheric contribution $\phi_{\mathrm{MT}}$ and an ionospheric contribution $\phi_{\mathrm{MI}}$,

$$
\phi_{\mathrm{M}}=\phi_{\mathrm{MT}}+\phi_{\mathrm{MI}} \text {. }
$$

If $\mathrm{L}_{\mathrm{T}}$ is the excess path length over vacuuo imposed on the phase link by the presence of the troposphere (the troposphere imposes roughly 2 meters of phase delay in the zenith direction over each antenna site) then

$$
\left|\frac{\mathrm{d} \phi_{\mathrm{MT}}}{\mathrm{dt}}\right|=\frac{\omega_{\mathrm{o}}}{\mathrm{c}}\left|\frac{\mathrm{dL}}{\mathrm{dt}}\right| \text { radians } \mathrm{sec}^{-1} .
$$

Since the phase link antennas pointing to Anik-B have reasonably large zenith angles, we will take $\mathrm{L}_{\mathrm{T}} \sim 8$ meters if we assume that $\mathrm{L}_{\mathrm{T}}$ can at most change by as much as 10 percent $(80 \mathrm{~cm})$ in an interval of the order of 1000 seconds ( 15 minutes), then

$$
\left|\frac{\mathrm{d} \phi_{\mathrm{MT}}}{\mathrm{dt}}\right| \leqslant 2.6 \times 10^{-12} \omega_{\mathrm{o}} \text { radians } \mathrm{sec}^{-1} \text {. }
$$

For an observing frequency of $3 \mathrm{GHz}\left(\omega_{\mathrm{o}}=6 \pi \times 10^{9}\right),\left|\mathrm{d} \phi_{\mathrm{MT}} / \mathrm{dt}\right| \leqslant 5 \times 10^{-2} \mathrm{radians} \mathrm{sec}^{-1}$ and a timing error of $\epsilon$ seconds introduces an error in the measurement of the order of $5 \times 10^{-2} \epsilon$ radians.

The ionospheric contribution $\phi_{\mathrm{MI}}$ is given [Rogers and Evans, 1968] by

$$
\phi_{\mathrm{MI}}=-\frac{2}{\omega_{\mathrm{o}}} \quad \frac{2 \pi \mathrm{e}^{2}}{\mathrm{~m}_{\mathrm{e}} \mathrm{c}} \int_{\mathrm{L}} \mathrm{N}_{\mathrm{e}} \mathrm{d} \mathrm{l},
$$

where $e$ is the charge on the electron (esu), $\mathrm{m}_{\mathrm{e}}$ is the mass of the electron, and $\mathrm{c}$ is the velocity of light; $\mathrm{N}_{\mathrm{e}}$ is the number of density of free electrons and the integral is taken along the path of the phase link. Ionospheric studies indicate that $\phi_{\mathrm{M} I}$ is generally in the range 


$$
-\frac{10^{11}}{\omega_{0}} \geqslant \phi_{\mathrm{MI}} \geqslant-\frac{3 \cdot 5 \times 10^{12}}{\omega_{\mathrm{o}}} \text { radians, }
$$

in the zenith direction at mid-latitudes. Since the phase link antennas pointing to Anik-B have reasonably large zenith angles, we will take as a worst case

$$
-\frac{4 \times 10^{11}}{\omega_{\mathrm{o}}} \geqslant \phi_{\mathrm{MI}} \geqslant-\frac{15 \times 10^{12}}{\omega_{\mathrm{o}}} \text { radians. }
$$

The ionosphere is spatially and temporally quite variable with travelling ionospheric disturbances (TID's) in the $F$ region and sporadic $E$ disturbances in the $E$ region modulating the integrated electron content by as much as \pm 2 percent in an interval as short as 10 minutes. In addition, most dramatically at sunrise, but also at sunset, the integrated electron content changes by as much as an order of magnitude in an interval as short as 3 hours.

It follows from this that

$$
\left|\frac{\mathrm{d} \phi_{\mathrm{MI}}}{\mathrm{dt}}\right| \stackrel{\sim}{\sim} \frac{1 \cdot 5 \times 10^{9}}{\omega_{\mathrm{o}}} \text { radians } \sec ^{-1}
$$

For an observing frequency of $3 \mathrm{GHz}\left(\omega_{\mathrm{O}}=6 \pi \times 10^{9}\right),\left|\mathrm{d} \phi_{\mathrm{MI}} / \mathrm{dt}\right| \approx 8 \times 10^{-2} \mathrm{radians} \mathrm{sec}^{-1}$ and a timing error of $\epsilon$ seconds introduces an error in the measurement of the order of $8 \times 10^{-2} \epsilon$ radians.

From the above analysis, it is apparent that synchronizing the data on playback to an accuracy of $\epsilon= \pm 10^{-4}$ seconds is sufficient to insure that errors due to timing do not contribute more than $10^{-2}$ radians $(\sim 0.5)$ of phase error to the measurement.

The phase rate $d \phi_{G}(t) / d t$ is caused by the motion of the satellite at time $t$, where $t$ refers to time kept by a set of synchronized clocks at rest in the rotating frame of the Earth. Phase link signals transitting Anik-B at time $t$ arrive at station $\# 1$ at time $t+d(t)$ and at station $\# 2$ at time $t+d(t)+$ $\delta(t)$, where $\delta(t)$ represents the inequality of the travel times from Anik-B to ground stations \# 1 and $\# 2$, respectively. Because of retarded electro-magnetic effects, the Doppler shift phase recorded at station \#1 $\varphi_{\mathrm{G}}^{1}$ is given by

$$
\phi_{\mathrm{G}}^{1}(\mathrm{t})=\int_{\mathrm{t}_{\mathrm{o}}}^{\mathrm{t}} \omega_{\mathrm{o}} \frac{\mathrm{dL}_{\mathrm{G}}}{\mathrm{dt}}\left(\mathrm{t}^{\prime}-\mathrm{d}\left(\mathrm{t}^{\prime}\right)\right) \mathrm{dt^{ \prime }}
$$

and the Doppler shift phase recorded at station $\# 2 \phi_{\mathrm{G}}^{2}$ is given by

$$
\phi_{\mathrm{G}}^{2}(\mathrm{t})=\int_{t_{\mathrm{o}}}^{\mathrm{t}} \omega_{\mathrm{o}} \frac{\mathrm{dL}_{\mathrm{G}}}{\mathrm{dt}}\left(\mathrm{t}^{\prime}-\mathrm{d}\left(\mathrm{t}^{\prime}\right)-\delta\left(\mathrm{t}^{\prime}\right)\right) d \mathrm{t}^{\prime},
$$

where $t_{o}$ is an arbitrary epoch and $L_{G}(t)$ is the geometric length of the phase link path. 
Clearly,

however,

$$
\begin{gathered}
\phi_{G}^{1}(t) \neq \phi_{G}^{2}(t) ; \\
\phi_{G}^{1}(t)=\phi_{G}^{2}(t+\delta(t)) .
\end{gathered}
$$

So, to cancel the effects of the satellite Doppler shift in the playback procedure, it is necessary to delay the signal from tape recorder \#1 relative to the signal from tape recorder \#2 by an amount $\delta(t)$. For simplicity, this delaying operation was omitted from figure 2 .

For ground stations in the beam of Anik-B, $\delta(t)$ is about 6 msec. However, we sce from the analysis above that $\delta(t)$ need only be known with an accuracy of the order of $\pm 0.1 \mathrm{msec}$. This implies that the location of Anik-B relative to the ground stations must be known with an accuracy of only $\pm 30 \mathrm{~km}$.

\section{Phase Link Performance Possibilities}

A discussion of the potential phase stability of the Anik-B phase link can be partitioned into two regimes: the first pertaining to time scales of roughly $10^{2}$ seconds or less over which environmental, atmospheric, and ionospheric conditions are relatively constant; and the second pertaining to time scales of roughly $10^{3}$ seconds or more over which environmental, atmospheric, and ionospheric conditions are changeable.

On short-time scales, the phase stability of the Anik-B phase link will be largely dependent on signal to noise ratios within the link itself and on the general stability of the phase lock loops in the electronics.

On long-time scales, the phase stability of the Anik-B phase link will be limited by environmental factors which, if left uncontrolled, will considerably degrade the long-term performance of the phase link. In the case of Anik-B, there are two principal limitations which will contribute to the degradation of the link's performance.

The first of these will be the largely temperature-dependent instrumental phase shifts occurring principally in the signal cables of the ground stations. These phase shifts will in general not be equal at both ground stations and so will not be cancelled by the overall operation of the phase link. Fortunately, a variety of techniques are available to either stabilize, eliminate, or calibrate these phase shifts, and it would seem that a persistent and systematic attack on this problem could eventually reduce these effects to something of the order of $\pm 5^{\circ}-10^{\circ}$ of phase rms.

The second long-term limitation on the performance of the Anik-B phase link is imposed by the ionosphere. The ionosphere degrades the performance of the phase link as the result of a small second order dispersive effect arising from the dual frequency nature of the two-way link. A dispersive phase shift developing between the two transmitted frequencies traversing the ionosphere on 
the up-link at $14 \mathrm{GHz}$ is in general not exactly cancelled by a corresponding dispersive phase shift between the two received frequencies transversing the ionosphere on the down-link at $12 \mathrm{GHz}$. The small non-cancelling dispersive phase shift denotes $\delta \phi_{M I}$ has been shown [Cannon, 1979] to be given by

$$
\delta \phi_{M I}(\mathrm{t})=\frac{\alpha}{1-\alpha} \cdot \frac{\mathrm{K}^{\mathrm{U}}-\mathrm{K}^{\mathrm{L}}}{\mathrm{K}^{\mathrm{U}} \mathrm{K}^{\mathrm{L}}} \cdot\left[\frac{\mathrm{A}_{1}(\mathrm{t})-\mathrm{A}_{2}(\mathrm{t})}{\omega_{\mathrm{o}}}\right],
$$

where

$$
\begin{gathered}
\mathrm{K}^{\mathrm{U}}=\frac{\mathrm{K}_{1}^{\mathrm{U}}+\mathrm{K}_{2}^{\mathrm{U}}}{2} \quad \mathrm{~K}^{\mathrm{L}}=\frac{\mathrm{K}_{1}^{\mathrm{L}}+\mathrm{K}_{2}^{\mathrm{L}}}{2} \\
\alpha=\frac{\mathrm{K}^{\mathrm{U}}+\mathrm{K}^{\mathrm{L}}}{\mathrm{K}^{\mathrm{U}} \mathrm{K}^{\mathrm{L}}} \frac{\omega_{T}}{\omega_{\mathrm{o}}}-\frac{1}{\mathrm{~K}^{\mathrm{U}} \mathrm{K}^{\mathrm{L}}}\left(\frac{\omega_{\mathrm{T}}}{\omega_{\mathrm{o}}}\right)^{2} \\
\mathrm{~A}_{1}(\mathrm{t})=\frac{2 \pi \mathrm{e}^{2}}{\mathrm{~m}_{\mathrm{c}} \mathrm{c}} \int_{\mathrm{L}_{1}} \mathrm{~N}_{\mathrm{e}} \mathrm{d} \ell \\
\mathrm{A}_{2}(\mathrm{t})=\frac{2 \pi \mathrm{e}^{2}}{\mathrm{~m}_{\mathrm{e}} \mathrm{c}} \int_{L_{2}} \mathrm{~N}_{\mathrm{e}} \mathrm{d} \ell .
\end{gathered}
$$

In the above formulas $\omega_{T}$ is the frequency of the onboard translation oscillator or equivalently the frequency difference between the up-link and down-link signals. The paths $L_{1}, L_{2}$ for the line integrals defining quantities $A_{1}(t), A_{2}(2)$ are taken along the phase link from the ground station to the satellite over stations \#1 and \#2, respectively.

In general, the quantity $\left[A_{1}(t)-A_{2}(t)\right]$ will be nonvanishing and will usually attain its largest values when the interferometer baseline straddles the day/night terminator on Earth. In this worst case situation, an order of magnitude difference may easily appear between $A_{1}(t)$ and $A_{2}(t)$. For typical values of $A_{1}(t), A_{2}(t)$, the second order ionospheric effect could produce a dispersive phase error $\delta \phi_{\mathrm{MI}}$ as large as $\pm \pi$ for several hours as the terminator passed through. However, aside from the day/night transition periods the quantities $A_{1}(t), A_{2}(t)$ are more likely to differ by factors of 2 to 3 or less. Under these circumstances, $\delta \phi_{\mathrm{MI}}$ can be expected to be typically of the order of $\pm \pi / 4$ rms. In addition, under both these circumstances, some portion of this second-order ionospheric effect is predictable on the basis of standard ionospheric models and may be removed in the data processing stage.

Finally, although the Anik-B program has no provision for it, it is of course possible to calibrate these dispersive ionospheric phase errors by the addition of a third frequency to the two-way phase link. With the bandwidth available (200 MHz) on Anik-B the dispersive ionospheric phase errors 
could be calibrated with an accuracy of only \pm 10 percent. However, this would reduce typical values of $\delta \phi_{\mathrm{MI}}$ to something of the order of $\pm 5^{\circ} \mathrm{rms}$.

These results are summarized in figure 3 which shows, for comparison, the operational and theoretical performance of the VLG-1 1 hydrogen maser [Vessot et al., 1977] and the operational performance of the Hermes (CTS) satellite phase link [Waltman, Knowles, et al., 1979]. It would appear that the performance of the Hermes (CTS) satellite phase link which degrades rather badly between time scales of $10^{2}$ seconds and $10^{3}$ seconds and longer is perhaps due to uncalibrated instrumental and environmental effects. If these are adequately remedied, a satellite phase link performance should be able to follow trends given by the solid lines in figure 3, achieving an equivalent frequency stability of the order of parts in $10^{16}$ on time scales of the order of 12 hours, and parts in $10^{17}$ on time scales of the order of 4 to 5 days.

\section{APPLICATION OF THE ANIK-B PHASE LINK TO THE MEASUREMENT OF VARIATIONS IN EARTH ROTATION}

\section{General Method of Measurement}

It is intended initially to apply the Anik-B phase link interferometer to the special purpose of measuring short-term variation in the Earth's rotation rate. This is a good application of a satellite

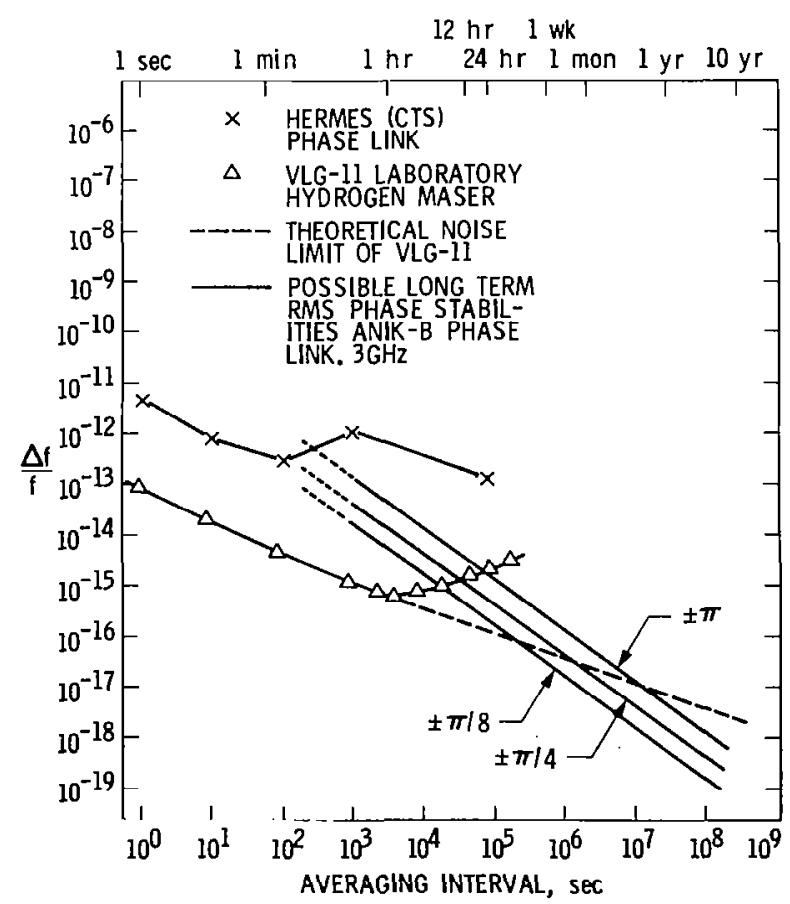

Figure 3. 
phase link interferometer as such measurements depend strongly on the longer-term phase stability of the interferometer rather than on its short-term phase stability [Broten, 1979].

The Earth's variable sidereal rotation rate $\Omega(t)$ is related to its mean sidcreal rotation rate $\Omega_{\mathrm{o}}$ by the small dimensionless parameter $\mathrm{m}_{3}(\mathrm{t})$

$$
\Omega(t)=\Omega_{0}\left[1+m_{3}(t)\right] .
$$

The quantity most readily measured by observing successive times of meridian transits of stars against an atomic clock is $\tau(t)$, the amount by which the variably rotating Earth lags behind a hypothetical uniformly rotating Earth.

$$
\tau(\mathrm{t})=-\int_{\mathrm{t}_{0}}^{\mathrm{t}} \mathrm{m}_{3}\left(\mathrm{t}^{\prime}\right) \mathrm{dt^{ \prime }}
$$

At any time $t$, the phase of the interferometer can be represented by $\Phi(t)$ where

$$
\Phi(t)=\phi_{\mathrm{g}}(\mathrm{t})+\phi_{\mathrm{c}}(\mathrm{t})+\phi_{\mathrm{A} 1}(\mathrm{t})+\phi_{\mathrm{A} 2}(\mathrm{t})+\phi_{\mathrm{I}}(\mathrm{t})
$$

and where

- $\phi_{c}(t)$ is the so-called clock phase arising as a result of relative phase shifts between two local oscillator signals

- $\phi_{\mathrm{A} 1}(\mathrm{t}), \phi_{\mathrm{A} 2}(\mathrm{t})$ are the result of relative phase delays on the astronomical signal due to the presence of the troposphere and ionosphere respectively

- $\phi_{\mathrm{I}}(\mathrm{t})$ is the so-called instrumental phase arising as a result of uncompensated phase delays between the receiving/recording systems at each interferometer terminus

- $\phi_{g}(t)$ is the geometric phase.

For an interferometer of baseline length $b$, east longitude $\lambda_{b}$, declination $\phi_{\mathrm{b}}{ }^{\prime}$, observing at frequency $\omega_{\mathrm{o}}$, a source with right ascension $\alpha_{\mathrm{s}}$ and declination $\delta_{\mathrm{s}}$ at Greenwich sidereal time $\theta$, the value of the geometric phase is given by

$$
\phi_{\mathrm{g}}=\frac{\omega_{\mathrm{o}} \mathrm{b}}{\mathrm{c}}\left[\sin \phi_{\mathrm{b}}{ }^{\prime} \sin \delta_{\mathrm{s}}+\cos \phi_{\mathrm{b}}{ }^{\prime} \cos \delta_{\mathrm{s}} \cos \left(\theta+\lambda_{\mathrm{b}}-\alpha_{\mathrm{s}}\right)\right] .
$$

Beginning at an epoch $t_{o}$ and carrying a continuous "fringe count" forward in time for an interval $\mathrm{t}_{\mathrm{o}} \leqslant \mathrm{t} \leqslant \mathrm{t}_{\mathrm{o}}+\tau$ yields the observable function of time

$$
\left[\Phi(\mathrm{t})-\Phi\left(\mathrm{t}_{\mathrm{o}}\right)\right]=\phi_{\mathrm{g}}(\mathrm{t})-\phi_{\mathrm{g}}\left(\mathrm{t}_{\mathrm{o}}\right)+\phi_{\mathrm{c}}(\mathrm{t})-\phi_{\mathrm{c}}\left(\mathrm{t}_{\mathrm{o}}\right)+\phi_{\mathrm{Al}}(\mathrm{t})-\phi_{\mathrm{A} 1}\left(\mathrm{t}_{\mathrm{o}}\right)+\phi_{\mathrm{A} 2}(\mathrm{t})-\phi_{\mathrm{A} 2}\left(\mathrm{t}_{\mathrm{o}}\right)+\phi_{1}(\mathrm{t})-\phi_{\mathrm{I}}\left(\mathrm{t}_{\mathrm{o}}\right)
$$


Repeating the measurement, an interval of $n$ sidereal days later for a corresponding interval $t_{o}+n T$ $\leqslant \mathrm{t} \leqslant \mathrm{t}_{\mathrm{o}}+\mathrm{nT}+\tau$ yields

$$
\begin{aligned}
{\left[\Phi(\mathrm{t}+\mathrm{nT})-\Phi\left(\mathrm{t}_{\mathrm{o}}+\mathrm{nT}\right)\right]=} & \phi_{\mathrm{g}}(\mathrm{t}+\mathrm{nT})-\phi_{\mathrm{g}}\left(\mathrm{t}_{\mathrm{o}}+\mathrm{nT}\right)+\phi_{\mathrm{c}}(\mathrm{t}+\mathrm{nT})-\phi_{\mathrm{c}}\left(\mathrm{t}_{\mathrm{o}}+\mathrm{nT}\right) \\
& +\phi_{\mathrm{Al}}(\mathrm{t}+\mathrm{nT})-\phi_{\mathrm{A} 1}\left(\mathrm{t}_{\mathrm{o}}+\mathrm{nT}\right)+\phi_{\mathrm{A} 2}(\mathrm{t}+\mathrm{nT})-\phi_{\mathrm{A} 2}\left(\mathrm{t}_{\mathrm{o}}+\mathrm{nT}\right) \\
& +\phi_{\mathrm{I}}(\mathrm{t}+\mathrm{nT})-\phi_{\mathrm{I}}\left(\mathrm{t}_{\mathrm{o}}+\mathrm{nT}\right),
\end{aligned}
$$

where the interval $\mathrm{T}$ represents the a priori estimate of the length of the sidereal day. Differencing these two data sets gives

$$
\begin{aligned}
& \left\{\left[\Phi(t+n T)-\Phi\left(t_{o}+n T\right)\right]-\left[\Phi(t)-\Phi\left(t_{o}\right)\right]\right\}-\left\{\left[\phi_{c}(t+n T)-\phi_{c}\left(t_{o}+n T\right)\right]\right. \\
& \left.-\left[\phi_{c}(t)-\phi_{c}\left(t_{o}\right)\right]\right\}-\left\{\left[\phi_{A 1}(t+n T)-\phi_{A 1}\left(t_{o}+n T\right)\right]-\left[\phi_{A 1}(t)-\phi_{A 1}\left(t_{o}\right)\right]\right\} \\
& -\left\{\left[\phi_{\Lambda 2}(t+n T)-\phi_{A 2}\left(t_{o}+n T\right)\right]-\left[\phi_{A 2}(t)-\phi_{\Lambda 2}\left(t_{o}\right)\right]\right\}-\left\{\left[\phi_{I}(t+n T)\right.\right. \\
& \left.\left.-\phi_{I}\left(t_{o}+n T\right)\right]-\left[\phi_{I}(t)-\phi_{I}\left(t_{o}\right)\right]\right\}=\phi_{g}(t+n T)-\phi_{g}(t)-K_{n}
\end{aligned}
$$

where the quantity $K_{n}$ is a constant for fixed $n$ given by

$$
\mathrm{K}_{\mathrm{n}}=\phi_{\mathrm{g}}\left(\mathrm{t}_{\mathrm{o}}+\mathrm{nT}\right)-\phi_{\mathrm{g}}\left(\mathrm{t}_{\mathrm{o}}\right) \text {. }
$$

Each term within the braces consists of the difference of two terms within brackets and the bracketed terms are all observable quantities within some experimental error.

- $\left[\Phi(t)-\Phi\left(t_{0}\right)\right]$ can be measured by counting fringes at the output of the correlator

- $\left[\phi_{\mathrm{c}}(\mathrm{t})-\phi_{\mathrm{c}}\left(\mathrm{t}_{\mathrm{o}}\right)\right]$ can be measured by the Anik-B phase link

- $\left[\phi_{\mathrm{Al}}(\mathrm{t})-\phi_{\mathrm{Al}}\left(\mathrm{t}_{\mathrm{o}}\right)\right]$ can be estimated using ground based pressure temperature and humidity measurements along with a standard atmospheric model

- $\left[\phi_{\mathrm{A} 2}(\mathrm{t})-\phi_{\mathrm{A} 2}\left(\mathrm{t}_{\mathrm{o}}\right)\right]$ can be measured by dual-frequency, $11 \mathrm{~cm}$ and $21 \mathrm{~cm}$, astronomical observations

- $\left[\phi_{\mathrm{I}}(\mathrm{t})-\phi_{\mathrm{I}}\left(\mathrm{t}_{\mathrm{o}}\right)\right]$ can be measured by the use of an instrumental phase calibrator system.

It can be shown that to first order in small quantities: 


$$
\begin{aligned}
\phi_{\mathrm{g}}(\mathrm{t}+\mathrm{nT})-\phi_{\mathrm{g}}(\mathrm{t})= & \frac{\omega_{\mathrm{o}} \mathrm{b}}{\mathrm{c}}\left\{-\Delta \theta(\mathrm{t}, \mathrm{nT}) \cos \phi_{\mathrm{b}}{ }^{\prime} \cos \delta_{\mathrm{s}} \sin \left(\theta+\lambda_{\mathrm{b}}-\alpha_{\mathrm{s}}\right)+\Delta \phi_{\mathrm{b}}{ }^{\prime}(\mathrm{t}, \mathrm{nT})\right. \\
& {\left[\cos \phi_{\mathrm{b}}{ }^{\prime} \sin \delta_{\mathrm{s}}-\sin \phi_{\mathrm{b}}{ }^{\prime} \cos \delta_{\mathrm{s}} \cos \left(\theta+\lambda_{\mathrm{b}}-\alpha_{\mathrm{s}}\right)\right]+\Delta \delta_{\mathrm{s}}(\mathrm{t}, \mathrm{nT}) } \\
& {\left[\sin \phi_{\mathrm{b}}{ }^{\prime} \cos \delta_{\mathrm{s}}-\cos \phi_{\mathrm{b}}{ }^{\prime} \sin \delta_{\mathrm{s}} \cos \left(\theta+\lambda_{\mathrm{b}}-\alpha_{\mathrm{s}}\right)\right]-\Delta \lambda_{\mathrm{b}}(\mathrm{t}, \mathrm{nT} \mathrm{T}) } \\
& \cos \phi_{\mathrm{b}}{ }^{\prime} \cos \delta_{\mathrm{s}} \sin \left(\theta+\lambda_{\mathrm{b}}-\alpha_{\mathrm{s}}\right)+\Delta \alpha_{\mathrm{s}}(\mathrm{t}, \mathrm{nT}) \cos \phi_{\mathrm{b}}{ }^{\prime} \cos \delta_{\mathrm{s}} \\
& \sin \left(\theta+\lambda_{\mathrm{b}}-\alpha_{\mathrm{s}}\right)+\Delta \mathrm{b}(\mathrm{t}, \mathrm{nT}) \frac{1}{\mathrm{~b}}\left[\sin \phi_{\mathrm{b}}{ }^{\prime} \sin \delta_{\mathrm{s}}+\cos \phi_{\mathrm{b}}{ }^{\prime} \cos \delta_{\mathrm{s}}\right. \\
& \left.\left.\cos \left(\theta+\lambda_{\mathrm{b}}-\alpha_{\mathrm{s}}\right)\right]\right\}
\end{aligned}
$$

where $\Delta \mathrm{b}(\mathrm{t}, \mathrm{nT}), \Delta \phi_{\mathrm{b}}{ }^{\prime}(\mathrm{t}, \mathrm{nT}), \Delta \lambda_{\mathrm{b}}(\mathrm{t}, \mathrm{nT})$ represent increments in the interval $\mathrm{t} \leqslant \mathrm{t}^{\prime} \leqslant \mathrm{t}+\mathrm{nT}$ to the baseline scalar length, declination, and east longitude respectively; and where $\Delta \alpha_{s}(t, n T), \Delta \delta_{s}(t, n T)$ represent increments in the interval $t \leqslant t^{\prime} \leqslant t+n T$ to the source coordinates of right ascension and declination respectively. The quantity $\Delta \theta(\mathrm{t}, \mathrm{nT})$ is defined in such a way that the total increment to the sidereal angle of the baseline which occurs in the interval $t \leqslant t^{\prime} \leqslant t+n T$ is $2 n \pi+\Delta \theta(t, n T)$. Hence,

$$
\Delta \theta(\mathrm{t}, \mathrm{nT})=-\Omega_{\mathrm{o}} \Delta \tau(\mathrm{t}, \mathrm{nT})
$$

or

$$
\Delta \theta(\mathrm{t}, \mathrm{nT})=\Omega_{\mathrm{o}} \int_{\mathrm{t}}^{\mathrm{t}+\mathrm{nT}} \mathrm{m}_{3}\left(\mathrm{t}^{\prime}\right) \mathrm{dt^{ \prime }}
$$

Table 3 summarizes the properties of the partial derivatives of the differenced interferometer phase with respect to the variations in the above parameters.

For measurement of variations $\Delta \theta$ in Earth rotation, it will be useful to adopt an observing strategy which concentrates on low $\left(\delta_{\mathrm{s}} \leqslant 10^{\circ}\right)$ declination sources. Since $\phi_{\mathrm{b}}{ }^{\prime} \cong-5^{\circ}$ on the baseline $\overrightarrow{\mathrm{DRAO}-\mathrm{ARO}}$ and $\phi_{\mathrm{b}}{ }^{\prime} \cong-14^{\circ}$ on the baseline $\overrightarrow{\mathrm{DRAO}-\mathrm{NRL}}$, it is clear that by observing low declination sources the interferometer can be rendered relatively insensitive to the variations $\Delta \phi_{\mathrm{b}}{ }^{\prime}$ and $\Delta \delta_{\mathrm{s}}$. The variations $\Delta \phi_{\mathrm{b}}{ }^{\prime}$ arise as a result of polar motion parallel to the meridian of the interferometer and the variations $\Delta \delta_{\mathrm{s}}$ arise as a result of precession and nutation in the interval $t \leqslant t^{\prime} \leqslant \mathrm{t}+\mathrm{nT}$. Furthermore, since polar motion $m_{1}(t) m_{2}(t)$ increments the interferometer baseline longitude by $\Delta \lambda_{\mathrm{b}}$ where

$$
\Delta \lambda_{\mathrm{b}}=\frac{\sin \phi_{\mathrm{b}}{ }^{\prime}}{\cos \phi_{\mathrm{b}}{ }^{\prime}}\left[\mathrm{m}_{1}(\mathrm{t}) \sin \lambda_{\mathrm{b}} \quad \mathrm{m}_{2}(\mathrm{t}) \cos \lambda_{\mathrm{b}}\right],
$$

we sec that these low declination basclines are also rather insensitive to variations $\Delta \lambda_{b}$ caused by polar motion. The values of the parameters $\Delta \phi_{b}{ }^{\prime}, \Delta \lambda_{b}, \Delta \delta_{s}$ to which the interferometer is relatively insensitive but which are required for data processing can be obtained from sources such as the Bureau International de l'Heure (BIH) and the standard IAU theory of the precession and nutation. 
Table 3

\begin{tabular}{|c|c|c|c|}
\hline \multirow{3}{*}{$\begin{array}{l}\text { ASTRONOMICAL } \\
\text { GEOPHYSICAL } \\
\text { PARAMETER } \\
\text { VARIATION }\end{array}$} & \multicolumn{3}{|c|}{ PARTIAL DERTVATIVE } \\
\hline & \multirow{2}{*}{$\begin{array}{l}\text { CONSTANT TERM } \\
\times \frac{\omega_{0} b}{c}\end{array}$} & \multicolumn{2}{|c|}{ TIME VARYING TERM $\times \frac{\omega_{0} b}{c}$} \\
\hline & & AMPLITUDE & $\begin{array}{l}\text { SIDEREAI TIME } \\
\text { DEPENDENCE }\end{array}$ \\
\hline$\Delta \theta$ & - & $-\cos \phi_{b}^{\prime} \cos \delta_{s}$ & $\sin \theta$ \\
\hline$\Delta \phi^{-}$ & $\cos \phi_{b}^{\prime} \sin \delta_{s}$ & $-\sin \phi_{\mathrm{b}}^{\prime} \cos \delta_{\mathrm{s}}$ & $\cos \theta$ \\
\hline$\Delta \delta_{S}$ & $\sin \phi_{b}^{\prime} \cos \delta_{s}$ & $-\cos \phi_{\mathrm{b}}^{-} \sin \delta_{\mathrm{s}}$ & $\cos \theta$ \\
\hline$\Delta \lambda_{\mathrm{b}}$ & & $-\cos \phi_{b}^{\prime} \cos \delta_{s}$ & $\sin \theta$ \\
\hline$\Delta \alpha_{s}$ & - & $\cos \phi_{b}^{\prime} \cos \delta_{s}$ & $\sin \theta$ \\
\hline$\Delta \mathrm{b}$ & $\frac{1}{b} \sin \phi_{b}^{\prime} \sin \delta_{s}$ & $\frac{1}{b} \cos \phi_{b}^{-} \cos \delta_{s}$ & $\cos \theta$ \\
\hline
\end{tabular}

Of the three remaining parameters to which this data is sensitive, we see that $\Delta \theta$ and $\Delta \alpha_{\mathrm{s}}$ are inseparable. It will therefore be necessary to use existing IAU models for the precession and nutation to theoretically compute the changes in right ascension $\Delta \alpha_{s}$ of the source position and finally to solve for quantities $\Delta \theta(t, n T)$ and $\Delta b(t, n T)$ separating them on the basis of their different sidereal time dependences.

The entire measurement procedure described above is insensitive to errors in the adopted a priori values for the parameters of the interferometer. The effect of such errors on the measurement is made to vanish by differencing the data from day to day.

\section{Performance Possibilities for Measurement of Earth Rotation}

The rms measurement errors $\sqrt{ } \delta \theta^{2}$ and $\sqrt{ } \delta \mathrm{b}^{2}$ in the parameters $\Delta \theta$ and $\Delta \mathrm{b}$ are dominated on the time scale of days to months by the rms error $\sqrt{ } \delta \phi^{2}$ in the measurement of the differenced interferometer phase rather than by systematic errors in the modelling of the quantities $\Delta \psi_{\mathrm{b}}{ }^{\prime} \Delta \lambda_{\mathrm{b}} \Delta \alpha_{\mathrm{s}}$ $\Delta \delta_{\mathrm{s}}$. The latter quantities, particularly $\Delta \alpha_{\mathrm{s}}, \Delta \delta_{\mathrm{s}}$, will become important sources of error on time scales of decades to centuries as errors in the models for the precession and nutation will inevitably propagate into errors in the measurement of earth rotation.

The parameters $\Delta \theta$ and $\Delta \mathrm{b}$ are quite well separable on the basis of 12 hours or more of differenced data; so, for data sets of 12 hours or more, we can neglect the covariances between $\Delta \theta$ and $\Delta \mathrm{b}$ and 
obtain a first order estimate of their respective measurement errors directly from their partial derivatives. We can take

$$
\sqrt{\overline{\delta \theta^{2}}} \cong \sqrt{2} \frac{\mathrm{c}}{\omega_{\mathrm{o}} \mathrm{b}} \sqrt{\overline{\delta \phi^{2}}}
$$

or equivalently

$$
\sqrt{\overline{\delta \tau^{2}}} \cong \sqrt{2} \frac{\mathrm{c}}{\Omega_{\mathrm{o}} \omega_{\mathrm{o}} \mathrm{b}} \sqrt{\overline{\delta \phi^{2}}}
$$

and

$$
\sqrt{\overline{\delta \mathrm{b}^{2}}} \cong \sqrt{2} \frac{\mathrm{c}}{\omega_{\mathrm{o}}} \sqrt{\overline{\delta \phi^{2}}}
$$

where the factor $\sqrt{2}$ is to approximately allow for the fact that the value of the partial derivative varies sinusoidally with sidereal time and where $\sqrt{\overline{\delta \phi^{2}}}$, given by

$$
\sqrt{\delta \phi^{2}}=\sqrt{2\left(\overline{\delta \phi^{2}}+\overline{\delta \phi_{\mathrm{c}}^{2}}+\overline{\delta \phi_{\mathrm{A} 1}^{2}}+\overline{\delta \phi_{\mathrm{A} 2}^{2}}+\overline{\delta \phi_{\mathrm{I}}^{2}}\right)}
$$

is the instantaneous (one sample) rms measurement error of the differenced interferometer phase. The factor $\sqrt{2}$ in the above formula is required to account for the fact that we are dealing with differenced data.

We shall assume that the stochastically varying phase errors in the measurements of $\left[\Phi(\mathrm{t})-\Phi\left(\mathrm{t}_{\mathrm{o}}\right)\right]$, $\left[\phi_{\mathrm{c}}(\mathrm{t})-\phi_{\mathrm{c}}\left(\mathrm{t}_{\mathrm{o}}\right)\right],\left[\phi_{\mathrm{A1}}(\mathrm{t})-\phi_{\mathrm{A1}}\left(\mathrm{t}_{\mathrm{o}}\right)\right],\left[\phi_{\mathrm{A} 2}(\mathrm{t})-\phi_{\mathrm{A} 2}\left(\mathrm{t}_{\mathrm{o}}\right)\right]$, and $\left[\phi_{\mathrm{I}}(\mathrm{t})-\phi_{\mathrm{I}}\left(\mathrm{t}_{\mathrm{o}}\right)\right]$, having variances

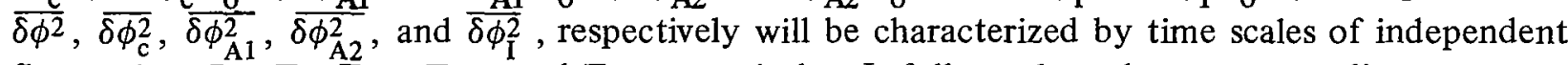
fluctuations $\mathrm{T}_{0}, \mathrm{~T}_{\mathrm{c}}, \mathrm{T}_{\mathrm{A} 1}, \mathrm{~T}_{\mathrm{A} 2}$, and $\mathrm{T}_{\mathrm{I}}$, respectively. It follows that a least squares adjustment to a data set of length $\tau$ will be corrupted by an rms phase error $\sqrt{\delta \phi^{2}}(\tau)$ given approximately by

$$
\sqrt{\overline{\delta \phi^{2}}}(\tau) \cong \sqrt{\frac{2}{\tau}\left(\mathrm{T}_{\mathrm{o}} \overline{\delta \phi^{2}}+\mathrm{T}_{\mathrm{c}} \overline{\delta \phi_{\mathrm{c}}^{2}}+\mathrm{T}_{\mathrm{Al}} \overline{\delta \phi_{\mathrm{A} 1}^{2}}+\mathrm{T}_{\mathrm{A} 2} \overline{\delta \phi_{\mathrm{A} 2}^{2}}+\mathrm{T}_{\mathrm{I}} \overline{\delta \phi_{\mathrm{I}}^{2}}\right.}
$$

$\sqrt{\sqrt{\delta \phi^{2}}}$ is the rms measurement error on the phase of the correlation function. For strong sources $\sqrt{\overline{\delta \phi^{2}}}$ is generally quite small, of the order of $\pm 10^{\circ}-15^{\circ}$ and since each integration provides an independent sample of this random variable the time scale $T_{0}$ is of the order of seconds.

$\sqrt{\delta \phi_{c}^{2}}$ is the rms measurement error on the clock phase and will be dominated on the time scales under consideration here by instrumental phase delay variations $\overline{\delta \phi_{\mathrm{c} 1}^{2}}$ in the cabling and electronics of the ground stations and by the dispersive ionospheric phase delay variations $\overline{\delta \phi_{\mathrm{c} 2}^{2}}$ which affect the phase link operation.

$$
\mathrm{T}_{\mathrm{c}} \overline{\delta \phi_{\mathrm{c}}^{2}}=\mathrm{T}_{\mathrm{c} 1} \overline{\delta \phi_{\mathrm{c} 1}^{2}}+\mathrm{T}_{\mathrm{c} 2} \overline{\delta \phi_{\mathrm{c} 2}^{2}}
$$


We have seen that $\sqrt{\delta \phi_{\mathrm{c} 2}^{2}}$ could be as large as $\pi$ when the day/night terminator passes through but could be expected under other conditions to be typically of the order of $\pi / 4$ with a time scale of fluctuations $T_{c 2}$ of the order of 30 minutes, the coherence time of the short-term temporal variations of the ionosphere.

In the absence of phase calibrators, the repetitive component of the diurnal variations in the instrumental phase delays at each ground station will be cancelled by the phase differencing procedure. The residual variations in the instrumental phase delays can be expected to be of the order of $2 \mathrm{nsec} \mathrm{rms}$ with a time scale of fluctuation of several hours. For a frequency of $3 \mathrm{GHz}$, we can take the uncalibrated rms variations $\sqrt{\delta \phi_{\mathrm{c} 1}^{2}}$ to be of the order of $12 \pi$ with a time scale of fluctuations of the order of 2 hours.

The addition of phase calibrator systems to the Anik-B phase link could be expected to greatly reduce this source of error, particularly since the operation of the phase link merely requires the stabilization of the instrumental phase delays; a much weaker requirement than their actual measurement. It would seem that phase calibrators could reduce $\sqrt{\delta \phi_{\mathrm{cl}}^{2}}$ to quite small values with something of the order of $\pi / 10$ reasonably attainable. In such a case, however, $T_{c 1}$ would remain the same, of the order of 2 hours.

To first order, we can apply these remarks to the error source $\sqrt{\delta \phi_{\mathrm{I}}^{2}}$ which arises from phase delay variations in the cabling and electronics of the astronomical receiver/recorder system. Thus, we can, for purposes of our estimate, take $\sqrt{\delta \phi_{I}^{2}} \cong 12 \pi$ without phase calibrators and $\sqrt{\delta \phi_{I}^{2}} \cong \pi / 10$ with phase calibrators and $T_{I} \cong 2$ hours in each case.

$\sqrt{\delta \overline{\phi_{\mathrm{A} 1}^{2}}}$ is the rms phase error arising from the failure to properly correct for the phase delay effects of the troposphere on the astronomical signal. Using ground based pressure, temperature, and humidity data along with a standard atmospheric model it is possible to compute the tropospheric phase delay with an accuracy of $5 \mathrm{~cm}$ rms at the zenith and $10 \mathrm{~cm}$ rms at a zenith angle of $60^{\circ}$. The additional use of dual frequency water vapor radiometers can apparently reduce these errors to something of the order of $2 \mathrm{~cm} \mathrm{rms}$ at the zenith and $4 \mathrm{~cm} \mathrm{rms}$ at a zenith angle of $60^{\circ}$. Since a long baseline interferometer almost always has one or other of its antennas pointing at a large zenith angle, we shall take the $60^{\circ}$ zenith angle figures as being typical. For a frequency of $3 \mathrm{GHz}$, we can assume $\sqrt{\delta \phi_{\mathrm{Al}}^{2}} \cong 2 \pi$ without the water vapor radiometer measurements and $\sqrt{\delta \phi_{\mathrm{A} 1}^{2}} \cong 0.8 \pi$ with the water vapor radiometer measurements. The time scale of fluctuations of these quantities will be taken to be 30 minutes, roughly the coherence time of the short-term variations of the atmosphere.

$\sqrt{\overline{\delta \phi_{\Lambda 2}^{2}}}$ is the rms phase error arising from the failure to properly correct for the phase delay effects of the ionosphere on the astronomical signal. Dual frequency observations at $11 \mathrm{~cm}$ and $21 \mathrm{~cm}$ allow removal of the effects of the variations in the differential ionospheric phase delay along the beams of the antennas with an rms error of roughly 1 percent. For a $3 \mathrm{GHz}$ signal, the magnitude of the ionospheric phase delay is of the order of 2 meters at zenith angles of $60^{\circ}$. Although large scale excursions in differential integrated electron content occur near sunrise and sunset, the differential integrated electron content can be expected to be typically much smaller. We shall assume a 
25 percent differential integrated electron content between the two antennas as being typical. If we again assume a $60^{\circ}$ zenith angle as being typical of the interferometer operation, then $\sqrt{\delta \phi_{\mathrm{A} 2}^{2}} \cong$ $0.1 \pi$ at $3 \mathrm{GHz}$. The time scale of independent fluctuations of this quantity can be taken to be of the order of 30 minutes, the coherence time of the short term ionospheric variations.

The results of this error budget are summarized in table 4 along with the quantity $\sqrt{\delta \phi^{2}}(\tau)$ which results. $\tau$ is measured in hours of data fitted.

Figures 4 and 5 based on the error budget of table 4 indicate that the addition of phase calibrator system to the astronomical receiver/recorders and to the phase link ground stations will greatly improve the performance of the interferometer. However, beyond this, the further addition of water vapor radiometers and a third frequency in the phase link to remove ionospheric effects can offer only marginal improvement by perhaps a further factor of two.

Table 4

\begin{tabular}{|c|c|c|c|c|c|}
\hline \multirow[b]{2}{*}{$\begin{array}{l}\text { ERROR } \\
\text { SOURCE }\end{array}$} & \multirow[b]{2}{*}{$\begin{array}{l}\text { TIME SCALE } \\
\text { OF } \\
\text { INDEPENDENT } \\
\text { ELUCTUATIONS }\end{array}$} & \multicolumn{4}{|c|}{ APPROXIMATE RMS AMPIITUDE } \\
\hline & & $\begin{array}{c}\text { DUAL } \\
\text { FREQUENCY } \\
\text { OPERATION }\end{array}$ & $\begin{array}{c}\text { PHASE } \\
\text { CALIBRATORS } \\
\text { ADDED }\end{array}$ & $\begin{array}{l}\text { WATER } \\
\text { VAPOR } \\
\text { RADIOMETERS } \\
\text { ADDED }\end{array}$ & $\begin{array}{l}\text { PHASE LINK } \\
\text { THIRD } \\
\text { FREQUENCY } \\
\text { ADDED }\end{array}$ \\
\hline$\overline{\delta \Phi^{2}}$ & $10 \mathrm{sec}$. & $0.08 \pi$ & $0.08 \pi$ & $0.08 \pi$ & $0.08 \pi$ \\
\hline$\overline{\delta \phi_{c l}^{2}}$ & $2 \mathrm{hr}$. & $12 \pi$ & $0.1 \pi$ & $0.1 \pi$ & $0.1 \pi$ \\
\hline$\overline{\delta \phi_{C 2}^{2}}$ & $2 \mathrm{hr}$. & $0.25 \pi$ & $0.25 \pi$ & $0.25 \pi$ & $0.02 \pi$ \\
\hline$\overline{\delta \phi_{\mathrm{A} I}^{2}}$ & $30 \mathrm{~min}$. & $2 \pi$ & $2 \pi$ & $0.8 \pi$ & $0.8 \pi$ \\
\hline$\overline{\delta \phi_{A}^{2}}$ & $30 \mathrm{~min}$. & $0.1 \mathrm{H}$ & $0.1 \pi$ & $0.1 \pi$ & $0.1 \pi$ \\
\hline$\overline{\delta \phi_{I}^{2}}$ & 2 hr. & $12 \pi$ & $0.1 \pi$ & $0.1 \pi$ & $0.1 \pi$ \\
\hline$\sqrt{\delta \phi^{2}}$ & & $\frac{34 \pi}{\sqrt{\tau}}$ & $\frac{2.1 \pi}{\sqrt{\tau}}$ & $\frac{1.3 \pi}{\sqrt{\tau}}$ & $\frac{1.2 \pi}{\sqrt{\tau}}$ \\
\hline
\end{tabular}




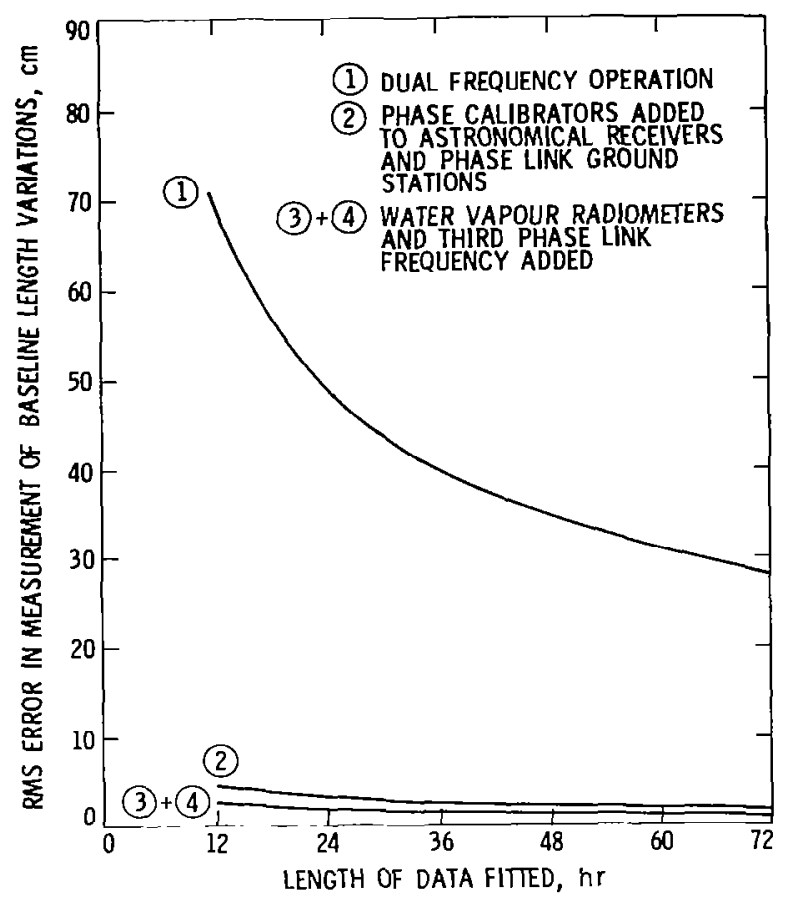

Figure 4.

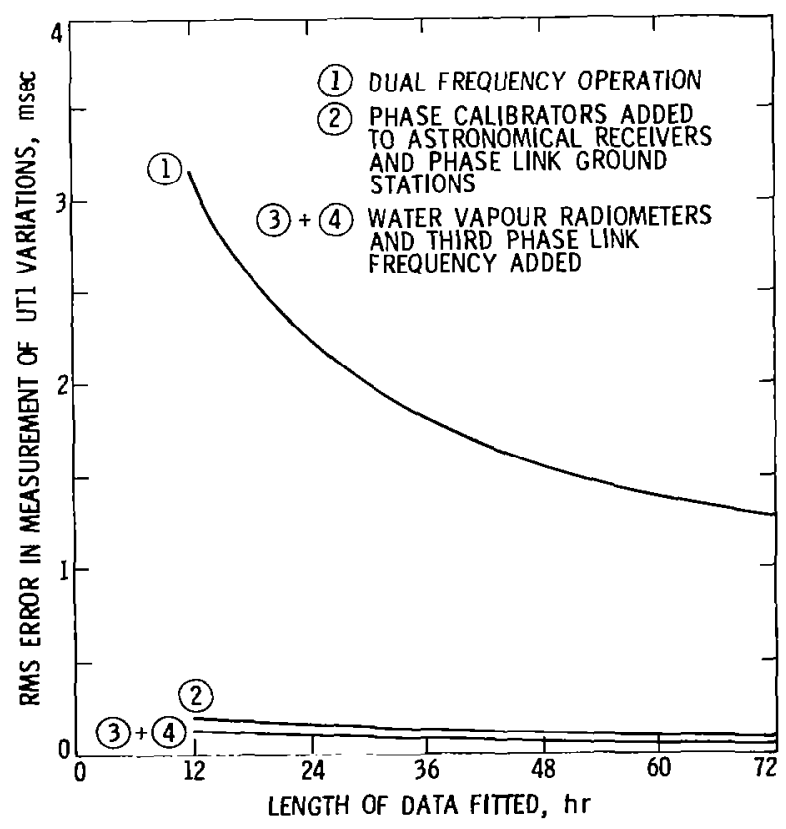

Figure 5. 
Broten, N. W., "The Role of Long Baseline Interferometry in the Measurements of Earth's Rotation", in Earthquake Displacement Fields and the Rotation of the Earth, 279-283, Mansinha, L., Smylie, D. E., Beck, A. E., eds. D. Reidel, 1970.

Cannon, W. H., "The Effects of the Ionosphere on a Satellite Phase Link" unpublished memorandum 1979.

Rogers, T. F., Evans, J. V., "Radio Wave Propagation Through the Earth's Neutral Atmosphere and Ionosphere", Chapt. II of Radar Astronomy, Evans, J. V., and Hayfors, T., ed. McGraw Hill, 1968.

Waltman, W. B., Knowles, S. H., Yen, J. L., Fort, D. N., Swenson, G. W. Cannon, W. H., Galt, J. A., "Phase Coherent VLBI Using a Satellite Link" NASA CP 21 15, "Radio Interferometry: Techniques for Geodesy", 1980.

Yen, J. L., Kellerman, K. I., Rayhrer, B., Broten, N. W., Fort, D. N., Knowles, S. H., Waltman, W.B., Swenson, G. W., "Real-Time Very Long Baseline Interferometry Based on the Use of a Communications Satellite" Science, 198, 289-291, 1977.

Yen, J. L. "Phase and Frequency Synchronization via a Satellite Link" unpublished memorandum 1977. 


\section{NASA GEODYNAMICS PROGRAM}

\section{E. A. Flinn}

National Aeronautics and Space Administration

Presented but not submitted 



\section{ATTENDEES}

$\longrightarrow$

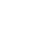




\section{ATTENDEES}

Leendert Aardoom

Onderafdeling der Geodesie

Observatorium Kootwijk

Postbus 581, Apeldoorn

The Netherlands

David W. Allan

Department of Commerce

National Bureau of Standards

325 Broadway,

Mail Code 524.06, Room 4035

Boulder, Colorado 80303

Telephone: (303) 499-1000 X3282

Allen Joel Anderson

Department of Solid Earth Physics

Department of Geodesy

Box 556 - The University of Uppsala

Uppsala, Sweden S-751 22

David Anthony

Air Force Geophysics Lab/LWH

Hanscom Air Force Base

Bedford, Massachusetts 01731

Telephone: (617) 861-3486

D. C. Backer

University of California

Radio Astronomy Lab

601 Campbell Hall

Berkeley, California 94720

John Ball

Harvard College Observatory

Harvard, Massachusetts 01451

Telephone: 456-3391 and 495-3919

John Bensen

National Radio Astronomy Observatory

Edgemont Road

Charlottsville, Virginia 22901

Telephone: (804) 296-0383
Winfried Beyer

Geodetic Institute, Bonn University

Nussallee 17, D-5300

Bonn, Germany

Harold Black

The Johns Hopkins University

Applied Physics Lab

Johns Hopkins Road

Laurel, Maryland 20810

Telephone: (301) 953-7100 X7158

J. A. R. Blais

Geodetic Survey of Canada

615 Booth Street

Ottawa, Canada K1A OE9

Telephone: 995-4345

Yehuda Bock

Ohio State University

Department of Geodetic Science

1958 Neil Avenue

Columbus, Ohio 43210

Telephone: (614) 422-2269

Claude Boucher

Institut Geographique National

2 Av. Pasteur

Saint-Mande, France 94160

Telephone: (1) 374-12-15 X2501

F. J. J. Brouwer

Computing Ctr. of the Delft

Geodetical Institute

Delft University of Technology

Thyssewes 11

2629 JA Delft

The Netherlands 
Gerry Cabaniss

Air Force Geophysics Lab/LWH

Hanscom Air Force Base

Bedford, Massachusetts 01731

James Campbell

Geodetic Institute, Bonn University

Nussallee 17, D-5300

Bonn, Germany

Telephone: 02221-733565

Wayne H. Cannon

Room 101, Physics Department

York University

4700 Keele Street

Toronto, Ontario, Canada M3J-1P3

Roger J. Cappallo

Massachusetts Institute of Technology

Department of Earth and Planetary

Sciences

Room 54-627

Cambridge, Massachusetts 02139

William E. Carter

National Geodetic Survey (C-133)

Rockville, Maryland 20852

Telephone: (301) 443-8171

G. W. Citters

Department of Astronomy

University of Texas

Austin, Texas 78712

Telephone: (512) 471-4477

E. Scott Claflin

Jet Propulsion Laboratory

4800 Oak Grove Drive

Pasadena, California 91103

Telephone: (213) 354-7468
Thomas A. Clark

Goddard Space Flight Center

Code 693

Greenbelt, Maryland 20771

Robert C. Clauss

Jet Propulsion Laboratory

4800 Oak Grove Drive

Pasadena, California 91103

Robert Coates

Goddard Space Flight Center

Code 900.0

Greenbelt, Maryland 20771

Telephone: (301) 344-8809

Nathaniel Cohen

National Astronomy and Ionosphere Center Cornell University

Space Sc. 206

Ithaca, New York 14853

Telephone: (607) 256-3376

Brian E. Corey

Department of Earth and Planetary Sciences

Massachusetts Institute of Technology

Room 54-420

Cambridge, Massachusetts 02139

Rene Cormier

Air Force Geophysics Lab/XOP

Hanscom Air Force Base

Bedford, Massachusetts 01731

Telephone: (617) 861-3486

William E. Cotton

Department of Earth and Planetary Sciences

Massachusetts Institute of Technology

Room 54-418

Cambridge, Massachusetts 02139 
Charles C. Counselman, III

Department of Earth and Planetary

Sciences

Massachusetts Institute of Technology

Room 54-626

Cambridge, Massachusetts 02139

Telephone: (617) 253-7902

Duncan B. Cox, Jr.

Draper Laboratory, MS-92

555 Technology Square

Cambridge, Massachusetts 02139

Telephone: (617) 258-4423

Patrick Crane

National Radio Astronomy Observatory

P.O. Box 2

Green Bank, West Virginia 24944

James Crooks/MZ 7-102

General Dynamics Electronics

P.O. Box 81127

San Diego, California 92138

Telephone: (714) 277-8900 X1636

David W. Curkendall

Jet Propulsion Laboratory

Mail Stop 264-748

4800 Oak Grove Drive

Pasadena, California 91103

Telephone: (213) 354-4199

D. A. Davidson

University of New Brunswick

Department Surveying

P.O. Box 4400

Fredericton, New Brunswick

Canada E3B 5A3

Telephone: (506) 453-4700

Edward Dobkowski

Air Force Geophysics Lab/XOP

Hanscom Air Force Base

Bedford, Massachusetts 01731

Telephone: (617) 861-3486
Donald Eckhardt

Air Force Geophysics Lab/LWG

Hanscom Air Force Base

Bedford, Massachusetts 01731

Telephone: (617) 861-3486

John Fanselow

Jet Propulsion Laboratory

4800 Oak Grove Drive

Pasadena, California 91103

Telephone: (213) 354-6329

Thomas Fischetti

NASA Headquarters

600 Independence Avenue, S.W.

Washington, D.C. 20546

Telephone: (202) 755-3848

E. A. Flinn

Code ERG-2

National Aeronautics and Space

Administration

Washington, D.C. 20546

Telephone: (202) 755-3848

D. N. Fort

Herzberg Institute of Astrophysics, NCR

100 Susses Drive

Ottawa, Ontario

Canada K1A OR6

Telephone: (613) 593-6060

John Galt

Herzberg Institute of Astrophysics

Box 248

Penticton, British Columbia

Canada V2A 6K3

Telephone: (604) 497-5321

E. Gaposchkin

Smithsonian Astrophysical Observatory

60 Garden Street

Cambridge, Massachusetts 02138

Telephone: (617) 495-7495 
Antanas Girnius

American Geophysical Union

40 Sunset Hill Road

Boston, Massachusetts 02131

Telephone: (617) 327-2092

Marc V. Gorenstein

Department of Earth and Planetary

Sciences

Massachusetts Institute of Technology

Room 54-418

Cambridge, Massachusetts 02139

Sergei Gourevitch

Department of Earth and Planetary

Sciences

Massachusetts Institute of Technology

Room 54-626

Cambridge, Massachusetts 02139

Richard L. Greenspan

C. S. Draper Laboratory

555 Technology Square

Cambridge, Massachusetts 02139

Telephone: (617) 258-4048

James Hammond

Air Force Geophysics Lab/LWG

Hanscom Air Force Base

Bedford, Massachusetts 01731

Telephone: (617) 861-3486

Stanley Hansen

National Radio Astronomy Observatory

Edgemont Road

Charlottesville, Virginia 22901

Telephone: (804) 296-0383

John N. Harris

M.I.T. Lincoln Lab

244 Wood Street

Lexington, Massachusetts 02173
Aubrey Haschick

Smithsonian Astrophysical Observatory

60 Garden Street

Cambridge, Massachusetts 02138

Telephone: (617) 495-7473

Warren Heller

The Analy tical Sciences Corporation

6 Jacob Way

Reading, Massachusetts 01867

Telephone: (617) 944-6850 X159

Samuel Hensel

Electronic Space Systems Corp. (ESSCO)

Old Powder Mill Road

West Concord, Massachusetts 01742

Telephone: (617) 369-7200 X215

Bruce R. Hermann

Naval Surface Weapons Center

K-13

Dahlgren, Virginia 22448

Thomas Herring

Department of Earth and Planetary Sciences

Massachusetts Institute of Technology

Room 54-611

Cambridge, Massachusetts 02139

Telephone: (617) 253-7949

Hans Hinteregger

NEROC/Haystack Observatory

Westford, Massachusetts 01886

Telephone: (617) 692-4764

Leonard E. Johnson

Earth Sciences Division

National Science Foundation

1800 G Street, N.W.

Washington, D.C. 20550

Telephone: (202) 632-1977 
Kenneth Johnston

Naval Research Laboratory

Code 7134

Washington, D.C. 20375

Telephone: (202) 767-2351

George Kaplan

U.S. Naval Observatory

34th Street and Mass. Avenue, N.W.

Washington, D.C. 20390

Telephone: (202) 254-4582

Vinod Kapoor

Jet Propulsion Laboratory

4800 Oak Grove Drive

Pasadena, California 91103

Robert W. King

Department of Earth and Planetary

Sciences

Massachusetts Institute of Technology

Room 54-627

Cambridge, Massachusetts 02139

Kenneth E. Kissell

AF Avionics Lab/RW

Building 22

Wright Patterson Air Force Base

Ohio 45433

Telephone: (513) 255-5771

William Klepczynski

U.S. Naval Observatory

Washington, D.C. 20390

C. A. Knight

NEROC/Haystack Observatory

Westford, Massachusetts 01886

Telephone: (617) 692-4764
Jan Kouba

Department of Energy, Mines and Resources

Earth Physics Branch

1 Observatory Crescent

Ottawa, Canada K1A OY3

Telephone: (613) 995-5302

Frank Kreimendahl

Department of Earth and Planetary Sciences

Massachusetts Institute of Technology

Room 54-611

Cambridge, Massachusetts 02139

Richard J. Lacassee

National Radio Astronomy Observatory

P.O. Box 2

Green Bank, West Virginia 24944

Telephone: (304) 456-2127

R. B. Langley

Department of Earth and Planetary Sciences

Massachusetts Institute of Technology

Room 54-422

Cambridge, Massachusetts 02139

Noel Lanham

Smithsonian Astrophysical Observatory

60 Garden Street

Cambridge, Massachusetts 02138

Telephone: (617) 495-7481

Douglas R. Larden

Joint Institute for Laboratory Astrophysics

University of Colorado

Boulder, Colorado 80309

Telephone: (303) 492-7857

James H. Latimer, II

Smithsonian Astrophysical Observatory

60 Garden Street

Cambridge, Massachusetts 02138

Telephone: (617) 495-7330 
Alfred Leick

University of Maine at Orono

103 Boardman Hall.

Orono, Maine 04469

Telephone: (207) 581-2561

James I. Levine

NEROC/Haystack Observatory

Westford, Massachusetts 01886

Telephone: (617) 692-4764

Philip Liebrecht

NASA/GSFC, Code 852.1

Glendale Road

Greenbelt, Maryland 20771

Telephone: (301) 344-7535

Roger Linfield

California Institute of Technology

105-24

Pasadena, California 91125

Telephone: (213) 795-6811 X1026

Chopo Ma

Goddard Space Flight Center

Greenbelt, Maryland 20771

Telephone: (301) 344-8809

Peter F. MacDoran

Jet Propulsion Laboratory

Cal Tech

4800 Oak Grove Drive

Pasadena, California 91103

Telephone: (213) 354-7118

Jon Marcaide

Massachusetts Institute of Technology 60 Wadsworth Street

Cambridge, Massachusetts 02142

Telephone: (617) 494-9129
Charles F. Martin

Defense Mapping Agency

Building 56, U.S. Naval Observatory

Washington, D.C. 20305

Telephone: (202) 254-4472

Norman Mason

Air Force Geophysics Lab/LWG

Hanscom Air Force Base

Bedford, Massachusetts 01731

Telephone: (617) 861-3486

William G. Melbourne

Jet Propulsion Laboratory, MS 264-748

4800 Oak Grove Drive

Pasadena, California 91103

James M. Moran

Center for Astrophysics

60 Garden Street

Cambridge, Massachusetts 02138

Telephone: (617) 495-7477

Ivan I. Mueller

Department of Geodetic Science

The Ohio State University

1958 Neil Avenue

Columbus, Ohio 43210

Telephone: (614) 422-2269

Edwin F. Nesman -

NEROC/Haystack Observatory

Westford, Massachusetts 01886

Arthur E. Niell

Jet Propulsion Laboratory

MS 264-765

4800 Oak Grove Drive

Pasadena, California 91103

Telephone: (213) 354-4633

FTS : 792-4633 
John Nolton

P.O. Box 2876

Van Nuys, California 91401

Telephone: (213) 883-3038

Klemens Nottarp

Institut für Angewandte Geodäsie,

Satellitenbeobach tungsstation Wettzell

D-8493 Kofetiny,

Federal Republic of Germany

Telephone: 09941-8643

Hyman Orlin

National Academy of Sciences

Comm. on Geodesy JH-413

2101 Constitution Avenue, N.W.

Washington, D.C. 20418

Telephone: (202) 389-6248

Hays Penfield

Smithsonsian Astrophysical Observatory

60 Garden Street

Cambridge, Massachusetts 02138

Telephone: (617) 495-7481

William T. Petrachenko

York University

4700 Keele Street

Toronto, Ontario

Canada M3J 1P3

Telephone: (416) 667-3860

Jay Pigg

Computer Sciences Corporation

c/o Goddard Space Flight Center

Greenbelt, Maryland 20771

Telephone: (301) 3446434

Joseph Popelar

Earll Physics Branch

Department of Energy, Mines and

Resources

1 Observatory Crescent

Ottowa, Ontario Canada KlA OY3

Telephone: (613) 995-5302
Robert A. Preston

Jet Propulsion Laboratory

4800 Oak Grove Drive

Pasadena, California 91103

Telephone: (213) 354-4471

George H. Purcell, Jr.

Jet Propulsion Laboratory

$\mathrm{M} / \mathrm{S}$ 264-765

4800 Oak Grove Drive

Pasadena, California 91103

Michael Ratner

Department of Earth and Planetary Sciences

Massachusetts Institute of Technology

Room 54-627

Cambridge, Massachusetts 02139

George Reams

Geodynamics Corporation

55 Hitchcock Way

Santa Barbara, Califormia 93105

Telephone: (805) 687-7747

Victor S. Reinhardt

NASA/GSFC

Greenbelt, Maryland 20760

Telephone: (301) 869-3085

Nicholas Renzetti

Jet Propulsion Laboratory

4800 Oak Grove Drive

Pasadena, California 91103

Telephone: (213) 354-4471

George M. Resch

Jet Propulsion Laboratory

M/S 264-765

4800 Oak Grove Drive

Pasadena, California 91103

Telephone: (213) 354-4786 
Roger Richard

National Research Council

Ottawa, Ontario

Canada K1A OR6

Donald Richardson

DMA Aerospace Center

St. Louis, Missouri 63118

Telephone: (314) 263-4742

Douglas S. Robertson

NGS, NOS, NOAA

$\mathrm{C} 133$

Rockville, Maryland 20852

Alan E. E. Rogers

NEROC/Haystack Observatory

Westford, Massachusetts 01886

Telephone: (617) 6924765

David H. Rogstad

Jet Propulsion Laboratory

4800 Oak Grove Drive

Pasadena, California 91103

Telephonc: (213) 354-2665

Jan Rolff

63 Ellery Street

Cambridge, Massachusetts 02138

Telephone: (617) 547-4797

Jonathan D. Romney

Max-Planck-Institut für

Radioastronomie

Auf dem Hügel 69

5300 Bonn 1

West Germany

Telephone: 02221-525237

Lauren J. Reuger

Johns Hopkins University

Applied Physics Laboratory

Johns Hopkins Road

Laurel, Maryland 20810

Telephone: (301) 953-7100

$\mathrm{X} 3078$ or $\mathrm{X} 3079$
James Ryan

Goddard Space Flight Center

Greenbelt, Maryland 20771

Telephone: (301) 344-8809

Tetsuo Sasao

CIRES, University of Colorado NOAA

University of Colorado at Boulder

Boulder, Colorado 80309

Telephone: (303) 492-8028

Richard T. Schilizzi

Netherlands Foundation for Radio

Astronomy

Radio Observatory

Dwingeloo

The Netherlands

Tclephone: (5219) 7244

Bruce R. Schupler

Computer Sciences Corporation

Code 922, Goddard Space Flight Center

Greenbelt, Maryland 20771

Telephone: (301) 3448605

Irwin I. Shapiro

Department of Earth and Planetary Sciences

Massachusetts Institute of Technology

Room 54-620

Cambridge, Massachusetts 02139

Elliott H. Sigman

Jet Propulsion Laboratory

M/S 264748

4800 Oak Grove Drive

Pasadena, California 91103

Telephone: (213) 354-6700

FTS : 792-6700

Martin A. Slade

Jet Propulsion Laboratory

M/S 264-720

4800 Oak Grove Drive

Pasadena, Califomia 91103

Telephone: (213) 354-3144 
John Spencer

Naval Research Laboratory

Code 7134.2

Washington, D.C. 20375

Telephone: (202) 767-3050

Byron Tapley

Department of Aerospace Engineering/

Engineering Mechanics

The University of Texas at Austin

Austin, Texas 78712

Telephone: (512) 471-1356

Guy L. Tarnstrom

MIT Lincoln Laboratory

P.O. Box 73

Lexington, Massachusetts 02173

Telephone: (617) 862-5500 X5701

J. B. Thomas

Jet Propulsion Laboratory

M/S 264-765

4800 Oak Grove Drive

Pasadena, California 91103

Telephone: (213) 354-6323

Nancy Vandenberg

Phoenix Corporation

1600 Anderson Road

McLean, Virginia 22102

Telephone: (703) 790-1450

Robert F. C. Vessot

Smithsonian Astrophysical Observatory

60 Garden Street

Cambridge, Massachusetts 02138

William B. Waltham

Naval Research Laboratory

Code 7136

Washington, D.C. 20375

Telephone: (202) 767-2668
Alan R. Whitney

NEROC/Haystack Observatory

Westford, Massachusetts 01886

Telephone: (617) 692-4764

Theodore Wirtanen

Air Force Geophysics Lab/LWG

Hanscom Air Force Base

Bedford, Massachusetts 01731

Telephone: (617) 861-3486

Dr. Jill J. Wittels

$\mathrm{M} / \mathrm{S} 65$

Honeywell Electro-Optics Center

2 Forbes Road

Lexington, Massachusetts 02173

J. L. Yen

University of Toronto

35 St. George Street

Department of Electrical Engineering

Toronto, Ontario

Canada M5S 1 A4

Telephone: (416) 978-8756

Lawrence E. Young

Jet Propulsion Laboratory

$\mathrm{M} / \mathrm{S} 264765$

4800 Oak Grove Drive

Pasadena, California 91103

Telephone: (213) 354-5082 


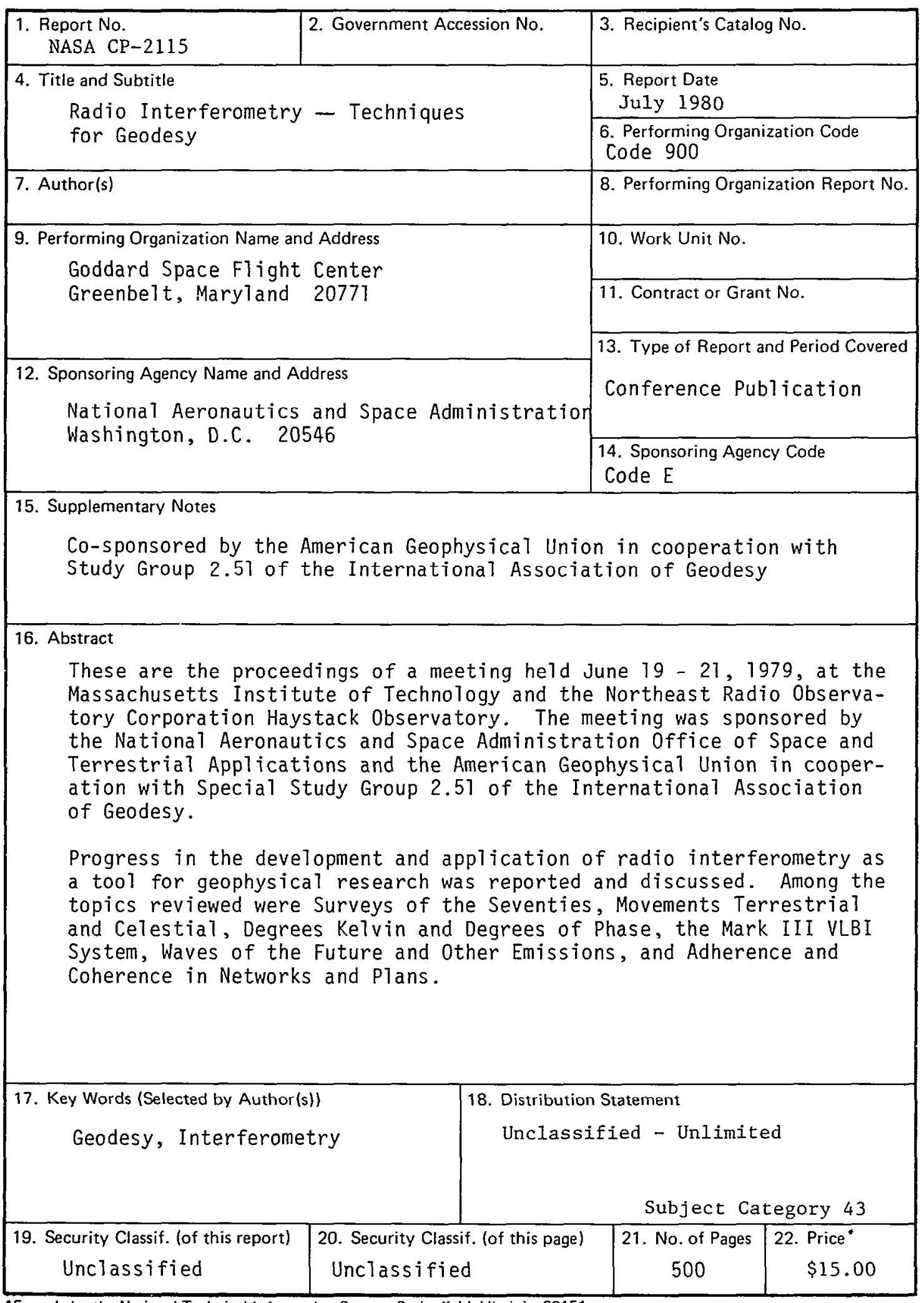

- For sale by the National Technical Information Service, Springfield, Virginia 22161. 